\title{
Plasmon excitation in waveguide and in metal particles embedded in glass
}

\author{
Alexander Andreyuk \\ M.Sc. (York), M.A.Sc. (Carleton) \\ A Thesis submitted to the \\ Faculty of Graduate and Postdoctoral Affairs \\ in partial fulfillment of the requirements for the \\ degree of Ph.D. in \\ Electrical and Computer Engineering \\ Ottawa-Carleton Institute for Electrical Engineering \\ Department of Electronics \\ Faculty of Engineering \\ Carleton University
}

(C) Alexander Andreyuk, Ottawa, Canada, 2014 


\begin{abstract}
Experimental part of this work dedicated to making a glass metal nanocomposite by forming silver nanoclusters in an ion-exchanged glass in a hydrogen atmosphere at elevated temperature followed by an investigation of electric field assisted dissolution of nanoclusters. The ultimate goal is building a waveguide in glass metal nanocomposite with volume Bragg grating, i.e. a Bragg grating that runs through the whole cross section of the waveguide.

A new type of phosphate glass, IOG-1 doped by rare earth ions, $E r^{+3}$ and $\mathrm{Yb}^{+3}$, designed for integrated optics application, was chosen as a substrate in this project for optimal optical properties of such waveguide and prospective active photonic devices.

The grating in a glass metal nanocomposite achieved by means of high DC voltage and periodically corrugated electrode thus activating electric field assisted dissolution process predominantly in the regions of a direct contact glass-electrode.

Since wavelength dependent refractive index of a glass metal nanocomposite determined by its absorption band through Kramers-Kronig relation, tuning parameters of surface plasmon resonance, related to a charge density oscillations in metal nanoclusters, controls the optical properties of glass metal nanocomposite even at a working wavelength of a waveguide which is far off plasmon resonance. Therefore tuning parameters of the silver nanoclusters in glass metal nanocomposite leads to optimizing grating contrast.

The rest part of this work concerned with building a computational model of a plasmon excitation in a waveguide with the coating in the form of periodic nanostructured metal distribution.

The standard approach used in an antenna theory, a wave equation with the source term, is applied for solving a problem of an optical waveguide coated by metal. A computational model was tested first on a slab waveguide illuminated by fields due to a finite current source. For such basic geometry experimental data is available which makes model verification a straightforward task.

Then the model was extended to the case of an optical fiber with the excitation fields due to a set of discrete current sources positioned in a fiber core and finally to a more realistic problem when plasmons are excited by a tilted fiber Bragg grating. Both, an uniform and $\pi$-shifted Bragg grating and metal distribution were included in the model.

In addition a mode solver for finding complex roots of an optical fiber was developed to a main model of plasmon excitation. The mode solver is based on a Galerkin wavelet method.
\end{abstract}




\section{Acknowledgements}

I would like to express my gratitude to my supervisor professor Jacques Albert for all his help during my study at Carleton university and for giving me opportunity to conduct research on this project in his group. I would like to thank people who helped me with this project especially Rob Vandusen, Angela and Rick from Microelectronics Fabrication Lab, Albane Laronche and all people from our Photonics Lab. I also want to thank the Department of Electronics and all professors whose courses I had a chance to take. 


\section{Dedication}

to my dear parents

моим дорогим любимым родителям,

моей дорогой маме, Андреюк Татьяне Михайловне и моему дорогому папе, Андреюку Терентию Тимофеевичу 


\section{Contents}

List of Tables $\quad$ viii

List of Figures $\quad$ X

1 Introduction and thesis outline $\quad 1$

1.1 Thesis outline. . . . . . . . . . . . . . . . . . 2

2 Technological background $\quad 4$

2.1 Silver clusters formation in a silica based glass. . . . . . . . . . . . . . 4

2.2 Spherical clusters reshaping. . . . . . . . . . . . . . . 8

2.3 Field assisted dissolution of silver clusters embedded in glass. . . . . . . . . 12

3 Field-assisted dissolution of silver nanoparticles in phosphate glass 22

3.1 Introduction . . . . . . . . . . . . . . . . . . 22

3.2 Formation of silver clusters in phosphate glass . . . . . . . . . . . . . . 23

3.2.1 Glass processing that leads to silver nanoclusters: ion-exchange, annealing in air followed by annealing in $H_{2}$. . . . . . . . . . . . 23

3.2.2 Sample measurements and observations . . . . . . . . . . . . . 25

3.3 Glass bleaching of samples with silver clusters and its patterning . . . . . . 30

3.3.1 Field-assisted dissolution of silver clusters . . . . . . . . . . . . 30

3.3.2 Sample measurements and observation . . . . . . . . . . . . . . 32

3.4 Absorption spectra simulation with Maxwell-Garnet theory . . . . . . . . . 33

3.4.1 Maxwell-Garnet mixing formula . . . . . . . . . . . . . . . 33

3.4.2 Dielectric constant for nanocluster sphere of radius $\mathrm{r}$. . . . . . . 36

3.4.3 Extended Maxwell-Garnet mixing formula . . . . . . . . . . . . . . 39

3.4.4 Maxwell Garnett formula for distributed cluster size . . . . . . . . . 41 
3.5 Discussion . . . . . . . . . . . . . . . . . . . . . . . . . . 42

3.5.1 Potential due to corrugated electrode . . . . . . . . . . . 42

3.5 .2 EDX measurements . . . . . . . . . . . . . . . . . 43

3.6 Conclusions . . . . . . . . . . . . . . . . . . . . . . . 46

3.7 Plan for future experimental work . . . . . . . . . . . . . . . . 48

4 Plasmon excitation in a waveguide with planar geometry 54

4.1 Probing plasmons by measuring reflectivity in Kretschmann experiment. . 54

4.1.1 Plasmon excitation by plane wave. . . . . . . . . . 56

4.1.2 Waveguide approach for plasmon in thin film . . . . . . . . 58

4.2 Inhomogeneous wave equation. General formalism for slab geometry. . . . 60

4.3 Plasmon excitation by divergent beam. . . . . . . . . . . . . 65

4.3.1 Scattering in a slab due to current source. . . . . . . . 65

4.3 .2 Excitation sources. . . . . . . . . . . . . . . . . . . 68

4.4 Choice of basis: electric and magnetic fields $(\vec{E}, \vec{H})$ versus vector potentials for solving wave equation. . . . . . . . . . . . . . 72

4.4.1 Electric and magnetic fields, $(\vec{E}, \vec{H}) \ldots \ldots \ldots \ldots$

4.4.2 Magnetic vector potential and scalar electric potential, $\left(\vec{A}, \varphi_{e}\right) . \ldots 73$

4.4.3 Electric Hertz vector and electric scalar potential, $\left(\vec{\Pi}_{e}, \varphi_{e}\right) \ldots \ldots 74$

4.4.4 Boundary conditions in terms of vector potentials. . . . . . . . 74

4.5 Simulation results and discussion. . . . . . . . . . . . . . . 75

4.5.1 Mode excitation in experimental setup. . . . . . . . . . . 75

4.5.2 Radiation mode and plasmon measurement in experimental setup. . 75

4.5.3 Excitation by a finite sheet of current. . . . . . . . . . 75

4.5.4 Excitation by a set of current sources. . . . . . . . . 78

4.6 Conclusions. . . . . . . . . . . . . . . . . . . . . . . . . . 80

5 Plasmon excitation in a waveguide with cylindrical geometry 83

5.1 Inhomogeneous wave equation. General formalism in cylindrical coordinates. 83

5.2 Scattering in fiber due to current source. . . . . . . . . . . . . . . 88

5.3 Excitation sources. . . . . . . . . . . . . . . . . . . . 93

5.3 .1 (A) Excitation by rings of current. . . . . . . . . . 95

5.3 .2 (B) Excitation by disc of current. . . . . . . . . . . . 98 
5.3.3 (C) Excitation by array of rings or discs of current. . . . . . . . . 98

5.4 Choice of basis: electric and magnetic fields $(\vec{E}, \vec{H})$ versus vector potentials for solving wave equation. . . . . . . . . . . . . . . 101

5.4.1 Electric and magnetic fields, $(\vec{E}, \vec{H}) . \ldots \ldots \ldots 10 \ldots$

5.4.2 Magnetic vector potential and scalar electric potential, $\left(\vec{A}, \varphi_{e}\right)$. . 102

5.4.3 Magnetic and electric vector potentials, $(\vec{A}, \vec{F})$. . . . . . . . . 102

5.4.4 Electric Hertz vector and electric scalar potential, $\left(\vec{\Pi}_{e}, \varphi_{e}\right) \ldots$. . 103

5.4.5 Pair of Hertz vectors, $\left(\vec{\Pi}_{e}, \vec{\Pi}_{m}\right) \ldots \ldots \ldots \ldots$

5.4.6 Boundary conditions in terms of vector potentials. . . . . . . . . . 104

5.5 Simulation results and discussion. . . . . . . . . . . . . . . . 104

5.6 Conclusion. . . . . . . . . . . . . . . . . . . 125

6 Plasmon excitation in a fiber by tilted Bragg grating 126

6.1 Introduction. . . . . . . . . . . . . . . . 126

6.2 Excitation by an uniform Bragg grating in fiber coated by metal film. . . . 127

6.3 Excitation by $\pi$-shifted Bragg grating in fiber coated by metal film. . . . . 129

6.4 Excitation by uniform Bragg grating in fiber coated by structured metal film.130

6.5 Simulation results and discussion. . . . . . . . . . . . . . 133

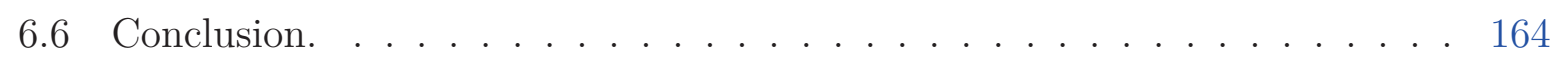

7 Wavelet-Galerkin analysis of metal coated step-index fiber 165

7.1 Piecewise cosine wavelets. . . . . . . . . . . . . . . . 166

7.2 Generalized eigenvalue wave equation. . . . . . . . . . . . . 166

7.3 Generalized eigenvalue wave equation. . . . . . . . . . . . . . . . 172

7.4 Fields at boundaries. . . . . . . . . . . . . . . . . . . . 174

7.5 Uniform vs. adaptive mesh. . . . . . . . . . . . . . . 175

7.6 Partitioning domain with Chebyshev nodes and Koslov map. . . . . . . . . 175

7.7 Simulation results and discussion. . . . . . . . . . . . . . 177

7.7 .1 Two layer fiber. . . . . . . . . . . . . . . . . 177

7.7.2 SMF fiber coated by thin metal layer. . . . . . . . . . . . . . . . 180

7.8 Treatment of spurious modes. . . . . . . . . . . . . . . . . . 182

7.9 The relevance of the method. . . . . . . . . . . . . . . 184

7.10 Conclusion. . . . . . . . . . . . . . . . . . 185 
$\begin{array}{ll}\text { APPENDICES } & 188\end{array}$

$\begin{array}{ll}\text { A Conformal mapping of corrugated plate } & 189\end{array}$

$\begin{array}{ll}\text { B } & 192\end{array}$

C Approximation of Bessel function through trigonometric series. 193

D Iterative scheme based on equivalence principle for finding scattered field in inhomogeneous waveguide.

$\mathbf{E}$

References 


\section{List of Tables}

7.1 Effective modal indices of two layer fiber for azimuthal index $m=4$ obtained by solving generalized eigenvalue equation for electric fields (columns 1,2), generalized eigenvalue equations for magnetic fields (columns 3,4) and by solving characteristic equation (column 5). $N_{1}, N_{2}$ are number of nodes in the core and outer layer respectively . . . . . . . . . . . . . . 178

7.2 Tangential fields in arbitrary units of each layer at the interface for the first mode where within at least first 55 digits the boundary conditions are satisfied.179

7.3 Tangential fields in arbitrary units of each layer at the interface for the last mode where within at least first 55 digits the boundary conditions are satisfied. 180 


\section{List of Figures}

2.1 Silver depth concentration profiles before and after annealing processes at constant time $(5 \mathrm{~h})$ and different temperatures, . . . . . . . . . . .

2.2 UV-visible absorption spectra for samples annealed at constant time (5h) and different temperatures, . . . . . . . . . . . . . . .

2.3 TEM micrographs (cross section) ofsilver nanoclusters, formed after annealing in hydrogen atmosphere for (a) 12 hours at $180^{\circ} \mathrm{C}$ and (b) 5 hours at $250^{\circ} \mathrm{C}$. Selected area diffraction patterns are reported in the insets, . . . .

2.4 Particles in the 'low-Ag' glass at a penetration depth of $1.5 \mu \mathrm{m}$; the inset shows, at higher magnification, the non-homogeneous image contrast of some particles due to twin defects, . . . . . . . . . . . . . . .

2.5 dependence of the mean particle size of the 'low-Ag' sample on the penetration depth, . . . . . . . . . . . . . . . . . . . .

2.6 The near-surface region of the 'high-Ag' glass in a cross-sectional view together with a profile plot of the relative image intensity showing the arrangement and extension of the Ag precipitate layers, . . . . . . . . . . . . 7

2.7 The distribution of Ag particles in one layer (n) of the 'high-Ag' glass, . . . 8

2.8 Typical optical spectra of glass containing silver nanoparticle before (solid line) and after irradiation with a single, linearly polarized femtosecond laser pulse at $400 \mathrm{~nm}$ (dashed lines), where the measuring light was linearly polarized, once parallel with respect to the laser polarization, once perpendicular to it, . . . . . . . . . . . . . . . . . .

2.9 TEM micrographs of silver particles in glass before (a) and after (b) irradiation with a single, linearly polarized femtosecond laser pulse at $400 \mathrm{~nm}$,

2.10 TEM pictures of silver particles in glass after irradiation with a single, linearly polarized femtosecond laser pulse at $400 \mathrm{~nm}$, numerically differentiated to enhance contrast. The bottom right picture corresponds to the inset of Fig. 2.9(b), . . . . . . . . . . . . . . . . . .

2.11 Optical spectra of silver particles in glass before and after irradiation with a single, circularly polarized femtosecond laser pulse at $400 \mathrm{~nm}$. Inset: TEM picture after irradiation, . . . . . . . . . . . . . . . . 
2.12 SEM pictures of cross sections of glass samples containing (a) spherical and (b) elongated silver nanoparticles (the Ag particles are reproduced as white spots). The fill factor gradient of the spherical nanoparticles is given by the inset, where the $\mathrm{x}$-axis was adjusted to the length scale of the picture. The ellipsoidal nanoparticles in part b, obtained by mechanical deformation of the sample, are located in a very thin surface layer of, with only very few large particles remaining deeper below the surface, . . . . . . . . . .

2.13 TABLE 1. Samples under test: $\mathrm{Ag}^{+}-\mathrm{Na}^{+}$ion exchanged soda-lime glass (substrate), soda-lime glass containing Ag nanoparticles of ellipsoidal shape (nanocomposite $E$ ), or spherical shape (nanocomposite $S$ ). L: Sample thickness, and $d$ : Thickness of the near-surface layer in which Ag nanoparticles are embedded. Color of the pristine glass and amount of poling-assisted bleaching of the glass are indicated. All samples (except No. 3) were poled with the nanoparticle layer facing the anode (cathode). All samples (except No. 8) were poled at $1 \mathrm{kV}$ and $280^{\circ} \mathrm{C}\left(4 \mathrm{kV}\right.$ and $\left.25^{\circ} \mathrm{C}\right), \ldots . . . .$.

2.14 Evolution of the current flowing through the sample as a function of time during thermal poling at $280^{\circ} \mathrm{C}$. Top chart: Ion-exchanged sample No. 10; middle chart: Samples containing ellipsoidal Ag nanoparticles (No. 1: Thick line, No. 3: Thin line); bottom chart: samples containing spherical Ag nanoparticles (No. 4: thin line, No. 4bis: thick line, No. 9: dotted line). The voltage was applied step by step up to a final value of $1.0 \mathrm{kV}$ (top chart No. 1: First step of $0.2 \mathrm{kV}$ during $2 \mathrm{~min}$, then steps of $0.1 \mathrm{kV}, 5 \mathrm{~min}$ each; middle and bottom charts: steps of $0.2 \mathrm{kV}, 10$ min each). Vertical dashed lines indicate the beginning of cooling of the sample with the voltage still applied . . . . . . . . . . . . . . . . . . .

2.15 (Color) Photograph image of samples No. 1 after poling. The glass became transparent under the anode after poling (i.e., PAB occurred). The sharp edges of the bleached region correspond perfectly to the electrode size $(6 \mathrm{x} 9$ $\mathrm{mm}), \ldots \ldots \ldots \ldots \ldots \ldots$

2.16 (a) Extinction spectra of a sample containing elongated Ag nanoparticles, before (dashed curves, observed with light polarized parallel and perpendicular to the long axis of the silver clusters) and after (solid curve, directly after process; dash-dotted curve, after additional heating) electric field-induced modification. SEM pictures of the surface of a sample with ellipsoidal Ag nanoparticles (b) before and (c) after dc electric field treatment, . . . . . .

2.17 Na, Ag, and $\mathrm{Si}$ distributions as a function of sample depth by local X-ray element analysis of vertical cross sections of samples (a) before and (b) after the electric field treatment; the horizontal length scales are identical. The Ag peak found in part a at $\approx 2.5 \mu \mathrm{m}$ corresponds to a single large silver particle seen in the SEM picture (not shown), . . . . . . . . . . . 
2.18 Microscopic photography of sub-micrometer lines made by electric field assisted dissolution, EFAD using an electrode scratched with a diamond marker (right-hand side) and calibrated length scale with same magnification (left-hand side),

2.19 Experimental setup for electric field thermal poling. (1) Tube furnace, (2) thermal couple, (3)dc power, (4) anode, (5) steel plate, (6) sample, (7) boron nitride $(\mathrm{BN})$ carrier with a mechanically patterned hole where the glass sample is fixed in, and (8) steel rod holder as cathode (fixed on 5), . .

2.20 Evolution of applied current and voltage as a function of time during electric field thermal poling, . . . . . . . . . . . . . . . .

2.21 (Color online) Absorption spectra of Au-doped silicate glass before (a) and after (b) annealing at $650^{\circ} \mathrm{C}$ without an electric field, . . . . . . . . . .

2.22 A selected current evolution cycle inset with the schematic image of three current conduction processes (I, II, and III), where $i$ denotes Au ions, $e$ denotes ejected electrons from $\mathrm{Au}$ particles, $e^{\prime}$ denotes electrons from the cathode, and $\vec{E}$ is the electric field, . . . . . . . . . . . . . .

2.23 The mapped absorption spectra (1) and the SEM images (2)-(7) of different areas on the surface of Au-sputtered glass after EFAD process, . . . . . . .

3.1 Images of IOG-1 glass before and after annealing in a furnace with continuous $\mathrm{H}_{2}$ flow. (a): glass containing silver ions after the ion-exchange $\mathrm{Na}^{+}-\mathrm{Ag}^{+}$process, (b): after annealing in $\mathrm{H}_{2}$ the samples are coated with silver film on the side where the ion-exchange was implemented, (c) and (d): samples after the surface is cleaned. . . . . . . . . . . . . . .

3.2 UV-vis absorption spectra (a) and TEM image (b) of the IOG-1 glass sample that underwent ion-exchange $(\mathrm{T}=95 \mathrm{C}, \mathrm{t}=28 \mathrm{hrs}, \mathrm{V}=380 \mathrm{~V})$, annealing in air $(\mathrm{T}=300 \mathrm{C}, \mathrm{t}=7 \mathrm{hrs})$ and reduction in $H_{2}(\mathrm{~T}=400 \mathrm{C}, \mathrm{t}=30 \mathrm{~min}$ followed by $\mathrm{T}=430 \mathrm{C}, \mathrm{t}=4 \mathrm{hrs}$ ). Black rounded spots in (b) are $20-30 \mathrm{~nm}$ silver clusters responsible for absorption peak in (a) in the range 402-426 nm due to plasmon resonance).

3.3 Absorption spectra of the samples with different ion-exchange time from 22 hrs (lowest curve) to $64 \mathrm{hrs}$ with $\mathrm{T}=95 \mathrm{C}$ and $\mathrm{V}=350 \mathrm{~V}$, other parameters, annealing in air: $\mathrm{T}=300 \mathrm{C}, \mathrm{t}=6 \mathrm{hrs}$, reduction in $H_{2}: \mathrm{T}=400 \mathrm{C} \mathrm{t}=1.5 \mathrm{hrs}$ followed by $\mathrm{T}=430 \mathrm{C}, \mathrm{t}=3 \mathrm{hrs} \ldots \ldots \ldots \ldots$

3.4 Absorption spectra of the samples with different ion-exchange temperature, $85 \mathrm{C}$ and $95 \mathrm{C}$ with $\mathrm{t}=25 \mathrm{hrs}$ and $\mathrm{V}=410 \mathrm{~V}$, other parameters, annealing in air: $\mathrm{T}=300 \mathrm{C}, \mathrm{t}=6 \mathrm{hrs}$, reduction in $H_{2}: \mathrm{T}=400 \mathrm{C} \mathrm{t}=1.5 \mathrm{hrs}$ followed by $\mathrm{T}=430 \mathrm{C}, \mathrm{t}=3$ hrs. . . . . . . . . . . . . . . . .

3.5 Absorption spectra of the samples with different treatment of annealing in $\mathrm{H}_{2}$ : 400C for $30 \mathrm{~min}$ followed by $430 \mathrm{C}$ for $4 \mathrm{hrs}$, 400C for $1.5 \mathrm{hrs}$ followed by $430 \mathrm{C}$ for $3 \mathrm{hrs}, 450 \mathrm{C}$ for $4 \mathrm{hrs}, 415 \mathrm{C}$ for $4 \mathrm{hrs}$. Other parameters, ionexchange: $\mathrm{T}=95 \mathrm{C}, \mathrm{t}=28 \mathrm{hrs}, \mathrm{V}=350 \mathrm{~V}$, annealing in air: $\mathrm{T}=300 \mathrm{C}, \mathrm{t}=6 \mathrm{hrs}$. 
3.6 Absorption spectra of the samples with different treatment of annealing in air: 300C for $6 \mathrm{hrs}, 350 \mathrm{C}$ for $3 \mathrm{hrs}$ and 350C for $6 \mathrm{hrs}$. Other parameters, ion-exchange: $\mathrm{T}=105 \mathrm{C}, \mathrm{t}=45 \mathrm{hrs}, \mathrm{V}=400 \mathrm{~V}$, annealing in $H_{2}: \mathrm{T}=400 \mathrm{C}$, $\mathrm{t}=30$ min followed by $\mathrm{T}=430 \mathrm{C}, \mathrm{t}=4 \mathrm{hrs} . \ldots \ldots \ldots$

3.7 Absorption spectra before and after bleaching of the sample with the following parameters, ion-exchange: $\mathrm{T}=95 \mathrm{C}, \mathrm{t}=70 \mathrm{hrs}, \mathrm{V}=370 \mathrm{~V}$, annealing in air: $\mathrm{T}=300 \mathrm{C}, \mathrm{t}=6 \mathrm{hrs}$, and annealing in $\mathrm{H}_{2}: \mathrm{T}=400 \mathrm{C}, \mathrm{t}=30 \mathrm{~min}$ followed by $\mathrm{T}=430 \mathrm{C}, \mathrm{t}=4$ hrs. Parameters of bleaching in Fig. 3.10. . . . . . . . . .

3.8 SEM image of periodic Si electrode produced by plasma etching, double bar

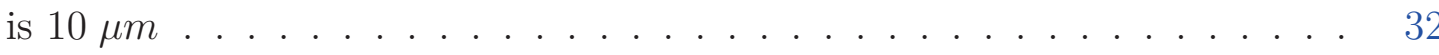

3.9 SEM image of IOG-1 glass bleached with periodic electrode. . . . . . . . . 33

3.10 Bleaching voltage (a) and current (b) vs. time of IOG-1 sample whose absorption plot is in Fig. 3.7. . . . . . . . . . . . . . . . .

3.11 Bleaching voltage (a) and current (b) vs. time of silicate glass (microscope slide) sample. . . . . . . . . . . . . . . . . . . . .

3.12 Models tested for absorption spectra, where $n_{M G}$ refractive index calculated by Maxwell-Garnet mixing formula

3.13 Calculated absorption spectra, $\alpha(\lambda)$ for models in Fig. 3.12. Parameters: $f_{v}=0.1, \mathrm{r}=12 \mathrm{~nm}, d=4 \mu m . \ldots \ldots \ldots$

3.14 absorption spectra, $\alpha(\lambda, r)$ for homogeneous interface of two mediums vs. cluster radius $\mathrm{r}$ and wavelength $\lambda \ldots \ldots \ldots$. . . . . . . .

3.15 absorption spectra, $\alpha\left(\lambda, f_{v}\right)$ for homogeneous interface of two mediums vs. wavelength $\lambda$ and fill factor $f_{v}$ with discrete normal distribution of cluster size. Parameters: $\mathrm{r}=12 \mathrm{~nm}, \sigma=2 \mathrm{~nm} \ldots \ldots \ldots \ldots$

3.16 Equipotential lines above corrugated electrode (a) for triangular saw tooth, (b) for rectangular saw tooth, all dimensions in microns; (c) potential modulation for capacitor with one electrode corrugated (rectangular saw tooth) at 4 microns from corrugated side. Voltage on electrodes: $V_{1}=1500 \mathrm{~V}$, $V_{2}=600 \mathrm{~V}$, inner distance between electrodes $20 \mu \mathrm{m}$. Vertical and horizontal axes displayed in units of volts and microns respectively. . . . . . . .

3.17 Fill factor distribution for bleached and unbleached region of the grating, distribution approximated by exponentially decaying function from the surface, bleached region differs from unbleached by a different constant factors:

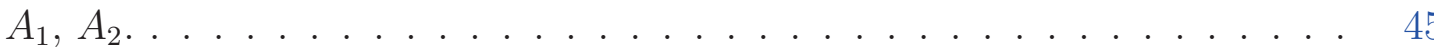

3.18 EDX measurements at polished level $x_{1}$. . . . . . . . . . . . . . . . . 45

3.19 Refractive index change (a) real part and (b) imaginary part vs. wavelength of bleached IOG-1 glass with corrugated electrode based on EDX measurements, the wavelength axis in units of nm. . . . . . . . . . . . 
3.20 SEM images of self-organized periodic nanoplanes. A: The light propagation direction $k$, the writing direction $S$. B: $E$ is parallel to $S$. Panel C: $E$ is perpendicular to $S$. Nominal separation of the grating planes is $250 \mathrm{~nm}, \ldots$

3.21 Pulse energy dependence of the experimental nanograting period of silica glass and tellurium dioxide single crystal. Theoretical curves of nanograting period versus electron temperature are also shown, . . . . . . . . . . .

3.22 Schematic steps of building a volume Bragg grating in IOG-1 glass with femtosecond laser exposure, ion-exchange and low temperature bonding processes. 51

3.23 Schematic diagram explaining the Low Temperature Bonding process as applied to mass production of hybrid substrates for integrated optic photonic

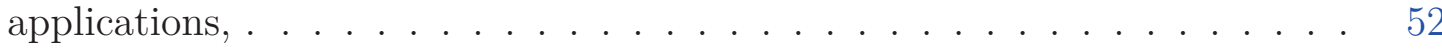

3.24 Photograph showing a hybrid preform and several hybrid substrates prepared from active Er/Yb doped phosphate glass (orange) and passive phosphate glass (clear) . . . . . . . . . . . . . . . . .

3.25 Single mode waveguide though a Low Temperature Bonding interface between active (i.e. Er doped) and passive phosphate glass IOG-1 (VIS microscope image $), \ldots \ldots \ldots \ldots \ldots \ldots \ldots \ldots$

4.1 Plasmon excitation in Kretchmann experiment by laser beam and plasmon probing on reflection $\ldots \ldots \ldots \ldots \ldots \ldots \ldots$

4.2 (top) Contour plot of reflectivity versus incident angle of plane wave and thickness of silver film in Kretchmann experiment; (bottom) its zoomed version near the angle $42.6^{\circ}-43.1^{\circ} \ldots \ldots \ldots \ldots \ldots \ldots$

4.3 Plot of reflectivity versus incident angle at film thickness $d=52 \mathrm{~nm} \ldots \quad . \quad 58$

4.4 Field distribution along y axis of a symmetric bound SPP mode with a film thickness as parameter: at a critical thickness $d_{0}=52 \mathrm{~nm}$ the field in the glass is a plane wave, at thickness greater than critical $d>d_{0}$ the field in the glass is evanescent, at thickness below the critical $d<d_{0}$ the field in the glass exponentially grows away from glass-metal interface. . . . . . . . .

4.5 (top) Real and imaginary parts of effective index for symmetric bound mode (green dashed line) and leaky wave mode (solid line) versus film thickness; (bottom) zoomed plot of imaginary parts. . . . . . . . . . . .

4.6 Effective index for symmetric bound mode (green dashed line) and leaky wave mode (solid line) versus film thickness on a complex plane. . . . . . . .

4.7 (top) Imaginary part of for symmetric bound mode (green dashed line) and leaky wave mode (solid line) versus film thickness; (bottom) zoomed plot

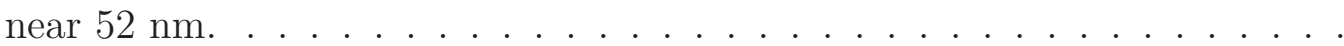

4.8 Primary and scattered fields in a 3-layer waveguide with the impressed current source in layer $1 \ldots \ldots \ldots \ldots \ldots \ldots \ldots$ 
4.9 Excitation of plasmon in a 3-layer silver film waveguide by a finite sheet of current. . . . . . . . . . . . . . . . . . 70

4.10 Excitation of plasmon in a 3-layer silver film waveguide by several equidistant rods of current positioned on a single sheet. . . . . . . . . . . . .

4.11 Excitation of plasmon in a 3-layer silver film waveguide by an array of finite

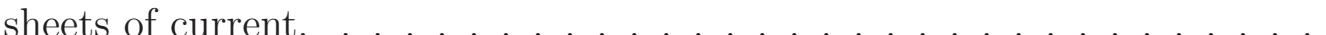

4.12 Plot of power carried by a plasmon (symmetric leaky wave) versus incident angle at silver thickness $d=40 \mathrm{~nm}$. Excitation is due to a finite sheet of current whose width $W=40 \mu \mathrm{m}$ and distance from the origin $R_{0}=40 \mu \mathrm{m}$ (as in Fig. 4.9). (top) The power in the air, layer 3 is positive, indicating it is carried in the positive $z$ direction. (bottom) The power inside of the silver film, layer 2 is negative, indicating it is carried in the negative $z$ direction or opposite to the modal propagation constant, i.e. inside of the film the power flux is opposite to the phase velocity. . . . . . . . . . . . . .

4.13 (top) Contour plot of power carried forward (positive $z$ direction) by a plasmon (symmetric leaky wave) in layer 3 (air) versus incident angle and silver thickness. Excitation is due to a finite sheet of current whose width $W=40 \mu \mathrm{m}$ and distance from the origin $R_{0}=40 \mu \mathrm{m}$ (as in Fig. 4.9). (bottom) Zoomed contour plot near the angle $42^{\circ}-43^{\circ}$. . . . . . . . . .

4.14 Contour plot of power carried backward (negative direction) by a plasmon (symmetric leaky wave) in layer 2 (silver film) versus incident angle and silver thickness. Excitation is due to a finite sheet of current whose width $W=40 \mu m$ and distance from the origin $R_{0}=40 \mu m$ (as in Fig. 4.9). . . .

4.15 Contour plot of power carried forward (positive $z$ direction) by a plasmon (symmetric leaky wave) in layer 3 (air) versus incident angle and silver thickness. Excitation is due to several rods of current positioned on a single sheet with width $W=40 \mu \mathrm{m}$ and distance from the origin $R_{0}=40 \mu \mathrm{m}$ (as in Fig. 4.10). (top) Number of rods $N=3$. (bottom) Number of rods $N=30.79$

4.16 Contour plot of power carried forward (positive $z$ direction) by a plasmon (symmetric leaky wave) in layer 3 (air) versus incident angle and silver thickness. Excitation is due to an array of current sheets with array width $W=40 \mu \mathrm{m}$ and distance from the origin $R_{0}=40 \mu \mathrm{m}$ (as in Fig. 4.11). (top) a uniform array with total of $N=8$ sheets. (bottom) a $\pi$ phase shifted array with total of $N=8$ sheets. . . . . . . . . . . . . . .

4.17 Contour plot of power carried forward (positive $z$ direction) by a plasmon (symmetric leaky wave) in layer 3 (air) versus incident angle and silver thickness. Excitation is due to several rods of current positioned on a single sheet with width $W=40 \mu \mathrm{m}$ and distance from the origin $R_{0}=40 \mu \mathrm{m}$ (as in Fig. 4.11). (top) number of rods $N=3$. (bottom) number of rods $N=30.82$ 
5.1 Scattering fields in a cylindrical 3 layer waveguide with the current source, shown as an array of tilted elements, in the first layer (in the core). $E^{p}$ represents a primary field due to current source, $E^{s}$ is a scattered field in the waveguide. . . . . . . . . . . . . . . .

5.2 Current source as a set of rings with common center positioned on a single tilted plane. A tilt is done in $(y z)$-plane with respect to $x$-axis. Tilt angle $\alpha$ measured between $y$-axis and tilted axis $y^{\prime}$.

5.3 Current source as a disc positioned on a single tilted plane. A tilt is done in (yz)-plane with respect to $x$-axis. Tilt angle $\alpha$ measured between $y$-axis and tilted axis $y^{\prime} \ldots \ldots \ldots \ldots \ldots \ldots \ldots$

5.4 Current source as an array of tilted elements located in the fiber core. Array is either uniform or $\pi$-shifted with element type (a): set of rings with common center on a single plane or, (b): disc. . . . . . . . . . . . .

5.5 Phase shifted array of tilted elements with parameters: $N_{1}, N_{2}$ number of elements in each part of the array; $z_{s}$ shift distance between two parts; $\Delta_{z}$ spacing along $z$-axis in each part of the array; $\Delta$ spacing along normal vector in each part of the array; $\alpha$ tilt angle. . . . . . . . . . . . . .

5.6 Line integral convolution plot of a tangential component of current source distribution in a tilted disc located in the fiber core. The disc is an interior of some ellipse whose longer axis is $y^{\prime}$. Tilt angle $\alpha$ is measured between axis $y$ of the fiber and tilted axis $y^{\prime}$. (a): alignment, (b): orientation. . .

5.7 Contour plot of power carried by plasmon mode excited by an uniform array of tilted discs of current in the core of a fiber coated by thin Au layer positioned in air environment versus wavelength and tilt angle. Simulation parameters: azimuthal index of the mode $m=3$, layer thickness $d=35 \mathrm{~nm}$, an array is tuned for coupling plasmon mode and core mode at resonance wavelength $\lambda_{\text {res }}=1550 \mathrm{~nm}$, effective index $n_{e f 1}$ shown in Fig. 5.12. Computation window for wavelength and angle range respectively (a): (1549 $\mathrm{nm}, 1551 \mathrm{~nm}),\left(0^{\circ}, 12^{\circ}\right),(\mathrm{b}):(1549.9 \mathrm{~nm}, 1550.1 \mathrm{~nm}),\left(0^{\circ}, 12^{\circ}\right),(\mathrm{c}):(1549.99$ $\mathrm{nm}, 1550.01 \mathrm{~nm}),\left(0^{\circ}, 12^{\circ}\right) \ldots \ldots \ldots \ldots \ldots \ldots \ldots$

5.8 Contour plot of power carried by plasmon mode excited by a $\pi$-shifted array of tilted discs of current in the core of a fiber coated by thin Au layer positioned in air environment versus wavelength and tilt angle. Simulation parameters: azimuthal index of the mode $m=3$, layer thickness $d=35 \mathrm{~nm}$, an array is tuned for coupling plasmon mode and core mode at resonance wavelength $\lambda_{\text {res }}=1550 \mathrm{~nm}$, effective index $n_{e f 1}$ shown in Fig. 5.11. Computation window for wavelength and angle range respectively (a): (1549 $\mathrm{nm}, 1551 \mathrm{~nm}),\left(0^{\circ}, 12^{\circ}\right),(\mathrm{b}):(1549.9 \mathrm{~nm}, 1550.1 \mathrm{~nm}),\left(0^{\circ}, 12^{\circ}\right),(\mathrm{c}):(1549.99$ $\mathrm{nm}, 1550.01 \mathrm{~nm}),\left(0^{\circ}, 12^{\circ}\right) \ldots \ldots \ldots \ldots \ldots \ldots$ 
5.9 Contour plot of power carried by plasmon mode excited by a $\pi$-shifted array of tilted discs of current in the core of a fiber coated by thin Au layer positioned in air environment versus wavelength and tilt angle. Simulation parameters: azimuthal index of the mode $m=3$, layer thickness $d=35 \mathrm{~nm}$, an array is tuned for coupling plasmon mode and core mode at resonance wavelength $\lambda_{\text {res }}=1550 \mathrm{~nm}$, effective index $n_{\text {ef2 }}$ shown in Fig. 5.11. Computation window for wavelength and angle range respectively (a): (1549 $\mathrm{nm}, 1551 \mathrm{~nm}),\left(0^{\circ}, 12^{\circ}\right),(\mathrm{b}):(1549.9 \mathrm{~nm}, 1550.1 \mathrm{~nm}),\left(0^{\circ}, 12^{\circ}\right),(\mathrm{c}):(1549.99$ $\mathrm{nm}, 1550.01 \mathrm{~nm}),\left(0^{\circ}, 12^{\circ}\right) \ldots \ldots \ldots \ldots \ldots$

5.10 Contour plot of power carried by plasmon mode excited by a $\pi$-shifted array of tilted discs of current in the core of a fiber coated by thin Pt layer positioned in air environment versus wavelength and tilt angle. Simulation parameters: azimuthal index of the mode $m=3$, layer thickness $d=35 \mathrm{~nm}$, an array is tuned for coupling plasmon mode and core mode at resonance wavelength $\lambda_{\text {res }}=1550 \mathrm{~nm}$, effective index $n_{\text {ef3 }}$ shown in Fig. 5.11. Computation window for wavelength and angle range respectively (a): (1549 $\mathrm{nm}, 1551 \mathrm{~nm}),\left(0^{\circ}, 12^{\circ}\right),(\mathrm{b}):(1549.9 \mathrm{~nm}, 1550.1 \mathrm{~nm}),\left(0^{\circ}, 12^{\circ}\right) . \quad \ldots .$.

5.11 Plot of effective indices for plasmon modes excited by a $\pi$-shifted array of tilted discs of current in the core of a fiber coated by thin metal layer positioned in air environment versus wavelength. Simulation parameters: azimuthal index of the mode $m=3$, layer thickness $d=35 \mathrm{~nm}$, grating is tuned for coupling plasmon mode and core mode at resonance wavelength $\lambda_{\text {res }}=1550 \mathrm{~nm}$. Effective indices as follows: solid line $n_{e f 1}$ (Au coating), short dashed line $n_{\text {ef } 2}$ (Au coating), large dashed line $n_{e f 3}$ (Pt coating). (a): real parts, (b): imaginary parts. . . . . . . . . . . .

5.12 Contour plot of power carried by plasmon mode excited by a $\pi$-shifted unbalanced array of tilted discs of current in the core of a fiber coated by thin $\mathrm{Au}$ layer positioned in air environment versus wavelength and tilt angle. Simulation parameters: azimuthal index of the mode $m=3$, layer thickness $d=35 \mathrm{~nm}$, array is tuned for coupling plasmon mode and core mode at resonance wavelength $\lambda_{\text {res }}=1550 \mathrm{~nm}$, effective index $n_{e f 2}$ shown in Fig. 5.11. The array is unbalanced by half of an element: $N_{2}=N_{1}+1 / 2$, where $N_{1}, N_{2}$ number of discs in the first and second halves of the array respectively. Computation window for wavelength and angle range respectively (a): $(1549 \mathrm{~nm}, 1551 \mathrm{~nm}),\left(0^{\circ}, 12^{\circ}\right),(\mathrm{b}):(1549.9 \mathrm{~nm}, 1550.1 \mathrm{~nm}),\left(0^{\circ}, 12^{\circ}\right)$, (c): $(1549.99 \mathrm{~nm}, 1550.01 \mathrm{~nm}),\left(0^{\circ}, 12^{\circ}\right) \ldots \ldots \ldots$. . . . . . . . 
5.13 Contour plot of power carried by plasmon mode excited by a $\pi$-shifted unbalanced array of tilted discs of current in the core of a fiber coated by thin Pt layer positioned in air environment versus wavelength and tilt angle. Simulation parameters: azimuthal index of the mode $m=3$, layer thickness $d=35 \mathrm{~nm}$, array is tuned for coupling plasmon mode and core mode at resonance wavelength $\lambda_{\text {res }}=1550 \mathrm{~nm}$, effective index $n_{\text {ef3 }}$ shown in Fig. 5.11. The array is unbalanced by half of an element: $N_{2}=N_{1}+1 / 2$, where $N_{1}, N_{2}$ number of discs in the first and second halves of the array respectively. Computation window for wavelength and angle range respectively (a): $(1549 \mathrm{~nm}, 1551 \mathrm{~nm}),\left(0^{\circ}, 12^{\circ}\right),(\mathrm{b}):(1549.9 \mathrm{~nm}, 1550.1 \mathrm{~nm}),\left(0^{\circ}, 12^{\circ}\right)$.

5.14 Contour plot of power carried by plasmon mode excited by $\pi$-shifted array of current sources where each element of an array is a set of tilted $N_{\text {rings }}$ rings or a tilted disc shown in Fig. 5.4(a). The array is located in a core of fiber coated by thin $\mathrm{Au}$ layer positioned in air environment. Contour plot computed versus wavelength $\lambda$ and tilt angle $\alpha$. Simulation parameters: azimuthal index of the mode $m=3$, layer thickness $d=35 \mathrm{~nm}$, array is tuned for coupling plasmon mode with azimuthal index $m=3$ and core mode at resonance wavelength $\lambda_{\text {res }}=1550 \mathrm{~nm}$ and resonance film thickness $d_{\text {res }}=$ $30 \mathrm{~nm}$, effective index $n_{\text {ef } 2}$ shown in Fig. 5.11. Modified source parameter respectively (A): rings with $N_{\text {rings }}=10,(\mathrm{~B})$ : rings with $N_{\text {rings }}=50,(\mathrm{C})$ : rings with $N_{\text {rings }}=70$, (D): discs. Computation window for wavelength and angle range respectively (a): $(1549 \mathrm{~nm}, 1551 \mathrm{~nm}),\left(0^{\circ}, 12^{\circ}\right),(\mathrm{b}):(1549.9$ $\mathrm{nm}, 1550.1 \mathrm{~nm}),\left(0^{\circ}, 12^{\circ}\right) \ldots \ldots \ldots \ldots$

5.15 Plot of effective index for plasmon mode excited by a $\pi$-shifted tilted grating in a fiber coated by Au layer positioned in air environment versus thickness. Simulation parameters: azimuthal index of the mode $m=3$, grating is tuned for coupling plasmon mode (calculated with Au thickness $d_{\text {res }}=30 \mathrm{~nm}$ ) and core mode at resonance wavelength $\lambda_{\text {res }}=1550 \mathrm{~nm}$. Modulation parameter is a wavelength, $\lambda$ : on both plots solid blue line represents $\lambda=1547 \mathrm{~nm}$ and dashed green line represents $\lambda=1555 \mathrm{~nm}$, increment in wavelength $\Delta \lambda=0.5 \mathrm{~nm}$ (a): real parts, (b): imaginary parts. . . . . . . . . . . .

5.16 Contour plot of power carried by plasmon mode excited by an array of tilted discs of current in the core of a fiber coated by thin Au layer positioned in air environment versus coating thickness and tilt angle. Simulation parameters: azimuthal index of the mode $m=3$, wavelength $\lambda=1550 \mathrm{~nm}$, array is tuned for coupling plasmon mode and core mode at resonance wavelength $\lambda_{\text {res }}=1550 \mathrm{~nm}$, effective indices shown in Fig. 5.15. (left): uniform array, (right): $\pi$-shifted array. Modulation parameter is a wavelength, $\lambda$ starting at $\lambda=1547 \mathrm{~nm}$ and finishing at $\lambda=1555 \mathrm{~nm}$ incrementing by $\Delta \lambda=1.0 \mathrm{~nm}$ in each contour plot. . . . . . . . . . . . . . . . . . . 
5.17 Polar plot of an absolute value of a normal component of cumulative electric field $\left|E_{r}\right|$ over 6 azimuthal indices, $m=0 \ldots 5$ of plasmons excited by a source of $\pi$-shifted array of tilted discs current located in a core of fiber coated by thin $\mathrm{Au}$ layer positioned in air environment versus azimuthal angle $\theta$ with the following parameters: tilt angle $\alpha$, film thickness $d$ and wavelength $\lambda$. Array is tuned for coupling plasmon mode with azimuthal index $m=3$ and core mode at resonance wavelength $\lambda_{\text {res }}=1550 \mathrm{~nm}$ and resonance film thickness $d_{\text {res }}=30 \mathrm{~nm}$. Field is measured at the middle of an array. Scale is arbitrary, but fixed within the same wavelength. . . . . .

5.18 Contour plot of power carried by plasmon mode excited by a $\pi$-shifted array of tilted discs of current in the core of a fiber coated by thin Au layer positioned in air environment versus wavelength and tilt angle. Simulation parameters: azimuthal index of the mode $m=3$, layer thickness $d=35 \mathrm{~nm}$, an array is tuned for coupling plasmon mode and core mode at resonance wavelength $\lambda_{\text {res }}=1550 \mathrm{~nm}$, effective index $n_{\text {ef } 1}$ shown in Fig. 5.11, array period in $z$-axis is $\Delta_{z}=\Delta / \cos \alpha$. Computation window for wavelength and angle range respectively (a): $(1549 \mathrm{~nm}, 1568 \mathrm{~nm}),\left(0^{\circ}, 12^{\circ}\right),(\mathrm{b}):(155159$ $\mathrm{nm}, 1552.5 \mathrm{~nm}),\left(0^{\circ}, 3.9^{\circ}\right)$. . . . . . . . . . . . . . . . . . .

5.19 Polar plot of an absolute value of a normal component of cumulative electric field $\left|E_{r}\right|$ over 6 azimuthal indices, $m=0 \ldots 5$ of plasmons excited by a source of $\pi$-shifted array of tilted discs current located in a core of fiber coated by thin Au layer positioned in air environment versus azimuthal angle $\theta$ the following parameters: tilt angle $\alpha$, film thickness $d$ and wavelength $\lambda$. Array is tuned for coupling plasmon mode with azimuthal index $m=3$ and core mode at resonance wavelength $\lambda_{\text {res }}=1550 \mathrm{~nm}$ and resonance film thickness $d_{\text {res }}=30 \mathrm{~nm}$. Array period in $z$-axis is $\Delta_{z}=\Delta / \cos \alpha$. Field is measured at the middle of an array. Scale is arbitrary, but fixed within the same wavelength. . . . . . . . . . . . . . . . . . .

6.1 A fiber with a realistic Bragg grating in the core as continuosly modulated tilted sine function. . . . .

6.2 A fiber with continuously modulated core by an uniform sine function along tilted axis $z^{\prime}$. . . . . . . . . . . . . . . . . . . . . . . . 128

6.3 A fiber with continuously modulated core by a $\pi$-shifted sine function along tilted axis $z^{\prime}$

6.4 Inhomogenuity of the fiber core (Bragg grating) and of metal distribution (structured coating) are introduced in an originally homogeneous cylindrical waveguide. . . . . . . . . . . . . . . . . . .

6.5 Geometry of metal distribution on a fiber with different tuning parameters of a distribution. Top left: set of patches; top right: distribution approaches to a strip like; bottom left: distribution approaches to a set of coils; bottom right: distribution approaches to a tube like. . . . . . . . . . . . . . 132 
6.6 Contour plot of power carried by plasmon mode excited by a uniform tilted grating in a fiber coated by thin Au layer positioned in air environment versus wavelength and tilt angle. Simulation parameters: azimuthal index of the mode $m=3$, layer thickness $d=35 \mathrm{~nm}$, grating is tuned for coupling plasmon mode and core mode at resonance wavelength $\lambda_{\text {res }}=1550 \mathrm{~nm}$, effective index $n_{e f 1}$ shown in Fig. 6.10. Computation window for wavelength and angle range respectively (a): $(1549 \mathrm{~nm}, 1565 \mathrm{~nm}),\left(0^{\circ}, 9^{\circ}\right),(\mathrm{b}):(1556.9$ $\mathrm{nm}, 1557.1 \mathrm{~nm}),\left(6.34^{\circ}, 6.44^{\circ}\right)$.

6.7 Contour plot of power carried by plasmon mode excited by a $\pi$-shifted tilted grating in a fiber coated by thin Au layer positioned in air environment versus wavelength and tilt angle. Simulation parameters: azimuthal index of the mode $m=3$, layer thickness $d=35 \mathrm{~nm}$, grating is tuned for coupling plasmon mode and core mode at resonance wavelength $\lambda_{\text {res }}=1550 \mathrm{~nm}$, effective index $n_{e f 1}$ shown in Fig. 6.10. Computation window for wavelength and angle range respectively (a): $(1549 \mathrm{~nm}, 1565 \mathrm{~nm}),\left(0^{\circ}, 9^{\circ}\right),(\mathrm{b}):(1556.9$ $\mathrm{nm}, 1557.1 \mathrm{~nm}),\left(6.34^{\circ}, 6.44^{\circ}\right)$

6.8 Contour plot of power carried by plasmon mode excited by a $\pi$-shifted tilted grating in a fiber coated by thin Au layer positioned in air environment versus wavelength and tilt angle. Simulation parameters: azimuthal index of the mode $m=3$, layer thickness $d=35 \mathrm{~nm}$, grating is tuned for coupling plasmon mode and core mode at resonance wavelength $\lambda_{\text {res }}=1550 \mathrm{~nm}$, effective index $n_{e f 2}$ shown in Fig. 6.10. Computation window for wavelength and angle range respectively (a): $(1549 \mathrm{~nm}, 1565 \mathrm{~nm}),\left(0^{\circ}, 9^{\circ}\right),(\mathrm{b}):(1556.9$ $\mathrm{nm}, 1557.1 \mathrm{~nm}),\left(6.34^{\circ}, 6.44^{\circ}\right) \ldots \ldots \ldots \ldots$

6.9 Contour plot of power carried by plasmon mode excited by a $\pi$-shifted tilted grating in a fiber coated by thin Pt layer positioned in air environment versus wavelength and tilt angle. Simulation parameters: azimuthal index of the mode $m=3$, layer thickness $d=35 \mathrm{~nm}$, grating is tuned for coupling plasmon mode and core mode at resonance wavelength $\lambda_{\text {res }}=1550 \mathrm{~nm}$, effective index $n_{\text {ef } 3}$ shown in Fig. 6.10. Computation window for wavelength and angle range respectively (a): $(1549 \mathrm{~nm}, 1570 \mathrm{~nm}),\left(0^{\circ}, 10^{\circ}\right),(\mathrm{b}):(1552$ $\mathrm{nm}, 1555 \mathrm{~nm}),\left(3.4^{\circ}, 5.3^{\circ}\right),(\mathrm{c}):(1552 \mathrm{~nm}, 1552.8 \mathrm{~nm}),\left(3.4^{\circ}, 4.4^{\circ}\right) \ldots \ldots$

6.10 Plot of effective indices for plasmon modes excited by a $\pi$-shifted array of tilted discs of current in the core of a fiber coated by thin metal layer positioned in air environment versus wavelength. Simulation parameters: azimuthal index of the mode $m=3$, layer thickness $d=35 \mathrm{~nm}$, grating is tuned for coupling plasmon mode and core mode at resonance wavelength $\lambda_{\text {res }}=1550 \mathrm{~nm}$. Effective indices as follows: solid line $n_{\text {ef } 1}$ (Au coating), short dashed line $n_{e f 2}$ (Au coating), large dashed line $n_{e f 3}$ (Pt coating). (a): real parts, (b): imaginary parts. . . . . . . . . . . 138 
6.11 Contour plot of power carried by plasmon mode excited by a $\pi$-shifted tilted grating in a fiber coated by thin Pt layer positioned in air environment versus wavelength and tilt angle. Simulation parameters: azimuthal index of the mode $m$, layer thickness $d=35 \mathrm{~nm}$, grating is tuned for coupling plasmon mode and core mode at resonance wavelength $\lambda_{\text {res }}=1550 \mathrm{~nm}$. Azimuthal indices: (a) $m=2$, (b) $m=3$, (c) $m=4$, (d) $m=5$. . . . . . . . . .

6.12 Plot of effective index for plasmon mode excited by a $\pi$-shifted tilted grating in a fiber coated by $\mathrm{Au}$ layer positioned in air environment versus thickness. Simulation parameters: azimuthal index of the mode $m=3$, grating is tuned for coupling plasmon mode (calculated with Au thickness $d_{r e s}=30 \mathrm{~nm}$ ) and core mode at resonance wavelength $\lambda_{\text {res }}=1550 \mathrm{~nm}$. (a): real parts, (b): imaginary parts. . . . . . . . . . . . . . .

6.13 Contour plot of power carried by plasmon mode excited by a $\pi$-shifted tilted grating in a fiber coated by thin $\mathrm{Au}$ layer positioned in air environment versus coating thickness and tilt angle. Simulation parameters: azimuthal index of the mode $m=3$, wavelength $\lambda=1550 \mathrm{~nm}$, grating is tuned for coupling plasmon mode and core mode at resonance wavelength $\lambda_{\text {res }}=$ $1550 \mathrm{~nm}$, effective index shown in Fig. 6.12. . . . . . . . . . . . . . . . .

6.14 Contour plot of power carried by plasmon excited by a (uniform and $\pi$ shifted) tilted grating in a fiber coated by $\mathrm{Au}$ distribution positioned in an index matching oil (with refractive index $n_{4}=1.42$ ) environment versus wavelength and tilt angle. Simulation parameters: azimuthal index of the mode $m=3$; wavelength $\lambda=1550 \mathrm{~nm}$; grating is tuned for coupling plasmon mode and core mode at resonance wavelength $\lambda_{\text {res }}=1550 \mathrm{~nm}$; thickness of $\mathrm{Au}$ distribution $d=100 \mathrm{~nm}$; angular width of distribution $\left(\theta_{\min }, \theta_{\max }\right): \theta_{\min }=-\pi / 10, \theta_{\max }=\pi / 10$, array length $2 z_{0}=2 \mathrm{~cm}$; distribution type: uniform array of patches; AC grating $\Delta \varepsilon_{A C}=3 \times 10^{-4}$. Au distribution parameters: $\delta z=50 \mathrm{~nm}, \Delta z=5000 \mu \mathrm{m} . \ldots . . . .$.

6.15 Contour plot of power carried by plasmon excited by a (uniform and $\pi$ shifted) tilted grating in a fiber coated by $\mathrm{Au}$ distribution positioned in an index matching oil (with refractive index $n_{4}=1.42$ ) environment versus wavelength and tilt angle. Simulation parameters: azimuthal index of the mode $m=3$; wavelength $\lambda=1550 \mathrm{~nm}$; grating is tuned for coupling plasmon mode and core mode at resonance wavelength $\lambda_{\text {res }}=1550 \mathrm{~nm}$; thickness of $\mathrm{Au}$ distribution $d=100 \mathrm{~nm}$; angular width of distribution $\left(\theta_{\min }, \theta_{\max }\right): \theta_{\min }=-\pi / 10, \theta_{\max }=\pi / 10$, array length $2 z_{0}=2 \mathrm{~cm}$; distribution type: uniform array of patches; AC grating $\Delta \varepsilon_{A C}=3 \times 10^{-4}$. Au distribution parameters: $\delta z=50 \mathrm{~nm}, \Delta z=52 \mu \mathrm{m} . \ldots \ldots . . \ldots 144$ 
6.16 Contour plot of power carried by plasmon excited by a (uniform and $\pi$ shifted) tilted grating in a fiber coated by $\mathrm{Au}$ distribution positioned in an index matching oil (with refractive index $n_{4}=1.42$ ) environment versus wavelength and tilt angle. Simulation parameters: azimuthal index of the mode $m=3$; wavelength $\lambda=1550 \mathrm{~nm}$; grating is tuned for coupling plasmon mode and core mode at resonance wavelength $\lambda_{\text {res }}=1550 \mathrm{~nm}$; thickness of $\mathrm{Au}$ distribution $d=100 \mathrm{~nm}$; angular width of distribution $\left(\theta_{\min }, \theta_{\max }\right): \theta_{\min }=-\pi / 10, \theta_{\max }=\pi / 10$, array length $2 z_{0}=2 \mathrm{~cm}$; distribution type: uniform array of patches; AC grating $\Delta \varepsilon_{A C}=3 \times 10^{-4}$. Au distribution parameters: $\delta z=50 \mathrm{~nm}, \Delta z=1 \mathrm{~cm} . \ldots . . . .$.

6.17 Contour plot of power carried by plasmon excited by a (uniform and $\pi$ shifted) tilted grating in a fiber coated by $\mathrm{Au}$ distribution positioned in an index matching oil (with refractive index $n_{4}=1.42$ ) environment versus wavelength and tilt angle. Simulation parameters: azimuthal index of the mode $m=3$; wavelength $\lambda=1550 \mathrm{~nm}$; grating is tuned for coupling plasmon mode and core mode at resonance wavelength $\lambda_{\text {res }}=1550 \mathrm{~nm}$; thickness of $\mathrm{Au}$ distribution $d=100 \mathrm{~nm}$; angular width of distribution $\left(\theta_{\min }, \theta_{\max }\right): \theta_{\min }=-\pi / 10, \theta_{\max }=\pi / 10$, array length $2 z_{0}=2 \mathrm{~cm}$; distribution type: uniform array of patches; AC grating $\Delta \varepsilon_{A C}=3 \times 10^{-4}$. Au distribution parameters: $\delta z=0.5 \mathrm{~cm}, \Delta z=1 \mathrm{~cm} . \ldots \ldots$

6.18 Contour plot of power carried by plasmon excited by a (uniform and $\pi$ shifted) tilted grating in a fiber coated by $\mathrm{Au}$ distribution positioned in an index matching oil (with refractive index $n_{4}=1.42$ ) environment versus wavelength and tilt angle. Simulation parameters: azimuthal index of the mode $m=3$; wavelength $\lambda=1550 \mathrm{~nm}$; grating is tuned for coupling plasmon mode and core mode at resonance wavelength $\lambda_{\text {res }}=1550 \mathrm{~nm}$; thickness of $\mathrm{Au}$ distribution $d=100 \mathrm{~nm}$; angular width of distribution $\left(\theta_{\min }, \theta_{\max }\right): \theta_{\min }=-\pi / 10, \theta_{\max }=\pi / 10$, array length $2 z_{0}=2 \mathrm{~cm}$; distribution type: uniform array of patches; AC grating $\Delta \varepsilon_{A C}=3 \times 10^{-4}$. Au distribution parameters: $\delta z=0.9 \mathrm{~cm}, \Delta z=1 \mathrm{~cm} . \ldots . . . . .$.

6.19 Contour plot of power carried by plasmon excited by a (uniform and $\pi$ shifted) tilted grating in a fiber coated by $\mathrm{Au}$ distribution positioned in an index matching oil (with refractive index $n_{4}=1.42$ ) environment versus wavelength and tilt angle. Simulation parameters: azimuthal index of the mode $m=3$; wavelength $\lambda=1550 \mathrm{~nm}$; grating is tuned for coupling plasmon mode and core mode at resonance wavelength $\lambda_{\text {res }}=1550 \mathrm{~nm}$; thickness of $\mathrm{Au}$ distribution $d=100 \mathrm{~nm}$; angular width of distribution $\left(\theta_{\min }, \theta_{\max }\right): \theta_{\min }=-\pi / 10, \theta_{\max }=\pi / 10$, array length $2 z_{0}=2 \mathrm{~cm}$; distribution type: uniform array of patches; AC grating $\Delta \varepsilon_{A C}=3 \times 10^{-4}$. Au distribution parameters: $\delta z=\Delta z-60 \mathrm{~nm}, \Delta z=1000 \mu \mathrm{m} \ldots \ldots \ldots$ 
6.20 Contour plot of power carried by plasmon excited by a (uniform and $\pi$ shifted) tilted grating in a fiber coated by $\mathrm{Au}$ distribution positioned in an index matching oil (with refractive index $n_{4}=1.42$ ) environment versus wavelength and tilt angle. Simulation parameters: azimuthal index of the mode $m=3$; wavelength $\lambda=1550 \mathrm{~nm}$; grating is tuned for coupling plasmon mode and core mode at resonance wavelength $\lambda_{\text {res }}=1550 \mathrm{~nm}$; thickness of $\mathrm{Au}$ distribution $d=100 \mathrm{~nm}$; angular width of distribution $\left(\theta_{\min }, \theta_{\max }\right): \theta_{\min }=-\pi / 10, \theta_{\max }=\pi / 10$, array length $2 z_{0}=2 \mathrm{~cm}$; distribution type: uniform array of patches; AC grating $\Delta \varepsilon_{A C}=3 \times 10^{-4}$. Au distribution parameters: $\delta z=\Delta z, \Delta z=1000 \mu m$

6.21 Contour plot of power carried by plasmon excited by a (uniform and $\pi$ shifted) tilted grating in a fiber coated by $\mathrm{Au}$ distribution positioned in an index matching oil (with refractive index $n_{4}=1.42$ ) environment versus wavelength and tilt angle. Simulation parameters: azimuthal index of the mode $m=3$; wavelength $\lambda=1550 \mathrm{~nm}$; grating is tuned for coupling plasmon mode and core mode at resonance wavelength $\lambda_{\text {res }}=1550 \mathrm{~nm}$; thickness of $\mathrm{Au}$ distribution $d=100 \mathrm{~nm}$; angular width of distribution $\left(\theta_{\min }, \theta_{\max }\right): \theta_{\min }=-\pi / 10, \theta_{\max }=\pi / 10$, array length $2 z_{0}=2 \mathrm{~cm}$; distribution type: uniform array of patches; AC grating $\Delta \varepsilon_{A C}=3 \times 10^{-4}$. Au distribution parameters: $\delta z=0.95 \mathrm{~cm}, \Delta z=1 \mathrm{~cm}$. . . . . . . . . .

6.22 Contour plot of power carried by plasmon excited by a (uniform and $\pi$ shifted) tilted grating in a fiber coated by $\mathrm{Au}$ distribution positioned in an index matching oil (with refractive index $n_{4}=1.42$ ) environment versus wavelength and tilt angle. Simulation parameters: azimuthal index of the mode $m=3$; wavelength $\lambda=1550 \mathrm{~nm}$; grating is tuned for coupling plasmon mode and core mode at resonance wavelength $\lambda_{\text {res }}=1550 \mathrm{~nm}$; thickness of $\mathrm{Au}$ distribution $d=100 \mathrm{~nm}$; angular width of distribution $\left(\theta_{\min }, \theta_{\max }\right): \theta_{\min }=-\pi / 10, \theta_{\max }=\pi / 10$, array length $2 z_{0}=2 \mathrm{~cm}$; distribution type: $\pi$-shifted array of patches; AC grating $\Delta \varepsilon_{A C}=3 \times 10^{-4}$. Au distribution parameters: $\delta z=50 \mathrm{~nm}, \Delta z=5000 \mu \mathrm{m} . . . . . . . .$.

6.23 Contour plot of power carried by plasmon excited by a (uniform and $\pi$ shifted) tilted grating in a fiber coated by $\mathrm{Au}$ distribution positioned in an index matching oil (with refractive index $n_{4}=1.42$ ) environment versus wavelength and tilt angle. Simulation parameters: azimuthal index of the mode $m=3$; wavelength $\lambda=1550 \mathrm{~nm}$; grating is tuned for coupling plasmon mode and core mode at resonance wavelength $\lambda_{\text {res }}=1550 \mathrm{~nm}$; thickness of $\mathrm{Au}$ distribution $d=100 \mathrm{~nm}$; angular width of distribution $\left(\theta_{\min }, \theta_{\max }\right): \theta_{\min }=-\pi / 10, \theta_{\max }=\pi / 10$, array length $2 z_{0}=2 \mathrm{~cm}$; distribution type: $\pi$-shifted array of patches; AC grating $\Delta \varepsilon_{A C}=3 \times 10^{-4}$. Au distribution parameters: $\delta z=50 \mathrm{~nm}, \Delta z=1 \mathrm{~cm} . \ldots . . . . . . .160$

6.24 Dependence intensity vs. wavelength $I(\lambda)$ suitable for detecting small external perturbation. . . . . . . . . . . . . . . . . 
6.25 Polar plot of an absolute value of a normal component of cumulative electric field $\left|E_{r}\right|$ over 4 azimuthal indices, $m=2 \ldots 5$ of plasmons excited by a source of $\pi$-shifted tilted grating of a fiber coated by thin Au layer positioned in air environment versus azimuthal angle $\theta$ with the following parameters: tilt angle $\alpha$, film thickness $d$ and wavelength $\lambda$. The grating is tuned for coupling plasmon mode with azimuthal index $m=3$ and core mode at resonance wavelength $\lambda_{\text {res }}=1550 \mathrm{~nm}$ and resonance film thickness $d_{\text {res }}=$ $30 \mathrm{~nm}$. Field is measured at the middle of an the grating. Scale is arbitrary, but fixed within the same wavelength. . . . . . . . . . . . . . .

7.1 Wavelet based on cosine function where number of segments (grids) spanned by wavelet. . . . . . . . . . . . . . . . . . 166

7.2 Train of wavelets over the computational domain where $N$ number of grids and $(N+1-s)$ number of wavelets of the whole domain. . . . . . . . 167

7.3 Overlap interval between two wavelets in the integrals $\widehat{L}_{j i}$. . . . . . . . . . 173

7.4 Wavelets adapted for different azimuthal index $m . \ldots . . . . . . .176$

7.5 Transversal electric and magnetic fields calculated with generalized eigenvalue equations for the first mode of Table $7.1, r_{1}$ is a core radius in $\mu m$. .

7.6 Transversal electric and magnetic fields calculated with generalized eigenvalue equations for the last mode of Table $7.1, r_{1}$ is a core radius in $\mu \mathrm{m}$.

7.7 z-component of Poynting vector calculated with generalized eigenvalue equations (a) and (b) for the first and last modes of Table 1 respectively. Dimensions in microns. . . . . . . . . . . . . . . . . . . . . . . . . . 181

7.8 Modal indices in complex plane $\left(\operatorname{Re}\left(n_{e f}\right), \operatorname{Im}\left(n_{e f}\right)\right)$ for step index fiber SMF28 coated by $35 \mathrm{~nm}$ of metal in water environment at wavelength $1550 \mathrm{~nm}$. . . . . . . . . . . . . . . . . . . . .

7.9 Modal indices of step index fiber SMF28 coated by $\mathrm{Au}$ in $\mathrm{H}_{2} \mathrm{O}$ environment with variable thickness of $A u$ layer at wavelength $1550 \mathrm{~nm}$ and azimuthal index $m=5$. (a) real parts vs. thickness in nanometers; (b) imaginary parts vs. thickness in nanometers. The real and imaginary parts of indices belonging to the same mode and the same metal thickness represented in (a) and (b) with the same color. . . . . . . . . . . . . .

7.10 Modal indices of step index fiber SMF28 coated by $\mathrm{Au}$ in $\mathrm{H}_{2} \mathrm{O}$ environment with variable wavelength and fixed $A u$ layer at thickness $35 \mathrm{~nm}$ and azimuthal index $m=5$. (a) real parts vs. wavelength in nanometers; (b) imaginary parts vs. wavelength in nanometers. The real and imaginary parts of indices belonging to the same mode and the same wavelength represented in (a) and (b) with the same color. . . . . . . . . . . .

A.1 Conformal mapping upper plane z to corrugated upper plane w (triangular saw tooth). 
A.2 Conformal mapping upper plane $\mathrm{z}$ to corrugated upper plane w (rectangular saw tooth). . . . . . . . . . . . . . . . . . . . 190 


\section{Chapter 1}

\section{Introduction and thesis outline}

An optical waveguide is a basic element of telecommunication system in general or integrated optical circuits in particular. For the integrated optics purpose it can be implemented either based on semiconductor technology or simply based on a glass substrate. A general waveguide should consist of a high refractive index guiding layer, a core, surrounded by low refractive index cladding layer. The signal propagates in a guiding layer and being confined in transverse direction because of the total internal reflection phenomena. Losses involved in delivering signal between optical elements such as scattering, absorption, coupling losses etc. require miniaturization and collecting many elements on a single integrated optical circuit. Glass has naturally high transmission over wide range of wavelengths, can be made with high homogeneity and low impurity of different shapes and sizes, therefore it is a good material for passive optical elements on a circuits.

For active optical devices the glass should be doped by elements that provide an optical gain. Such elements usually are the trivalent rare earth ions. The rare earth ions governed by $4 \mathrm{f}$ wavefunctions that lie within completely closed $5 \mathrm{~s}$ and $5 \mathrm{p}$ shells, electrons of these shells shield the $4 \mathrm{f}$ electrons from the disturbing effects of the environment, this causes only small influence of the host lattice on the wavelengths, bandwidth and cross sections of the corresponding optical transitions, [1]. The pump and lasing transitions are only weakly allowed transitions (i.e. are not completely forbidden) with small oscillation strength. As a result of this the upper state lifetimes can be long, approaching to milliseconds, which makes amount of energy stored in the media substantial. In a mixture of erbiumytterbium ions the dipole-dipole interactions allow energy transfer between them, i.e. pump energy absorbed by ytterbium ions and transferred to erbium ions. The erbium ions have transitions at $1.54 \mu \mathrm{m}$, which is a standard wavelength used in optical telecommunication, $[2]$.

An additional advantage of a glass waveguide is that the glass substrate is a good host material suitable for accommodation of rare earth ions as a gain material in an optically pumped laser cavity or optical amplifier. To achieve optical gain with active devices based on rare earth ions the optical pump should be strong enough to make a population inversion between two levels, i.e. population on a lasing level exceeds the population on a ground 
level. Then the signal that propagates thorough such device induces a stimulated transition and gets amplified.

Also, the glass contains alkali elements and therefore permits refractive index change through an ion-exchange process where host alkali ions are replaced by other type of ions. Thus, in the glass waveguide the core, a layer with higher refractive index can be built.

To suitably modify properties of the optical devices, the nanocomposites such as metal particles can be implanted in the glass matrix. The spectral response of the glass media with metal nanoparticles embedded under appropriate tuning is controlled by a surface plasmon resonance a density oscillation of the electrons in the metal coupled to the electromagnetic field. The surface plasmon resonance is responsible for many optical phenomena where strong local field is crucial such as large value of nonlinearity (second harmonic generation, four wave mixing, third order susceptability) due to locally increased field, one the theory for example explains mechanism of surface enchanced Raman scattering, SERS by a localized surface plasmons. The proof of principle of a single molecule sensitivity of SERS has been demonstrated, [3]. Therefore the ability to implement metal nanoparticles in the host media of the optical devices opens/widens sensor like waveguide applications.

There is a possibility of further patterning these nanoparticles in the media, it could be done for example by means of applying DC voltage to the surface of the glass. As a result of a strong external field, the metal nanoparticles can be ionized and eventually dissolved away from the surface. By making electrode spacially modulated, the dissolution process can be controlled locally. The ability of patterning of embedded metal nanoparticles in the glass can be used to build up a laser cavity by creating gratings on both ends.

\subsection{Thesis outline.}

This thesis organized as follows.

The Chapter 1 reviews the literature that describes processes of forming metal nanoclusters in the glass media followed by process of a field assisted dissolving of a nanoclusters embedded in a glass. The emphasis is made on ability of patterning nanoclusters distribution in glass substrate that would allow to built up various optical circuits on a glass substrates and possible make a laser cavity by forming deep (volume) gratings.

In Chapter 2 the experimental part of the thesis aims to build a deep volume grating with the silver ion-exchange process in a phosphate glass IOG-1 doped by Er/Yt ions, i.e. with ions $\mathrm{Na}^{+}-\mathrm{Ag}^{+}$substitution, and subsequent field assisted silver cluster dissolution applied locally through periodic electrode with the final goal in the future of achieving Bragg grating suitable for making laser cavity. Though the actual grating period considered in this chapter is not small enough for a Bragg grating, it demonstrates the proof of such possibility. Silver nano sized clusters posses strong plasmon resonance, the high contrast grating build up by this method is due to a plasmon excitation even for off resonance wavelengths.

The Chapter 3 sketches the approache for a future experimental work to achieve the goal of a Bragg grating with this technique. Plasmon excitation also considered in a 
configuration other than metal nanoparticles in a glass, namely in a metal nanostructures deposited on fiber. The computational model of a plasmon excitation was developed in steps and tested on a slab waveguide with thin metal film illuminated by a finite sheet of current in Chapter 4, fiber coated by thin metal film excited by discrete sources of current in the fiber core in Chapter 5 and finally fiber with nanostructured metal coating excited by a tilted Bragg grating in Chapter 6. For a plasmon excitation by tilted Bragg grating in a fiber the knowledge of the complex modal indices of a fiber is crucial. Chapter 7 provides a numerical procedure based on Galerkin method for finding complex roots of a fiber. 


\section{Chapter 2}

\section{Technological background}

This chapter presents a brief review of the literature on silver nanoclusters embedded in glass and subsequent modification of such clusters.

\subsection{Silver clusters formation in a silica based glass.}

Silver nanoclustering formation was reported for the first time in ion-exchanged glass as a host media by ion $\mathrm{Ag}^{+}$reduction in hydrogen atmosphere, [4]. Light waveguides made by ion-exchange $\mathrm{Ag}^{+}-\mathrm{Na}^{+}$process in soda-lime glass, has been annealed in hydrogen atmosphere at temperatures in the $120^{\circ}-250^{\circ}$ range. The annealing time was 5 hours.

Annealings stimulate a near-surface precipitation of metallic silver to form nanometersize clusters with good uniformity in size and spatial distribution. Clustering formation occurs because solubility of silver atoms in glass is very low while concentration of silver ions in an ion-exchanged glass is relatively high. Therefore precipitation of silver atoms in glass above the level of solubility leads to a clustering.

Fig. 2.1 shows the silver depth distribution after 5 hours annealing at different temperatures.

Annealing in hydrogen atmosphere results a silver migration towards the surface which is evident from the plot on Fig. 2.1 where the peak concentration of the silver located just below the glass surface.

When silver atoms migrate to the surface, they form nanoclusters. Their presence is indicated by optical absorption band with maximum at $410 \mathrm{~nm}$, see Fig. 2.2. Such absorption band is predicted by Mie theory for silver spherical nanoclusters with radius of a few nanometers. The intensity of the absorption peak increases with annealing temperature, without broadening and shifting. Such behavior is due to increase volume fraction of the silver.

TEM micrographs of samples annealed in hydrogen at $180^{\circ} \mathrm{C}$ of 12 hours and at $250^{\circ} \mathrm{C}$ for 5 hours are reported in Fig. 2.3. Bright-field and dark-field imaging were used to 


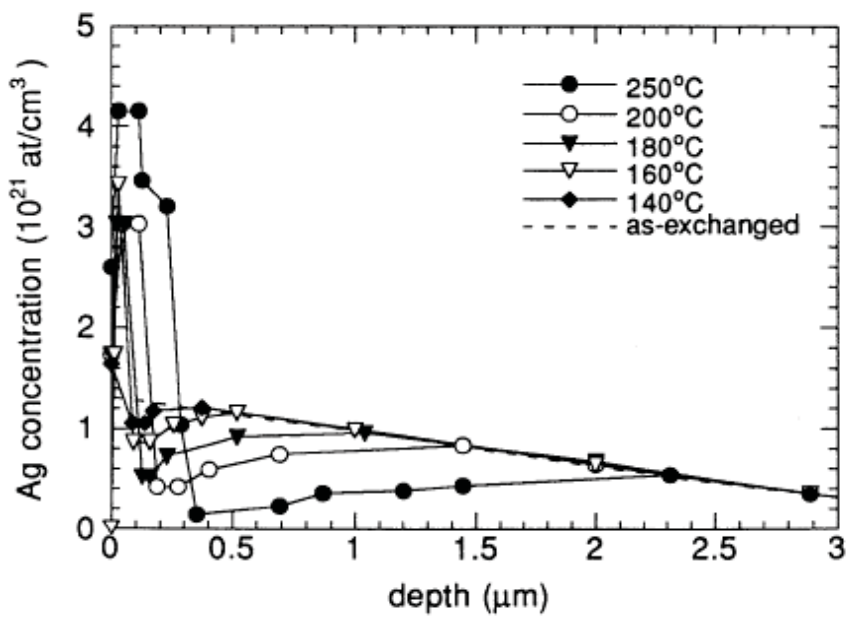

Figure 2.1: Silver depth concentration profiles before and after annealing processes at constant time (5h) and different temperatures, [4].

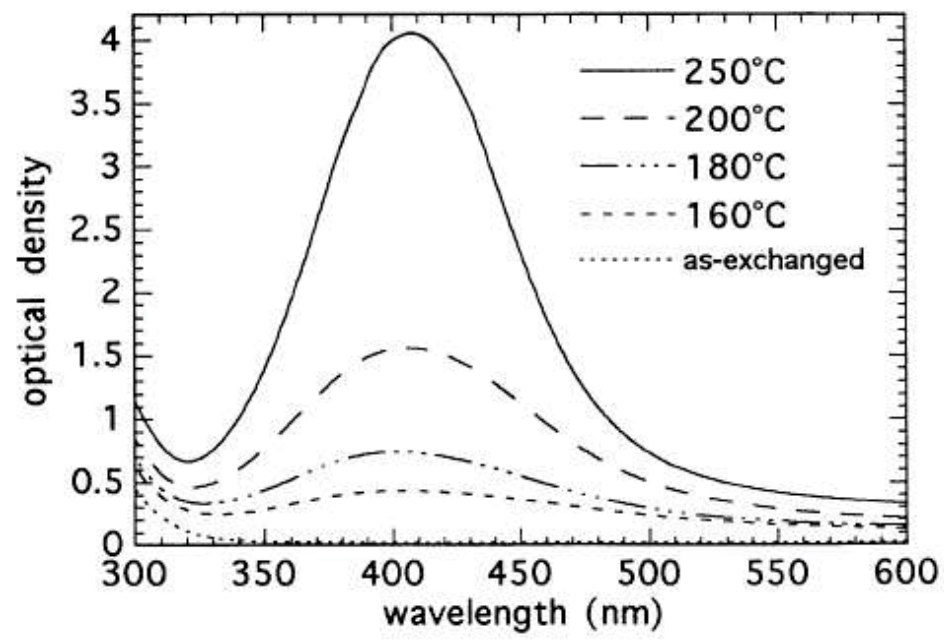

Figure 2.2: UV-visible absorption spectra for samples annealed at constant time (5h) and different temperatures, [4].

characterize the shape and geometrical structure of the nanoclusters. Cluster diameters are about 2-4 $\mathrm{nm}$ for the lower temperature sample and 4-6 $\mathrm{nm}$ for the higher temperature one. Selected area electron diffraction patterns are reported as insets of Fig. 2.3.

Treatment of ion-exchange glass in a hydrogen atmosphere can also result in a periodic precipitation of silver clusters, [5].

Ion-exchanged soda-lime glass slides of thickness, with homogeneous distribution of silver ions, were treated in hydrogen at atmospheric pressure and at $500^{\circ} \mathrm{C}$ for 5 hours. For the 'low-Ag' glass $\left(0.8 \mathrm{~mol}_{\%} \mathrm{Ag}_{2} \mathrm{O}\right)$ this treatment resulted in the formation of silver particles within a region of $3 \mu \mathrm{m}$ thickness below the glass surface. These particles have nearly spherical shape as shown in TEM micrographs in Fig. 2.4.

Density of silver particles and their size decrease with penetration depth. Near the 

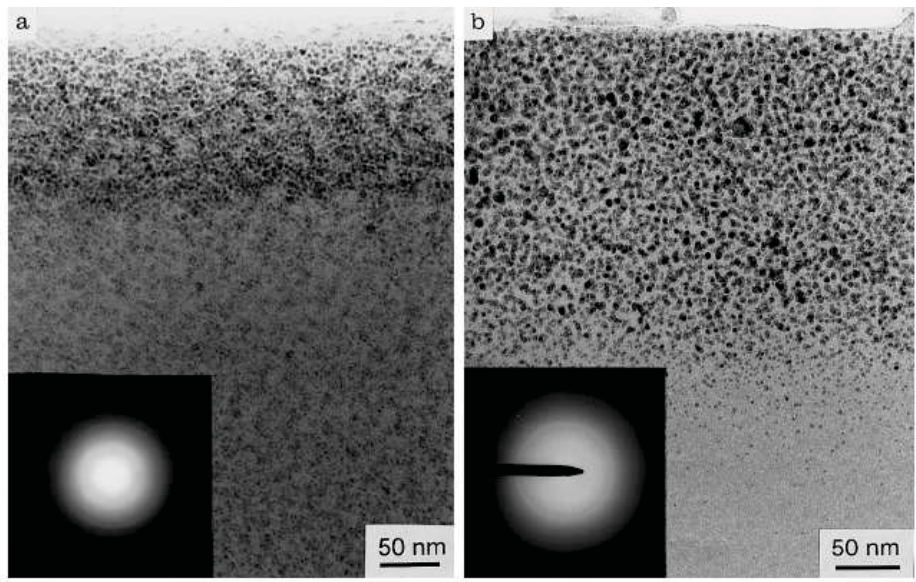

Figure 2.3: TEM micrographs (cross section) ofsilver nanoclusters, formed after annealing in hydrogen atmosphere for (a) 12 hours at $180^{\circ} \mathrm{C}$ and (b) 5 hours at $250^{\circ} \mathrm{C}$. Selected area diffraction patterns are reported in the insets, [4].

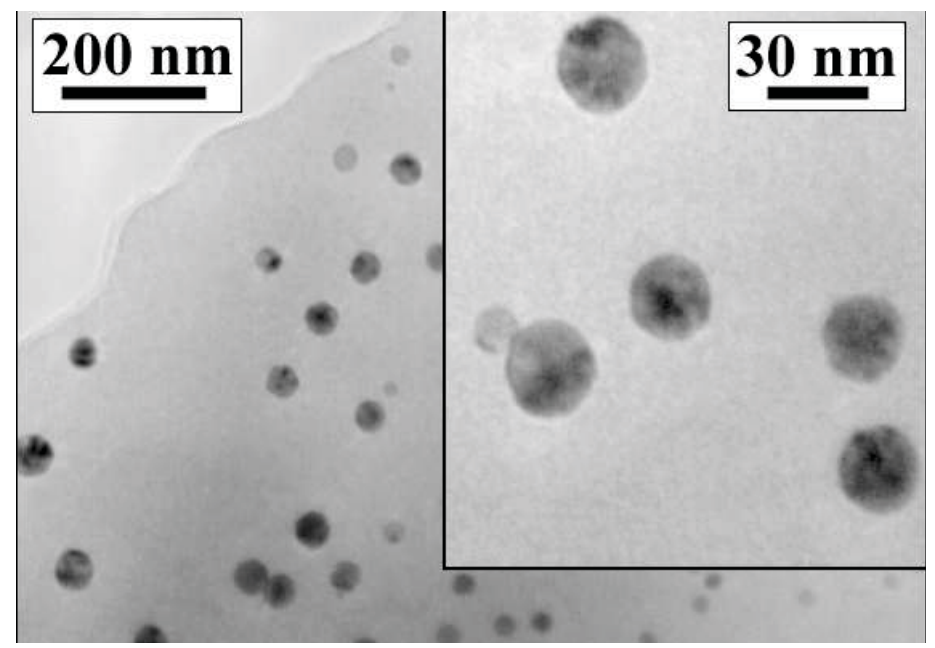

Figure 2.4: Particles in the 'low-Ag' glass at a penetration depth of $1.5 \mu \mathrm{m}$; the inset shows, at higher magnification, the non-homogeneous image contrast of some particles due to twin defects, [5].

surface particle diameter is $\approx 25 \mathrm{~nm}$ diameter and $\approx 5 \mathrm{~nm}$ at about $2.5 \mu \mathrm{m}$ penetration depth, see Fig. 2.5. Beyond $3 \mu \mathrm{m}$, no Ag particles were found in this sample.

In the 'high-Ag' glass (11.0 mol\% $\mathrm{Ag}_{2} \mathrm{O}$ ), after annealing in hydrogen periodic layers of silver particles were formed within a region of about thickness below the glass surface.

The electron micrograph of Fig. 2.6 demonstrates periodic layers of silver clusters in the region thickness below the surface. They are parallel to the surface. Fig. 2.4 shows one of this layer made up of silver particles. Below the $(n+7)$ th layer, no further layer-like particle distribution was observed, but around penetration depth a second region of silver particles having a nearly homogeneous spatial distribution was found. This region ranges 


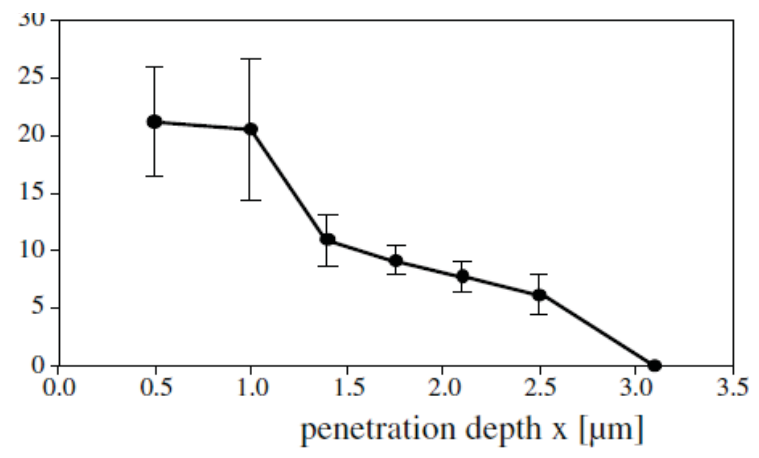

Figure 2.5: dependence of the mean particle size of the 'low-Ag' sample on the penetration depth, [5].

throughout the whole glass thickness until it meets the mirrored counterpart of the abovementioned layer-like particle arrangement near the other surface of the thick glass plate. The layer-like distribution of silver particles near the surface explained by the interdiffusion of two mobile species, hydrogen and silver ions, when reaction product of both, i.e. silver atoms, exceeds a certain supersaturation. Thus, formation of periodic layers is a sequence of a high enough initial concentrations of hydrogen and silver ions near the surface. The spacial distribution of the particles inside of one layer is shown in Fig. 2.7.

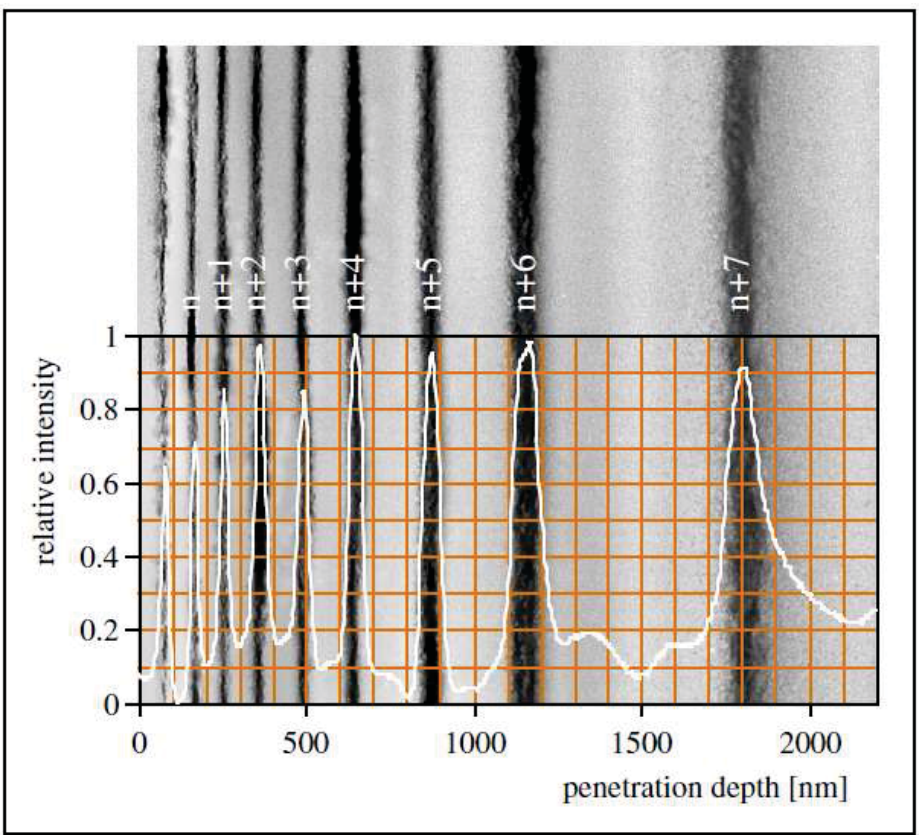

Figure 2.6: The near-surface region of the 'high-Ag' glass in a cross-sectional view together with a profile plot of the relative image intensity showing the arrangement and extension of the Ag precipitate layers, [5].

The basic mechanism of hydrogen-induced particle formation consists of two steps. It starts with silver ions which, due to ion exchange, replace sodium ions and are bonded to 


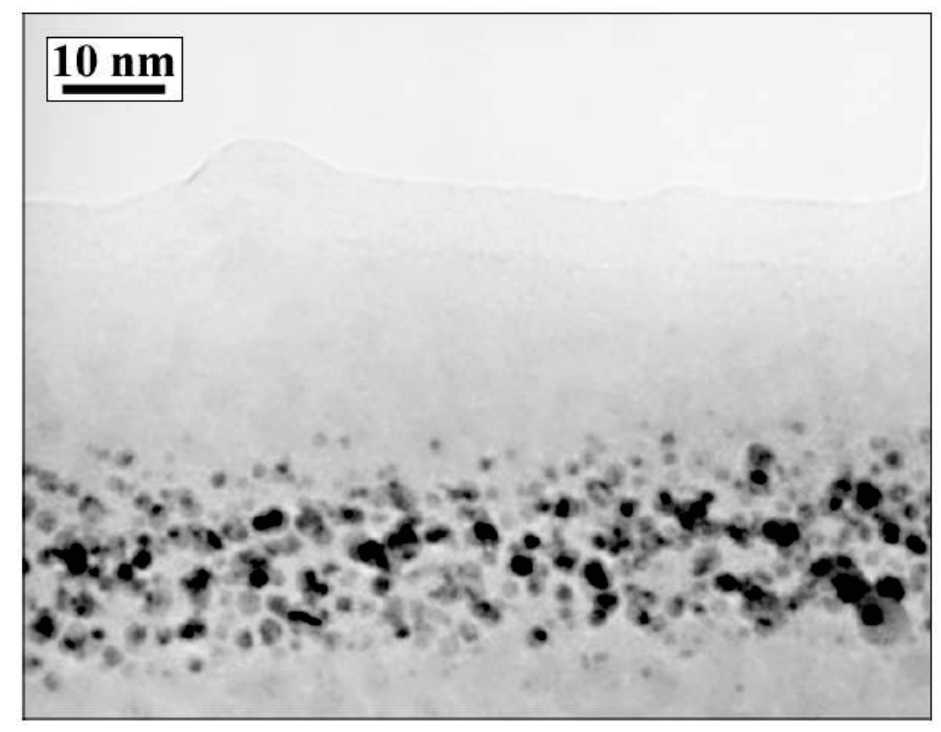

Figure 2.7: The distribution of Ag particles in one layer (n) of the 'high-Ag' glass, [5].

non-bridging oxygens. First, silver ions will be reduced by atomic hydrogen:

$$
\equiv S i-O^{-} A g^{+}+(1 / 2) H_{2} \rightarrow \equiv S i-O H+A g^{0}
$$

These free silver atoms then diffuse down and form nanometer size clusters. Because hydrogen concentration is high, all silver ions are reduced to atoms.

The silver particles in regions deeper than about in the 'high-Ag' glass have size about close to the surface and about in the central region.

The mechanism of particle formation in these regions without hydrogen permeation is expected to be based on $\mathrm{Fe}^{2+}$ ions as reducing agents:

$$
\mathrm{Ag}^{+}+\mathrm{Fe}^{2+} \rightarrow \mathrm{Ag}^{0}+\mathrm{Fe}^{3+}
$$

\subsection{Spherical clusters reshaping.}

Other methods to form Ag-clustering in glass without using hydrogen as a reduction agent are also reported. High-power excimer-laser irradiation at $248 \mathrm{~nm}$ wavelength and energy densities up to $4.6 \mathrm{~J} / \mathrm{cm}^{2}$ has been performed on silver-exchanged soda-lime glass. Silver nanoclusters have been obtained with average size depending on the energy density of the laser pulses. The excimer laser pulses induce either the reduction of the silver ions or the heating of the irradiated glass matrix, [6]. Laser irradiation, thermal annealing, ion irradiation and various combination of these factors are led to Ag-clustering in ionexchanged soda lime glass, [7]. Precipitation of silver particles in silver doped glass in a 
controlled location was achieved with femtosecond laser pulses, [8], [9]. Fractal dendritic silver microstructures on the surface of the $\mathrm{Ag}^{+}$-doped glasses as a result of a photothermal interaction with a focused multiline cw high-power $(\operatorname{Pmax}=8 \mathrm{~W}) A r^{+}$laser beam is reported recently, [10].

Once silver clusters are embedded in a glass, it is possible to modify their basic spherical shape from basic spherical to elongated with polarized laser pulse 150 fs at wavelength 400 $\mathrm{nm}$ close to plasmon resonance in $\mathrm{Ag}$, [11].

The samples used for the experiments prepared from standard glass by an ion exchange in a $\mathrm{AgNO}_{3}: \mathrm{NaNO}_{3}$ mixed melt. Heating to temperatures $600^{\circ} \mathrm{C}$ results in a chemical reduction of the silver ions and formation of spherical silver nanoparticles, shown in the top of Fig. 2.9. The mean radius of particles is $\approx 12 \mathrm{~nm}$, a size distribution of about $\pm 30 \%$ and a volume fill factor of $10^{-4}$.

These samples were irradiated with single laser pulses from an amplified, frequencydoubled titanium:sapphire laser (wavelength $400 \mathrm{~nm}$, pulse duration $\approx 150 \mathrm{fs}$, pulse intensity in the order of $10^{11} \mathrm{~W} / \mathrm{cm}^{2}$, linear or circular polarization). Optical extinction spectra were taken both in not irradiated and in irradiated areas of the sample with linearly polarized light, whose polarization was either parallel or perpendicular to the polarization of the laser pulse. Fig. 2.8 shows optical spectra of the samples probed by linearly polarized light.

Solid curve represents sample spectra before irradiation, with its maximum at $415 \mathrm{~nm}$ and a full bandwidth at half height $(\mathrm{FWHH})$ of $34 \mathrm{~nm}$. This band is caused by the surface plasmon resonance (SPR) of the silver particles.

Spectra after irradiation is anisotropic (dichroitic), i.e. it depends on probing light polarization. New peak is shifted towards longer wavelengths: $510 \mathrm{~nm}$ for parallel polarization (dotted curve) and $575 \mathrm{~nm}$ for perpendicular (dashed curve). Spectral width is significantly larger, more than $200 \mathrm{~nm}$.

Images from transmission electron microscope (TEM) of sample before and after irradiation are shown in Fig. 2.9.

Fig. 2.9(a) shows a region of the original sample that contains spherical particles of crystalline silver with a radius of $15 \pm 1 \mathrm{~nm}$. These particles do not interact with each other since average distance between them around ten particle diameters.

After laser irradiation shape of the particles acquired elongated shape (see Fig. 2.9(b)). The size of the long axis remained unchanged, i.e. of about $30 \mathrm{~nm}$. Long axes of particles are approximately parallel to each other and perpendicular to the linear polarization of the laser.

Fig. 2.10 shows pictures of additional particles from this sample. These pictures show that not all particles have an elongated shape after laser irradiation, but if they do show an aspherical form, the axes of the longest extension are all nearly parallel to each other and perpendicular to the laser polarization.

For comparison spectra of circularly polarized light and particle image are shown in Fig. 2.11. Shape of the particle is about the same, spectral curve is shifted and broadened, peak at $460 \mathrm{~nm}$, bandwidth $\approx 180 \mathrm{~nm}$ maximum is also shifted. 


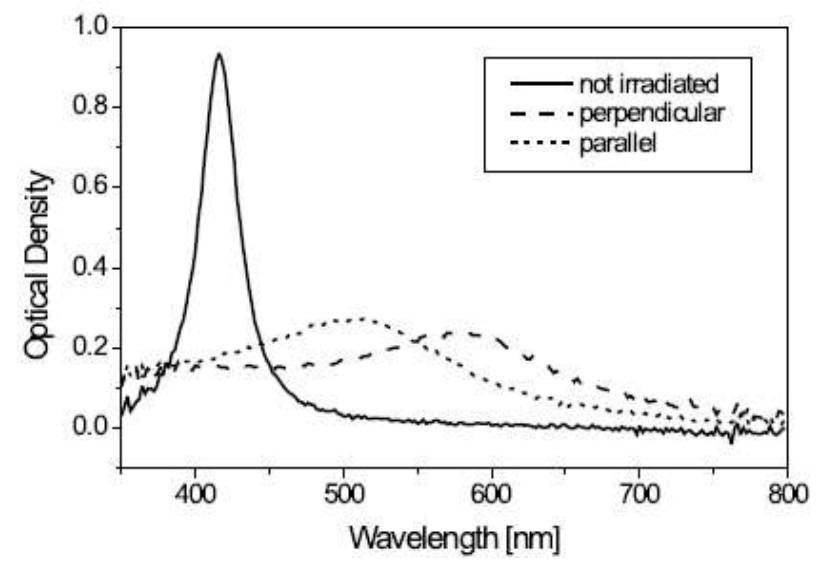

Figure 2.8: Typical optical spectra of glass containing silver nanoparticle before (solid line) and after irradiation with a single, linearly polarized femtosecond laser pulse at $400 \mathrm{~nm}$ (dashed lines), where the measuring light was linearly polarized, once parallel with respect to the laser polarization, once perpendicular to it, [11].
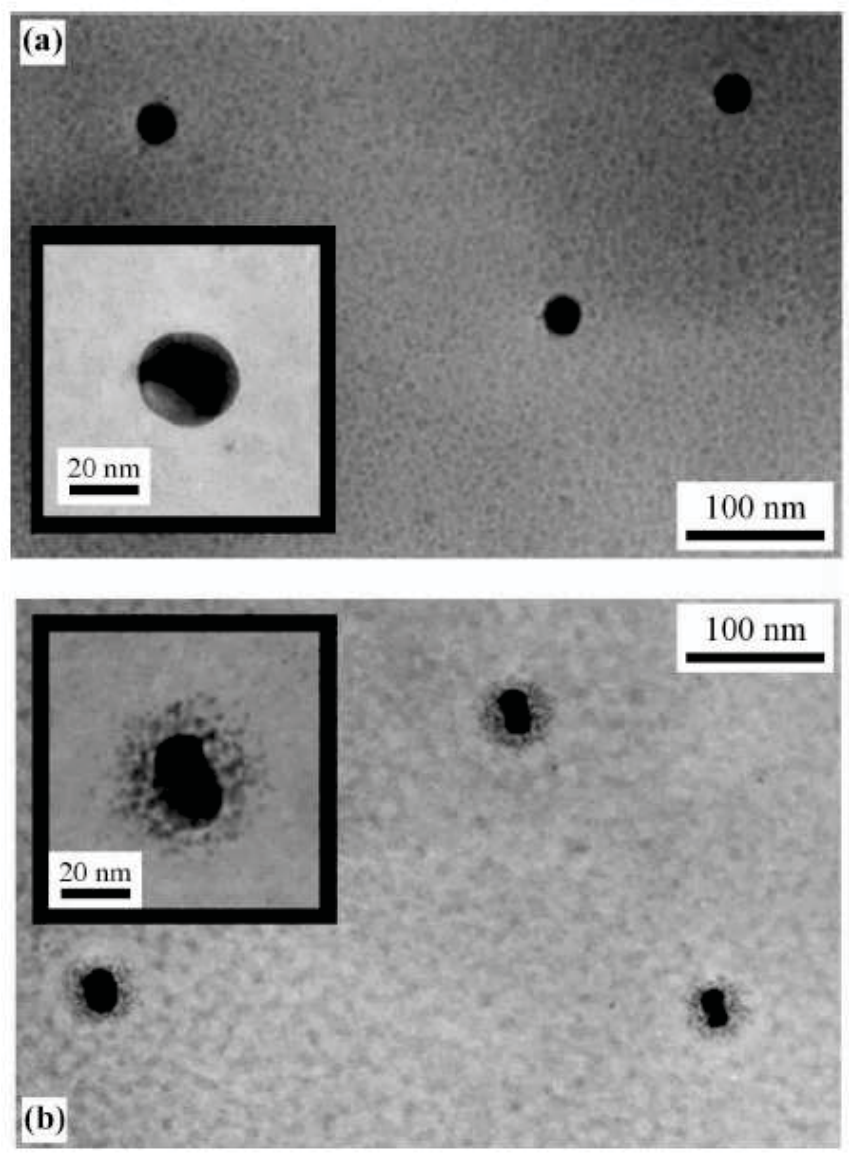

Figure 2.9: TEM micrographs of silver particles in glass before (a) and after (b) irradiation with a single, linearly polarized femtosecond laser pulse at $400 \mathrm{~nm}$, [11]. 

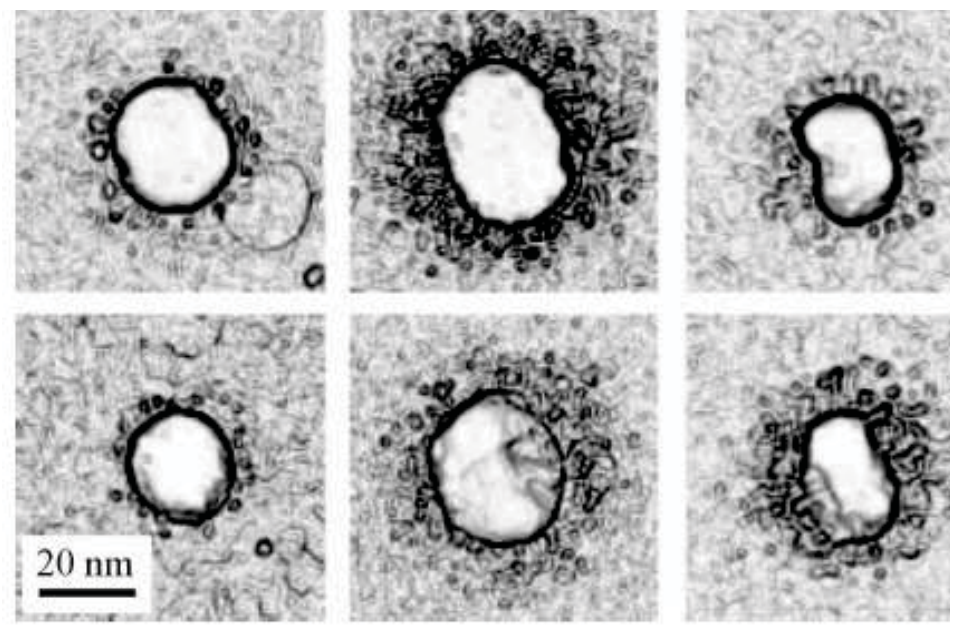

Figure 2.10: TEM pictures of silver particles in glass after irradiation with a single, linearly polarized femtosecond laser pulse at $400 \mathrm{~nm}$, numerically differentiated to enhance contrast. The bottom right picture corresponds to the inset of Fig. 2.9(b), [11].

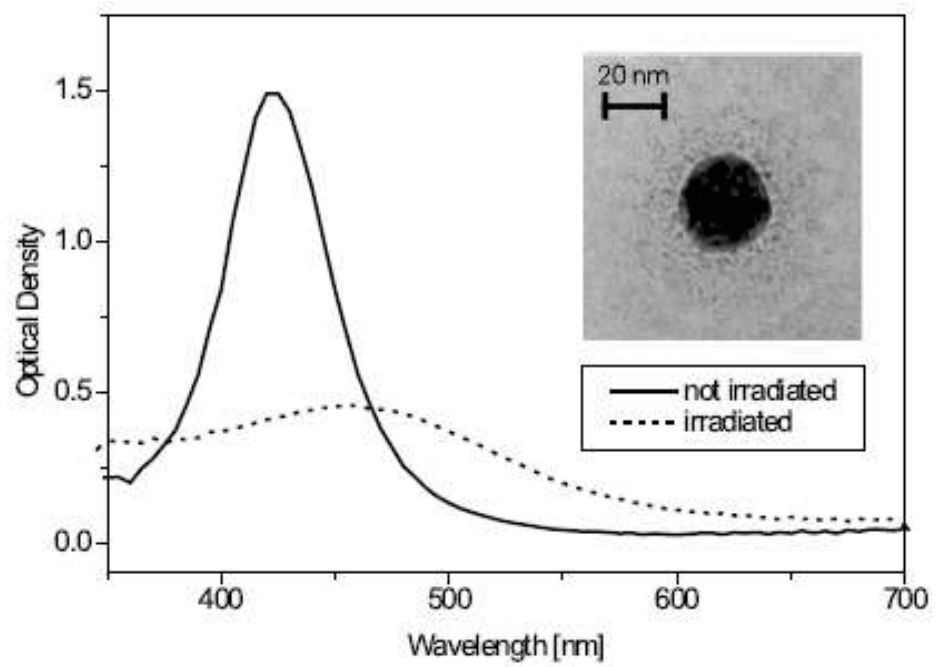

Figure 2.11: Optical spectra of silver particles in glass before and after irradiation with a single, circularly polarized femtosecond laser pulse at $400 \mathrm{~nm}$. Inset: TEM picture after irradiation, [11].

From the TEM images it follows that laser irradiation can modify shape of the silver particle from spherical into elongated and also it forms the halo around particle. Shape modification can occur only after irradiation by linearly polarized light, with longer axis perpendicular to laser polarization. Both linearly and circularly polarized light cause the halo. The mechanism for the formation of the halo and the particle deformation have not been found so far. 
Later work of the same group, [12] shows that three-dimensional (3D) anisotropic modifications are also possible in a glass where the volume fill factor significantly decreases from the surface. The idea is based on the fact that Ag nanoparticles with different fill factor exhibit different spectral width and position of their surface plasmon band. Therefore, they can selectively be excited by different laser wavelengths.

They used for the irradiation linearly polarized pulses at three different wavelengths (400, 500 and $550 \mathrm{~nm}$ ), with an energy of $20 \mu \mathrm{J}$ and a temporal width of $150 \mathrm{fs}$ in each case derived from a mode-locked Ti:sapphire laser operating at $\lambda=800 \mathrm{~nm}$ with pulse repetition rate $1 \mathrm{kHz}$. This time they reported elongation of originally spherical silver particles oriented parallel to the laser polarization.

Irradiation of the Ag nanoparticles at wavelengths sufficiently far away from the SP resonance did not evoke any measurable extinction changes. The preferential orientation of the ellipsoidal Ag particles is due to the high electric field of the laser pulse, which leads to a photoemission of electrons along the direction of the electric field, and further on to ejection of $\mathrm{Ag}$ ions into the glass matrix.

The following requirements for the laser pulses should be satisfied for shape modifications to occur: (i) sufficiently high intensity to achieve field-driven electron emission, (ii) energy input mainly into the Ag particles (e.g., via SP resonance), and (iii) limited input of total energy per pulse to avoid relaxation of the deformed particles to spherical shapes by macroscopic heating above the glass transition temperature.

These requirements can only be met by pulses shorter than some tens of picoseconds, while nanosecond or longer pulses act mainly as a localized heat source leading to relaxation of the particles to spherical shape.

The shape modification cases continuous shift of plasmon resonance peak. At some point it will go out of the laser wavelength and stops the modification (elongation).

\subsection{Field assisted dissolution of silver clusters embedded in glass.}

A pioneering work on field assisted metal cluster dissolution described in [13], [14]. High DC voltage and elevated temperature were applied to the glass doped by metal particle. They observed bleaching of the glass under anodic surface.

Initially soda lime glass underwent $\mathrm{Ag}^{+}-\mathrm{Na}^{+}$ion-exchange process. Then annealing in $\mathrm{H}_{2}$ atmosphere resulted in formation of silver nanoparticles of spherical shape with average diameter $30-40 \mathrm{~nm}$ under the surface. The volume filling factor of silver cluster was estimated at 0.8 just near the surface and 0.001 at its full depth, $6 \mu \mathrm{m}$. See SEM image of Fig. 2.12(a) below.

Total thickness of glass is $1 \mathrm{~mm}$. Six different samples were tested, parameters of the samples and final results are characterized in Table 1. Two samples, 1 and 3 were heated and deformed before the experiment, this caused in reduction of total thickness from $1 \mathrm{~mm}$ 


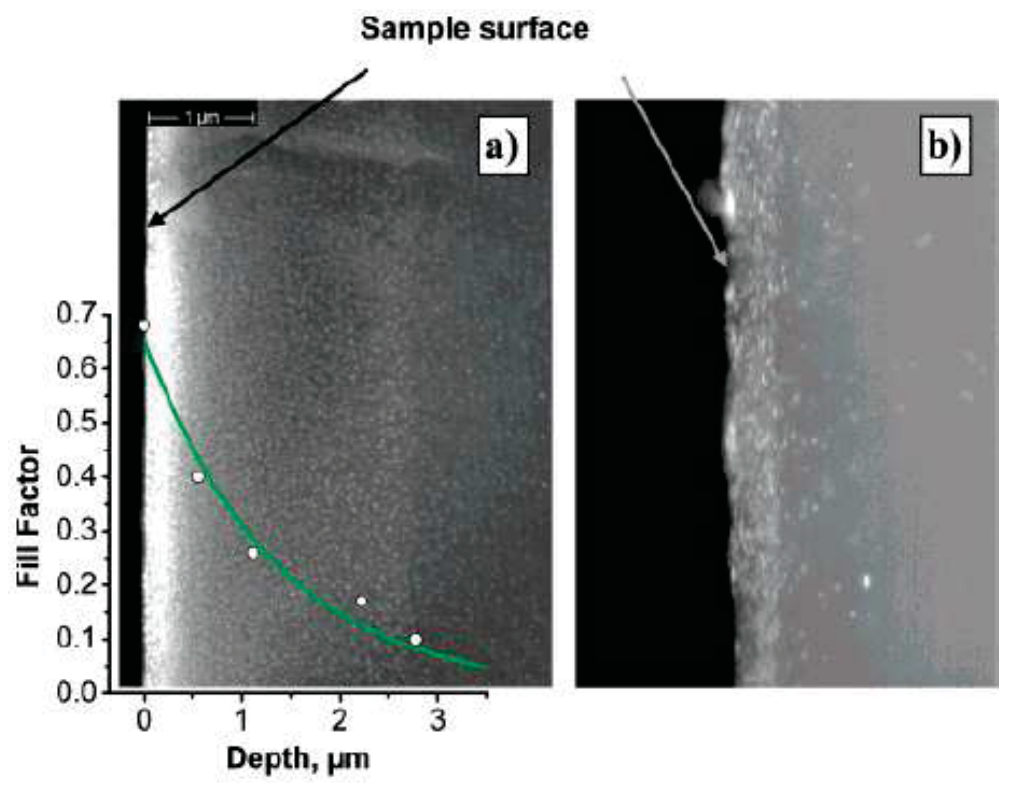

Figure 2.12: SEM pictures of cross sections of glass samples containing (a) spherical and (b) elongated silver nanoparticles (the Ag particles are reproduced as white spots). The fill factor gradient of the spherical nanoparticles is given by the inset, where the $\mathrm{x}$-axis was adjusted to the length scale of the picture. The ellipsoidal nanoparticles in part b, obtained by mechanical deformation of the sample, are located in a very thin surface layer of , with only very few large particles remaining deeper below the surface, [13].

to $0.2 \mathrm{~mm}$ and consequently of silver layer from $6 \mu \mathrm{m}$ to $1 \mu \mathrm{m}$. Also, originally spherical silver nanoparticles became elongated. SEM image of this sample is shown in Fig. 2.12(b). Sample 9 was etched out from the surface so that silver layer thickness became $1 \mu \mathrm{m}$.

Thermal poling was carried out at $280^{\circ} \mathrm{C}$ and $1 \mathrm{kV}$ in an air atmosphere inside an oven. Applied voltage was increased in steps of $0.2 \mathrm{kV}$ such that peak current was always less than $250 \mu \mathrm{A}$ with a total time of around $60 \mathrm{~min}$. During each step the current is increases and then decays, see Fig. 2.14. Top plot in Fig. 2.14 shows measured current of the sample with no silver cluster in it (sample 10).

In samples, doped with silver nanoparticles, the nearsurface layer contains isolated clusters of metallic silver. Sodium ions in this layer are depleted. The electrode polarity was chosen in such a way that the silver-doped layer faced the anode. During the first two steps of the poling the ionic conductivity in that layer is very low and current is smaller than in sample 10, see middle and bottom part of Fig. 2.14. In the next few steps peak current increases substantially.

After poling of a sample doped with ellipsoidal particles (No. 1), the glass region under the positive electrode had become completely transparent with sharp edges corresponding perfectly to the electrode size (Fig. 2.15). The original dark green color of the glass was due to the presence of silver particles. So the bleaching indicates the dissolution of these particles. 


\begin{tabular}{cccccc}
\hline \hline $\begin{array}{c}\text { Sample } \\
\text { No. }\end{array}$ & Type of material & $\begin{array}{c}L \\
(\mathrm{~mm})\end{array}$ & $\begin{array}{c}d \\
(\mu \mathrm{m})\end{array}$ & Color & $\begin{array}{c}\text { Poling-assisted } \\
\text { bleaching }\end{array}$ \\
\hline 10 & Substrate & 1.0 & - & Transparent & $\ldots$ \\
1 & Nanocomposite $E$ & 0.2 & $\sim 1.0$ & Dark green & Total \\
3 & Nanocomposite $E$ & 0.2 & $\sim 1.0$ & Dark grey & Absent \\
4 & Nanocomposite $S$ & 1.0 & $\sim 6.0$ & Dark orange & Partial \\
4 bis & Nanocomposite $S$ & 0.2 & $\sim 6.0$ & Dark orange & Partial \\
9 & Nanocomposite $S$ & 1.0 & $<1.0$ & Faint yellow & Absent \\
8 & Nanocomposite $S$ & 1.0 & $\sim 6.0$ & Orange & Absent \\
\hline \hline
\end{tabular}

Figure 2.13: TABLE 1. Samples under test: $\mathrm{Ag}^{+}-\mathrm{Na}^{+}$ion exchanged soda-lime glass (substrate), soda-lime glass containing Ag nanoparticles of ellipsoidal shape (nanocomposite $E$ ), or spherical shape (nanocomposite $S$ ). L: Sample thickness, and $d$ : Thickness of the near-surface layer in which Ag nanoparticles are embedded. Color of the pristine glass and amount of poling-assisted bleaching of the glass are indicated. All samples (except No. 3 ) were poled with the nanoparticle layer facing the anode (cathode). All samples (except No. 8) were poled at $1 \mathrm{kV}$ and $280^{\circ} \mathrm{C}\left(4 \mathrm{kV}\right.$ and $\left.25^{\circ} \mathrm{C}\right)$, [14].
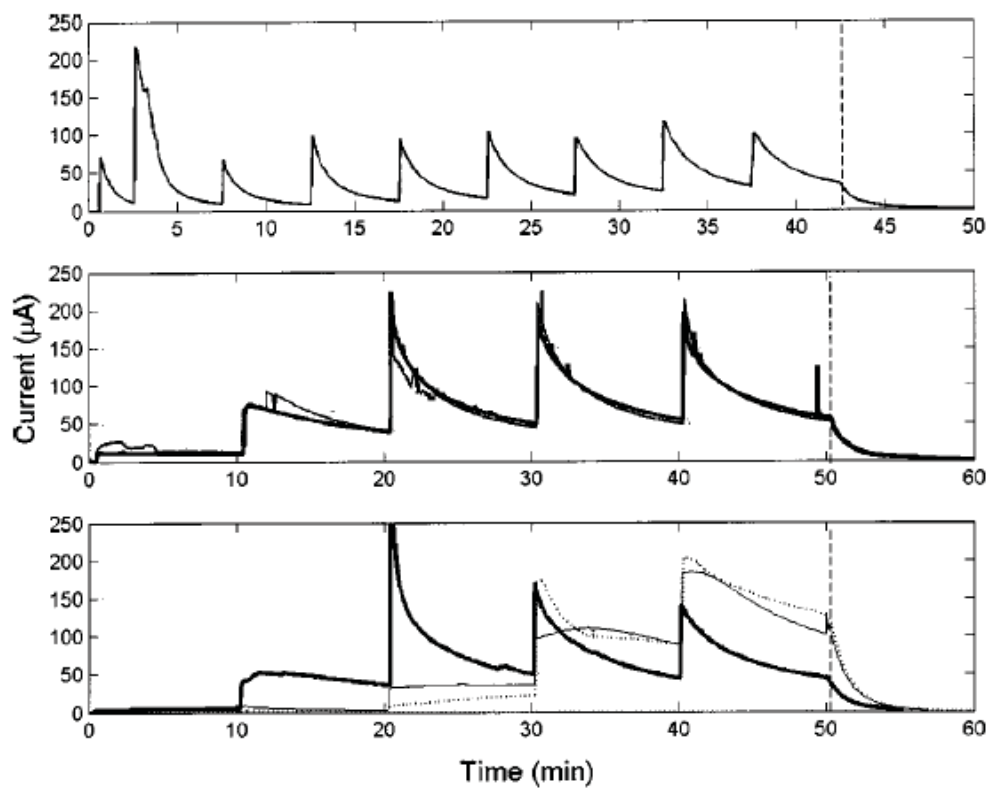

Figure 2.14: Evolution of the current flowing through the sample as a function of time during thermal poling at $280^{\circ} \mathrm{C}$. Top chart: Ion-exchanged sample No. 10; middle chart: Samples containing ellipsoidal Ag nanoparticles (No. 1: Thick line, No. 3: Thin line); bottom chart: samples containing spherical Ag nanoparticles (No. 4: thin line, No. 4bis: thick line, No. 9: dotted line). The voltage was applied step by step up to a final value of $1.0 \mathrm{kV}$ (top chart No. 1: First step of $0.2 \mathrm{kV}$ during $2 \mathrm{~min}$, then steps of $0.1 \mathrm{kV}, 5$ min each; middle and bottom charts: steps of $0.2 \mathrm{kV}, 10 \mathrm{~min}$ each). Vertical dashed lines indicate the beginning of cooling of the sample with the voltage still applied, [14]. 


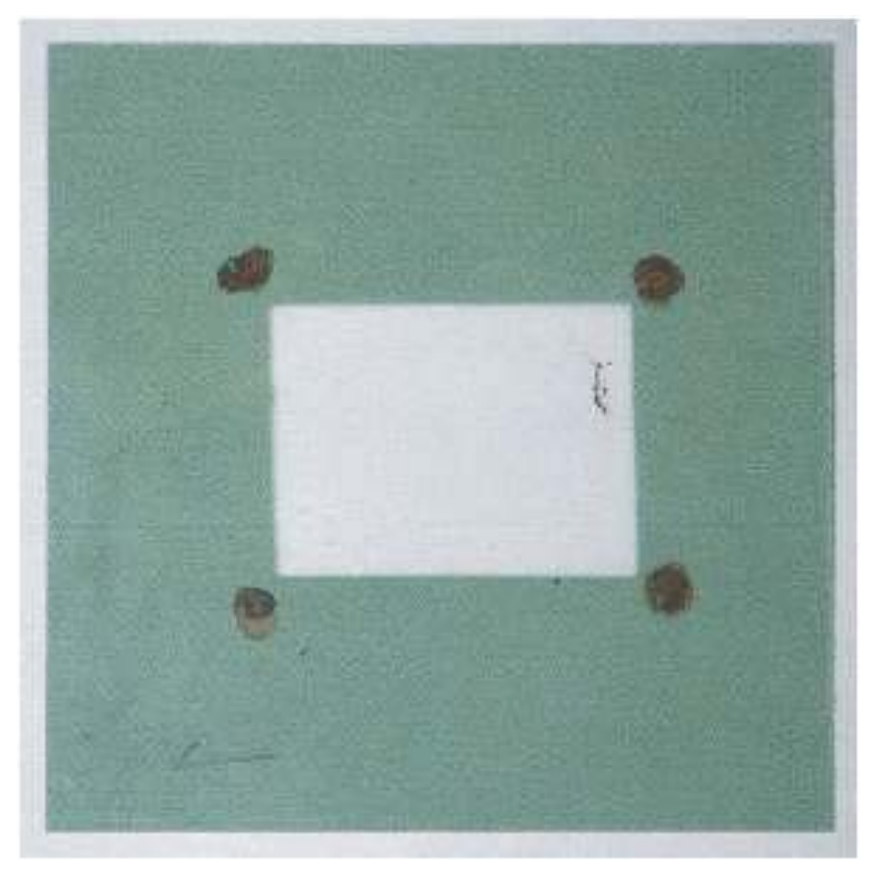

Figure 2.15: (Color) Photograph image of samples No. 1 after poling. The glass became transparent under the anode after poling (i.e., PAB occurred). The sharp edges of the bleached region correspond perfectly to the electrode size $(6 \mathrm{x} 9 \mathrm{~mm})$, [14].

Scanning electron microscope (SEM) images of the cross section of the sample 1 showed that there was no more silver nonoparticles, compare Fig. 2.16(c) and Fig. 2.16(b). Uniformly oriented silver ellipsoids of the original sample (reproduced in white or light gray in Fig. 2.16(b) are no longer present in the optically transparent area after DC electric field treatment. Silver particles have been destroyed during the procedure without any (micro)damage of the glass matrix or surface. Optical spectra of a sample 1 is presented in Fig. 2.16(a). Surface plasmon resonance of dashed curves (before treatment) indicating presence of silver atoms $A g^{0}$ disappeared on solid curve (after treatment).

An additional thermal annealing at $500^{\circ} \mathrm{C}$ after the treatment however can convert some silver ions into atoms, this is evident from small surface plasmon resonance on a dotted dashed curve. Authors explain partially assisted bleaching, PAB effect as a sequence of events: ionization of silver atoms in the nanoclusters, removal them from the clusters and the drift of the silver ions deeper under the surface. Those processes are due to high electric field which is created underneath the anodic surface during poling and elevated temperature.

X-ray spectral analysis of cross section of sample 1 is shown in Fig. 2.17 (left-hand side, original sample; right-hand side, modified sample). The actual position of the sample surface is indicated by the Si curves.

Concentration of $\mathrm{Ag}$ and $\mathrm{Na}$ has decreased close to the surface and increased in the depth of a few microns (the Ag peak at $\approx 2.5 \mu \mathrm{m}$ in Fig. 2.17(a) is caused by a single, 

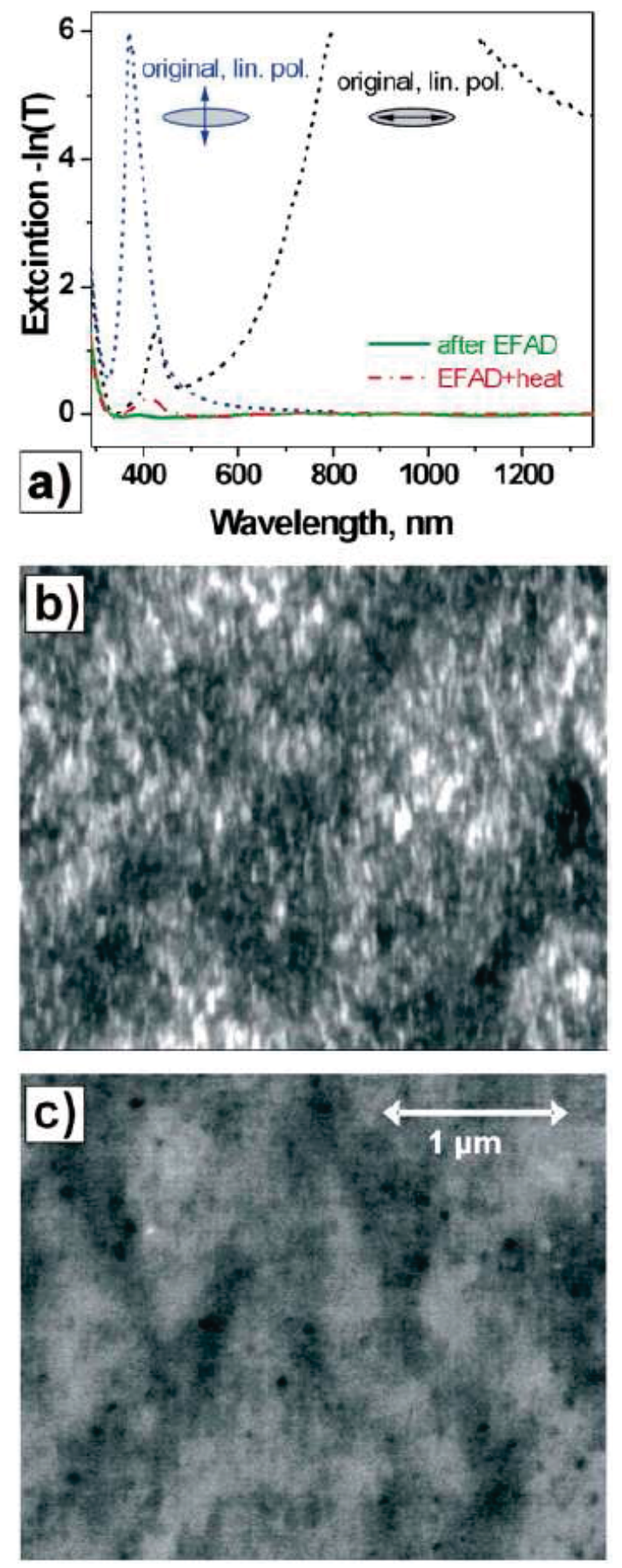

Figure 2.16: (a) Extinction spectra of a sample containing elongated Ag nanoparticles, before (dashed curves, observed with light polarized parallel and perpendicular to the long axis of the silver clusters) and after (solid curve, directly after process; dash-dotted curve, after additional heating) electric field-induced modification. SEM pictures of the surface of a sample with ellipsoidal Ag nanoparticles (b) before and (c) after dc electric field treatment, [13]. 
large nanoparticle). The silver distribution after treatment has a maximum at a distance of $\approx 2.5 \mu \mathrm{m}$ from the sample surface. Atoms cannot be distinguished from ions in the X-ray signal. However, only ions $\mathrm{Ag}^{+}$can migrate in the direction of applied electric field. Therefore, the $\mathrm{Ag}$ nanoparticles have been converted to $\mathrm{Ag}^{+}$ions.
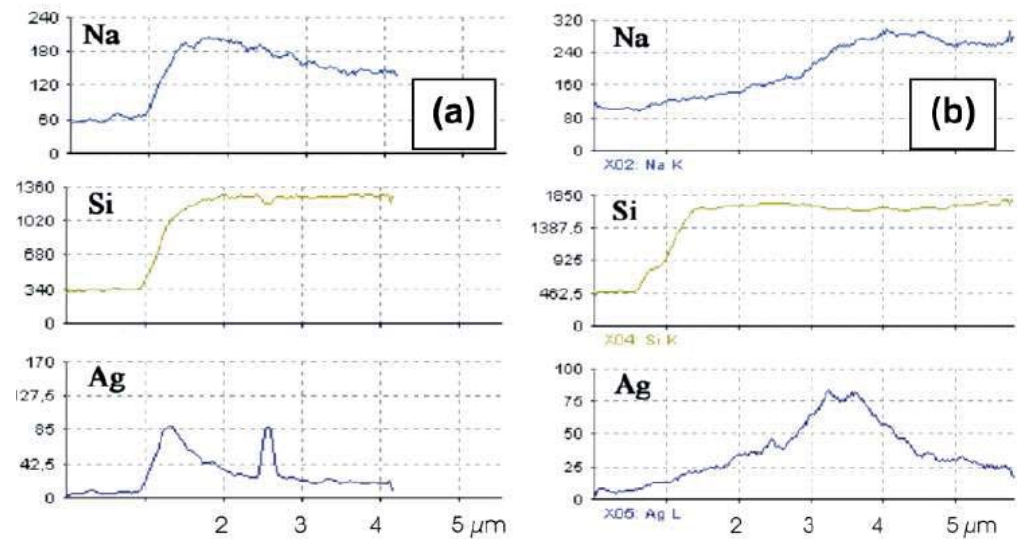

Figure 2.17: Na, Ag, and Si distributions as a function of sample depth by local X-ray element analysis of vertical cross sections of samples (a) before and (b) after the electric field treatment; the horizontal length scales are identical. The Ag peak found in part a at $\approx 2.5 \mu \mathrm{m}$ corresponds to a single large silver particle seen in the SEM picture (not shown), [13].

Different conditions of the poling were tested with other samples. The sample doped with ellipsoidal particles (No. 3) was poled with the silver-doped layer facing the cathode instead of the anode. No change of color was observed. For samples doped with spherical particles (Nos. 4 and 4bis) only partial bleaching was observed but also with the sharp edges. Bleaching effect does not depend on glass thickness: it was equal for sample 4 (1 mm) and for sample $4 \mathrm{bis}(0.2 \mathrm{~mm})$. No change was observed when fill factor of silver particles was very low, $f<0.01$ (sample 9 ). To achieve bleaching effect with such conditions (low f) required higher voltage. Room temperature $\left(25^{\circ} \mathrm{C}\right)$ and higher voltage $(4 \mathrm{kV}$ instead of $1 \mathrm{kV}$ ) resulted in no change as well, (sample 8).

The field assisted bleaching of the glass can be used to build submicron optical microstructures. Right-hand side of Fig. 2.18 shows microscope image of the dark horizontal lines produced with this technique. The dark lines (less than $1 \mu m$ thickness), were produced by making scratches in the steel anode with a diamond marker before the treatment. After the treatment, the whole area under the anode was transparent, with the exception of the scratches.

In a more recent work [15] a group from China reported dissolution of $\mathrm{Au}$ rods in gold-doped silicate glass during the dc electric field thermal poling at the optimized temperature of $500^{\circ} \mathrm{C}$. Scanning electron microscopy characterizations show that some $\mathrm{Au}$ rods with a high aspect ratio are dissolved to spherelike particles. The mechanism for dissolution of Au particles is attributed to electron tunneling conduction and Au cationic 


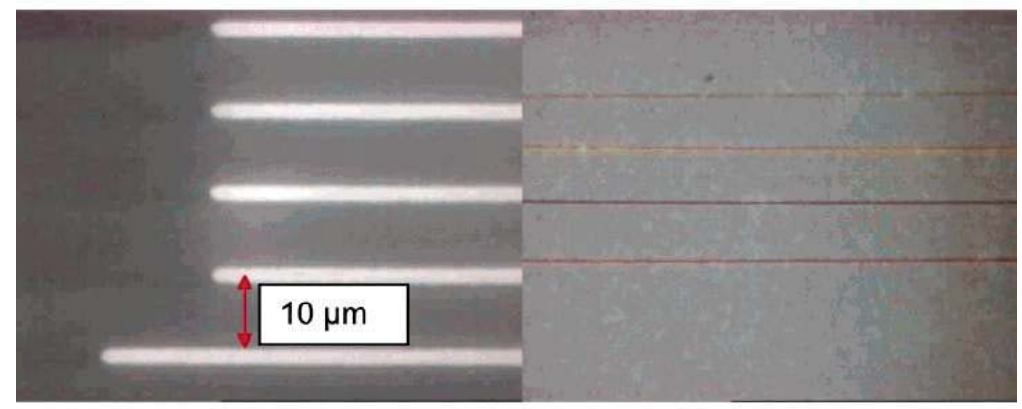

Figure 2.18: Microscopic photography of sub-micrometer lines made by electric field assisted dissolution, EFAD using an electrode scratched with a diamond marker (right-hand side) and calibrated length scale with same magnification (left-hand side), [13].

conduction, based on electrical measurements during the electric field assisted dissolution process. Experimental setup schematically shown in Fig. 2.19.

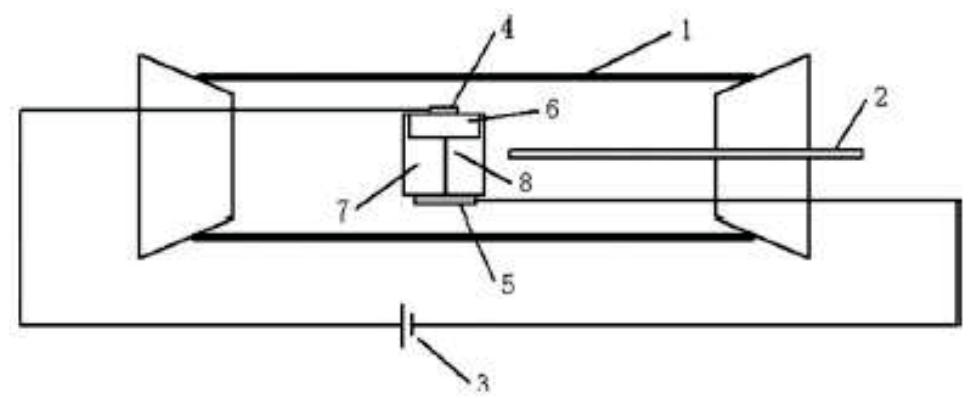

Figure 2.19: Experimental setup for electric field thermal poling. (1) Tube furnace, (2) thermal couple, (3)dc power, (4) anode, (5) steel plate, (6) sample, (7) boron nitride (BN) carrier with a mechanically patterned hole where the glass sample is fixed in, and (8) steel rod holder as cathode (fixed on 5), [15].

An applied voltage vs. time during dissolution process and appropriate current evolution shown in Fig. 2.20.

Absorption spectra of as-prepared sample before and after annealing are shown in Fig. 2.21.

The dissolution mechanism, formulated in subsequent work [16], assumed three electrical conduction processes illustrated in Fig. 2.22: ejected electrons from Au particles tunneling (process I) to anode will initiate the following Au cationic conduction (process II) and electron conduction from the cathode into glass matrix (process III). At the voltage step of $700 \mathrm{~V}$, Au ions charge Q2 is rather small, indicating little dissolution of $\mathrm{Au}$ particles, which indicates the dissolution is mainly finished at the voltage step of 800 and $900 \mathrm{~V}$ because Q2 is much larger; while at increased step of $1000 \mathrm{~V}$ Q2 is lowered, which indicates almost total dissolution of most Au particles.

According to one of the theory, [17], field assisted dissolution process leads to forming nanovoids of the same size as metal nanoparticles at the beginning, i.e. spharical 

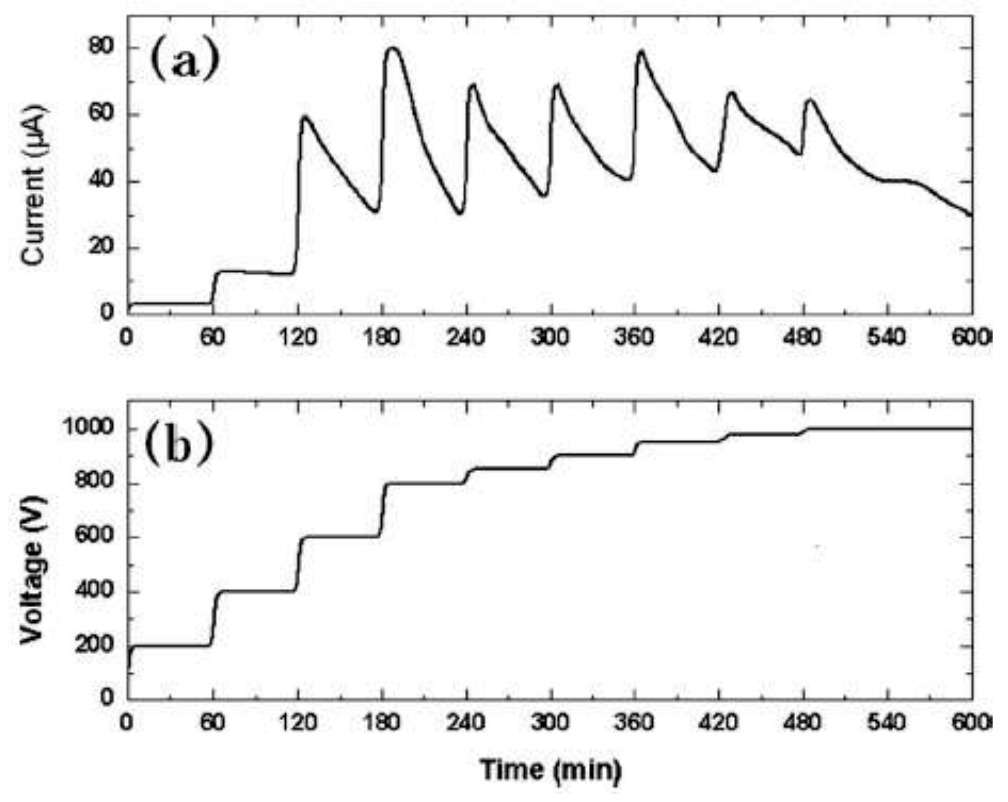

Figure 2.20: Evolution of applied current and voltage as a function of time during electric field thermal poling, [15].

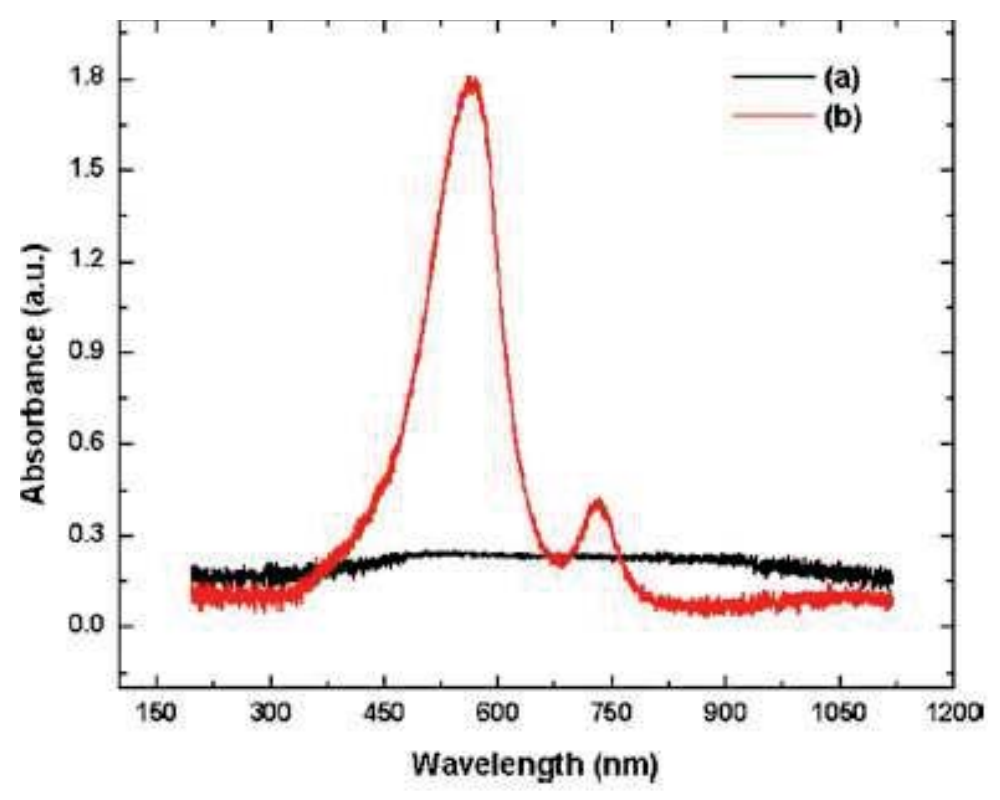

Figure 2.21: (Color online) Absorption spectra of Au-doped silicate glass before (a) and after (b) annealing at $650^{\circ} \mathrm{C}$ without an electric field, [15].

nanoparticle after dissolution left an empty spherical space in the glass.

Several authors reported nanovoids formation, i.e. a spot, previuosly ocupied by a spherical metal nanoparticle converted, as a result of cluster dissolution, into a spherical empty space, so called nanovoid, [16,17]. Evolution of spherical Au nanoclusters during 


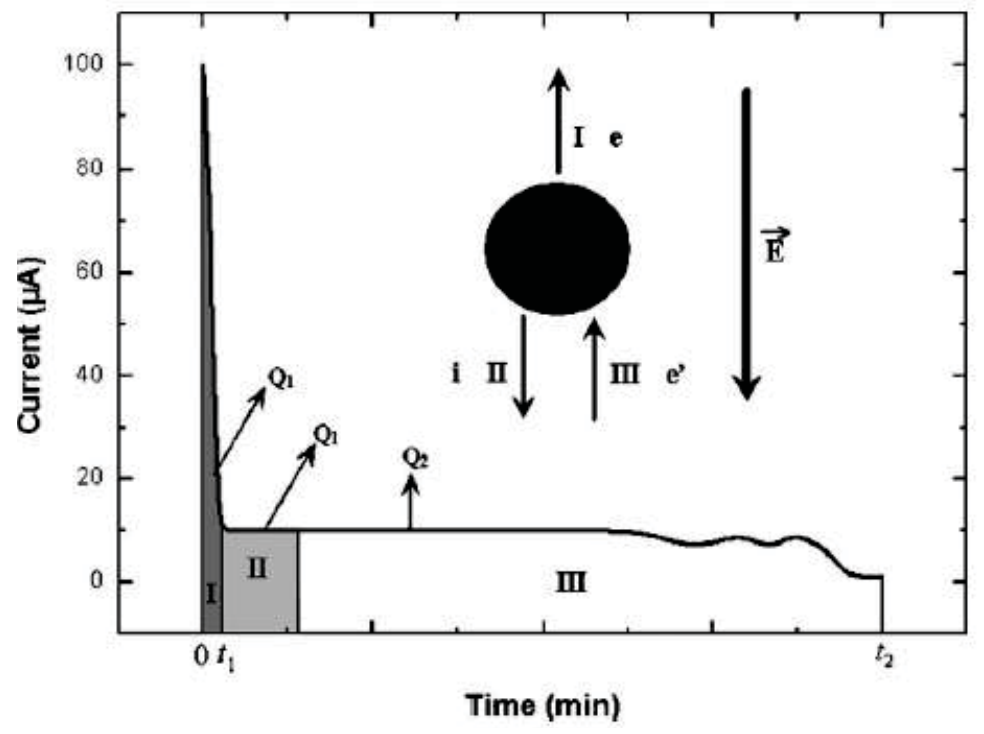

Figure 2.22: A selected current evolution cycle inset with the schematic image of three current conduction processes (I, II, and III), where $i$ denotes Au ions, $e$ denotes ejected electrons from $\mathrm{Au}$ particles, $e^{\prime}$ denotes electrons from the cathode, and $\vec{E}$ is the electric field, [16].

field assisted dissolution process that eventually leads to complete nanovoids is shown in Fig. 2.22. 


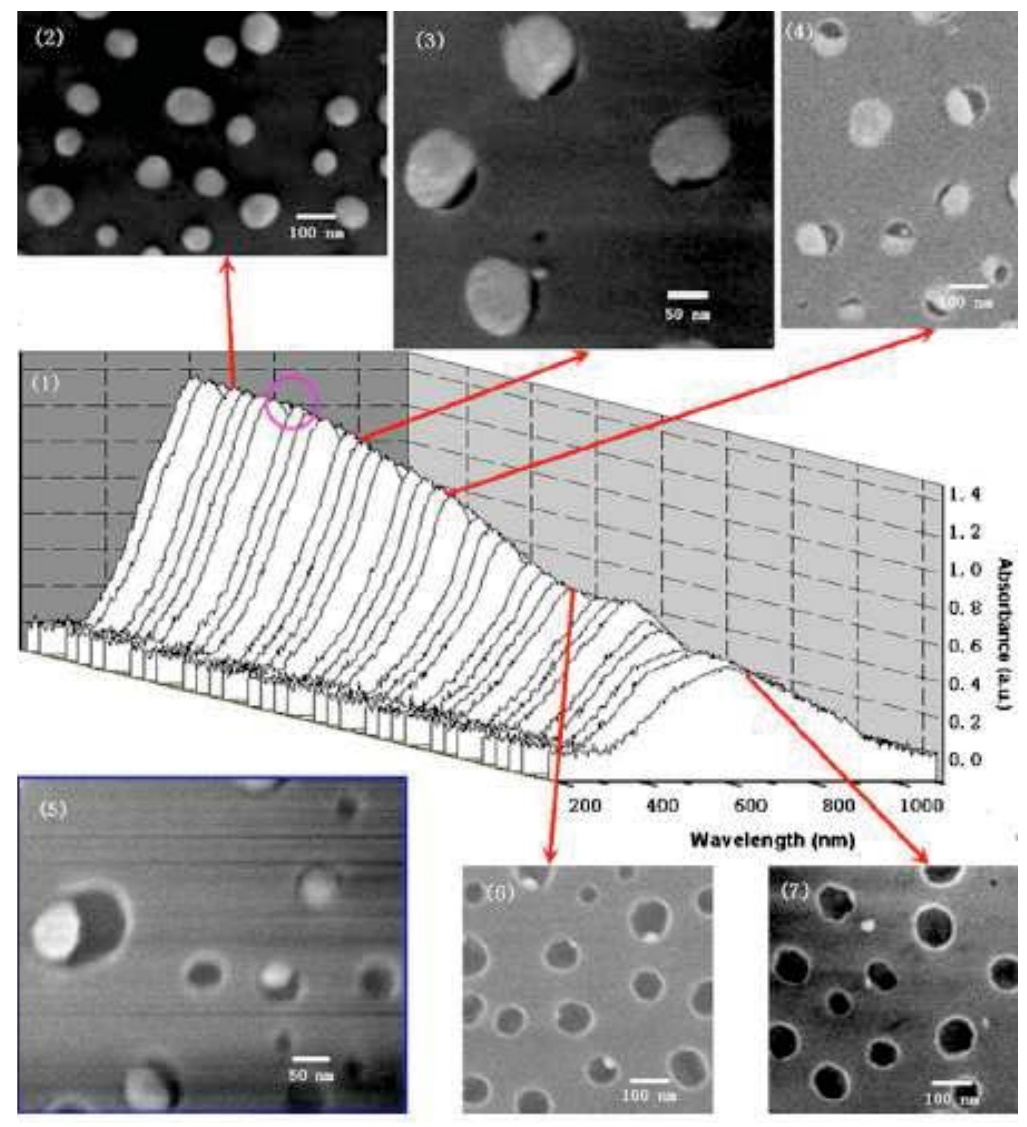

Figure 2.23: The mapped absorption spectra (1) and the SEM images (2)-(7) of different areas on the surface of Au-sputtered glass after EFAD process, [16]. 


\section{Chapter 3}

\section{Field-assisted dissolution of silver nanopar- ticles in phosphate glass}

Phosphate glass samples doped with silver ions through a $\mathrm{Na}^{+}-\mathrm{Ag}^{+}$ion-exchange process were treated in a hydrogen atmosphere at temperatures near $430^{\circ} \mathrm{C}$ for durations ranging from 4 to 5 hours. Such treatment causes metallic silver precipitation at the surface as well as nanoclustering of silver atoms under the surface under conditions very similar to those used for silicate glasses. The presence of silver clusters resulted in a characteristic coloring of the glass and this was verified by the observation of a plasmon resonance peak near 410-420 nm in the absorption spectra of the treated glass. Applying a DC voltage between 1.4-2 $\mathrm{kV}$ at temperatures between $120^{\circ} \mathrm{C}$ and $130^{\circ} \mathrm{C}$ led to dissolution of the clusters in the area under the positive electrode, thereby bleaching the glass color. The use of a patterned doped-silicon electrode further led to the formation of a $300 \mathrm{~nm}$ thick surface relief on the glass surface and of a volume optical grating extending at least $4 \mu \mathrm{m}$ under the surface.

\subsection{Introduction}

Phosphate glass meets several material requirements for integrated optics design. Due to the high solubility of its glass matrix it can accommodate high concentrations of rare earth ions $\left(E r^{+}, Y b^{+}\right)$homogeneously, i.e. without forming aggregates and thus keeping scattering at a low level $[18,19]$. The typical rare earth ion concentrations that can be achieved without clustering are 10 to 20 times higher than in silicate glasses. This feature is important because Er/Yb co-doped glasses can transfer $980 \mathrm{~nm}$ pump photon energy into the $1550 \mathrm{~nm}$ emission band with a long upper state lifetime, $10 \mathrm{~ms}$ compared to nanoseconds in semiconductors. This makes Er/Yb doped glass fiber and waveguide lasers more favorable than semiconductor lasers in telecommunication application [20,21], and the emission linewidths of such lasers can be as narrow as $10 \mathrm{kHz}$ [22]. The higher concentrations of active material (the rare earth ions) that can be achieved in phosphate glasses provide more gain per unit length and thus allow compact device sizes and high power output. In 
addition, phosphate glass has low optical loss in the near infrared and can also be modified by ion-exchange to form waveguides [19,23].

In order to fabricate a waveguide laser in a glass substrate, a pair of low loss narrowband reflectors must be created to form a laser cavity. The best way to do this is to use volume refractive index gratings, a well-developed technique in certain types of silica glasses [2428]. However, in phosphate glass the standard method of making grating with UV exposure through a diffractive phase mask does not work well because this glass does not contain photosensitive molecules $[29,30]$. While gratings have been successfully produced by a related thermally-assisted UV irradiation process [31,32], the index modulation amplitudes of the gratings remained small.

Here, we present work that investigates the possibility of achieving a relatively large periodic modulation in both the real and imaginary parts of the phosphate glass bulk permittivity by means of dissolved and partially clustered silver nanoparticles inside the glass matrix. We used ion-exchange to introduce silver into the glass, precipitated some of it into clusters and then used field assisted bleaching with a patterned electrode to locally bleach the nanoparticles and hence the complex permittivity of the material.

Field assisted bleaching of a soda-lime glass doped with silver clusters has been known since 2004 [13,14], and later in other types of silicate-based glasses such as sol-gel, borosilicate N-BK7, K8, and VO73 [33-35]. Furthermore, other embedded nanoparticles such as gold and copper were successfully dissolved with high electric fields [16,33-38] in those glasses. We report on similar effects and the conditions required for observing them in a silica-free phosphate glass especially designed for integrated optics applications, especially waveguide and waveguide laser formation.

\subsection{Formation of silver clusters in phosphate glass}

\subsubsection{Glass processing that leads to silver nanoclusters: ion-exchange, annealing in air followed by annealing in $\mathrm{H}_{2}$}

The glass substrate used is an active (Er/Yb doped) IOG-1 phosphate glass from Schott Glass Technologies Inc. with a mole $\%$ composition of: $60 \% \mathrm{P}_{2} \mathrm{O}_{5}, 13 \% \mathrm{Al}_{2} \mathrm{O}_{3}, 24 \% \mathrm{Na}_{2} \mathrm{O}$, $1.1 \% \mathrm{La}_{2} \mathrm{O}_{3}, 1.5 \% \mathrm{Yb}_{2} \mathrm{O}_{3}$ and $0.4 \% \mathrm{Er}_{2} \mathrm{O}_{3}$ [39]. Silver ions were introduced into the glass by the dry silver-film ion-exchange technique, also known as field-assisted ion diffusion and electromigration (see [40-42] for descriptions of these techniques). The goal in preparing the sample is to optimize the size distribution of silver clusters embedded in the glass matrix, i.e. the mean value of the cluster diameter and its standard deviation that leads to the strongest plasmon resonance.

The sample was first prepared by depositing silver films on both sides of a $1.5 \mathrm{~mm}$ thick IOG-1 phosphate glass, $400 \mathrm{~nm}$ of Ag on the side towards the positive electrode (top side) and $100 \mathrm{~nm}$ on the side towards the negative electrode (bottom side). Upon heating with the imposition of a high voltage between the electrodes, silver ions from the top side 
diffuse into the substrate by ion-exchange, replacing sodium ions that move towards the negative bottom electrode. An ion-exchanged layer results under the top surface, with sodium accumulating on the bottom side. The use of deposited films on both sides ensures good electrical contact between the glass surfaces and the electrodes. Depositions on both sides of the sample were performed individually by E-beam evaporation using a Balzers BA 510 system. The deposited thickness of the silver film is controlled by the duration of the process, as calibrated beforehand on dummy samples.

For the ion-exchange process, the sample was sandwiched between two $10 \mathrm{~cm}$ diameter circular polished aluminum electrodes weighting $1.7 \mathrm{~kg}$. The pressure from the top electrode ensured good electrical contact between the electrodes and the silver films on both sides of the sample. The sample assembly with electrodes was placed in an oven and kept at $95^{\circ} \mathrm{C}$ with an applied DC voltage of $380 \mathrm{~V}$ for 28 hours, such long lasted ion-exchange needed for precipitation silver in the form of clusters. After this period the oven was turned off to let the ion-exchanged glass cool down to room temperature over 4-5 hrs with the DC voltage kept on. Then the DC voltage was switched off, the electrodes removed, any remaining silver film dissolved chemically by standard cleaning solution (developed at RCA laboratories) made up of 6 parts of deionized water (DI), 1 part of $30 \%$ ammonium hydroxide $\left(\mathrm{NH}_{4} \mathrm{OH}\right)$ and 1 part of $30 \%$ hydrogen peroxide $\left(\mathrm{H}_{2} \mathrm{O}_{2}\right)$, and the sample was annealed in air at $300^{\circ} \mathrm{C}$ for 7 hours to allow silver to diffuse and homogenize further down under the glass surface. Finally the sample was annealed a second time but in a $H_{2}$ atmosphere in the furnace with a constant hydrogen flow to ensure $100 \%$ hydrogen concentration at $400^{\circ} \mathrm{C}$ for 30 minutes followed by 4 hours at $430^{\circ} \mathrm{C}$. These particular conditions were chosen (after several trials, some of which are described below) to maximize the absorption peak of the processed glass at the wavelengths of the plasmon resonance. During annealing in $\mathrm{H}_{2}$, it was observed that silver atoms precipitate on the top side of the glass surface (where the positive electrode was located), forming a shiny silver layer. It is assumed that the silver ions nearest to the surface capture an electron from the $\mathrm{H}_{2}$ gas and neutralize into a smooth layer of pure $A g^{0}$ [4], thereby leaving room in the glass for other ions to diffuse back towards the top surface. Reduction of silver ions to metallic (neutral) silver atoms may be represented as:

$$
2 \mathrm{Ag}^{+}+\mathrm{H}_{2} \rightarrow 2 \mathrm{Ag}^{0}+2 \mathrm{H}^{+}
$$

It was observed in separate experiments that such precipitation does not occur in silver ion-exchanged silicate glasses (such as microscope slide glass). Silver precipitation on the surface is an undesired effect that lowers the amount of silver available inside the glass for cluster formation. The precipitation of silver out of the glass is thought to be one of the reasons why the ion-exchange process has to be prolonged from 1.5-2 hours (typical for silicate type glasses [43] to 20-40 hours for phosphate glass in order to keep enough silver in the glass to get significant clustering. Finally, the glass surfaces were cleaned in a standard cleaning solution to remove the precipitated silver from the surface and any residue on the other side. The final appearance of the initially transparent samples was yellowish, thus confirming visually the formation of nanoscale silver clusters inside the glass since yellowish discoloration of the glass is due to absorption by localised surface plasmons on silver nanoclusters. 


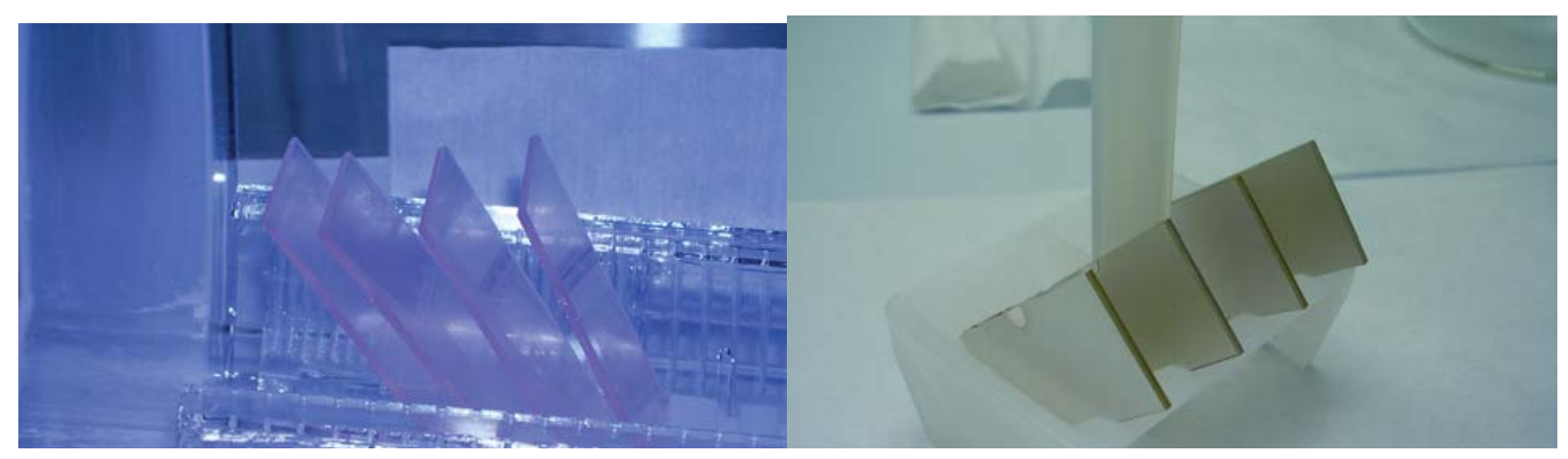

(a)

(b)

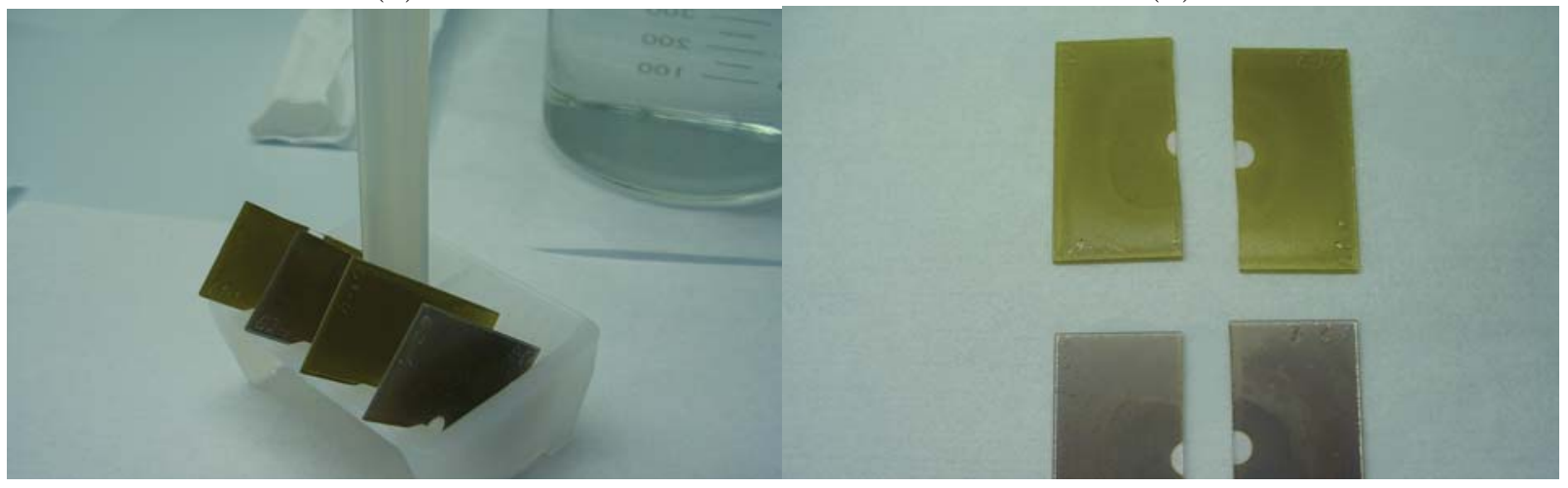

(c)

(d)

Figure 3.1: Images of IOG-1 glass before and after annealing in a furnace with continuous $\mathrm{H}_{2}$ flow. (a): glass containing silver ions after the ion-exchange $\mathrm{Na}^{+}-\mathrm{Ag}^{+}$process, (b): after annealing in $H_{2}$ the samples are coated with silver film on the side where the ion-exchange was implemented, (c) and (d): samples after the surface is cleaned.

\subsubsection{Sample measurements and observations}

\section{Spectroscopy and TEM image of the sample}

Absorbance spectrum of this glass specimen, measured with a Cary 3 UV-Visible spectrophotometer in the range from 200 to $900 \mathrm{~nm}$, is presented in Fig. 3.2(a). The absorbance was not corrected for reflection off the sample faces. As can be seen, the strength of the surface plasmon resonance (SPR) in the range of wavelengths (402 $\mathrm{nm}, 426 \mathrm{~nm}$ ) is so high that its peak can not be measured. The plasmon resonance indicates a presence of silver atom nanoclusters in the glass. Outside of a peak band the absorbance quickly drops out. The resonance width is related to cluster concentration (filling factor) in the glass matrix as well as to distribution of cluster sizes. The broadening associated with concentration rise is due to electromagnetic coupling among neighboring clusters when distance between them is less than the wavelength. Such coupling is responsible for substantial raise of absorption band as shown in [44]. The TEM image of the sample reveals rounded silver clusters of diameter around 20-30 nm, see Fig. 3.2(b).

The shape of absorption spectra is controlled by various parameters of the glass treat- 
ment presented below.

\section{Influence of ion-exchange time and temperature}

The optimum conditions described above were obtained following several tests to be described presently. One set of samples was prepared with ion-exchange durations from 22 hrs up to $64 \mathrm{hrs}$ under the same remaining parameters: $T=95^{\circ} \mathrm{C}$ and $\mathrm{V}=350 \mathrm{~V}$, followed by annealing in air at $T=300^{\circ} \mathrm{C}$ for $\mathrm{t}=6 \mathrm{hrs}$, then reduction in $H_{2}$ at $T=400^{\circ} \mathrm{C}$ for $\mathrm{t}=1.5$ hrs followed by a ramping temperature to $430^{\circ} \mathrm{C}$, which was kept for $3 \mathrm{hrs}$ (Fig. 3.3). The general trend of absorption spectra evolution with increasing ion-exchange time, revealed from this figure, is increasing peak value ( $22 \mathrm{hrs}, 25 \mathrm{hrs})$ and then, when the maximum is reached, peak widening ( $28 \mathrm{hrs}-64 \mathrm{hrs}$ ). Another pair of samples was used to check the effect of the ion-exchange temperature between $85^{\circ} \mathrm{C}$ and $95^{\circ} \mathrm{C}$, with the other parameters of ion-exchange set as: $\mathrm{t}=25 \mathrm{hrs}$ and $\mathrm{V}=410 \mathrm{~V}$, annealing in air: at $T=300^{\circ} \mathrm{C}$ for $\mathrm{t}=6$ hrs and reduction in $H_{2}$ : at $T=400^{\circ} \mathrm{C}$ for $\mathrm{t}=1.5 \mathrm{hrs}$ followed by $T=430^{\circ} \mathrm{C}, \mathrm{t}=3 \mathrm{hrs}$ (Fig. 3.4).

\section{Influence of annealing parameters in $H_{2}$ and in air}

Absorption spectra with different times and temperatures of annealing in $H_{2}$ are shown in Fig. 3.5, with other parameters fixed (ion-exchange: $T=95^{\circ} \mathrm{C}, \mathrm{t}=28 \mathrm{hrs}, \mathrm{V}=350 \mathrm{~V}$, annealing in air: $T=300^{\circ} \mathrm{C}, \mathrm{t}=6 \mathrm{hrs}$ ). There is an optimal temperature of annealing in $H_{2}$ that maximizes resonance peak, below and above of which the peak value drops. Another observation is that pair time-temperature can be used to adjust not only peak height (similar to time, temperature of ion-exchange) but peak position as well.

Absorption spectra for different time, temperature pair of annealing in air shown in Fig. 3.6, other parameters are fixed, ion-exchange $T=105^{\circ} \mathrm{C}, \mathrm{t}=45 \mathrm{hrs}, \mathrm{V}=400 \mathrm{~V}$, annealing in $H_{2}: T=400^{\circ} \mathrm{C}, \mathrm{t}=30 \mathrm{~min}$ followed by $T=430^{\circ} \mathrm{C}, \mathrm{t}=4 \mathrm{hrs}$. Annealing in air parameters can be used to adjust peak height (similar to time, temperature of ionexchange).

\section{Discussion of the clustering results}

All the samples have a single well-defined peak centered near $410 \mathrm{~nm}$, with absorbances often exceeding 6 (the limit of our measurement system), and no other notable spectral features between 250 and $900 \mathrm{~nm}$. The $410 \mathrm{~nm}$ peak is known to correspond to a localized plasmon resonance of nanoscale silver aggregates in glass [4,13], and this was verified by Transmission Electron Microscope (TEM) images of cleaved slivers from the sample shown in Fig. 3.2, that reveals the presence of rounded silver clusters with diameters near 20-30 $\mathrm{nm}$. The plasmon peak increases with ion-exchange time or temperature, likely because such increases lead to larger amounts of silver ions being introduced in the glass. Results further indicate that it is not beneficial to increase temperature of annealing in $\mathrm{H}_{2}$ beyond $400^{\circ} \mathrm{C}$, otherwise the plasmon peak starts to decrease and shift towards longer wavelengths 


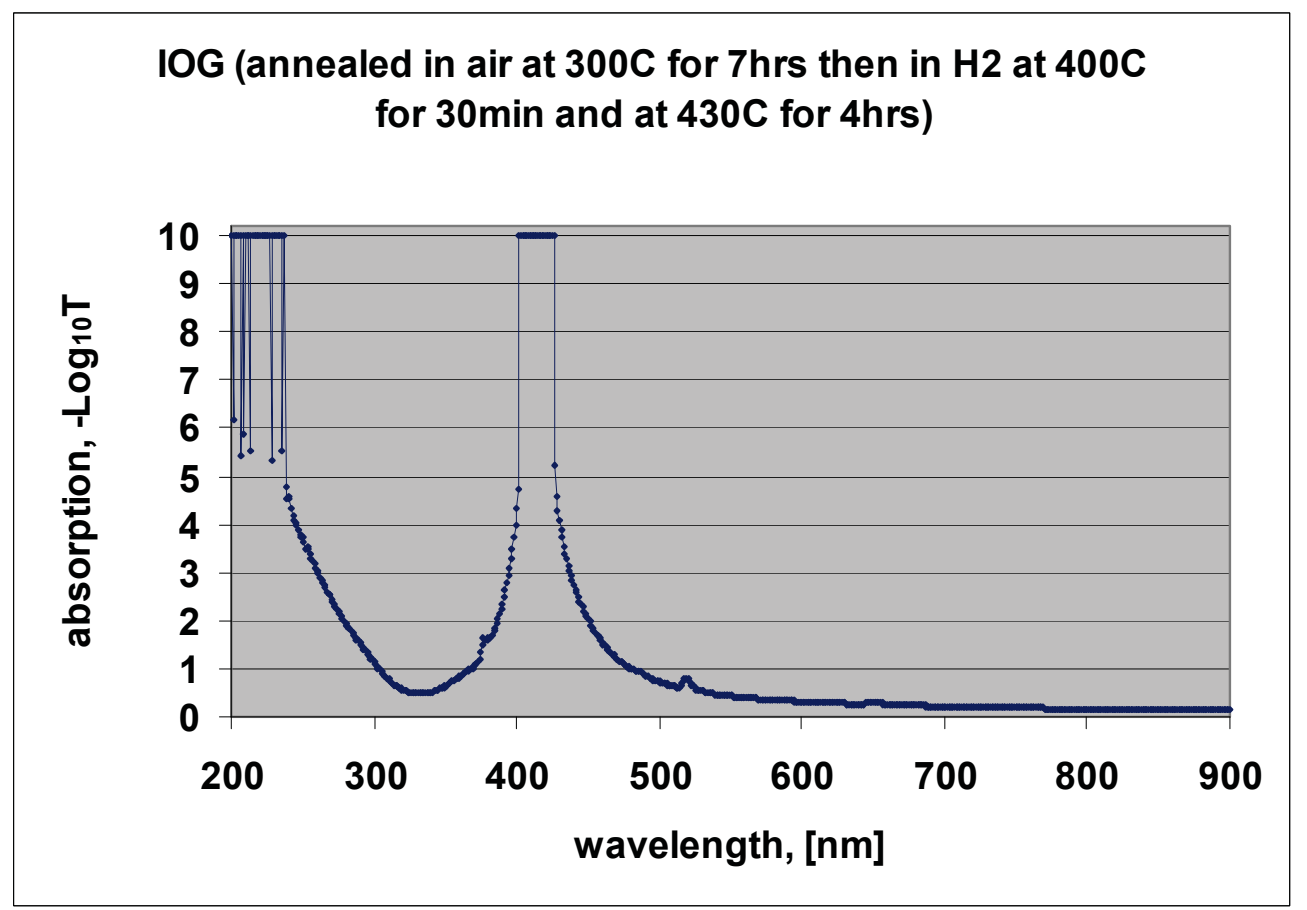

(a)

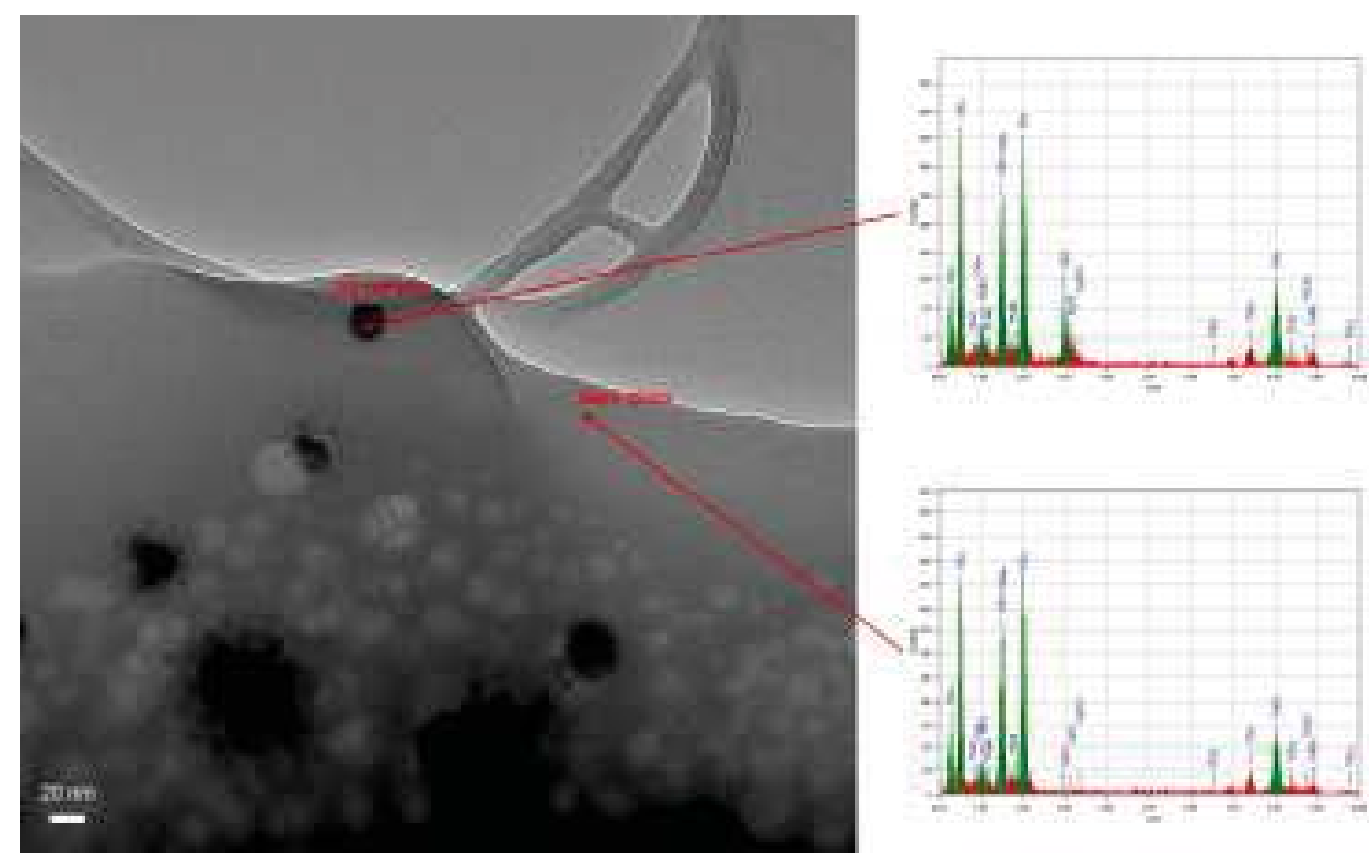

(b)

Figure 3.2: UV-vis absorption spectra (a) and TEM image (b) of the IOG-1 glass sample that underwent ion-exchange $(\mathrm{T}=95 \mathrm{C}, \mathrm{t}=28 \mathrm{hrs}, \mathrm{V}=380 \mathrm{~V})$, annealing in air $(\mathrm{T}=$ $300 \mathrm{C}, \mathrm{t}=7 \mathrm{hrs})$ and reduction in $H_{2}(\mathrm{~T}=400 \mathrm{C}, \mathrm{t}=30 \mathrm{~min}$ followed by $\mathrm{T}=430 \mathrm{C}, \mathrm{t}=$ $4 \mathrm{hrs}$ ). Black rounded spots in (b) are 20-30 $\mathrm{nm}$ silver clusters responsible for absorption peak in (a) in the range 402-426 $\mathrm{nm}$ due to plasmon resonance). 


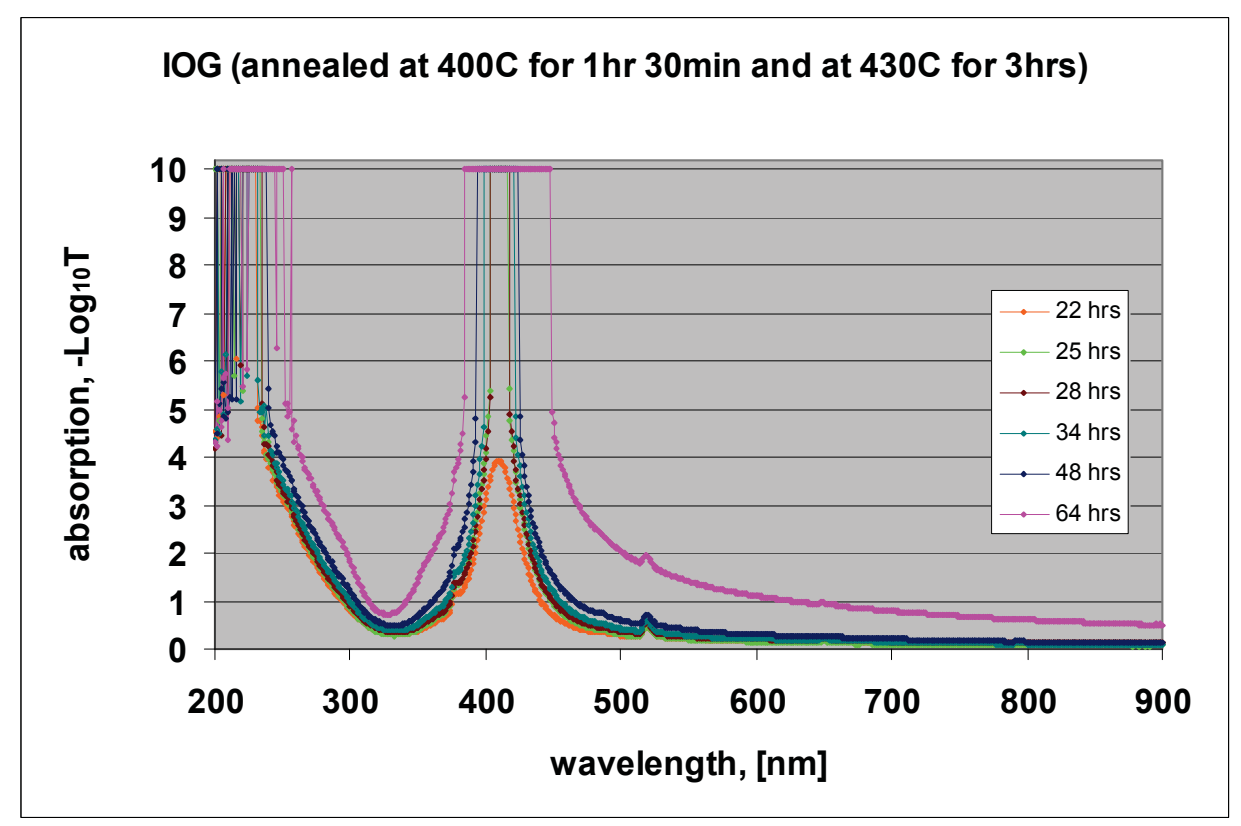

Figure 3.3: Absorption spectra of the samples with different ion-exchange time from 22 hrs (lowest curve) to $64 \mathrm{hrs}$ with $\mathrm{T}=95 \mathrm{C}$ and $\mathrm{V}=350 \mathrm{~V}$, other parameters, annealing in air: $\mathrm{T}=300 \mathrm{C}, \mathrm{t}=6 \mathrm{hrs}$, reduction in $H_{2}: \mathrm{T}=400 \mathrm{C} \mathrm{t}=1.5 \mathrm{hrs}$ followed by $\mathrm{T}=430 \mathrm{C}, \mathrm{t}=3 \mathrm{hrs}$.

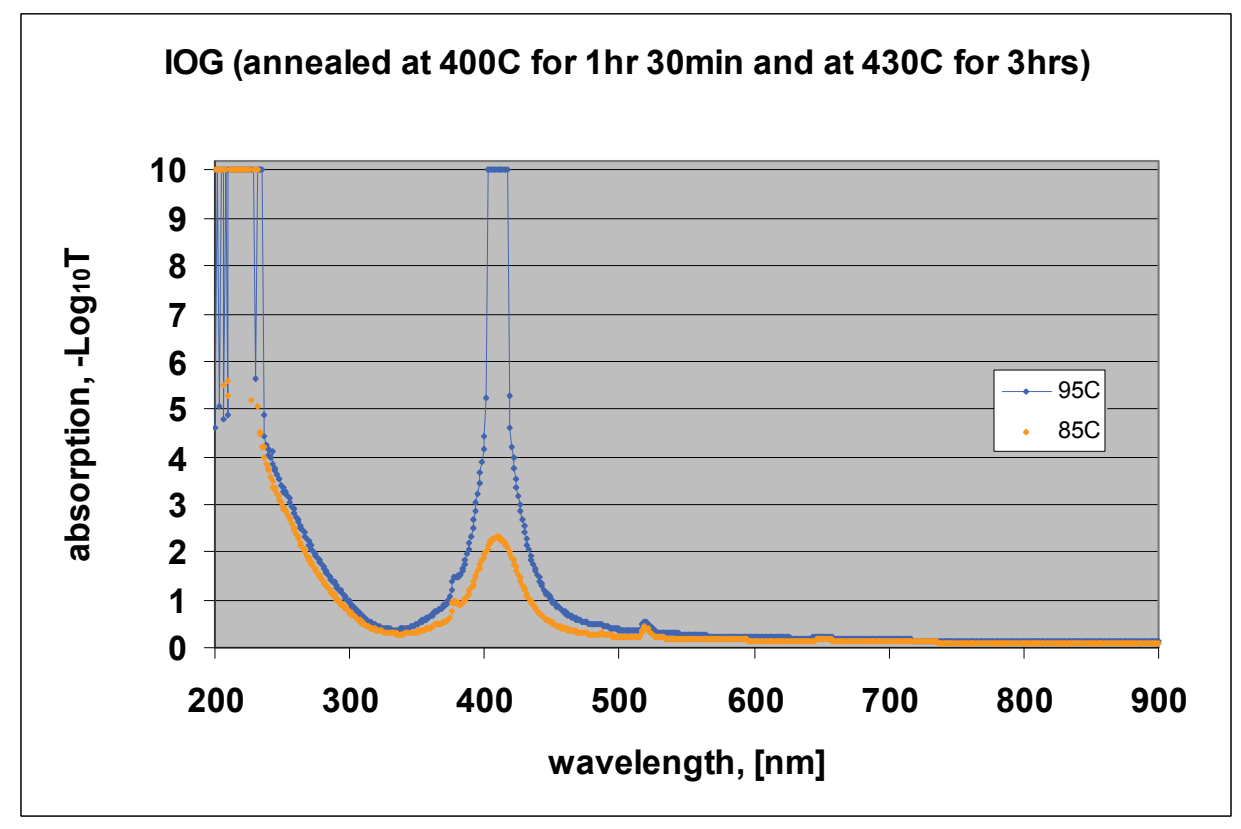

Figure 3.4: Absorption spectra of the samples with different ion-exchange temperature, $85 \mathrm{C}$ and $95 \mathrm{C}$ with $\mathrm{t}=25 \mathrm{hrs}$ and $\mathrm{V}=410 \mathrm{~V}$, other parameters, annealing in air: $\mathrm{T}=300 \mathrm{C}$, $\mathrm{t}=6 \mathrm{hrs}$, reduction in $\mathrm{H}_{2}: \mathrm{T}=400 \mathrm{C} \mathrm{t}=1.5 \mathrm{hrs}$ followed by $\mathrm{T}=430 \mathrm{C}, \mathrm{t}=3 \mathrm{hrs}$.

(corresponding to smaller densities of larger clusters). Finally, the absorption spectra for different time-temperature pairs the annealing in air show that temperature has a strong 


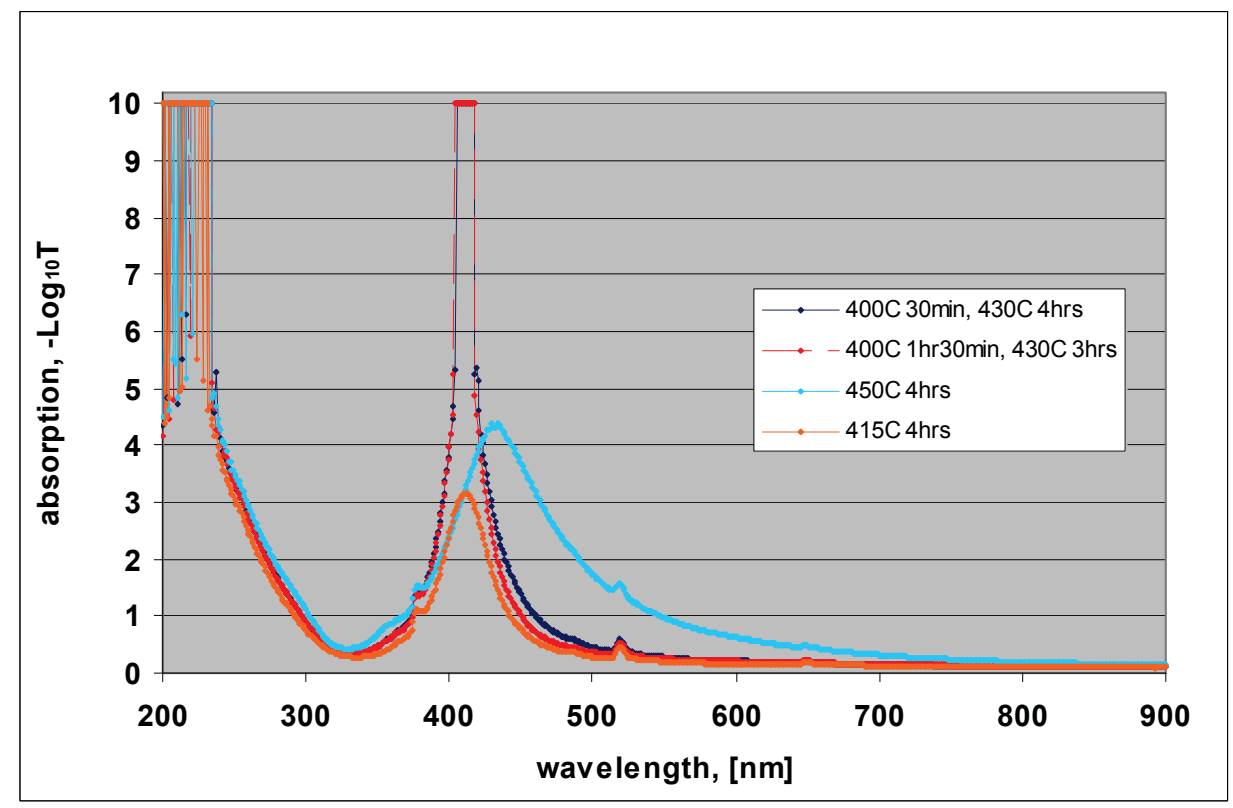

Figure 3.5: Absorption spectra of the samples with different treatment of annealing in $H_{2}$ : 400C for $30 \mathrm{~min}$ followed by $430 \mathrm{C}$ for $4 \mathrm{hrs}, 400 \mathrm{C}$ for $1.5 \mathrm{hrs}$ followed by $430 \mathrm{C}$ for 3 hrs, $450 \mathrm{C}$ for $4 \mathrm{hrs}, 415 \mathrm{C}$ for $4 \mathrm{hrs}$. Other parameters, ion-exchange: $\mathrm{T}=95 \mathrm{C}$, t=28 hrs, $\mathrm{V}=350 \mathrm{~V}$, annealing in air: $\mathrm{T}=300 \mathrm{C}, \mathrm{t}=6 \mathrm{hrs}$.

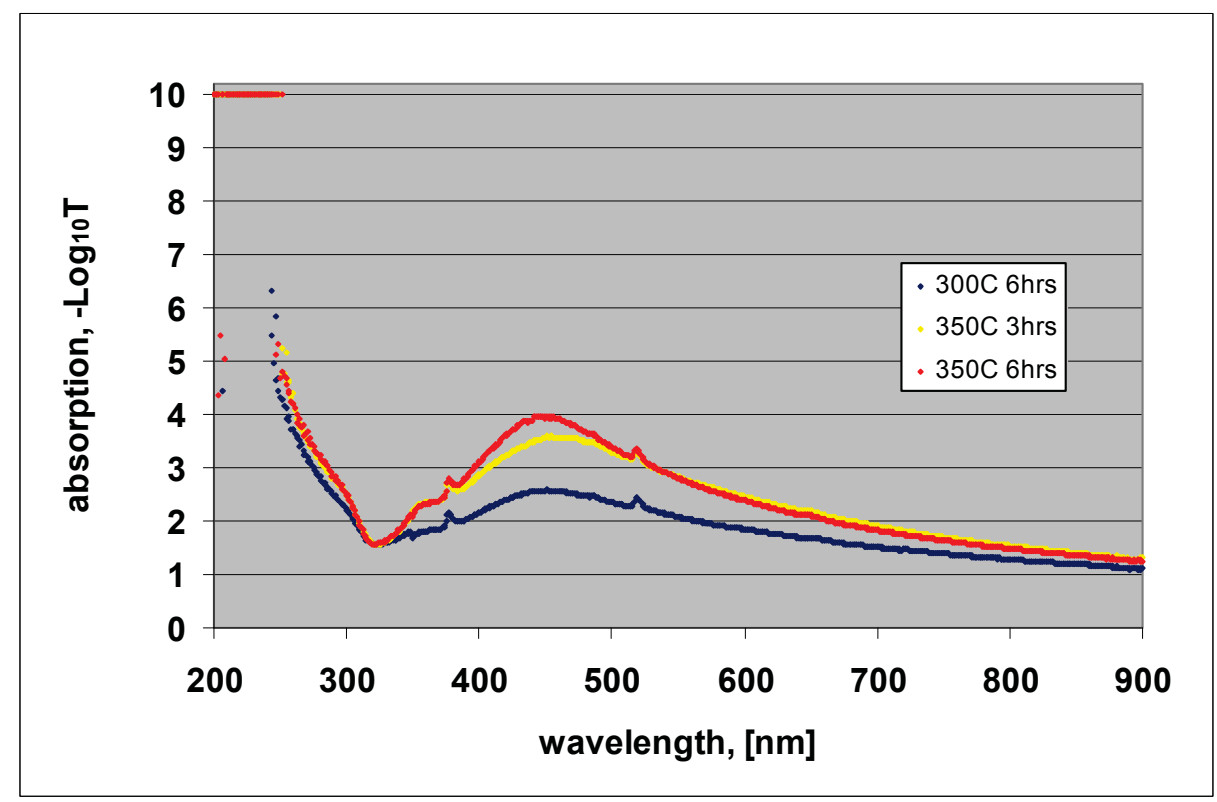

Figure 3.6: Absorption spectra of the samples with different treatment of annealing in air: 300C for $6 \mathrm{hrs}, 350 \mathrm{C}$ for $3 \mathrm{hrs}$ and 350C for $6 \mathrm{hrs}$. Other parameters, ion-exchange: $\mathrm{T}=105 \mathrm{C}, \mathrm{t}=45 \mathrm{hrs}, \mathrm{V}=400 \mathrm{~V}$, annealing in $H_{2}: \mathrm{T}=400 \mathrm{C}, \mathrm{t}=30 \mathrm{~min}$ followed by $\mathrm{T}=430 \mathrm{C}$, $\mathrm{t}=4 \mathrm{hrs}$. 
effect but not so much time, in the range studied.

In summary, by properly adjusting the parameters of the three phases of the clustering processes (ion-exchange, annealing in air and annealing in $H_{2}$ ) the shape of the induced absorption can be controlled, namely the height, width, and to a certain extent, position of the plasmon resonance peak. This was more or less expected and led us to the conditions necessary to prepare samples with the largest possible concentration of silver clusters, to be used in bleaching experiments. Such sample is shown in Fig. 3.7 (ion-exchange: $T=95^{\circ} \mathrm{C}, \mathrm{t}=70 \mathrm{hrs}, \mathrm{V}=370 \mathrm{~V}$, annealing in air: $T=300^{\circ} \mathrm{C}, \mathrm{t}=6 \mathrm{hrs}$, and annealing in $H_{2}: T=400^{\circ} \mathrm{C}, \mathrm{t}=30 \mathrm{~min}$ followed by $T=430^{\circ} \mathrm{C}, \mathrm{t}=4 \mathrm{hrs}$ ). As we can see later, bleaching of the sample can be achieved efficiently only for glass sample with large area of absorption spectra, i.e. with the large height and large width of the resonance like the one in Fig. 3.7 with absorption peak value spanned almost hundred nanometers, (399-497 nm).

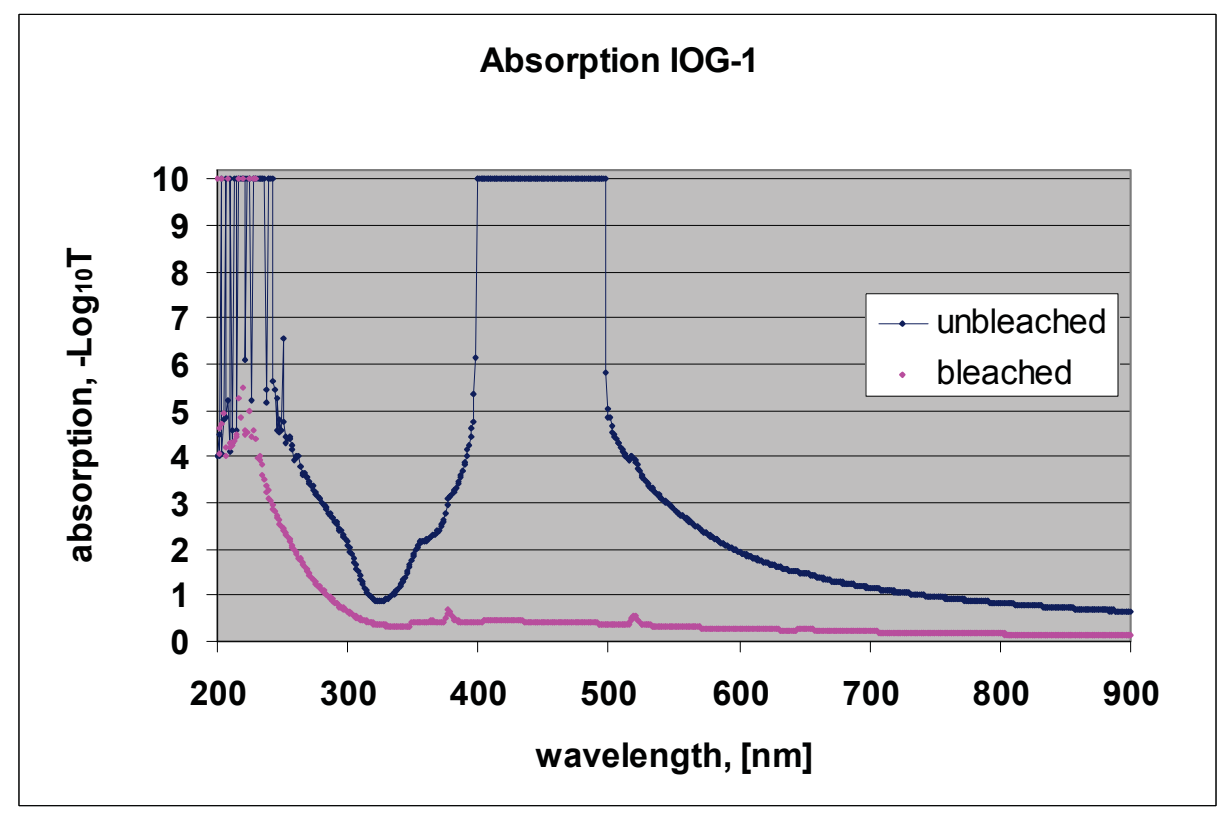

Figure 3.7: Absorption spectra before and after bleaching of the sample with the following parameters, ion-exchange: $\mathrm{T}=95 \mathrm{C}, \mathrm{t}=70 \mathrm{hrs}, \mathrm{V}=370 \mathrm{~V}$, annealing in air: $\mathrm{T}=300 \mathrm{C}, \mathrm{t}=6$ hrs, and annealing in $H_{2}: \mathrm{T}=400 \mathrm{C}, \mathrm{t}=30 \mathrm{~min}$ followed by $\mathrm{T}=430 \mathrm{C}, \mathrm{t}=4 \mathrm{hrs}$. Parameters of bleaching in Fig. 3.10.

\subsection{Glass bleaching of samples with silver clusters and its patterning}

\subsubsection{Field-assisted dissolution of silver clusters}

Metal clusters can be fully or partially dissolved if a glass sample containing such clusters is subjected to high voltage electric field at an elevated temperature $[13,14]$. The cluster 
dissolution process starts with electron tunneling through the metal-dielectric barrier which leads to atomic silver ionization and in turn to a silver ion leaving the cluster. It was shown that tunneling sharply depends on distance between clusters and therefore on their filling factor, as well as on the temperature and voltage applied [13]. Full cluster dissolution is achieved when the glass becomes transparent again, or "bleached". This is shown in Fig. 3.7, with bleaching conditions shown in Fig. 3.10.

It is therefore possible to dissolve the clusters locally if a patterned electrode is used, as was shown in $[45,46]$. In particular, since electron tunneling strongly depends on the voltage applied, there is a threshold voltage that triggers the clusters dissolution. Therefore, a periodically corrugated anode may lead to a potential modulation around the threshold voltage over a certain region of the glass and thus to a spatially modulated dissolution. Our goal is to investigate how deep such periodic modulation of the dissolution reaches under the surface and hence to investigate the possibility of making volume permittivity gratings.

A n-type silicon wafer was patterned with a periodic array of rectangular grooves made by anisotropic plasma etching (Fig. 3.8). The grating period was $7.4 \mu \mathrm{m}$ and the groove depth $5.7 \mu \mathrm{m}$. The patterned wafer was placed groove side down on the glass surface containing the silver clusters and then the wafer-glass pair was pressed between two stainless electrodes with the wafer on the positive electrode side. The whole assembly was placed in the oven. When the temperature in the oven reached $120-140^{\circ} \mathrm{C}$, the voltage was increased in steps of $100 \mathrm{~V}$ every $15 \mathrm{~min}$ up until reaching the 1.3-1.8 kV levels. Such gradual voltage increase is necessary to prevent the excessive current that otherwise would permanently damage the glass sample if the high voltage was applied instantaneously. It is worth noting, that conductivity of the glass is mainly ionic with primary contribution from highly mobile ions such as sodium ions, . Therefore DC voltage, applied across the ion-exchanged glass containing metal nanoclusters, is not distributed uniformly under the surface. Since such glass can be viewed as a voltage divider, a substantial part of DC voltage drops across depleted layer only (whose depth a couple of microns). It turns out that corresponding electric field in depleted layer can reach the value that triggers tunneling of electrons from the metal nanoclusters which initiates the bleaching process.

The side view SEM images shown in Fig. 3.9 also reveal that a surface relief pattern remains after the bleaching process, as was previously observed when a microstructured anode was used to pattern the dissolution of a silicate glass-metal nanocomposite sample $[45,46]$. The field-assisted dissolution of silver clusters in phosphate glass matrix results in similar surface relief and its depth is estimated to be $300 \mathrm{~nm}$, almost 10 times larger than reported in $[45,46]$. This difference is likely due to the fact that the periodicity of our electrodes is also about one order of magnitude larger than in those references.

Fig. 3.10 present the evolution of the electrical properties of the glass sample, bleached with patterned electrode with period $8 \mu \mathrm{m}$, whose absorption plots was presented in Fig. 3.7 during the bleaching process. At each voltage increment the current experienced a jump, starting from the 5th increment, and immediately after each voltage step the current decayed until the next step up. When the voltage reached its maximum value of $1800 \mathrm{~V}$ the peak current reached $90 \mu \mathrm{A}$ then monotonically decayed. At 5 hours of treatment the 


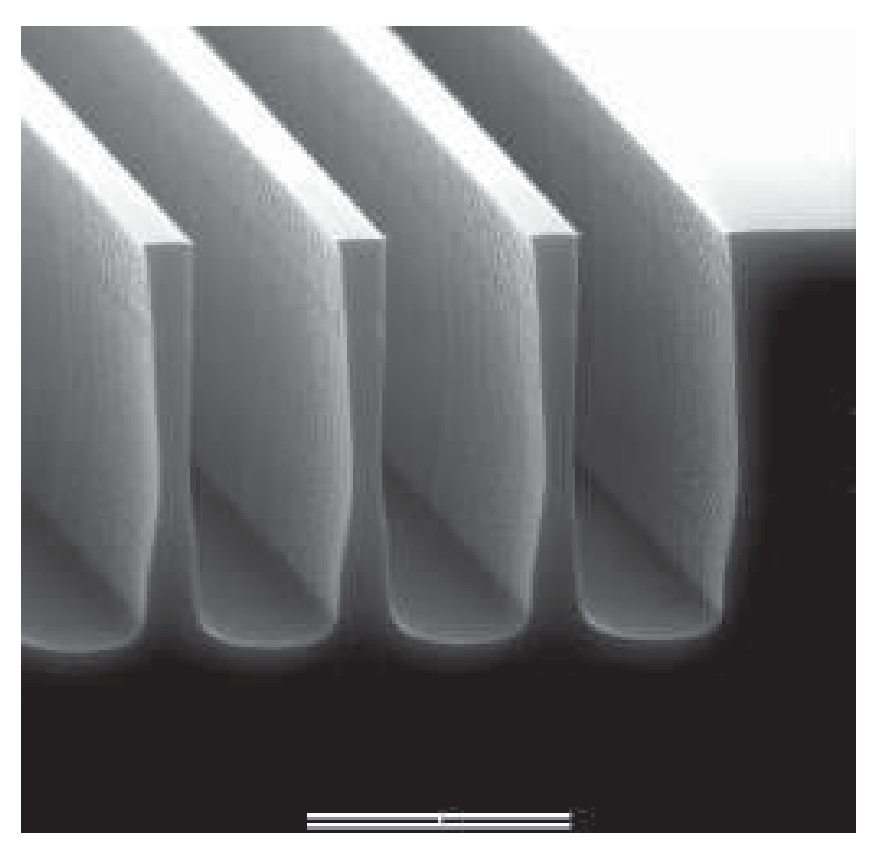

Figure 3.8: SEM image of periodic Si electrode produced by plasma etching, double bar is $10 \mu \mathrm{m}$

decay rate increased because the oven was turned off and sample started cooling, thereby decreasing the ionic conductivity of the glass. At 9.4 hours the voltage starts to drop off with the rate every $6 \mathrm{~s}$ by $6 \mathrm{~V}$ and reached zero at 10 hours. For comparison the bleaching dynamics of a silicate glass sample containing silver clusters is shown in Fig. 3.11 where the current decay after voltage increment is more pronounced and overall current value is larger (electrode area the same for both cases). So while the conductivity of the phosphate glass is significantly lower than that of the comparative silicate glass, the overall effect of bleaching is similar. Silicate glass sample was prepared as follows: $1 \mathrm{hr}$. of ion-exchange at $130^{\circ} \mathrm{C}$ and $400 \mathrm{~V}, 6 \mathrm{hrs} 30 \mathrm{~min}$. of annealing in air at $300^{\circ} \mathrm{C}$ and finally annealing in hydrogen at $400^{\circ} \mathrm{C}$ for $30 \mathrm{~min}$ ramped to $430^{\circ} \mathrm{C}$ for $4 \mathrm{hrs}$.

\subsubsection{Sample measurements and observation}

Relative concentrations of the local chemical content of the sample were determined at several locations of the patterned samples by scanning electron microscope (SEM) equipped with an energy dispersive X-ray spectrum analyzer (EDX). This allows the identification and concentration measurement of the chemical compounds within a depth of about $1 \mu \mathrm{m}$, with a lateral accuracy also of the order of $1 \mu \mathrm{m}$. The theoretical detection limit of the EDX measurements is $0.08 \mathrm{wt} \%$ while in practice the limit is estimated to $0.1 \mathrm{wt} \%$ according to [47]. EDX analyzer uses the intensity of characteristic X-rays emitted by the elements within the beam to quantify the concentrations of those elements. The electron probe size is only several nanometers however due to electron scattering off the sample, the detected beam diameter and overall spacial resolution of the measurement is around $1 \mu \mathrm{m}$ which 


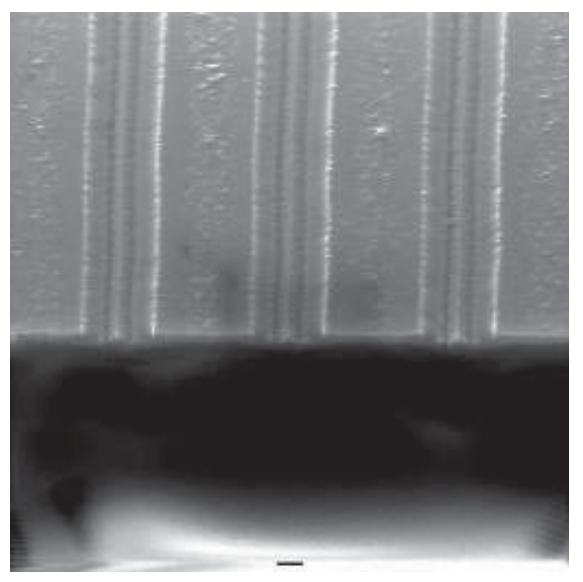

(a)

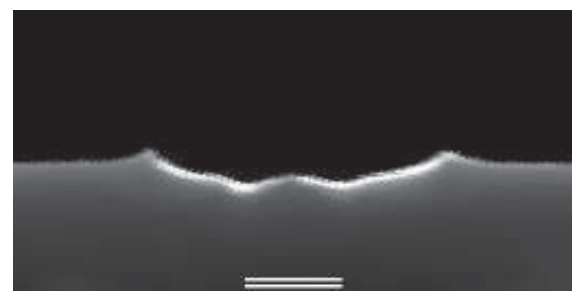

(c) double bar is $1 \mu \mathrm{m}$

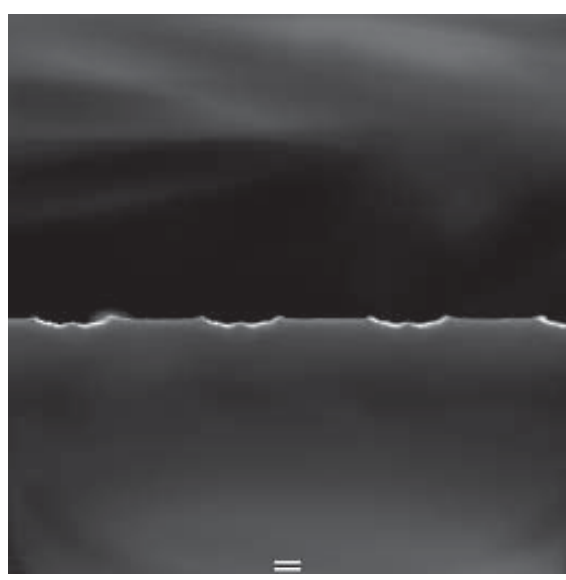

(b) double bar is $1 \mu \mathrm{m}$

Figure 3.9: SEM image of IOG-1 glass bleached with periodic electrode.

still acceptable for our purpose because the groove region and space between the grooves is not smaller than $2 \mu \mathrm{m}$. Performing measurements in various locations as indicated in Fig. 3.17 allows to experimentally determine the spacial concentration changes across the sample and therefore grating contrast at the surface and at polished level which was about $4 \mu \mathrm{m}$ below the surface. SEM image of glass sample and corresponding EDX spectroscopy at point specified in Fig. 3.17 as 4 (polished level) is shown in Fig. 3.18.

\subsection{Absorption spectra simulation with Maxwell-Garnet theory}

\subsubsection{Maxwell-Garnet mixing formula}

Effective dielectric constant medium that has two components, host medium and spherical particle inclusions described by Maxwell Garnett mixing formula as [48]:

$$
\varepsilon_{e f f}=\varepsilon_{h}+3 f \varepsilon_{h} \frac{\varepsilon_{i}-\varepsilon_{h}}{\varepsilon_{i}+2 \varepsilon_{h}-f\left(\varepsilon_{i}-\varepsilon_{h}\right)}
$$




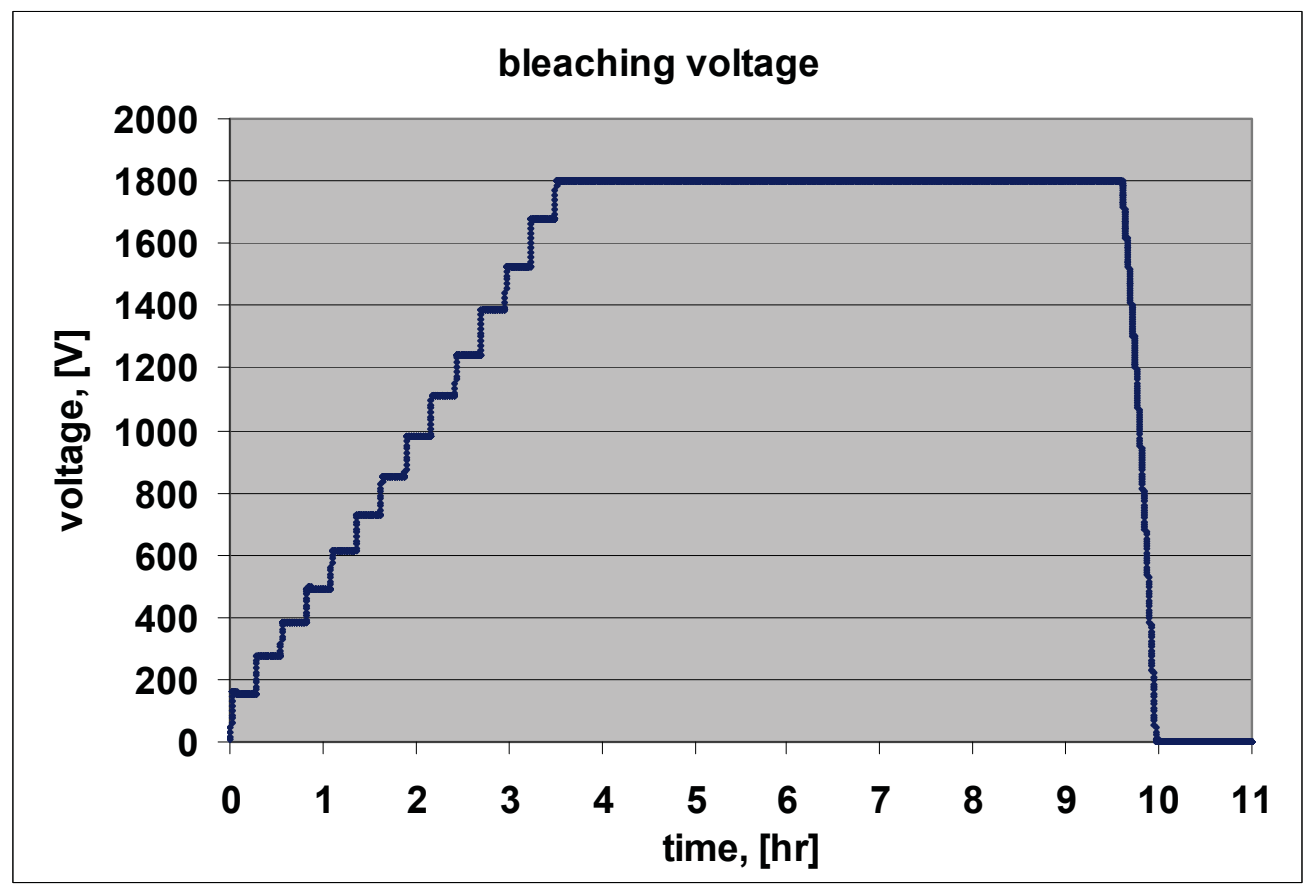

(a)

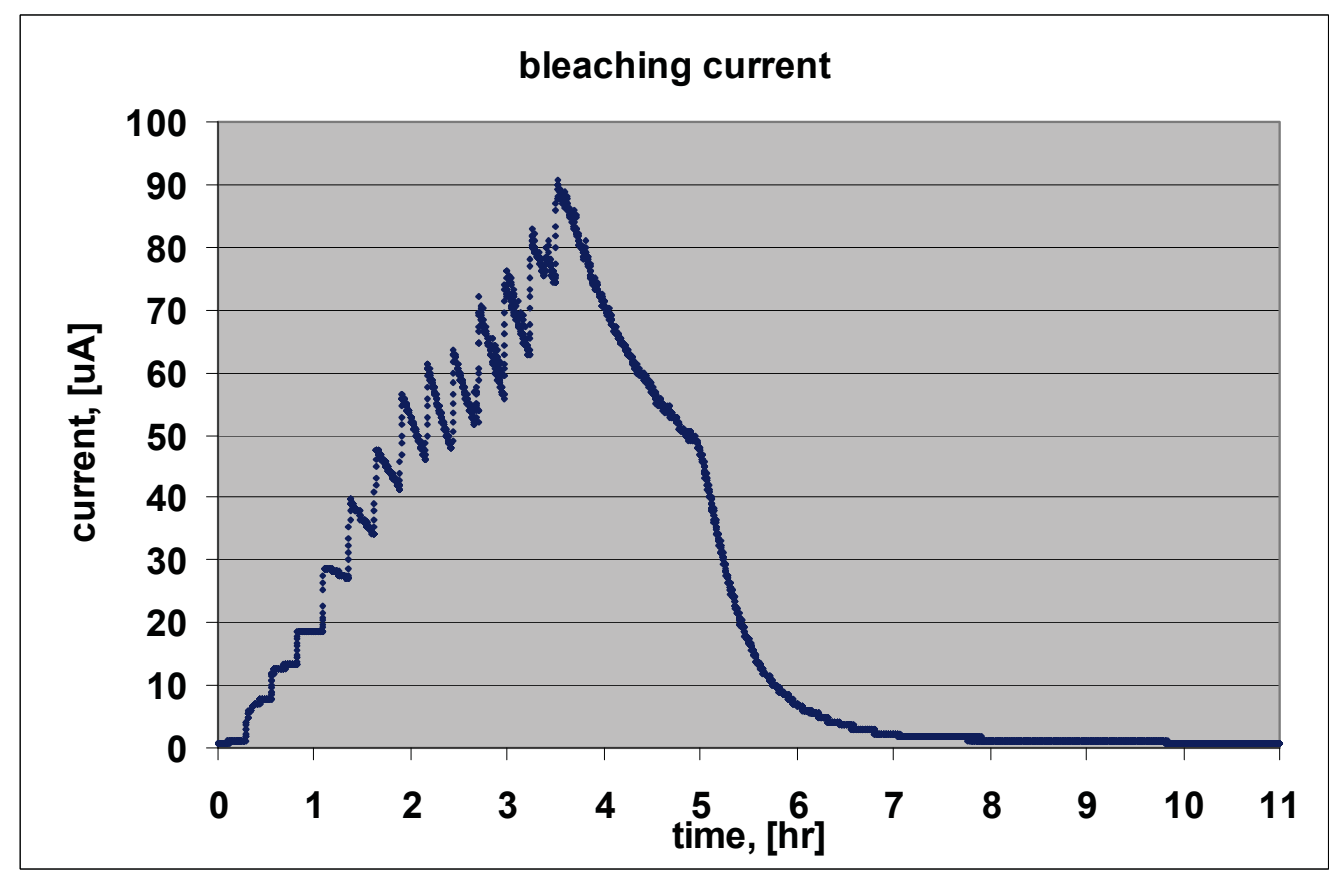

(b)

Figure 3.10: Bleaching voltage (a) and current (b) vs. time of IOG-1 sample whose absorption plot is in Fig. 3.7.

where: 


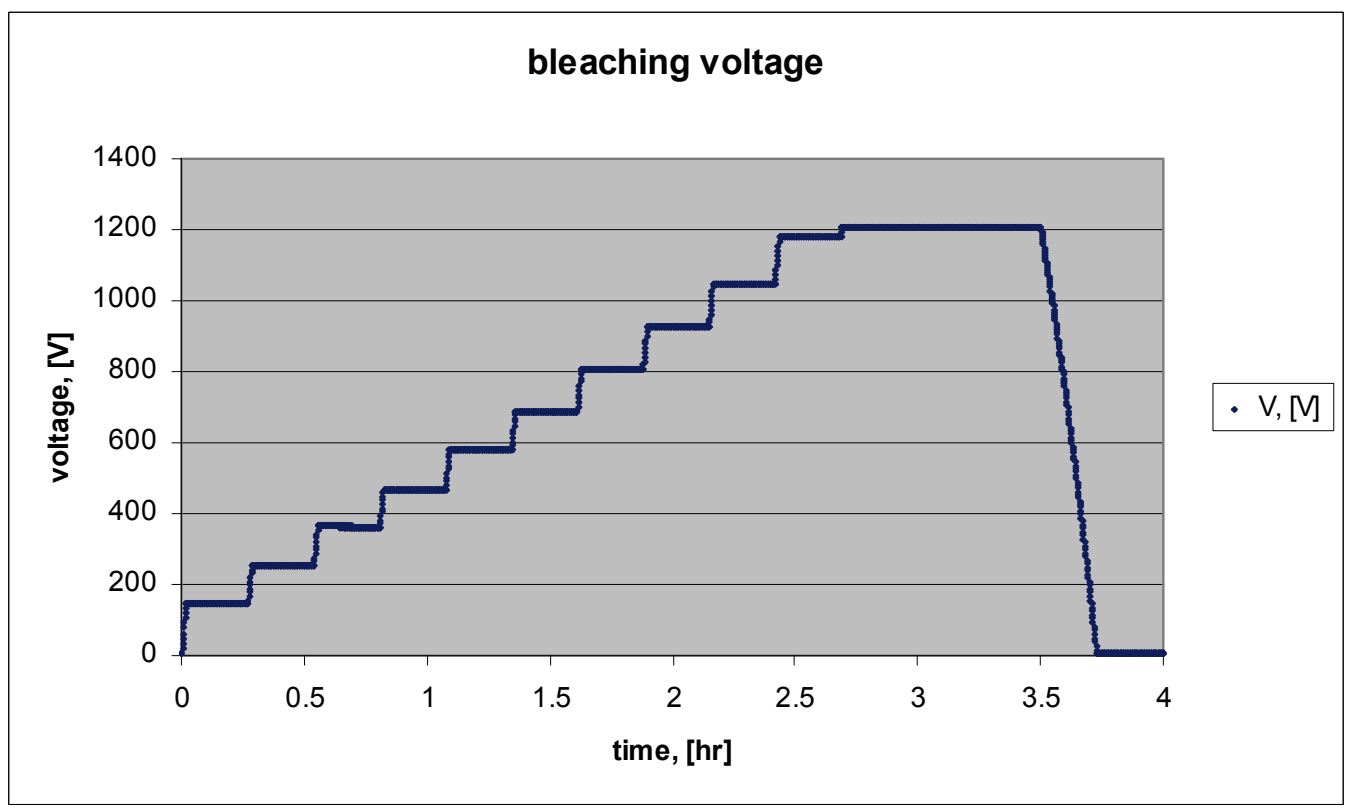

(a)

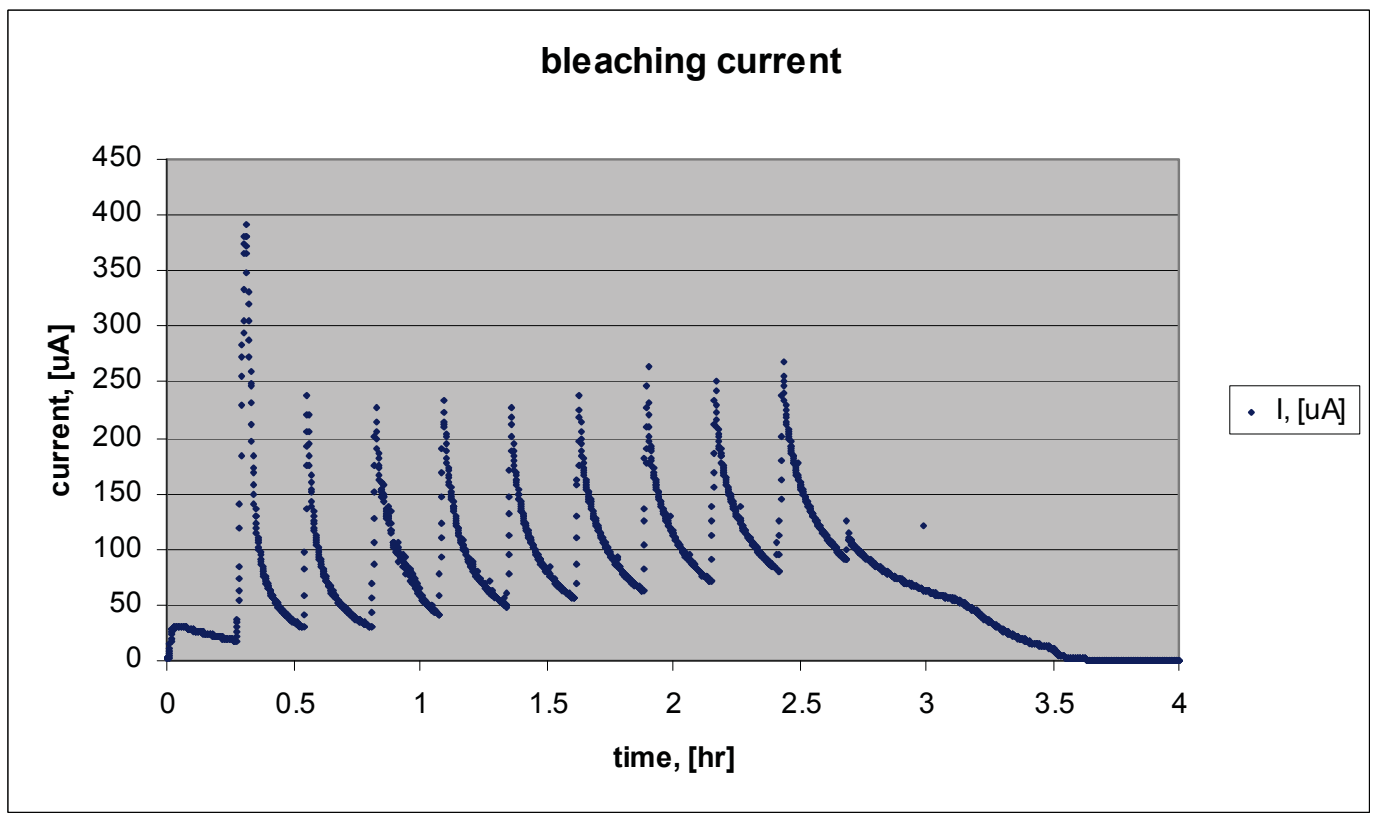

(b)

Figure 3.11: Bleaching voltage (a) and current (b) vs. time of silicate glass (microscope slide) sample. 
$\varepsilon_{h}$ dielectric constant of host medium

$\varepsilon_{i}$ dielectric constant of particle embedded in host medium

$f$ volume density of embedded particle in a mixed medium

Maxwell Garnet mixing formula assumes particles of spherical shape and various sizes with the condition that size of each particle much smaller than wavelength of the incident light, even though the diameter does not enter the formula. Polarizability of the particles assumed to be electrostatic.

\subsubsection{Dielectric constant for nanocluster sphere of radius $r$}

Size dependent dielectric constant for silver based on Drude model and mean free path effect is given by [49]:

$$
\varepsilon_{A g}(\omega, r)=\varepsilon_{b u l k}+\frac{\omega_{p}^{2}}{\omega^{2}+i \omega \gamma_{0}}-\frac{\omega_{p}^{2}}{\omega^{2}+i \omega\left(\gamma_{0}+A v_{F} / r\right)}
$$

where

$\omega_{p}$ plasma frequency, $\omega_{p}=9.17 \mathrm{eV}[50]$

$\gamma_{0}$ damping constant, $\gamma_{0}=0.021 \mathrm{eV}[50]$

$A$ size factor, $A \simeq 1$

$v_{F}$ Fermi velocity for $\mathrm{Ag}, v_{F}=1.39 \times 10^{6} \mathrm{~m} / \mathrm{s}[51]$

$r$ silver cluster radius

$\varepsilon_{\text {bulk }}$ dielectric constant of bulk silver, data from [52]
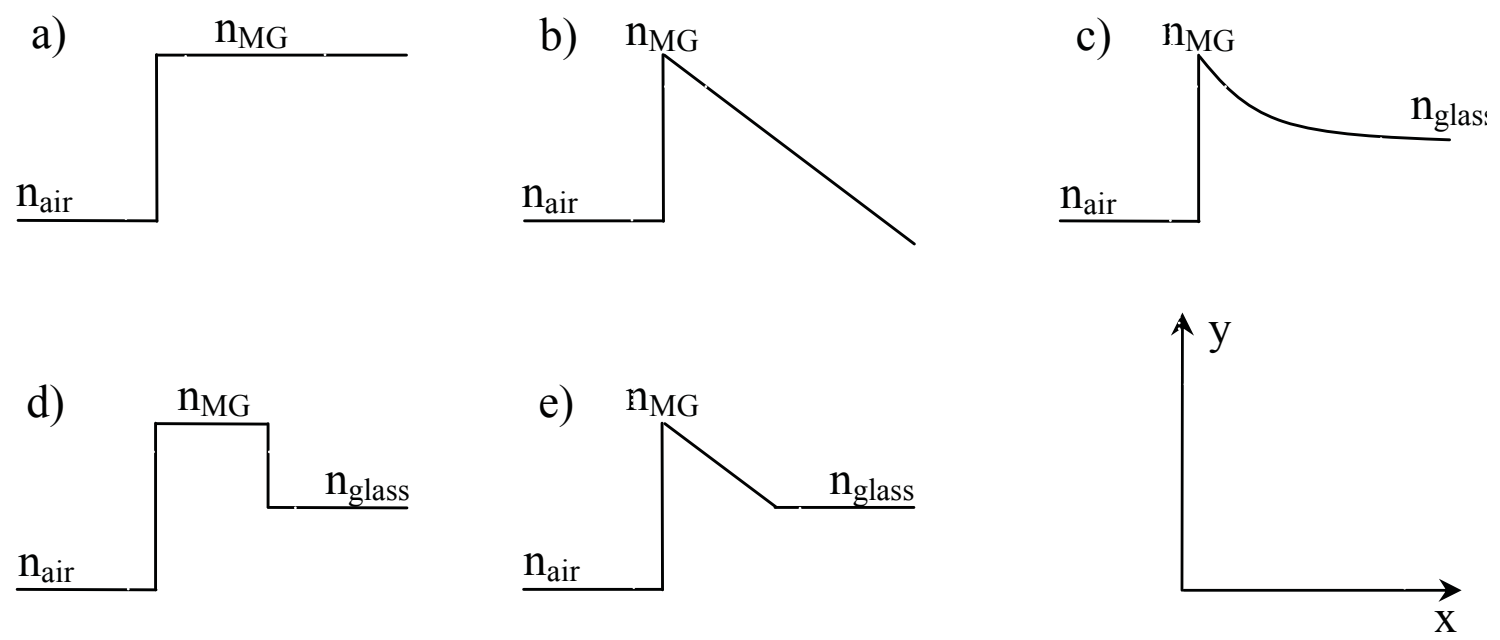

Figure 3.12: Models tested for absorption spectra, where $n_{M G}$ refractive index calculated by Maxwell-Garnet mixing formula . 


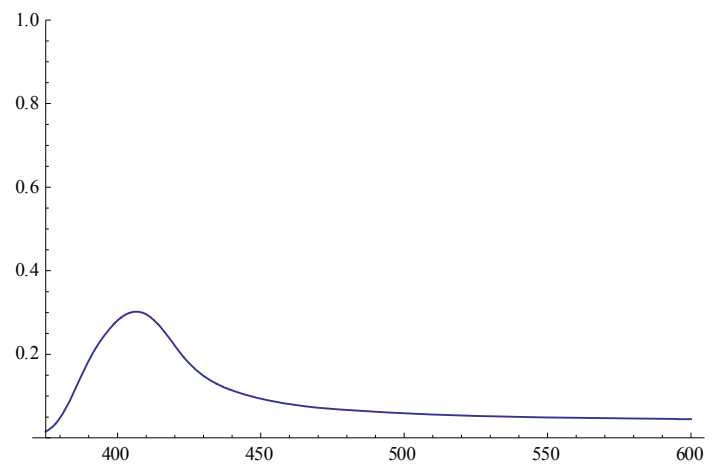

(a)

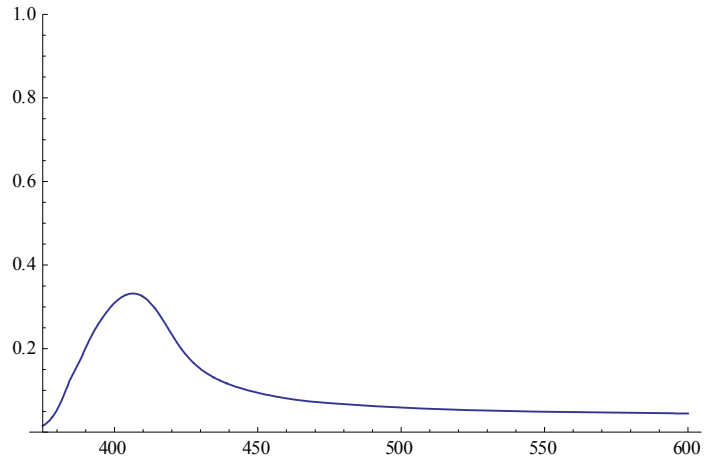

(c)

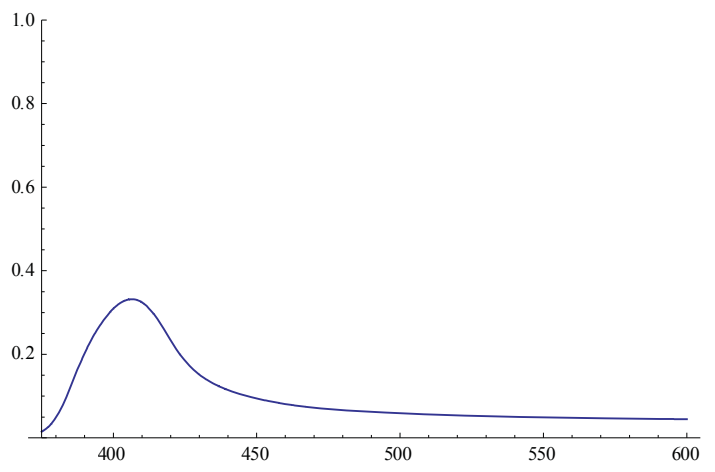

(b)

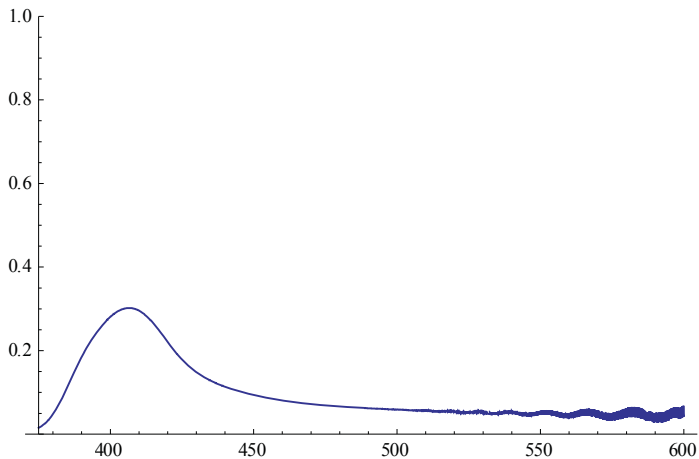

(d)

Figure 3.13: Calculated absorption spectra, $\alpha(\lambda)$ for models in Fig. 3.12. Parameters: $f_{v}=0.1, \mathrm{r}=12 \mathrm{~nm}, d=4 \mu \mathrm{m}$. 


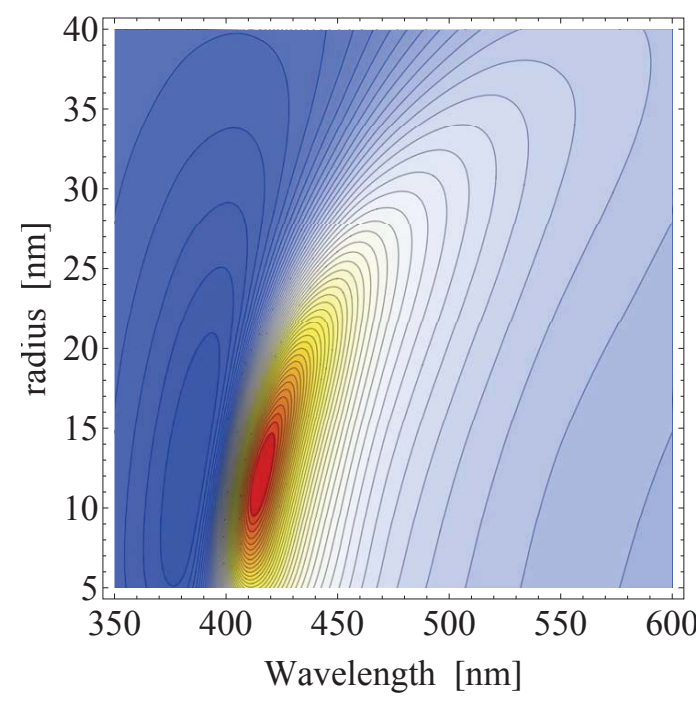

(a) $f_{v}=0.05$

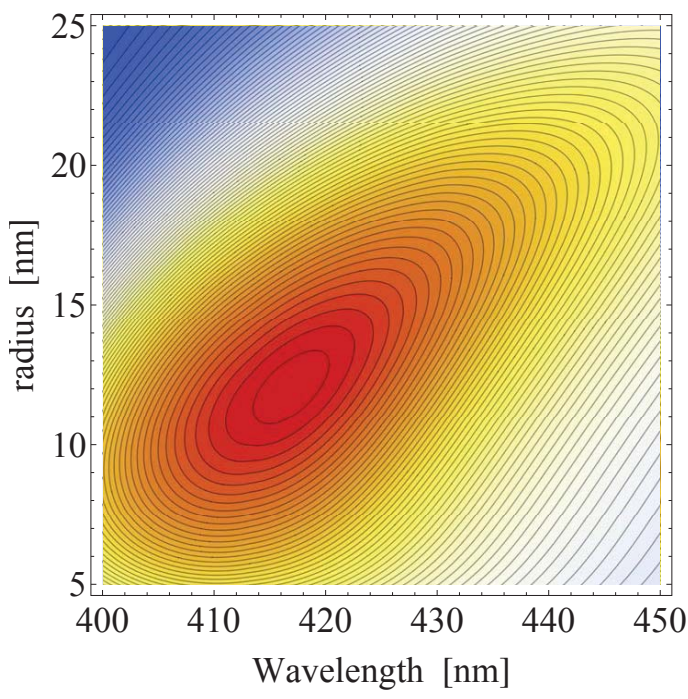

(c) $f_{v}=0.1$

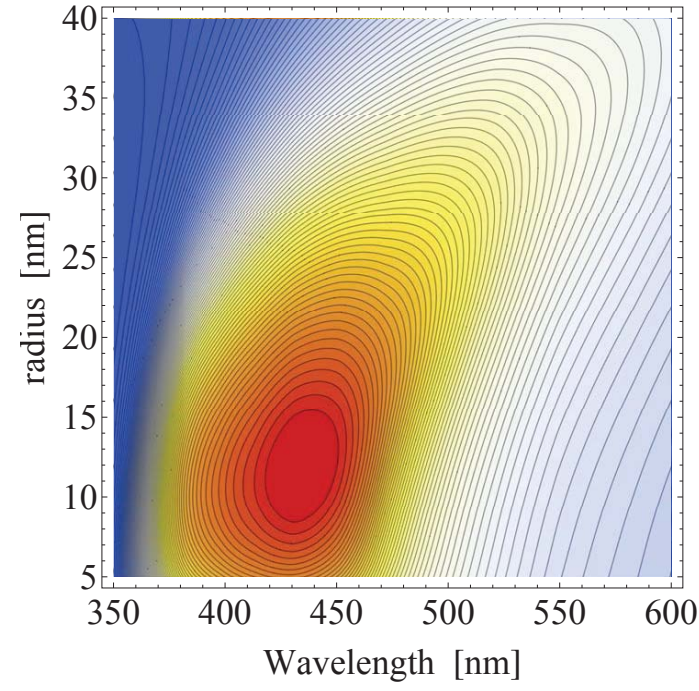

(e) $f_{v}=0.3$
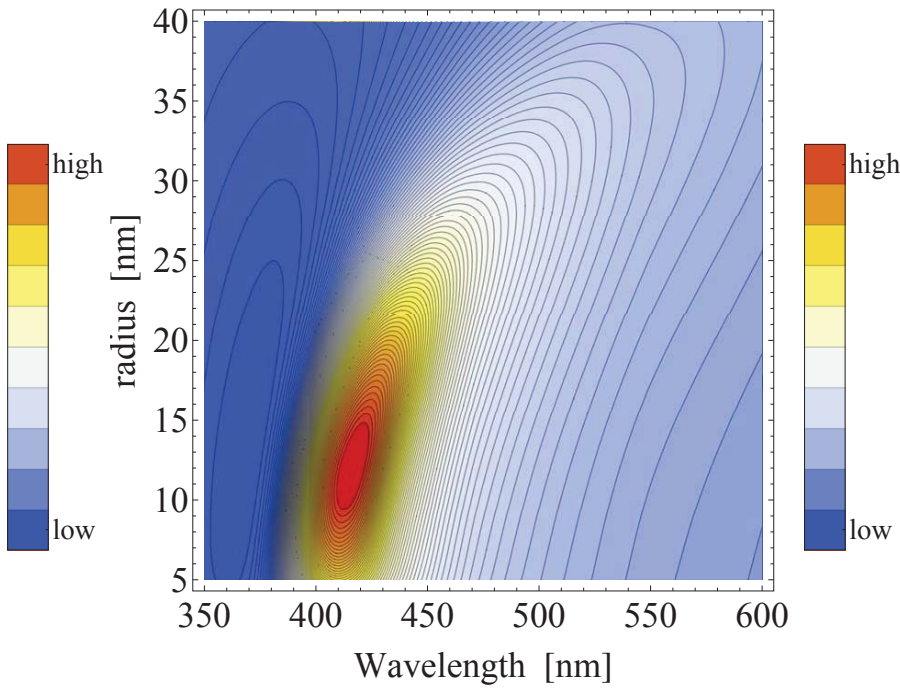

(b) $f_{v}=0.1$
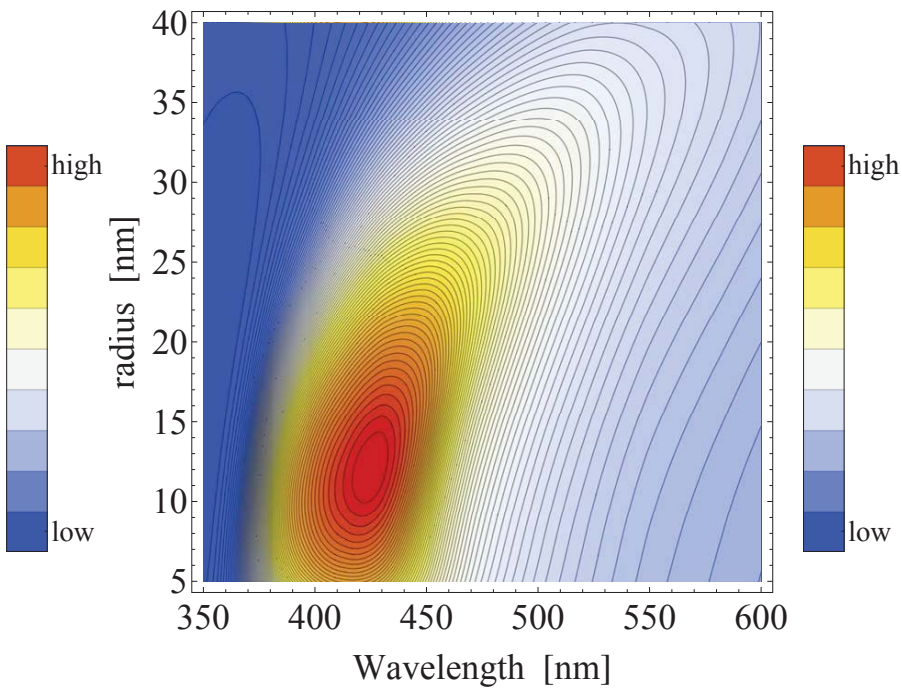

(d) $f_{v}=0.2$

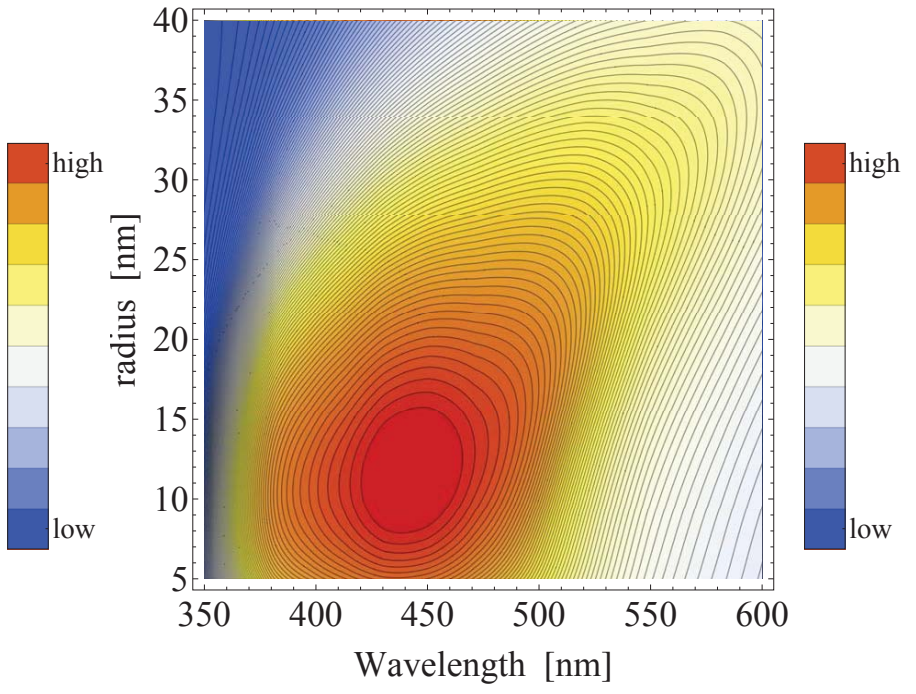

38

(f) $f_{v}=0.4$ 


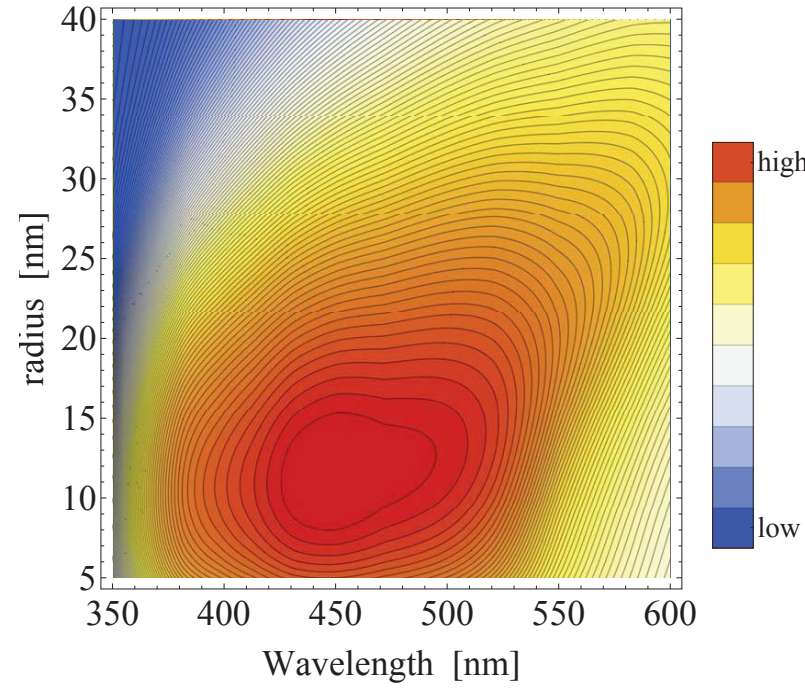

(g) $f_{v}=0.5$

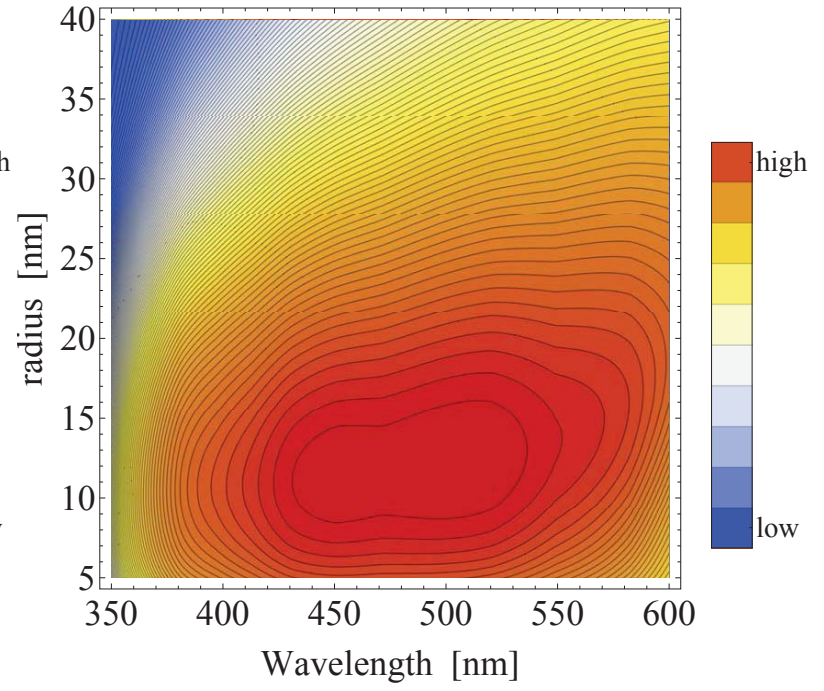

(h) $f_{v}=0.6$

Figure 3.14: absorption spectra, $\alpha(\lambda, r)$ for homogeneous interface of two mediums vs. cluster radius $\mathrm{r}$ and wavelength $\lambda$.

In UV-vis spectrometry absorption is evaluated with Lambert-Beer's low trough transmission as:

$$
\alpha_{a b s}=-\log _{10} T
$$

For a simple model when media with effective permittivity $\varepsilon_{e f f}$ is treated as semiinfinite with constant permittivity, the transmission is found from Fresnel equation for a normal incidence as,

$$
T=1-\left|\frac{\sqrt{\varepsilon_{a i r}}-\sqrt{\varepsilon_{e f f}}}{\sqrt{\varepsilon_{a i r}}+\sqrt{\varepsilon_{e f f}}}\right|^{2}
$$

A few more realistic models that take into account finite thickness of the media and its permittivity dependence with distance away from the surface, $\varepsilon_{\text {eff }}(x)$ have analytical solution for corresponding one dimensional wave equation and therefore for absorption in 3.3. For example uniform slab model, slab model with linear dependence $\varepsilon_{\text {eff }}(x)$ and slab model with exponential dependence $\varepsilon_{\text {eff }}(x)$, shown schematically in Fig. 3.12, have known analytical solutions. However, it turns out that position of the absorption peak and absorption width does not differ significantly from the simple model based on Fresnel equation. Absorption plots for models in Fig. 3.12 are shown in Fig. 3.13. Therefore for simplicity, in the remaining part only 3.4 will be used.

\subsubsection{Extended Maxwell-Garnet mixing formula}

Extended Maxwell-Garnet mixing formula [53] replaces electrostatic polarizability of the sphere with size dependent electric dipole polarizability $\alpha$ calculated through Mie scattering 


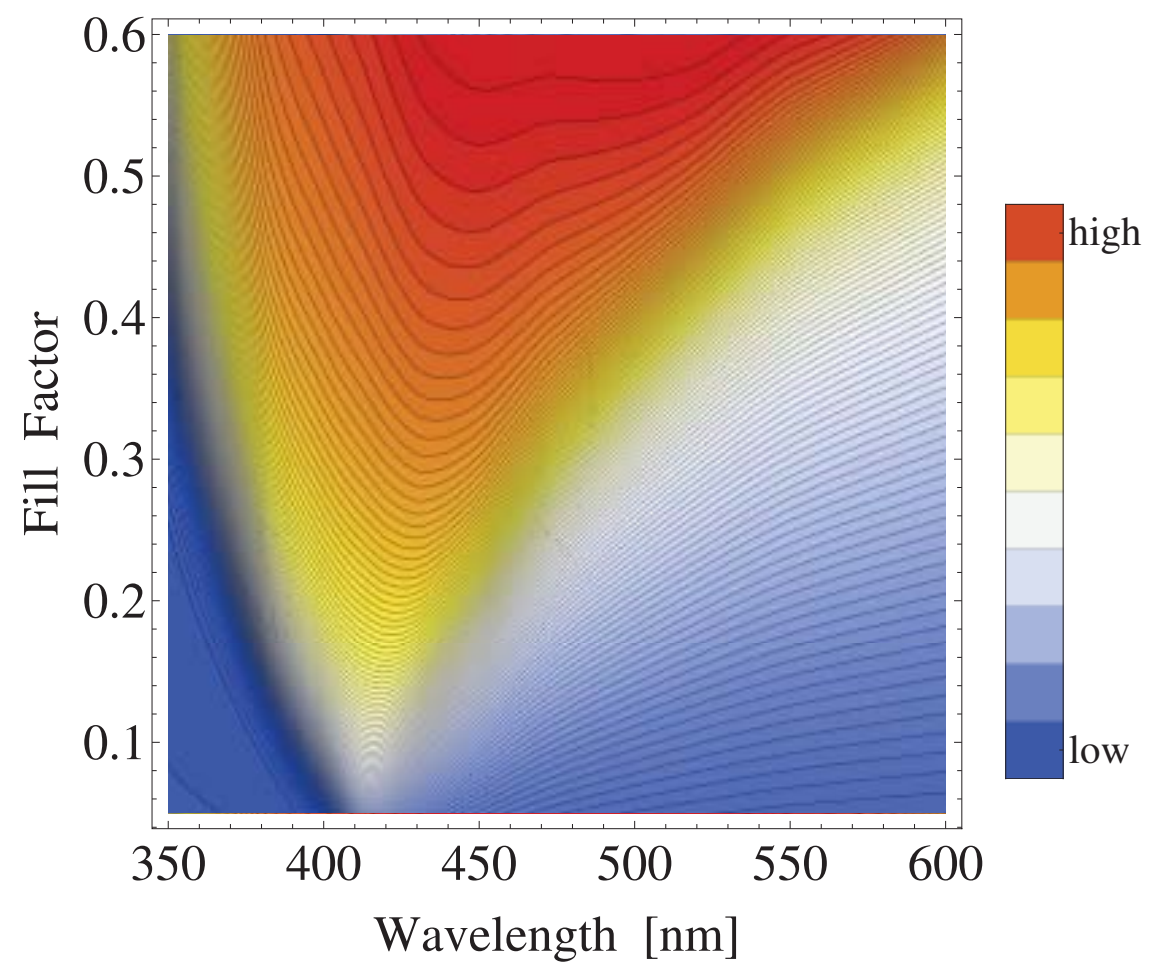

Figure 3.15: absorption spectra, $\alpha\left(\lambda, f_{v}\right)$ for homogeneous interface of two mediums vs. wavelength $\lambda$ and fill factor $f_{v}$ with discrete normal distribution of cluster size. Parameters: $\mathrm{r}=12 \mathrm{~nm}, \sigma=2 \mathrm{~nm}$.

coefficient $a_{1}$ :

$$
\begin{gathered}
\alpha=i \frac{3 r^{3}}{2 x^{3}} a_{1} \\
a_{1}=\frac{m \psi_{1}(m x) \psi_{1}^{\prime}(x)-\psi_{1}(x) \psi_{1}^{\prime}(m x)}{m \psi_{1}(m x) \xi_{1}^{\prime}(x)-\xi_{1}(x) \psi_{1}^{\prime}(m x)}
\end{gathered}
$$

where

$\alpha$ sphere polarizability

$r$ sphere radius

$i=\sqrt{-1}$

$x=k_{0} \sqrt{\varepsilon_{h}} r$ size parameter

$a_{1}$ Mie scattering coefficient

$m=\sqrt{\varepsilon_{i} / \varepsilon_{h}}$

Size dependent effective dielectric constant is given:

$$
\varepsilon_{e f f}=\varepsilon_{h} \frac{x^{3}+i 3 f a_{1}}{x^{3}-i(3 / 2) f a_{1}}
$$


where $\psi_{n}(x)=x j_{n}(x)$

$\xi_{n}(x)=x h_{n}^{(1)}(x)$

$j_{n}(x)$ spherical Bessel function of the first kind

$h_{n}^{(1)}(x)$ spherical Hankel function of the first kind

\section{Absorption coefficient dependence on cluster size and volume factor}

With the size dependent dielectric constant of the silver cluster and size dependent dielectric constant of the mixture (this dependence arises through dipole moment approximation of the cluster polarizability) we can investigate how absorption coefficient depends on $\mathrm{r}$, cluster radius. The contour plots of absorption versus wavelength and cluster radius presented in Fig. 3.14 where the volume factor varied as a parameter from 0.1 to 0.6. Resonance peak is shifting from 410-420 nm for smaller $\mathrm{f}(\mathrm{f}=0.1)$ towards longer wavelengths, $450 \mathrm{~nm}$ at $\mathrm{f}=0.4$ then splits at $\mathrm{f}=0.6$. The cluster radius that maximizes the peak is around $12 \mathrm{~nm}$. Another feature of the peaks behavior is that for a given volume factor it red shifts with the cluster size.

\subsubsection{Maxwell Garnett formula for distributed cluster size}

Assume cluster size is random variable with discrete normal distribution $N\left(r_{i}, r_{0}, \sigma\right)$, then weighted value of dielectric constant becomes:

$$
\begin{gathered}
\varepsilon_{M G}=\sum_{i} N\left(r_{i}, r_{0}, \sigma\right) \varepsilon_{\text {eff }}\left(r_{i}\right) \\
\varepsilon_{e f f}\left(r_{i}\right)=\varepsilon_{h} \frac{\left(k_{0} \sqrt{\varepsilon_{h}} r_{i}\right)^{3}+i 3 f a_{1}\left(r_{i}\right)}{\left(k_{0} \sqrt{\varepsilon_{h}} r_{i}\right)^{3}-i(3 / 2) f a_{1}\left(r_{i}\right)}
\end{gathered}
$$

the size-dependence in $a_{1}\left(r_{i}\right)$ is trough the size dependent dielectric constant of silver cluster $\varepsilon_{A g}\left(\omega, r_{i}\right)$ :

$$
a_{1}\left(r_{i}\right)=a_{1}\left(m\left(\varepsilon_{A g}\left(r_{i}\right)\right)\right)
$$

discrete normal distribution:

$$
N\left(r_{i}, r_{0}, \sigma\right)=\frac{1}{\sigma \sqrt{2 \pi}} \exp \left(-\frac{1}{2}\left(\frac{r_{i}-r_{0}}{\sigma}\right)^{2}\right)
$$

\section{Absorption coefficient dependence vs. wavelength and volume filling factor for dis- tributed cluster size mixture}

In this simulation we assume mixture of glass (host medium) and Ag-clusters of various

radiuses with discrete normal distribution around the radius that gives maximum absorption peak, $r_{0}=12 \mathrm{~nm}, \sigma=2 \mathrm{~nm}$. Contour plot in Fig. 3.15 shows substantial broadening 
of the absorption band with increasing volume fill factor. Since electromagnetic coupling of the clusters is due to multipolar interaction and since extended Maxwell-Garnet mixing formula is valid within the limits of dipole approximation, the simulation presented in contour plots captures the leading term of the multipolar interaction, i.e. dipolar. However, at high volume factor $f_{v}=0.5,0.6$ the simulation may not be valid, since at such high clusters density each cluster (nanoparticle) feels predominantly scattered field (spherical wave) rather than incident field (plane wave) at which point electric dipole approximation and whole Mie theory breaks down.

Simulation suggests a red shift of the peak with larger cluster size. Such shift has been experimentally observed in Fig. 3.5. This implies that temperature annealing in $\mathrm{H}_{2}$ may control the cluster size.

\subsection{Discussion}

\subsubsection{Potential due to corrugated electrode}

We want investigate potential distribution under periodically corrugated plate. This is needed to evaluate periodic modulation of the potential which leads (since tunneling depends on potential) to tunneling modulation and in turn creates periodic bleaching of the glass, i.e. region of undissolved (less dissolved) clusters followed by region of dissolved (more dissolved) clusters. Conformal map $w=f(z)$ is useful for evaluating potential distribution under periodic electrode since it maps equipotential lines in $z$ (flat electrode) into equipotential lines in $w$ (periodic electrode).

\section{Triangular groove}

Conformal mapping of a flat upper plane $z$ on a periodically corrugated upper plane $w$ with triangular grooves represented by formula (details in Appendix A):

$$
w=f(z)=i(-1)^{-p} B\left(a \sin ^{2}\left(\frac{\pi z}{2 b}\right), p, 1-p\right)
$$

where $B(\zeta, p, 1-p)$ is incomplete beta function.

\section{Rectangular groove}

Conformal mapping of a flat upper plane $z$ on a periodically corrugated upper plane $w$ with rectangular grooves represented by formula:

$$
w=f(z)=\frac{i 2}{\sqrt{a_{3}\left(a_{4}-1\right)}}\left(\left(a_{3}-1\right) F(\phi(z), m)+\Pi(n, \phi(z), m)\right)
$$




$$
\phi(z)=\arcsin \left(\frac{\sin ((\pi z) /(2 b)) \sqrt{\left(a_{4}-1\right)}}{\sqrt{\left(a_{4} \sin ^{2}((\pi z) /(2 b))-1\right)}}\right)
$$

where

$F(\phi, m)$ is elliptic integral of the first kind

$\Pi(n, \phi, m)$ is incomplete elliptic integral

Equipotential lines under periodically corrugated plate with triangular and rectangular groove are shown in Fig. 3.16, they calculated with formulas obtained through conformal mapping. As seen from the figure the spacial modulation, $\Delta y\left(\Phi_{i}, x\right)$ of equipotential lines decays with the distance and at $4 \mu \mathrm{m}$ from the electrode surface is very weak for both triangular and rectangular electrode geometry as seen from Fig. 3.16. The actual voltage modulation $\Delta V\left(y_{i}, x\right)$ at $y_{i}=4 \mu \mathrm{m}$ shown in Fig. 3.16(c) was calculated with finite difference method for capacitor with one electrode corrugated (rectangular geometry) is only a few volts given that the voltages at electrodes $V_{1}=1500 \mathrm{~V}, V_{2}=600 \mathrm{~V}$.

Despite weak modulation, as seen below the non-zero grating formed at 4 microns, it should imply that threshold model (for electron tunneling and dissolution) is working.

\subsubsection{EDX measurements}

Silver concentration was analyzed with Energy Dispersive X-ray Spectroscopy (EDX) measurements at four spots: on the surface and on the bottom of the groove. Additional two measurements are done on the bleached and unbleached region after removing upper part by polishing away specimen material at a polished level as schematically shown in Fig. 3.17. The atomic silver concentration measurements at the four spots shown in Fig. 3.17 are given as weight fractions $f_{m}$. The values at points $1,2,3,4$ are $0.0714,0.0883$, 0.0237, 0.0211 respectively and they are approximated with exponentially decaying functions from the surface, $A_{1} \exp (-x / d)$ and $A_{2} \exp (-x / d)$ respectively for the bleached and unbleached regions. A representative SEM image of the glass sample and corresponding EDX spectroscopy results at the point specified in Fig. 3.17 as 4 (polished level) is shown in Fig. 3.18. Bleaching voltage and current versus time of the sample measured with EDX unit is shown in Fig. X21, bleaching temperature is $130^{\circ} \mathrm{C}$. Knowing groove depth of 300 $\mathrm{nm}$ the distance of polished level (spots 3,4 ) is estimated as $x_{1} \approx 4 \mu \mathrm{m}$. Another estimation of polished level based on silver doping profile obtained by assumption of diffusion from an unlimited source [54], i.e.

$$
C(x, t)=C_{s}\left(1-\operatorname{erf}\left(\frac{x}{2 \sqrt{D t}}\right)\right)
$$

and concentration under unbleached and bleached region from the surface as $A_{1}(1-\operatorname{er} f(x / d))$ and $A_{2}(1-\operatorname{erf}(x / d))$ gives the value $x_{1} \approx 5.5 \mu \mathrm{m}$. 


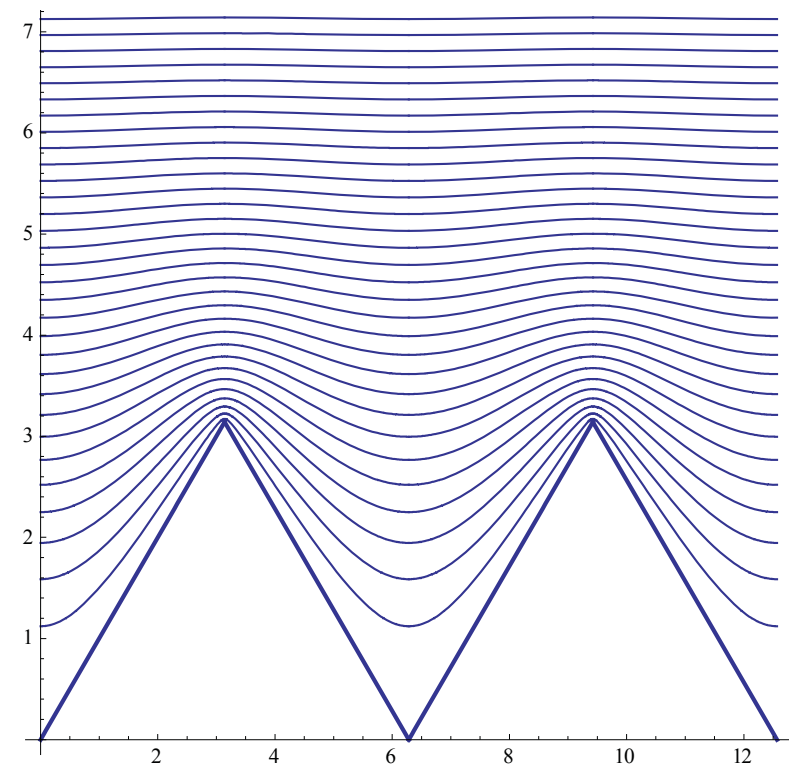

(a)

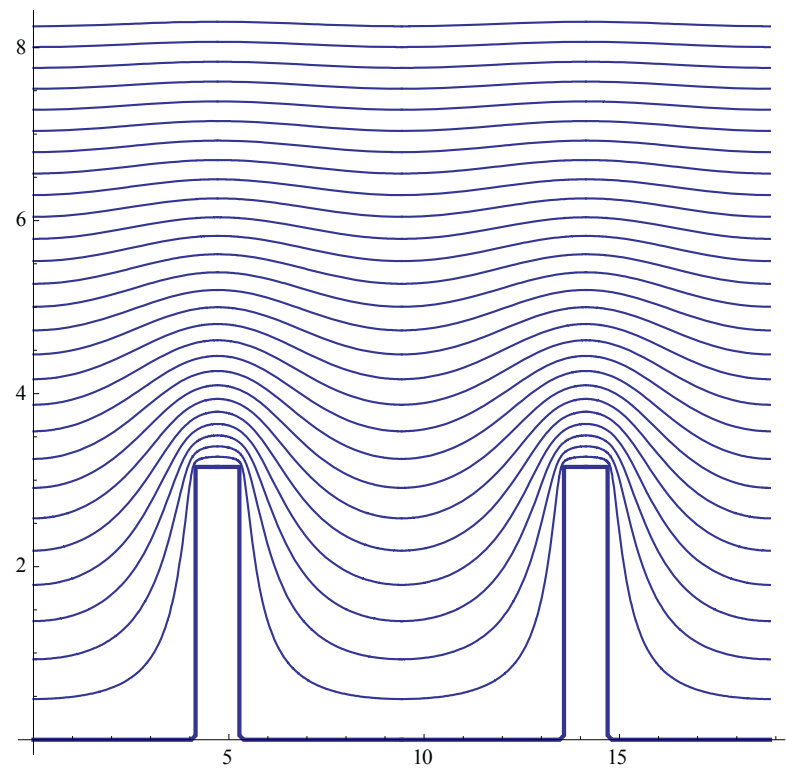

(b)

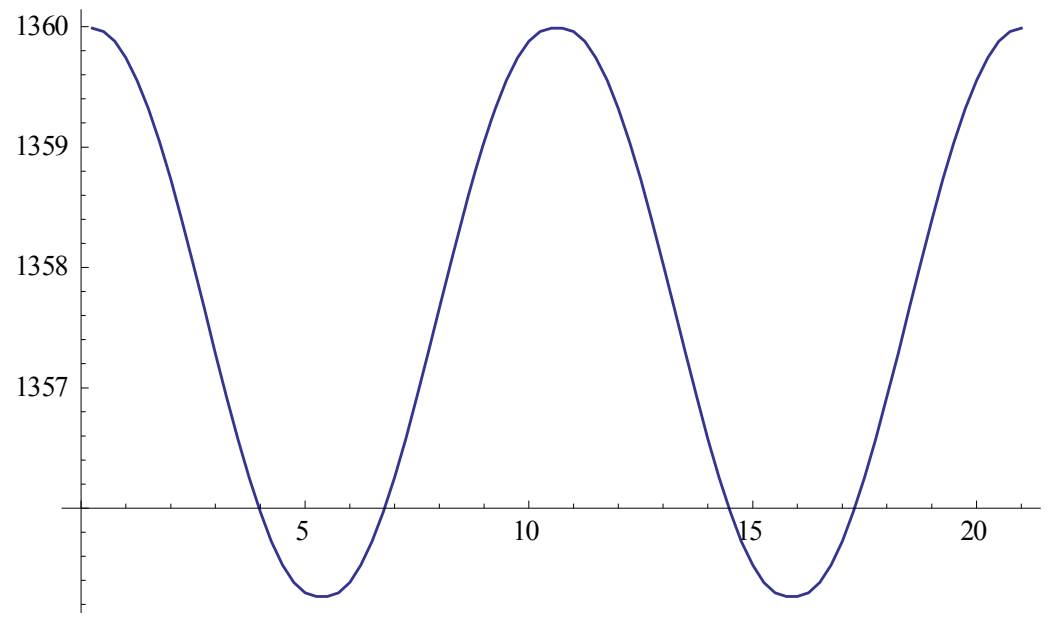

(c)

Figure 3.16: Equipotential lines above corrugated electrode (a) for triangular saw tooth, (b) for rectangular saw tooth, all dimensions in microns; (c) potential modulation for capacitor with one electrode corrugated (rectangular saw tooth) at 4 microns from corrugated side. Voltage on electrodes: $V_{1}=1500 \mathrm{~V}, V_{2}=600 \mathrm{~V}$, inner distance between electrodes $20 \mu \mathrm{m}$. Vertical and horizontal axes displayed in units of volts and microns respectively.

Dielectric constant of bleached and unbleached region at this distance can be evaluated by Maxwell Garnett mixing formula 3.1, where volume fraction $f_{v}$ is expressed through weight fraction $f_{m}$ as,

$$
f_{v}=\frac{f_{m}}{f_{m}+\left(1-f_{m}\right) \rho_{A g} / \rho_{I O G}}
$$




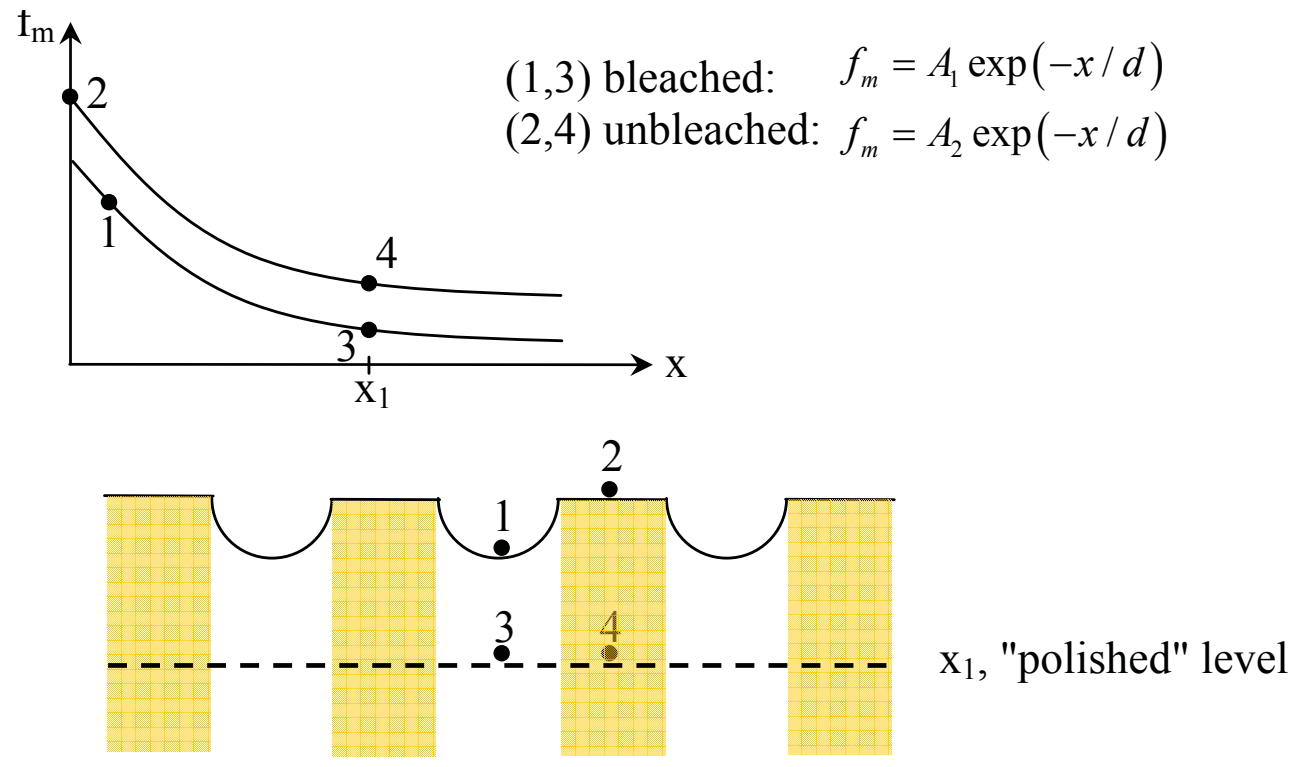

Figure 3.17: Fill factor distribution for bleached and unbleached region of the grating, distribution approximated by exponentially decaying function from the surface, bleached region differs from unbleached by a different constant factors: $A_{1}, A_{2}$.
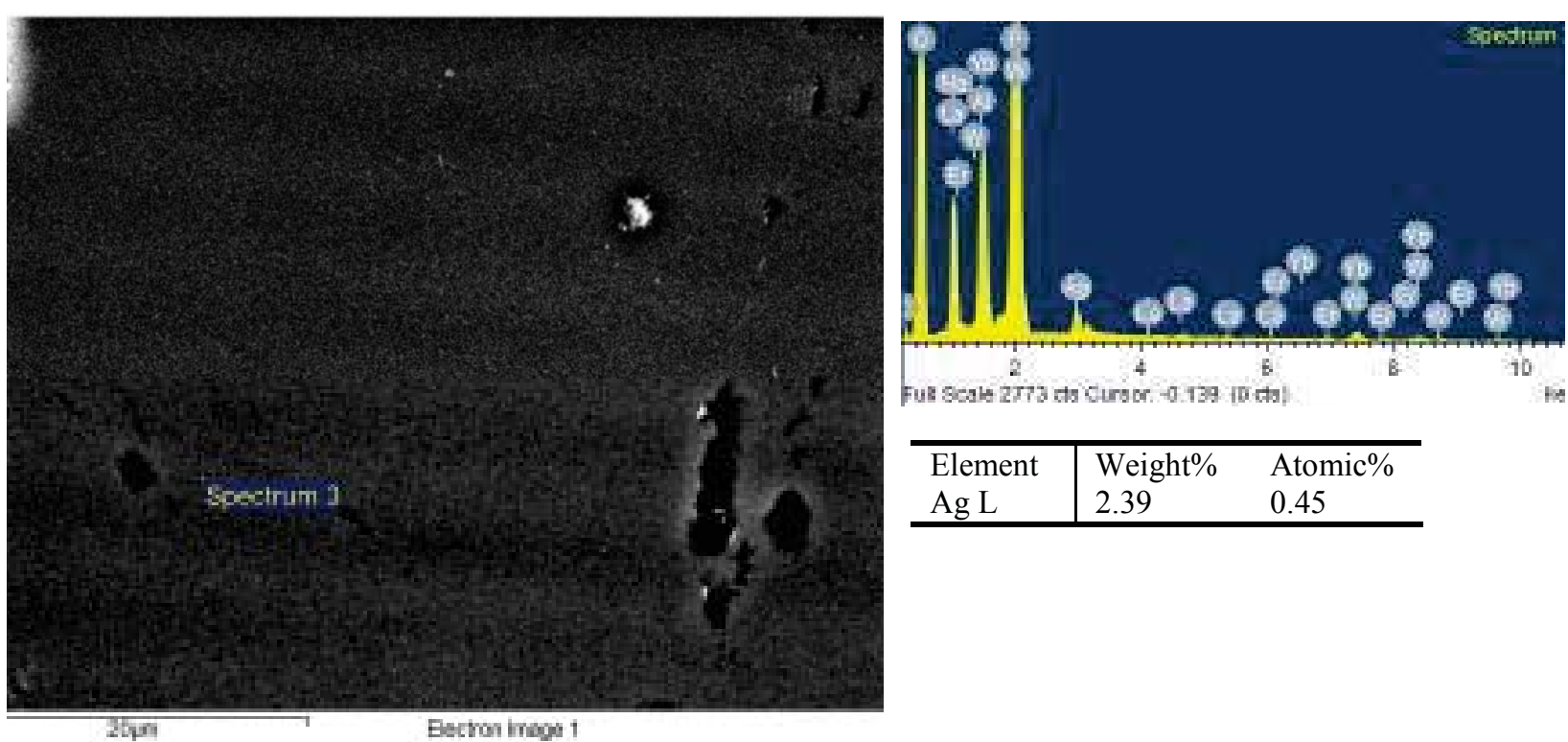

\begin{tabular}{l|ll}
\hline Element & Weight\% & Atomic\% \\
$\mathrm{Ag} \mathrm{L}$ & 2.39 & 0.45 \\
\hline
\end{tabular}

Figure 3.18: EDX measurements at polished level $x_{1}$.

$\rho_{A g}, \quad \rho_{I O G}$ are silver and IOG glass densities: $\rho_{A g}=10.49 \mathrm{~g} / \mathrm{cm}^{3}, \rho_{I O G}=2.74 \mathrm{~g} / \mathrm{cm}^{3}[55]$.

Knowing effective dielectric constant of bleached $\varepsilon_{e f f, b l}$ and unbleached $\varepsilon_{e f f, u n b}$ regions 
at $x_{1}$ from the surface we can calculate real and imaginary grating contrast,

$$
\begin{aligned}
& \operatorname{Re}(\Delta n(\lambda))=\operatorname{Re}\left(\sqrt{\varepsilon_{\text {eff,unb }}(\lambda)}\right)-\operatorname{Re}\left(\sqrt{\varepsilon_{\text {eff,bl}}(\lambda)}\right) \\
& \operatorname{Im}(\Delta n(\lambda))=\operatorname{Im}\left(\sqrt{\varepsilon_{\text {eff,unb }}(\lambda)}\right)-\operatorname{Im}\left(\sqrt{\varepsilon_{\text {eff,bl }}(\lambda)}\right)
\end{aligned}
$$

Plots of real and imaginary grating contrast versus wavelength is shown in Fig. 3.19, the peak values are 0.01 and 0.02 for real and imaginary parts respectively, they decay away from resonance, for telecom wavelength $1550 \mathrm{~nm}$ the values $1.7 \times 10^{-3}, 1.3 \times 10^{-5}$.

Permittivity of the host as a function of wavelength $\varepsilon_{h}(\lambda)$ was obtained form the Sellmeier coefficients of Er-doped phosphate glass provided by [56] that covers visible and near infrared range of wavelengths.

With extrapolated value of volume filling factor the refractive index change calculated at 10 microns for telecom wavelength $1550 \mathrm{~nm}$ is $2.6 \times 10^{-4}$ (real part) and $2.0 \times 10^{-6}$ (imaginary part) far less than at 4 microns, but still greater than the one photoinduced by UV-exposure.

\subsection{Conclusions}

In conclusion, we have tested experimentally periodically modulated electric field assisted dissolution of silver nanoclusters embedded in a phosphate glass.

1. In experimental part of this work the silver clusters formation in a hydrogen atmosphere followed by electric field assisted clusters dissolution was successfully demonstrated on a new type of phosphate glass, IOG-1, designed specifically for integrated optics application. Whereas most of related works, reported in the literature, were done on a more conventional silica based glass.

2. An attempt has been made to build a volume grating in a phosphate glass IOG-1 with field assisted silver clusters dissolution technique and periodically corrugated electrode. DC voltage modulation, periodic along the glass surface, is pretty weak at $4 \mu \mathrm{m}$ under the surface with amplitude modulation of a few volts, Fig.X. However, the electron and ion tunneling current from nanoclusters have a factor exponentially dependent on voltage potential. Therefore a weak modulation of the voltage may induce a moderate modulation of tunneling current and thus moderate modulation (spacially periodic) glass bleaching. The indirect estimation of spacial modulation of refractive index with period about $10 \mu \mathrm{m}$ based on EDX measurements shows $\operatorname{Re}(\Delta n) \approx 2.6 \times 10^{-4}$ at wavelength $1550 \mu \mathrm{m}$. Whether or not a true volume Bragg grating can be achieved with this approach remains an open question. The proper test would involve building channel waveguide, applying a bleaching technique with electrode period of about $0.5 \mu \mathrm{m}$ and final measurements of transmission or reflection of such waveguide at telecom wavelength.

3. Grating contrast estimation, based on Maxwell-Garnet extended mixing formula, predicts a Lorentzian type decay with wavelength. The resonance occurs at about $410 \mu \mathrm{m}$ 


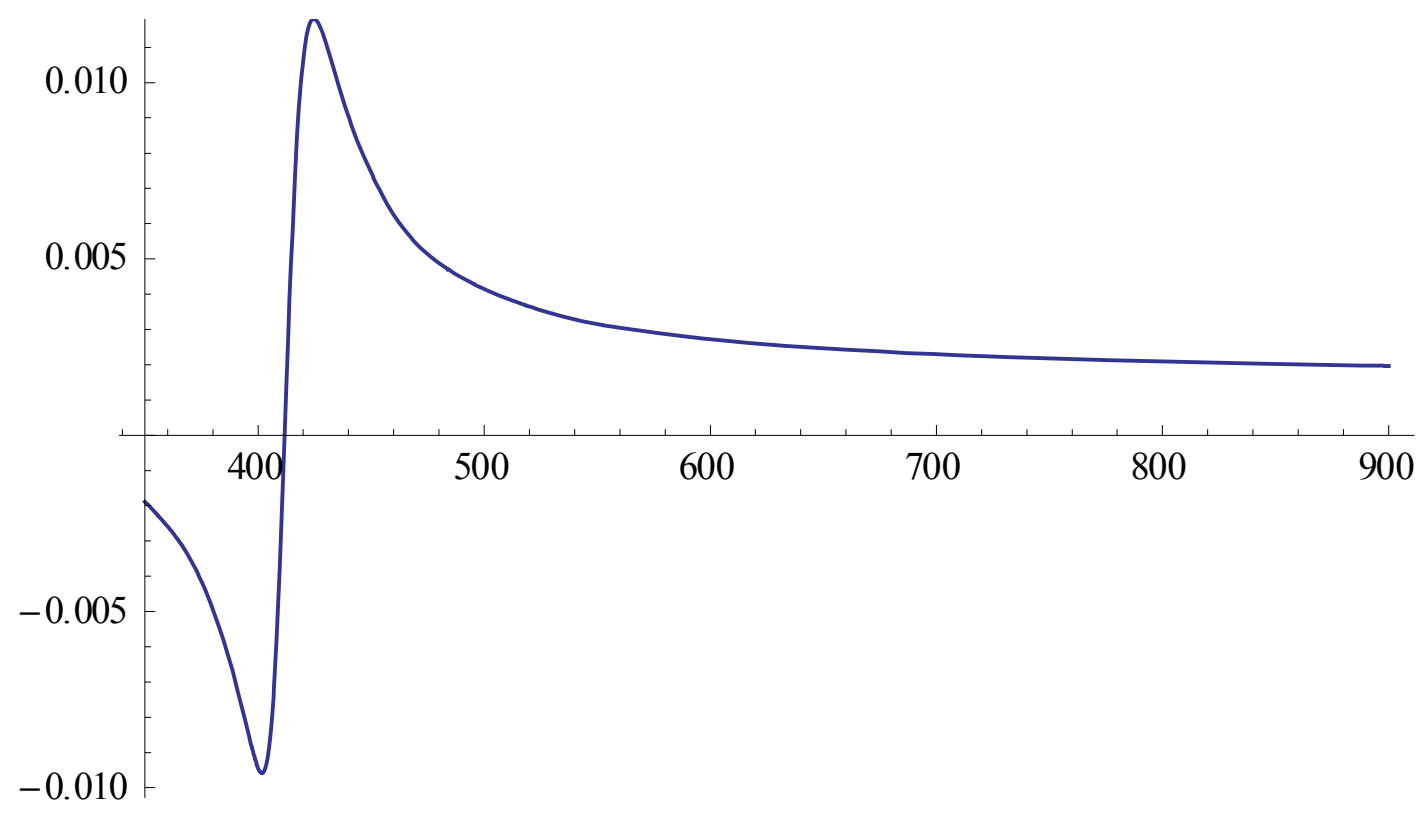

(a)

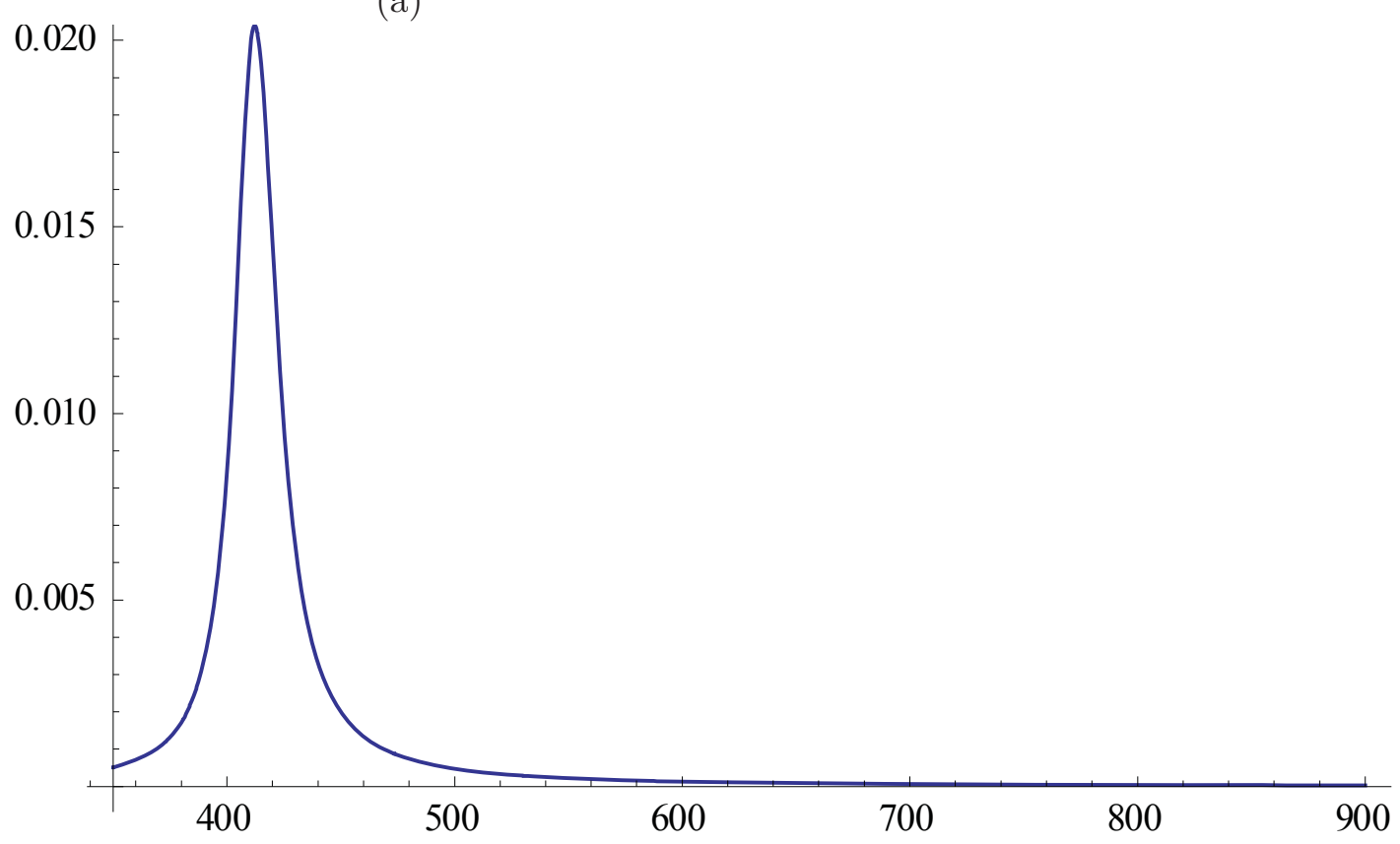

(b)

Figure 3.19: Refractive index change (a) real part and (b) imaginary part vs. wavelength of bleached IOG-1 glass with corrugated electrode based on EDX measurements, the wavelength axis in units of $\mathrm{nm}$.

which is also a wavelength of a plasmon resonance for a silver nanoclusters embedded in the glass. The advantage of a grating existing due to plasmon excitation but operating at a wavelength far off plasmon resonance is a two-fold. Since Lorentzian decay rate is 
much weaker than exponential, the real part refractive index change at a far off resonance wavelength, $1550 \mathrm{~nm}$ is still sufficient for Bragg grating. Yet decaying imaginary part of refractive index change results in reducing losses associated with plasmon resonance.

4. By choosing properly parameters of the procedures (ion-exchange, annealing in air, annealing in $H_{2}$ ) absorption spectra shape can be controlled, namely peak height, peak width and to some extent the peak wavelength.

5. Simulation largely underestimates the height of absorption peak compare to (with respect to) experimental data. However the cluster radius that maximizes absorption peak is estimated at around $r \approx 12 \mathrm{~nm}$ which is relevant to TEM image of the glass sample where cluster diameters are in the range of $20-30 \mathrm{~nm}$. It also predicts moderately well resonance wavelength but overestimates absorption width. However the trend of absorption broadening with fill factor represented correctly due to dipole approximation of extended Maxwell-Garnet formula that allows taking into account dipolar coupling. Model that assumes finite thickness of cluster layer fails to show any significant dependence of absorption band on that thickness.

6. Grating made with field assisted bleaching in glass metal nanocomposites is not only a grating of refractive index change but also a grating of nonlinearity change. The grating of nonlinearity has two contributions. One related to a grating of Kerr type nonlinearities due to silver concentration being modulated. The unbleached region, i.e. the region of the glass that was not in direct contact with electrode, has greater silver concentration and therefore greater Kerr type nonlinearity. The second contribution comes from the fact that high DC voltage applied to the glass breaks down the symmetry of the glass matrix and therefore introduces nonlinearity to a glass, such process called glass poling. Nonlinearity of the type second-harmonic generation, SHG of a poled glass IOG-1 was studied in [57]. The unbleached region of the glass has glass matrix poled less and therefore has smaller poled nonlinearity.

The grating of poled nonlinearity probably decays faster with the depth since exponential dependence on voltage is not likely to happen. Therefore two contributions to a grating of nonlinearity are out of phase to each other and one decays faster with the depth than the other. Periodic bleaching also could lead to periodic energy transfer from $\mathrm{Ag}$ to

$E r$ and $Y b$. Energy transfer from $A g$ to $E r$ in silicate glass discussed in [58] and to $Y b$ in oxyfluoride glass discussed in [59].

7. Grating with intrinsic losses related to absorption and scattering, might be considered as a saturable absorber in certain applications.

\subsection{Plan for future experimental work}

Volume Bragg grating based on electric field assisted bleaching of glass metal nanocomposites may or may not be implemented therefore we should explore some other alternatives.

In a paper [60] the physical limitations imposed on a grating properties made by means of ion-exchange process and electrically stimulated ion-exchange are theoretically analyzed. 
Grating made by thermal ion-exchange in glass is of the type Raman Nath, i.e. grating doesn't penetrate deep under the surface. The reason behind is that diffusion coefficient in transverse direction is the same as in lateral. Therefore grating made by ion-exchange under the surface deeper than roughly a grating period is simply washed out. For an ion-exchange process stimulated by electric field the ions migration is not only due to diffusion (driven by temperature) but due to drift (driven by electric field) as well. However, to achieve volume grating (deep grating) requires substantially increase drift factor and corresponding electric field in glass should reach the value larger than typical dielectric strength of the glass. I.e. most of the glass can not sustain electric field needed for making deep grating with field assisted ion-exchange.

This suggests that a bypass of existing limitations can be accomplished by breaking down locally isotropy of the glass near the surface. This would make diffusion process anisotropic, i.e. predominantly directed.

It was found that silica based glass that undergoes femtosecond laser exposure acquired characteristic modifications in the glass structure, $[61,62]$. The energy bandgap of a fused silica is $7.5 \mathrm{eV}$ while the energy of a photon at $\lambda=800 \mathrm{~nm}$ is $1.55 \mathrm{eV}$, therefore one photon ionization of a silica is not possible, however for a femtosecond laser beam intensity is high enough so that a multiphoton ionization of a silica takes place. Such ionization leads to a plasma creation in the glass and subsequent plasma coupling with incident light. The interference pattern of such coupling can make permanent modification of the glass structure in the form of nanocracks (nanogratings) on and under the surface of the glass sample, [61]. The orientation of nanocracks depend on light polarization and direction of moving laser beam with respect to glass which is illustrated in Fig. 3.20, [62].
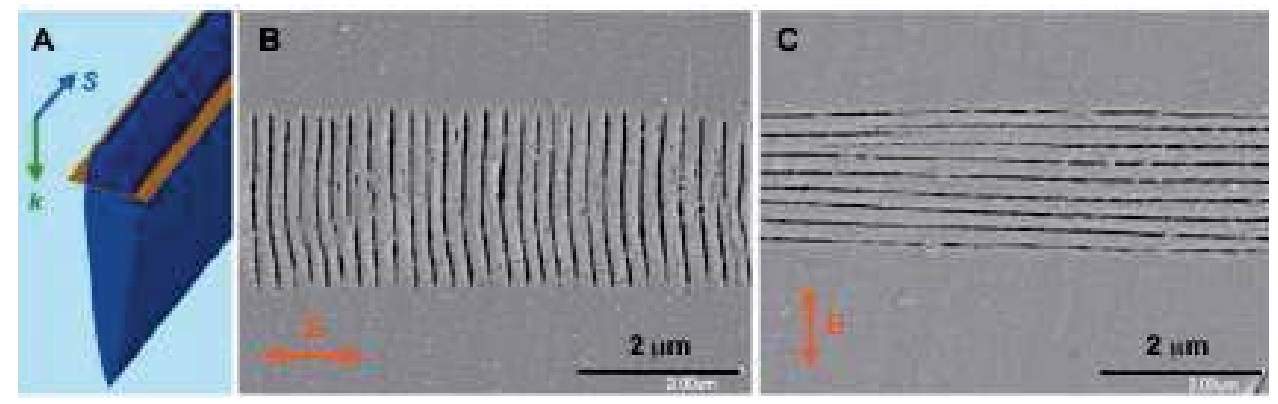

Figure 3.20: SEM images of self-organized periodic nanoplanes. A: The light propagation direction $k$, the writing direction $S$. B: $E$ is parallel to $S$. Panel C: $E$ is perpendicular to $S$. Nominal separation of the grating planes is $250 \mathrm{~nm}$, [62].

Typical geometrical parameters of nanocracks are: thickness 5 - $30 \mathrm{~nm}$ [62], width depends on light polarization and writing direction, [62], Fig. 3.20; depth $10-15 \mu m$ [62], $4 \mu \mathrm{m}$ [63]; nominal period $250 \mathrm{~nm}$ [62], can be adjusted by energy pulse [61], Fig. 3.21.

Assuming that femtosecond laser pulse exposure can modify IOG-1 glass in a similar fashion, the following steps can be employed to locally induce anisotropy under the glass surface and build a volume Bragg grating. 


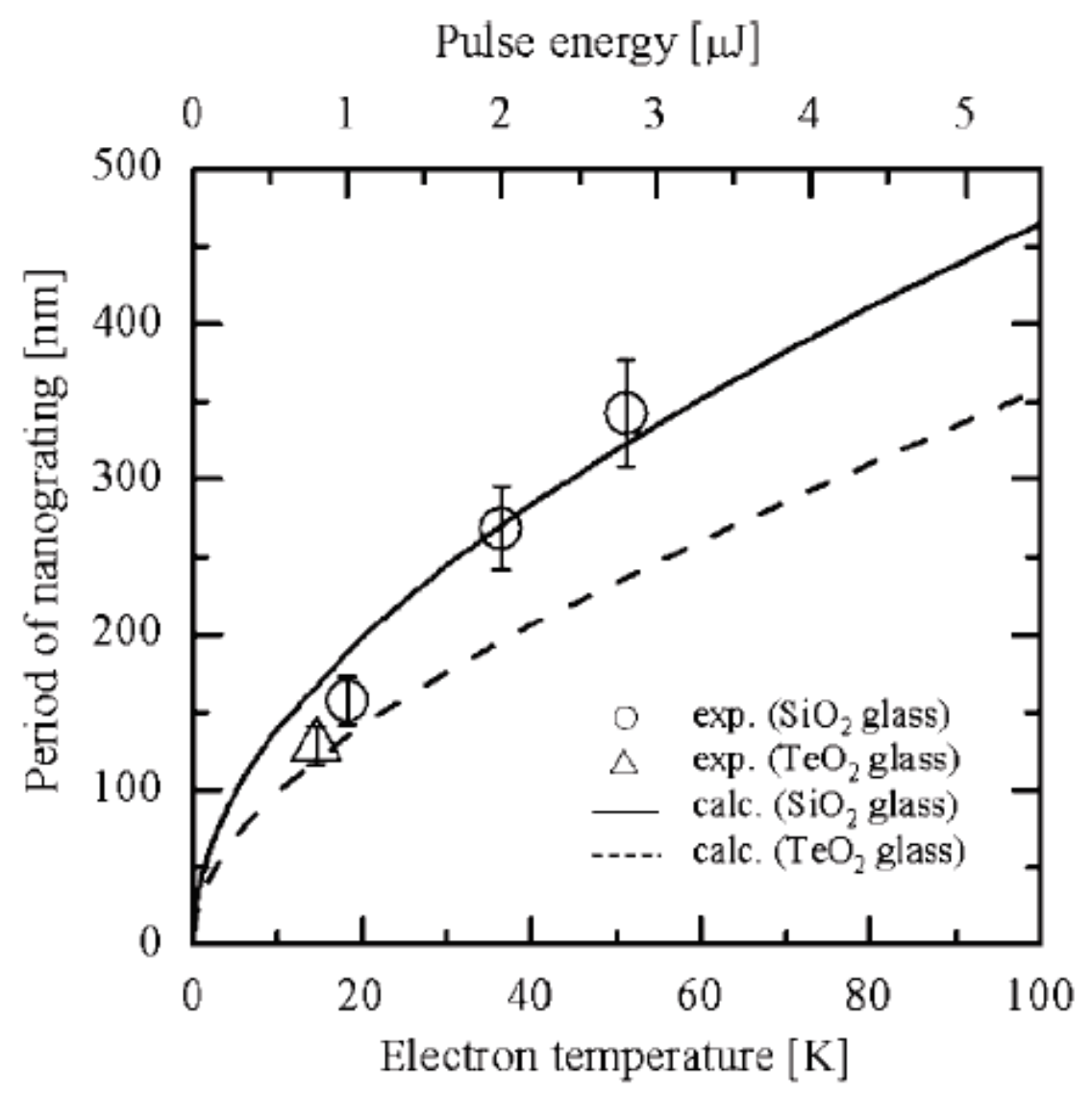

Figure 3.21: Pulse energy dependence of the experimental nanograting period of silica glass and tellurium dioxide single crystal. Theoretical curves of nanograting period versus electron temperature are also shown, [61].

The process consists of masking surface with period of Bragg grating (1), femtosecond laser beam exposure that induces nanocracks in the mask openings (2), coating with silver film (3), performing ion-exchange (4) and surface cleaning (5). Two samples prepared with the same mask aligned together (6) and placed in the oven for annealing in air (7). Schematic diagram of these steps is shown in Fig. 3.22. In the last step silver ions redistributed down the surface in each sample. In addition the silver ions diffuse from the sample with larger concentration into another. Such cross diffusion essentially makes bonding between two glass samples and combines two separate waveguides in a single one. The bonding might occur in the glass region outside of the waveguide region if two samples initially would have slightly different composition, say with amount of $\mathrm{Na}_{2} \mathrm{O}$ or $\mathrm{Al}_{2} \mathrm{O}_{3}$ different by $0.5 \%$, then interdiffusion between these ions across two samples would bond them in the cladding region as well.

The bonding ability of IOG-1 glass was demonstrated by Schott company in [64]. Polishing both surfaces, applying pressure and moderate heat makes two surfaces bonded, this technique, low temperature bonding, developed for the purpose of making hybrid substrate 


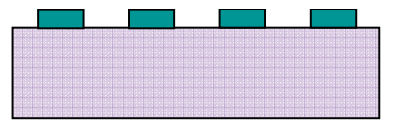

(1) mask

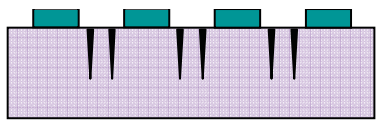

(2) femtosecond laser exposure

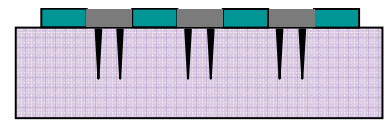

(3) Ag film

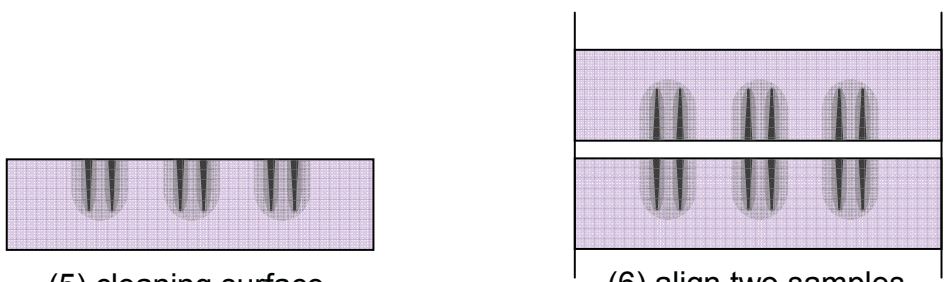

(6) align two samples

(4) ion exchange, $\mathrm{T}=95 \mathrm{C}$

(5) cleaning surface

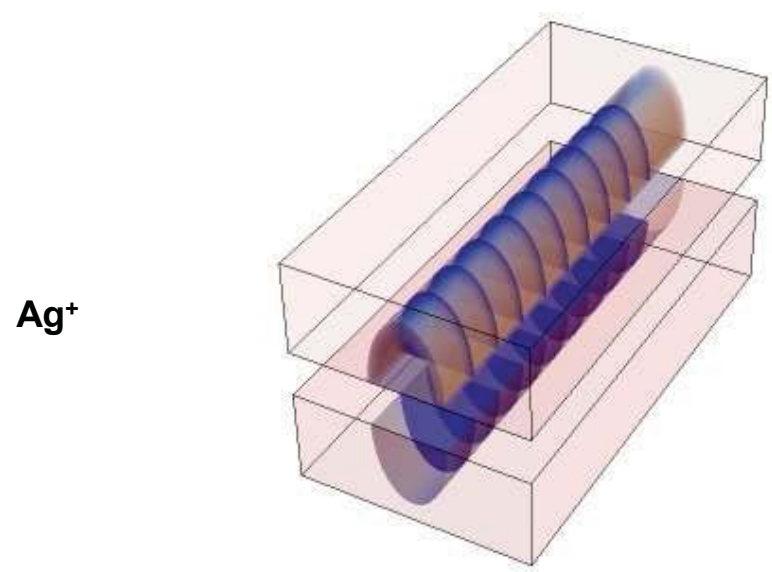

(7) annealing in air, $\mathrm{T}=300 \mathrm{C}$

Figure 3.22: Schematic steps of building a volume Bragg grating in IOG-1 glass with femtosecond laser exposure, ion-exchange and low temperature bonding processes.

where active and passive glass joined together, Fig. 3.23.

Mechanical quality of the joint is so good that glass can sustain cutting into several slices and polishing each slice. The images of two row blocks of active and passive glass and final hybrid substrate presented in Fig. 3.24.

The waveguide built on a hybrid substrate that runs across the joint is shown in Fig. 3.25. To verify high optical quality of the joint the laser beam at wavelength coupled in waveguide. The light propagates in the passive part of the waveguide and remains invisible, while in the active side of the waveguide it causes fluorescence.

Therefore, inducing nanocracks by femtosecond laser pulse, applying ion-exchange and low temperature bonding processes probably a good way of building volume Bragg grating in IOG-1 glass. 

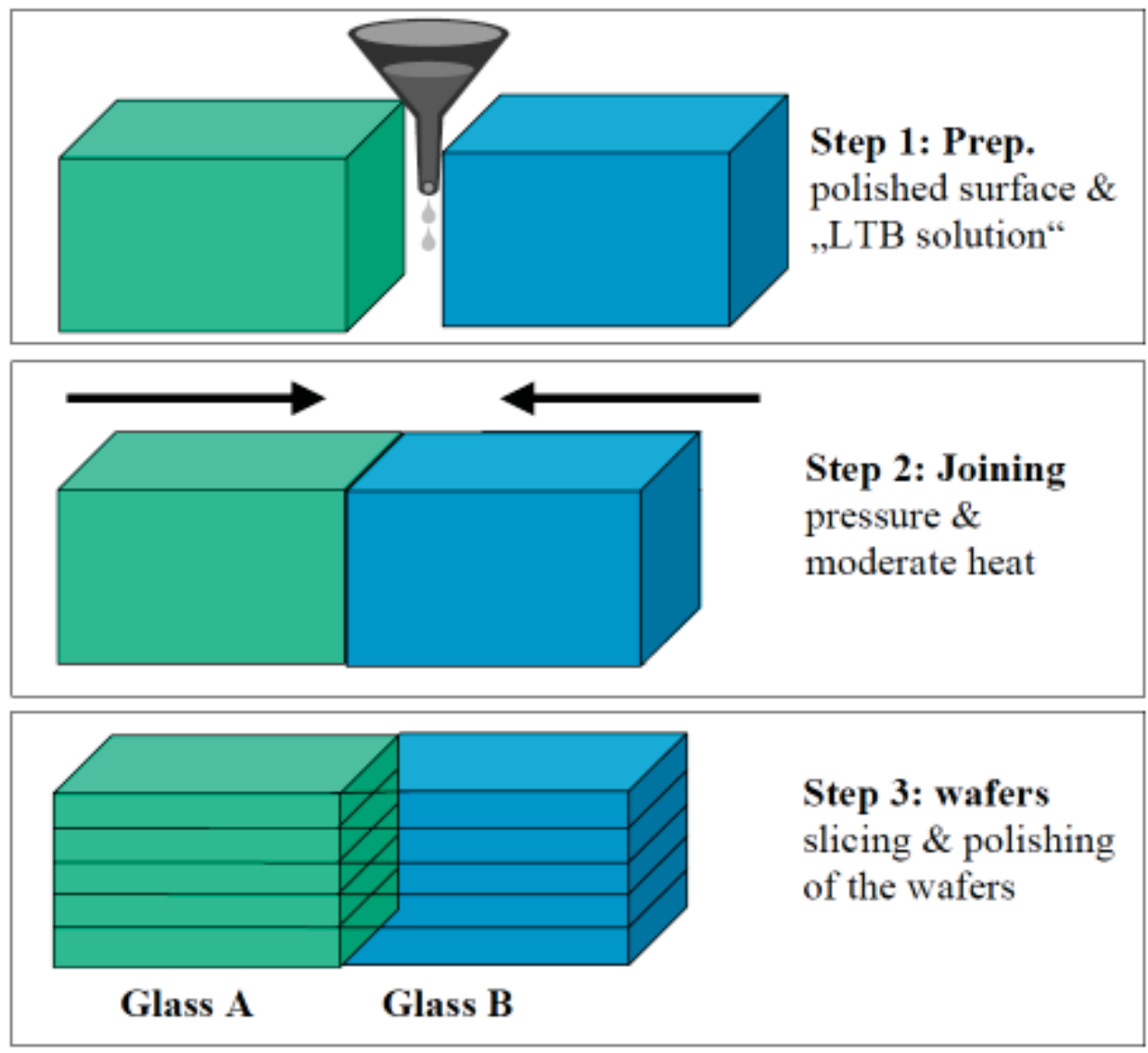

Figure 3.23: Schematic diagram explaining the Low Temperature Bonding process as applied to mass production of hybrid substrates for integrated optic photonic applications, [64]. 


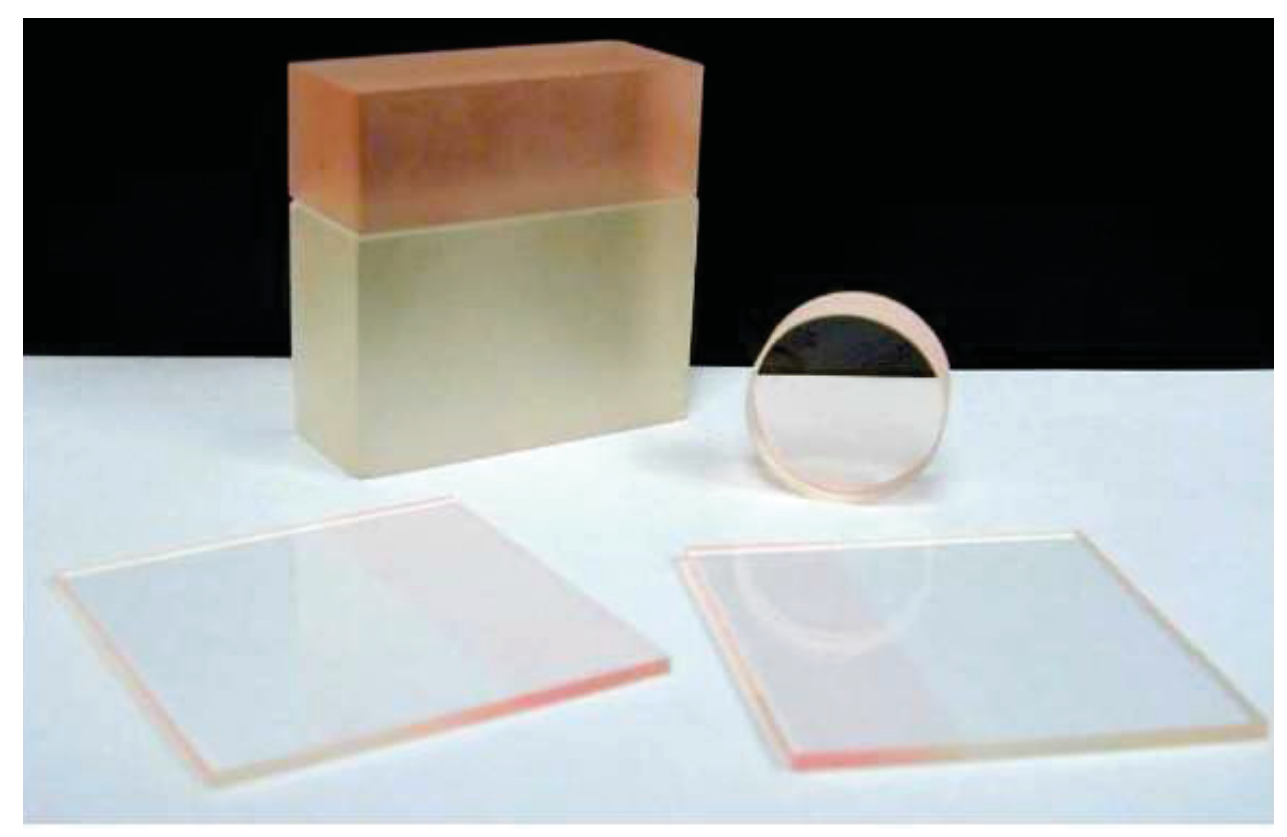

Figure 3.24: Photograph showing a hybrid preform and several hybrid substrates prepared from active Er/Yb doped phosphate glass (orange) and passive phosphate glass (clear), [64].

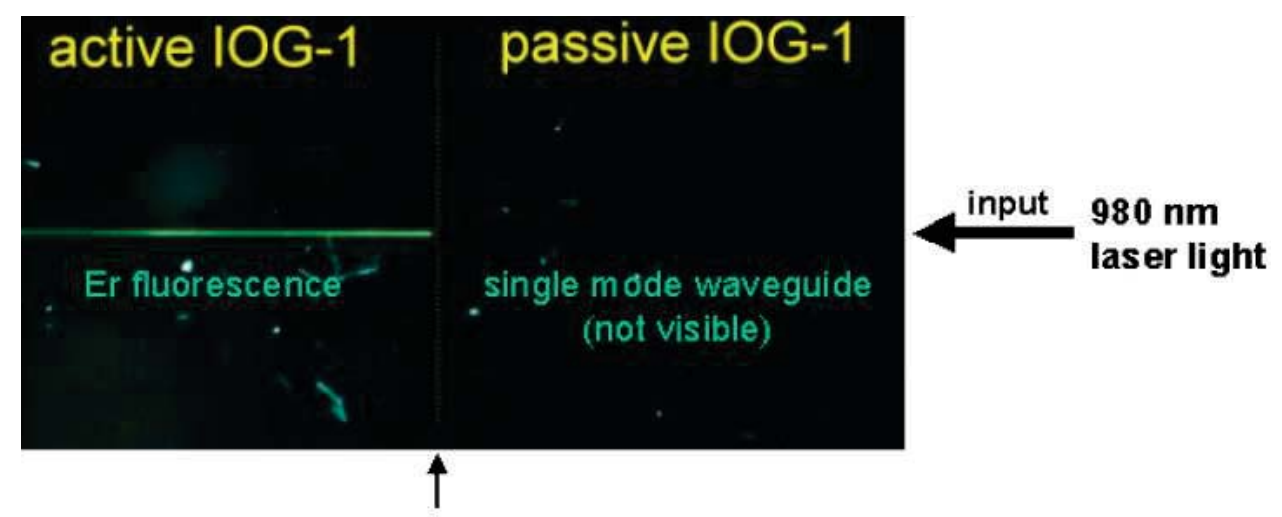

LTB joint

Figure 3.25: Single mode waveguide though a Low Temperature Bonding interface between active (i.e. Er doped) and passive phosphate glass IOG-1 (VIS microscope image), [64]. 


\section{Chapter 4}

\section{Plasmon excitation in a waveguide with planar geometry}

In this chapter a model of plasmon excitation in a metal slab waveguide illuminated by an optical antenna, i.e. by a finite size of current source oscillating at optical frequencies, is developed and tested against well known experimental data.

Scattering due to a set of tilted sheets as an impressed current source placed under the metal slab is analyzed with Green's function formulation. Scattered field then applied for a plasmon excitation in a thin metal film.

\subsection{Probing plasmons by measuring reflectivity in Kretschmann experiment.}

Surface Plasmon polariton (SPP) excitation by optical means is done in the Kretschmann type experimental setup [65] schematically depicted in Fig. 4.1. With the following materials: glass BK7 for a prism, silver for a metal film and air for environmental background under room temperature and normal pressure and with the incident light at the He-Ne laser wavelength $\lambda_{0}=632.8 \mathrm{~nm}$ the refractive indices of each media in the setup are given by $[66-68]$,

$$
\begin{aligned}
& n_{1}=n_{B K 7}=1.51509 \\
& \varepsilon_{A g}=-19+i 0.53, \quad n_{2}=\sqrt{\varepsilon_{A g}} \\
& n_{3}=n_{\text {air }}=1.0002718
\end{aligned}
$$

The thin silver film in experiment is illuminated by a p-polarized laser beam from the optically denser media (glass BK7). The excited plasmon appears propagating on the film interface with the media of a lesser optical density (air). Assuming for a moment no metal film present in the setup, the interface of two mediums glass-air is illuminated by a laser beam approximated as a plane wave of a gradually increasing incident angle $\alpha_{i n}$ from zero 


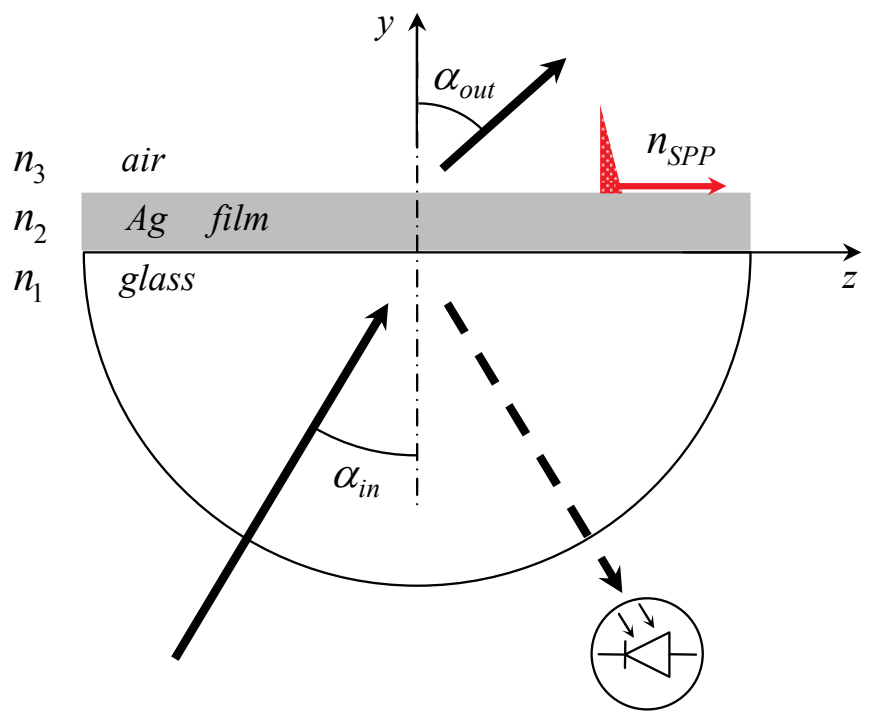

Figure 4.1: Plasmon excitation in Kretchmann experiment by laser beam and plasmon probing on reflection

until it reaches the critical angle $\alpha_{c}$ at which point the transmitted light propagates along the interface of two mediums, i.e. the transmitted angle $\alpha_{\text {out }}$ matches 90 degrees. From the Snell's law,

$$
n_{1} \sin \alpha_{\text {in }}=n_{3} \sin \alpha_{\text {out }}
$$

with the given above refractive indices the critical angle is found to be $\alpha_{c}=41.3^{\circ}$. Past this angle due to a total internal reflection (TIR) all the incident energy reflected back into the incident media, however the transmitted field

$$
\vec{E}_{t}=\vec{E}_{t 0} \exp \left[i\left(\vec{k}_{3} \cdot \vec{r}-\omega t\right)\right]
$$

is not identically equals to zero. Eliminating angle $\alpha_{\text {out }}$ from the phase factor $\vec{k}_{3} \cdot \vec{r}$,

$$
\vec{k}_{3} \cdot \vec{r}=k_{3}\left(z \sin \alpha_{\text {out }}+y \cos \alpha_{\text {out }}\right)
$$

via Snell's law 4.2, the transmitted field is found to be given by [69],

$$
\vec{E}_{t}=\vec{E}_{t 0} \exp \left[i k_{3} z \frac{n_{1}}{n_{3}} \sin \alpha_{i n}\right] \exp \left[\mp k_{3} y \sqrt{\left(\frac{n_{1}}{n_{3}} \sin \alpha_{i n}\right)^{2}-1}\right] \exp [-i \omega t]
$$

It follows from 4.5 that above the critical angle the transmitted field in media 3 is evanescent, i.e. exponentially decaying with $y$. If now the metal film is introduced back 
between two mediums its presence allows sustain the evanescent field via the plasmon mode whose field is known to be evanescent in y therefore plasmon must be excited at the incident angle greater than critical angle. It is known from experiment that such incident angle is only slightly above the critical angle, thus a rough estimation for the real part of the plasmon effective index can be done through the Snell's law,

$$
\operatorname{Re}\left(n_{e f}\right)=n_{1} \sin \alpha_{i n}=n_{1} \sin \left(\alpha_{c}+\delta \alpha\right), \quad 0<\delta \alpha \ll 1
$$

Since sine function is monotonically increasing in the interval $(0, \pi / 2)$ it follows,

$$
n_{1} \sin \left(\alpha_{c}+\delta \alpha\right)=n_{1} \sin \alpha_{c}+\delta n=n_{3}+\delta n, \quad 0<\delta n \ll 1
$$

Therefore the real part of the plasmon effective index in Kretschmann experimental setup is just above the environmental refractive index $n_{3}$,

$$
\operatorname{Re}\left(n_{e f}\right)=n_{3}+\delta n, \quad 0<\delta n \ll 1
$$

\subsubsection{Plasmon excitation by plane wave.}

Reflectivity $R\left(\alpha_{i n}, d\right)$ in the setup versus an incident angle $\alpha_{i n}$ and versus film thickness $d$ for this setup under assumption that incident field is a plane wave is given by [70,71],

$$
R\left(\alpha_{i n}, d\right)=\left|\frac{r_{12}\left(\alpha_{i n}\right)+r_{23}\left(\alpha_{i n}\right) \exp \left(i 2 d k_{z}\left(\alpha_{i n}\right)\right)}{1+r_{12}\left(\alpha_{i n}\right) r_{23}\left(\alpha_{i n}\right) \exp \left(i 2 d k_{z}\left(\alpha_{i n}\right)\right)}\right|^{2}
$$

where $r_{12}$ and $r_{23}$ reflection coefficients from interface 1-2 and 2-3 respectively given by,

$$
\begin{aligned}
& r_{12}\left(\alpha_{i n}\right)=\frac{n_{2} \cos \alpha_{i n}-n_{1} \sqrt{1-\left(\left(n_{1} / n_{2}\right) \sin \alpha_{i n}\right)^{2}}}{n_{2} \cos \alpha_{i n}+n_{1} \sqrt{1-\left(\left(n_{1} / n_{2}\right) \sin \alpha_{i n}\right)^{2}}} \\
& r_{23}\left(\alpha_{i n}\right)=\frac{n_{3} \sqrt{1-\left(\left(n_{1} / n_{2}\right) \sin \alpha_{i n}\right)^{2}}-n_{2} \sqrt{1-\left(\left(n_{1} / n_{3}\right) \sin \alpha_{i n}\right)^{2}}}{n_{3} \sqrt{1-\left(\left(n_{1} / n_{2}\right) \sin \alpha_{i n}\right)^{2}}+n_{2} \sqrt{1-\left(\left(n_{1} / n_{3}\right) \sin \alpha_{i n}\right)^{2}}} \\
& k_{z}\left(\alpha_{i n}\right)=\left(2 \pi / \lambda_{0}\right) n_{2} \sqrt{1-\left(\left(n_{1} / n_{2}\right) \sin \alpha_{i n}\right)^{2}}
\end{aligned}
$$

Derivation $R\left(\alpha_{i n}, d\right)$ is based on Fresnel's coefficients for each single interface and, optionally, on transfer matrix approach.

Plot of reflectivity with indices in 4.1 is shown in Fig. 4.2, where a strong absorption observed in the parameter window $\left(\alpha:\left(42.6^{\circ}, 43.1^{\circ}\right), d:(30 \mathrm{~nm}, 70 \mathrm{~nm})\right)$. The maximum reflectivity with $d=52 \mathrm{~nm}$ is plotted in Fig. 4.3. Experimental data $[72,73]$ agrees well with this model, call it M1. The model based on Fresnel's coefficients relies in plasmon probing entirely on measuring reflectivity of a thin metal film and not distinguishing between plasmon modes that may coexist in the film. 

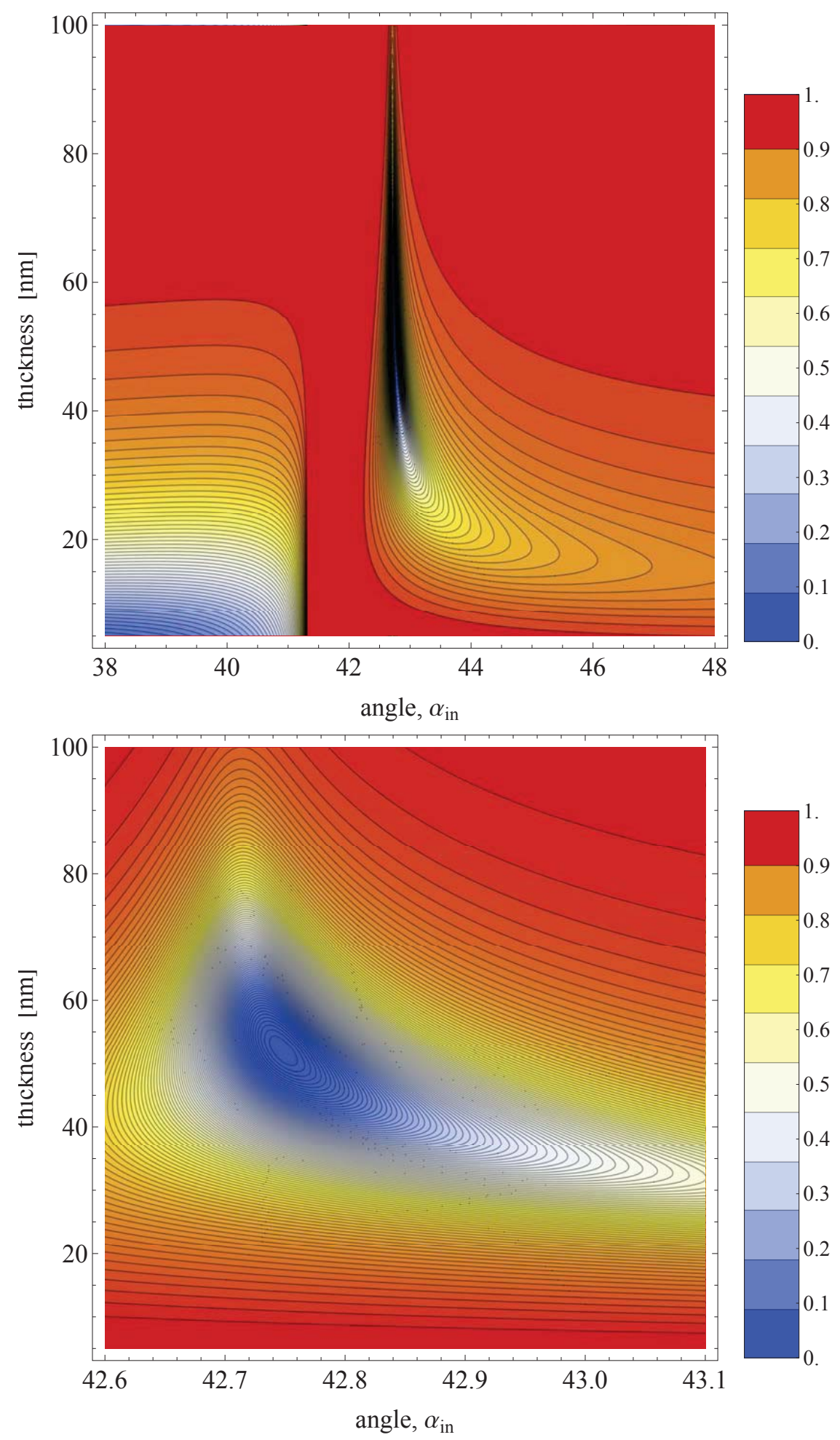

Figure 4.2: (top) Contour plot of reflectivity versus incident angle of plane wave and thickness of silver film in Kretchmann experiment; (bottom) its zoomed version near the angle $42.6^{\circ}-43.1^{\circ}$ 


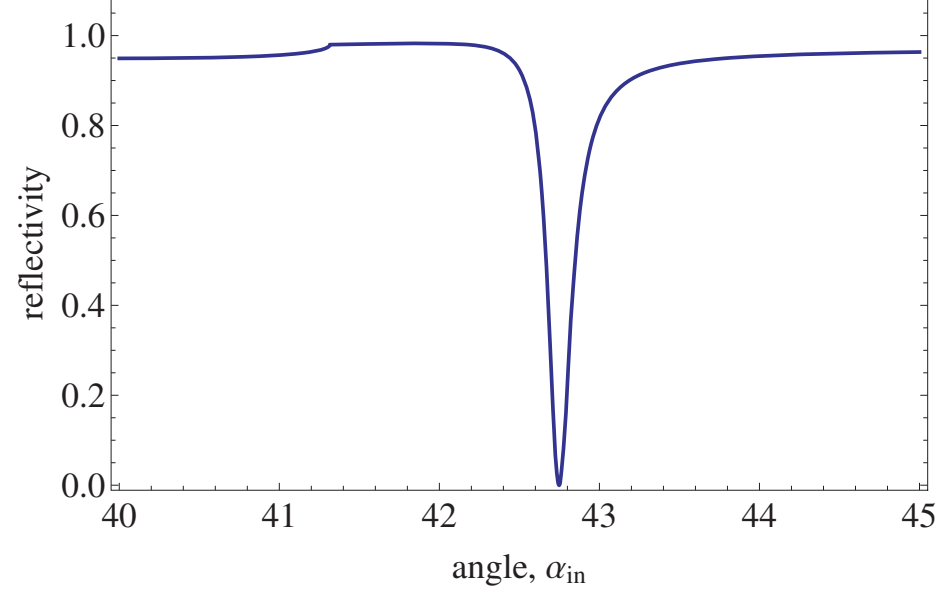

Figure 4.3: Plot of reflectivity versus incident angle at film thickness $d=52 \mathrm{~nm}$

\subsubsection{Waveguide approach for plasmon in thin film}

Another model (call it model M2) of plasmons in a film treats the thin film as a slab waveguide and is capable of finding plasmon modes by solving dispersion equation. Detailed comprehensive analysis of SPP guided by thin film taking into account losses is covered in [67]. Dispersion equation for bounded TM slab modes based on Maxwell's equations and matching boundary conditions for TM mode at each interface is given by [67],

$$
\gamma_{2} \varepsilon_{r 2}\left(\gamma_{3} \varepsilon_{r 1}+i q_{1} \varepsilon_{r 3}\right)+\left(i q_{1} \gamma_{3} \varepsilon_{r 2}^{2}+\gamma_{2}^{2} \varepsilon_{r 1} \varepsilon_{r 3}\right) \tanh \left(d \gamma_{2}\right)=0
$$

where $q_{1}^{2}=k_{0}^{2}\left(n_{1}^{2}-n_{e f}^{2}\right), \gamma_{2}^{2}=k_{0}^{2}\left(n_{e f}^{2}-n_{2}^{2}\right), \gamma_{3}^{2}=k_{0}^{2}\left(n_{e f}^{2}-n_{3}^{2}\right), k_{0}=2 \pi / \lambda_{0}, \varepsilon_{r i}=n_{i}^{2}$ and $n_{e f}$ mode index.

Dispersion equation 4.10 has several roots. Only the roots near refractive index of media 3 (media of lesser density) considered here as the potential modes that can be optically excited. In deriving 4.10 it was implicitly assumed that the mode is bounded (localized) at both interfaces, however this is not guaranteed and in fact it may not be so over the whole range of parameters as seen later. In media 1 the field distribution has the factor $e^{i q_{1} y}=e^{i \operatorname{Re}\left(q_{1}\right) y} e^{-\operatorname{Im}\left(q_{1}\right) y}$. Whether or not the field is bounded depends on the sign of imaginary part of $q_{1}$, which in turns depends on the mode effective index $n_{e f}=\operatorname{Re} n_{e f}+i \operatorname{Im} n_{e f}$. If an imaginary part of effective index $\operatorname{Im} n_{e f}$ crosses zero, then the imaginary part of $q_{1}$ crosses zero as well. Essentially, $\operatorname{Im} n_{\text {ef }}$ behavior controls the filed distribution in media 1. It turns out that $\operatorname{Im} n_{e f}$ an imaginary part of effective index $n_{e f}$, calculated from dispersion equation SPP 4.10 with film thickness decreasing from 100 to 10 $\mathrm{nm}$, changes the sign and so does $\operatorname{Im} q_{1}$ an imaginary part of $q_{1}$. This means that the field in media $1, e^{i \operatorname{Re}\left(q_{1}\right) y} e^{-\operatorname{Im}\left(q_{1}\right) y}$ varies from being localized near the interface to being unbounded, 
i.e. growing away from the interface. The actual transition happens at a critical thickness $d=d_{0}$ whose value found to be equal to $52 \mathrm{~nm}$. This is schematically illustrated in Fig. 4.4. The field distribution remains fully localized only for the thicknesses greater than the critical value $d>d_{0}$ standing for the SPP mode bounded at both interfaces, then turns into pure plane wave in media 1 right at the critical thickness $d=d_{0}$ and becomes exponentially unbounded in media 1 for the thicknesses less than critical $d<d_{0}$ representing the SPP mode bounded on one side only. This branch of solution 4.10 is known as symmetric bound wave [67]. It behaves in a similar fashion in the lateral direction as well, i.e. exponential growth along $z$ axis until film thickness reaches $d_{0}$ followed by exponential decay for thickness greater than $d_{0}$.

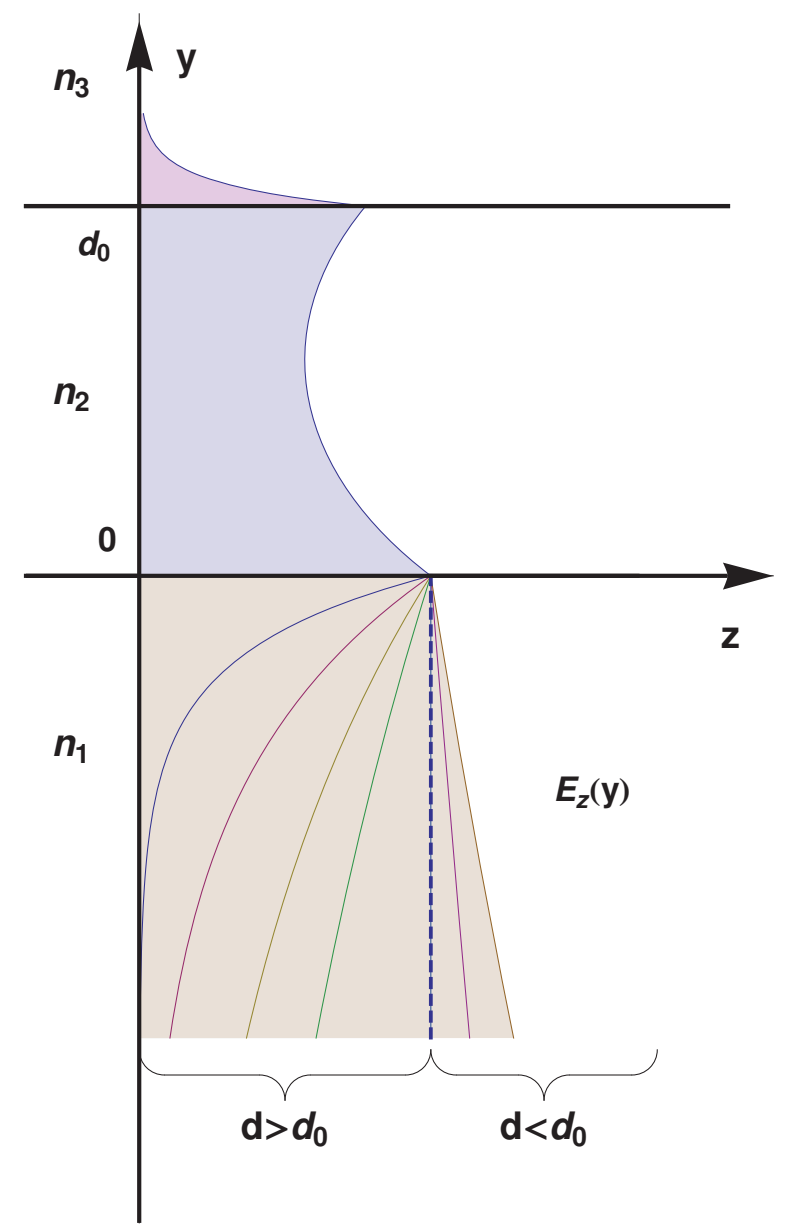

Figure 4.4: Field distribution along y axis of a symmetric bound SPP mode with a film thickness as parameter: at a critical thickness $d_{0}=52 \mathrm{~nm}$ the field in the glass is a plane wave, at thickness greater than critical $d>d_{0}$ the field in the glass is evanescent, at thickness below the critical $d<d_{0}$ the field in the glass exponentially grows away from glass-metal interface.

Another possible independent field distribution at media 1 has a factor $e^{-i q_{1} y}=e^{-i \operatorname{Re}\left(q_{1}\right) y} e^{\operatorname{Im}\left(q_{1}\right) y}$. Such field distribution has its own dispersion equation obtained 
from 4.10 where each term $q_{1}$ replaced by $-q_{1}$. Imaginary part of an effective index $\operatorname{Im} n_{e f}$ of newly obtained dispersion equation does not change the sign over whole possible range of film thickness, for this reason the field distribution in media 1 in this case remains unbounded throughout. This case represents a symmetric leaky wave [67]. Behavior of an effective mode index for symmetric bound and symmetric leaky wave branches of real and imaginary parts over film thickness range from $10 \mathrm{~nm}$ to $110 \mathrm{~nm}$ and a zoomed version of imaginary parts only (the ones that dictate field distribution by changing the sign at $d_{0}=52.2 \mathrm{~nm}$ ) is shown in Fig. 5. Effective indices of both branches on a complex plane versus film thickness are shown in Fig. 6. The imaginary parts, $\operatorname{Im} q_{1}$ for symmetric bound and symmetric leaky waves have shown in Fig. 4.7 (top), the zoomed version of it with crossing zero at $d_{0}=52.2 \mathrm{~nm}$ shown in Fig. 4.7 (bottom).

Unlike symmetric bound wave the symmetric leaky wave grows exponentially in transverse direction in media 1 while decays exponentially in the lateral direction regardless of film thickness. Since leaky mode is not confined sideways it seems unphysical, however the leaky modes have been confirmed experimentally over finite distance from the waveguide [74], therefore leaky modes is a measurable quantity and can not be ignored. The unbounded field in media 1 for symmetric leaky wave can be interpreted as a reflected field due to a fictitious source field even though the source field is not present in dispersion equation. In general, unbounded behavior of the mode is valid only over a limited range in space, i.e. exponential growth lasts until the energy supplied to the mode from the source can sustain it.

The side effect of this model is, even though it does have a mode dependence on the film thickness $d$, it does not have the angle of the incident field as part of the model and the incident field itself which induces the modes.

Yet another possible way of modeling process of plasmon excitation optically is to keep the waveguide approach while including explicitly the incident field. The full version of Maxwell's equations can admit the incident field as the field radiated by non-zero source terms. Solving these equations would allow evaluate mode evolution (symmetric bound wave, symmetric leaky wave) versus source parameters (incident field polarization, angle of incidence $\alpha_{i n}$ ) and waveguide parameters (film thickness $d$, medium permittivity).

\subsection{Inhomogeneous wave equation. General formalism for slab geometry.}

Finding the modes supported by a waveguide requires solving Maxwell's equations with zero right hand side. Such modes admitted but may or may not exist in a waveguide, i.e. they might not be excited. An excitation problem involves solving Maxwell's equations with nonzero right hand side, i.e. with the source terms. Maxwell's equations with the source terms responsible for excitation and constitutive relations shown below,

$$
\nabla \vec{D}=\rho_{f}
$$



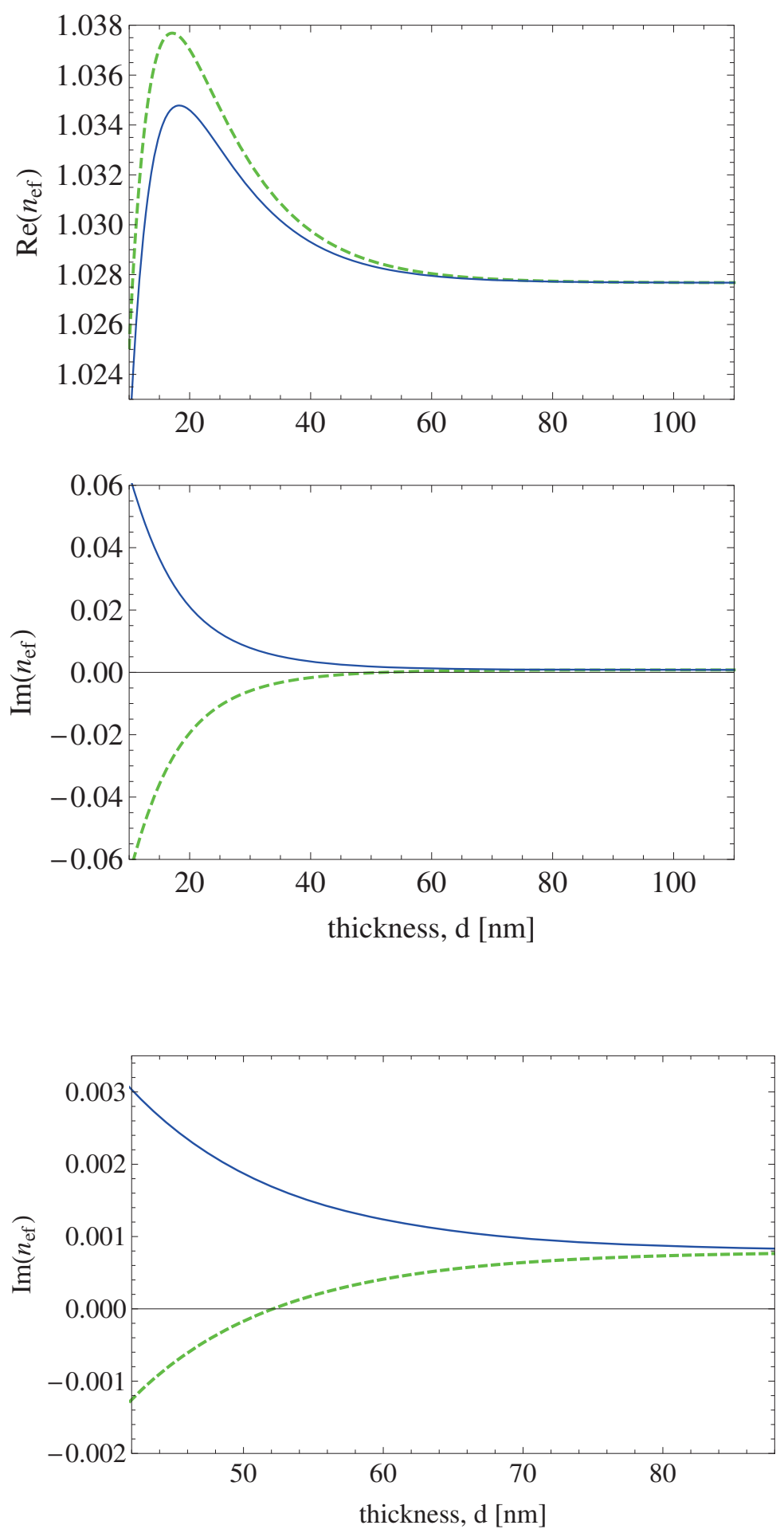

Figure 4.5: (top) Real and imaginary parts of effective index for symmetric bound mode (green dashed line) and leaky wave mode (solid line) versus film thickness; (bottom) zoomed plot of imaginary parts. 


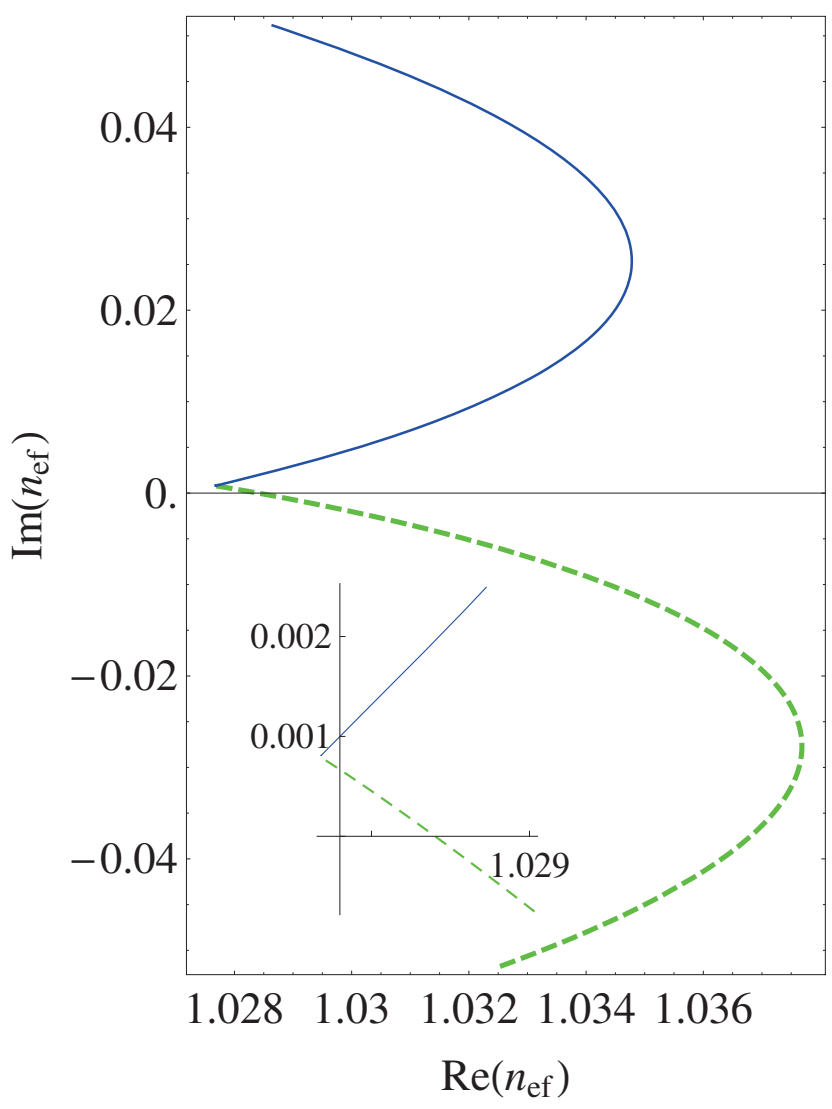

Figure 4.6: Effective index for symmetric bound mode (green dashed line) and leaky wave mode (solid line) versus film thickness on a complex plane.

$$
\begin{gathered}
\nabla \vec{B}=0 \\
\nabla \times \vec{E}=-\frac{\partial \vec{B}}{\partial t} \\
\nabla \times \vec{H}=\frac{\partial \vec{D}}{\partial t}+\vec{J} \\
\vec{D}=\varepsilon \vec{E} \\
\vec{B}=\mu \vec{H}
\end{gathered}
$$

Eliminating charge density, which relates to current density through continuity equation, eliminating magnetic fields in 4.11-4.16 and assuming harmonic time dependence $e^{-i \omega t}$ leads to a vector wave equation for electric field with the source term,

$$
\nabla^{2} \vec{E}+k^{2} \vec{E}=-i \omega \mu\left(\vec{J}+\frac{1}{k^{2}} \nabla \nabla \cdot \vec{J}\right)
$$

here $\vec{J}$ is an impressed current density. Solving it involves introducing an auxiliary vector function $\vec{F}$ satisfying the following equation, 

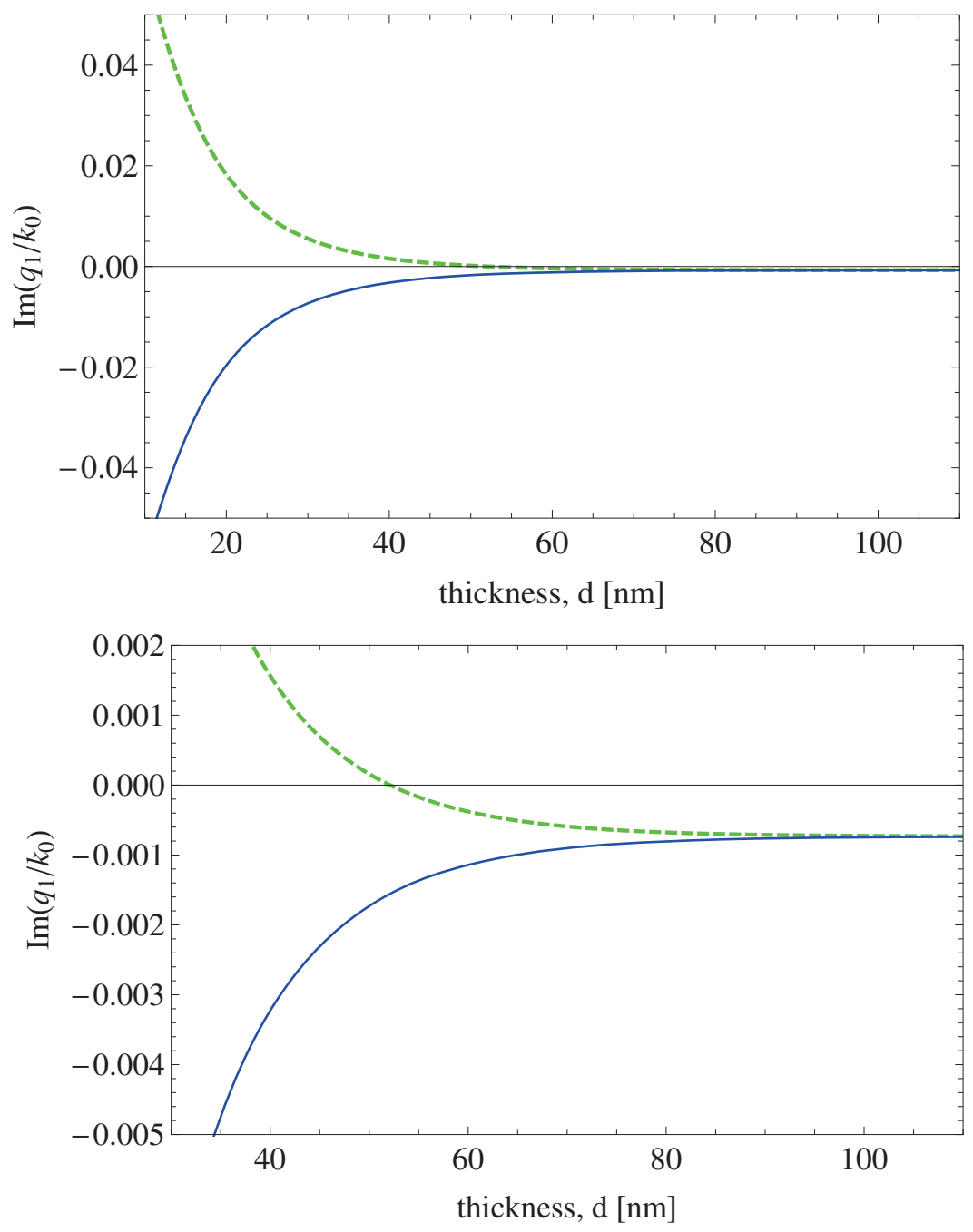

Figure 4.7: (top) Imaginary part of for symmetric bound mode (green dashed line) and leaky wave mode (solid line) versus film thickness; (bottom) zoomed plot near $52 \mathrm{~nm}$.

$$
\nabla^{2} \vec{F}+k^{2} \vec{F}=-i \omega \mu \vec{J}
$$

Since operators $\nabla^{2}$ and $(\nabla \nabla \cdot)$ commute, the solution of 4.17 can be found from solution of 4.18 as,

$$
\vec{E}=\vec{F}+\frac{1}{k^{2}} \nabla \nabla \cdot \vec{F}
$$

In Cartesian coordinates 4.18 is split in three uncoupled scalar equations, one of which for z-component is given below,

$$
\nabla^{2} F_{z}+k^{2} F_{z}=-i \omega \mu J_{z}
$$


For a slab waveguide and current source both uniform in $\mathrm{x}$-axis the derivative with respect to $\mathrm{x}$ vanishes,

$$
\left(\frac{\partial^{2}}{\partial y^{2}}+\frac{\partial^{2}}{\partial z^{2}}\right) F_{z}+k^{2} F_{z}=-i \omega \mu J_{z}
$$

Solving a scalar equation requires carrying out a Fourier transform in since the waveguide is uniform along $z$. Below is the transformation pair for some function $Q$ and its Fourier image $q$,

$$
\begin{aligned}
q(y, v) & =\int_{-\infty}^{\infty} Q(y, z) e^{-i v z} d z \\
Q(y, z) & =\frac{1}{2 \pi} \int_{-\infty}^{+\infty} q(y, v) e^{i v z} d v
\end{aligned}
$$

In transformed coordinates $(y, v)$ the scalar equation is,

$$
\frac{\partial^{2} f_{z}}{\partial y^{2}}+p^{2} f_{z}=-i \omega \mu j_{z}
$$

where $f_{z}(y, v)$ and $j_{z}(y, v)$ are Fourier transforms of $F_{z}(y, z)$ and $J_{z}(y, z)$ respectively and $p^{2}=k^{2}-v^{2}$. Solution in the form of integral is straightforward provided an appropriate Green's function is known,

$$
f_{\alpha}(y, v)=i \omega \mu \int_{S} g(y \mid s) j_{\alpha}(s, v) d s, \quad \alpha=y, z
$$

the integration is done over position of the current source, $g\left(y \mid y_{0}\right)$ is Green's function of one dimensional Helmholtz equation,

$$
\begin{gathered}
g\left(y \mid y_{0}\right)=\frac{i}{2 p} e^{i p\left|y-y_{0}\right|} \\
\frac{d^{2} g}{d y^{2}}+p^{2} g=-\delta\left(y-y_{0}\right)
\end{gathered}
$$

The transformed field function $\vec{e}(y, v)$ then can be found by Fourier transform of equation 4.19,

$$
\vec{e}(y, v)=\vec{f}(y, v)+\frac{1}{k^{2}} \nabla^{\prime} \nabla^{\prime} \cdot \vec{f}(y, v)
$$

where $\vec{e}(y, v)$ and $\vec{f}(y, v)$ are Fourier transforms of $\vec{E}(y, z)$ and $\vec{F}(y, z)$ respectively, the 
symbol $\nabla^{\prime}$ denotes an operator $\nabla$ in which, as a result of taking Fourier integral by parts, the derivative $d / d z$ replaced by $i v$. Once electric field is known the magnetic field is calculated from 4.13,

$$
\vec{H}=\frac{1}{i \omega \mu} \nabla \times \vec{E}
$$

or in transformed space,

$$
\vec{h}(y, v)=\frac{1}{i \omega \mu} \nabla^{\prime} \times \vec{e}(y, v)
$$

\subsection{Plasmon excitation by divergent beam.}

\subsubsection{Scattering in a slab due to current source.}

Assume the source term in 4.17 is due a current source located in layer 1 . The primary fields $\vec{e}^{p}$ and $\vec{h}^{p}$, i.e. the fields directly due to current source, exist in layer 1 only, as indicated in Fig. 8. They obey Maxwell's and wave equations with the source term. The scattered fields, $\vec{e}^{s}$ and $\vec{h}^{s}$ exist in each layer including layer 1 . They are due to primary fields scattered off boundary. They can be calculated from Maxwell's equations with zero source terms. The total field in layer 1 is the sum of primary and scattered ones. Imposing the boundary conditions at each interface couples the primary and the scattered field at each layer.

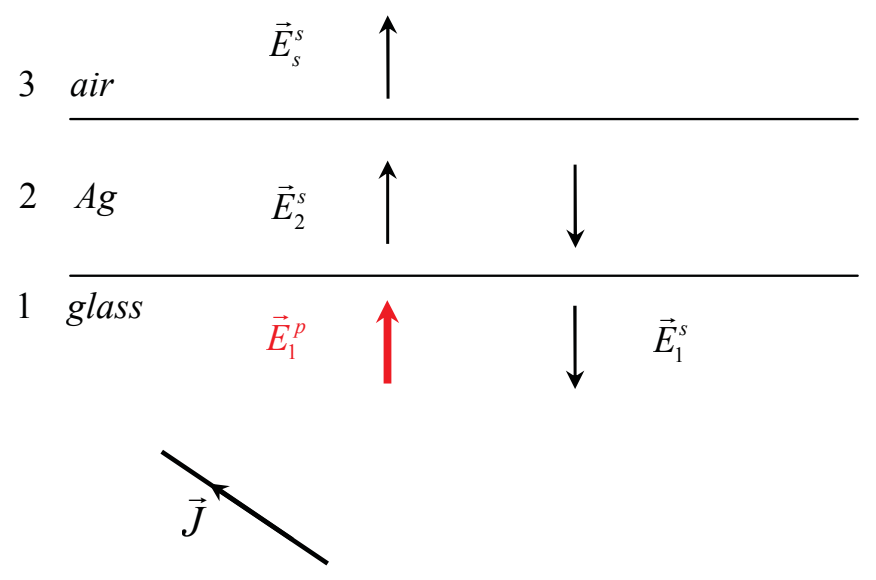

Figure 4.8: Primary and scattered fields in a 3-layer waveguide with the impressed current source in layer 1.

In TM mode, responsible for plasmon excitation, all non-zero scattered field components can be calculated from single component, say $e_{z}^{s}$. In the transformed space $(y, v)$ the two curl Maxwell's equations are, 


$$
\begin{gathered}
\nabla^{\prime} \times \vec{e}^{s}=i \omega \mu \vec{h}^{s} \\
\nabla^{\prime} \times \vec{h}^{s}=-i \omega \varepsilon \vec{e}^{s}
\end{gathered}
$$

Solving them for $h_{x}^{s}$ and $e_{y}^{s}$ gives,

$$
\begin{aligned}
h_{x}^{s} & =\frac{-i \omega \varepsilon}{\omega^{2} \mu \varepsilon-v^{2}} \frac{\partial e_{z}^{s}}{\partial y} \\
e_{y}^{s} & =\frac{i v}{\omega^{2} \mu \varepsilon-v^{2}} \frac{\partial e_{z}^{s}}{\partial y}
\end{aligned}
$$

where $e_{z}^{s}$ can be found from the usual scalar wave equation with zero source term. Below are z-components $e_{z}^{s}$ of each layer respectively,

$$
e_{z 1}^{s}=A_{1}(v) \exp \left(i q_{1} y\right)
$$

for bound wave and

$$
e_{z 1}^{s}=A_{1}(v) \exp \left(-i q_{1} y\right)
$$

for leaky wave

$$
\begin{gathered}
e_{z 2}^{s}=A_{2} \exp \left(\gamma_{2} y\right)+B_{2} \exp \left(-\gamma_{2} y\right) \\
e_{z 3}^{s}=A_{3} \exp \left(-\gamma_{3} y\right)
\end{gathered}
$$

where unknown coefficients $A_{1}, A_{2}, B_{2}, A_{3}$ don't depend on $y$. Applying boundary conditions for tangential field components at each interface $(y=0, \quad y=d)$ leads to 4 linear equations for 4 unknown coefficients $A_{1}, A_{2}, B_{2}, A_{3}$,

$$
\begin{aligned}
& e_{z 1}^{s}(0)-e_{z 2}^{s}(0)=-e_{z 1}^{p}(0) \\
& h_{x 1}^{s}(0)-h_{z 2}^{s}(0)=-h_{z 1}^{p}(0) \\
& e_{z 2}^{s}(d)-e_{z 3}^{s}(d)=0 \\
& h_{x 2}^{s}(d)-h_{x 3}^{s}(d)=0
\end{aligned}
$$

where $d$ metal thickness. In matrix form these equations written as,

$$
M_{0} A=S
$$

where $A$ is a column vector, made up of unknown coefficients,

$$
A=\left[A_{1}, A_{2}, B_{2}, A_{3}\right]^{T}
$$

$S$ is column vector of source terms, i.e. made up of primary fields, 


$$
S=\left[-e_{z 1}^{p}(0),-h_{z 1}^{p}(0), 0,0\right]^{T}
$$

$M_{0}$ is a matrix whose elements are appropriate factors in front of each coefficient $A_{1}, A_{2}, B_{2}, A_{3}$ for each equation in 4.39. Since the plasmon modes predominantly localized near the interface at outer layer, only coefficient $A_{3}$ is needed (for the fields due to plasmon). Follow Cramer's rule it can be evaluated as,

$$
A_{3}=\frac{\operatorname{det}\left(M_{A 3}\right)}{\operatorname{det}\left(M_{0}\right)}
$$

here $M_{A 3}$ is a matrix formed from $M_{0}$ where column 4 replaced by column vector of the source terms $S$. To reconstruct the original scattered fields in physical space $(y, z)$ the inverse Fourier transform applied to the scattered fields in transformed space $(y, z)$, for layer 3 these fields are,

$$
\begin{gathered}
E_{z 3}(y, z)=\frac{1}{2 \pi} \int_{-\infty}^{+\infty} e_{z 3}^{s}(y, v) e^{i v z} d v=\frac{1}{2 \pi} \int_{-\infty}^{+\infty} \frac{\operatorname{det}\left(M_{A 3}\right)}{\operatorname{det}\left(M_{0}\right)} e^{\gamma_{3} y} e^{i v z} d v \\
E_{y 3}(y, z)=\frac{1}{2 \pi} \int_{-\infty}^{+\infty} e_{y 3}^{s}(y, v) e^{i v z} d v=\frac{1}{2 \pi} \int_{-\infty}^{+\infty} \frac{i v}{\gamma_{3}} \frac{\operatorname{det}\left(M_{A 3}\right)}{\operatorname{det}\left(M_{0}\right)} e^{\gamma_{3} y} e^{i v z} d v \\
H_{x 3}(y, z)=\frac{1}{2 \pi} \int_{-\infty}^{+\infty} h_{x 3}^{s}(y, v) e^{i v z} d v=\frac{1}{2 \pi} \int_{-\infty}^{+\infty} \frac{-i \omega \varepsilon_{3}}{\gamma_{3}} \frac{\operatorname{det}\left(M_{A 3}\right)}{\operatorname{det}\left(M_{0}\right)} e^{\gamma_{3} y} e^{i v z} d v
\end{gathered}
$$

The contribution to general Sommerfeld integral 4.44 comes from residues associated with discrete modes and from continuous integration along the branch cuts associated with radiation modes $[75,76]$, i.e. integral represents general spectra of all possible waveguide modes. The radiation modes carry energy far away from a waveguide, therefore in a layer 3 at incident angle past the critical angle $\alpha_{c}$ such contribution vanishes and evaluation the integrals 4.42-4.44 involves calculation of residues due to poles, i.e. zeros of $\operatorname{det}\left(M_{0}\right)$ which are the guided modes of homogeneous waveguide. For some fractional function $f / g$ with isolated poles at $z_{0 j}$ the integral over real axis can be evaluated with the help of Cauchy's residue theorem, provided the function vanishes at infinity on complex plane,

$$
\int_{-\infty}^{+\infty} \frac{f(z)}{g(z)} d z=i 2 \pi \sum_{j} \operatorname{Res}\left(\frac{f(z)}{g(z)}\right)=i 2 \pi \sum_{j} \frac{f\left(z_{0 j}\right)}{g^{\prime}\left(z_{0 j}\right)}
$$

provided,

$$
g\left(z_{0 j}\right)=0, \quad \lim _{|z| \rightarrow \infty} \frac{f(z)}{g(z)}=0
$$


Derivative of matrix determinant can be evaluated as a sum over rows of determinants of a matrix where one row replaced by its derivative, for example for $3 \mathrm{x} 3$ matrix and its rows $R_{1}, R_{2}, R_{3}$ the formula is,

$$
\frac{d}{d x} \operatorname{det}\left|\begin{array}{l}
R_{1} \\
R_{2} \\
R_{3}
\end{array}\right|=\operatorname{det}\left|\begin{array}{l}
R_{1}^{\prime} \\
R_{2} \\
R_{3}
\end{array}\right|+\operatorname{det}\left|\begin{array}{l}
R_{1} \\
R_{2}^{\prime} \\
R_{3}
\end{array}\right|+\operatorname{det}\left|\begin{array}{l}
R_{1} \\
R_{2} \\
R_{3}^{\prime}
\end{array}\right|
$$

where $R_{1}^{\prime}, R_{2}^{\prime}, R_{3}^{\prime}$ derivatives of $R_{1}, R_{2}, R_{3}$ respectively. Power carried in outer layer (layer 3) through plane $z=0$ by a plasmon mode propagating in z-direction per unit length in $\mathrm{x}$-direction is given by,

$$
P=\left[\int_{d}^{\infty} P_{z 3} d y\right]_{z=0}
$$

where $P_{z 3}$ is $z$-component of time-averaged Poynting vector which for TM mode in layer 3 is given by,

$$
P_{z 3}=\operatorname{Re}\left(-E_{y 3} H_{x 3}^{*}\right)
$$

\subsubsection{Excitation sources.}

Consider the following current sources shown in Fig. 4.9- 4.11: (A) a plane sheet of current finite in $(y, z)$ and infinite length in $x,(\mathrm{~B})$ a set of several rods positioned in a single plane with equidistant spacing $\Delta$, where each rod is infinite in $x$ and $(\mathrm{C})$ a set of plane sheets each as in (A) arranged either in a uniform or a $\pi$-shifted phased array. The current density for general case can be described as,

$$
\vec{J}(y, z)=I_{0} L(y, z) \hat{J}(y, z)
$$

where $I_{0}$ is a constant scale factor, $L(y, z)$ a function describing source location and $\hat{J}(y, z)$ a unit vector that represents orientation of the current density. For TM mode which is responsible for plasmons in the slab the current source in $(A),(B)$ and $(C)$ should have $y-$ and z-components, i.e.

$$
\hat{J}=\hat{y} \sin \alpha-\hat{z} \cos \alpha
$$

\section{(A) Primary fields due to finite sheet of current.}

Function $L(y, z)$ describing the source location in $(y, z)$ defined as, 


$$
L(y, z)=\delta\left(z-f_{J}(y, \alpha)\right) \Pi\left(y, y_{1}, y_{2}\right)
$$

where $\delta(x)$ Dirac delta function, $\Pi\left(x, x_{1}, x_{2}\right)$ box function whose plot resembles symbol $\Pi$ and whose definition is given by,

$$
\Pi\left(x, x_{1}, x_{2}\right)=\left\{\begin{array}{cc}
1 & x_{1} \leq x \leq x_{2} \\
0 & \text { otherwise }
\end{array}\right.
$$

$f_{J}(y, \alpha)$ describes plane where the sheet of current positioned,

$$
f_{J}(y, \alpha)=-y \frac{1}{\tan \alpha}-R_{0} \frac{1}{\sin \alpha}
$$

$W, R_{0}, \alpha$ sheet width, distance from the origin and tilt angle respectively.

$y_{m}=-R_{0} \cos \alpha$

$y_{1}=y_{m}-(W / 2) \sin \alpha$

$y_{2}=y_{m}+(W / 2) \sin \alpha$

All geometrical parameters $W, R_{0}, y_{m}, y_{1}, y_{2}, \alpha$ also defined in Fig. 9. Density current source then becomes a product,

$$
\vec{J}(y, z)=I_{0} L(y, z) \hat{J}(\alpha)
$$

where $I_{0}$ scale factor and $\hat{J}(\alpha)$ a unit vector of current density, $\hat{J}(\alpha)=\hat{y} \sin \alpha-\hat{z} \cos \alpha$.

Fourier transform of the source term then evaluated as,

$$
\vec{j}(y, v)=I_{0} \hat{J}(\alpha) \Pi\left(y, y_{1}, y_{2}\right) e^{-i v f_{J}(y, \alpha)}
$$

A vector function $\vec{f}$ for a region above the current sheet found from 4.25,

$$
\vec{f}(y, v)=i \omega \mu I_{0 A} \hat{J}(\alpha) \frac{1}{2 q_{1}} e^{i q_{1} y} e^{i v \frac{R_{0}}{\sin \alpha}} \frac{1}{\left(v \cot \alpha-q_{1}\right)}\left(e^{i\left(v \cot \alpha-q_{1}\right) y_{2}}-e^{i\left(v \cot \alpha-q_{1}\right) y_{1}}\right)
$$

With known $\vec{f}$ all the primary fields $\left(h_{x}^{p}, e_{y}^{p}, e_{z}^{p}\right)$ due to a finite sheet of current and power flowing along $z$ axis are readily evaluated with 4.28, 4.30 and 4.48.

\section{(B) Primary fields due to set of rods with current.}

Function $L(y, z)$ defined as a sum of point sources, i.e. sum of products of two delta functions,

$$
L(y, z)=\sum_{j=0}^{N-1} \delta\left(z-z_{j}\right) \delta\left(y-y_{j}\right)
$$




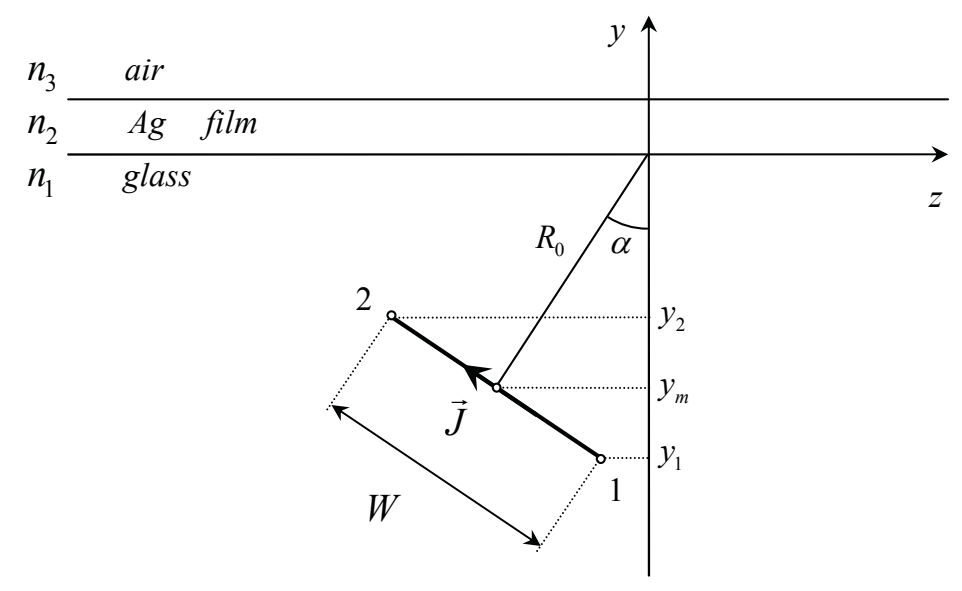

Figure 4.9: Excitation of plasmon in a 3-layer silver film waveguide by a finite sheet of current.

where

$$
\begin{aligned}
& z_{j}=z_{1}-j \Delta \cos \alpha \\
& z_{1}=z_{m}+(W / 2) \cos \alpha \\
& z_{m}=-R_{0} \sin \alpha \\
& y_{j}=y_{1}+j \Delta \sin \alpha \\
& y_{1}=y_{m}-(W / 2) \sin \alpha \\
& y_{m}=-R_{0} \cos \alpha \\
& \Delta=W /(N-1)
\end{aligned}
$$

$N$ a number of point sources (assumed odd number)

All geometrical parameters also defined in Fig. 4.10. Density current source becomes a product,

$$
\vec{J}(y, z)=I_{0} L(y, z) \hat{J}(\alpha)
$$

where current flows as in (A), i.e. in plane $(y, z)$.

Fourier transform of the source term then evaluated as,

$$
\vec{j}(y, v)=I_{0 B} \hat{J}(\alpha) \sum_{j=0}^{N-1} e^{-i v z_{j}} \delta\left(y-y_{j}\right)
$$

A vector function $\vec{f}$ in a region above the set of rods found from 4.25,

$$
\vec{f}(y, v)=i \omega \mu I_{0} \hat{J}(\alpha) \frac{i}{2 q_{1}} e^{i q_{1} y} e^{-i\left(v z_{1}+q_{1} y_{1}\right)} A F
$$


where $A F$ is an array factor,

$$
A F=\frac{1-e^{i N \Delta\left(v \cos \alpha-q_{1} \sin \alpha\right)}}{1-e^{i \Delta\left(v \cos \alpha-q_{1} \sin \alpha\right)}}
$$

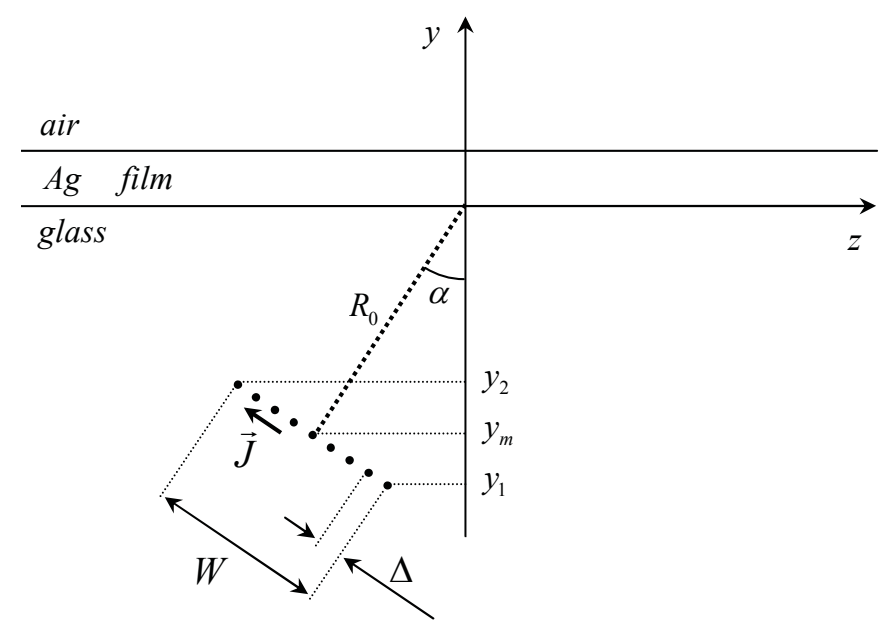

Figure 4.10: Excitation of plasmon in a 3-layer silver film waveguide by several equidistant rods of current positioned on a single sheet.

\section{(C) Primary fields due to array of finite sheets of current.}

In this case the excitation is due to a set of current sheets each as in (A) with equidistant spacing $\Delta$ along $z$ axis over the whole set forming a uniform phased array or two uniform halves with spacing $\Delta$ in each while the second half shifted by $\Delta / 2$ from the first one forming a phase shifted array. Location function $L(y, z)$ defined as a sum of $2 N$ finite current sheets,

$$
L(y, z)=\sum_{j=0}^{2 N-1} \delta\left(z-h_{j}(y, \alpha)\right) \Pi_{j}
$$

where

$\Pi_{j}=\Pi\left(y, y_{1 j}, y_{2 j}\right)$

$h_{j}(y, \alpha)=-y \frac{1}{\tan \alpha}-R_{j} \frac{1}{\sin \alpha}$

$y_{m j}=-R_{j} \cos \alpha$

$y_{1 j}=y_{m j}-(W / 2) \sin \alpha$

$y_{2 j}=y_{m j}+(W / 2) \sin \alpha$

$\alpha$ tilt angle. 
All geometrical parameters also defined in Fig. 4.11. Density current source is,

$$
\vec{J}(y, z)=I_{0} L(y, z) \hat{J}(\alpha)
$$

Fourier transform of the source term then evaluated as,

$$
\vec{j}(y, v)=I_{0} \hat{J}(\alpha) \sum_{j=0}^{2 N-1} e^{-i v h_{j}(y, \alpha)} \Pi_{j}
$$

A vector function $\vec{f}$ for a region above the set is given by,

$$
\vec{f}(y, v)=\left\{\begin{array}{l}
\vec{f}_{A}(y, v) A F_{u} \quad \text { uniform array } \\
\vec{f}_{A}(y, v) A F_{\pi} \quad \pi-\text { shifted array }
\end{array}\right.
$$

where

$\vec{f}_{A}(y, v)$ vector function as in $(\mathrm{A})$

$A F_{u}$ array factor for a uniform array of $2 N$ current sheets,

$$
A F_{u}=\frac{1-e^{i 2 N \Delta\left(v \sin \alpha+q_{1} \cos \alpha\right)}}{1-e^{i \Delta\left(v \sin \alpha+q_{1} \cos \alpha\right)}}
$$

$A F_{\pi}$ array factor for a $\pi$ phase shifted array of $2 N$ current sheets,

$$
A F_{\pi}=\frac{1-e^{i N \Delta\left(v \sin \alpha+q_{1} \cos \alpha\right)}}{1-e^{i \Delta\left(v \sin \alpha+q_{1} \cos \alpha\right)}}\left(1+e^{i(N-1 / 2) \Delta\left(v \sin \alpha+q_{1} \cos \alpha\right)}\right)
$$

Spacing $\Delta$ of an array defined through parameter $n_{\gamma}$ as,

$$
\Delta=n_{\gamma} \frac{\lambda_{0}}{\sqrt{\varepsilon_{r 1}}}
$$

\subsection{Choice of basis: electric and magnetic fields $(\vec{E}, \vec{H})$ versus vector potentials for solving wave equation.}

For a certain type of problems the choice of vector magnetic and scalar potentials $\left(\vec{A}, \varphi_{e}\right)$ is advantageous since it has only 4 scalar variables rather than 6 as is the case with electric and magnetic fields, however it is usually harder to implement boundary conditions in terms of potentials. 


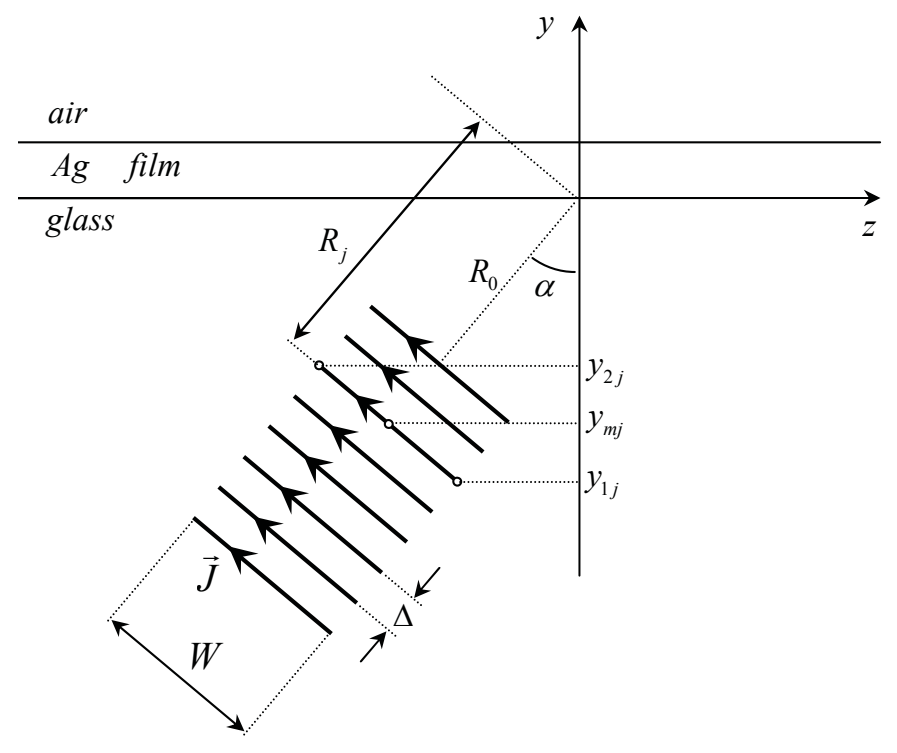

Figure 4.11: Excitation of plasmon in a 3-layer silver film waveguide by an array of finite sheets of current.

\subsubsection{Electric and magnetic fields, $(\vec{E}, \vec{H})$.}

For TM mode one independent scalar field, say longitudinal field $E_{z}$, is sufficient to describe all the fields in the slab. Therefore 4 unknown coefficients is sufficient for describing all the fields: one for each longitudinal field of the first and third layer and two for each longitudinal field of the second layer, thus it matches the total number of independent boundary conditions with two interfaces. The matrix size for characteristic waveguide equation det $M=0$ is $4 \times 4$. The same task of finding all the fields of TM mode in a slab waveguide through one independent scalar quantities can be solved in terms of properly chosen non-zero components of vector and scalar potentials $\left(\vec{A}, \varphi_{e}\right)$.

\subsubsection{Magnetic vector potential and scalar electric potential, $\left(\vec{A}, \varphi_{e}\right)$.}

Vector and scalar potentials $\left(\vec{A}, \varphi_{e}\right)$ defined through electric and magnetic fields $(\vec{E}, \vec{H})$ as follows, $\vec{E}=-\nabla \varphi_{e}+i \omega \vec{A}$ and $\vec{H}=\frac{1}{\mu} \nabla \times \vec{A}$. In addition, Lorenz gauge condition is assumed, $\nabla \cdot \vec{A}=i \omega \mu \varepsilon \varphi_{e}$. TM mode of the slab geometry can be treated in terms of vector and scalar potentials $\left(\vec{A}, \varphi_{e}\right)$ by keeping only one component of the vector potential non-zero, either $A_{z}$ or $A_{y}$. Eliminating scalar potential from TM fields via Lorenz gauge condition leaves single independent component, $A_{z}$ or $A_{y}$ that describes all TM fields of the slab waveguide. Therefore size of the matrix $M$ with vector potential remains $4 \times 4$ 
as in the case of $(\vec{E}, \vec{H})$ base. The basis with a minimal non-zero scalar components is $\left(\vec{A}, \varphi_{e}\right)=\left(0, A_{y}, 0, \varphi_{e}\right)$ or $\left(\vec{A}, \varphi_{e}\right)=\left(0,0, A_{z}, \varphi_{e}\right)$.

For a superposition of TM and TE modes when all 6 components of the fields are non-zero any component of the vector potential can be set to zero with remaining two being independent that describe all the fields of the slab. The corresponding matrix size is $8 \times 8$. The corresponding matrix $M$ in this case is a two block diagonal matrix each block representing its own mode, TE and TM, the determinant of is a product of two determinants.

\subsubsection{Electric Hertz vector and electric scalar potential, $\left(\vec{\Pi}_{e}, \varphi_{e}\right)$.}

Another possible choice is an electric Hertz vector $\vec{\Pi}_{e}$ defined as in [77], $\vec{A}=-i \omega \mu \varepsilon \vec{\Pi}_{e}$ can be used for slab waveguide with non-zero, either $\Pi_{e z}$ or $\Pi_{e y}$. The fields $\vec{E}$ and $\vec{H}$ expressed in terms of as,

$$
\begin{gathered}
\vec{E}=\nabla\left(\nabla \cdot \vec{\Pi}_{e}\right)+\omega^{2} \mu \varepsilon \vec{\Pi}_{e} \\
\vec{H}=-i \omega \varepsilon \nabla \times \vec{\Pi}_{e}
\end{gathered}
$$

The basis with a minimal non-zero scalar components is $\left(\vec{\Pi}_{e}, \varphi_{e}\right)=\left(\Pi_{e r}, \Pi_{e \theta}, 0, \varphi_{e}\right)$ similar to $\left(\vec{A}, \varphi_{e}\right)$ basis. Any one component of $\vec{\Pi}_{e}$ can be set to zero with remaining two fully describing a superposition of TE and TM modes in a slab, for instance $\vec{\Pi}_{e}=$ $\left(0, \Pi_{e y}, \Pi_{e z}\right)$.

\subsubsection{Boundary conditions in terms of vector potentials.}

The implementation of boundary conditions in terms of vector potentials is somewhat involved. Sometimes to simplify the calculations the boundary conditions for normal components of the fields are ignored, usually normal component of electric field for non-magnetic mediums or normal components of both electric and magnetic fields for magnetic mediums. For example in [78] the boundary conditions for normal electric field left unsatisfied. Such approach justified with an uniqueness theorem arguments that electromagnetic fields in a given region are uniquely defined if all sources are given and either the tangential components of electric field, $\vec{E}_{t}$ or the tangential components of magnetic field, $\vec{H}_{t}$ are specified at the boundary of the region. However such argumentation of ignoring normal components has a flaw which was investigated and treated in details by X. L. Zhou in a series of articles [79-81]. It was shown that usual textbook formulation of uniqueness theorem for electromagnetic fields that requires only tangential components of the fields is not complete. Such formulation steams from the proving theorem by method of contradiction where only one particular case out of three possible was considered and conclusion 
has been drawn based on a particular case. Considering remaining cases, which is a necessary condition for a proving by method of contradiction and which makes a formulation of uniqueness theorem complete, requires the knowledge of tangential as well as normal components of the fields. Therefore boundary conditions for normal components can not be ignored.

\subsection{Simulation results and discussion.}

\subsubsection{Mode excitation in experimental setup.}

3 layer slab waveguide with a metal film admits several modes (see [67]) which may or may not be present in the waveguide under a particular measurement. An example of a mode which is perfectly valid, may exist in a waveguide but not accessible, i.e. can not be directly excited (because of its pole laying in a very close proximity from the branch cut) is Zenneck mode, a surface wave propagating along the interface between dielectric and conducting medium at a range of radio frequencies, [75]. Antisymmetric modes of the metal film whose real part of effective index is slightly greater than refractive index of the glass can not be excited in Kretschmann setup, because of the phase matching condition constrain, i.e. a wave vector of the incident field remains always less than propagation constant of these modes. Since mode excitation in this model is done by scattering a primary field $e^{i q_{1} y}$ in media 1 (glass) off the glass-metal interface as indicated in Fig. 8, the scattered field is the mode's field. Because primary field propagates forward $(+y)$, the scattered field has to propagate backward $(-y)$. Therefore only symmetric leaky wave whose field $e^{-i q_{1} y}$ in media 1 propagates in the negative $y$ direction can be excited. While excitation of symmetric bound wave, whose field $e^{i q_{1} y}$ in media 1 propagates forward and requires direct coupling to the primary field rather than coupling through the scattering, can not be done in a current setup (see also [67]).

\subsubsection{Radiation mode and plasmon measurement in experimental setup.}

A radiation mode due to the branch cut associated with square root in $q_{1}$ is responsible for delivering energy away from a waveguide in the far field zone of layer 1 where it can be detected by a photodiode as in Kretschmann experimental setup in Fig. 4.1. Symmetric leaky wave experience exponential growth only over finite distance from the waveguide, it drops in the far field where the measuring by a photodiode is done.

\subsubsection{Excitation by a finite sheet of current.}

Power carried by a symmetric leaky wave plasmon due to a finite sheet of current presented in Fig. 12-14. It has a peak for the angle $42.9^{\circ}$ which agrees with experimental data and with calculation based on plane wave excitation in reflection 4.9. However the thickness dependence is monotonic unlike plane wave excitation with maximum at $d=52 \mathrm{~nm}$. 
Primary field propagates forward $(+z)$ along the waveguide axis, so the propagation constant of the plasmon in waveguide is positive and plasmon power in layer 3 carried forward, see Fig. 4.12 (top). Since most energy of plasmon concentrated in dielectric, the global power carried forward as well, [82]. Nevertheless inside of the metal film power transmitted backward, see Fig. 4.12 (bottom).
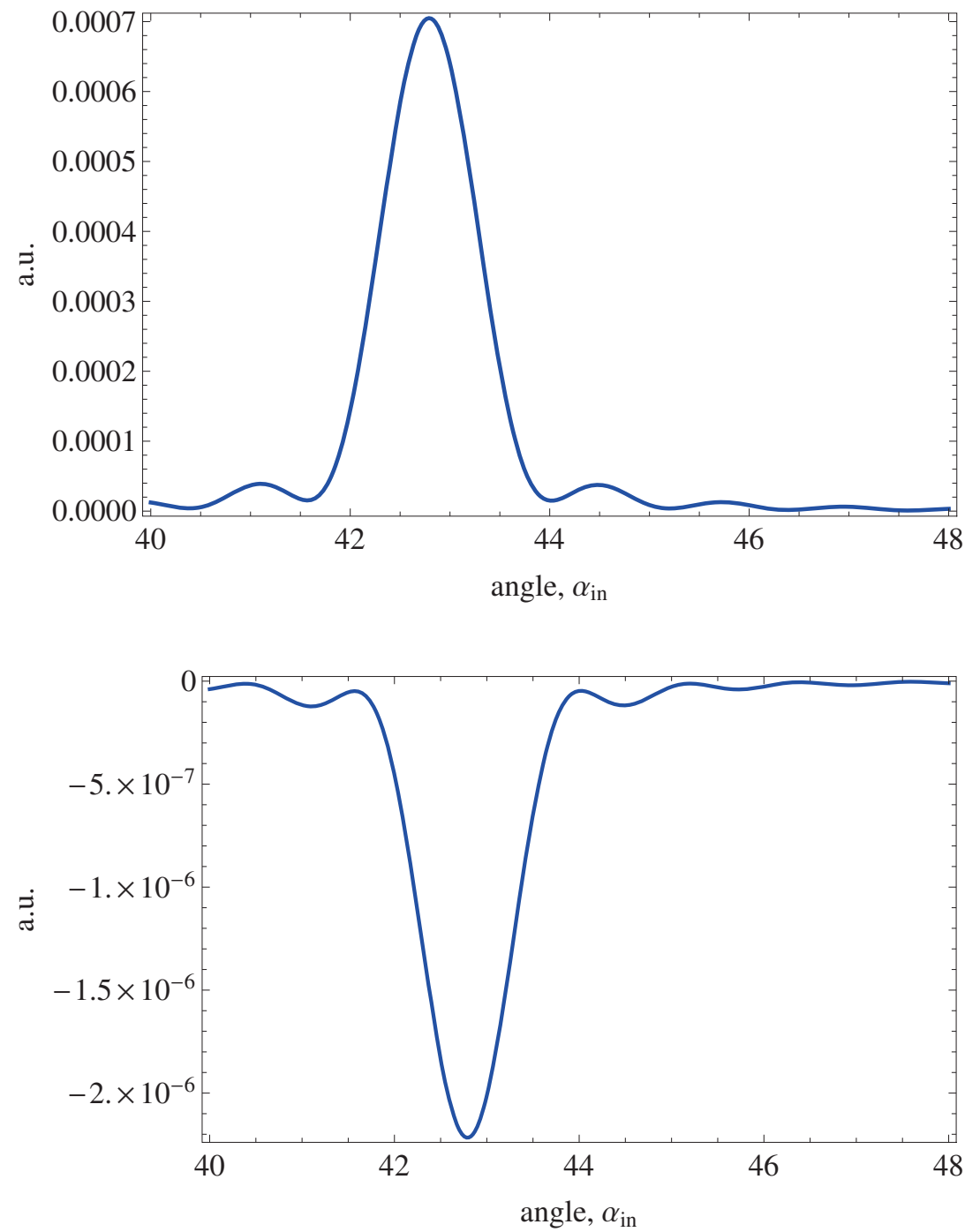

Figure 4.12: Plot of power carried by a plasmon (symmetric leaky wave) versus incident angle at silver thickness $d=40 \mathrm{~nm}$. Excitation is due to a finite sheet of current whose width $W=40 \mu \mathrm{m}$ and distance from the origin $R_{0}=40 \mu \mathrm{m}$ (as in Fig. 4.9). (top) The power in the air, layer 3 is positive, indicating it is carried in the positive $z$ direction. (bottom) The power inside of the silver film, layer 2 is negative, indicating it is carried in the negative $z$ direction or opposite to the modal propagation constant, i.e. inside of the film the power flux is opposite to the phase velocity. 

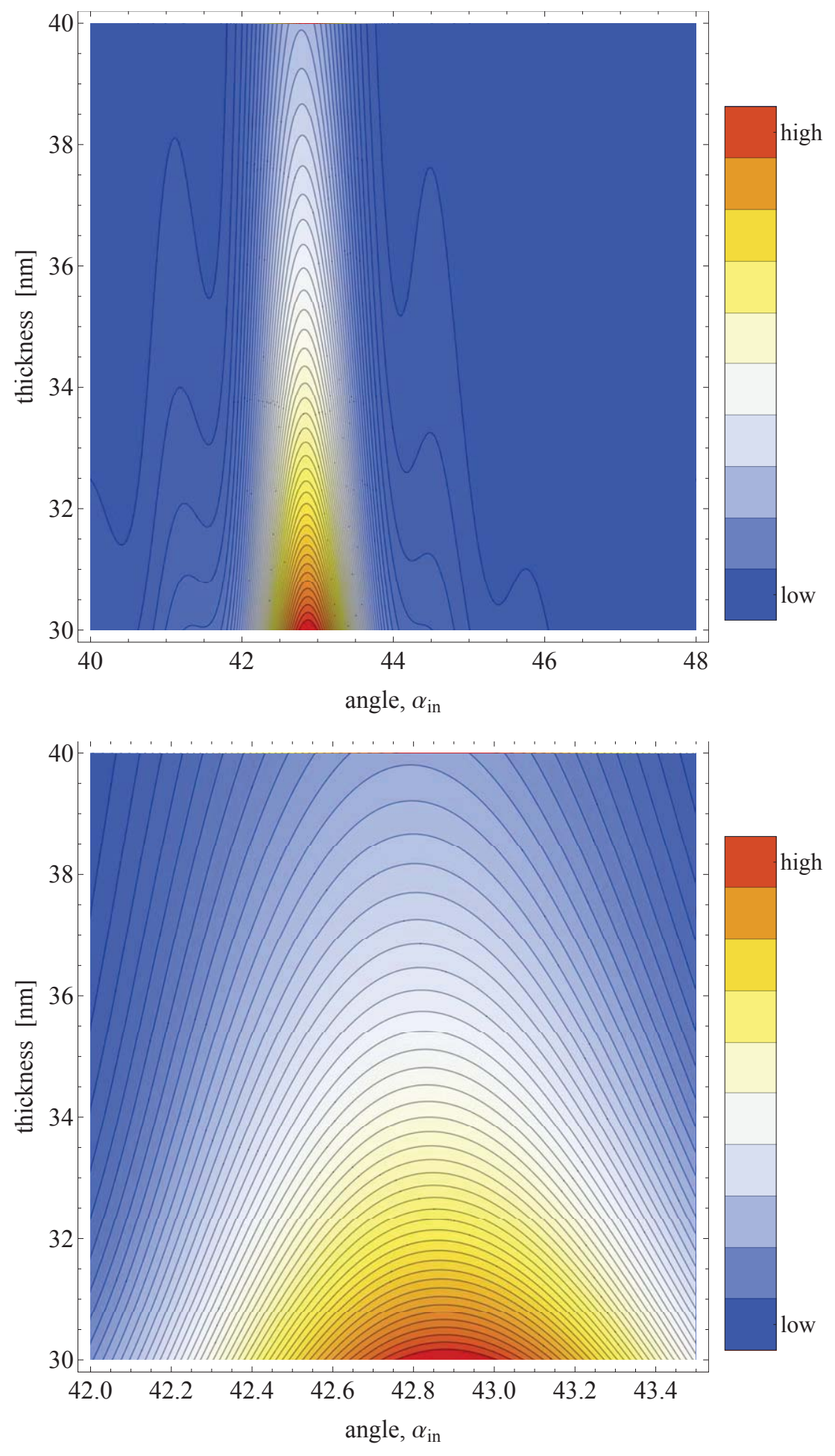

Figure 4.13: (top) Contour plot of power carried forward (positive $z$ direction) by a plasmon (symmetric leaky wave) in layer 3 (air) versus incident angle and silver thickness. Excitation is due to a finite sheet of current whose width $W=40 \mu \mathrm{m}$ and distance from the origin $R_{0}=40 \mu \mathrm{m}$ (as in Fig. 4.9). (bottom) Zoomed contour plot near the angle $42^{\circ}-43^{\circ}$. 


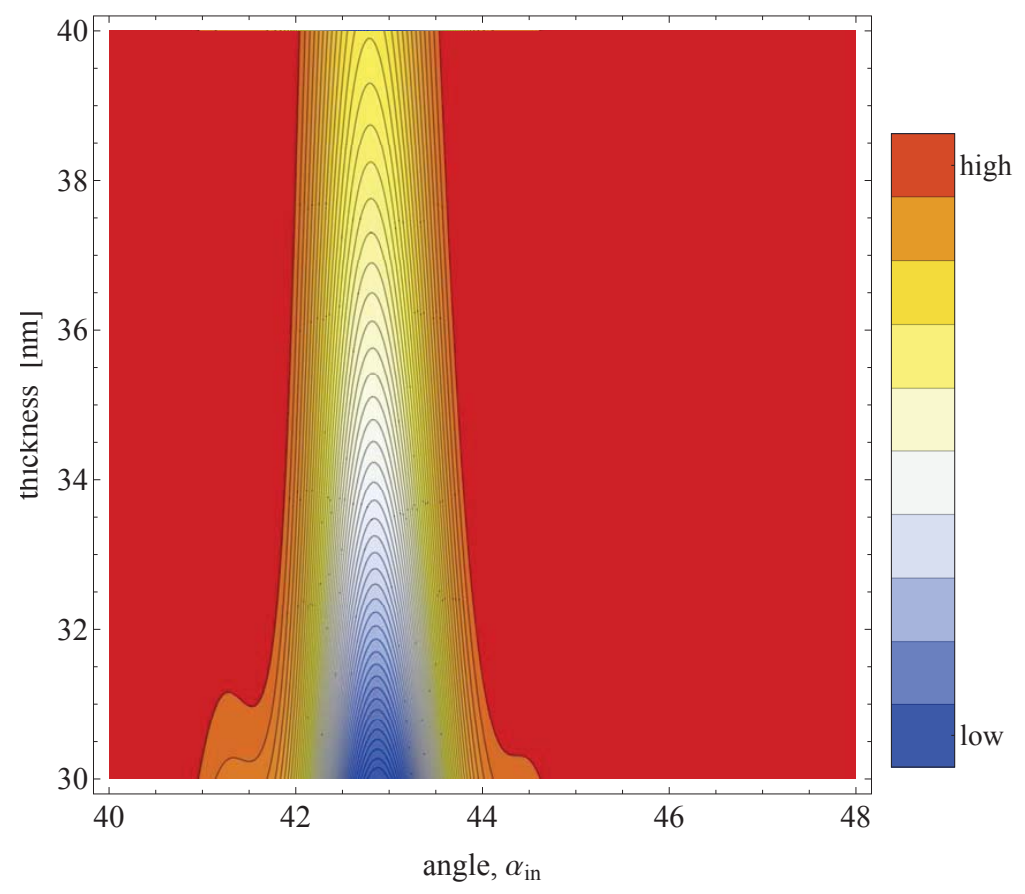

Figure 4.14: Contour plot of power carried backward (negative direction) by a plasmon (symmetric leaky wave) in layer 2 (silver film) versus incident angle and silver thickness. Excitation is due to a finite sheet of current whose width $W=40 \mu \mathrm{m}$ and distance from the origin $R_{0}=40 \mu \mathrm{m}$ (as in Fig. 4.9).

\subsubsection{Excitation by a set of current sources.}

For plasmon excitation by a couple of rods $(N)$ spread over $W=40 \mu m$ (spacing $\Delta=$ $W / N$ ) the incident field is not well defined, i.e. it consists of a couple of fields each due to a point-like source with its own angle of incidence. As a result a few peaks are observed as in Fig. 4.15 where $N=3, \Delta=13.3 \mu \mathrm{m}, R_{0}=40 \mu \mathrm{m}$. It gets more definition with larger $N$ (smaller spacing). The excitation by $N=30 \operatorname{rods}(\Delta=1.33 \mu \mathrm{m})$ is very similar to the excitation by a finite sheet of current, compare Fig. 4.15 (bottom) and Fig. 4.13 (top).

The excitation by an array of current sheets differs from the excitation by a single sheet of current by an array factor. A uniform array factor attains its peak value at $n_{\gamma}=1$ when spacing between adjacent current sheets is equal to a wavelength in media 1 (see Fig. 4.16 (top)). A $\pi$-shifted array can be viewed as two identical uniform arrays shifted with respect to each other by $\pi$, i.e. the second array starts at the end of the first one plus half wavelength in the media, $\lambda_{0} /\left(2 n_{1}\right)$. So that at $n_{\gamma}=1$ the spacing in each array is matching a wavelength in media 1 and both array factors have their maximum, however their fields are out of phase to each other and therefore are canceled out since both arrays shifted by $\pi$. As a result a narrow needle-like deep cuts the original peak right in the middle. Visually it appears as a single peak of a uniform array factor splits in two peaks of a $\pi$-shifted array factor as indicated in Fig. 4.16. For a larger number of sheets the peaks 

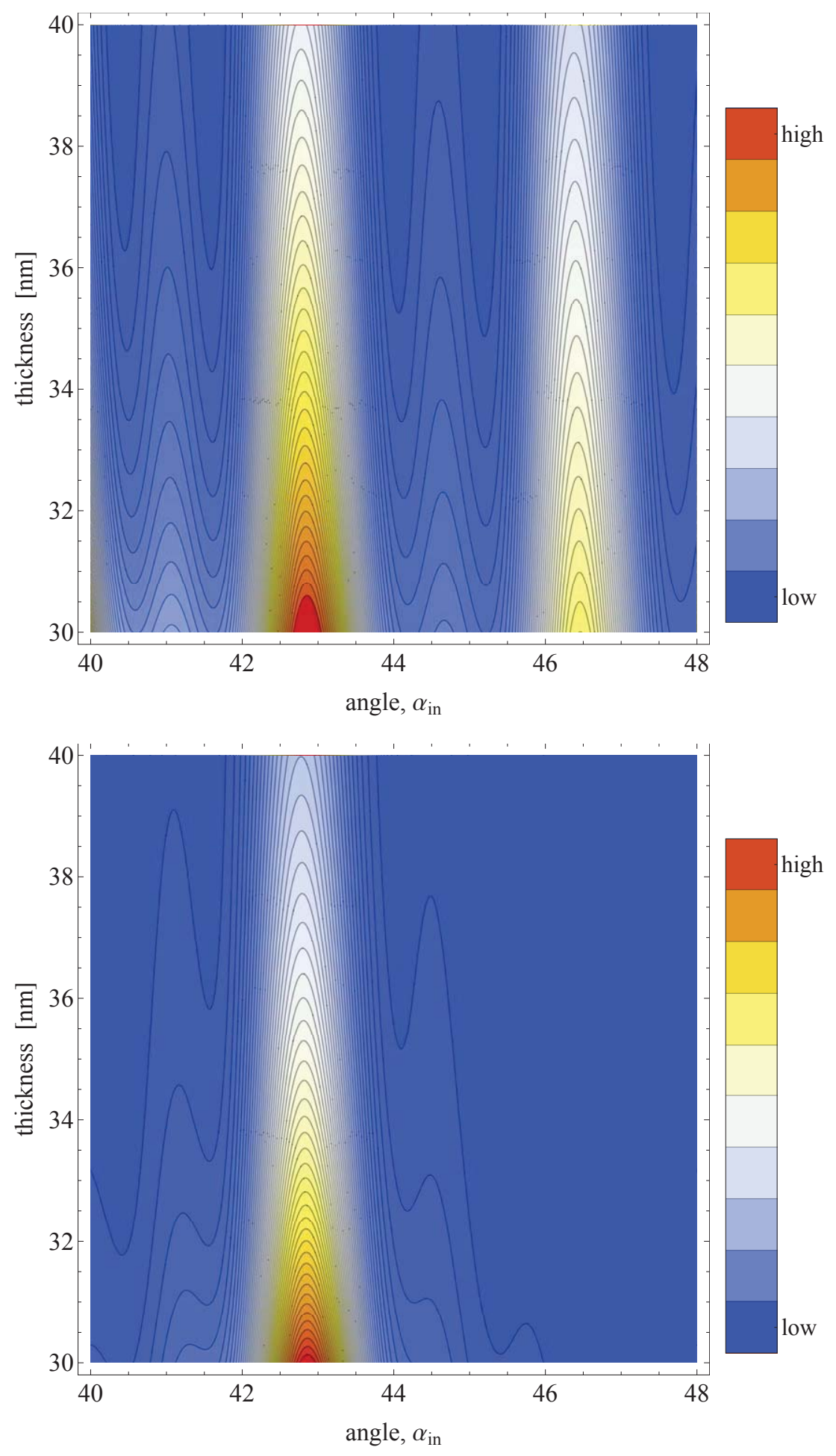

Figure 4.15: Contour plot of power carried forward (positive $z$ direction) by a plasmon (symmetric leaky wave) in layer 3 (air) versus incident angle and silver thickness. Excitation is due to several rods of current positioned on a single sheet with width $W=40 \mu \mathrm{m}$ and distance from the origin $R_{0}=40 \mu m$ (as in Fig. 4.10). (top) Number of rods $N=3$. (bottom) Number of rods $N=30$. 
and a deep get sharper, see Fig. 4.17.

\subsection{Conclusions.}

The problem of plasmon excitation in planar slab geometry by a current sheet of finite size derived from the first principle, i.e. from the Maxwell's equations. The simulation results found to be in a good agreement with experimental data as well as with a simplified simulation where the incident field replaced by a plane wave. This approach can be extended to the case of a focused beam excitation with replacing a single planar current sheet by several planar current sheets inscribed in a parabolic surface, i.e. transforming flat current sheet into shape of polygon whose vertices lie on some parabola so that its radiated field would imitate a convergent Gaussian beam. Also it can be extended to the case of other geometrical structures where the simplified version of the simulation is not available, for example plasmon excitation in cylindrical layered waveguide by planar discs of current. 

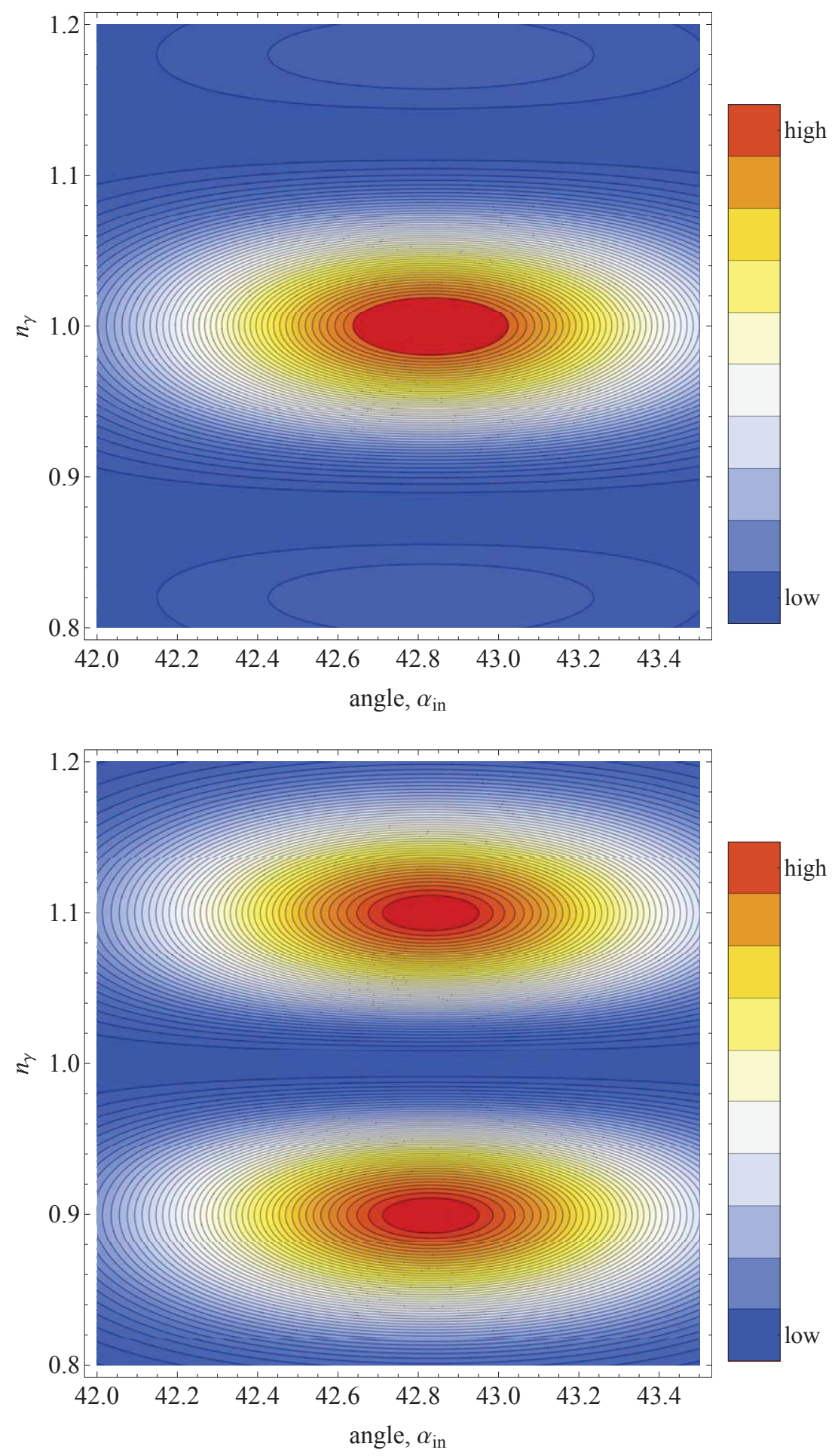

Figure 4.16: Contour plot of power carried forward (positive $z$ direction) by a plasmon (symmetric leaky wave) in layer 3 (air) versus incident angle and silver thickness. Excitation is due to an array of current sheets with array width $W=40 \mu \mathrm{m}$ and distance from the origin $R_{0}=40 \mu \mathrm{m}$ (as in Fig. 4.11). (top) a uniform array with total of $N=8$ sheets. (bottom) a $\pi$ phase shifted array with total of $N=8$ sheets. 

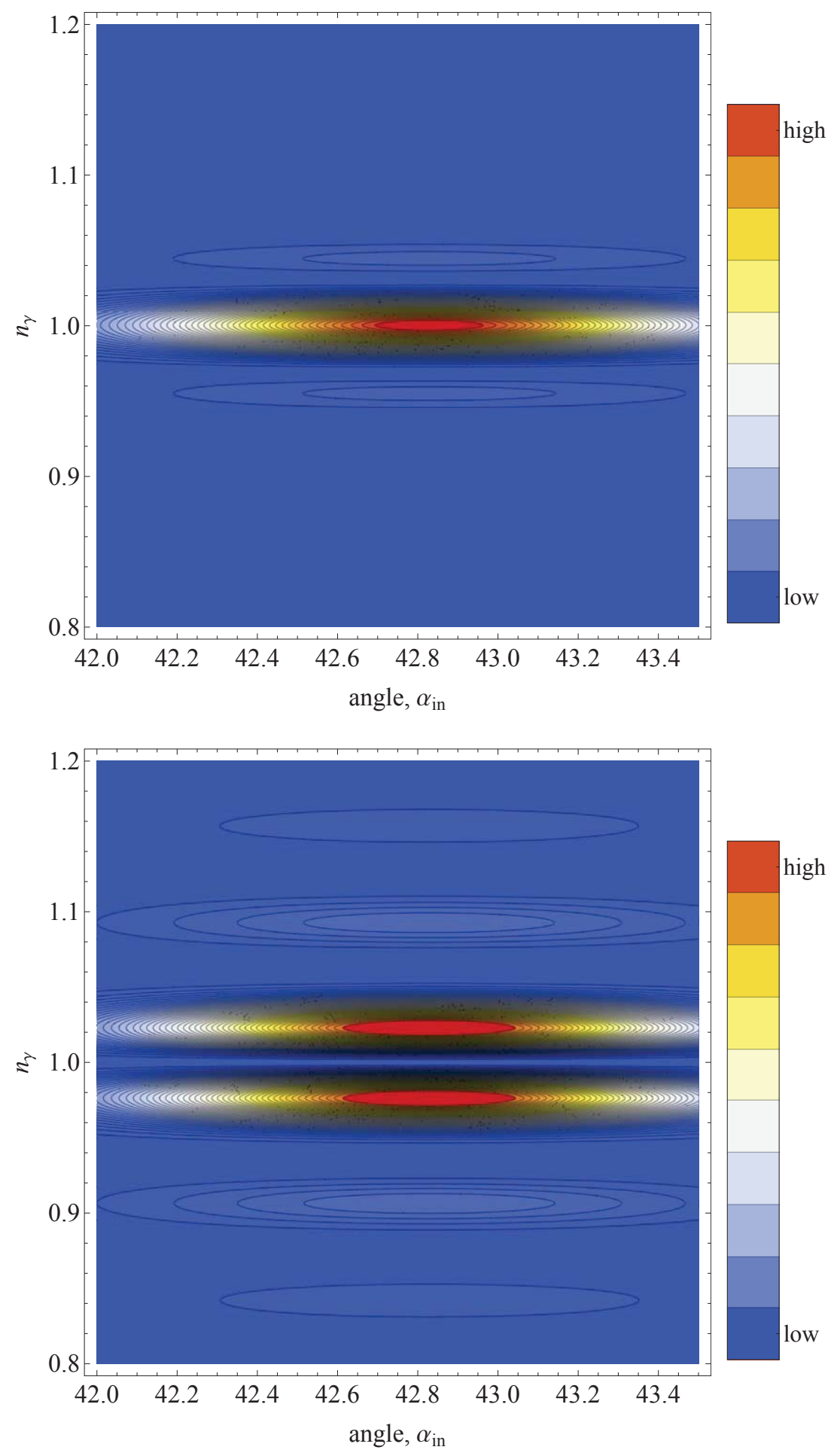

Figure 4.17: Contour plot of power carried forward (positive $z$ direction) by a plasmon (symmetric leaky wave) in layer 3 (air) versus incident angle and silver thickness. Excitation is due to several rods of current positioned on a single sheet with width $W=40 \mu \mathrm{m}$ and distance from the origin $R_{0}=40 \mu \mathrm{m}$ (as in Fig. 4.11). (top) number of rods $N=3$. (bottom) number of rods $N=30$. 


\section{Chapter 5}

\section{Plasmon excitation in a waveguide with cylindrical geometry}

In this chapter a model of plasmon excitation by an optical antenna, developed in the previous chapter, extended to the case of cylindrical waveguide (optical fiber), An optical antenna in this case is the set of tilted discs of current distributed in the a fiber core either uniformly or with phase shift ( $\pi$-shifted distribution).

Scattering due to a set of tilted rings and disks as an impressed source of current placed in a dielectric cylinder is analyzed with Green's function formulation. Scattered field then applied for a plasmon excitation in a fiber coated by thin metal film.

\subsection{Inhomogeneous wave equation. General formalism in cylindrical coordinates.}

Finding the modes supported by a waveguide requires solving Maxwell's equations with zero right hand side. Such modes may or may not exist in a waveguide, i.e. they might not be excited. Excitation problem involves solving Maxwell's equations with nonzero right hand side, i.e. with the source terms. Maxwell's equations with the source terms responsible for excitation and constitutive relations shown below,

$$
\begin{gathered}
\nabla \vec{D}=\rho_{f} \\
\nabla \vec{B}=0 \\
\nabla \times \vec{E}=-\frac{\partial \vec{B}}{\partial t}
\end{gathered}
$$




$$
\begin{aligned}
\nabla \times \vec{H} & =\frac{\partial \vec{D}}{\partial t}+\vec{J} \\
\vec{D} & =\varepsilon \vec{E} \\
\vec{B} & =\mu \vec{H}
\end{aligned}
$$

Eliminating charge density which relates to current density through continuity equation, eliminating magnetic fields in 5.1-5.6 and assuming harmonic time dependence $e^{-i \omega t}$ leads to a vector wave equation for electric field with the source term,

$$
\nabla^{2} \vec{E}+k^{2} \vec{E}=-i \omega \mu\left(\vec{J}+\frac{1}{k^{2}} \nabla \nabla \cdot \vec{J}\right)
$$

where $\vec{J}$ is an impressed current density. An impressed current source here has the following context: a current driven by a fixed external source not affected by fields $\vec{E}, \vec{H}$ under investigation, for example an ideal current generator as in the circuit theory. (i.e. conductor whose terminals connected to voltage source) An alternative to impressed current source is an induced current source which can be represented by a source of free charges (a conductor with finite conductivity) driven by fields $\vec{E}, \vec{H}$ (i.e. conductor not connected to a voltage source). If both current sources are present (conductors connected to voltage source and conductor not connected to voltage source), the total current is the sum of both. Solving it involves introducing an auxiliary vector function $\vec{F}$ satisfying the following equation,

$$
\nabla^{2} \vec{F}+k^{2} \vec{F}=-i \omega \mu \vec{J}
$$

By inspection both right hand sides it is seen that an original wave equation 5.7 differs from an auxiliary equation 5.8 by an operator $\left(1+\frac{1}{k^{2}} \nabla \nabla \cdot\right)$, therefore relation between $\vec{E}$ and $\vec{F}$ can be found from,

$$
\nabla^{2} \vec{E}+k^{2} \vec{E}=\left(1+\frac{1}{k^{2}} \nabla \nabla \cdot\right)\left(\nabla^{2} \vec{F}+k^{2} \vec{F}\right)
$$

Since operators $\nabla^{2}$ and $\nabla \nabla \cdot$ commute (see Appendix B), the right hand side of 5.9 can be rewritten as,

$$
\begin{aligned}
& \left(1+\frac{1}{k^{2}} \nabla \nabla \cdot\right)\left(\nabla^{2} \vec{F}+k^{2} \vec{F}\right)=\nabla^{2} \vec{F}+k^{2} \vec{F}+\frac{1}{k^{2}} \nabla\left(\nabla \cdot\left(\nabla^{2} \vec{F}\right)\right)+\frac{1}{k^{2}} \nabla \nabla \cdot k^{2} \vec{F}= \\
& =\nabla^{2} \vec{F}+k^{2} \vec{F}+\frac{1}{k^{2}} \nabla^{2}(\nabla(\nabla \cdot \vec{F}))+\frac{1}{k^{2}} \nabla \nabla \cdot k^{2} \vec{F}= \\
& =\nabla^{2}\left\{\vec{F}+\frac{1}{k^{2}} \nabla(\nabla \cdot \vec{F})\right\}+k^{2}\left\{\vec{F}+\frac{1}{k^{2}} \nabla(\nabla \cdot \vec{F})\right\}=\nabla^{2} \vec{E}+k^{2} \vec{E}
\end{aligned}
$$

Therefore the solution of 5.7 can be found from an auxiliary solution of 5.8 by applying an operator $\left(1+\frac{1}{k^{2}} \nabla \nabla \cdot\right)$, 


$$
\vec{E}=\vec{F}+\frac{1}{k^{2}} \nabla \nabla \cdot \vec{F}
$$

Since the waveguide is inhomogeneous only in radial direction while uniform and homogeneous along $z$ and $\theta$, it is advantageous to apply a double Fourier transform in $(z, \theta)$ to the vector equations 5.7, 5.8 and 5.10. This is a standard technique of solving partial differential equations (PDE) that allows to lift all the derivatives with respect to $z$ and $\theta$ replacing them in a transformed space by a Fourier transform of original function with some scale factor.

Below are the transformation pair for some function $Q$ and its Fourier image $q$ where transform in is finite in the sense that integration limits $(-\pi, \pi)$ are finite which results in the inverse transform being summation rather than integration,

$$
\begin{gathered}
q(r, m, v)=\int_{-\infty}^{+\infty} \int_{-\pi}^{+\pi} Q(r, \theta, z) e^{-i(v z+m \theta)} d \theta d z, \quad m=0, \pm 1, \pm 2, \ldots \\
Q(r, \theta, z)=\frac{1}{(2 \pi)^{2}} \sum_{m=-\infty}^{\infty}\left(\int_{-\infty}^{+\infty} q(r, m, v) e^{i(v z+m \theta)} d v\right)
\end{gathered}
$$

Fourier transforms of derivatives with respect to $z$ and $\theta$ in a waveguide environment can be simplified and expressed through Fourier transforms of original function as follows,

$\int_{-\infty}^{+\infty} \int_{-\pi}^{+\pi} \frac{\partial Q}{\partial z} e^{-i(v z+m \theta)} d \theta d z=\left[\int_{-\pi}^{+\pi} Q(r, \theta, z) e^{-i(v z+m \theta)} d \theta\right]_{-\infty}^{+\infty}-(-i v) \int_{-\infty}^{+\infty} \int_{-\pi}^{+\pi} Q(r, \theta, z) e^{-i(v z+m \theta)} d \theta d z$

Assuming a small loss is always present in a waveguide, the integrand in the first term of the right hand side at infinity $z= \pm \infty$ vanishes, therefore Fourier transform of derivative with respect to $z$ is replaced by a function in transformed space and a factor $(i v)$,

$$
\begin{gathered}
\int_{-\infty}^{+\infty} \int_{-\pi}^{+\pi} \frac{\partial Q}{\partial z} e^{-i(v z+m \theta)} d \theta d z=(i v) q(r, m, v) \\
\int_{-\infty}^{+\infty} \int_{-\pi}^{+\pi} \frac{\partial Q}{\partial \theta} e^{-i(v z+m \theta)} d \theta d z=\left[\int_{-\infty}^{+\infty} Q(r, \theta, z) e^{-i(v z+m \theta)} d z\right]_{-\pi}^{+\pi}-(-i m) \int_{-\infty}^{+\infty} \int_{-\pi}^{+\pi} Q(r, \theta, z) e^{-i(v z+m \theta)} d \theta d z
\end{gathered}
$$

The first term in the right hand side is zero because points at $\theta=-\pi$ and $\theta=\pi$ are physically identical, therefore Fourier transform of derivative with respect to $\theta$ is replaced by a function in transformed space and a factor $(\mathrm{im})$, 


$$
\int_{-\infty}^{+\infty} \int_{-\pi}^{+\pi} \frac{\partial Q}{\partial \theta} e^{-i(v z+m \theta)} d \theta d z=(i m) q(r, m, v)
$$

Applying Fourier transform to equations 5.7, 5.8 and 5.10 leads to the following equations in a transformed space,

$$
\begin{gathered}
\left(\nabla^{\prime}\right)^{2} \vec{e}+k^{2} \vec{e}=-i \omega \mu\left(\vec{j}+\frac{1}{k^{2}} \nabla^{\prime} \nabla^{\prime} \cdot \vec{j}\right) \\
\left(\nabla^{\prime}\right)^{2} \vec{f}+k^{2} \vec{f}=-i \omega \mu \vec{j} \\
\vec{e}(r, m, v)=\vec{f}(r, m, v)+\frac{1}{k^{2}} \nabla^{\prime} \nabla^{\prime} \cdot \vec{f}(r, m, v)
\end{gathered}
$$

here the $\nabla^{\prime}$ is an operator $\nabla$ where, as a result of taking Fourier integral by parts, the derivatives $d / d z$ and $d / d \theta$ replaced by $i v$ and $i m$ respectively; the vector functions $\vec{e}(r, m, v), \vec{j}(r, m, v)$ and $\vec{f}(r, m, v)$ are Fourier transforms of vector functions $\vec{E}(r, \theta, z)$, $\vec{J}(r, \theta, z)$ and $\vec{F}(r, \theta, z)$ respectively. As a result, $\left(\nabla^{\prime}\right)^{2}$, Laplacian operator in transformed domain $(r, m, v)$ is given by,

$$
\left(\nabla^{\prime}\right)^{2}=\frac{\partial^{2}}{\partial r^{2}}+\frac{1}{r} \frac{\partial}{\partial r}-\frac{1}{r^{2}} m^{2}-v^{2}
$$

In a transformed domain an auxiliary vector equation 5.15 splits up in three coupled scalar equations,

$$
\begin{gathered}
\left(\nabla^{\prime}\right)^{2} f_{r}-\frac{1}{r^{2}} f_{r}-\frac{2}{r^{2}}(i m) f_{\theta}+k^{2} f_{r}=-i \omega \mu j_{r} \\
\left(\nabla^{\prime}\right)^{2} f_{\theta}-\frac{1}{r^{2}} f_{\theta}+\frac{2}{r^{2}}(i m) f_{r}+k^{2} f_{\theta}=-i \omega \mu j_{\theta} \\
\left(\nabla^{\prime}\right)^{2} f_{z}+k^{2} f_{z}=-i \omega \mu j_{z}
\end{gathered}
$$

Equations 5.19 and 5.20 have common components $f_{r}, f_{\theta}$ and therefore in such form difficult to solve. The decoupling can be done by the following substitution (see [83]),

$$
\begin{aligned}
& f_{+}=f_{r}+i f_{\theta} \\
& f_{-}=f_{r}-i f_{\theta}
\end{aligned}
$$


and the procedure 5.19 $\pm i 5.20$. The decoupled scalar wave equations for $f_{+}, f_{-}, f_{z}$ become,

$$
\begin{gathered}
\frac{\partial^{2} f_{+}}{\partial r^{2}}+\frac{1}{r} \frac{\partial f_{+}}{\partial r}-\frac{(m+1)^{2}}{r^{2}} f_{+}+p^{2} f_{+}=-i \omega \mu j_{+} \\
\frac{\partial^{2} f_{-}}{\partial r^{2}}+\frac{1}{r} \frac{\partial f_{-}}{\partial r}-\frac{(m-1)^{2}}{r^{2}} f_{-}+p^{2} f_{-}=-i \omega \mu j_{-} \\
\frac{\partial^{2} f_{z}}{\partial r^{2}}+\frac{1}{r} \frac{\partial f_{z}}{\partial r}-\frac{m^{2}}{r^{2}} f_{z}+p^{2} f_{z}=-i \omega \mu j_{z}
\end{gathered}
$$

where $p^{2}=k^{2}-v^{2}$ and substitution 5.18 for a transformed Laplacian $\left(\nabla^{\prime}\right)^{2}$ also applied. Solution of scalar equation 5.24 in the form of integral is straightforward provided an appropriate Green's function is known,

$$
f_{+}(r, m, v)=i \omega \mu \int_{S} g_{m+1}(r \mid s) j_{+}(s, m, v) s d s
$$

where the integration is done over position of the current source; $g_{n}\left(r \mid r_{0}\right)$ is Green's function for Bessel equation,

$$
\frac{\partial^{2} g_{n}}{\partial r^{2}}+\frac{1}{r} \frac{\partial g_{n}}{\partial r}-\frac{n^{2}}{r^{2}} g_{n}+\gamma^{2} g_{n}=-\frac{1}{r} \delta\left(r-r_{0}\right)
$$

$g_{n}\left(r \mid r_{0}\right)$ is well known and defined as,

$$
g_{n}\left(r \mid r_{0}\right)= \begin{cases}i \frac{\pi}{2} H_{n}^{(1)}\left(\gamma r_{0}\right) J_{n}(\gamma r), & r \leq r_{0} \\ i \frac{\pi}{2} J_{n}\left(\gamma r_{0}\right) H_{n}^{(1)}(\gamma r), & r \geq r_{0}\end{cases}
$$

where $J_{n}(x), H_{n}^{(1)}(x)$ are Bessel and Hankel functions. The remaining scalar equations 5.25 and 5.26 are treated in a similar fashion, their solutions represented by the integrals below,

$$
\begin{gathered}
f_{-}(r, m, v)=i \omega \mu \int_{S} g_{m-1}(r \mid s) j_{-}(s, m, v) s d s \\
f_{z}(r, m, v)=i \omega \mu \int_{S} g_{m}(r \mid s) j_{z}(s, m, v) s d s
\end{gathered}
$$

The $r$ and $\theta$ components of a vector function $\vec{f}$ are,

$$
f_{r}=\frac{1}{2}\left(f_{+}+f_{-}\right)
$$




$$
f_{\theta}=\frac{1}{i 2}\left(f_{+}-f_{-}\right)
$$

The transformed electric field function $\vec{e}(r, m, v)$ then can be found by straightforward evaluation according to 5.17. Once electric field is known the magnetic field is calculated from 5.3,

$$
\vec{H}=\frac{1}{i \omega \mu} \nabla \times \vec{E}
$$

or in transformed space,

$$
\vec{h}(r, m, v)=\frac{1}{i \omega \mu} \nabla^{\prime} \times \vec{e}(r, m, v)
$$

In this section an inhomogeneous vector Helmholtz equation in circular cylindrical coordinates were split up into three uncoupled scalar equations, i.e. into three partial differential equations with one unknown scalar function in each equation which was solved with the help of one dimensional Green's function for Bessel equation. The splitting achieved by a Fourier transform followed by substitutions 5.22, 5.23 introduced in [83] for untransformed domain . Another approach that allows solving non-scalar Helmholtz equation in a more general cylindrical coordinates (that includes circular based as well as parabolic and elliptical) involves spin-weighted cylindrical harmonics which can be viewed as a generalization of Bessel functions. This method described in details in $[84,85]$.

\subsection{Scattering in fiber due to current source.}

The simulation model is the step index fiber with cladding coated by a thin metal layer followed by an outer layer of air. In order to simplify the calculation the boundary between the core and cladding ignored, i.e. core and cladding assumed to have the same refractive index as for cladding. This assumption is justified since the plasmon modes essentially excited by cladding modes and cladding modes of a fiber with core-cladding boundary do not significantly differ from cladding modes of a fiber without core-cladding boundary. Also, the plasmon modes are localized near the metal layer which is far away from the fiber core. This assumption is done just for the sake of simplifying numerical simulation, it is not a principle one.

The current source, assembled as an array of planes, is located in the fiber core. The primary fields $\vec{e}^{p}, \vec{h}^{p}$, i.e. the fields directly due to current source, exist in a cladding layer only, as indicated in Fig. 5.1. They obey Maxwell's and wave equations with the source term. The scattered fields, $\vec{e}^{s}, \vec{h}^{s}$ exist in each layer including cladding. They are due to primary fields scattered off boundary. They can be calculated from Maxwell's equations

with zero source terms. The total field in the cladding is the sum of primary and scattered 


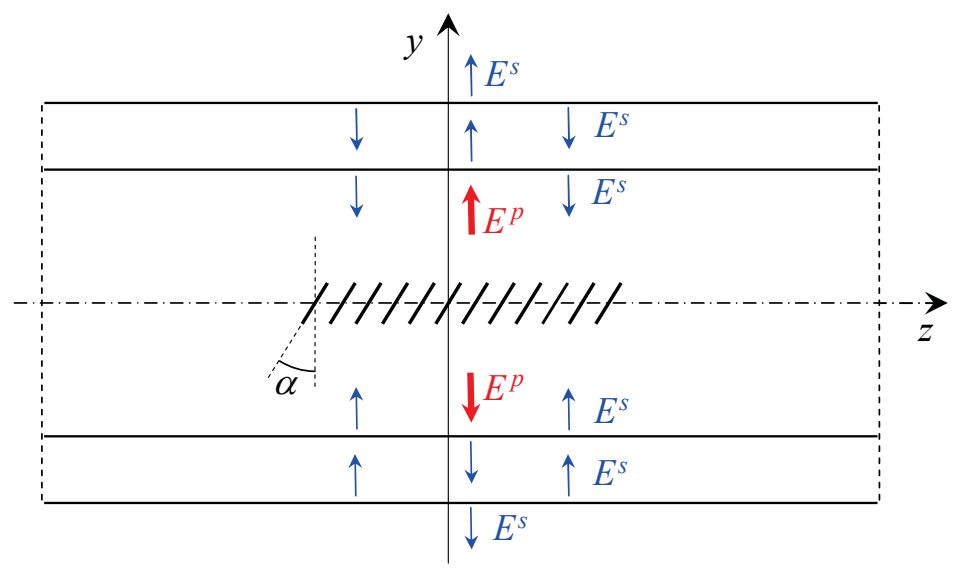

Figure 5.1: Scattering fields in a cylindrical 3 layer waveguide with the current source, shown as an array of tilted elements, in the first layer (in the core). $E^{p}$ represents a primary field due to current source, $E^{s}$ is a scattered field in the waveguide.

ones. Imposing the boundary conditions at each interface couples the primary and the scattered field at each layer.

All the scattered field components can be calculated from two independent field components say $e_{z}^{s}, h_{z}^{s}$. In the transformed space $(r, m, v)$ the two curl Maxwell's equations are,

$$
\begin{gathered}
\nabla^{\prime} \times \vec{e}^{s}=i \omega \mu \vec{h}^{s} \\
\nabla^{\prime} \times \vec{h}^{s}=-i \omega \varepsilon \vec{e}^{s}
\end{gathered}
$$

Solving them for $e_{r}^{s}, e_{\theta}^{s}, h_{r}^{s}, h_{\theta}^{s}$ gives,

$$
\begin{aligned}
e_{r}^{s} & =\frac{i}{\omega^{2} \mu \varepsilon-v^{2}}\left(v \frac{\partial e_{z}^{s}}{\partial r}+\omega \mu \frac{1}{r} i m h_{z}^{s}\right) \\
e_{\theta}^{s} & =\frac{i}{\omega^{2} \mu \varepsilon-v^{2}}\left(v \frac{1}{r} i m e_{z}^{s}-\omega \mu \frac{\partial h_{z}^{s}}{\partial r}\right) \\
h_{r}^{s} & =\frac{i}{\omega^{2} \mu \varepsilon-v^{2}}\left(-\omega \varepsilon \frac{1}{r} i m e_{z}^{s}+v \frac{\partial h_{z}^{s}}{\partial r}\right) \\
h_{\theta}^{s} & =\frac{i}{\omega^{2} \mu \varepsilon-v^{2}}\left(\omega \varepsilon \frac{\partial e_{z}^{s}}{\partial r}+v \frac{1}{r} i m h_{z}^{s}\right)
\end{aligned}
$$


where $e_{z}^{s}, h_{z}^{s}$ can be found from the scalar wave equations with zero source term. Below are z-components $e_{z}^{s}, h_{z}^{s}$ of cladding, metal and outer layer respectively,

$$
\begin{gathered}
e_{z 2}^{s}=A_{2} J_{m}\left(p_{2} r\right) \\
h_{z 2}^{s}=C_{2} J_{m}\left(p_{2} r\right) \\
e_{z 3}^{s}=A_{3} K_{m}\left(q_{3} r\right)+B_{3} I_{m}\left(q_{3} r\right) \\
h_{z 3}^{s}=C_{3} K_{m}\left(q_{3} r\right)+D_{3} I_{m}\left(q_{3} r\right) \\
e_{z 4}^{s}=B_{4} K_{m}\left(q_{4} r\right) \\
h_{z 4}^{s}=D_{4} K_{m}\left(q_{4} r\right)
\end{gathered}
$$

where unknown coefficients $A_{2}, B_{2}, A_{3}, B_{3}, C_{3}, D_{3}, B_{4}, D_{4}$ don't depend on $r$,

$$
\begin{aligned}
& p_{2}=\sqrt{\omega^{2} \mu \varepsilon_{2}-v^{2}} \\
& q_{3}=\sqrt{v^{2}-\omega^{2} \mu \varepsilon_{3}} \\
& q_{4}=\sqrt{v^{2}-\omega^{2} \mu \varepsilon_{4}}
\end{aligned}
$$

$J_{m}(x), K_{m}(x), I_{m}(x)$ appropriate Bessel functions. Applying boundary conditions for tangential field components at each interface $\left(r=r_{2}, \quad r=r_{2}+d\right)$ leads to 8 linear inhomogeneous equations for 8 unknown coefficients $A_{2}, B_{2}, A_{3}, B_{3}, C_{3}, D_{3}, B_{4}, D_{4}$,

$$
\begin{aligned}
& e_{z 2}^{s}\left(r_{2}\right)-e_{z 3}^{s}\left(r_{2}\right)=-e_{z 2}^{p}\left(r_{2}\right) \\
& h_{z 2}^{s}\left(r_{2}\right)-h_{z 3}^{s}\left(r_{2}\right)=-h_{z 2}^{p}\left(r_{2}\right) \\
& e_{\theta 2}^{s}\left(r_{2}\right)-e_{\theta 3}^{s}\left(r_{2}\right)=-e_{\theta 2}^{p}\left(r_{2}\right) \\
& h_{\theta 2}^{s}\left(r_{2}\right)-h_{\theta 3}^{s}\left(r_{2}\right)=-h_{\theta 2}^{p}\left(r_{2}\right)
\end{aligned}
$$




$$
\begin{aligned}
& e_{z 3}^{s}\left(r_{2}+d\right)-e_{z 4}^{s}\left(r_{2}+d\right)=0 \\
& h_{z 3}^{s}\left(r_{2}+d\right)-h_{z 4}^{s}\left(r_{2}+d\right)=0 \\
& e_{\theta 3}^{s}\left(r_{2}+d\right)-e_{\theta 4}^{s}\left(r_{2}+d\right)=0 \\
& h_{\theta 3}^{s}\left(r_{2}+d\right)-h_{\theta 4}^{s}\left(r_{2}+d\right)=0
\end{aligned}
$$

where $r_{2}$ cladding radius, $d$ metal thickness. In matrix form these equations written as,

$$
M_{0} A=S
$$

where $A$ is a column vector, made up of unknown coefficients,

$$
A=\left[A_{2}, B_{2}, A_{3}, B_{3}, C_{3}, D_{3}, B_{4}, D_{4}\right]^{T}
$$

$S$ is column vector of source terms, i.e. made up of primary fields,

$$
S=\left[-e_{z 2}^{p}\left(r_{2}\right),-h_{z 2}^{p}\left(r_{2}\right),-e_{\theta 2}^{p}\left(r_{2}\right),-h_{\theta 2}^{p}\left(r_{2}\right), 0,0,0,0\right]^{T}
$$

$M_{0}$ is a matrix whose elements are appropriate factors in front of each coefficient $A_{2}, B_{2}$, $A_{3}, B_{3}, C_{3}, D_{3}, B_{4}, D_{4}$ for each equation in 5.51-5.58.

Since the plasmon modes predominantly localized near the interface at outer layer, only coefficients $B_{4}, D_{4}$ are needed (for the fields due to plasmon). Follow Cramer's rule they can be evaluated as,

$$
\begin{aligned}
& B_{4}=\frac{\operatorname{det}\left(M_{B 4}\right)}{\operatorname{det}\left(M_{0}\right)} \\
& D_{4}=\frac{\operatorname{det}\left(M_{D 4}\right)}{\operatorname{det}\left(M_{0}\right)}
\end{aligned}
$$

here $M_{B 4}, M_{D 4}$ are matrices formed from $M_{0}$ by replacing columns 7,8 respectively by column vector of the source terms $S$. To reconstruct the original scattered fields in physical space $(r, \theta, z)$ the inverse Fourier transform applied to the scattered fields in transformed space $(r, m, v)$, for layer 4 the radial component of electric field is,

$$
E_{r 4}(r, \theta, z)=\frac{1}{(2 \pi)^{2}} \sum_{m=-\infty}^{\infty}\left(\int_{-\infty}^{+\infty} e_{r 4}^{s}(r, m, v) e^{i(v z+m \theta)} d v\right)
$$




$$
\begin{aligned}
& E_{r 4}(r, \theta, z)= \\
& =\frac{1}{(2 \pi)^{2}} \sum_{m=-\infty}^{\infty}\left(\int_{-\infty}^{+\infty} \frac{i}{-p_{4}^{2}}\left(\frac{\operatorname{det}\left(M_{B 4}\right)}{\operatorname{det}\left(M_{0}\right)} v q_{4} K_{m}{ }^{\prime}\left(q_{4} r\right)+\frac{\operatorname{det}\left(M_{D 4}\right)}{\operatorname{det}\left(M_{0}\right)} \omega \mu \frac{1}{r} i m K_{m}\left(q_{4} r\right)\right) e^{i(v z+m \theta)} d v\right)
\end{aligned}
$$

The integral above belongs to the family of Sommerfeld integrals with integration path along the real axis on a complex plane closed by an infinite semicircle on upper half plane. According to Cauchy's residue theorem the contribution to the integral comes from isolated special points, poles, and from special points of type branch points enclosed by integration path.

The poles, or the zeros of $\operatorname{det}\left(M_{0}\right)$ in 5.63 , contributing to the integral by discrete sum of their residues; they are associated with the isolated modes of a source free waveguide. While the branch points contributing by continuous integration along the branch cuts; they are associated with radiation modes responsible for delivering energy of the waveguide in the far field zone, (see $[76,86]$ ).

Since the main goal here is investigation of plasmon modes under various excitation parameters, only contribution from the plasmon mode with particular azimuthal index $m$ to the integral is considered.

$$
\begin{aligned}
& E_{r 4, S P P}= \\
& =\frac{1}{(2 \pi)^{2}} \int_{-\infty}^{+\infty} \frac{i}{-p_{4}^{2}}\left(\frac{\operatorname{det}\left(M_{B 4}\right)}{\operatorname{det}\left(M_{0}\right)} v q_{4} K_{m}{ }^{\prime}\left(q_{4} r\right)+\frac{\operatorname{det}\left(M_{D 4}\right)}{\operatorname{det}\left(M_{0}\right)} \omega \mu \frac{1}{r} i m K_{m}\left(q_{4} r\right)\right) e^{i(v z+m \theta)} d v
\end{aligned}
$$

For some fractional function $f / g$ with isolated pole at $z_{0}$ the integral over real axis can be evaluated with the help of Cauchy's residue theorem, provided the function vanishes at infinity on complex plane,

$$
\int_{-\infty}^{+\infty} \frac{f(z)}{g(z)} d z=i 2 \pi \operatorname{Res}\left(\frac{f(z)}{g(z)}\right)=i 2 \pi \frac{f\left(z_{0}\right)}{g^{\prime}\left(z_{0}\right)}
$$

provided,

$$
g\left(z_{0}\right)=0, \quad \lim _{|z| \rightarrow \infty} \frac{f(z)}{g(z)}=0
$$

The field in 5.64 for a plasmon mode with certain values $\left(v_{0}, m_{0}\right)$ then reduces to, 


$$
\begin{aligned}
& E_{r 4, S P P}= \\
& =\frac{i}{2 \pi}\left[\operatorname{Res}\left(\frac{i}{-p_{4}^{2}}\left(\frac{\operatorname{det}\left(M_{B 4}\right)}{\operatorname{det}\left(M_{0}\right)} v q_{4} K_{m}^{\prime}\left(q_{4} r\right)+\frac{\operatorname{det}\left(M_{D 4}\right)}{\operatorname{det}\left(M_{0}\right)} \omega \mu \frac{1}{r} i m K_{m}\left(q_{4} r\right)\right) e^{i(v z+m \theta)}\right)\right]_{\substack{v=v_{0} \\
m=m_{0}}}
\end{aligned}
$$

where $m_{0}$ is a chosen azimuthal index and a propagation constant of the mode $v_{0}$ defined from condition,

$$
\left[\operatorname{det}\left(M_{0}\right)\right]_{\substack{v=v_{0} \\ m=m_{0}}}=0
$$

Derivative of matrix determinant can be evaluates as a sum over rows of determinants of a matrix where one row replaced by its derivative, for example for 3x3 matrix and its rows $R_{1}, R_{2}, R_{3}$ the formula is,

$$
\frac{d}{d x} \operatorname{det}\left|\begin{array}{l}
R_{1} \\
R_{2} \\
R_{3}
\end{array}\right|=\operatorname{det}\left|\begin{array}{c}
R_{1}^{\prime} \\
R_{2} \\
R_{3}
\end{array}\right|+\operatorname{det}\left|\begin{array}{c}
R_{1} \\
R_{2}^{\prime} \\
R_{3}
\end{array}\right|+\operatorname{det}\left|\begin{array}{c}
R_{1} \\
R_{2} \\
R_{3}^{\prime}
\end{array}\right|
$$

where $R_{1}^{\prime}, R_{2}^{\prime}, R_{3}^{\prime}$ derivatives of $R_{1}, R_{2}, R_{3}$ respectively.

All other fields components can be calculated in similar fashion, this allows evaluate power carried in outer layer (layer 4 ) through plane $z=0$ by a plasmon mode propagating in z-direction,

$$
P=\left[\int_{r_{3}}^{\infty} \int_{0}^{2 \pi} P_{z 4} r d \theta d r\right]_{z=0}
$$

where $P_{z 4}$ is $z$-component of time-averaged Poynting vector which for the mode in layer 4 is given by,

$$
P_{z 4}=\operatorname{Re}\left(E_{r 4} H_{\theta 4}^{*}-E_{\theta 4} H_{r 4}^{*}\right)
$$

With asymptotic approximation of Bessel function for large argument the integral can be evaluated analytically.

\subsection{Excitation sources.}

Assume an incident field $\vec{E}^{i}$ propagates in a fiber and scatters off a non-magnetic inhomogeneity inserted in a fiber core. The scattering is viewed as a reradiation, i.e. an excitation caused by the incident field followed by emission with the same frequency as in the case 
of a two level atom material. The excitation, modeled by a current, limited spacially by inhomogeneity region. Since excitation is due to an incident field, the current is proportional to the incident field, i.e. equal up to a scale factor. The scattered field then is due to this current, therefore to calculate the scattered field involves solving an inhomogeneous wave equation 5.7 with the current, flowing in inhomogeneity, as a source term. Such oversimplified intuitive model of a scattering can be justified as a first order approximation of some iterative scheme. The incident field is the field of a core mode $\vec{E}_{\text {core }}$ in a fiber SMF28 (see [87]) surrounded by some material, typically air, water or index matching oil. For simplicity a presence of the metal coating is ignored in evaluating $\vec{E}_{\text {core }}$, because most energy of the core mode concentrated in the fiber core which is far away from the metal layer and therefore weakly affected by it. Such simplification is for numerical calculation ease only. The SMF28 fiber admits two core modes, one with azimuthal index $m_{C}=1$ and other with $-m_{C}$, both of them having identical effective index $n_{e f f}$. The third core mode with $m_{C}=0$ (TE, TM modes) also exist for wavelength below $1310 \mathrm{~nm}$ which is not considered here since the wavelength range of interest is above $1310 \mathrm{~nm}$ where TE, TM modes suffer cutoff condition. Therefore the general electric field of the core mode is given by,

$$
\vec{E}_{\text {core }}(r, \theta, z)=\vec{R}_{\text {core }}(r) e^{i \beta z}\left(e^{i m_{C} \theta}+e^{-i m_{C}\left(\theta+\theta_{0}\right)}\right)
$$

where

$\vec{R}_{\text {core }}(r)=\left(R_{r}(r), R_{\theta}(r), R_{z}(r)\right)$

$R_{r}(r)=\frac{i}{q_{1}^{2}}\left(\beta q_{1} A_{1} J_{m_{C}}^{\prime}\left(q_{1} r\right)+\omega \mu \frac{1}{r} i m_{C} C_{1} J_{m_{C}}\left(q_{1} r\right)\right)$

$R_{\theta}(r)=\frac{i}{q_{1}^{2}}\left(\beta \frac{1}{r} i m_{C} A_{1} J_{m_{C}}\left(q_{1} r\right)-\omega \mu q_{1} C_{1} J_{m_{C}}^{\prime}\left(q_{1} r\right)\right)$

$R_{z}(r)=A_{1} J_{m_{C}}\left(q_{1} r\right)$

$R_{+}(r)=R_{r}(r)+i R_{\theta}(r)$

$R_{-}(r)=R_{r}(r)-i R_{\theta}(r)$

$q_{1}=\sqrt{\omega^{2} \mu \varepsilon_{1}-\beta^{2}}$

$\beta=k_{0} n_{\text {eff }}$

$A_{1}, C_{1}$ constant coefficients scaled with some arbitrary factor, the ratio $A_{1} / C_{1}$ is determined by satisfying the boundary conditions at the mode's effective index $n_{\text {eff }}$

$\theta_{0}$ is phase shift between two modes. The current density source is proportional to the field,

$$
\vec{J}(r, \theta, z)=C_{0} L(r, \theta, z) \vec{E}_{\text {core }}(r, \theta, z)
$$

where $C_{0}$ is a constant, and $L(r, \theta, z)$ is a function describing source location.

The following current sources are treated below.

(A) a set of rings, as in Fig. 5.2, with common center all positioned in the fiber core on a single tilted plane. A tilt is performed in $(y z)$-plane with respect to $x$-axis. Tilt angle $\alpha$ is measured between $y$-axis and tilted axis $y^{\prime}$. For a non-zero tilt angle each ring is an ellipse. 


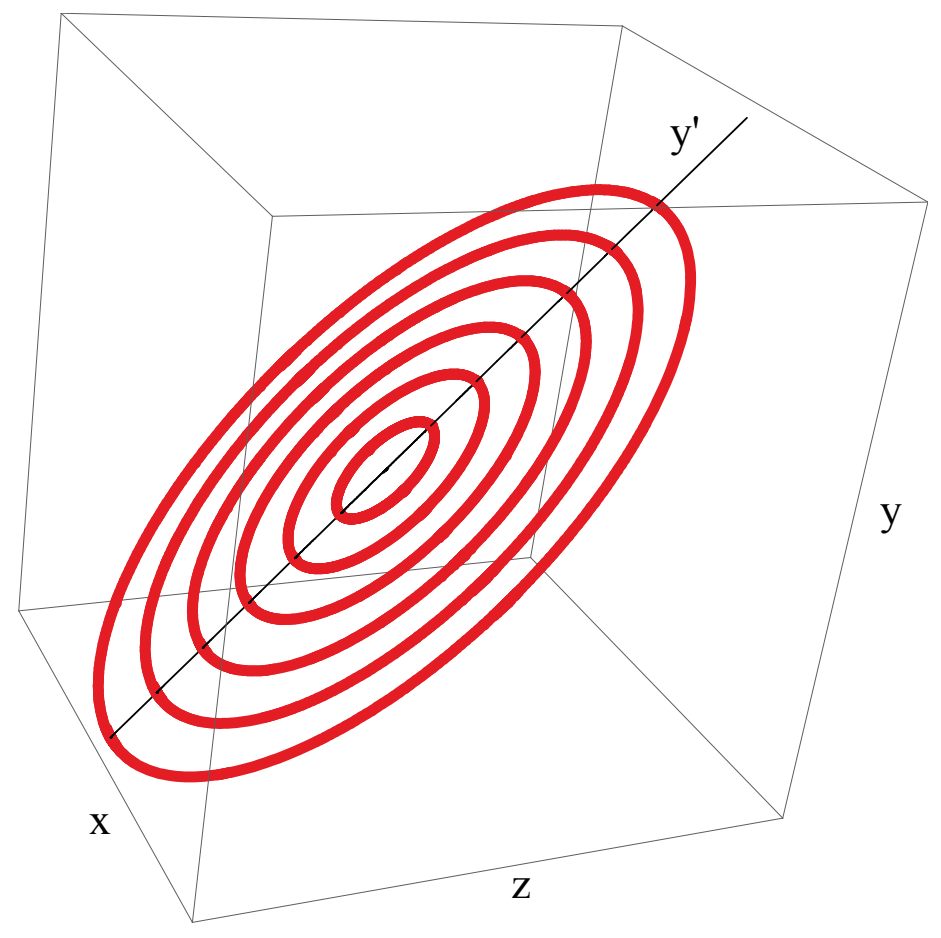

Figure 5.2: Current source as a set of rings with common center positioned on a single tilted plane. A tilt is done in $(y z)$-plane with respect to $x$-axis. Tilt angle $\alpha$ measured between $y$-axis and tilted axis $y^{\prime}$.

(B) a disc positioned in the fiber core on a tilted plane. For a non-zero tilt angle a disc is an interior of some ellipse. A few tilted discs with different tilt angle schematically illustrated in Fig. 5.3.

(C) an array either uniform or $\pi$-shifted made of elements as in (A) or in (B). Illustration of such array presented in Fig. 5.4.

\subsection{1 (A) Excitation by rings of current.}

A single ring with radius $r_{0}$ tilted in $(y z)$-plane at an angle $\alpha$ with respect to $y$-axis and intersecting $z$-axis at $z_{0}$ described by the following function,

$$
L(r, \theta, z)=\delta\left(z-r \sin \theta \tan \alpha-z_{0}\right) \delta\left(r-r_{0}\right)
$$

while series of $N_{R}$ rings in this plane with common center and equidistant radiuses described by the function,

$$
L(r, \theta, z)=\delta\left(z-r \sin \theta \tan \alpha-z_{0}\right) D c(r, \Delta r)
$$




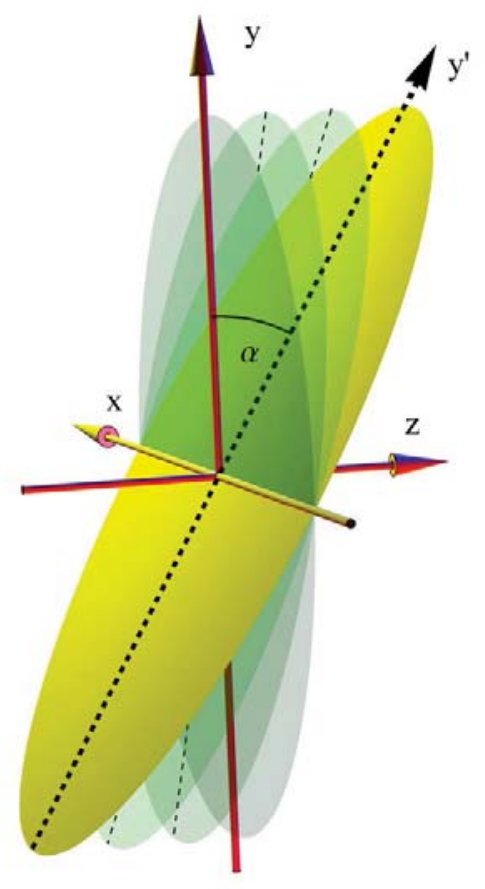

Figure 5.3: Current source as a disc positioned on a single tilted plane. A tilt is done in $(y z)$-plane with respect to $x$-axis. Tilt angle $\alpha$ measured between $y$-axis and tilted axis $y^{\prime}$.

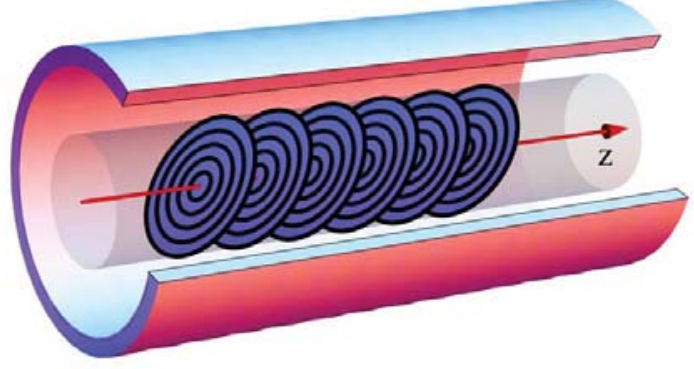

(a)

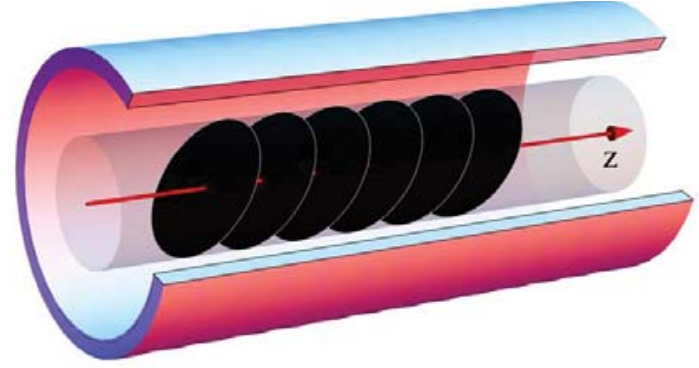

(b)

Figure 5.4: Current source as an array of tilted elements located in the fiber core. Array is either uniform or $\pi$-shifted with element type (a): set of rings with common center on a single plane or, (b): disc.

where $\delta(x)$ Dirac delta function, $D c(r, \Delta r)$ Dirac comb with the finite number of terms,

$$
D c(r, \Delta r)=\sum_{j=1}^{N_{R}} \delta(r-j \Delta r)
$$

Exploiting integral representation of Bessel function, 


$$
J_{n}(x)=\frac{1}{2 \pi} \int_{-\pi}^{\pi} e^{-i(n \varphi-x \sin \varphi)} d \varphi
$$

the Fourier transform of current density can be calculated analytically as follows,

$$
\begin{gathered}
j_{z}(r, m, v)=\int_{-\infty}^{+\infty} \int_{-\pi}^{+\pi} J_{z}(r, \theta, z) e^{-i(v z+m \theta)} d \theta d z \\
j_{z}=C_{0} D c(r, \Delta r) R_{z}(r) 2 \pi e^{i(\beta-v) z_{0}} B
\end{gathered}
$$

where,

$$
B=\left(J_{m-m_{C}}((\beta-v) r \tan \alpha)+e^{-i m_{C} \theta_{0}} J_{m+m_{C}}((\beta-v) r \tan \alpha)\right)
$$

Expressions for $j_{+}, j_{-}$obtained from expression for $j_{z}$ by replacing $R_{z}(r)$ with $R_{+}(r)$, $R_{-}(r)$ respectively.

The presence of Dirac comb in $j_{z}, j_{+}, j_{-}$allows evaluate components of a vector function $f_{z}, f_{+}, f_{-}$in $5.31,5.27$ and 5.30 respectively in closed form,

$$
\begin{gathered}
f_{z}=-\omega \mu \pi^{2} C_{0} H_{m}^{(1)}\left(p_{2} r\right) e^{i(\beta-v) z_{0}} \sum_{j=1}^{N_{R}} J_{m}\left(p_{2} j \Delta r\right) B_{j} R_{z}(j \Delta r) j \Delta r \\
f_{+}=-\omega \mu \pi^{2} C_{0} H_{m+1}^{(1)}\left(p_{2} r\right) e^{i(\beta-v) z_{0}} \sum_{j=1}^{N_{R}} J_{m+1}\left(p_{2} j \Delta r\right) B_{j} R_{+}(j \Delta r) j \Delta r \\
f_{-}=-\omega \mu \pi^{2} C_{0} H_{m-1}^{(1)}\left(p_{2} r\right) e^{i(\beta-v) z_{0}} \sum_{j=1}^{N_{R}} J_{m-1}\left(p_{2} j \Delta r\right) B_{j} R_{-}(j \Delta r) j \Delta r
\end{gathered}
$$

where,

$$
\begin{gathered}
B_{j}=\left(J_{m-m_{C}}((\beta-v) j \Delta r \tan \alpha)+e^{-i m_{C} \theta_{0}} J_{m+m_{C}}((\beta-v) j \Delta r \tan \alpha)\right) \\
p_{2}^{2}=\omega^{2} \mu \varepsilon_{2}-v^{2}
\end{gathered}
$$

With known $\vec{f}$ all the primary fields $\left(\vec{e}^{p}, \vec{h}^{p}\right)$ due to a set of rings of current and power flowing along $z$-axis in the outer layer are readily evaluated with 5.17, 5.35 and 5.71, 5.70. 


\subsection{2 (B) Excitation by disc of current.}

A disc with radius $r_{0}$ tilted in $(y z)$-plane at an angle $\alpha$ with respect to $y$-axis and intersecting $z$-axis at $z_{0}$ described by the following function,

$$
L(r, \theta, z)=\delta\left(z-r \sin \theta \tan \alpha-z_{0}\right) \Pi\left(r, 0, r_{0}\right)
$$

where $\Pi\left(x, x_{1}, x_{2}\right)$ box function defined as,

$$
\Pi\left(x, x_{1}, x_{2}\right)=\left\{\begin{array}{cc}
1 & x_{1} \leq x \leq x_{2} \\
0 & \text { otherwise }
\end{array}\right.
$$

The Fourier images of the current density components become,

$$
j_{z}=C_{0} \Pi\left(r, 0, r_{0}\right) R_{z}(r) 2 \pi e^{i(\beta-v) z_{0}} B
$$

where $B$ is given by 5.82. Expressions for $j_{+}, j_{-}$obtained from expression for $j_{z}$ by replacing $R_{z}(r)$ with $R_{+}(r), R_{-}(r)$ respectively.

Unlike the case (A) components of vector function $\vec{f}$ can not be calculated analytically because of a lack of delta function in $j_{z}, j_{+}, j_{-}$. The integrands in 5.31, 5.27 and 5.30 now have the product of three Bessel functions of the first kind. For evaluating corresponding integrals each Bessel function involved should be simplified. Approximation of Bessel function $J_{n}(x)$ can be done by Taylor expansion in power series,

$$
J_{n}(x) \approx \sum_{j=0}^{j=N} \frac{(-1)^{j}}{j !(j+n) !}\left(\frac{x}{2}\right)^{2 j+n}
$$

Another alternative is trigonometric expansion (see [88] and Appendix C),

$$
J_{n}(x) \approx \frac{1}{N} \sum_{j=1}^{j=N} e^{-i\left(n \frac{2 \pi}{N} j-x \sin \left(\frac{2 \pi}{N} j\right)\right)}
$$

It turns out that trigonometric expansion has larger convergence radius and for this

reason is more favorable to calculate all components of $\vec{f}$ and eventually primary fields $\left(\vec{e}^{p}, \vec{h}^{p}\right)$ and power carried by mode along $z$-axis in outer layer.

\subsection{3 (C) Excitation by array of rings or discs of current.}

In this case the excitation is due to a set of sources each as in (A) or (B) with equidistant spacing $\Delta_{z}$ along $z$-axis over the whole set forming an uniform array or two uniform halves with spacing $\Delta_{z}$ in each while the second half shifted by $\Delta_{z} / 2$ from the first one forming a $\pi$-phase shifted array. 


\section{Uniform array.}

A $z$ dependent part of position function for rings as in (A) or disc as in (B) in a single tilted plane intersecting $z$-axis at $z_{0}$ is described by Dirac delta function,

$$
\delta\left(z-r \sin \theta \tan \alpha-z_{0}\right)
$$

therefore a $z$ dependent part of position of a series of $2 N_{1}$ of such equidistant objects along $z$-axis, where $N_{1}$ planes located at $z \leq z_{0}$ and remaining $N_{1}$ at $z>z_{0}$ with spacing step $\Delta_{z}$, is described by Dirac comb function,

$$
D c\left(z, \Delta_{z}\right)=\sum_{j=1-N_{1}}^{N_{1}} \delta\left(z-r \sin \theta \tan \alpha-\left(z_{0}-j \Delta_{z}\right)\right)
$$

Hence, integration over $z$ instead of factor $e^{i(\beta-v) z_{0}}$, as is the case for a single source, results in the factor of the following geometric series,

$$
e^{i(\beta-v) z_{0}} \sum_{j=1-N_{1}}^{N_{1}} e^{i(\beta-v) j \Delta_{z}}=e^{i(\beta-v) z_{0}} \frac{e^{i(\beta-v)\left(1-N_{1}\right) \Delta_{z}}-e^{i(\beta-v)\left(N_{1}+1\right) \Delta_{z}}}{1-e^{i(\beta-v) \Delta_{z}}}
$$

Thus a vector function $\vec{f}$ for an uniform array in a region $r>r_{1}\left(r_{1}\right.$ is a core radius) is given by,

$$
\overrightarrow{f_{u}}=A F_{u} \vec{f}_{A B}
$$

where

$\vec{f}_{A B}$ a vector function $\vec{f}$ as in $(\mathrm{A})$ for a series of set of rings or a vector function $\vec{f}$ as in (B) for a series of discs,

$A F_{u}$ an array factor for a uniform array of $2 N_{1}$ discs or sets of rings,

$$
A F_{u}=\frac{e^{i(\beta-v)\left(1-N_{1}\right) \Delta_{z}}-e^{i(\beta-v)\left(N_{1}+1\right) \Delta_{z}}}{1-e^{i(\beta-v) \Delta_{z}}}
$$

\section{$\pi$-shifted array.}

A phase shifted array can be viewed as two uniform arrays with number of elements in each $N_{1}$ and $N_{2}$ shifted with respect to each other by a certain distance $z_{s}$, as shown in Fig. 5.5. For a $\pi$-shifted array these two uniform arrays are identical, i.e. $N_{1}=N_{2}$ with equal spacing $\Delta_{z}$ for both and with shift distance equal to half of a spacing, $z_{s}=\Delta_{z} / 2$.

The function that describes position of a $\pi$-shifted array of $2 N_{1}$ elements, either discs or set of rings, with $N_{1}$ located at $z \leq z_{0}$ and remaining $N_{1}$ at $z>z_{0}$ and with spacing step $\Delta_{z}$ is given by the following Dirac comb function, 


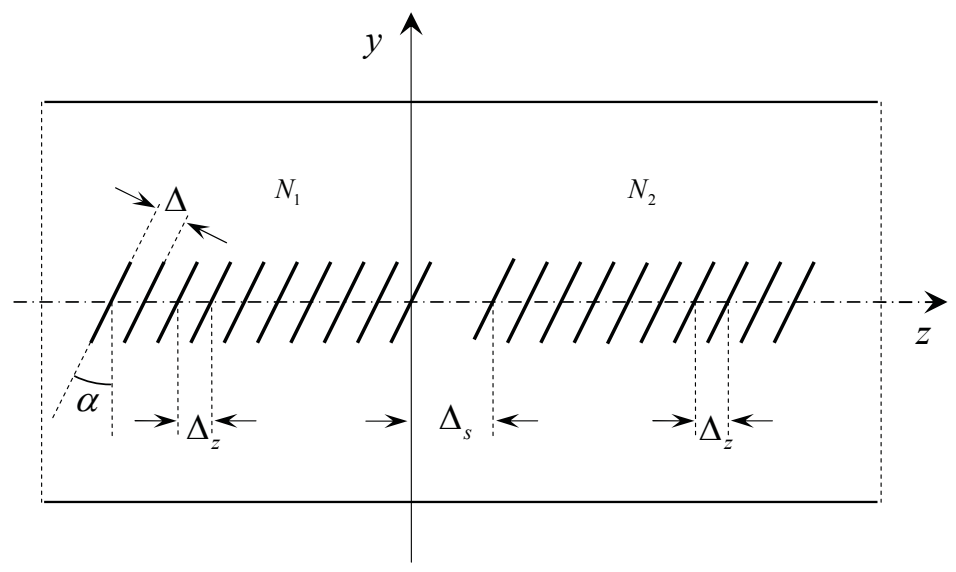

Figure 5.5: Phase shifted array of tilted elements with parameters: $N_{1}, N_{2}$ number of elements in each part of the array; $z_{s}$ shift distance between two parts; $\Delta_{z}$ spacing along $z$-axis in each part of the array; $\Delta$ spacing along normal vector in each part of the array; $\alpha$ tilt angle.

$$
\begin{array}{r}
D c\left(z, \Delta_{z}\right)=\sum_{j=0}^{N_{1}-1} \delta\left(z-r \sin \theta \tan \alpha-\left(z_{0}-j \Delta_{z}\right)\right)+ \\
+\sum_{j=0}^{N_{1}-1} \delta\left(z-r \sin \theta \tan \alpha-\left(z_{0}+\left(j+\frac{1}{2}\right) \Delta_{z}\right)\right)
\end{array}
$$

Hence, integration over $z$ instead of factor $e^{i(\beta-v) z_{0}}$, as is the case for a single source, results in the factor contributing from two geometric series,

$$
\begin{array}{r}
e^{i(\beta-v) z_{0}}\left(\sum_{j=0}^{N_{1}-1} e^{-i(\beta-v) j \Delta_{z}}+\sum_{j=0}^{N_{1}-1} e^{i(\beta-v)\left(j+\frac{1}{2}\right) \Delta_{z}}\right)= \\
=e^{i(\beta-v) z_{0}} \frac{\left(1-e^{i(\beta-v) N_{1} \Delta_{z}}\right)\left(e^{i(\beta-v)\left(1-N_{1}\right) \Delta_{z}}+e^{i(\beta-v) \frac{\Delta_{z}}{2}}\right)}{1-e^{i(\beta-v) \Delta_{z}}}
\end{array}
$$

Thus a vector function $\vec{f}$ for a $\pi$-shifted array in a region $r>r_{1}\left(r_{1}\right.$ is a core radius) is given by,

$$
\overrightarrow{f_{\pi}}=A F_{\pi} \vec{f}_{A B}
$$

where

$\vec{f}_{A B}$ a vector function $\vec{f}$ as in $(\mathrm{A})$ for a series of set of rings or a vector function $\vec{f}$ as in 
(B) for a series of discs,

$A F_{\pi}$ an array factor for a $\pi$-shifted array of $2 N_{1}$ discs or sets of rings,

$$
A F_{\pi}=\frac{\left(1-e^{i(\beta-v) N_{1} \Delta_{z}}\right)\left(e^{i(\beta-v)\left(1-N_{1}\right) \Delta_{z}}+e^{i(\beta-v) \frac{\Delta_{z}}{2}}\right)}{1-e^{i(\beta-v) \Delta_{z}}}
$$

The power of a mode excited with an array of sources differs from the power of the same mode excited with a single source by an additional factor $\left|A F_{u, \pi}\right|^{2}$ where $A F_{u, \pi}$ is either $A F_{u}$ for a uniform array or $A F_{\pi}$ for a $\pi$-shifted array. By choosing appropriate sign in front of propagation constants for core mode, $\beta$ and for plasmon mode, $v$ both type of scattering can be calculated: backward when signs of $\beta$ and $v$ are different or forward when both signs are the same.

\subsection{Choice of basis: electric and magnetic fields $(\vec{E}, \vec{H})$ versus vector potentials for solving wave equation.}

For a certain type of problems the choice of vector magnetic and scalar potentials $\left(\vec{A}, \varphi_{e}\right)$ is advantageous since it has only 4 scalar variables rather than 6 as is the case with electric and magnetic fields, however it is usually harder to implement boundary conditions in terms of potentials.

\subsubsection{Electric and magnetic fields, $(\vec{E}, \vec{H})$.}

From Maxwell's curl equations for a cylindrical waveguide, assuming $z, \theta$ and $t$-dependences as $e^{i \beta z}, e^{i m \theta}$ and $e^{-i \omega t}$ it follows that the normal field components can be written as,

$$
\omega \varepsilon E_{r}=-\frac{1}{r} m H_{z}+\beta H_{\theta}
$$

and

$$
\omega \mu H_{r}=\frac{1}{r} m E_{z}-\beta E_{\theta}
$$

which implies that boundary conditions for normal field components reduced to a linear combination of boundary conditions for tangential field components. Thus boundary conditions for tangential fields form a complete set of boundary conditions for cylindrical dielectric waveguide. All the transverse fields, $E_{r}, E_{\theta}, H_{r}$ and $H_{\theta}$ are found through Maxwell's curl equations in terms of two longitudinal fields, $E_{z}$ and $H_{z}$ which can be viewed as two independent field components.

Therefore in a 3 layer cylindrical waveguide 8 unknown coefficients is sufficient for describing all the fields: one for each longitudinal field of the first and third layer and two for each longitudinal field of the second layer, thus it matches the total number of 
independent boundary conditions with two interfaces. The matrix size for characteristic waveguide equation $\operatorname{det} M=0$ is $8 \times 8$.

The same task of finding all 6 fields in cylindrical waveguide through two independent scalar quantities can be solved in terms of properly chosen non-zero components of vector and scalar potentials $\left(\vec{A}, \varphi_{e}\right)$.

\subsubsection{Magnetic vector potential and scalar electric potential, $\left(\vec{A}, \varphi_{e}\right)$.}

Vector and scalar potentials $\left(\vec{A}, \varphi_{e}\right)$ defined through electric and magnetic fields $(\vec{E}, \vec{H})$ as follows,

$$
\vec{E}=-\nabla \varphi_{e}+i \omega \vec{A}
$$

and

$$
\vec{H}=\frac{1}{\mu} \nabla \times \vec{A}
$$

In addition, Lorenz gauge condition is assumed,

$$
\nabla \cdot \vec{A}=i \omega \mu \varepsilon \varphi_{e}
$$

The transversal components of vector potential are coupled by a wave equation, therefore letting anyone of them $A_{r}$ or $A_{\theta}$ be zero leads to being zero both $A_{r}$ and $A_{\theta}$. In this case $z$-component of magnetic field $H_{z}$ calculated to identical zero, hence choice of $A_{r}=0$ or $A_{\theta}=0$ is not acceptable. On the other hand, the choice of $A_{r}, A_{\theta}$ as two independent scalar quantities of vector potential and its $z$-component being zero $A_{z}=0$ allows calculate all 6 fields. The non-zero electric scalar potential $\varphi_{e}$ is not an independent since it is related to $A_{r}$ and $A_{\theta}$ via Lorenz gauge condition. Therefore basis $\left(\vec{A}, \varphi_{e}\right)$ with minimal nonzero components reduced to $\left(\vec{A}, \varphi_{e}\right)=\left(A_{r}, A_{\theta}, 0, \varphi_{e}\right)$. The size of matrix in characteristic waveguide equation is the same as with basis $(\vec{E}, \vec{H})$.

\subsubsection{Magnetic and electric vector potentials, $(\vec{A}, \vec{F})$.}

Electric and magnetic fields related to vector potentials $(\vec{A}, \vec{F})$ as follows ( [89]),

$$
\begin{aligned}
\vec{H} & =\frac{1}{\mu} \nabla \times \vec{A}-\nabla \cdot \varphi_{m}+i \omega \vec{F} \\
\vec{E} & =-\frac{1}{\varepsilon} \nabla \times \vec{F}-\nabla \cdot \varphi_{e}+i \omega \vec{A}
\end{aligned}
$$

with Lorenz gauge condition for vector electric potential, 


$$
\nabla \cdot \vec{F}=i \omega \mu \varepsilon \varphi_{m}
$$

Choosing one $z$-component of each vector potential is suffice to describe all 6 fields components, i.e. $\vec{A}=\left(0,0, A_{z}\right)$ and $\vec{F}=\left(0,0, F_{z}\right)$. Since scalar potentials related to vector potentials via Lorenz gauge conditions, there are two independent scalar variables $A_{z}, F_{z}$ describing all 6 fields. The basis with minimal components reduced to $\left(0,0, A_{z}, \varphi_{e}\right)$ and $\left(0,0, F_{z}, \varphi_{m}\right)$. The size of matrix in characteristic waveguide equation is $8 \times 8$, the same as with basis $(\vec{E}, \vec{H})$.

\subsubsection{Electric Hertz vector and electric scalar potential, $\left(\vec{\Pi}_{e}, \varphi_{e}\right)$.}

Electric Hertz vector defined as ( [77]),

$$
\vec{A}=-i \omega \mu \varepsilon \vec{\Pi}_{e}
$$

can be used for cylindrical waveguide with zero z-component,

$$
\left(\vec{\Pi}_{e}, \varphi_{e}\right)=\left(\Pi_{e r}, \Pi_{e \theta}, 0, \varphi_{e}\right)
$$

similar to $\left(\vec{A}, \varphi_{e}\right)$ basis.

\subsubsection{Pair of Hertz vectors, $\left(\vec{\Pi}_{e}, \vec{\Pi}_{m}\right)$.}

Magnetic Hertz vector $\vec{\Pi}_{m}$ defined as

$$
\vec{F}=-i \omega \mu \varepsilon \vec{\Pi}_{m}
$$

Pair of electric and magnetic Hertz vectors, $\left(\vec{\Pi}_{e}, \vec{\Pi}_{m}\right)$ can form a basis for cylindrical waveguide similar to $(\vec{A}, \vec{F})$ pair as $\left(0,0, \Pi_{e z}, \varphi_{e}\right)$ and $\left(0,0, \Pi_{m z}, \varphi_{m}\right)$. Electric and magnetic fields related to vector potentials $\left(\vec{\Pi}_{e}, \vec{\Pi}_{m}\right)$ as follows,

$$
\begin{aligned}
& \vec{F}=-i \omega \mu \varepsilon \vec{\Pi}_{m} \\
& \vec{F}=-i \omega \mu \varepsilon \vec{\Pi}_{m}
\end{aligned}
$$

Choosing one component of each vector potential is suffice to describe all 6 fields components, i.e. $\vec{\Pi}_{e}=\left(0,0, \Pi_{e z}\right)$ and $\vec{\Pi}_{m}=\left(0,0, \Pi_{m z}\right)$. 


\subsubsection{Boundary conditions in terms of vector potentials.}

The implementation of boundary conditions in terms of vector potentials is somewhat involved. Sometimes to simplify the calculations the boundary conditions for normal components of the fields are ignored, usually normal component of electric field for non-magnetic mediums or normal components of both electric and magnetic fields for magnetic mediums. For example in [78] the boundary conditions for normal electric field left unsatisfied. Such approach justified with an uniqueness theorem arguments that electromagnetic fields in a given region are uniquely defined if all sources are given and either the tangential components of electric field, $\vec{E}_{t}$ or the tangential components of magnetic field, $\vec{H}_{t}$ are specified at the boundary of the region. However such argumentation of ignoring normal components has a flaw which was investigated and treated in details by X. L. Zhou in a series of articles [79-81]. It was shown that usual textbook formulation of uniqueness theorem for electromagnetic fields that requires only tangential components of the fields is not complete. Such formulation steams from the proving theorem by method of contradiction where only one particular case out of three possible was considered and conclusion has been drawn based on a particular case. Considering remaining cases, which is a necessary condition for a proving by method of contradiction and which makes a formulation of uniqueness theorem complete, requires the knowledge of tangential as well as normal components of the fields. Therefore boundary conditions for normal components can not be ignored.

\subsection{Simulation results and discussion.}

To visualize a tangential component of current distribution in the tilted disc its line integral convolution plot, frequently used for plotting vector field, is shown in Fig. 5.6. For a field imaging it is informative provide tiny geometrical details of the field distribution as well as the local direction. To capture spacial details of the distribution the current is convolved with the white noise in a gray scale resulted in a distribution alignment, presented in Fig. 5.6(a), where a distribution direction is ignored to maximize the spacial resolution. Such picture resembles small metallic chips spread on a sheet with the magnet just below the sheet. The local orientation of the current distribution illustrated in Fig. 5.6(b) where coarse stream lines are added to the plot. Line integral convolution plot does not carry out information about local strength (absolute value) of a vector field. Introduction to a line integral convolution and its use covered in [90].

Power carried by a plasmon mode excited by an array of current sources tilted by an angle $\alpha$ positioned in the core of a fiber coated with thin metal layer versus tilted angle and wavelength is demonstrated in the plots below. The role of a $\pi$-shift in an array of current sources can be examined by comparison Fig. 5.7 and Fig. 5.8 where the plots of power carried by plasmon mode excited with uniform array and phase shifted array respectively are shown. Predictably, the resonance power peak due to an uniform array (Fig. 5.7) is divided in two halves when an array acquired a $\pi$-phase shift (Fig. 5.8). 


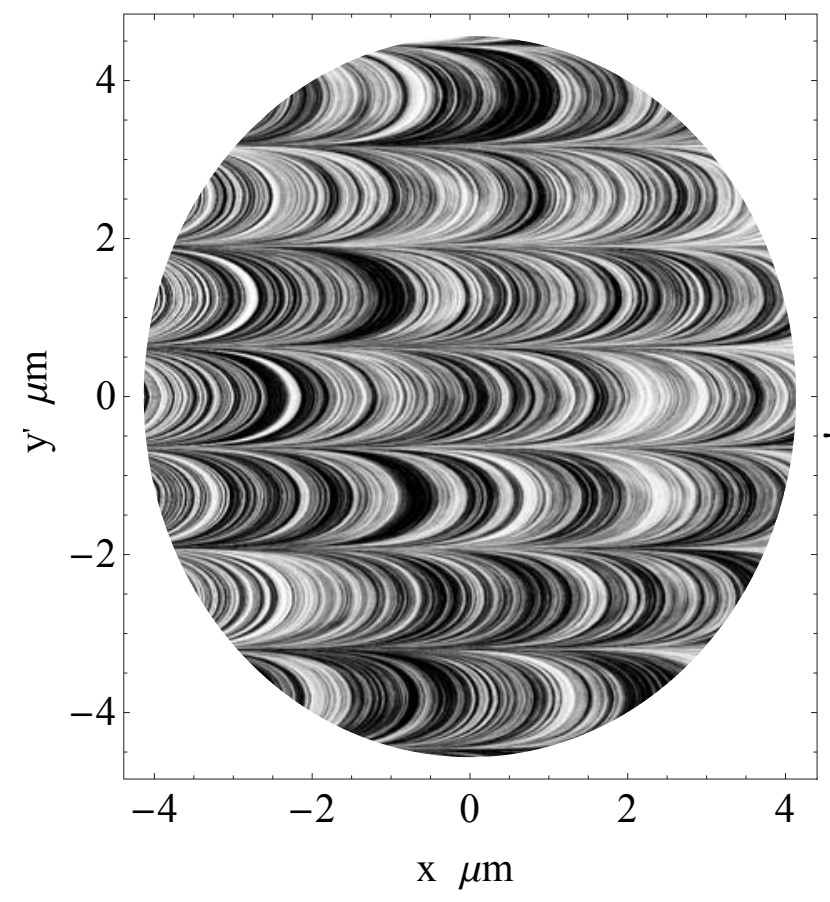

(a)

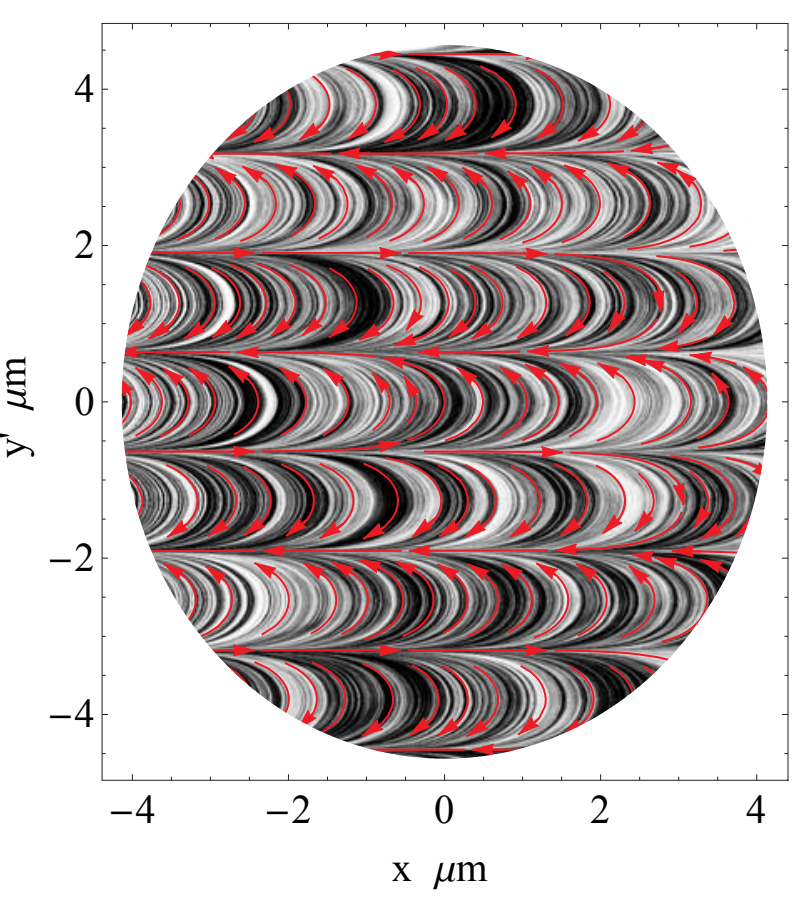

(b)

Figure 5.6: Line integral convolution plot of a tangential component of current source distribution in a tilted disc located in the fiber core. The disc is an interior of some ellipse whose longer axis is $y^{\prime}$. Tilt angle $\alpha$ is measured between axis $y$ of the fiber and tilted axis $y^{\prime}$. (a): alignment, (b): orientation.

The depth of the dip, that separates two halves of the power peak and that is due to a $\pi$-shift, strongly depends on imaginary part of effective index. This is evident by comparing Fig. 5.8 and Fig. 5.9 where two plasmon modes with a slightly different imaginary part of effective index are excited. The mode with larger imaginary part has slightly shallower dip of the peak. Replacing coating layer from Au to platinum Pt whose imaginary part of refractive index (whose loss, damping rate) considerably larger than of $\mathrm{Au}$, allows excite the modes with quite a large imaginary part of effective index. As a result, the dip of the peak becomes so shallow that the difference between uniform and $\pi$-shifted grating essentially vanishes. Plot of power carried by a plasmon mode with Pt coating excited by uniform and $\pi$-shifted array is shown in Fig. 5.10. Effective indices of modes with Au coating, (two modes), and with Pt coating (one mode) versus wavelength are shown in Fig. 5.11.

As shown in Fig. 5.8 and Fig. 5.9, a moderately large imaginary part of an effective index of a plasmon mode makes a dip on the resonance peak visibly shallower. It is worth mentioning however, that the effect of a larger imaginary part of effective index on a dip can be compensated by some unbalancing of the $\pi$-shifted array, i.e. by making the second half of the array (the half that is shifted by $\pi$ ), slightly different from the first half. For example, adding a fraction of a disc to the second half of the array, $N_{2}=N_{1}+1 / 2$ almost restores the depth of the dip to original depth as with a small imaginary part of effective index. 


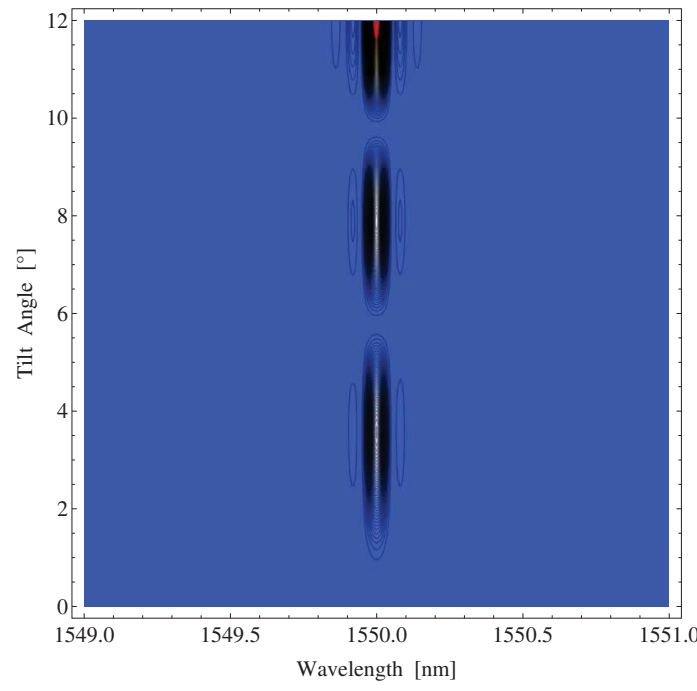

(a)

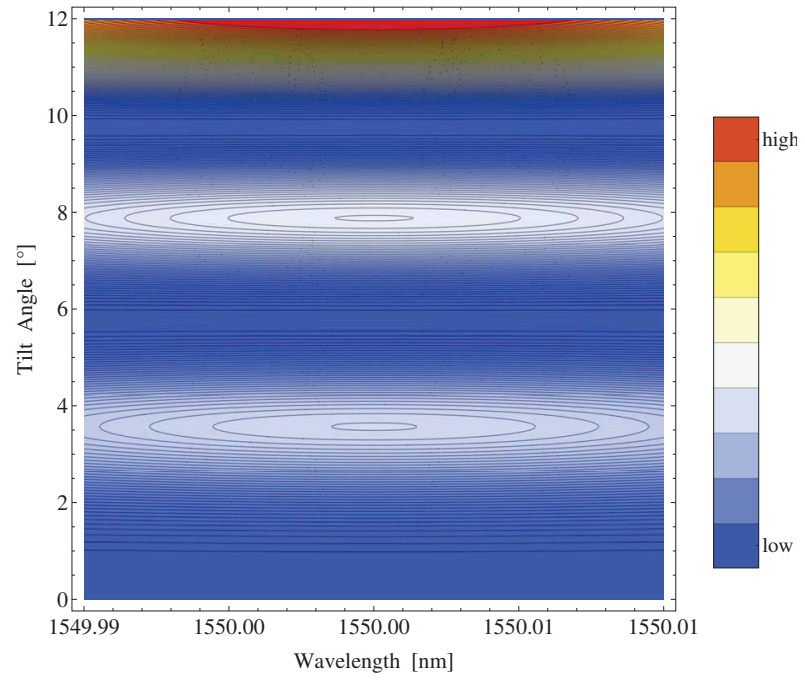

(c)

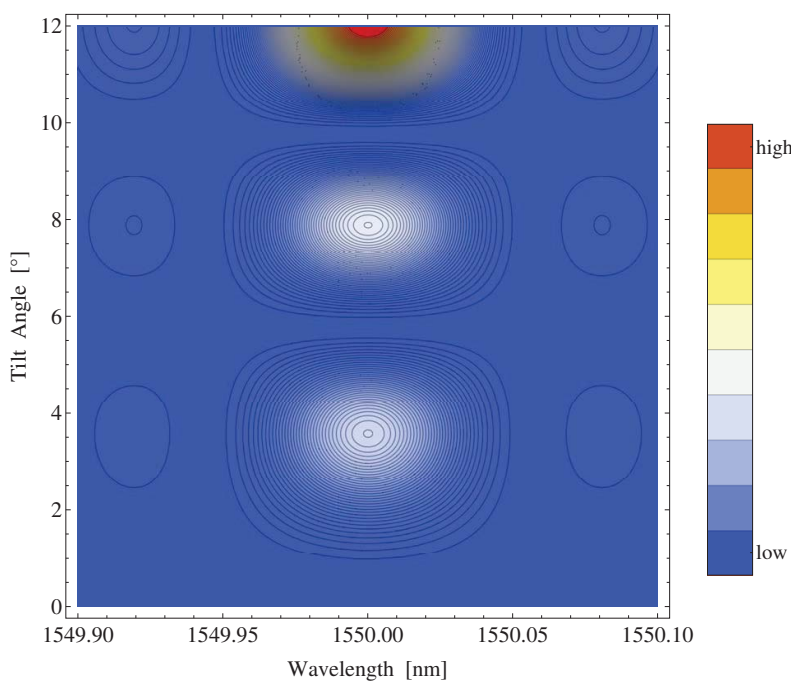

(b)

Figure 5.7: Contour plot of power carried by plasmon mode excited by an uniform array of tilted discs of current in the core of a fiber coated by thin Au layer positioned in air environment versus wavelength and tilt angle. Simulation parameters: azimuthal index of the mode $m=3$, layer thickness $d=35 \mathrm{~nm}$, an array is tuned for coupling plasmon mode and core mode at resonance wavelength $\lambda_{\text {res }}=1550 \mathrm{~nm}$, effective index $n_{e f 1}$ shown in Fig. 5.12. Computation window for wavelength and angle range respectively (a): (1549 $\mathrm{nm}, 1551 \mathrm{~nm}),\left(0^{\circ}, 12^{\circ}\right),(\mathrm{b}):(1549.9 \mathrm{~nm}, 1550.1 \mathrm{~nm}),\left(0^{\circ}, 12^{\circ}\right),(\mathrm{c}):(1549.99 \mathrm{~nm}, 1550.01$ $\mathrm{nm}),\left(0^{\circ}, 12^{\circ}\right)$.

This is illustrated in Fig. 5.12 by comparing it with Fig. 5.9. However such unbalancing approach can not compensate an effect of a much larger imaginary part of effective index as with Pt coating case, the dip is invisible with $\pi$-shifted balanced array (Fig. 5.10) as 


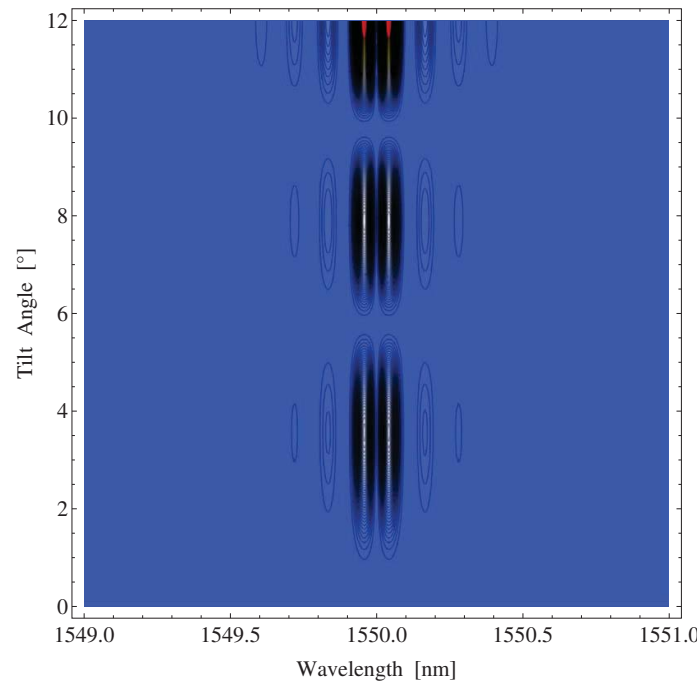

(a)

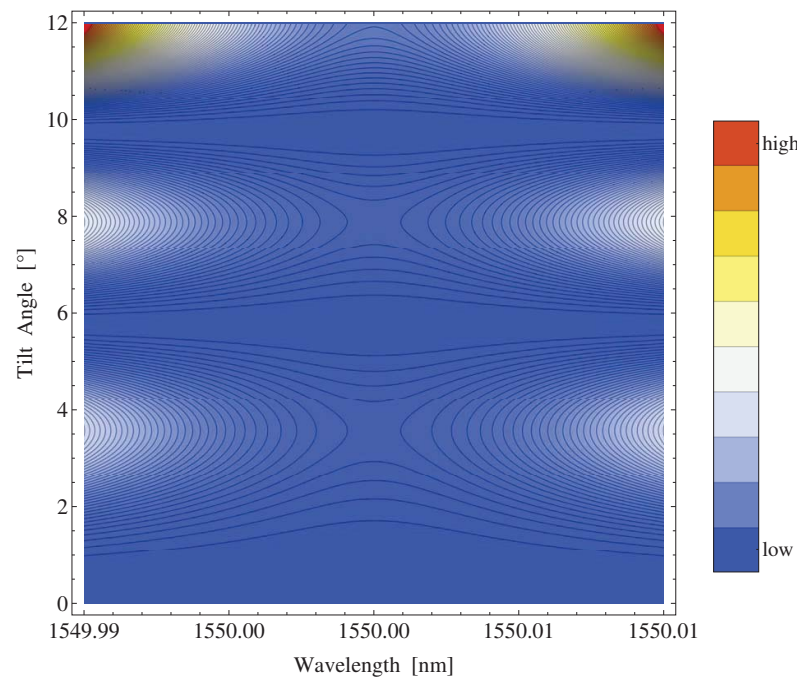

(c)

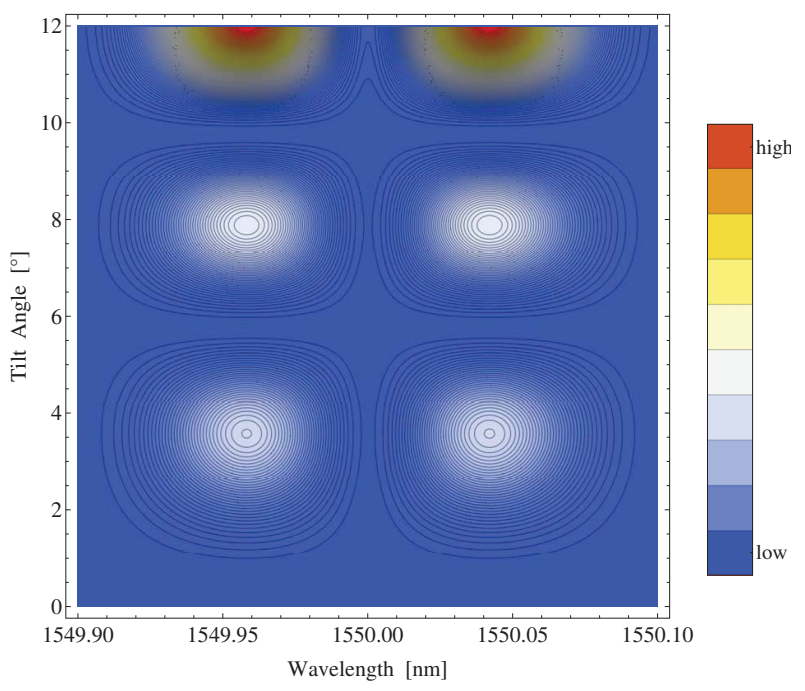

(b)

Figure 5.8: Contour plot of power carried by plasmon mode excited by a $\pi$-shifted array of tilted discs of current in the core of a fiber coated by thin Au layer positioned in air environment versus wavelength and tilt angle. Simulation parameters: azimuthal index of the mode $m=3$, layer thickness $d=35 \mathrm{~nm}$, an array is tuned for coupling plasmon mode and core mode at resonance wavelength $\lambda_{\text {res }}=1550 \mathrm{~nm}$, effective index $n_{e f 1}$ shown in Fig. 5.11. Computation window for wavelength and angle range respectively (a): (1549 $\mathrm{nm}, 1551 \mathrm{~nm}),\left(0^{\circ}, 12^{\circ}\right),(\mathrm{b}):(1549.9 \mathrm{~nm}, 1550.1 \mathrm{~nm}),\left(0^{\circ}, 12^{\circ}\right),(\mathrm{c}):(1549.99 \mathrm{~nm}, 1550.01$ $\mathrm{nm}),\left(0^{\circ}, 12^{\circ}\right)$.

well as with $\pi$-shifted unbalanced array (Fig. 5.13).

Here is a comparison of two type of sources, a solid disc of current versus a set of rings with a common center and arranged in a single plane. It should be noted that the current 


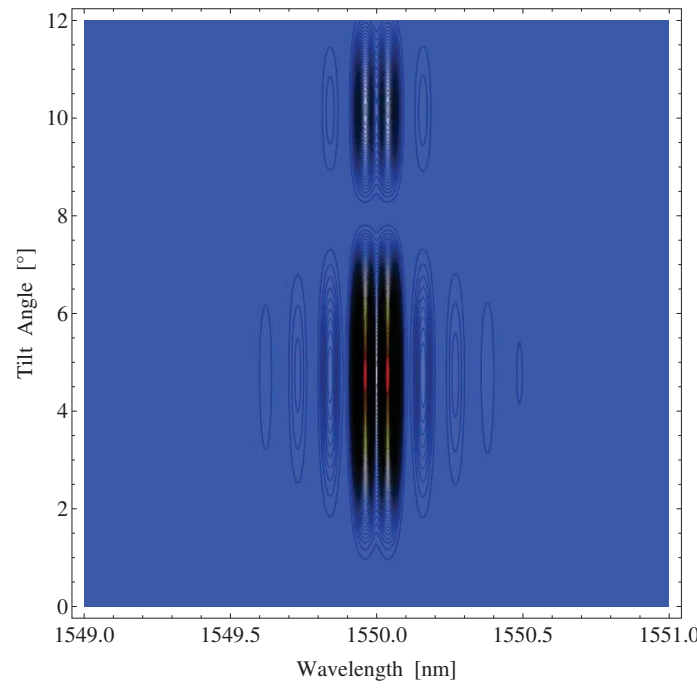

(a)

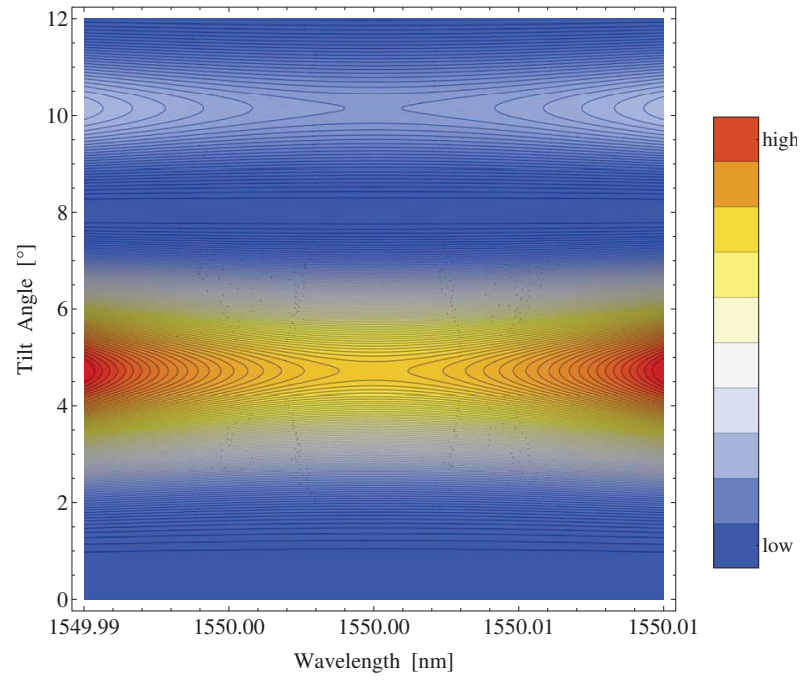

(c)

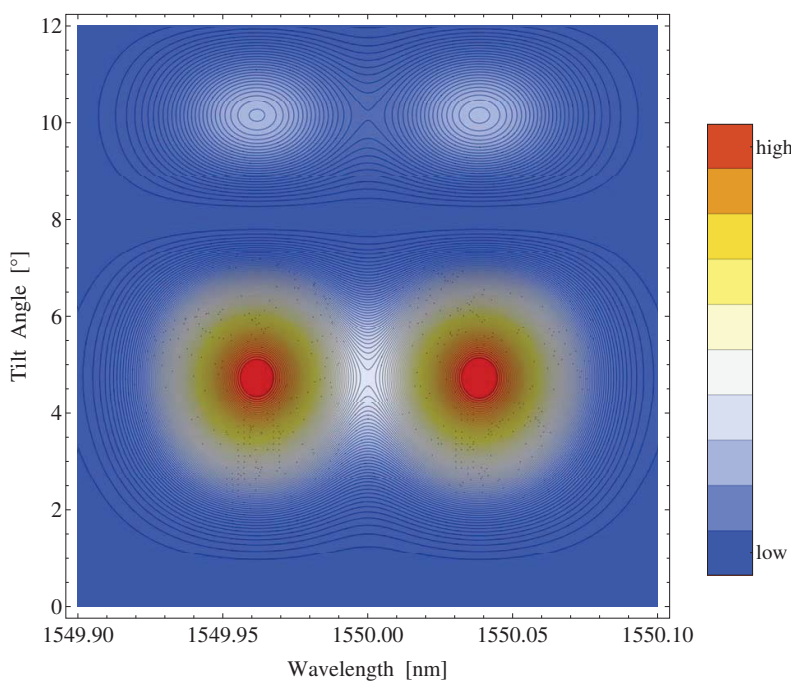

(b)

Figure 5.9: Contour plot of power carried by plasmon mode excited by a $\pi$-shifted array of tilted discs of current in the core of a fiber coated by thin Au layer positioned in air environment versus wavelength and tilt angle. Simulation parameters: azimuthal index of the mode $m=3$, layer thickness $d=35 \mathrm{~nm}$, an array is tuned for coupling plasmon mode and core mode at resonance wavelength $\lambda_{\text {res }}=1550 \mathrm{~nm}$, effective index $n_{e f 2}$ shown in Fig. 5.11. Computation window for wavelength and angle range respectively (a): (1549 $\mathrm{nm}, 1551 \mathrm{~nm}),\left(0^{\circ}, 12^{\circ}\right),(\mathrm{b}):(1549.9 \mathrm{~nm}, 1550.1 \mathrm{~nm}),\left(0^{\circ}, 12^{\circ}\right),(\mathrm{c}):(1549.99 \mathrm{~nm}, 1550.01$ $\mathrm{nm}),\left(0^{\circ}, 12^{\circ}\right)$.

on a disc is not a typical surface current and the current on a ring is not a typical line current in the sense that they both have not only tangential components (tangential to the disc and tangential to the ring respectively) but normal components as well. This 


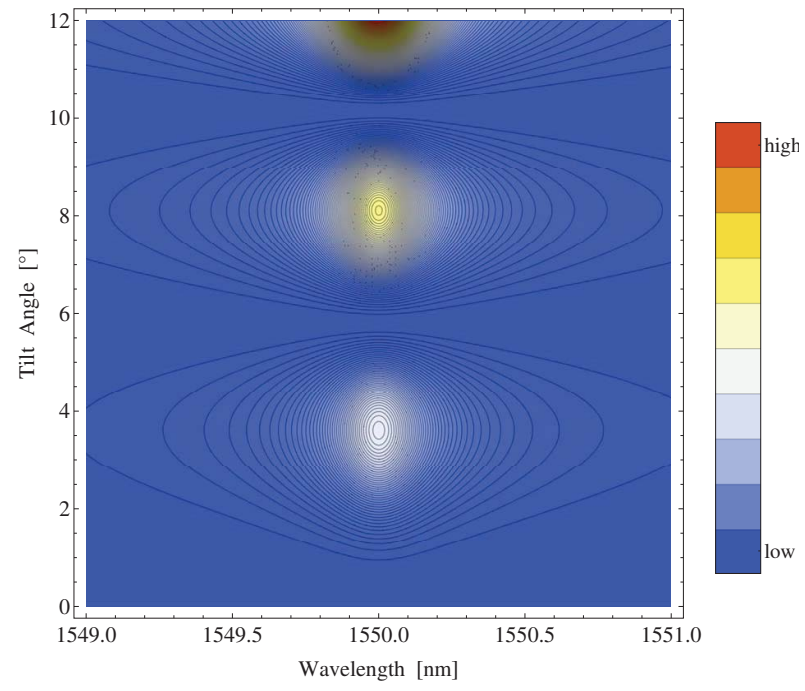

(a)

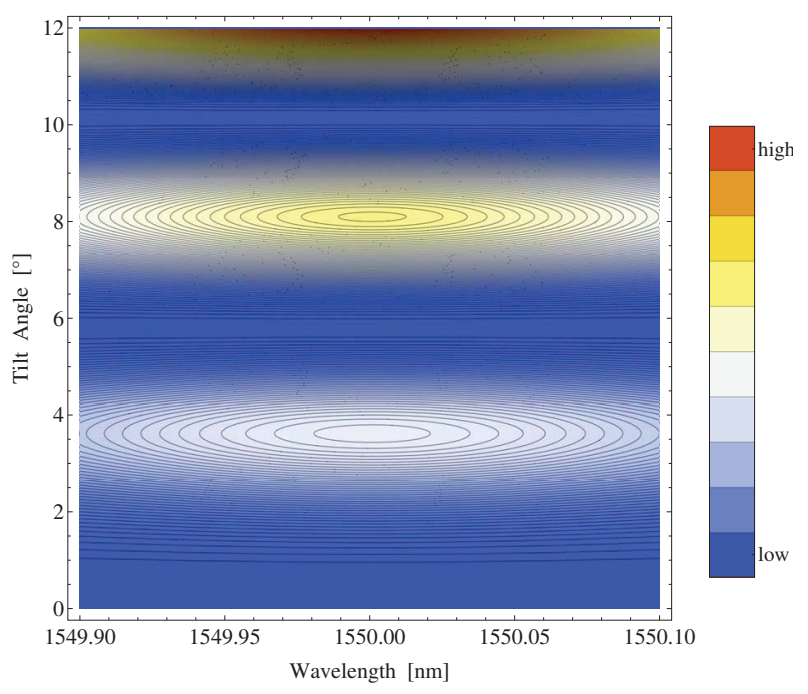

(b)

Figure 5.10: Contour plot of power carried by plasmon mode excited by a $\pi$-shifted array of tilted discs of current in the core of a fiber coated by thin Pt layer positioned in air environment versus wavelength and tilt angle. Simulation parameters: azimuthal index of the mode $m=3$, layer thickness $d=35 \mathrm{~nm}$, an array is tuned for coupling plasmon mode and core mode at resonance wavelength $\lambda_{\text {res }}=1550 \mathrm{~nm}$, effective index $n_{e f 3}$ shown in Fig. 5.11. Computation window for wavelength and angle range respectively (a): (1549 $\mathrm{nm}, 1551 \mathrm{~nm}),\left(0^{\circ}, 12^{\circ}\right),(\mathrm{b}):(1549.9 \mathrm{~nm}, 1550.1 \mathrm{~nm}),\left(0^{\circ}, 12^{\circ}\right)$.

originates from the fact that current sources in question just mimics the electric field of the core mode which has all three non-zero components $E_{r}, E_{\theta}, E_{z}$. The calculation is done with different number of rings $N_{\text {rings }}$ in a single plane as a parameter. The calculated power $P_{z}(\lambda, \alpha)$, versus wavelength $\lambda$ and tilt angle $\alpha$, of a plasmon mode excited by $\pi$-shifted array of sources positioned in a core of a fiber coated by thin Au film looks similar, apart from the scale factor, for a different number of rings $N_{\text {rings }}$ starting from $N_{\text {rings }}=10$ and for discs sources. This is illustrated in Fig. 5.14. 


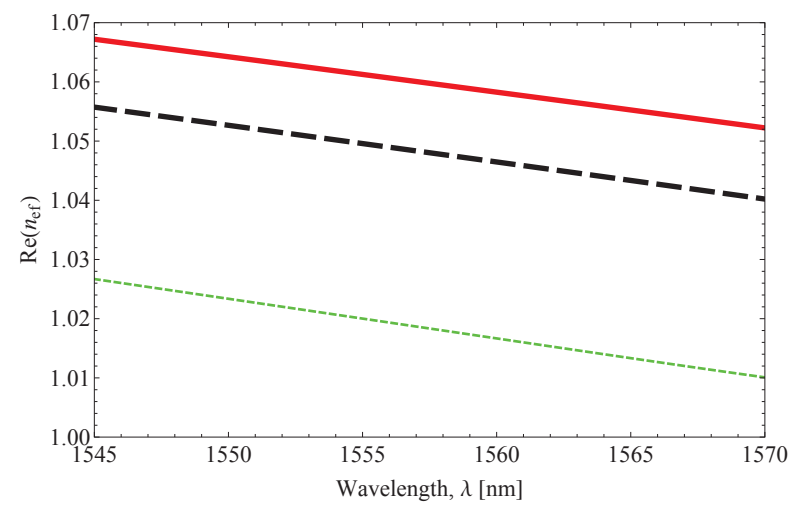

(a)

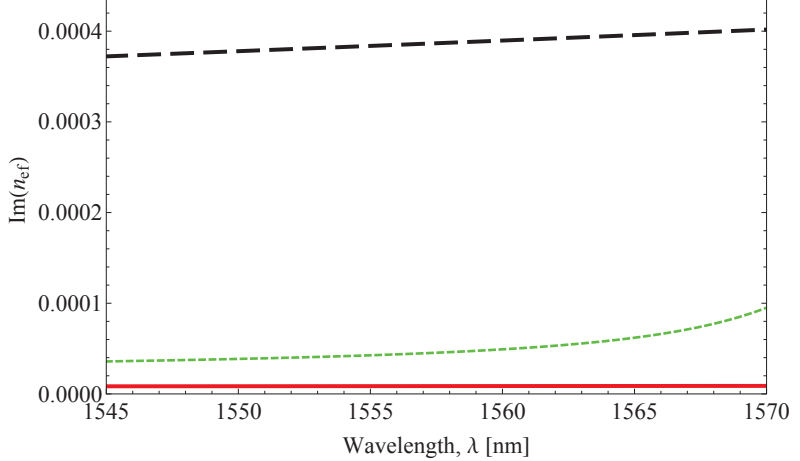

(b)

Figure 5.11: Plot of effective indices for plasmon modes excited by a $\pi$-shifted array of tilted discs of current in the core of a fiber coated by thin metal layer positioned in air environment versus wavelength. Simulation parameters: azimuthal index of the mode $m=3$, layer thickness $d=35 \mathrm{~nm}$, grating is tuned for coupling plasmon mode and core mode at resonance wavelength $\lambda_{\text {res }}=1550 \mathrm{~nm}$. Effective indices as follows: solid line $n_{e f 1}$ (Au coating), short dashed line $n_{\text {ef } 2}$ (Au coating), large dashed line $n_{e f 3}$ (Pt coating). (a): real parts, (b): imaginary parts. 


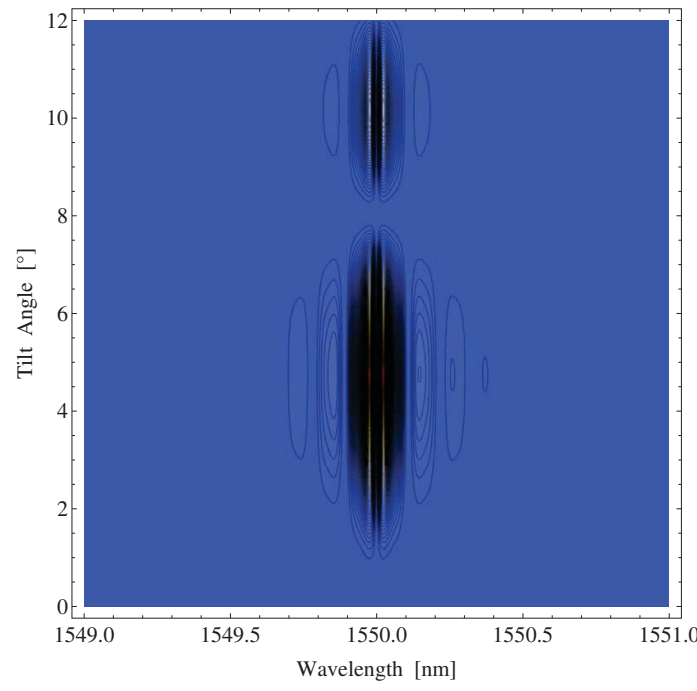

(a)

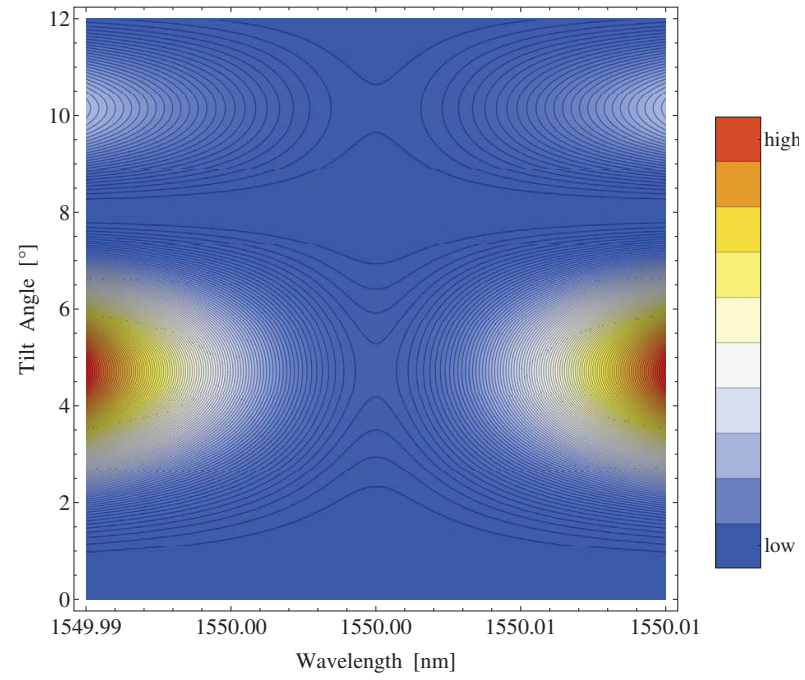

(c)

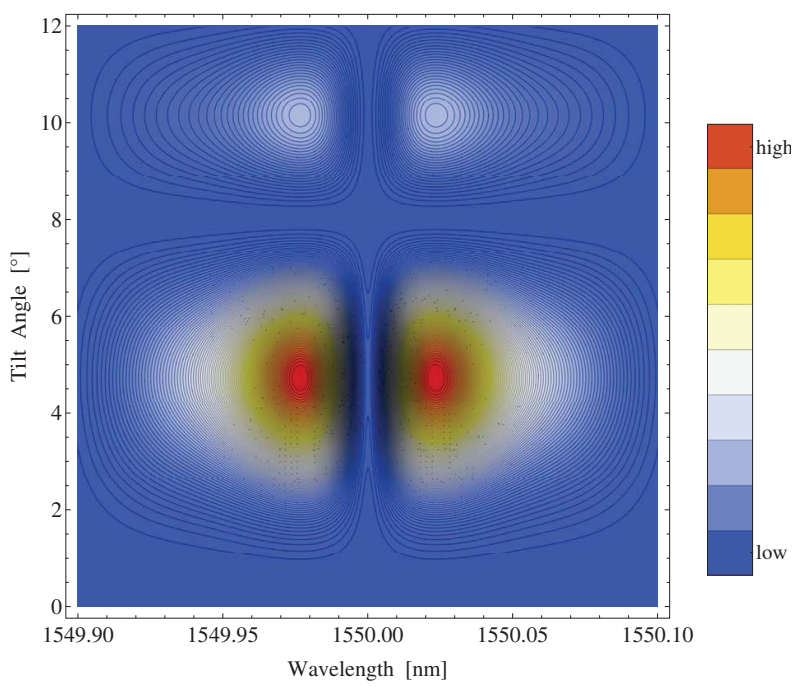

(b)

Figure 5.12: Contour plot of power carried by plasmon mode excited by a $\pi$-shifted unbalanced array of tilted discs of current in the core of a fiber coated by thin Au layer positioned in air environment versus wavelength and tilt angle. Simulation parameters: azimuthal index of the mode $m=3$, layer thickness $d=35 \mathrm{~nm}$, array is tuned for coupling plasmon mode and core mode at resonance wavelength $\lambda_{\text {res }}=1550 \mathrm{~nm}$, effective index $n_{\text {ef } 2}$ shown in Fig. 5.11. The array is unbalanced by half of an element: $N_{2}=N_{1}+1 / 2$, where $N_{1}, N_{2}$ number of discs in the first and second halves of the array respectively. Computation window for wavelength and angle range respectively (a): (1549 nm,1551 nm), $\left(0^{\circ}, 12^{\circ}\right),(\mathrm{b}):(1549.9 \mathrm{~nm}, 1550.1 \mathrm{~nm}),\left(0^{\circ}, 12^{\circ}\right),(\mathrm{c}):(1549.99 \mathrm{~nm}, 1550.01 \mathrm{~nm}),\left(0^{\circ}, 12^{\circ}\right)$. 


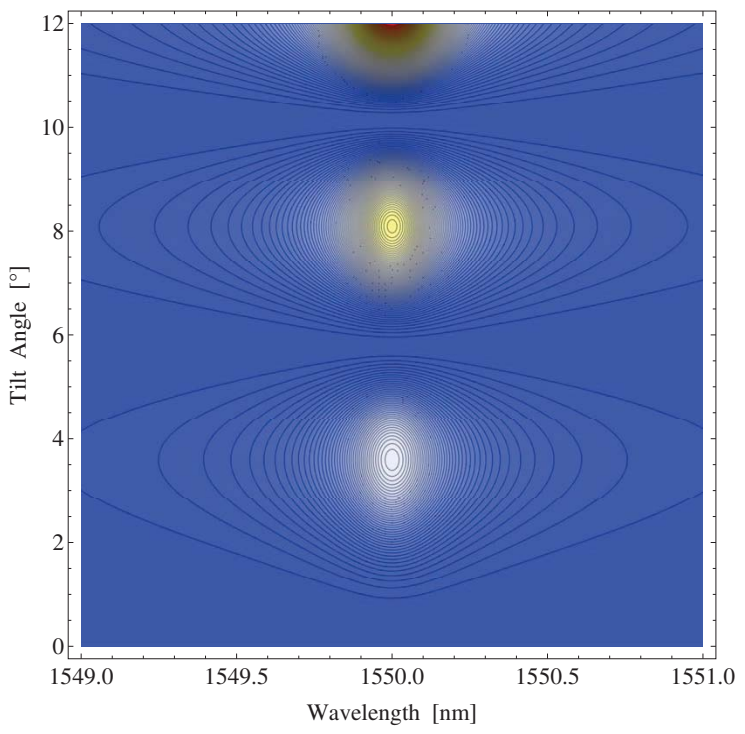

(a)

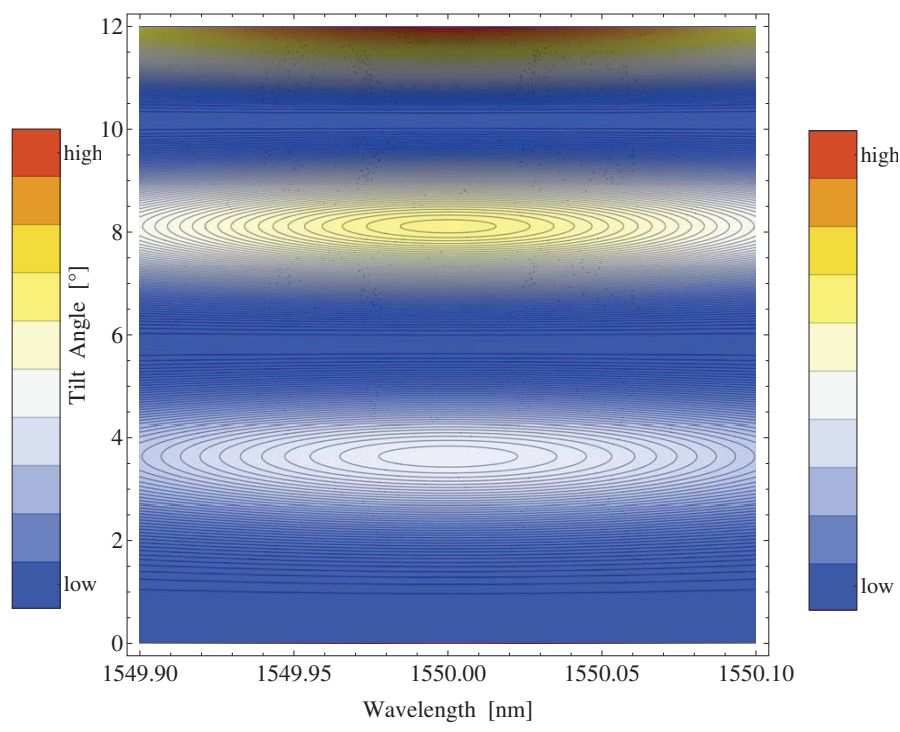

(b)

Figure 5.13: Contour plot of power carried by plasmon mode excited by a $\pi$-shifted unbalanced array of tilted discs of current in the core of a fiber coated by thin Pt layer positioned in air environment versus wavelength and tilt angle. Simulation parameters: azimuthal index of the mode $m=3$, layer thickness $d=35 \mathrm{~nm}$, array is tuned for coupling plasmon mode and core mode at resonance wavelength $\lambda_{\text {res }}=1550 \mathrm{~nm}$, effective index $n_{\text {ef } 3}$ shown in Fig. 5.11. The array is unbalanced by half of an element: $N_{2}=N_{1}+1 / 2$, where $N_{1}, N_{2}$ number of discs in the first and second halves of the array respectively. Computation window for wavelength and angle range respectively (a): (1549 nm,1551 nm), $\left(0^{\circ}, 12^{\circ}\right)$, (b): (1549.9 nm,1550.1 nm), $\left(0^{\circ}, 12^{\circ}\right)$. 


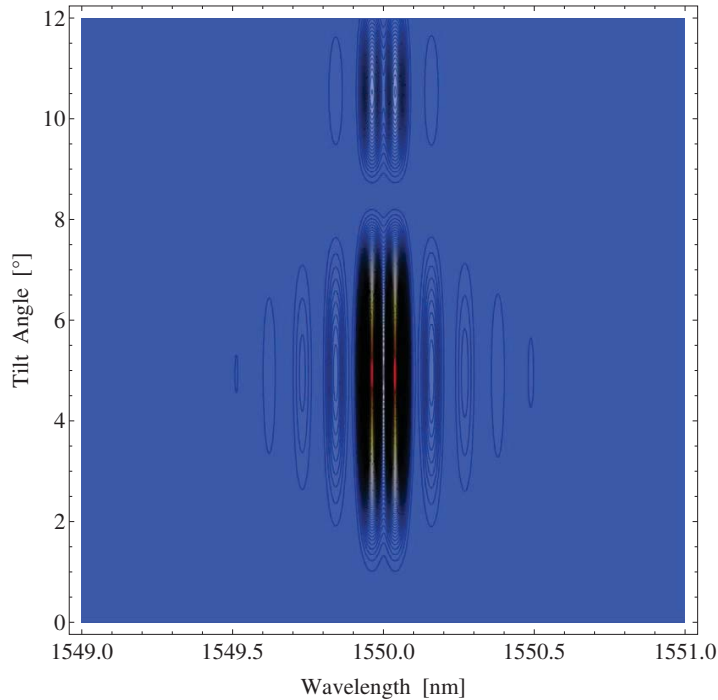

(a) $\mathrm{Aa}$

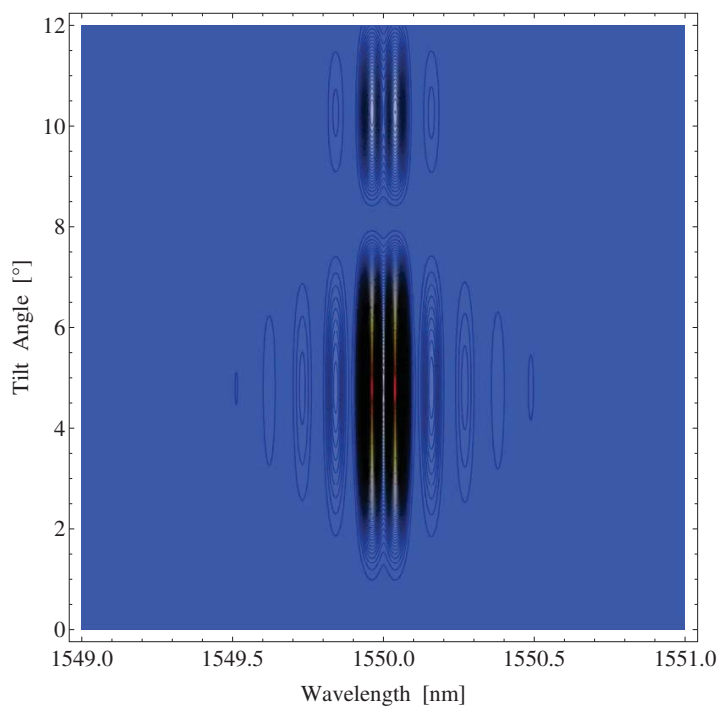

(c) $\mathrm{Ba}$

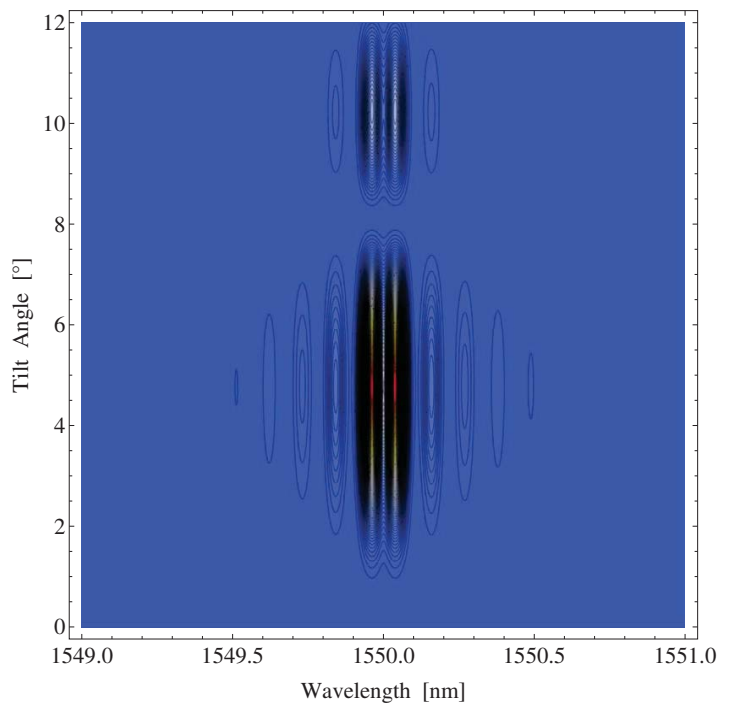

(e) $\mathrm{Ca}$

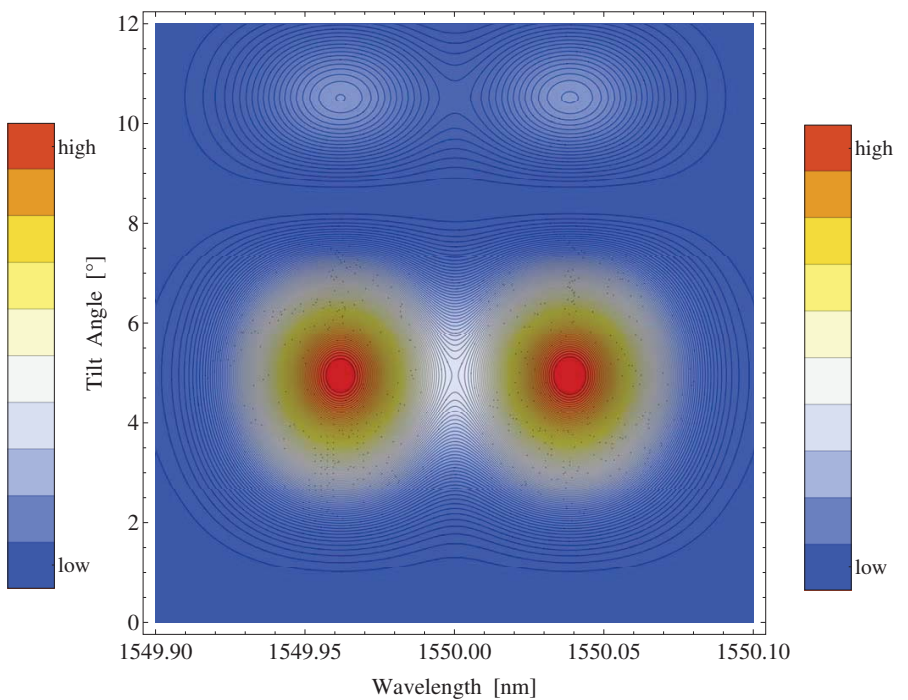

(b) $\mathrm{Ab}$

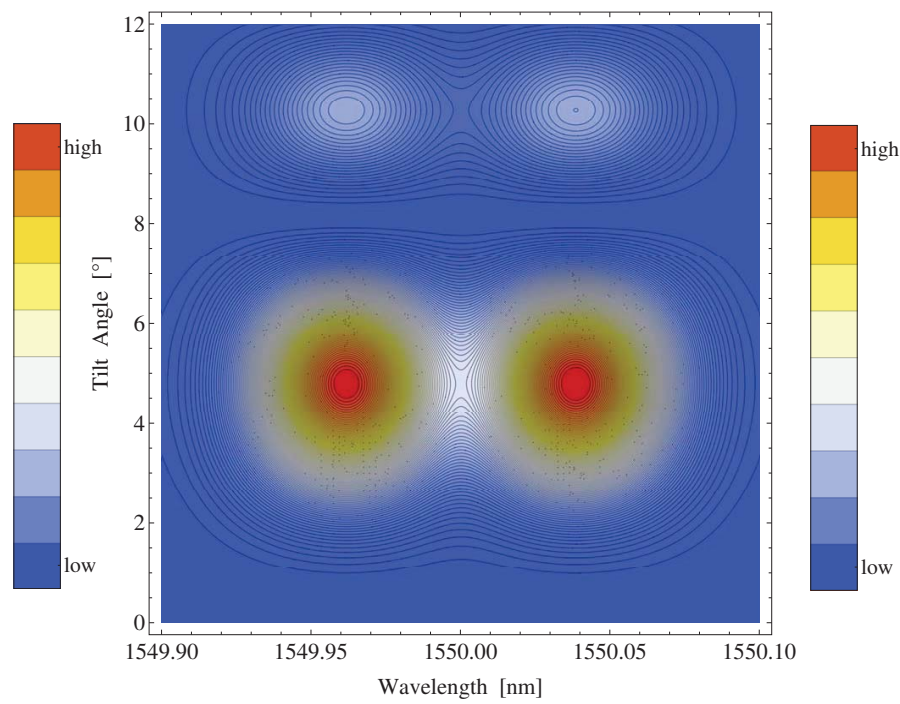

(d) $\mathrm{Bb}$

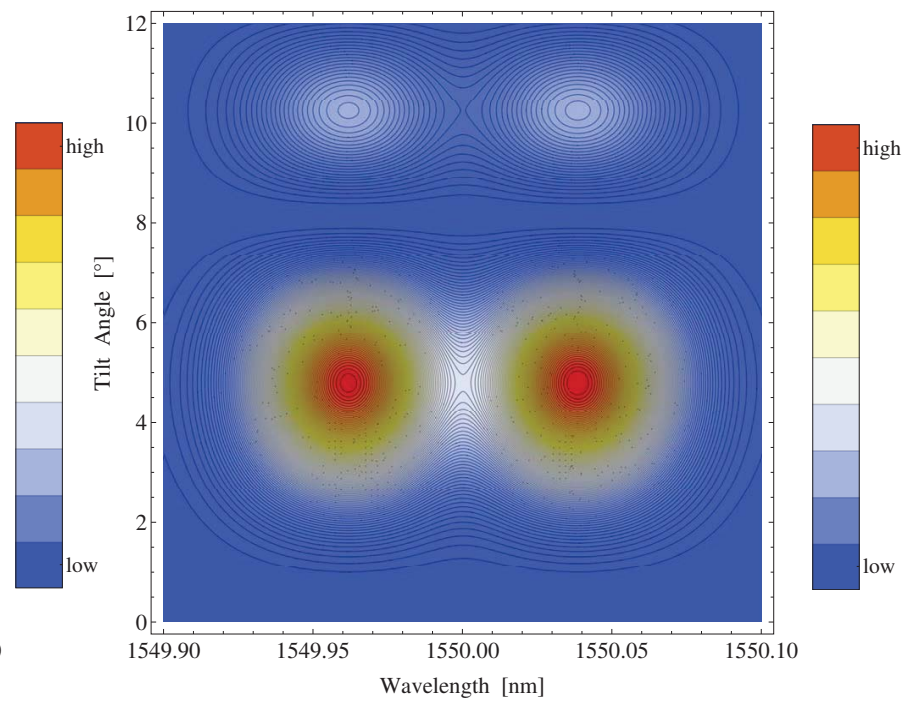

113

(f) $\mathrm{Cb}$ 


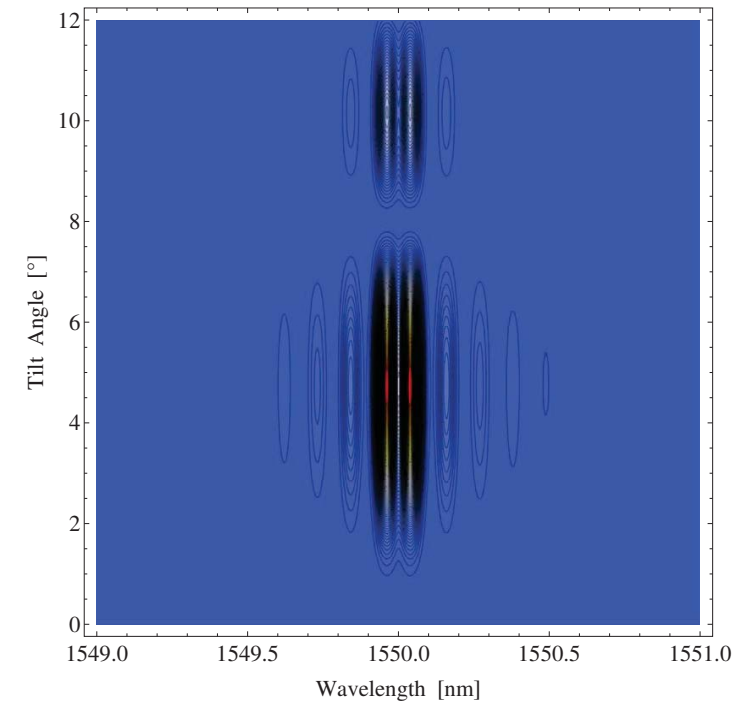

(g) $\mathrm{Da}$

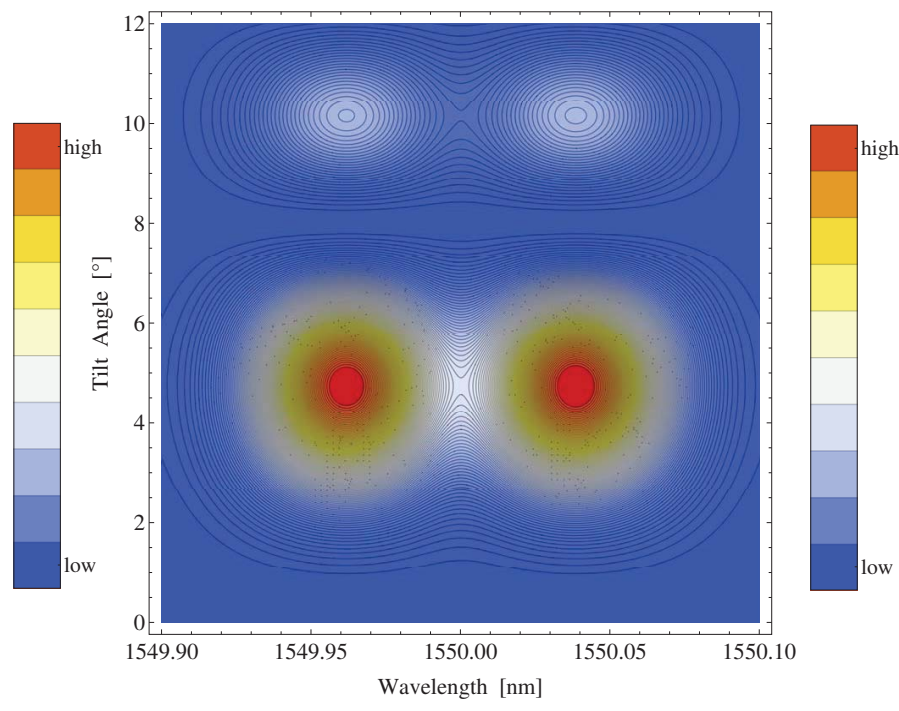

(h) $\mathrm{Db}$

Figure 5.14: Contour plot of power carried by plasmon mode excited by $\pi$-shifted array of current sources where each element of an array is a set of tilted $N_{\text {rings }}$ rings or a tilted disc shown in Fig. 5.4(a). The array is located in a core of fiber coated by thin Au layer positioned in air environment. Contour plot computed versus wavelength $\lambda$ and tilt angle $\alpha$. Simulation parameters: azimuthal index of the mode $m=3$, layer thickness $d=35 \mathrm{~nm}$, array is tuned for coupling plasmon mode with azimuthal index $m=3$ and core mode at resonance wavelength $\lambda_{\text {res }}=1550 \mathrm{~nm}$ and resonance film thickness $d_{\text {res }}=30 \mathrm{~nm}$, effective index $n_{\text {ef2 } 2}$ shown in Fig. 5.11. Modified source parameter respectively (A): rings with $N_{\text {rings }}=10$, (B): rings with $N_{\text {rings }}=50$, (C): rings with $N_{\text {rings }}=70$, (D): discs. Computation window for wavelength and angle range respectively (a): (1549 nm,1551 $\mathrm{nm}),\left(0^{\circ}, 12^{\circ}\right),(\mathrm{b}):(1549.9 \mathrm{~nm}, 1550.1 \mathrm{~nm}),\left(0^{\circ}, 12^{\circ}\right)$.

Modifying imaginary part of effective index can be done by tuning coating thickness. Example of effective index versus thickness with wavelength as a modulation parameter in the range $(1547 \mathrm{~nm}, 1555 \mathrm{~nm})$ with increment $0.5 \mathrm{~nm}$ is shown in Fig. 5.15. It is evident from the plot that most significant change in imaginary part of effective index observed in the range of thickness $d$ approximately below $50 \mathrm{~nm}$, while above this, $d>50 \mathrm{~nm}$ imaginary part of effective index goes on a plato and essentially doesn't change.

Evolution of a plasmon power for effective indices, with wavelength as a modulation parameter, is shown in Fig. 5.15 in a series of contour plots versus Au thickness $d$ and tilt angle $\alpha$ and also presented in Fig. 5.16 for both an uniform and a $\pi$-shifted array of discs of current. 


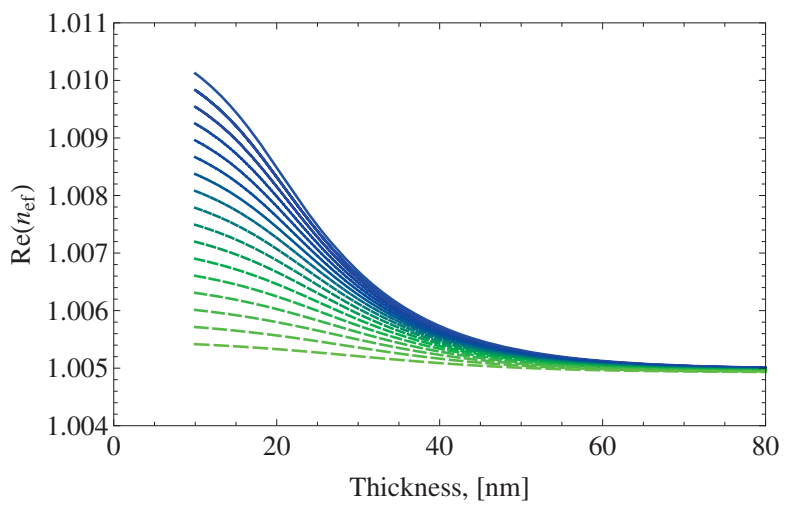

(a)

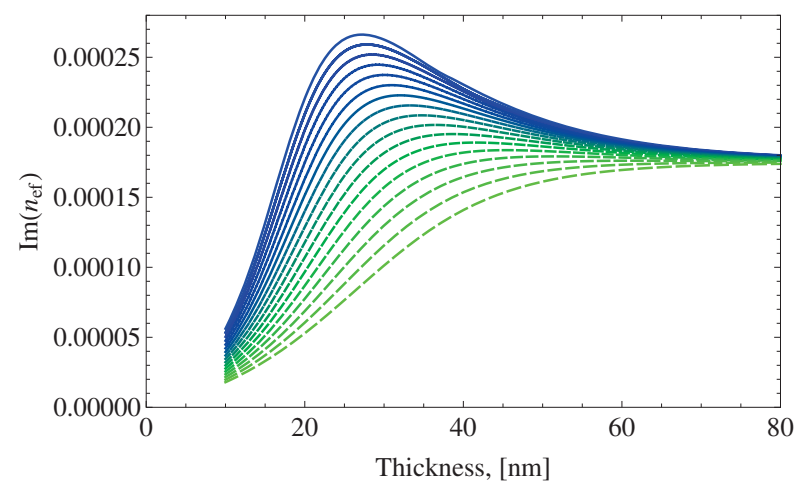

(b)

Figure 5.15: Plot of effective index for plasmon mode excited by a $\pi$-shifted tilted grating in a fiber coated by Au layer positioned in air environment versus thickness. Simulation parameters: azimuthal index of the mode $m=3$, grating is tuned for coupling plasmon mode (calculated with Au thickness $d_{\text {res }}=30 \mathrm{~nm}$ ) and core mode at resonance wavelength $\lambda_{\text {res }}=1550 \mathrm{~nm}$. Modulation parameter is a wavelength, $\lambda$ : on both plots solid blue line represents $\lambda=1547 \mathrm{~nm}$ and dashed green line represents $\lambda=1555 \mathrm{~nm}$, increment in wavelength $\Delta \lambda=0.5 \mathrm{~nm}$ (a): real parts, (b): imaginary parts. 


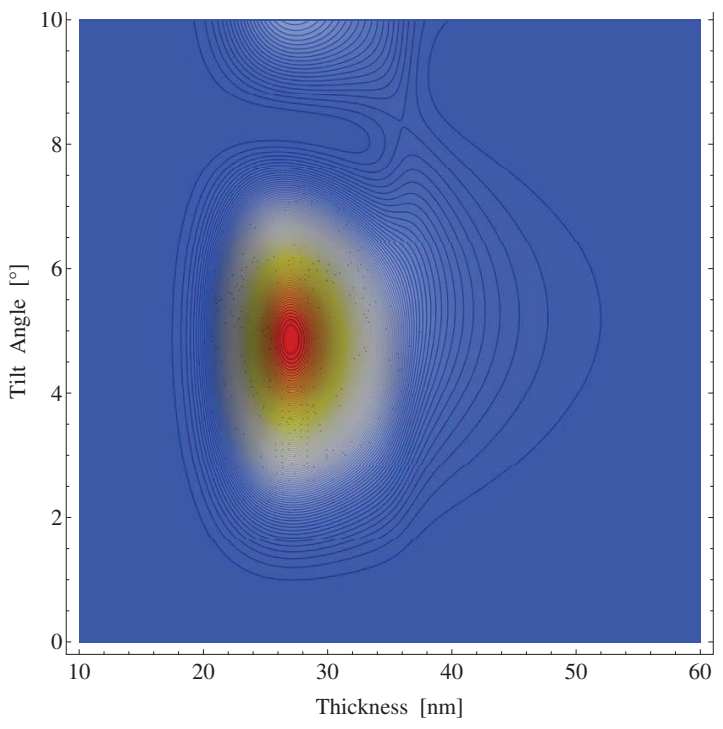

(a) $\lambda=1547 \mathrm{~nm}$

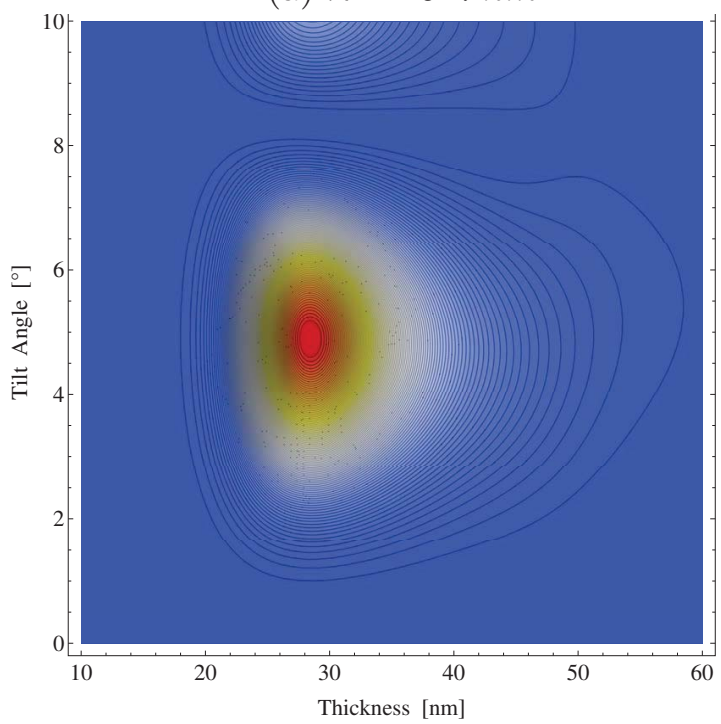

(c) $\lambda=1548 \mathrm{~nm}$

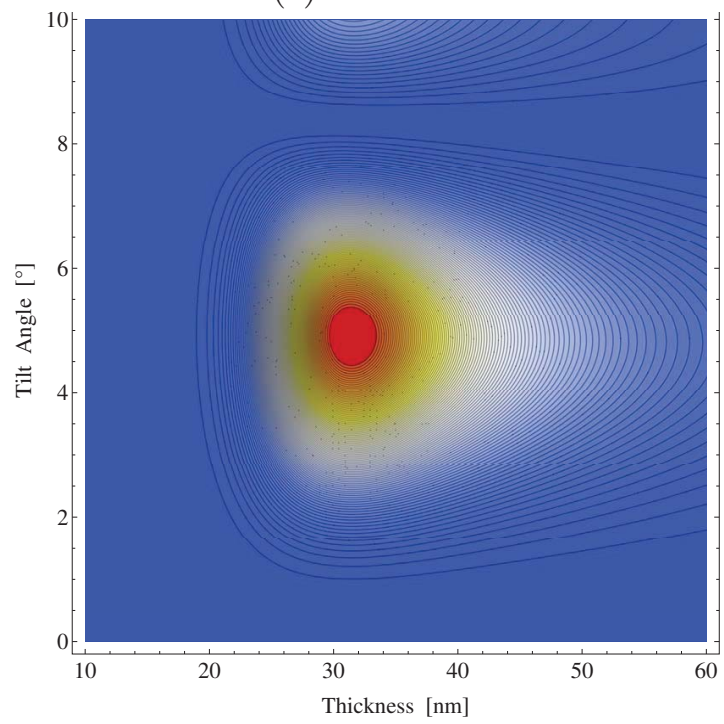

(e) $\lambda=1549 \mathrm{~nm}$

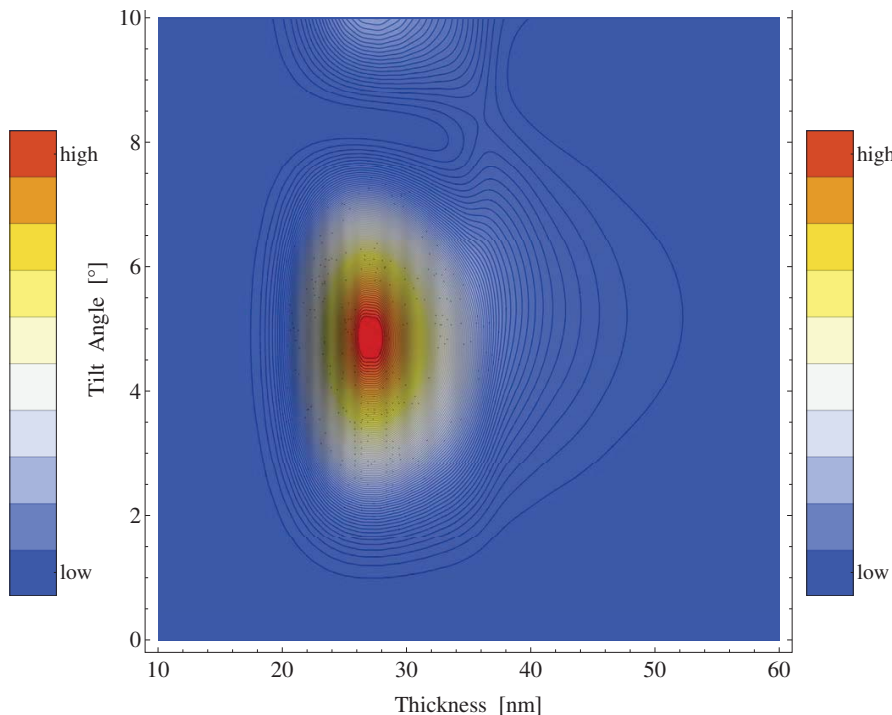

(b) $\lambda=1547 \mathrm{~nm}$

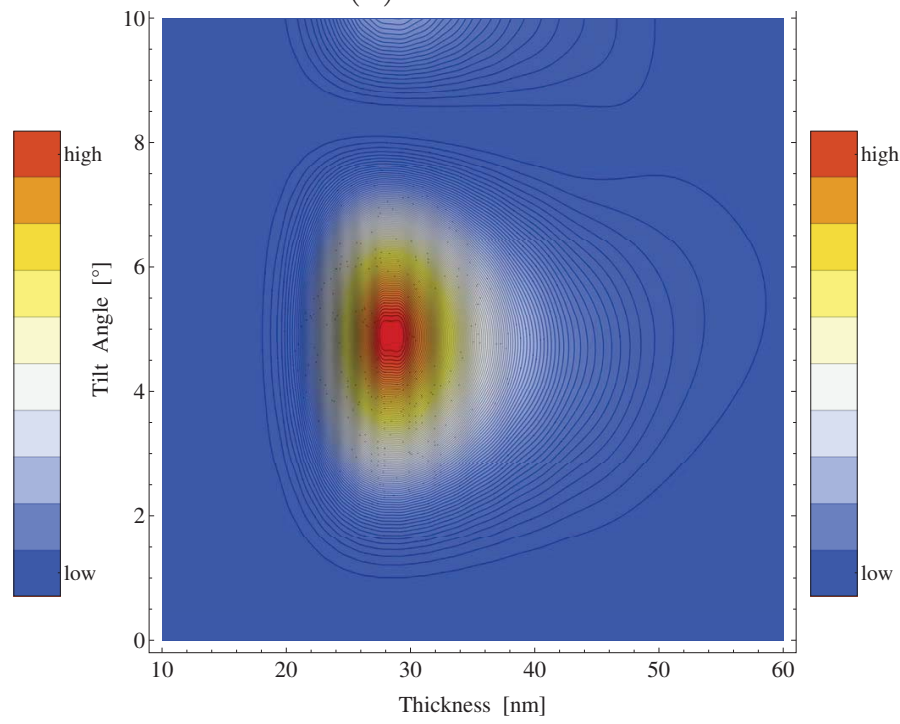

(d) $\lambda=1548 \mathrm{~nm}$

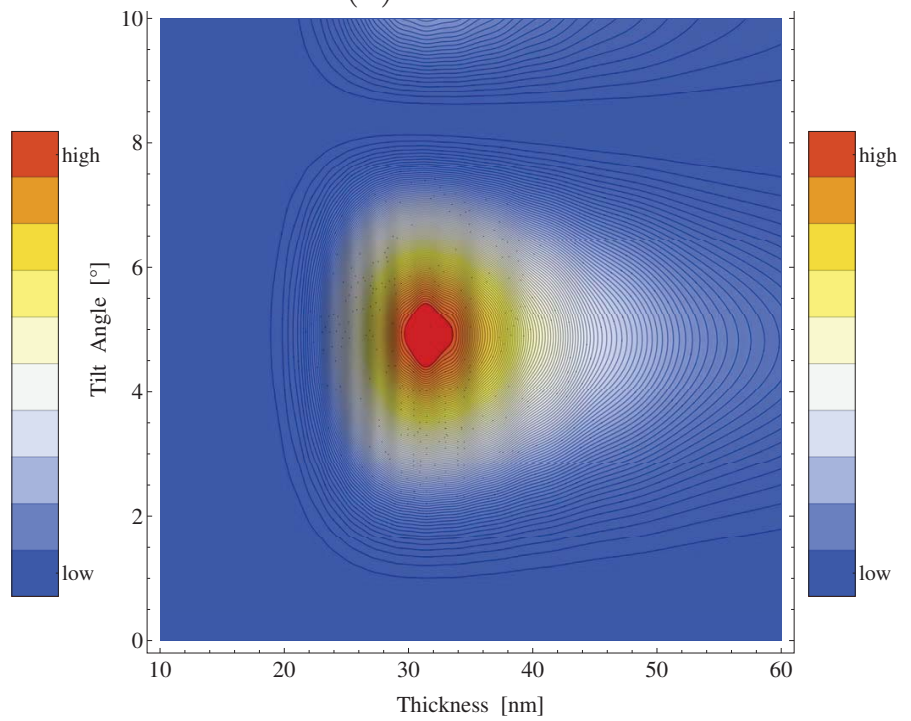

(f) $\lambda=1549 \mathrm{~nm}$ 


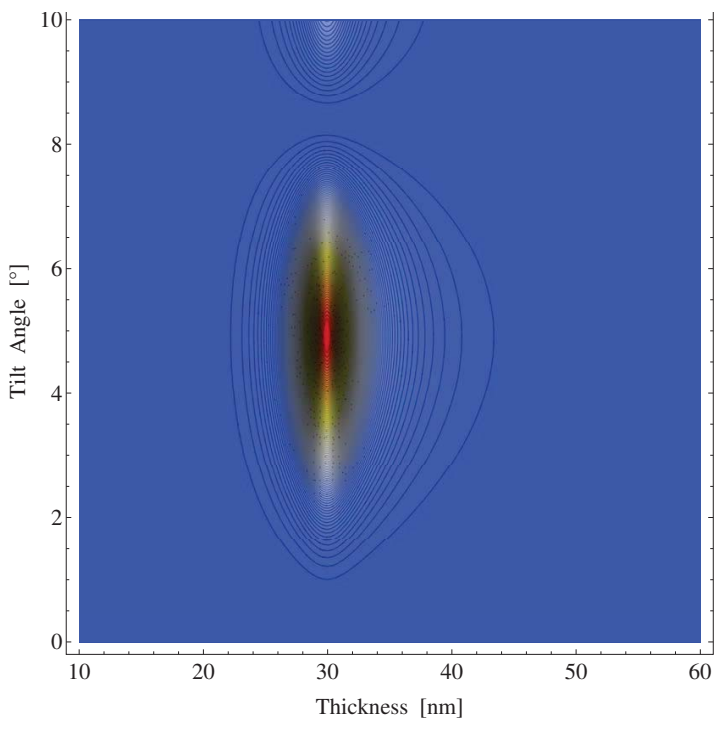

(g) $\lambda=1550 \mathrm{~nm}$

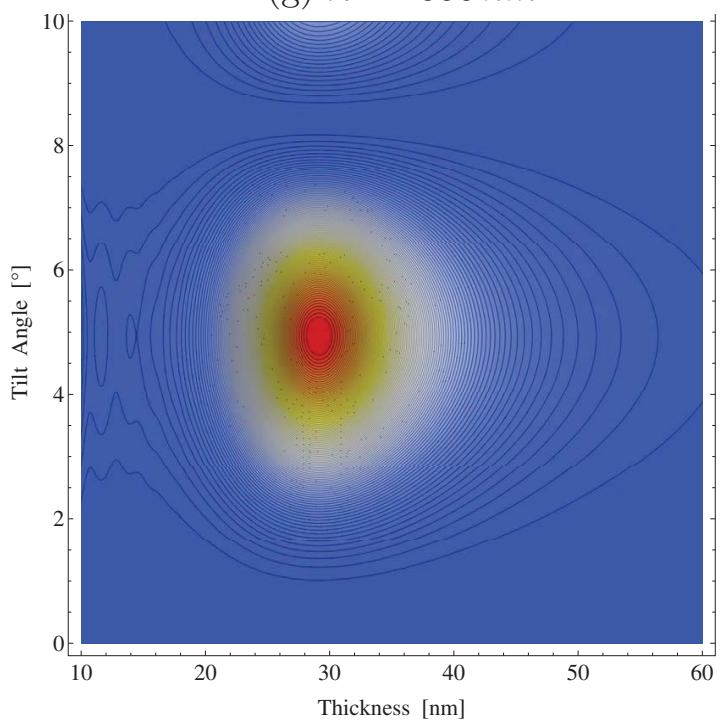

(i) $\lambda=1551 \mathrm{~nm}$

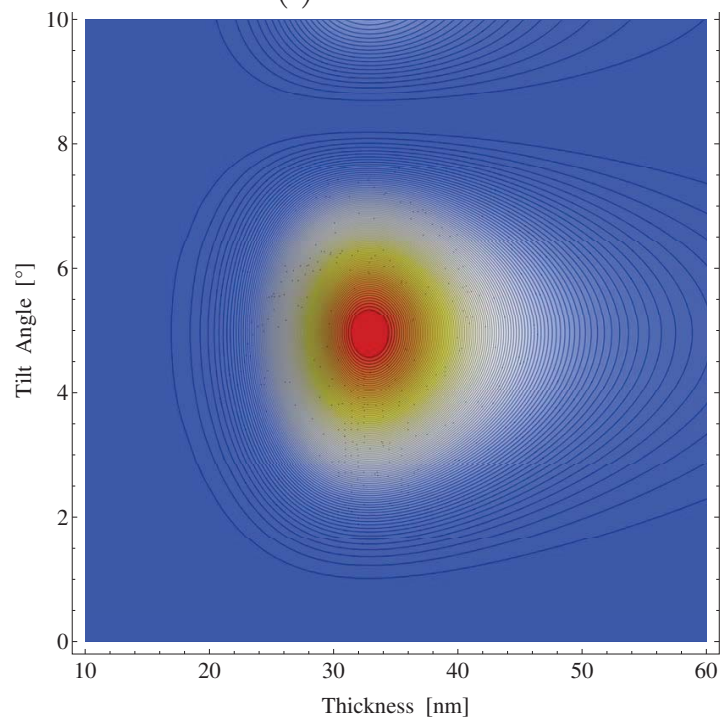

(k) $\lambda=1552 \mathrm{~nm}$

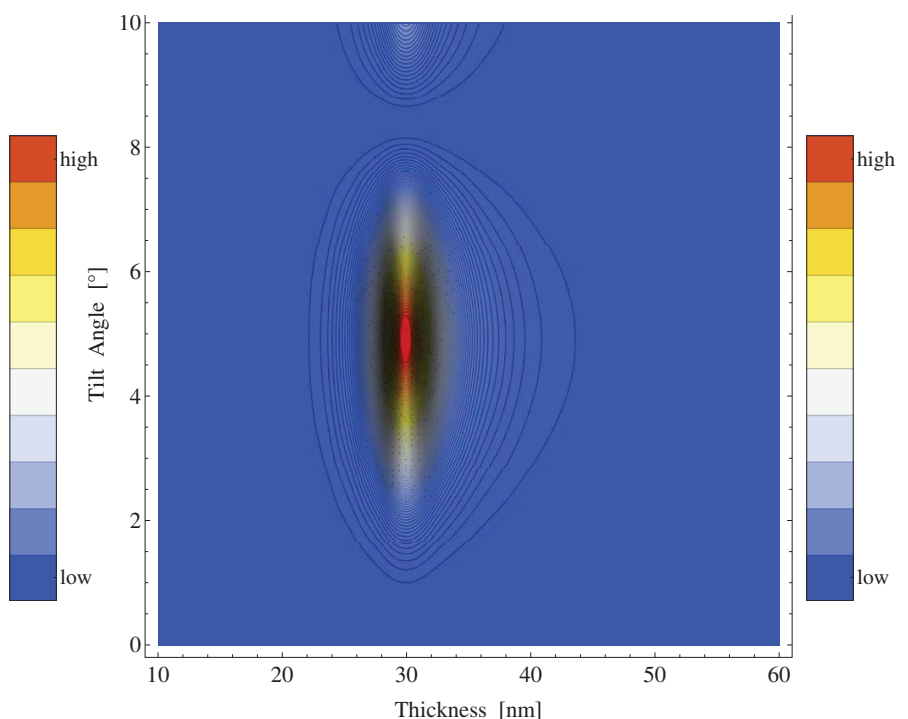

(h) $\lambda=1550 \mathrm{~nm}$

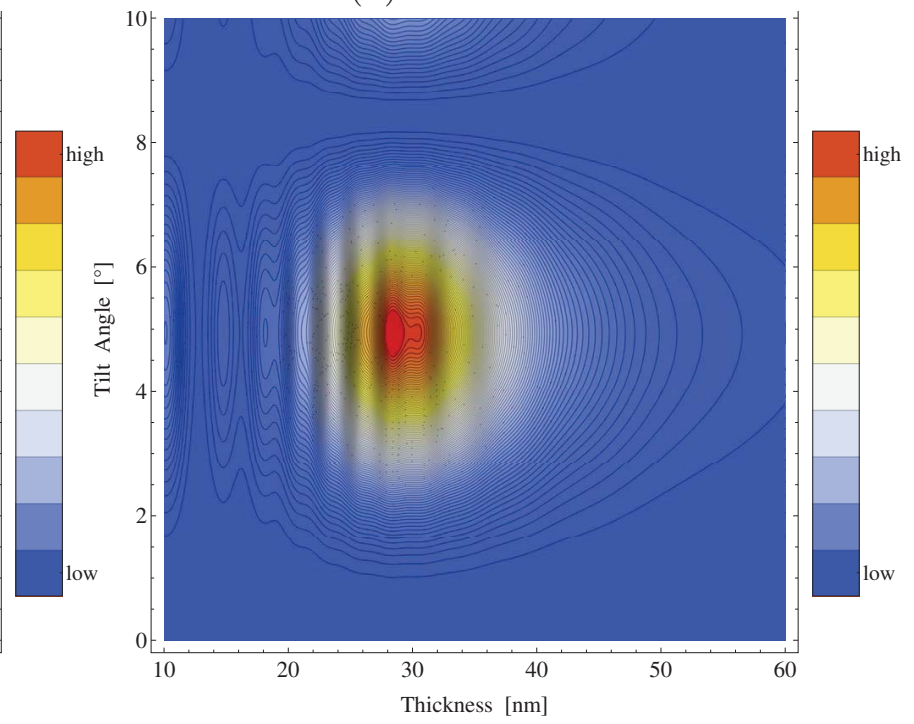

(j) $\lambda=1551 \mathrm{~nm}$

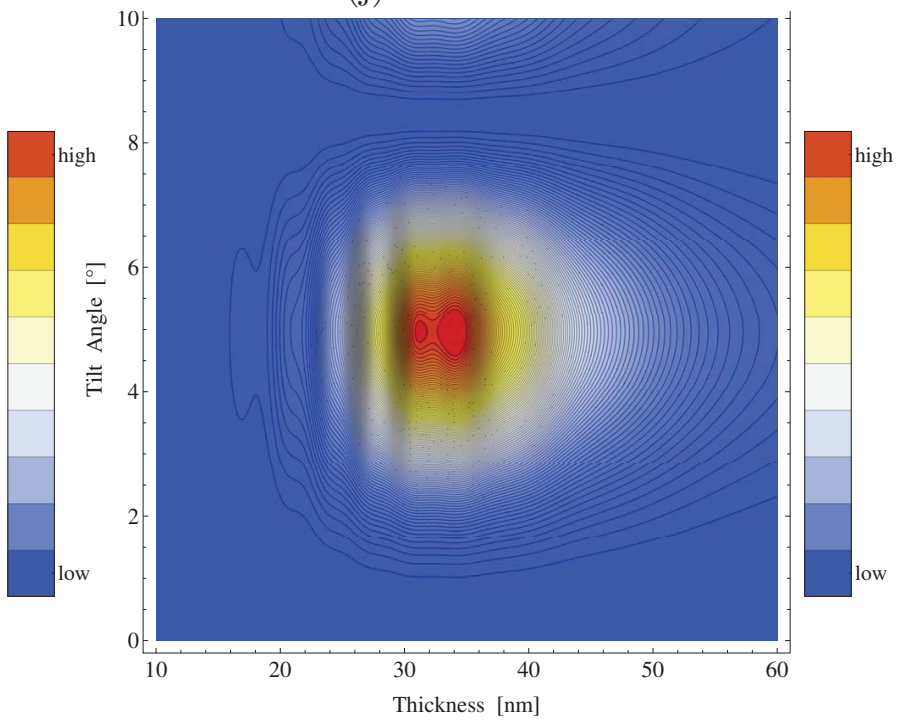

117

(l) $\lambda=1552 \mathrm{~nm}$ 


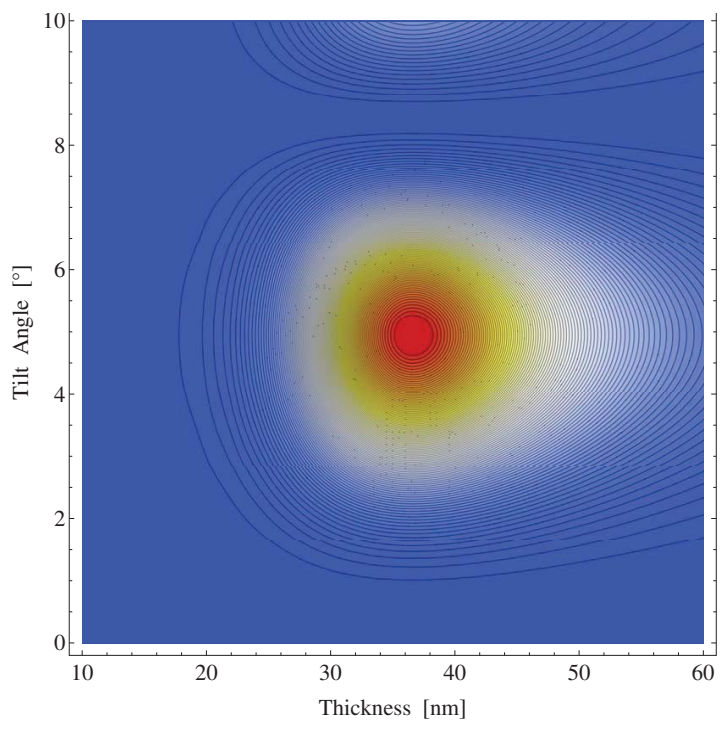

(m) $\lambda=1553 \mathrm{~nm}$

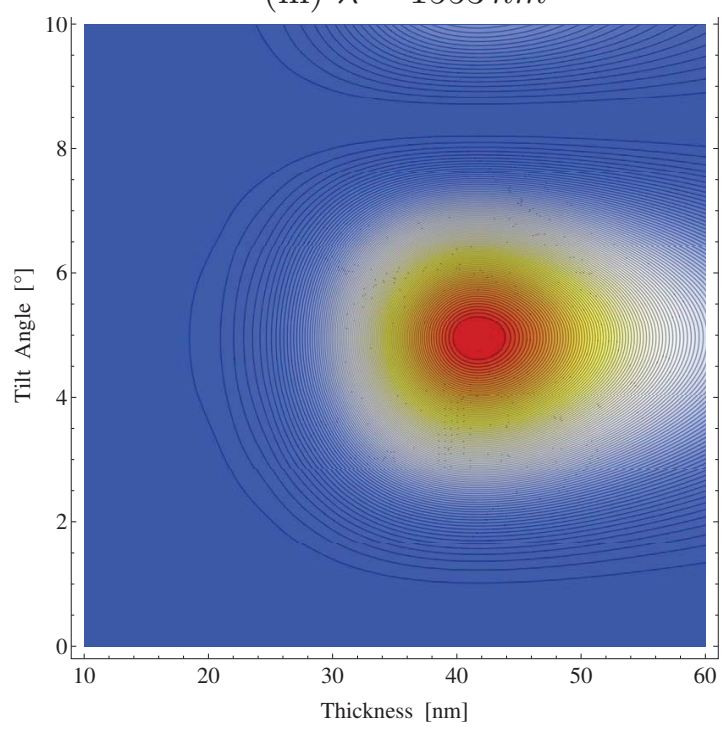

(o) $\lambda=1554 \mathrm{~nm}$

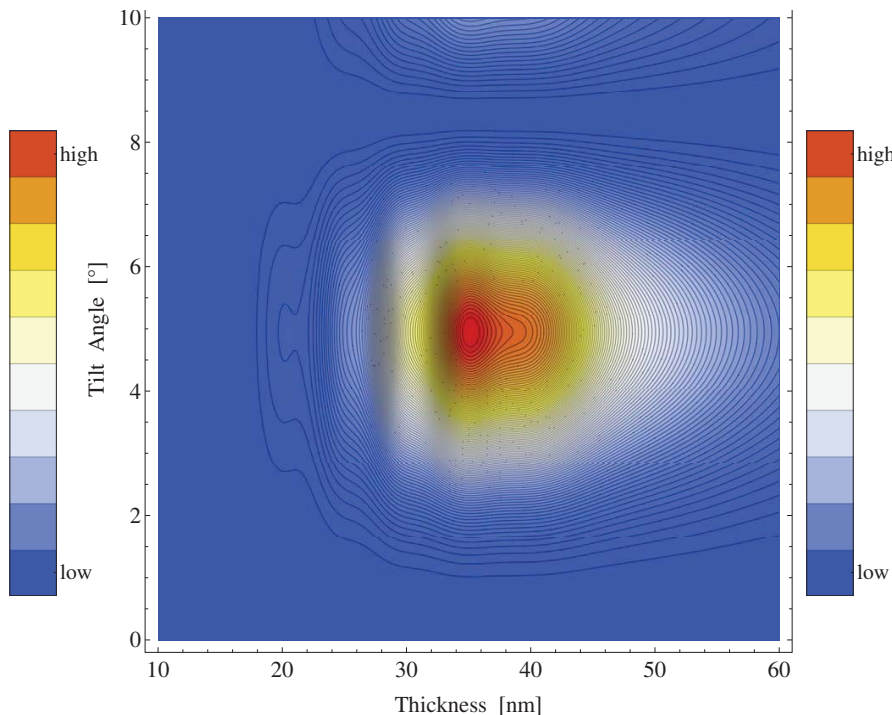

(n) $\lambda=1553 \mathrm{~nm}$

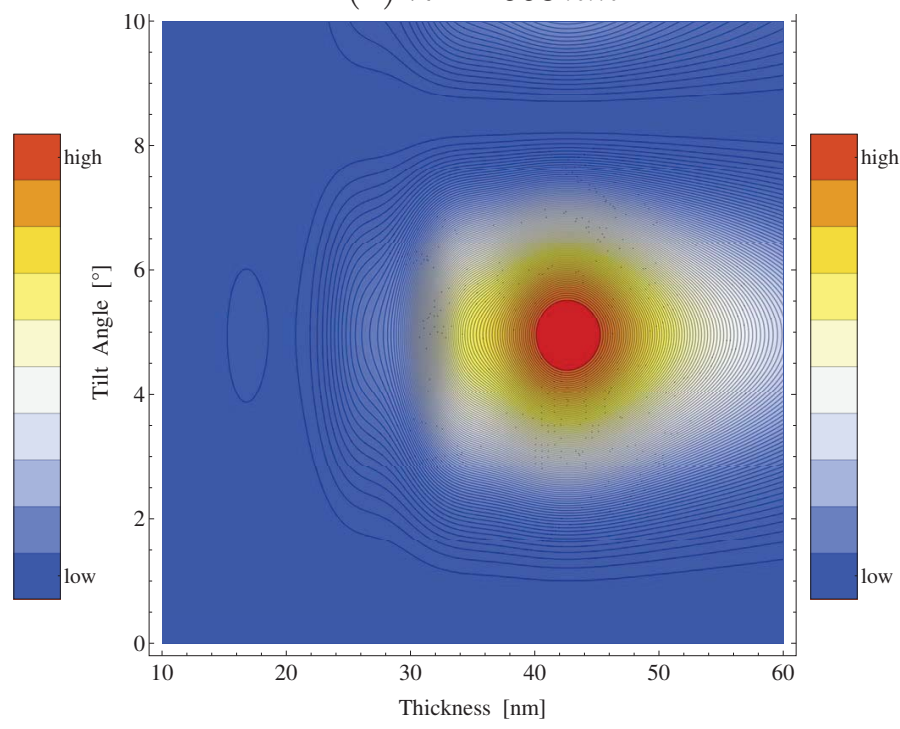

(p) $\lambda=1554 \mathrm{~nm}$ 


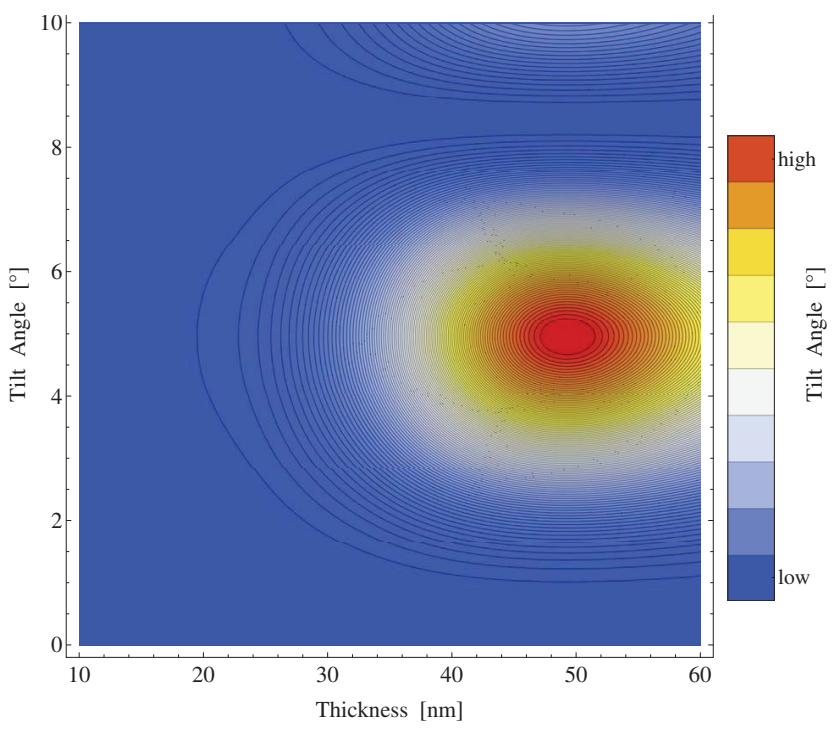

(q) $\lambda=1555 \mathrm{~nm}$

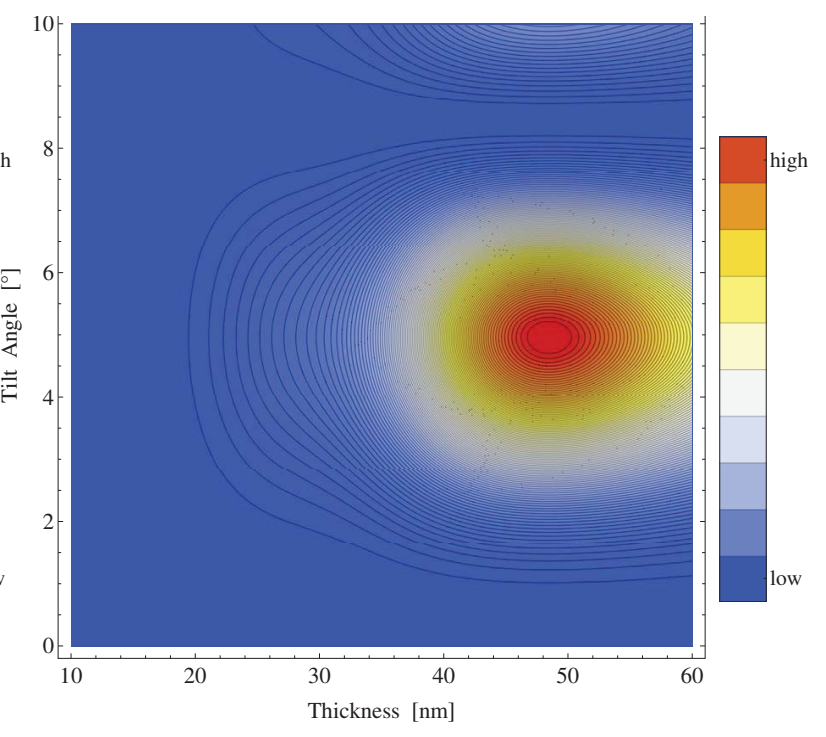

(r) $\lambda=1555 \mathrm{~nm}$

Figure 5.16: Contour plot of power carried by plasmon mode excited by an array of tilted discs of current in the core of a fiber coated by thin Au layer positioned in air environment versus coating thickness and tilt angle. Simulation parameters: azimuthal index of the mode $m=3$, wavelength $\lambda=1550 \mathrm{~nm}$, array is tuned for coupling plasmon mode and core mode at resonance wavelength $\lambda_{\text {res }}=1550 \mathrm{~nm}$, effective indices shown in Fig. 5.15. (left): uniform array, (right): $\pi$-shifted array. Modulation parameter is a wavelength, $\lambda$ starting at $\lambda=1547 \mathrm{~nm}$ and finishing at $\lambda=1555 \mathrm{~nm}$ incrementing by $\Delta \lambda=1.0 \mathrm{~nm}$ in each contour plot.

For plasmon modes in a biosensor application it is a normal component of electric field $E_{r}$ that interacts with the biosample under investigation and possibly polarizes it. Therefore it is of great interest evaluate behaviour of $E_{r}$ strength versus azimuthal angle and tilt angle of the grating. The polar plot of normal component of cumulative electric field over the six modes $(m=0 \ldots 5)$ is shown in Fig. 5.17. 

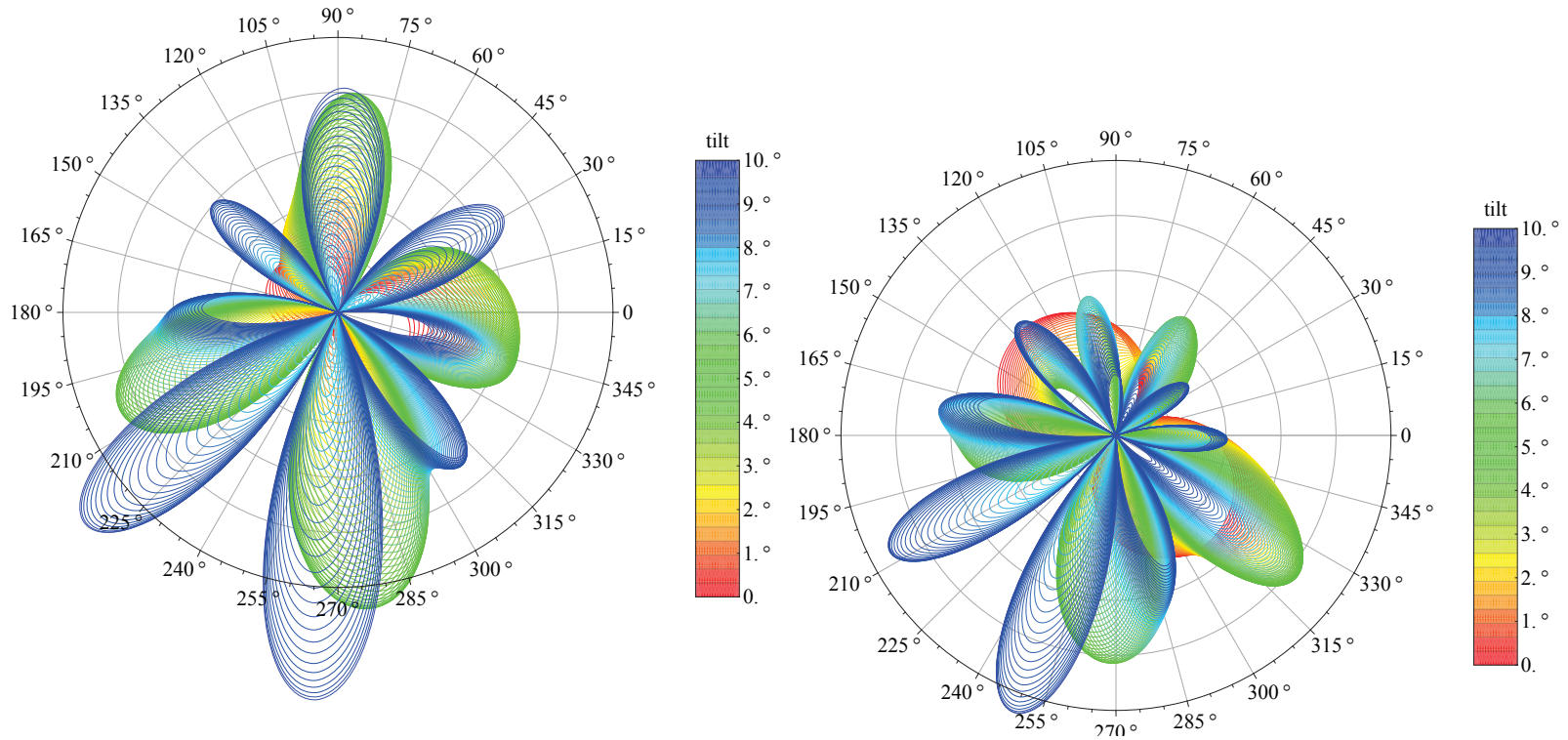

(a) $\lambda=1549 \mathrm{~nm}, d=25 \mathrm{~nm}$
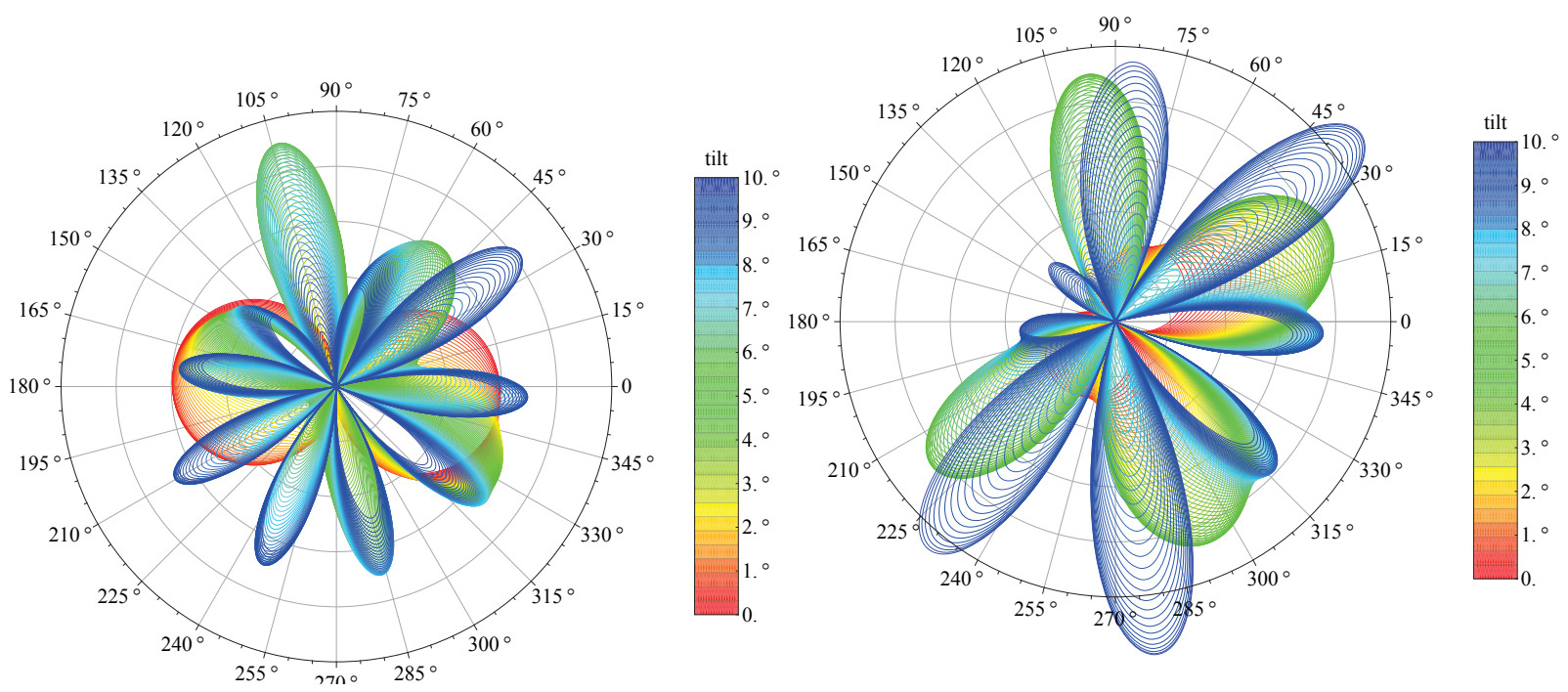

(c) $\lambda=1549 \mathrm{~nm}, d=35 \mathrm{~nm}$

(d) $\lambda=1550 \mathrm{~nm}, d=25 \mathrm{~nm}$
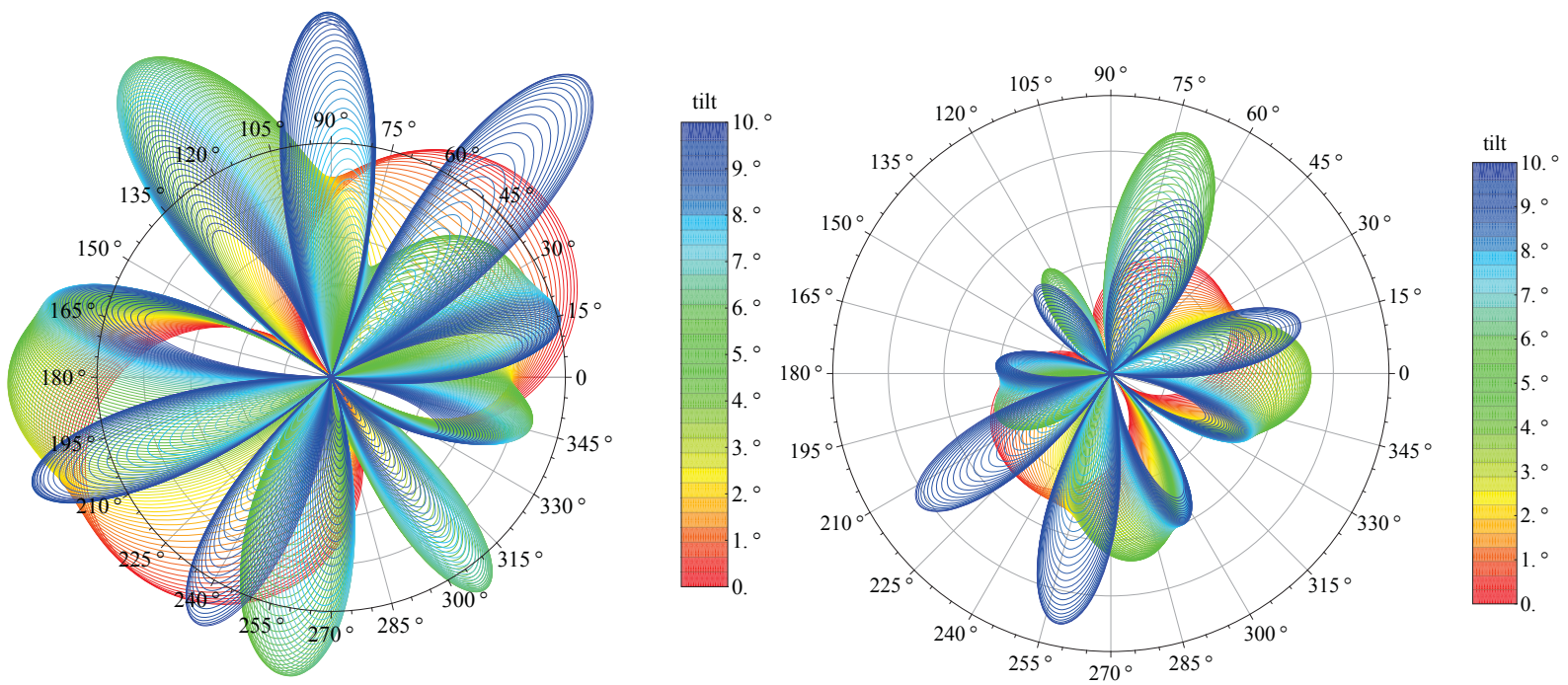

(e) $\lambda=1550 \mathrm{~nm}, d=30 \mathrm{~nm}$

120

(f) $\lambda=1550 \mathrm{~nm}, d=35 \mathrm{~nm}$ 


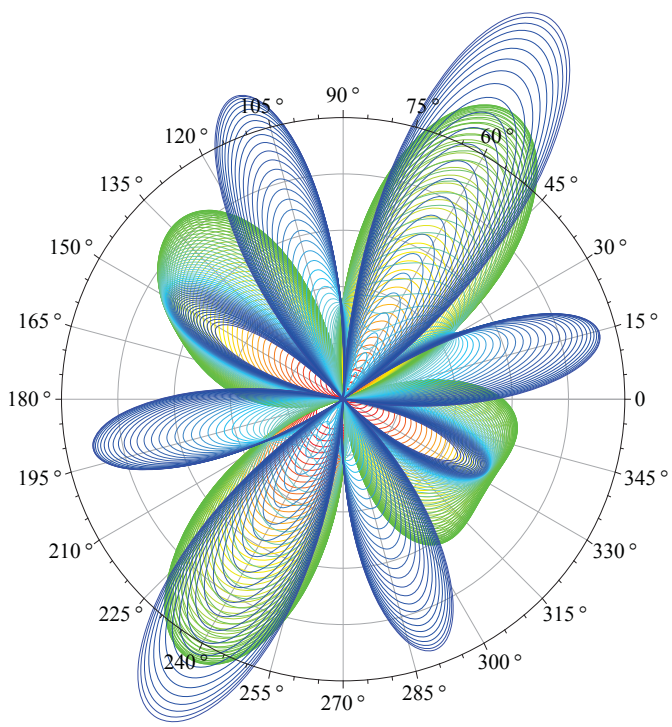

(g) $\lambda=1551 \mathrm{~nm}, d=25 \mathrm{~nm}$

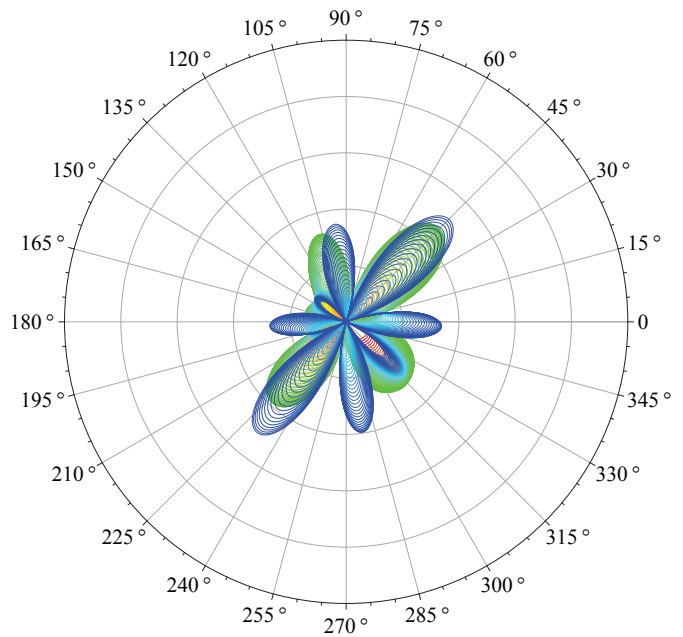

(i) $\lambda=1551 \mathrm{~nm}, d=35 \mathrm{~nm}$

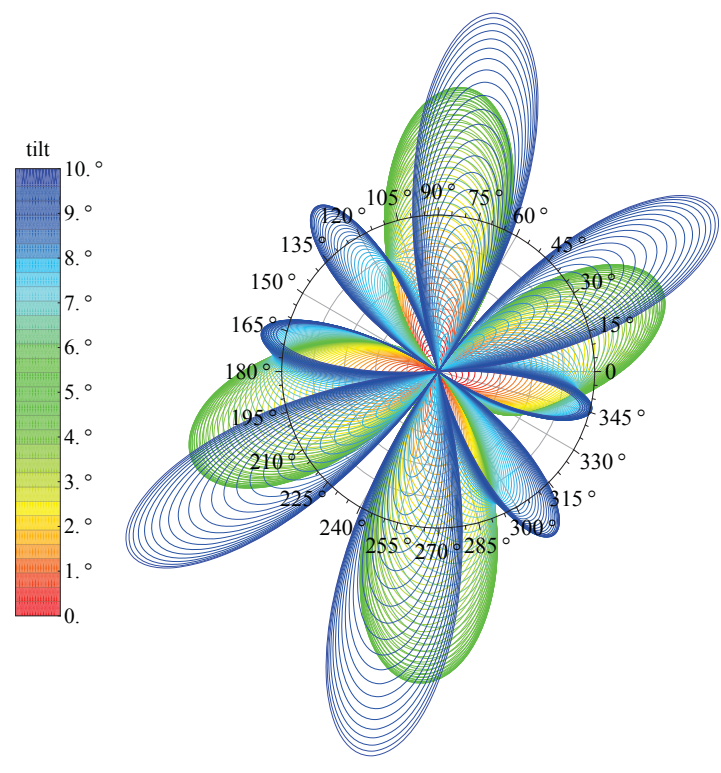

(h) $\lambda=1551 \mathrm{~nm}, d=30 \mathrm{~nm}$

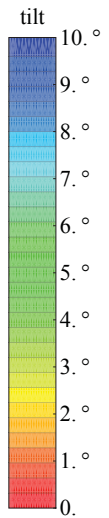

Figure 5.17: Polar plot of an absolute value of a normal component of cumulative electric field $\left|E_{r}\right|$ over 6 azimuthal indices, $m=0 \ldots 5$ of plasmons excited by a source of $\pi$-shifted array of tilted discs current located in a core of fiber coated by thin Au layer positioned in air environment versus azimuthal angle $\theta$ with the following parameters: tilt angle $\alpha$, film thickness $d$ and wavelength $\lambda$. Array is tuned for coupling plasmon mode with azimuthal index $m=3$ and core mode at resonance wavelength $\lambda_{\text {res }}=1550 \mathrm{~nm}$ and resonance film thickness $d_{\text {res }}=30 \mathrm{~nm}$. Field is measured at the middle of an array. Scale is arbitrary, but fixed within the same wavelength.

It is assumed, that tilting of a current source is done in such a way that for each disc (or ring) its center stay unchanged. In this case a translational period over $z$-axis, $\Delta_{z}$ for an array remains fixed during tilting. Another way of tilting is to keep the spacing $\Delta$ 


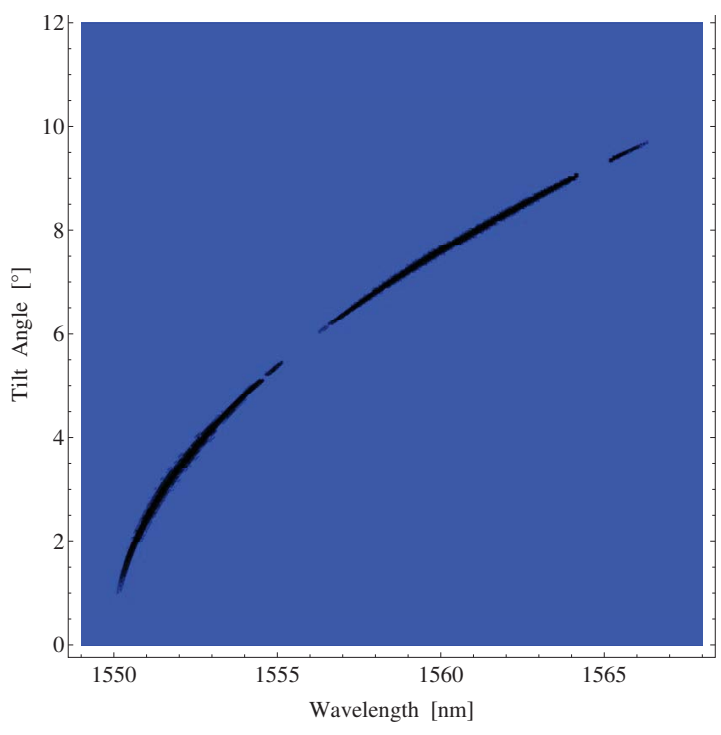

(a)

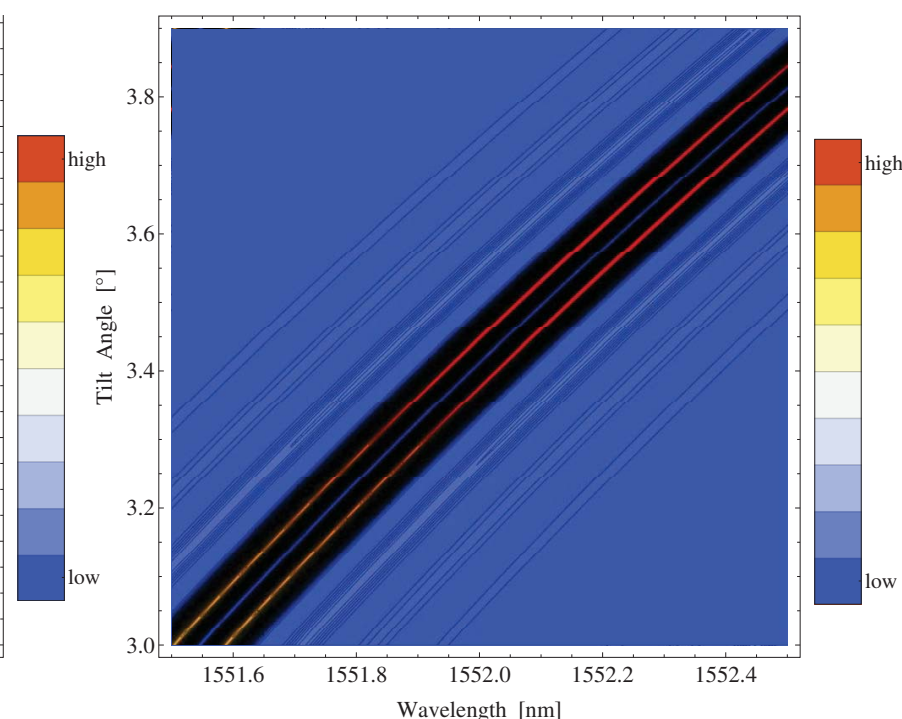

(b)

Figure 5.18: Contour plot of power carried by plasmon mode excited by a $\pi$-shifted array of tilted discs of current in the core of a fiber coated by thin Au layer positioned in air environment versus wavelength and tilt angle. Simulation parameters: azimuthal index of the mode $m=3$, layer thickness $d=35 \mathrm{~nm}$, an array is tuned for coupling plasmon mode and core mode at resonance wavelength $\lambda_{\text {res }}=1550 \mathrm{~nm}$, effective index $n_{e f 1}$ shown in Fig. 5.11, array period in z-axis is $\Delta_{z}=\Delta / \cos \alpha$. Computation window for wavelength and angle range respectively (a): (1549 nm,1568 nm), $\left(0^{\circ}, 12^{\circ}\right)$, (b): (155159 nm,1552.5 $\mathrm{nm}),\left(0^{\circ}, 3.9^{\circ}\right)$.

between adjacent discs in an array unchanged, where the spacing $\Delta$ is measured along the normal to the disc while $\Delta_{z}$ is evaluated as $\Delta_{z}=\Delta / \cos \alpha$. Since period of an array over $z$-axis now increases with a tilt angle $\alpha$, so does the resonance wavelength of an array. As a result, the power peak in contour plot versus wavelength $\lambda$ and tilt angle $\alpha$ appears bent when $\Delta_{z}=\Delta / \cos \alpha$ compare to being elongated vertically when $\Delta_{z}$ is unchanged with tilt angle, $\Delta_{z}=$ const $(\alpha)$. See for comparison Fig. 5.18 and Fig. 5.8.

If array period along normal $\Delta$ is kept fixed and therefore $\Delta_{z}$ is angle dependent, a small perturbation of a tilt angle $\alpha$ leads to a new resonance condition for the array which causes significant change of azimuthal dependence of the field $\left|E_{r}(\theta)\right|$ and results in a more sensitive polar plot modulation shown in Fig. 5.19. 


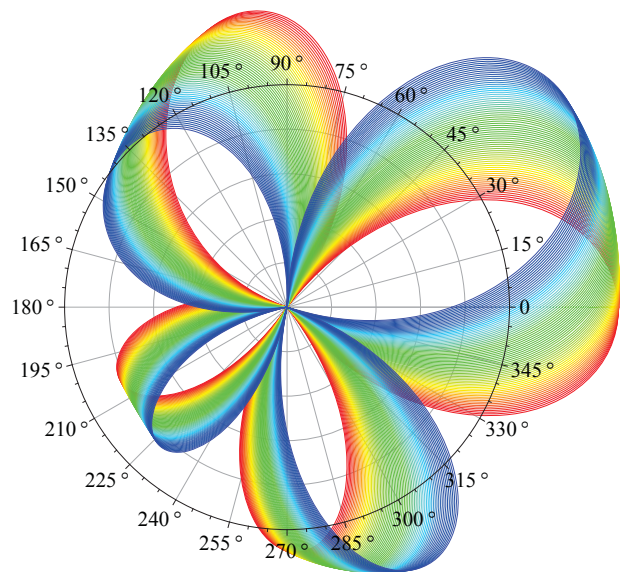

(a) $\lambda=1549 \mathrm{~nm}, d=25 \mathrm{~nm}$

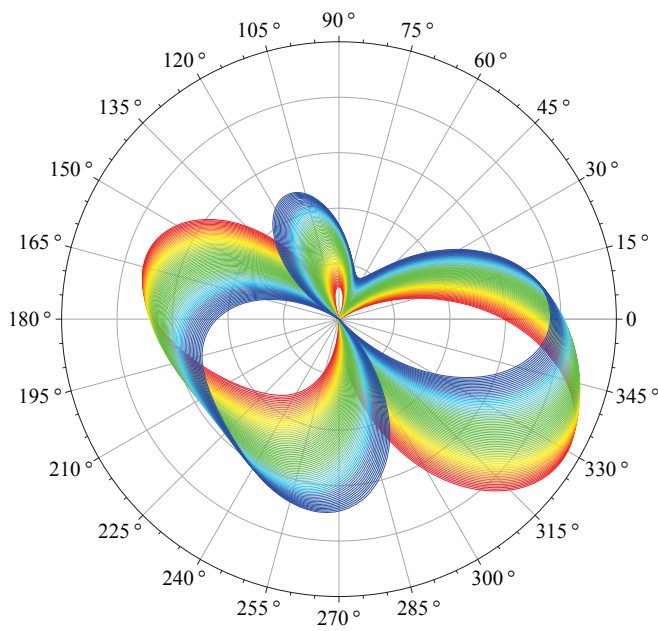

(c) $\lambda=1549 \mathrm{~nm}, d=35 \mathrm{~nm}$

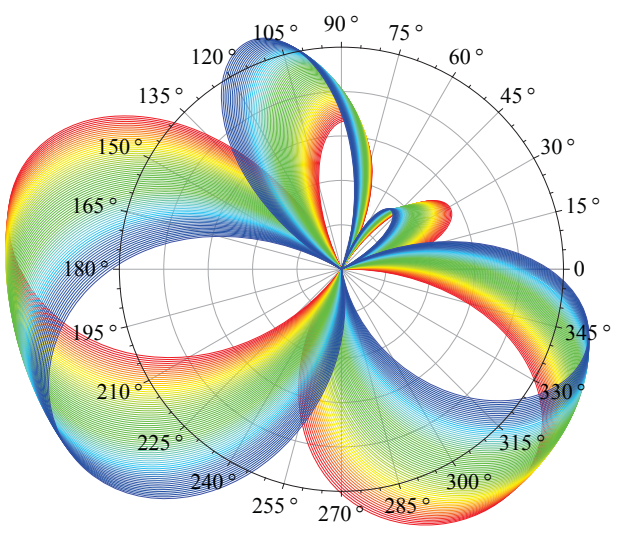

(e) $\lambda=1550 \mathrm{~nm}, d=30 \mathrm{~nm}$

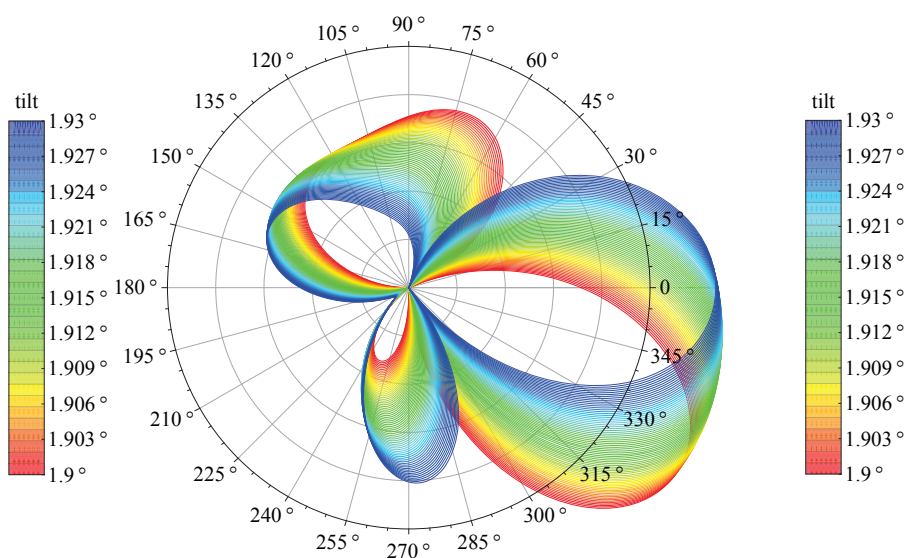

(b) $\lambda=1549 \mathrm{~nm}, d=30 \mathrm{~nm}$

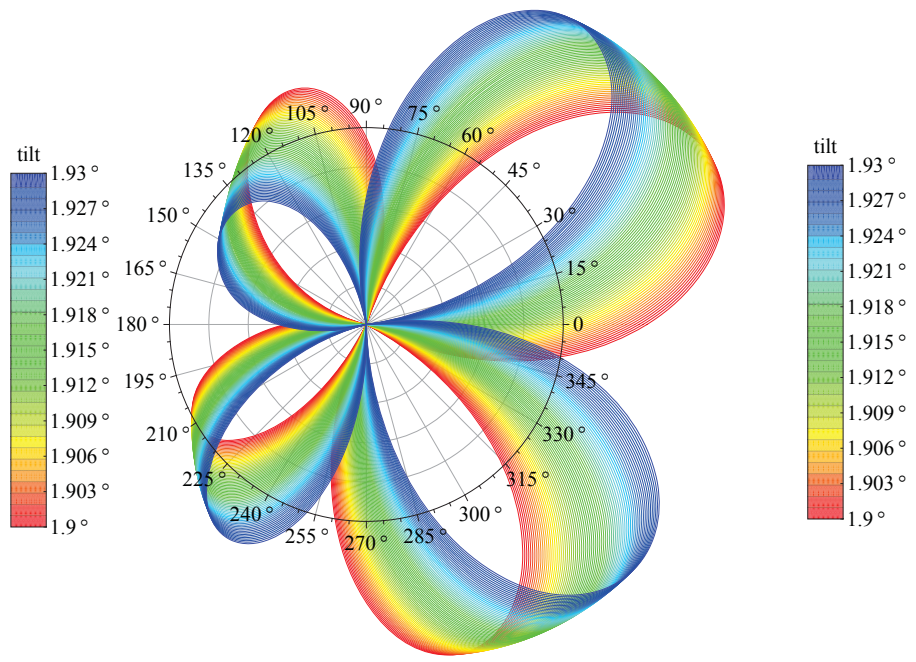

(d) $\lambda=1550 \mathrm{~nm}, d=25 \mathrm{~nm}$

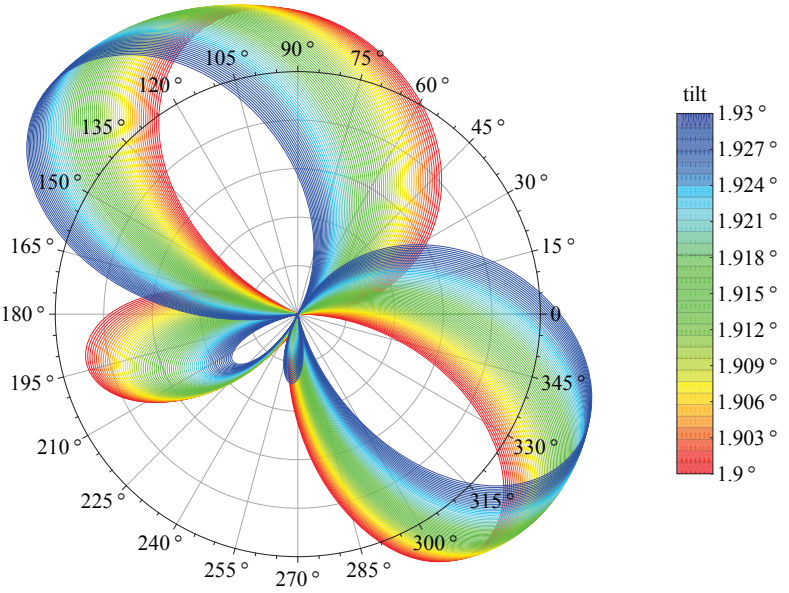

(f) $\lambda=1550 \mathrm{~nm}, d=35 \mathrm{~nm}$ 


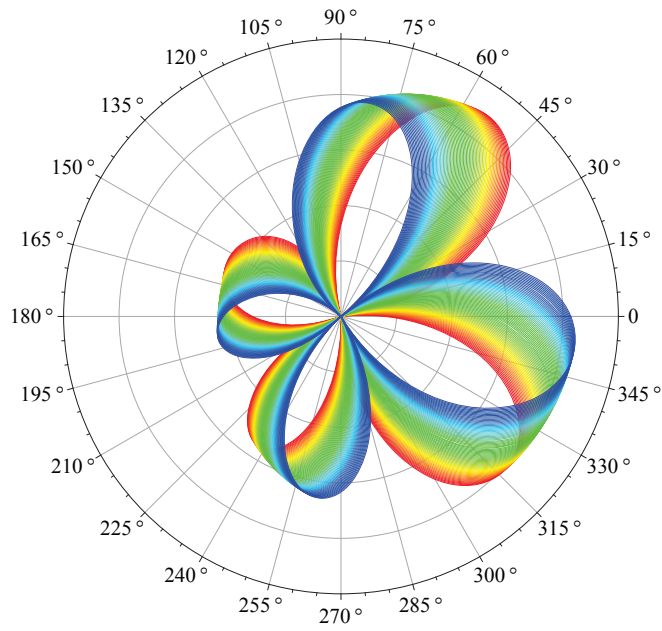

(g) $\lambda=1551 \mathrm{~nm}, d=25 \mathrm{~nm}$

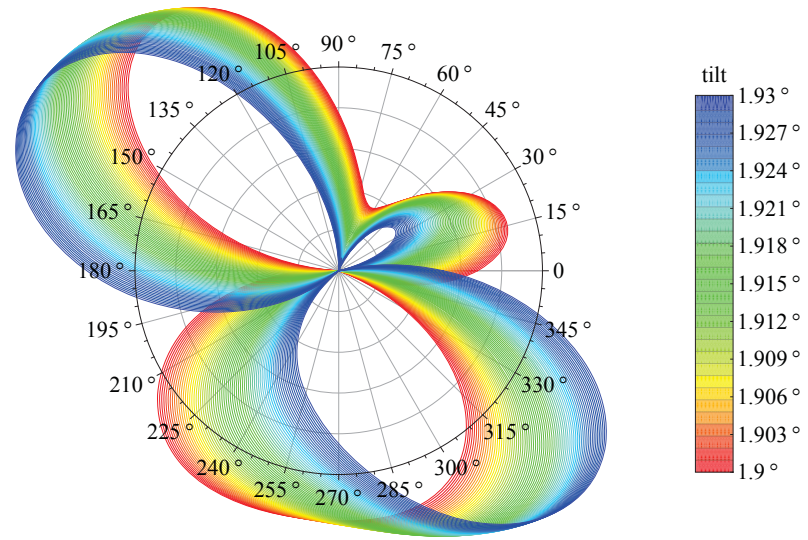

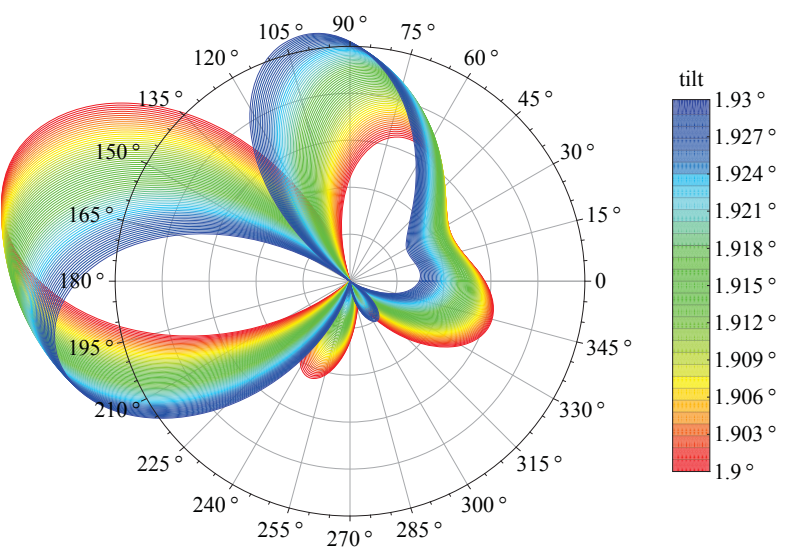

(h) $\lambda=1551 \mathrm{~nm}, d=30 \mathrm{~nm}$

(i) $\lambda=1551 \mathrm{~nm}, d=35 \mathrm{~nm}$

Figure 5.19: Polar plot of an absolute value of a normal component of cumulative electric field $\left|E_{r}\right|$ over 6 azimuthal indices, $m=0 \ldots 5$ of plasmons excited by a source of $\pi$-shifted array of tilted discs current located in a core of fiber coated by thin Au layer positioned in air environment versus azimuthal angle $\theta$ the following parameters: tilt angle $\alpha$, film thickness $d$ and wavelength $\lambda$. Array is tuned for coupling plasmon mode with azimuthal index $m=3$ and core mode at resonance wavelength $\lambda_{\text {res }}=1550 \mathrm{~nm}$ and resonance film thickness $d_{\text {res }}=30 \mathrm{~nm}$. Array period in $z$-axis is $\Delta_{z}=\Delta / \cos \alpha$. Field is measured at the middle of an array. Scale is arbitrary, but fixed within the same wavelength.

It was assumed in numerical simulation that refractive indices of the fiber core, cladding and metal layer are wavelength dependent, while refractive index of the outer layer is constant. The data for wavelength dependent refractive index of gold and platinum was taken from [52]. Wavelength dependent refractive index of fused silica calculated with Sellmeier coefficients from [91]. 


\subsection{Conclusion.}

Plasmon excitation problem due to a discrete set of sources in the core of a fiber coated by thin metal layer was solved from the Maxwell's equations. This approach can be extended to a more realistic model such as fiber with a tilted Brag grating and can be found useful for a sensor like fiber application. 


\section{Chapter 6}

\section{Plasmon excitation in a fiber by tilted Bragg grating}

A model, developed previously, extended in this chapter to the case of plasmon excitation in a fiber by a tilted Bragg grating. A metal coating in this case is a nanostructured metal distributed over finite region on a fiber surface. Both, Bragg grating and metal distribution considered in this case are either uniform or $\pi$-shifted.

Scattering due to a tilted Bragg gratings spacially modulated by a sine function in dielectric cylinder is analyzed with Green's function formulation and equivalence principle. Scattered field due to gratings then applied for a plasmon excitation in a fiber coated by a continuous thin metal film as well as in a fiber coated by a metal distribution in the form of periodic patches.

\subsection{Introduction.}

In previous chapter a plasmon excitation by an array of discretely distributed sources placed in a core of a fiber SMF28 coated by a thin metal film is considered. Such an array resembles a Bragg grating, however in a realistic fiber Bragg grating the core material is continuously modulated with a sine function as shown in Fig. 6.1. Therefore it is of interest to take into account a periodic continuous material variation rather than an array of discrete sources. This can be done with the help of equivalence principle introduced by Schelkunoff in 1936, [92]. An equivalence principle can imply for example the following: when field outside of a closed region is calculated, a certain current source positioned on the surface of this region is equivalent to the field inside of the region and therefore they can replace (substitute) each other to simplify a particular problem. Several variations of equivalence principle have been formulated, [93]. The one exploited here uses current source inside of the closed region rather than on its surface, [89] (see also Appendix D for brief introduction). 


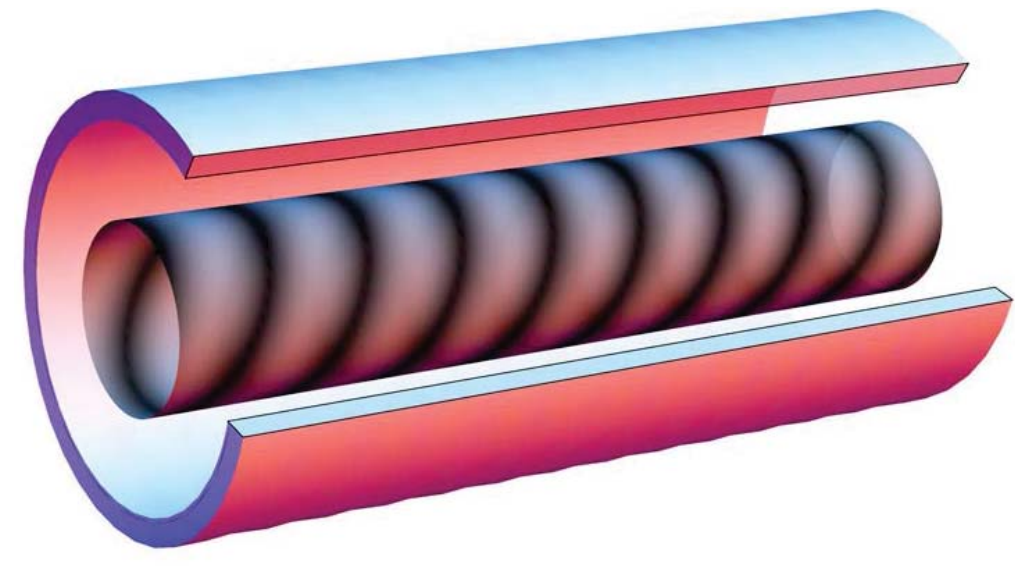

Figure 6.1: A fiber with a realistic Bragg grating in the core as continuosly modulated tilted sine function.

\subsection{Excitation by an uniform Bragg grating in fiber coated by metal film.}

Assume permittivity of the fiber core is modulated by sine function along tilted axis as,

$$
\varepsilon_{D C}+\varepsilon_{A C} \sin \left(\frac{2 \pi}{\Lambda} z^{\prime}+\varphi_{s}\right)
$$

where,

$\varepsilon_{D C}, \varepsilon_{A C}$ are $\mathrm{DC}$ and $\mathrm{AC}$ components of the modulation, $\Lambda$ modulation period along $z^{\prime}$, $z^{\prime}$ a tilted axis transformed as,

$$
z^{\prime}=z \cos \alpha-r \sin \theta \sin \alpha
$$

$\varphi_{s}$ some phase shift. A constant $\varphi_{s}$ over the whole grating keeps it uniform. An Illustration of a fiber with continuously modulated core by an uniform sine function along tilted axis is given in Fig. 6.2.

To model the first order approximation of the scattered field due to grating the equivalent current density of zero order $\vec{J}^{e q},(0)$ is employed as an impressed current density source in wave equation 5.7 ,

$$
\vec{J}^{e q},(0)=-i \omega \Delta \varepsilon \vec{E}^{h}
$$

where $\Delta \varepsilon$ is a difference between permittivity of a modulated fiber core by a grating and permittivity of a homogeneous fiber core, 


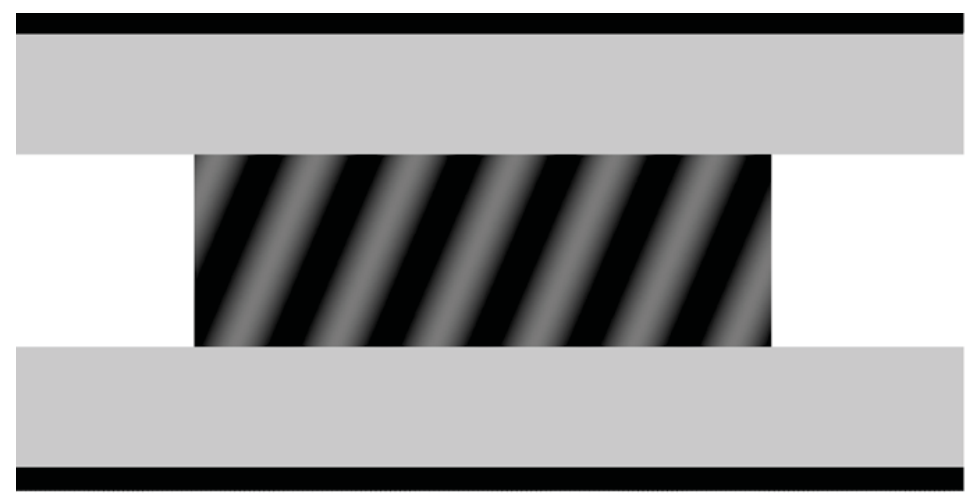

Figure 6.2: A fiber with continuously modulated core by an uniform sine function along tilted axis $z^{\prime}$.

$$
\Delta \varepsilon=\left(\varepsilon_{D C}+\varepsilon_{A C} \sin \left(\frac{2 \pi}{\Lambda} z^{\prime}+\varphi_{s}\right)\right) \Pi\left(r, 0, r_{1}\right) \Pi\left(z,-z_{1}, z_{1}\right)
$$

where $\vec{E}^{h}$ is a field of the core mode of a single mode fiber SMF28 defined in a previous chapter,

$$
\vec{E}^{h}=\vec{E}_{\text {core }}(r, \theta, z)
$$

The Fourier transform of $z$ component current density

$$
j_{z}^{(0)}=\int_{-\infty}^{+\infty} \int_{-\pi}^{+\pi} J_{z}^{e q,(0)} e^{-i(v z+m \theta)} d \theta d z
$$

becomes,

$$
j_{z}^{(0)}=(-1) i \omega R_{z}(r) \Pi\left(r, 0, r_{1}\right) 4 \pi z_{1}\left(\psi_{1}+\psi_{2} \psi_{3}\right)
$$

where

$$
\begin{gathered}
\psi_{1}=\varepsilon_{D C}\left\{\operatorname{sinc}\left(\left(m-m_{C}\right) \pi\right)+e^{-i m_{C} \theta_{0}} \operatorname{sinc}\left(\left(m+m_{C}\right) \pi\right)\right\} \operatorname{sinc}\left((v-\beta) z_{1}\right) \\
\psi_{2}=\frac{1}{i 2} \varepsilon_{A C}\left(J_{m-m_{C}}\left(\frac{2 \pi}{\Lambda} r \sin \alpha\right)+e^{-i m_{C} \theta_{0}} J_{m+m_{C}}\left(\frac{2 \pi}{\Lambda} r \sin \alpha\right)\right) \\
\psi_{3}=e^{i \varphi_{s}}(-1)^{m-m_{C}} \operatorname{sinc}\left(\left((v-\beta)-\frac{2 \pi}{\Lambda} \cos \alpha\right) z_{1}\right)+ \\
+(-1) e^{-i \varphi_{s}} \operatorname{sinc}\left(\left((v-\beta)+\frac{2 \pi}{\Lambda} \cos \alpha\right) z_{1}\right)
\end{gathered}
$$




\subsection{Excitation by $\pi$-shifted Bragg grating in fiber coated by metal film.}

A $\pi$-phase shifted grating is implemented as two identical uniform gratings, one is written from $z=-z_{1}$ to $z=0$ with phase shift $\varphi_{s 1}=0$ and the second from $z=0$ to $z=z_{1}$ with phase shift $\varphi_{s 2}=\pi$, i.e.

$$
\Delta \varepsilon=\Delta \varepsilon_{1}+\Delta \varepsilon_{2}
$$

where

$$
\begin{aligned}
\Delta \varepsilon_{1} & =\left(\varepsilon_{D C}+\varepsilon_{A C} \sin \left(\frac{2 \pi}{\Lambda} z^{\prime}+\varphi_{s 1}\right)\right) \Pi\left(r, 0, r_{1}\right) \Pi\left(z,-z_{1}, 0\right) \\
\Delta \varepsilon_{2} & =\left(\varepsilon_{D C}+\varepsilon_{A C} \sin \left(\frac{2 \pi}{\Lambda} z^{\prime}+\varphi_{s 2}\right)\right) \Pi\left(r, 0, r_{1}\right) \Pi\left(z, 0, z_{1}\right)
\end{aligned}
$$

An Illustration of a fiber with continuously modulated core by a $\pi$-shifted sine function along tilted axis is given in Fig. 6.3.

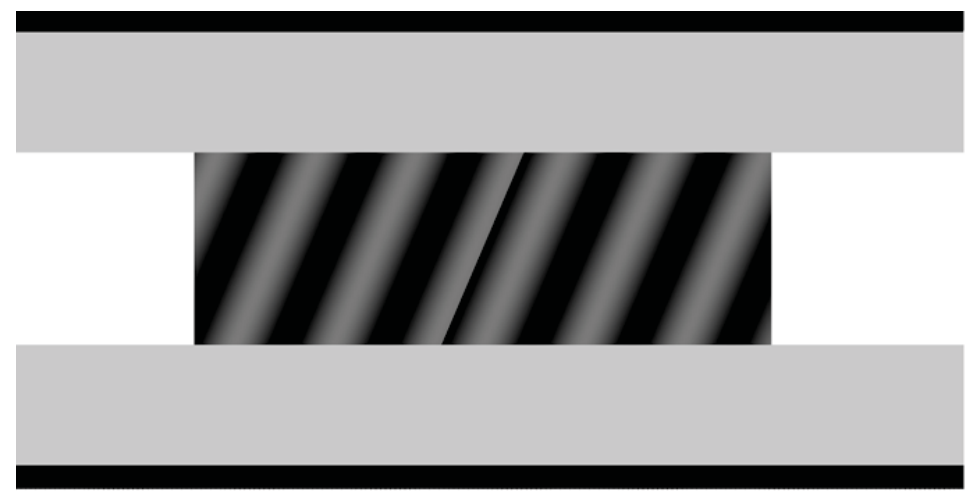

Figure 6.3: A fiber with continuously modulated core by a $\pi$-shifted sine function along tilted axis $z^{\prime}$.

The Fourier transform of $z$ component of the current density then becomes,

$$
j_{z}^{(0)}=(-1) i \omega R_{z}(r) \Pi\left(r, 0, r_{1}\right)\left(\xi_{1}+\left(\xi_{2}+\xi_{3}+\xi_{4}+\xi_{5}\right) \xi_{6}\right)
$$

where

$$
\xi_{1}=\varepsilon_{D C} 2 \pi z_{1} \operatorname{sinc}\left((v-\beta) z_{1}\right)\left\{\operatorname{sinc}\left(\left(m-m_{C}\right) \pi\right)+e^{-i m_{C} \theta_{0}} \operatorname{sinc}\left(\left(m+m_{C}\right) \pi\right)\right\}
$$




$$
\begin{gathered}
\xi_{2}=\frac{1-e^{i\left((v-\beta)-\frac{2 \pi}{\Lambda} \cos \alpha\right) z_{1}}}{(v-\beta)-\frac{2 \pi}{\Lambda} \cos \alpha} e^{i \varphi_{s 1}}(-1)^{m-m_{C}} \\
\xi_{3}=\frac{1-e^{i\left((v-\beta)+\frac{2 \pi}{\Lambda} \cos \alpha\right) z_{1}}}{(v-\beta)+\frac{2 \pi}{\Lambda} \cos \alpha}\left(-e^{-i \varphi_{s 1}}\right) \\
\xi_{4}=\frac{e^{-i\left((v-\beta)-\frac{2 \pi}{\Lambda} \cos \alpha\right) z_{1}}-1}{(v-\beta)-\frac{2 \pi}{\Lambda} \cos \alpha} e^{i \varphi_{s 2}}(-1)^{m-m_{C}} \\
\xi_{5}=\frac{e^{-i\left((v-\beta)+\frac{2 \pi}{\Lambda} \cos \alpha\right) z_{1}}-1}{(v-\beta)+\frac{2 \pi}{\Lambda} \cos \alpha}\left(-e^{-i \varphi_{s 2}}\right) \\
\xi_{6}=\varepsilon_{A C} \pi\left\{J_{m-m_{C}}\left(\frac{2 \pi}{\Lambda} r \sin \alpha\right)+e^{-i m_{C} \theta_{0}} J_{m+m_{C}}\left(\frac{2 \pi}{\Lambda} r \sin \alpha\right)\right\}
\end{gathered}
$$

Expressions for $j_{+}^{(0)}, j_{-}^{(0)}$ are obtained from expression for $j_{z}^{(0)} 6.6$ for an uniform grating or 6.13 for a $\pi$-shifted grating with $R_{z}(r)$ replaced by $R_{+}(r), R_{-}(r)$ respectively. To find all components $f_{z}, f_{+}, f_{-}$of a vector function $\vec{f}$ in 5.26, 5.24 and 5.25 every Bessel function under the integrals 5.31, 5.27 and 5.30, suited for each case, is expanded in series according to 5.87. With known $\vec{f}$ the primary fields $\left(\vec{e}^{p}, \vec{h}^{p}\right)$ and the power carried by a mode along $z$-axis in outer layer evaluated with 5.17, 5.35 and 5.70 .

\subsection{Excitation by uniform Bragg grating in fiber coated by structured metal film.}

The metal layer so far has been treated as a part of homogeneous waveguide, i.e. it was assumed uniform and of infinite extent in $z$-axis. However in practical use the metal coating has a short length, also for some sensor type applications a metal distribution on the fiber should be made in the form of a certain pattern. Taking into account finite and structured nature of coating can be done with the help of equivalence principle which allows treating metal coating as a part of inhomogeneous waveguide.

Two types of inhomogenuitiy, Bragg grating in the core and metal distribution in the outer layer, are introduced to the homogeneous waveguide one after the other. In step one the field $\vec{E}^{h}$ of a homogeneous waveguide, which is a fiber SMF28 placed in some environmental media, is calculated. In step two a grating is placed in the core; electric

field from the previous step is used to calculate an equivalent current density due to the grating,

$$
\vec{J}_{g r}^{e q,(0)}=-i \omega \Delta \varepsilon_{g r} \vec{E}^{h}
$$


Solving wave equation with the source term $\overrightarrow{J_{g r}^{e q}(0)}$ gives a scattered field due to grating, $\vec{E}_{g r}^{s,(1)}$. Solution, obtained by inverse Fourier transform integral 5.12, can be brought in the following form,

$$
\vec{E}_{g r}^{s,(1)}(r, \theta, z)=\vec{R}_{g r}^{s,(1)}(r) e^{i v_{0} z} e^{i m_{0} \theta}
$$

where $v_{0}, m_{0}$ is a propagation constant and azimuthal index of some cladding mode excited by a fiber Bragg grating.

In step three a metal coating in a form of certain pattern deposited on a fiber; an equivalent current density due to a metal distribution is calculated utilizing scattered field due to a grating found in previous step,

$$
\vec{J}_{m}^{e q,(0)}=-i \omega \Delta \varepsilon_{m} \vec{E}_{g r}^{s,(1)}
$$

Finding scattered fields when metal distribution and Bragg grating both present in the waveguide, is the goal which involves solving wave equation with both source terms, $\overrightarrow{J_{g r}^{e q},(0)}$ due to grating in the fiber core and $\overrightarrow{J_{m}^{e q},(0)}$ due to a metal distribution in the outer layer.

A core-cladding interface, existing in step one, is removed in steps two and three to simplify the calculations. Schematic diagram of these steps illustrated in Fig. 6.4. An equivalent current source due to a metal distribution is the source term in a wave equation and therefore it is treated as an impressed current source, i.e. driven externally. However metal coating also associated with induced current. Since metal has electrons in a conduction band, a metal coating of the waveguide has free charge density source $\rho_{f}$ and induced current density source $\vec{J}_{\text {ind }}$ which is related to the field in the waveguide through conductivity, $\vec{J}_{i n d}=\sigma \vec{E}$. The presence of free charge density $\rho_{f}$ and induced current density $\vec{J}_{\text {ind }}$ is taken into account by allowing an imaginary part of a metal permittivity,

$$
\varepsilon_{m}=\varepsilon_{m, \operatorname{Re}}-\frac{\sigma}{i \omega}
$$

For a metal distribution in the form of set of patches periodic in $z$-axis the permittivity difference is described by,

$$
\Delta \varepsilon_{m}=\left(\varepsilon_{3}-\varepsilon_{4}\right) \Pi\left(r, r_{2}, r_{3}\right) \Pi\left(\theta, \theta_{1}, \theta_{2}\right) \sum_{j=-N}^{N} \Pi\left(z, z_{1}+j \Delta, z_{2}+j \Delta\right)
$$

Such set of patches can be converted to different geometrical structures, displayed in Fig. 6.5, by turning its parameters:

to a set of coils, by letting $\theta_{1}=-\pi, \theta_{2}=\pi$,

to a continuous coating of finite length (tube like), by letting $\theta_{1}=-\pi, \theta_{2}=\pi, z_{2} \rightarrow z_{1}+\Delta$, to a strip of finite length, by letting $z_{2} \rightarrow z_{1}+\Delta$,

to a set of patches with different aspect ratio $\left(z_{2}-z_{1}\right) / \Delta$, by letting $z_{2}=z_{1}+\Delta-\delta$ or $z_{2}=z_{1}+\delta$ with $0<\delta \ll 1$. 
I

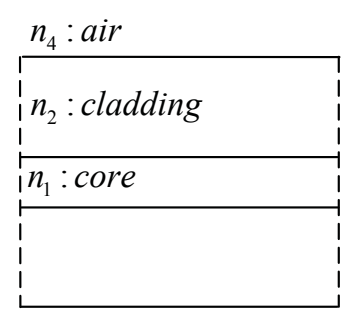

$\vec{E}^{h}$
II

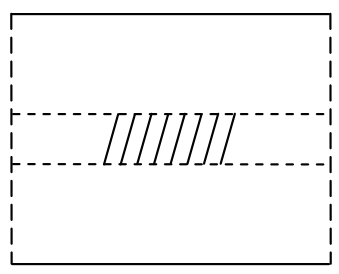

$$
\begin{gathered}
\vec{J}_{2}^{e q,(0)}=-i \omega \Delta \varepsilon_{g r} \vec{E}^{h} \\
\vec{E}_{g r}^{S,(1)}
\end{gathered}
$$

III

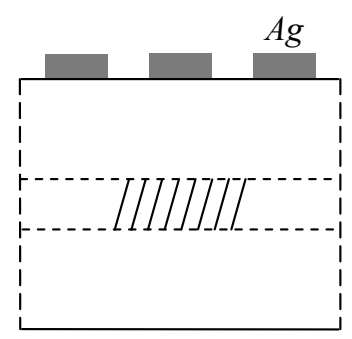

$\vec{J}_{2}^{e q,(0)}=-i \omega \Delta \varepsilon_{g r} \vec{E}^{h}$

$\vec{J}_{4}^{e q,(0)}=-i \omega \Delta \varepsilon_{m} \vec{E}_{g r, 4}^{S,(1)}$

$\vec{E}_{g r, m}^{S,(1)}$

Figure 6.4: Inhomogenuity of the fiber core (Bragg grating) and of metal distribution (structured coating) are introduced in an originally homogeneous cylindrical waveguide.
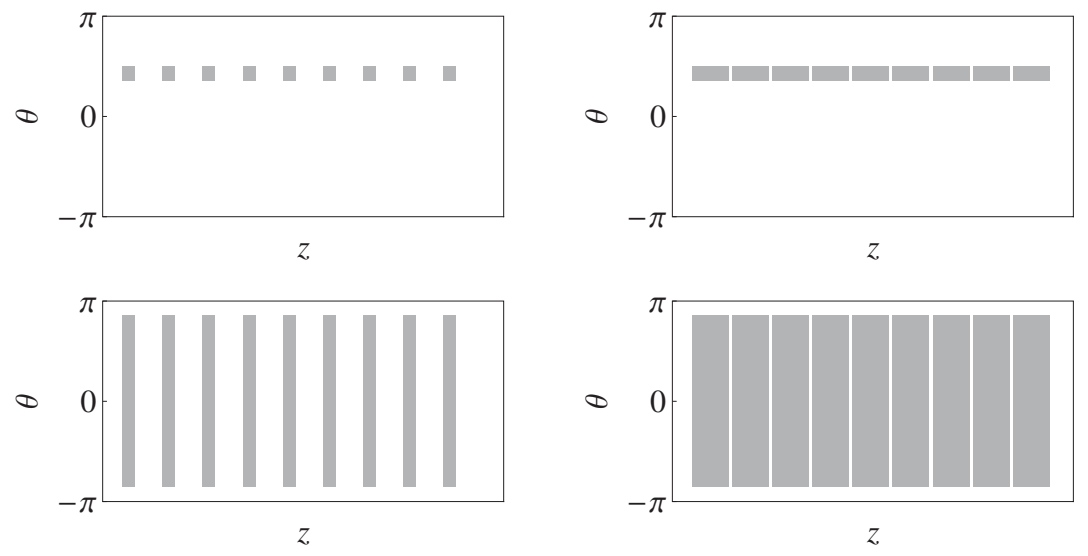

Figure 6.5: Geometry of metal distribution on a fiber with different tuning parameters of a distribution. Top left: set of patches; top right: distribution approaches to a strip like; bottom left: distribution approaches to a set of coils; bottom right: distribution approaches to a tube like.

The Fourier transform of $z$-component of equivalent current density due to metal distribution then becomes,

$$
j_{m, z}^{(0)}=-i \omega R_{g r, z}^{s,(1)}(r)\left(\varepsilon_{3}-\varepsilon_{4}\right) \Pi\left(r, r_{2}, r_{3}\right) \eta_{1} \eta_{2} \eta_{3}
$$


where,

$$
\begin{gathered}
\eta_{1}=\left(\frac{e^{-i\left(m-m_{0}\right) \theta_{2}}-e^{-i\left(m-m_{0}\right) \theta_{1}}}{m-m_{0}}\right) \\
\eta_{2}=(-1)\left(\frac{e^{-i\left(v-v_{0}\right) z_{2}}-e^{-i\left(v-v_{0}\right) z_{1}}}{v-v_{0}}\right) \\
\eta_{3}=\left(\frac{e^{-i\left(v-v_{0}\right)(1-N) \Delta}-e^{-i\left(v-v_{0}\right) N \Delta}}{1-e^{-i\left(v-v_{0}\right) \Delta}}\right)
\end{gathered}
$$

Expressions for $j_{m,+}^{(0)}, j_{m,-}^{(0)}$ are obtained from expression for $j_{m, z}^{(0)} 6.25$ with $R_{g r, z}^{s,(1)}$ replaced by $R_{g r,+}^{s,(1)}, R_{g r,-}^{s,(1)}$ respectively.

Corresponding vector functions $\vec{f}_{2}$ for cladding (layer 2) and $\vec{f}_{4}$ for outer layer (layer 4 ) obtained with $j_{g r, z}^{(0)}, j_{g r,+}^{(0)}, \quad j_{g r,-}^{(0)}$ and $j_{m, z}^{(0)}, j_{m,+}^{(0)}, j_{m,-}^{(0)}$ respectively through integrals 5.31, 5.27 and 5.30. With known $\vec{f}_{g r}$ and $\vec{f}_{m}$ the primary fields $\left(\vec{e}_{g r}^{p}, \vec{h}_{g r}^{p}\right),\left(\vec{e}_{m}^{p}, \vec{h}_{m}^{p}\right)$ evaluated with $5.17,5.35$. Power carried along axis in outer layer by a field scattered off air-cladding boundary evaluated with 5.70 .

Evaluation of a power in outer layer carried by primary fields requires evaluation of inverse Fourier transform integrals of the primary fields. Only part of the field due to a scattering off air-cladding interface is calculated, another part is a primary field due to equivalent current source of metal patches is present, its evaluation requires a Fourier transform which can be done numerically via fast Fourier transform, FFT procedure. Analytical evaluation is not available because integrand is too complicated. Since metal distribution is not part of a homogeneous waveguide any more, a plasmon can not be considered as a mode of a homogeneous waveguide.

\subsection{Simulation results and discussion.}

Having set a calculation model and its code for plasmon excitation by tilted Bragg grating in a fiber, its response to excitation and waveguide parameters can be examined. Contribution from DC component of the grating $\Delta \varepsilon_{D C}$ on scattered fields has a factor sinc $\left(\left(m-m_{c}\right) \pi\right)$, therefore for the cases of azimuthal indices $m$ other than $m_{c}$ ( with $\left.m_{c}= \pm 1\right)$ such contribution reduces to identical zero. Since azimuthal indices considered here are greater than 1 , in the results below DC component $\Delta \varepsilon_{D C}$ hasn't played any role. Power of excited plasmon mode is not a monotonic function in either argument: layer thickness, wavelength or tilt angle in the window parameters given, therefore each mode can be tuned-up to its optimal parameters that provide either local minima or local maxima of the mode's power. Power variation carried by a plasmon mode for the case of infinite metal coating versus wavelength and tilt angle is presented in Fig. 6.6-Fig. 6.9, Fig. 6.11 and versus thickness and tilt angle in Fig. 6.13. The impact of grating type, uniform versus $\pi$-shifted, can be seen by comparing Fig. 6.6 and Fig. 6.7 where the plots of power carried by plasmon mode excited with grating uniform and phase shifted grating respectively are 


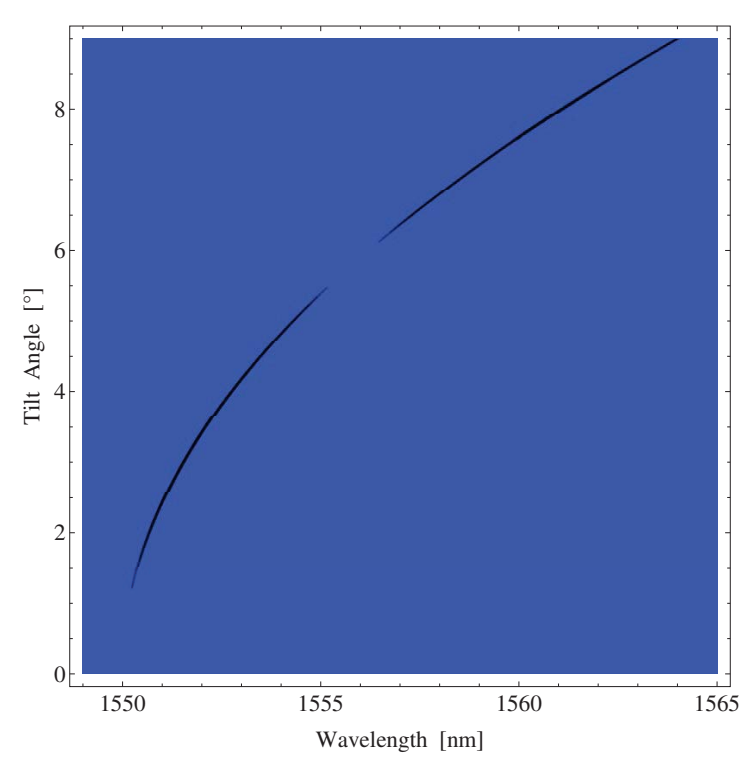

(a)

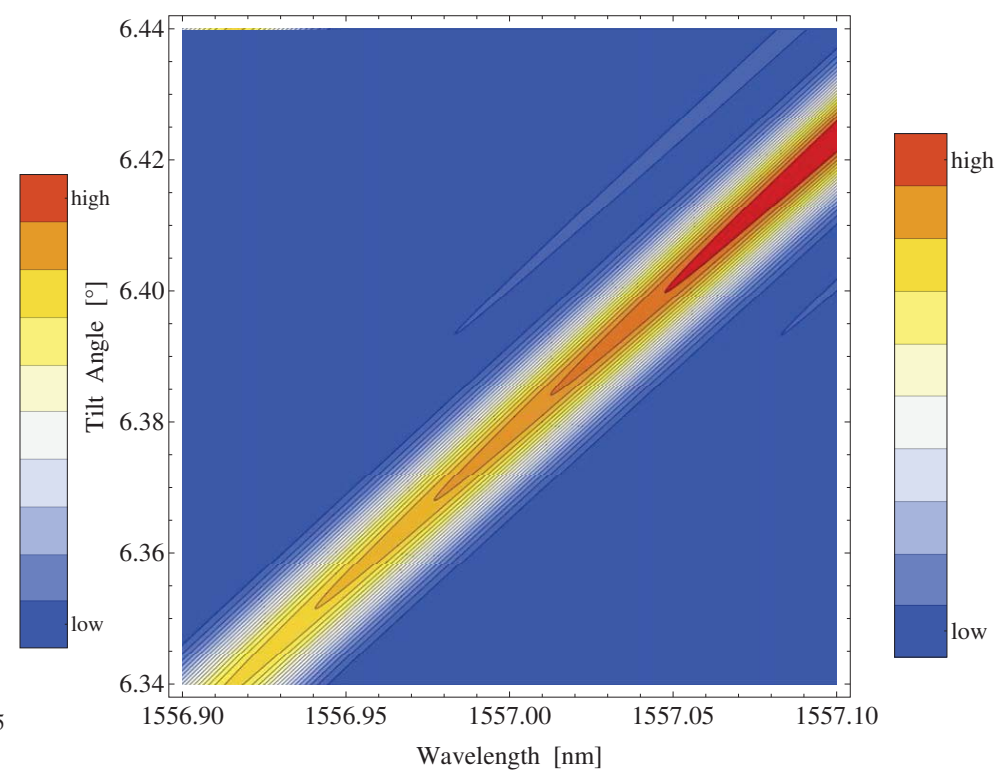

(b)

Figure 6.6: Contour plot of power carried by plasmon mode excited by a uniform tilted grating in a fiber coated by thin Au layer positioned in air environment versus wavelength and tilt angle. Simulation parameters: azimuthal index of the mode $m=3$, layer thickness $d=35 \mathrm{~nm}$, grating is tuned for coupling plasmon mode and core mode at resonance wavelength $\lambda_{\text {res }}=1550 \mathrm{~nm}$, effective index $n_{\text {ef } 1}$ shown in Fig. 6.10. Computation window

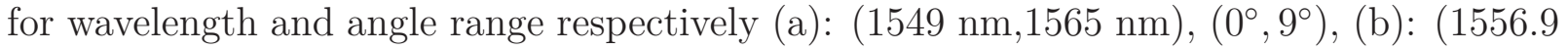
$\mathrm{nm}, 1557.1 \mathrm{~nm}),\left(6.34^{\circ}, 6.44^{\circ}\right)$.

shown. As expected, the plasmon peak observed with uniform grating (Fig. 6.6) is split in two halves when $\pi$-shift is introduced in the grating (Fig. 6.7). The dip between two halves of the peak runs all the way along curved line of the peak.

The depth of the dip, which is due to a $\pi$-shift and which separates a power peak in the middle, is controlled by imaginary part of effective index. This can be seen by comparing Fig. 6.7 and Fig. 6.8 where two modes with a slightly different imaginary part of effective index are excited. The mode with larger imaginary part has slightly shallower dip of the peak. Replacing coating layer from Au to platinum Pt whose imaginary part of refractive index (whose loss, damping rate) considerably larger than of $\mathrm{Au}$, allows excite the modes with quite a large imaginary part of effective index. As a result, the dip of the peak almost vanishes and the difference between uniform and $\pi$-shifted grating essentially disappears. Plot of power carried by a plasmon mode with Pt coating excited by uniform and $\pi$-shifted grating is shown in Fig. 6.9. Effective indices of modes with Au coating, (two modes), and with Pt coating (one mode) versus wavelength are shown in Fig. 6.10.

It seems the azimuthal index $m$ is coupled with the tilt angle $\alpha$ in the sense, that with larger $m$ the maximum power of plasmon mode shifted towards larger wavelength and larger tilt angle, as seen in Fg. 6.11.

Modifying imaginary part of effective index can be done by tuning coating thickness. 


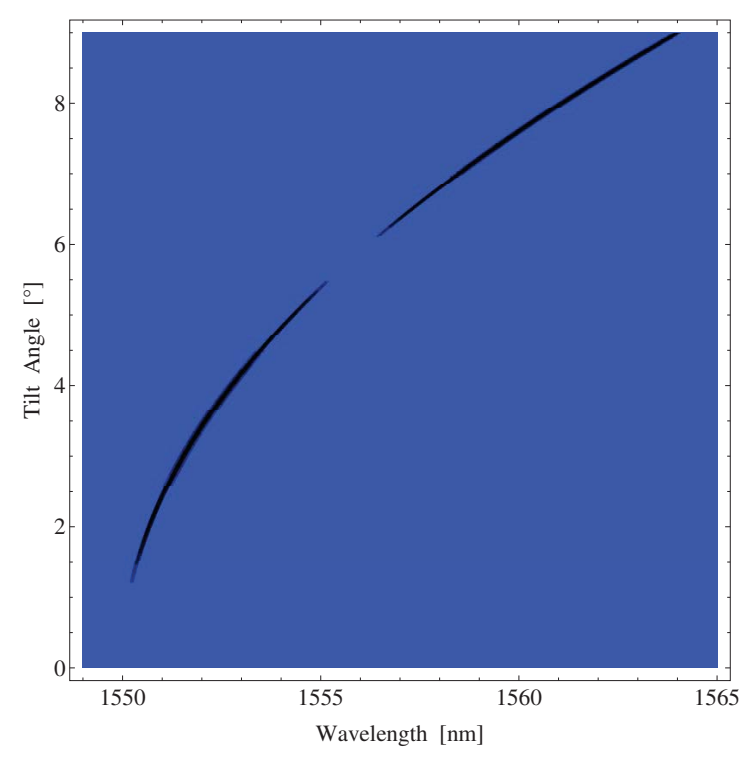

(a)

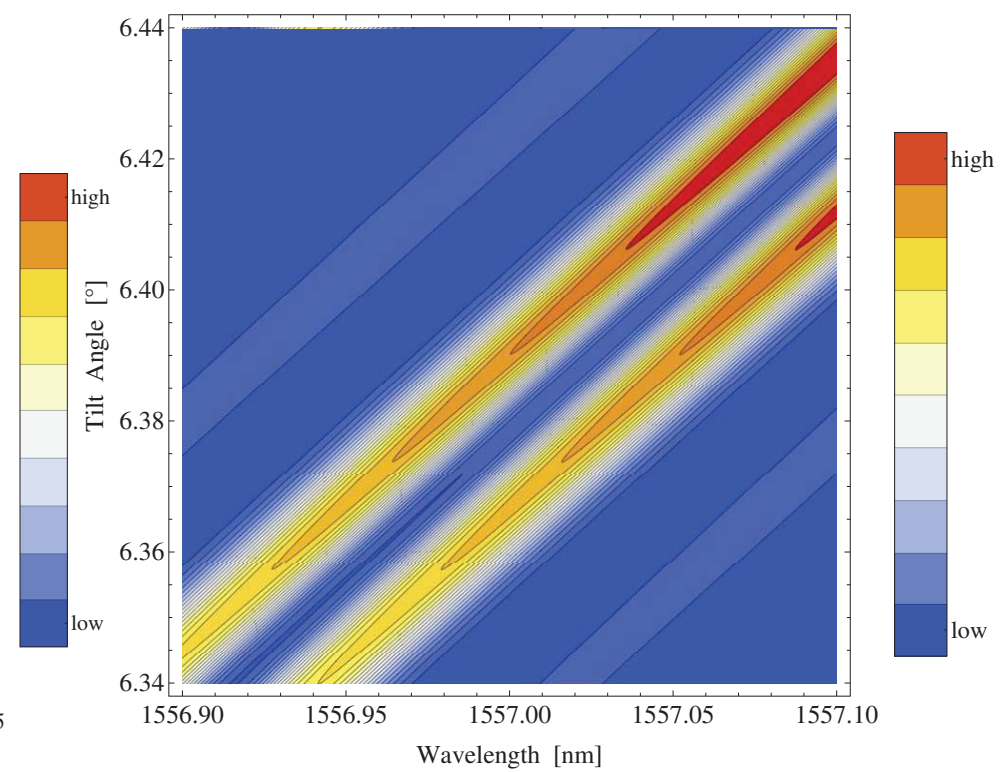

(b)

Figure 6.7: Contour plot of power carried by plasmon mode excited by a $\pi$-shifted tilted grating in a fiber coated by thin Au layer positioned in air environment versus wavelength and tilt angle. Simulation parameters: azimuthal index of the mode $m=3$, layer thickness $d=35 \mathrm{~nm}$, grating is tuned for coupling plasmon mode and core mode at resonance wavelength $\lambda_{\text {res }}=1550 \mathrm{~nm}$, effective index $n_{\text {ef } 1}$ shown in Fig. 6.10. Computation window for wavelength and angle range respectively (a): $(1549 \mathrm{~nm}, 1565 \mathrm{~nm}),\left(0^{\circ}, 9^{\circ}\right),(\mathrm{b}):(1556.9$ $\mathrm{nm}, 1557.1 \mathrm{~nm}),\left(6.34^{\circ}, 6.44^{\circ}\right)$.

Example of effective index versus thickness is shown in Fig. 6.12.

Plot of power dependence versus metal thickness and tilt angle presented in Fig. 6.13.

Power variation carried by a plasmon for the case of structured metal coating versus tilted angle and wavelength is presented in Fig. 6.14-Fig. 6.21. Plasmon for the case of infinite metal coating is a waveguide's mode, whereas now it is just a perturbation of some cladding modes since metal coating is a discrete finite distribution rather than an infinite uniform film. Tuning parameters of metal distribution: patch length $\delta z$, array period $\Delta z$, array length $2 z_{0}$ changes geometrical pattern of the coating as seen in the Fig. 6.5 which eventually should lead to a certain redistribution of the plasmon power in $(\lambda, \alpha)$-space. This is seen from Fig. 6.14-Fig. 6.21 where plasmon power computed for several distinct cases. Such power redistribution can be found useful in some sensor like application. 


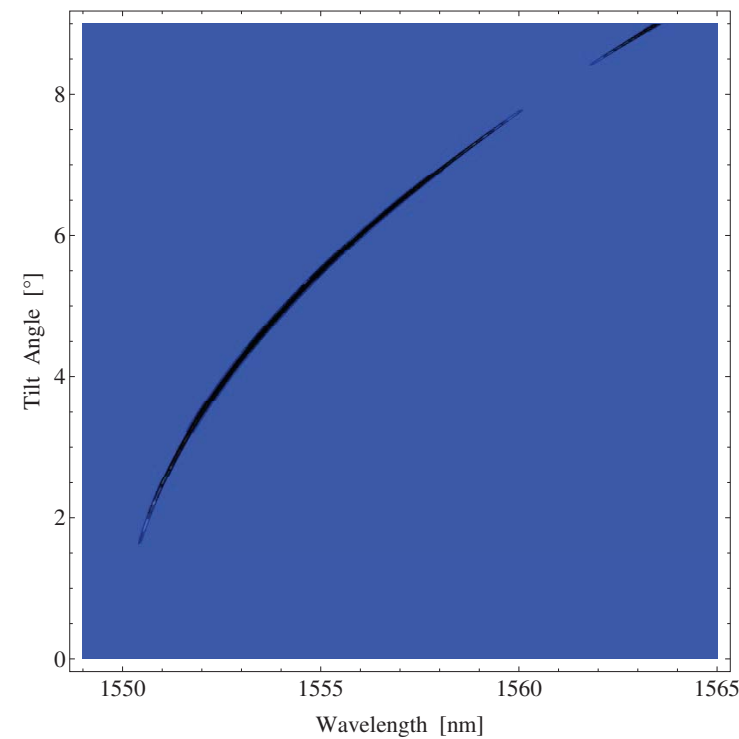

(a)

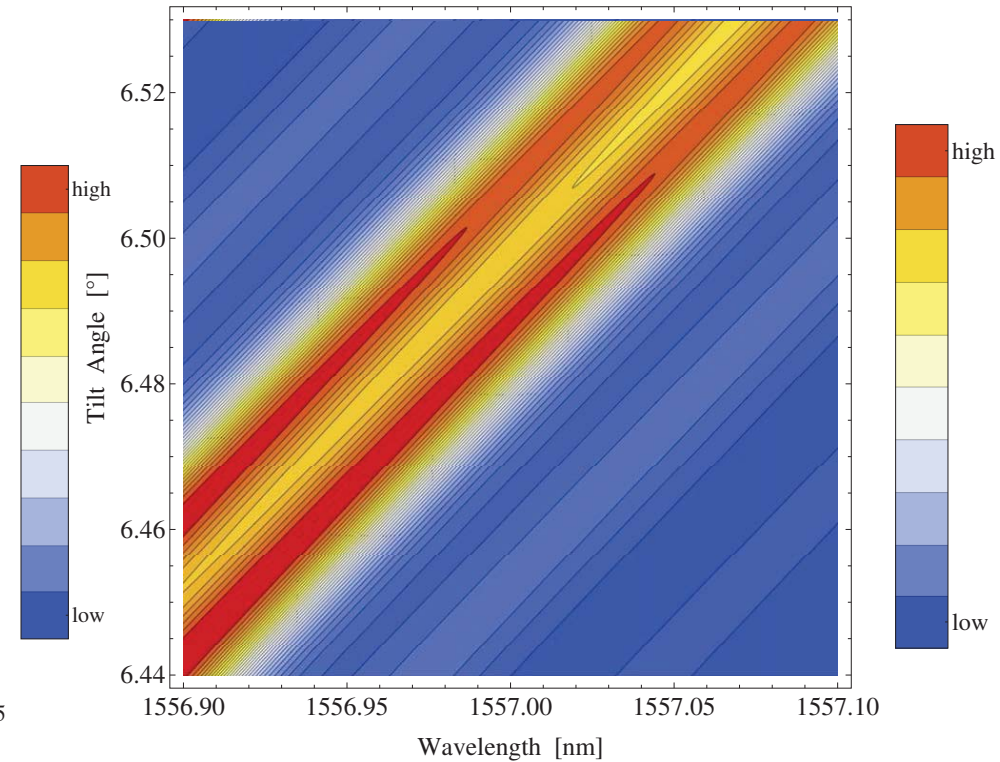

(b)

Figure 6.8: Contour plot of power carried by plasmon mode excited by a $\pi$-shifted tilted grating in a fiber coated by thin Au layer positioned in air environment versus wavelength and tilt angle. Simulation parameters: azimuthal index of the mode $m=3$, layer thickness $d=35 \mathrm{~nm}$, grating is tuned for coupling plasmon mode and core mode at resonance wavelength $\lambda_{\text {res }}=1550 \mathrm{~nm}$, effective index $n_{\text {ef2 }}$ shown in Fig. 6.10. Computation window for wavelength and angle range respectively (a): $(1549 \mathrm{~nm}, 1565 \mathrm{~nm}),\left(0^{\circ}, 9^{\circ}\right),(\mathrm{b}):(1556.9$ $\mathrm{nm}, 1557.1 \mathrm{~nm}),\left(6.34^{\circ}, 6.44^{\circ}\right)$. 


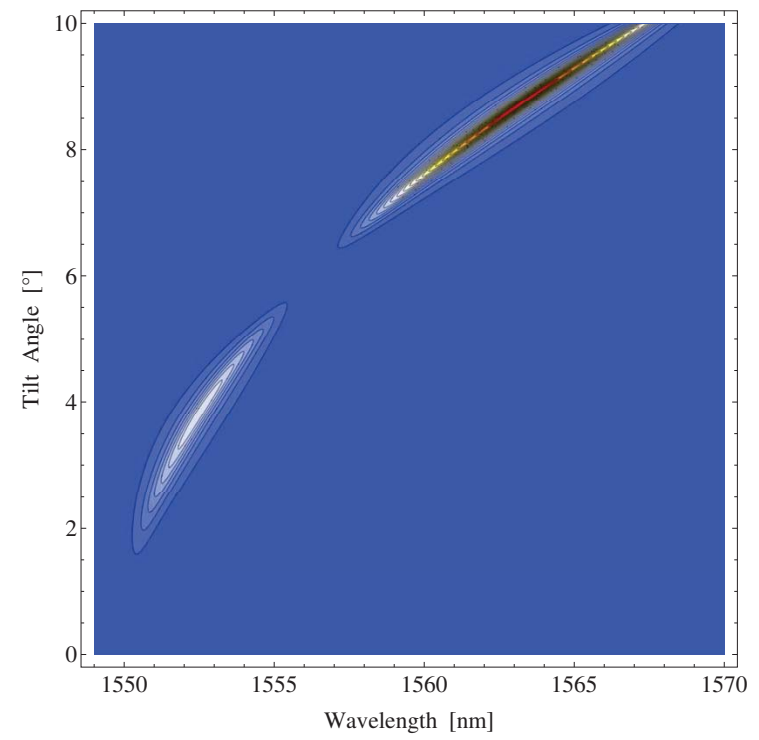

(a)

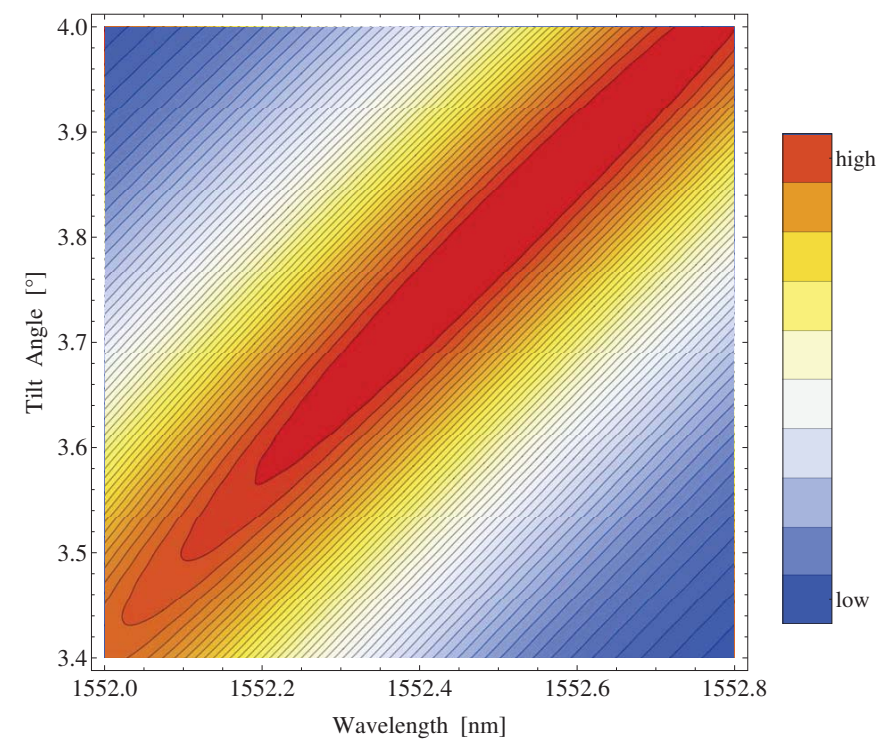

(c)

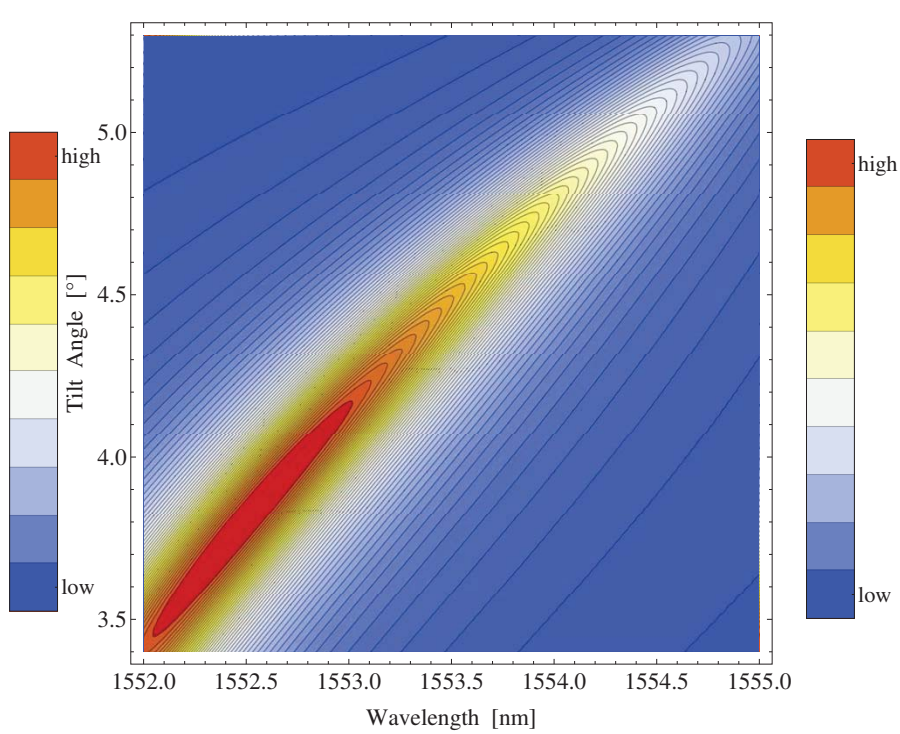

(b)

Figure 6.9: Contour plot of power carried by plasmon mode excited by a $\pi$-shifted tilted grating in a fiber coated by thin Pt layer positioned in air environment versus wavelength and tilt angle. Simulation parameters: azimuthal index of the mode $m=3$, layer thickness $d=35 \mathrm{~nm}$, grating is tuned for coupling plasmon mode and core mode at resonance wavelength $\lambda_{\text {res }}=1550 \mathrm{~nm}$, effective index $n_{\text {ef3 }}$ shown in Fig. 6.10. Computation window for wavelength and angle range respectively (a): $(1549 \mathrm{~nm}, 1570 \mathrm{~nm}),\left(0^{\circ}, 10^{\circ}\right),(\mathrm{b}):(1552$ $\mathrm{nm}, 1555 \mathrm{~nm}),\left(3.4^{\circ}, 5.3^{\circ}\right)$, (c): (1552 nm,1552.8 nm), $\left(3.4^{\circ}, 4.4^{\circ}\right)$. 


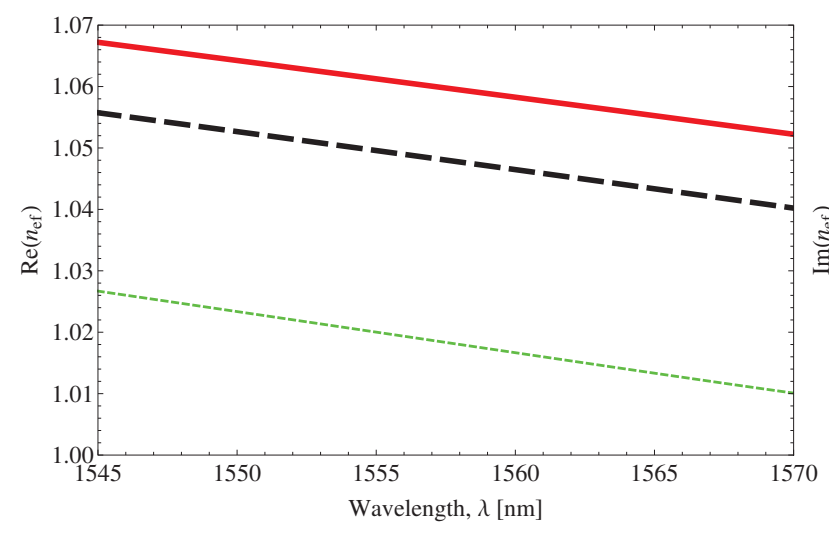

(a)

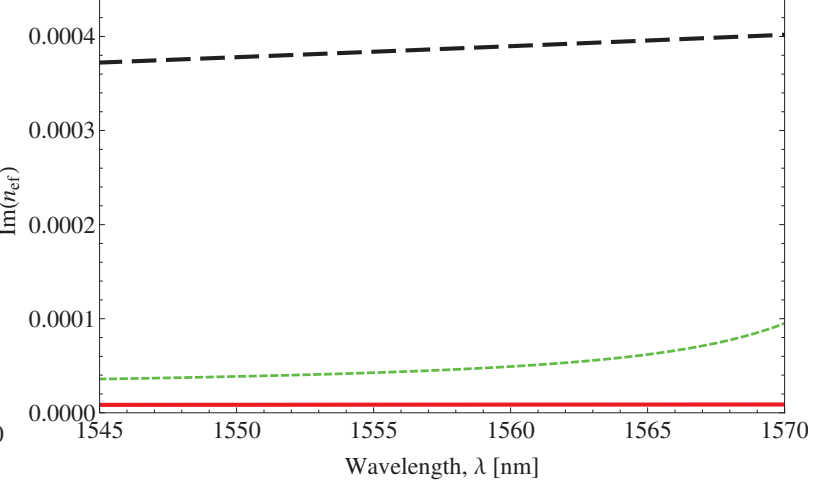

(b)

Figure 6.10: Plot of effective indices for plasmon modes excited by a $\pi$-shifted array of tilted discs of current in the core of a fiber coated by thin metal layer positioned in air environment versus wavelength. Simulation parameters: azimuthal index of the mode $m=3$, layer thickness $d=35 \mathrm{~nm}$, grating is tuned for coupling plasmon mode and core mode at resonance wavelength $\lambda_{\text {res }}=1550 \mathrm{~nm}$. Effective indices as follows: solid line $n_{e f 1}$ (Au coating), short dashed line $n_{\text {ef } 2}$ (Au coating), large dashed line $n_{\text {ef3 }}$ (Pt coating). (a): real parts, (b): imaginary parts. 


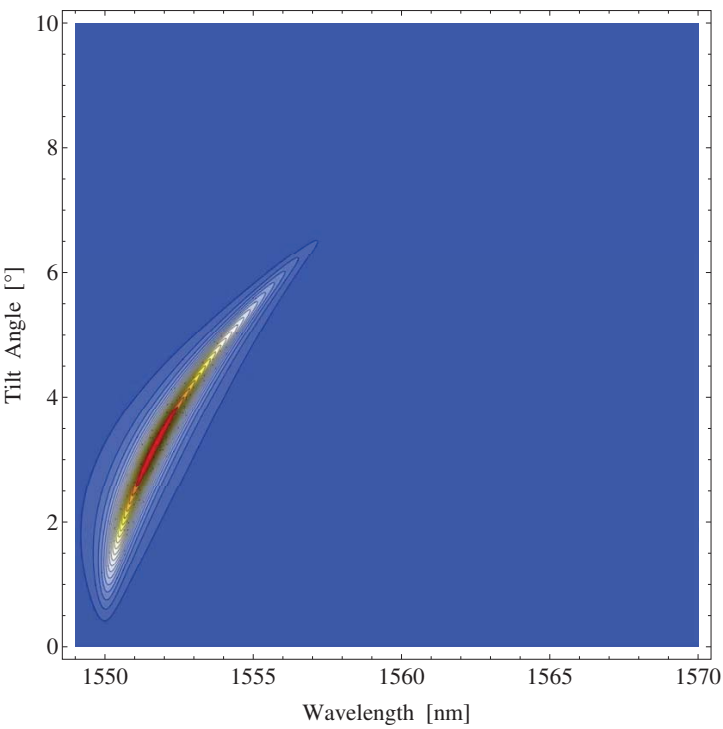

(a)

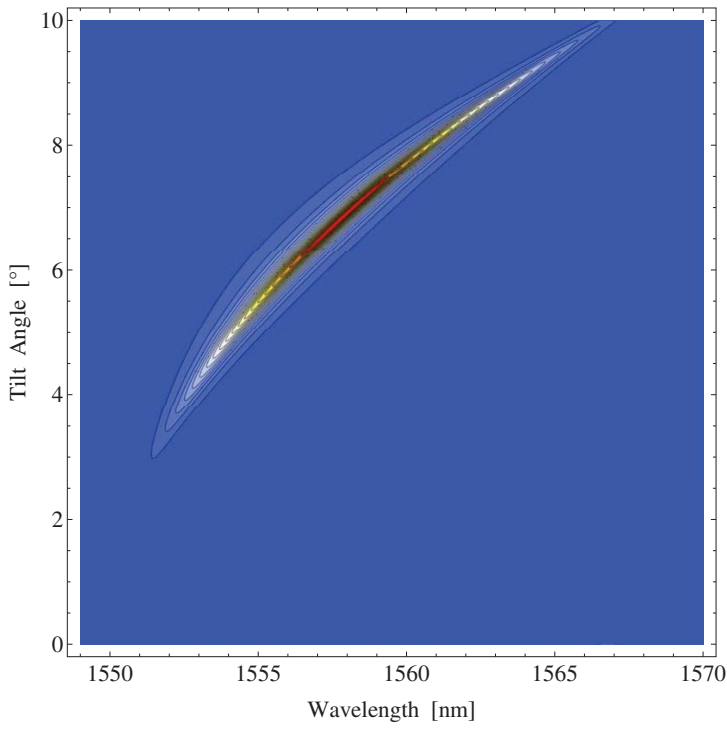

(c)

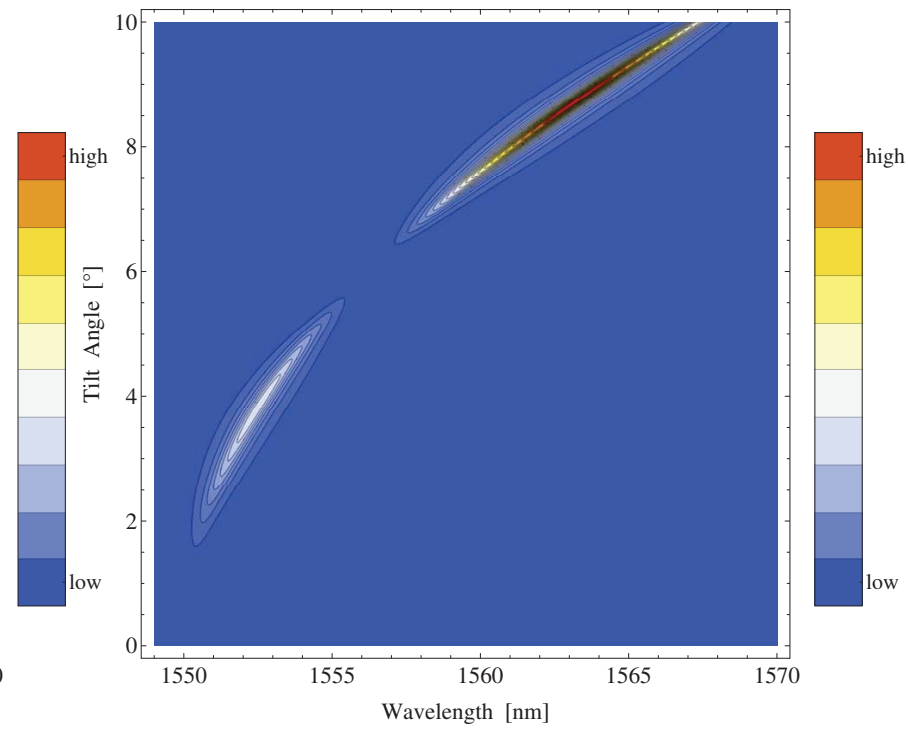

(b)

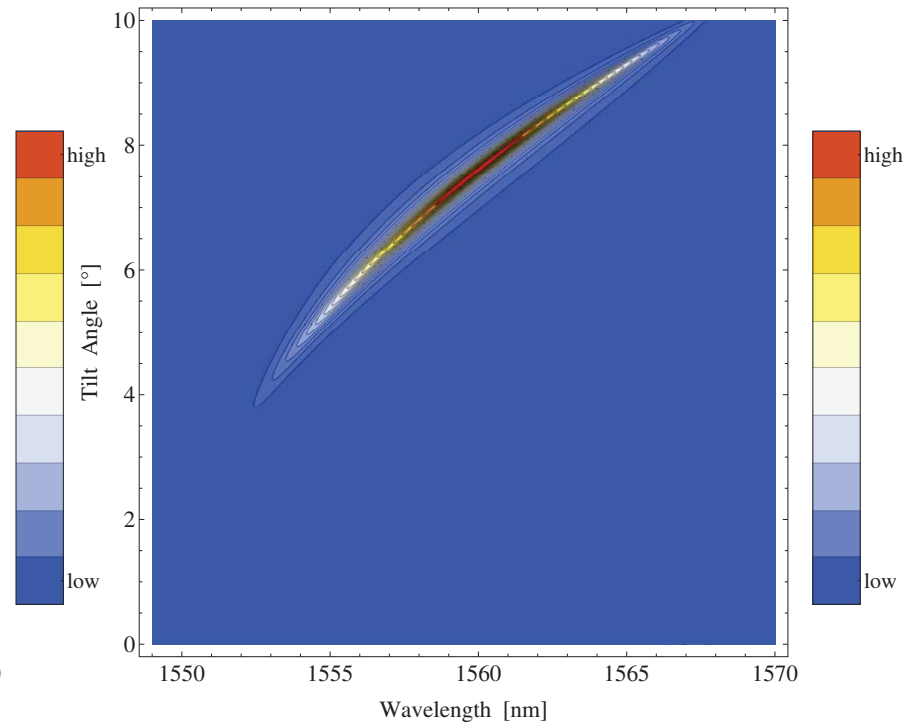

(d)

Figure 6.11: Contour plot of power carried by plasmon mode excited by a $\pi$-shifted tilted grating in a fiber coated by thin Pt layer positioned in air environment versus wavelength and tilt angle. Simulation parameters: azimuthal index of the mode $m$, layer thickness $d=35 \mathrm{~nm}$, grating is tuned for coupling plasmon mode and core mode at resonance wavelength $\lambda_{\text {res }}=1550 \mathrm{~nm}$. Azimuthal indices: (a) $m=2$, (b) $m=3$, (c) $m=4$, (d) $m=5$. 


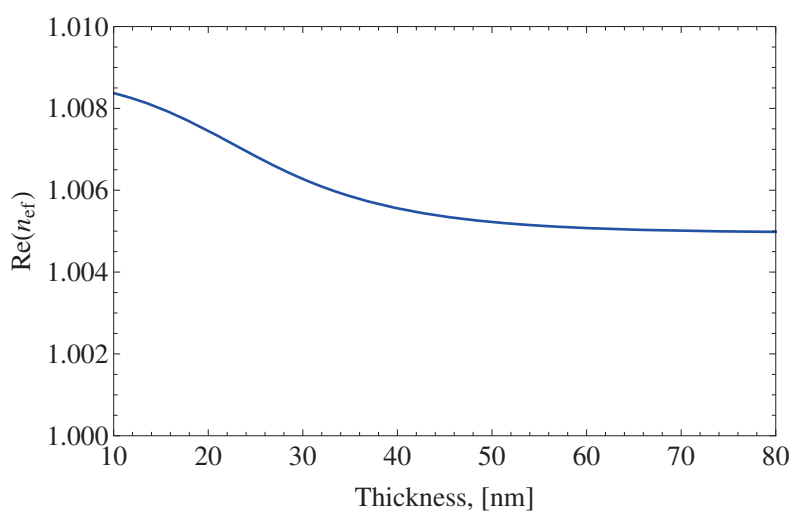

(a)

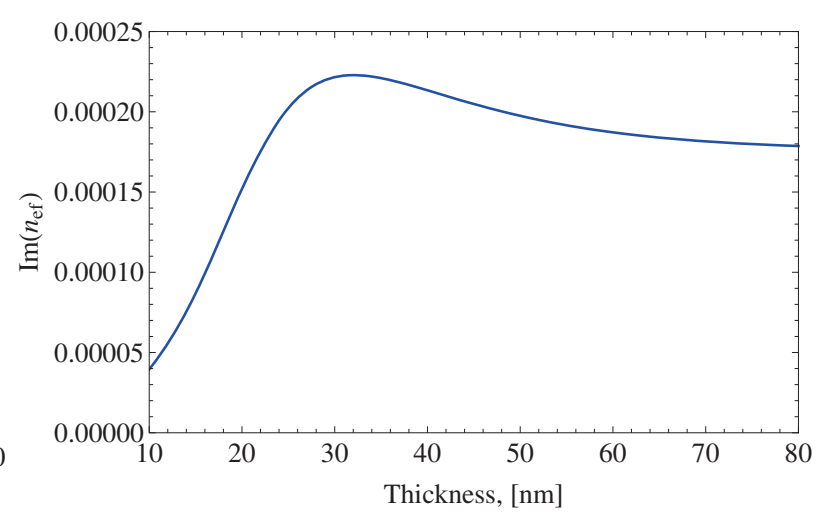

(b)

Figure 6.12: Plot of effective index for plasmon mode excited by a $\pi$-shifted tilted grating in a fiber coated by Au layer positioned in air environment versus thickness. Simulation parameters: azimuthal index of the mode $m=3$, grating is tuned for coupling plasmon mode (calculated with Au thickness $d_{\text {res }}=30 \mathrm{~nm}$ ) and core mode at resonance wavelength $\lambda_{\text {res }}=1550 \mathrm{~nm}$. (a): real parts, (b): imaginary parts.

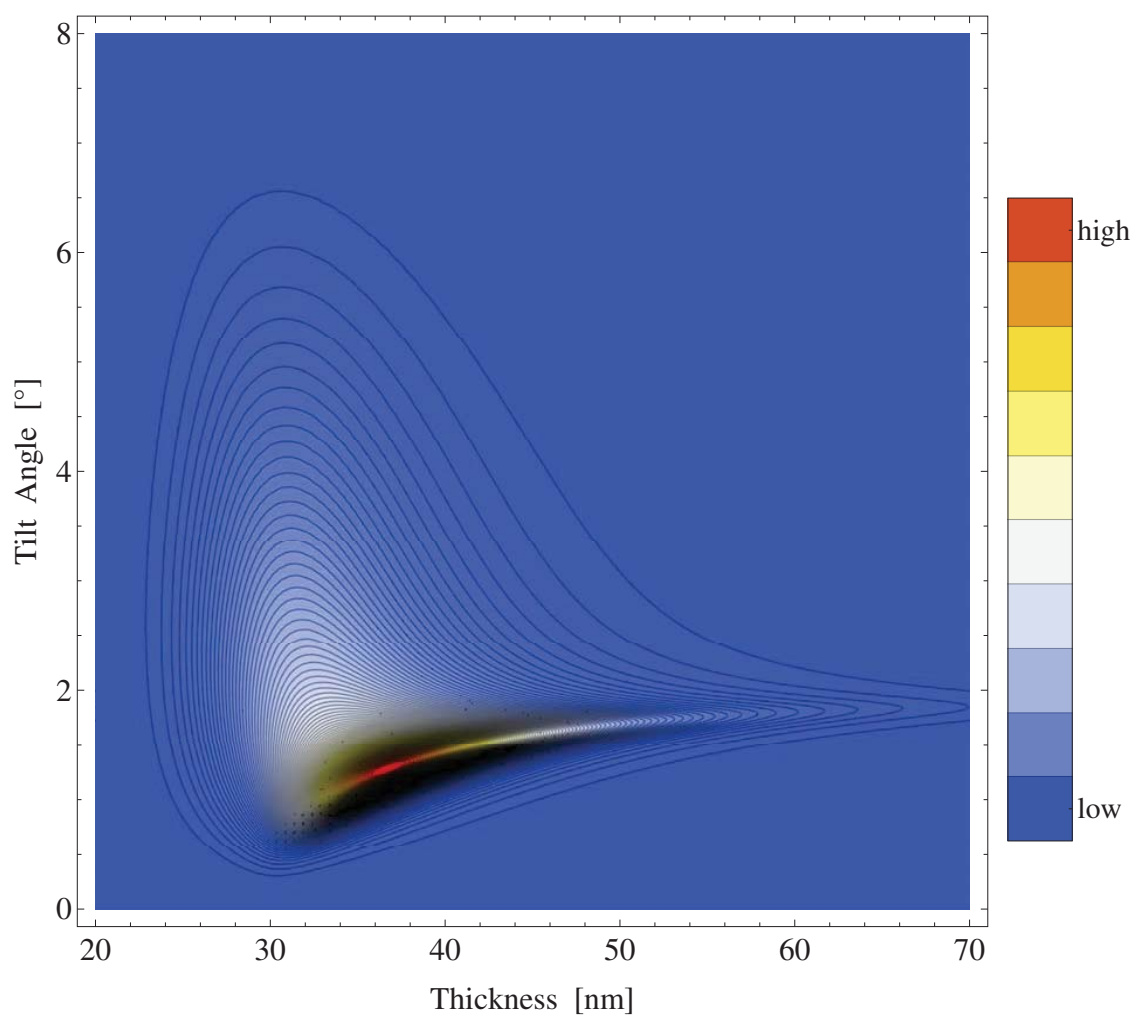

Figure 6.13: Contour plot of power carried by plasmon mode excited by a $\pi$-shifted tilted grating in a fiber coated by thin Au layer positioned in air environment versus coating thickness and tilt angle. Simulation parameters: azimuthal index of the mode $m=3$, wavelength $\lambda=1550 \mathrm{~nm}$, grating is tuned for coupling plasmon mode and core mode at resonance wavelength $\lambda_{\text {res }}=1550 \mathrm{~nm}$, effective index shown in Fig. 6.12. 


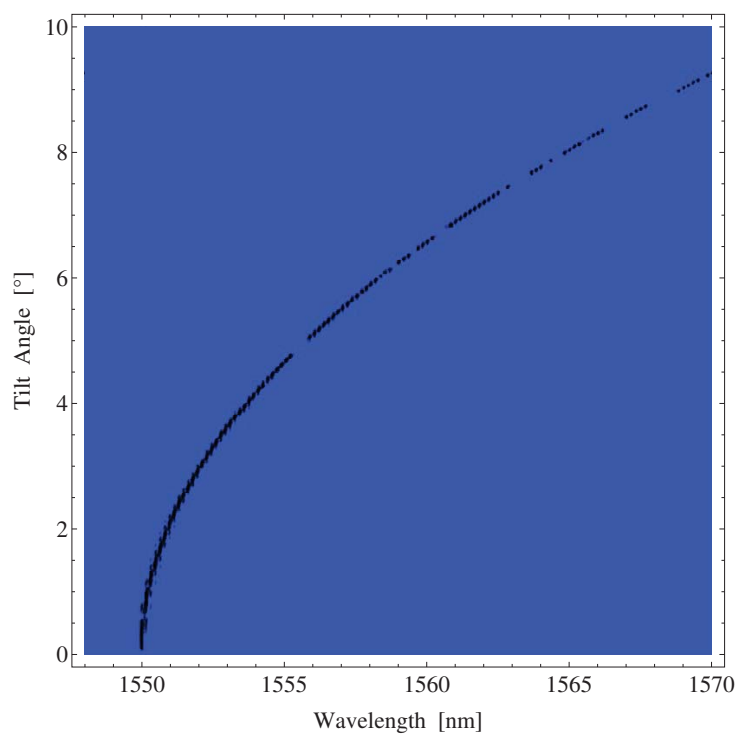

(a) $\pi$-shifted

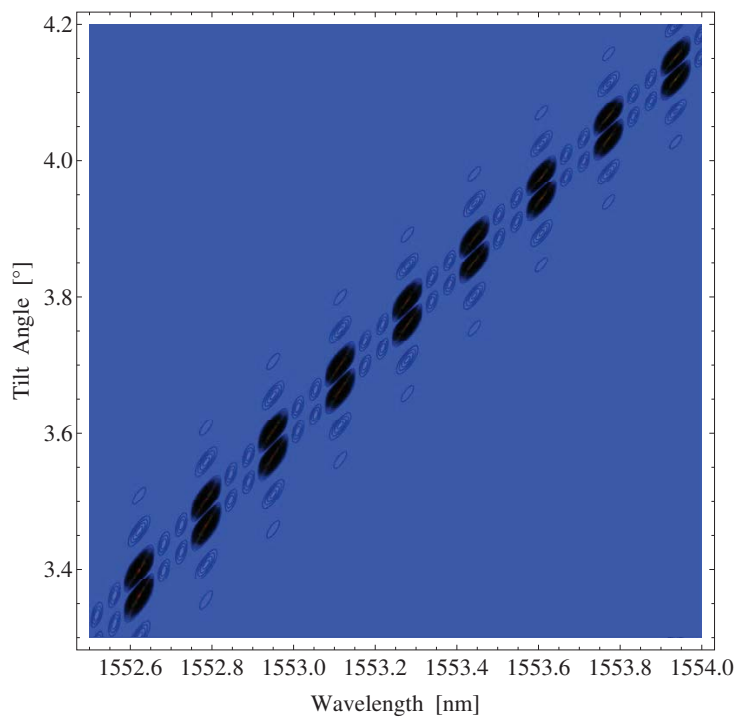

(c) $\pi$-shifted

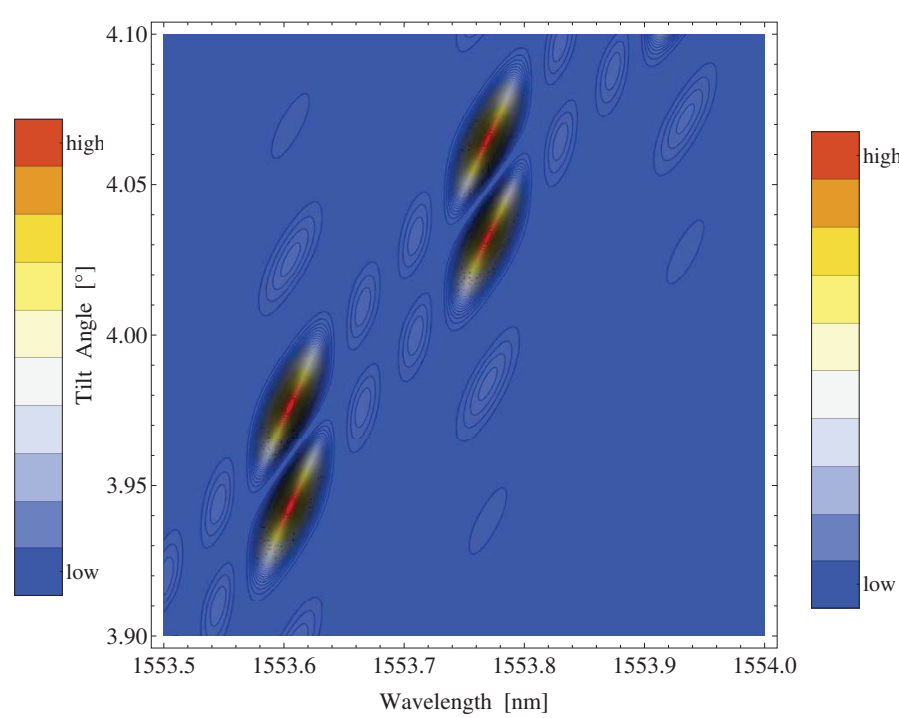

(b) $\pi$-shifted

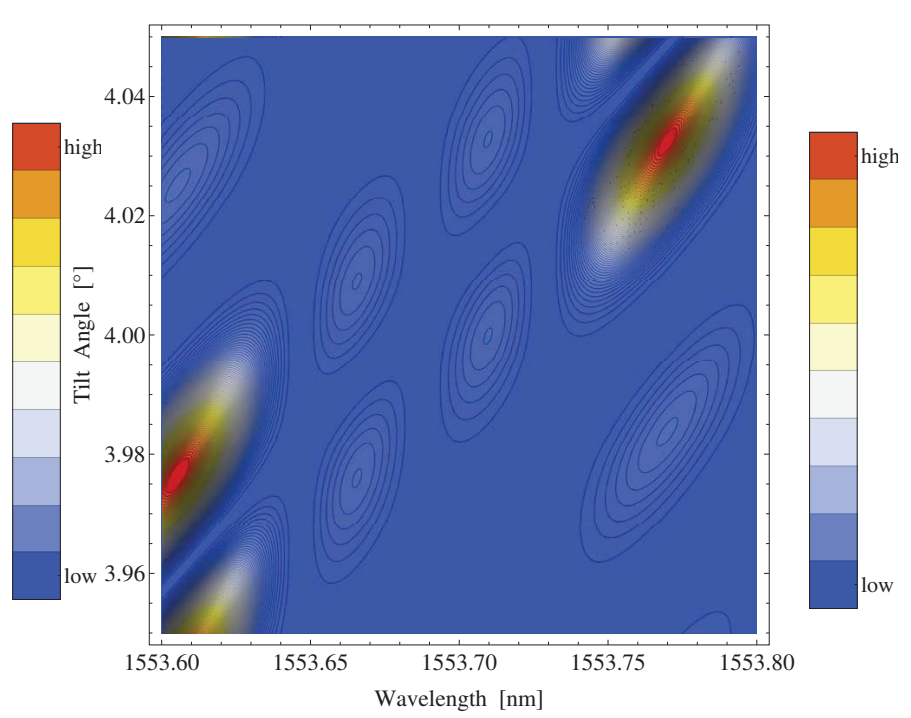

(d) $\pi$-shifted 


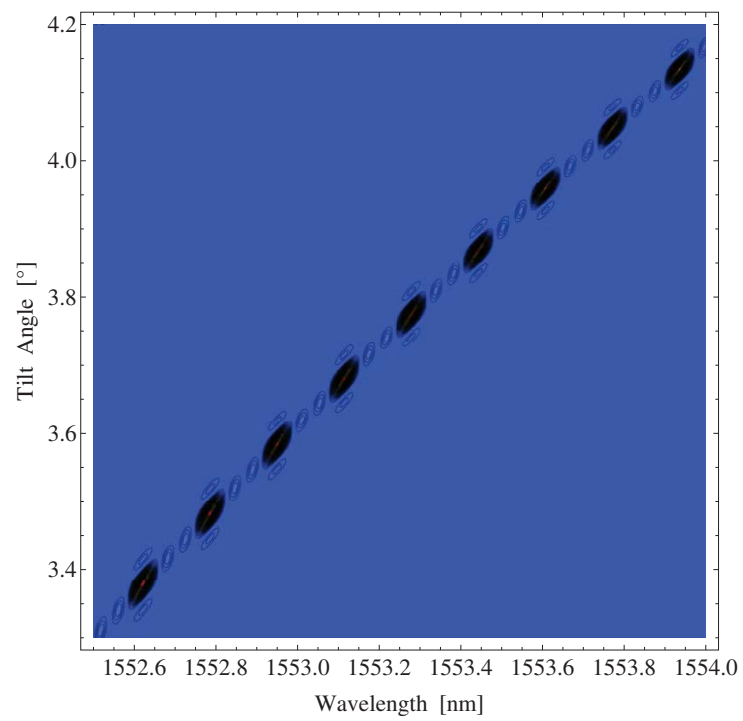

(e) uniform

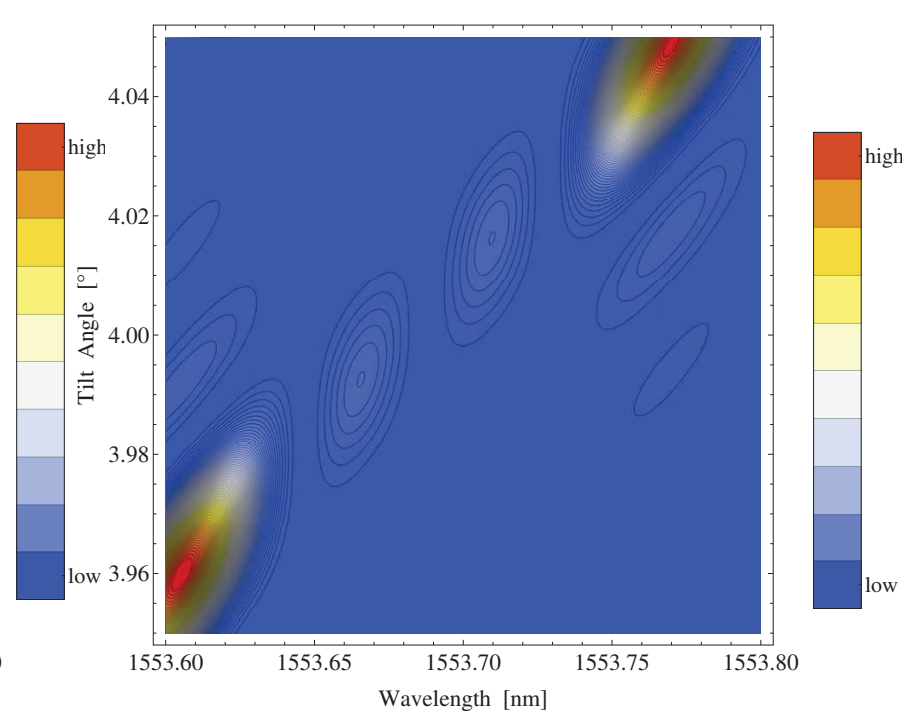

(f) uniform

Figure 6.14: Contour plot of power carried by plasmon excited by a (uniform and $\pi$-shifted) tilted grating in a fiber coated by Au distribution positioned in an index matching oil (with refractive index $n_{4}=1.42$ ) environment versus wavelength and tilt angle. Simulation parameters: azimuthal index of the mode $m=3$; wavelength $\lambda=1550 \mathrm{~nm}$; grating is tuned for coupling plasmon mode and core mode at resonance wavelength $\lambda_{\text {res }}=1550 \mathrm{~nm}$; thickness of $\mathrm{Au}$ distribution $d=100 \mathrm{~nm}$; angular width of distribution $\left(\theta_{\min }, \theta_{\max }\right)$ : $\theta_{\min }=-\pi / 10, \theta_{\max }=\pi / 10$, array length $2 z_{0}=2 \mathrm{~cm}$; distribution type: uniform array of patches; AC grating $\Delta \varepsilon_{A C}=3 \times 10^{-4}$. Au distribution parameters: $\delta z=50 \mathrm{~nm}$, $\Delta z=5000 \mu m$. 


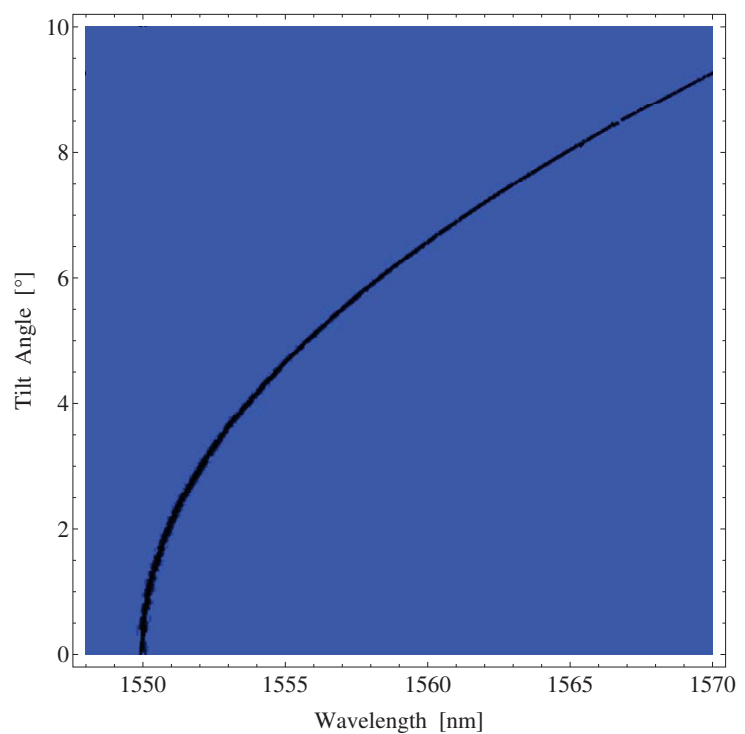

(a) $\pi$-shifted

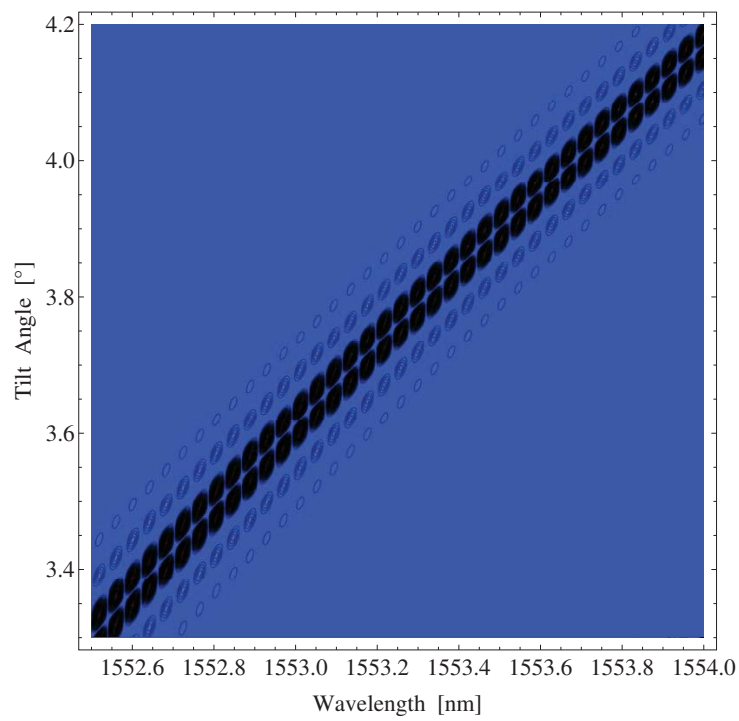

(c) $\pi$-shifted

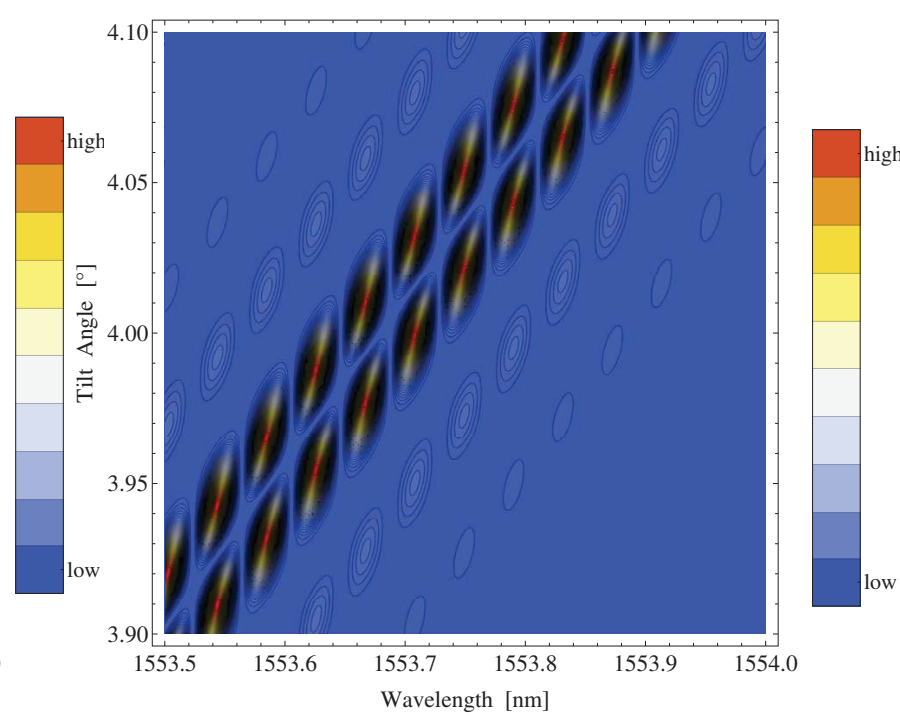

(b) $\pi$-shifted

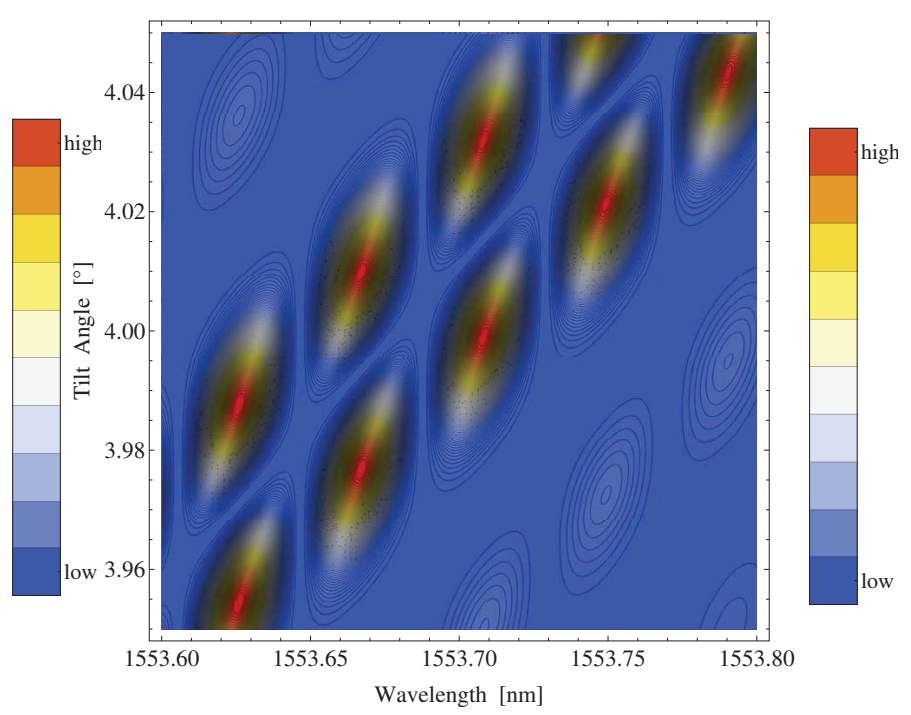

(d) $\pi$-shifted 


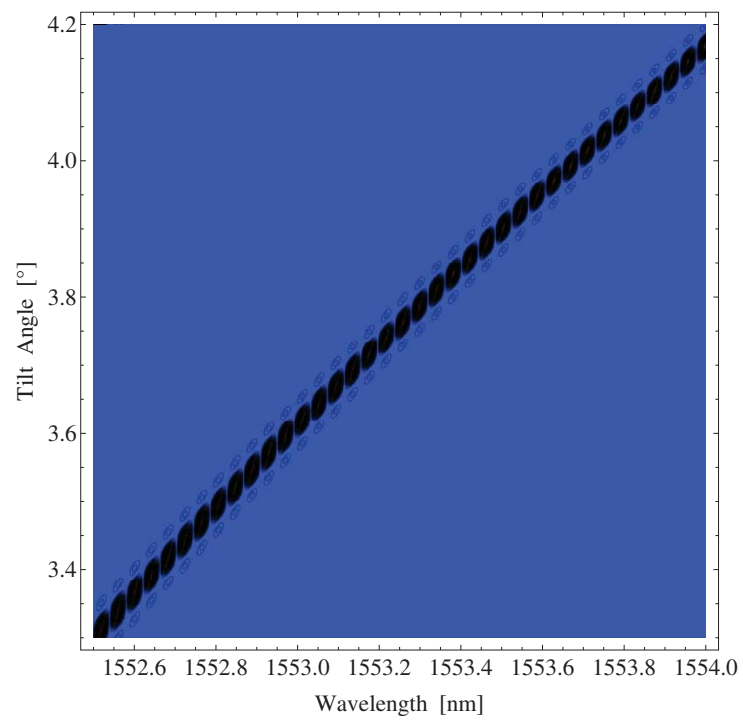

(e) uniform

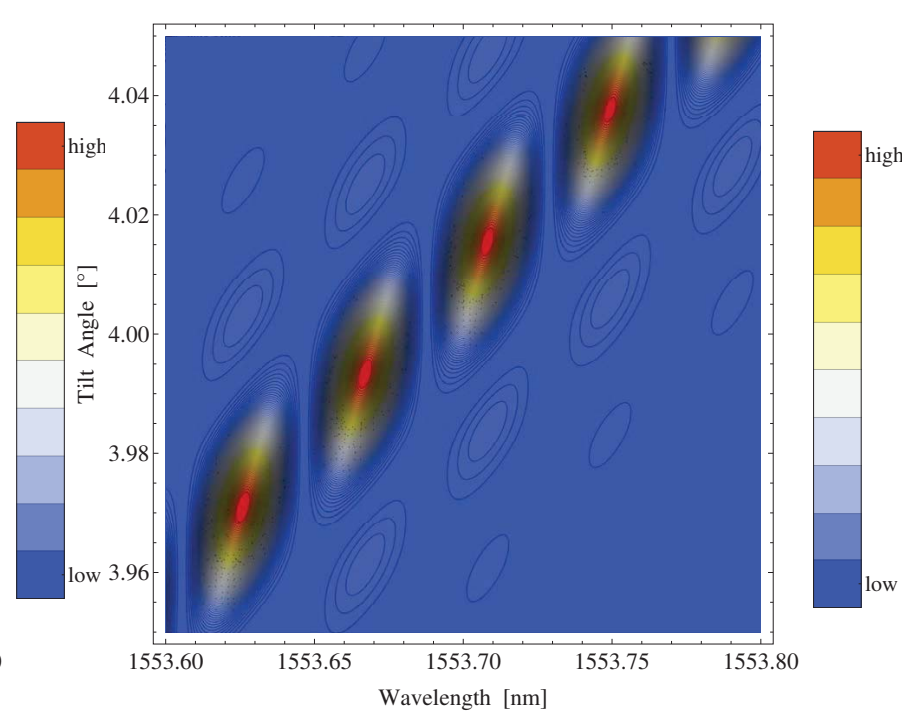

(f) uniform

Figure 6.15: Contour plot of power carried by plasmon excited by a (uniform and $\pi$-shifted) tilted grating in a fiber coated by Au distribution positioned in an index matching oil (with refractive index $n_{4}=1.42$ ) environment versus wavelength and tilt angle. Simulation parameters: azimuthal index of the mode $m=3$; wavelength $\lambda=1550 \mathrm{~nm}$; grating is tuned for coupling plasmon mode and core mode at resonance wavelength $\lambda_{\text {res }}=1550 \mathrm{~nm}$; thickness of $\mathrm{Au}$ distribution $d=100 \mathrm{~nm}$; angular width of distribution $\left(\theta_{\min }, \theta_{\max }\right)$ : $\theta_{\min }=-\pi / 10, \theta_{\max }=\pi / 10$, array length $2 z_{0}=2 \mathrm{~cm}$; distribution type: uniform array of patches; AC grating $\Delta \varepsilon_{A C}=3 \times 10^{-4}$. Au distribution parameters: $\delta z=50 \mathrm{~nm}$, $\Delta z=52 \mu \mathrm{m}$. 


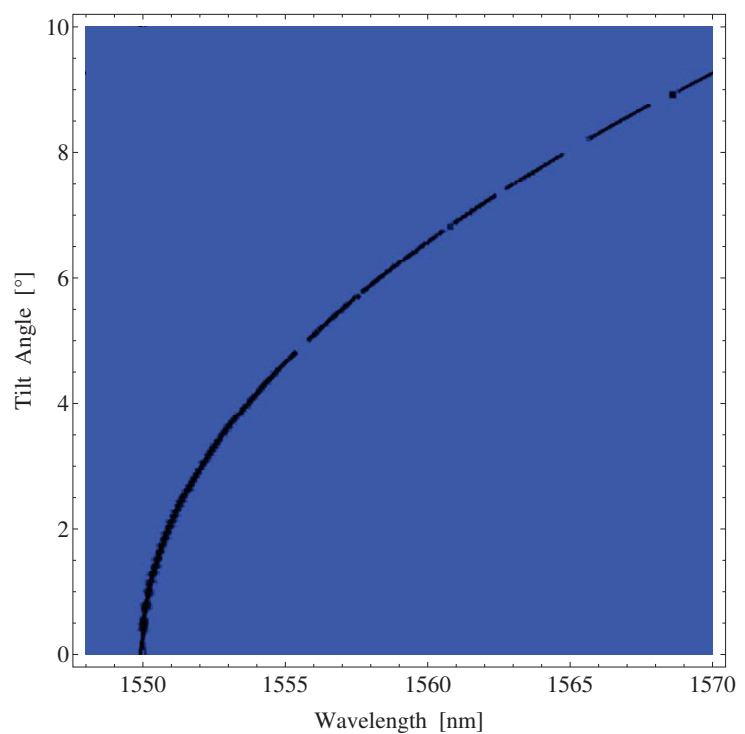

(a) $\pi$-shifted

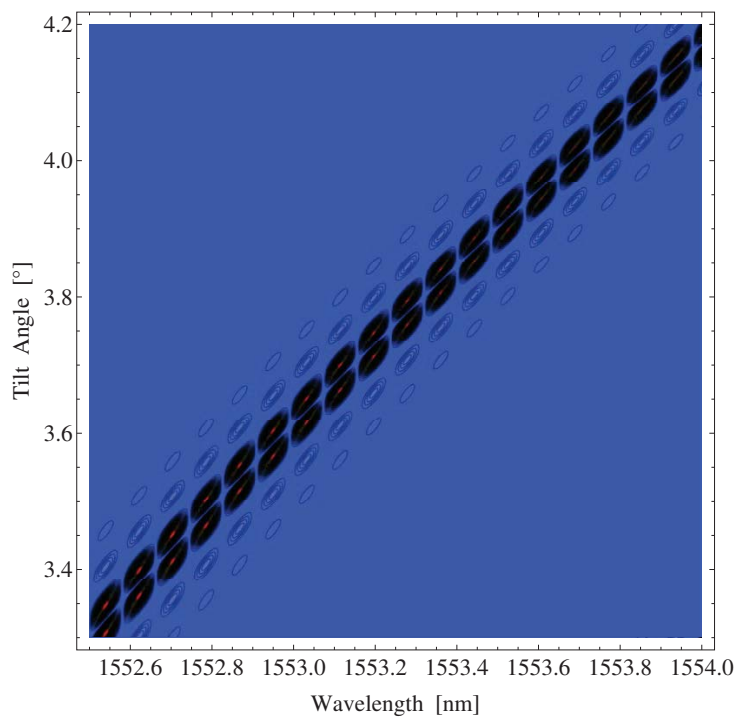

(c) $\pi$-shifted

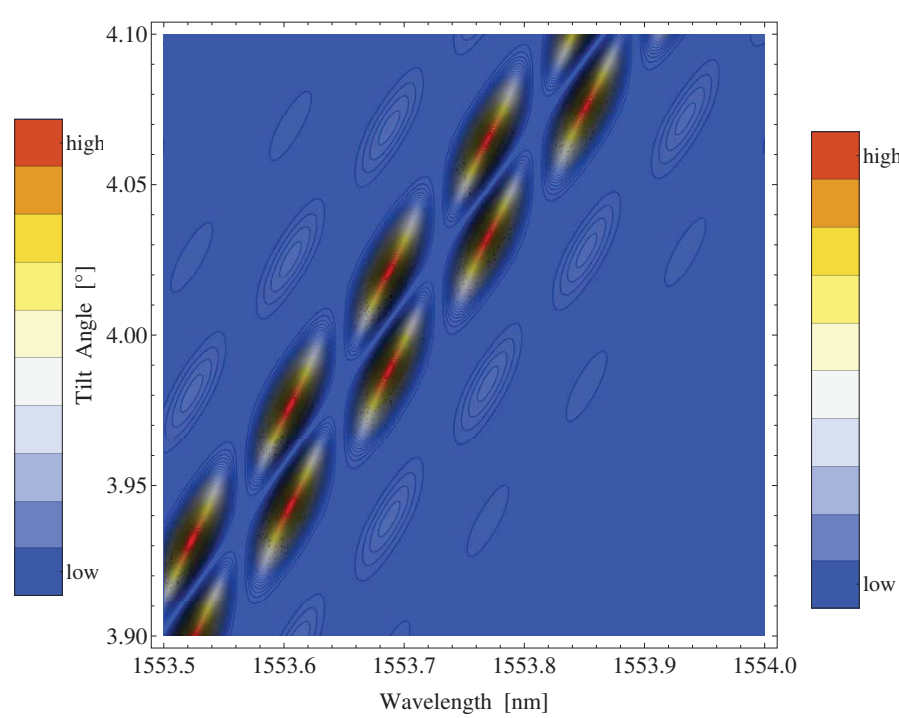

(b) $\pi$-shifted

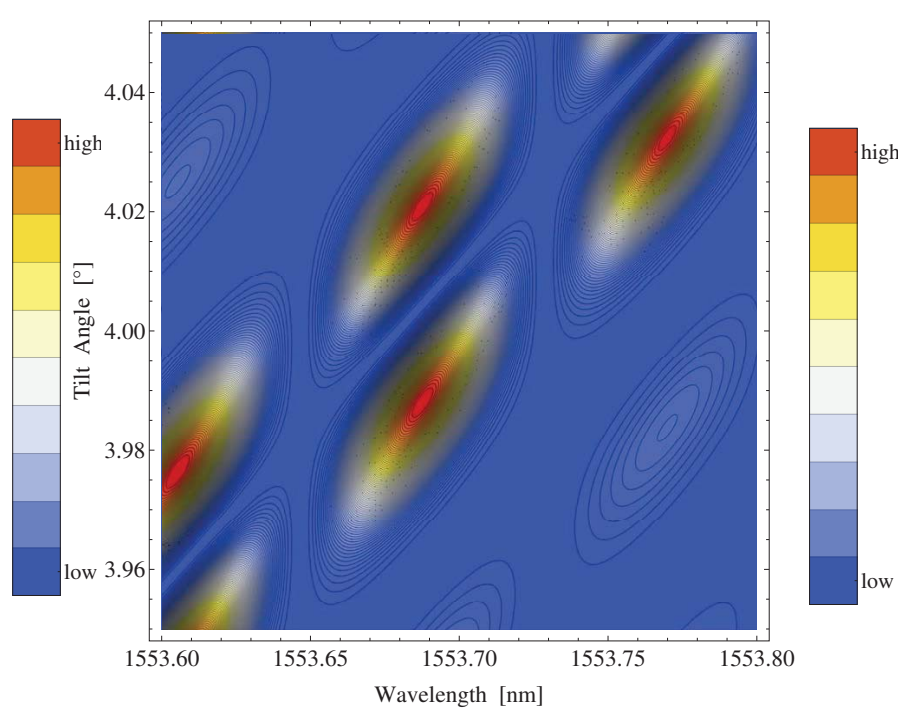

(d) $\pi$-shifted 


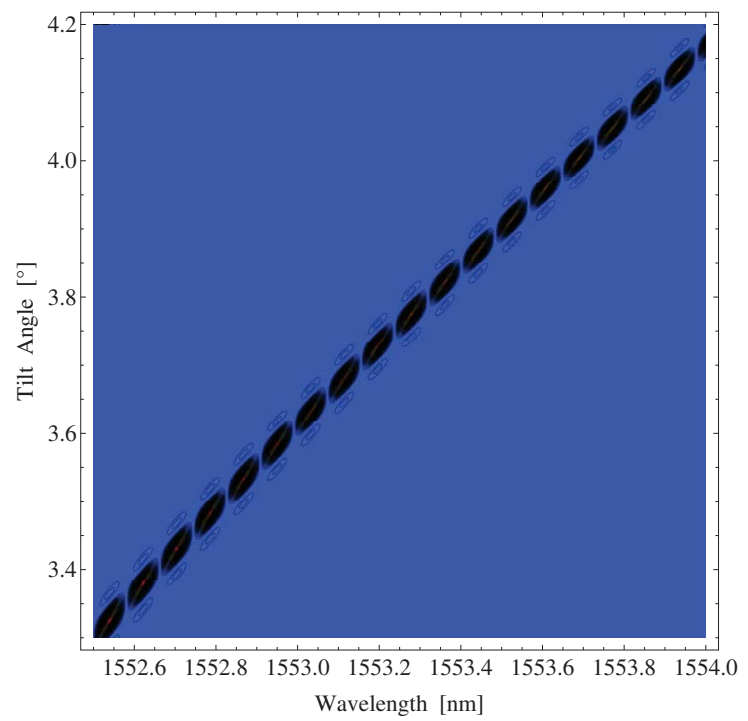

(e) uniform

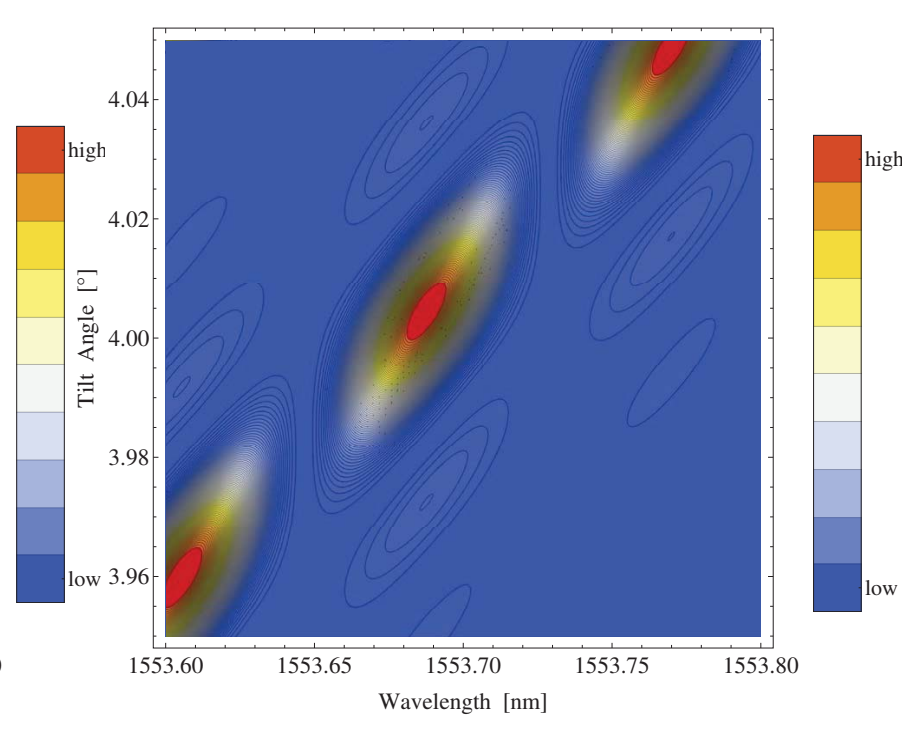

(f) uniform

Figure 6.16: Contour plot of power carried by plasmon excited by a (uniform and $\pi$-shifted) tilted grating in a fiber coated by Au distribution positioned in an index matching oil (with refractive index $n_{4}=1.42$ ) environment versus wavelength and tilt angle. Simulation parameters: azimuthal index of the mode $m=3$; wavelength $\lambda=1550 \mathrm{~nm}$; grating is tuned for coupling plasmon mode and core mode at resonance wavelength $\lambda_{\text {res }}=1550 \mathrm{~nm}$; thickness of $\mathrm{Au}$ distribution $d=100 \mathrm{~nm}$; angular width of distribution $\left(\theta_{\min }, \theta_{\max }\right)$ : $\theta_{\min }=-\pi / 10, \theta_{\max }=\pi / 10$, array length $2 z_{0}=2 \mathrm{~cm}$; distribution type: uniform array of patches; AC grating $\Delta \varepsilon_{A C}=3 \times 10^{-4}$. Au distribution parameters: $\delta z=50 \mathrm{~nm}$, $\Delta z=1 \mathrm{~cm}$. 


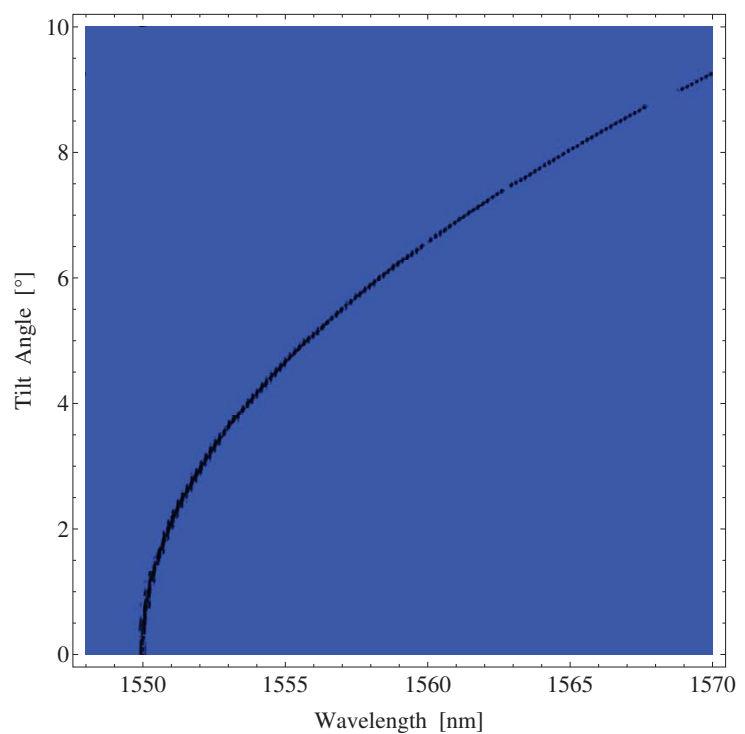

(a) $\pi$-shifted

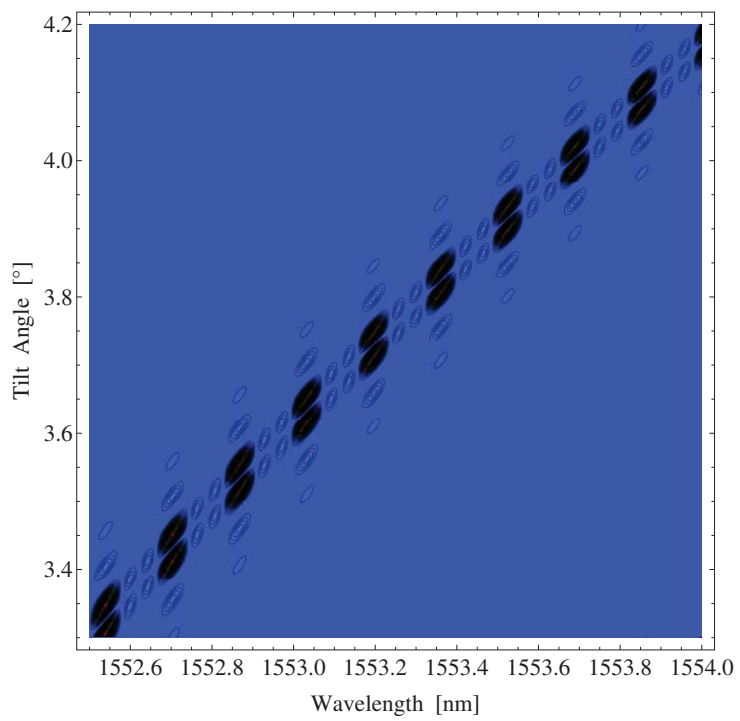

(c) $\pi$-shifted

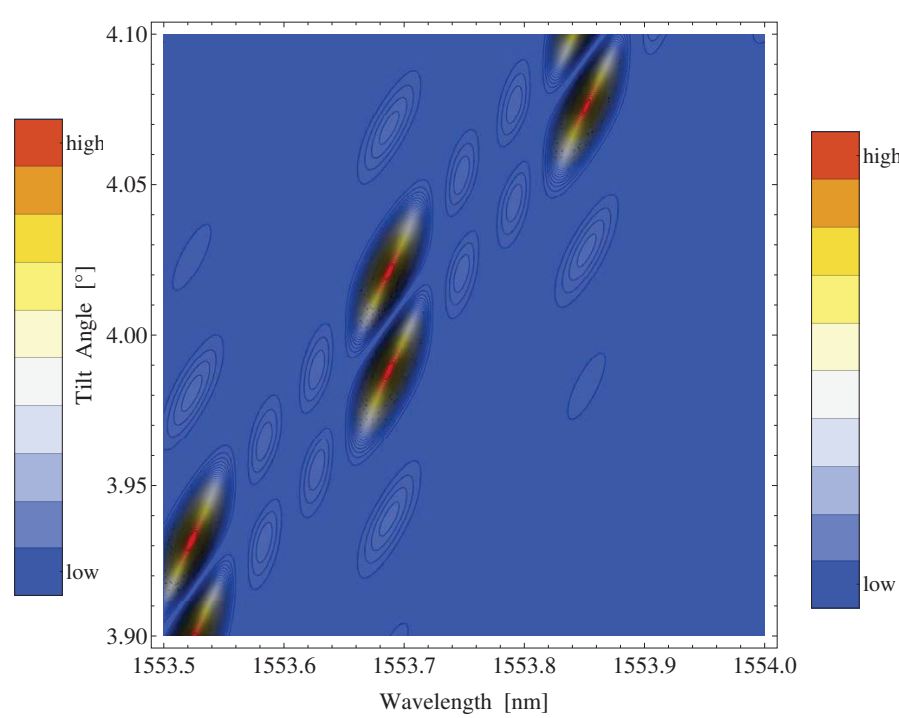

(b) $\pi$-shifted

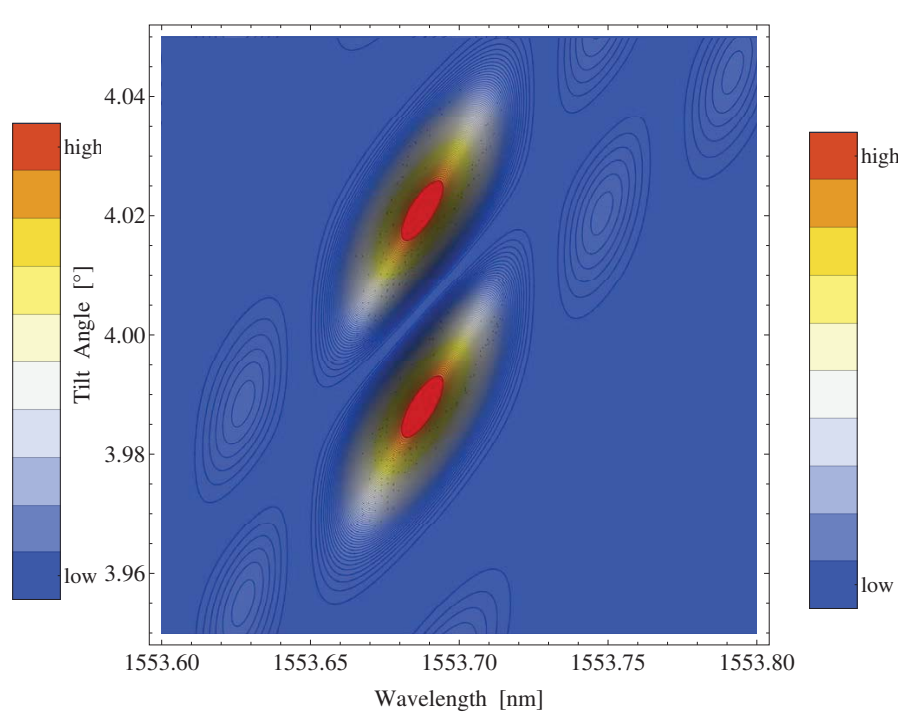

(d) $\pi$-shifted 


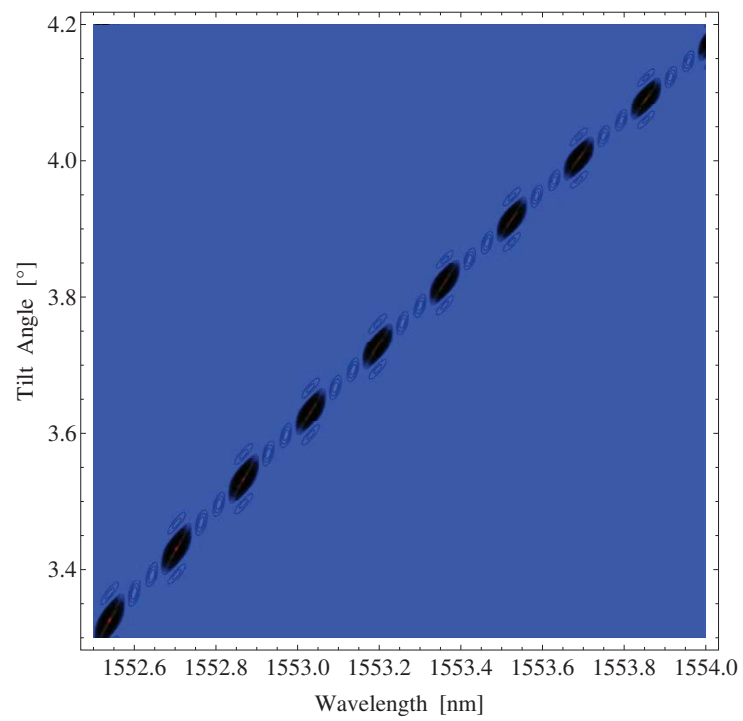

(e) uniform

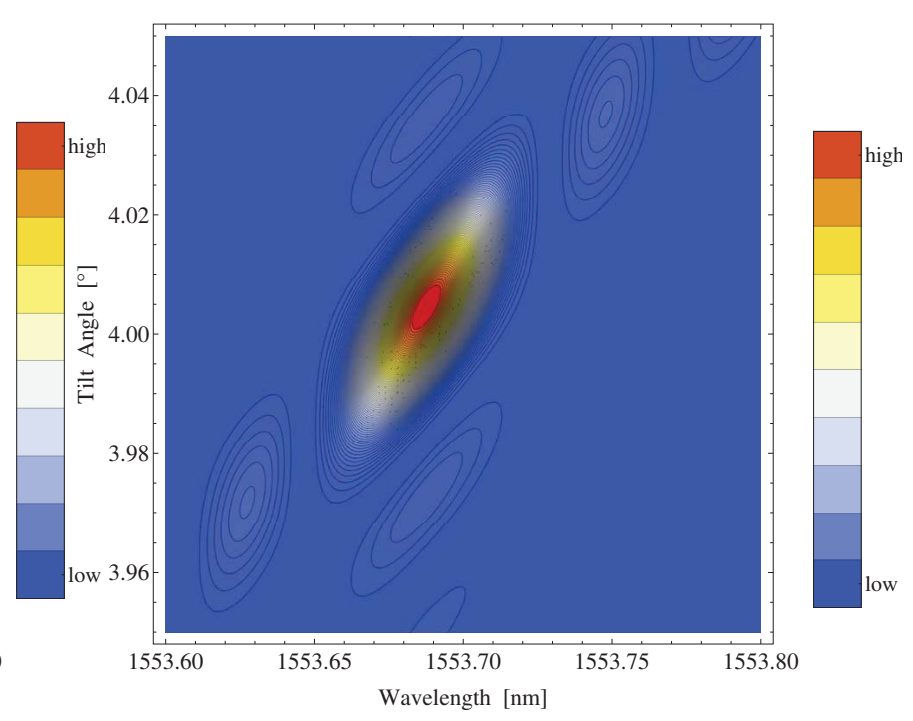

(f) uniform

Figure 6.17: Contour plot of power carried by plasmon excited by a (uniform and $\pi$-shifted) tilted grating in a fiber coated by Au distribution positioned in an index matching oil (with refractive index $n_{4}=1.42$ ) environment versus wavelength and tilt angle. Simulation parameters: azimuthal index of the mode $m=3$; wavelength $\lambda=1550 \mathrm{~nm}$; grating is tuned for coupling plasmon mode and core mode at resonance wavelength $\lambda_{\text {res }}=1550 \mathrm{~nm}$; thickness of $\mathrm{Au}$ distribution $d=100 \mathrm{~nm}$; angular width of distribution $\left(\theta_{\min }, \theta_{\max }\right)$ : $\theta_{\min }=-\pi / 10, \theta_{\max }=\pi / 10$, array length $2 z_{0}=2 \mathrm{~cm}$; distribution type: uniform array of patches; AC grating $\Delta \varepsilon_{A C}=3 \times 10^{-4}$. Au distribution parameters: $\delta z=0.5 \mathrm{~cm}$, $\Delta z=1 \mathrm{~cm}$. 


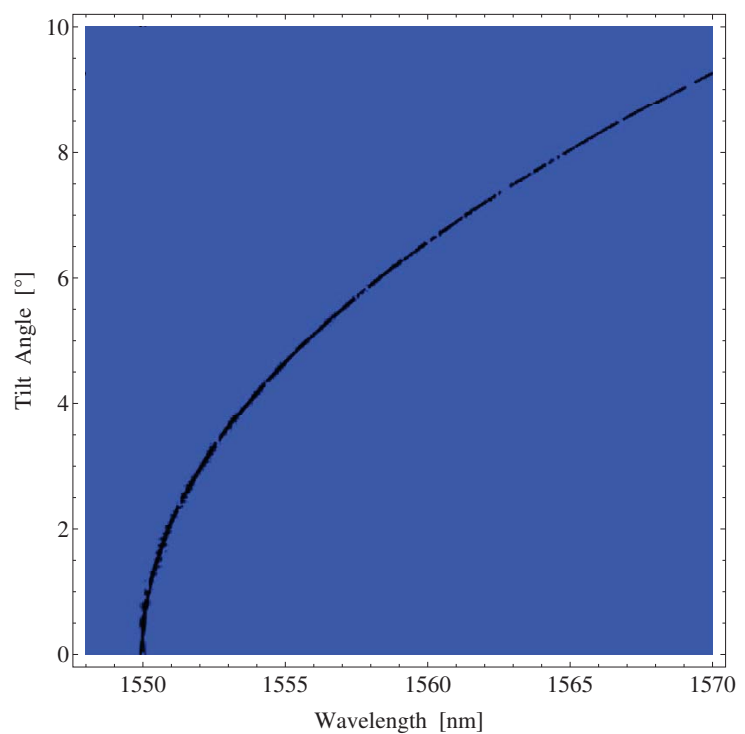

(a) $\pi$-shifted

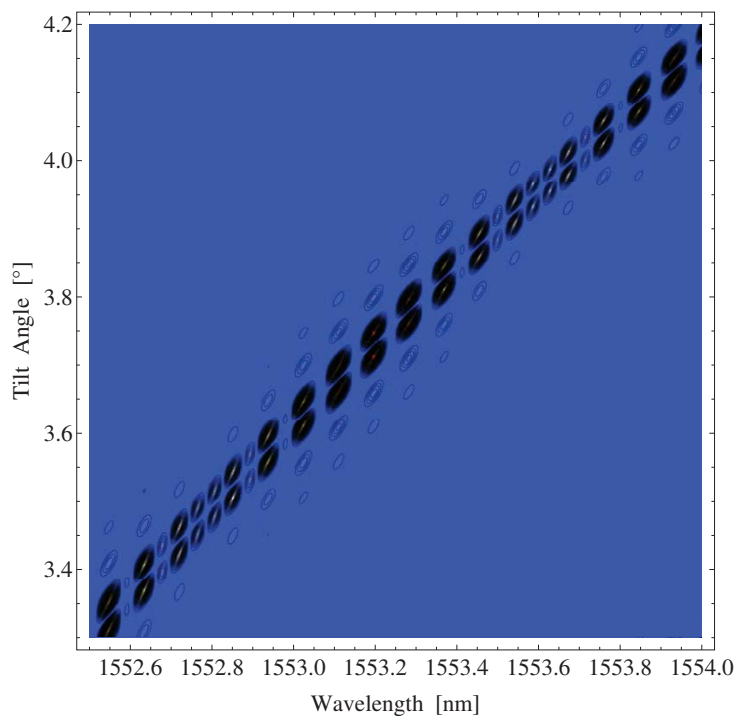

(c) $\pi$-shifted

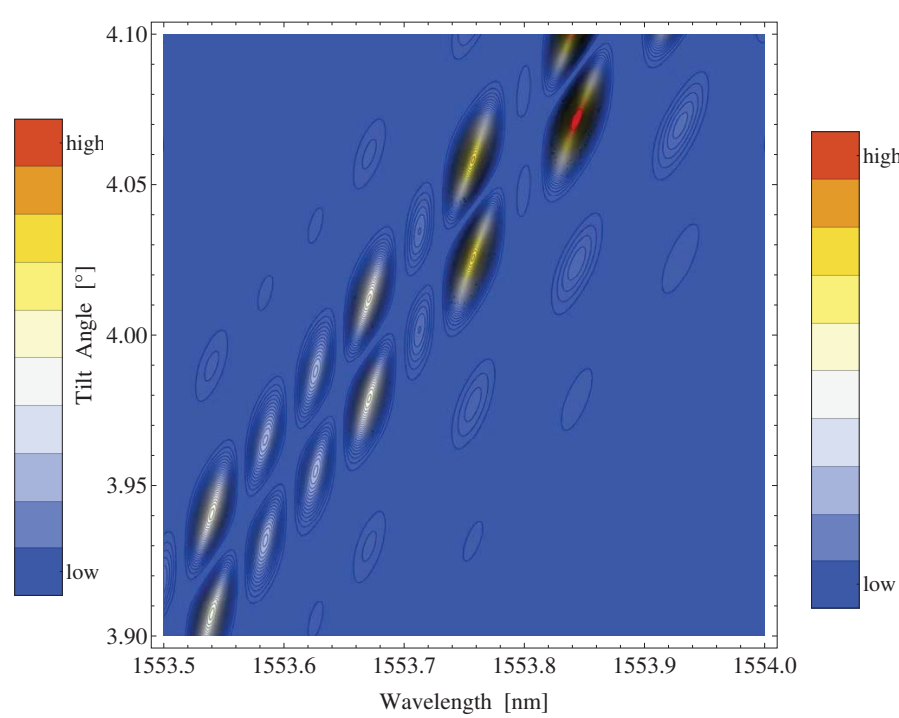

(b) $\pi$-shifted

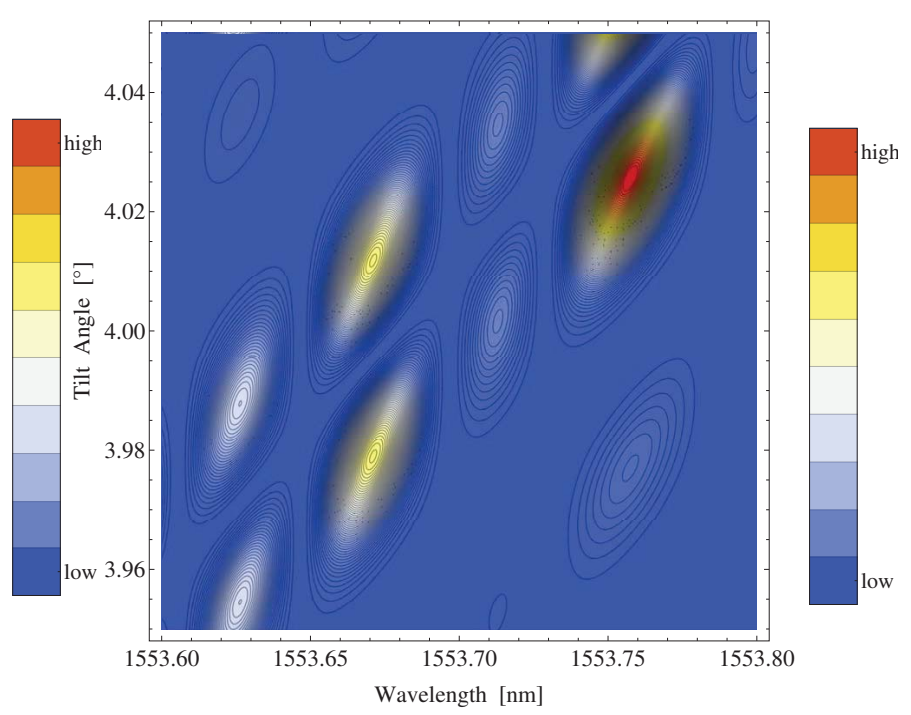

(d) $\pi$-shifted 


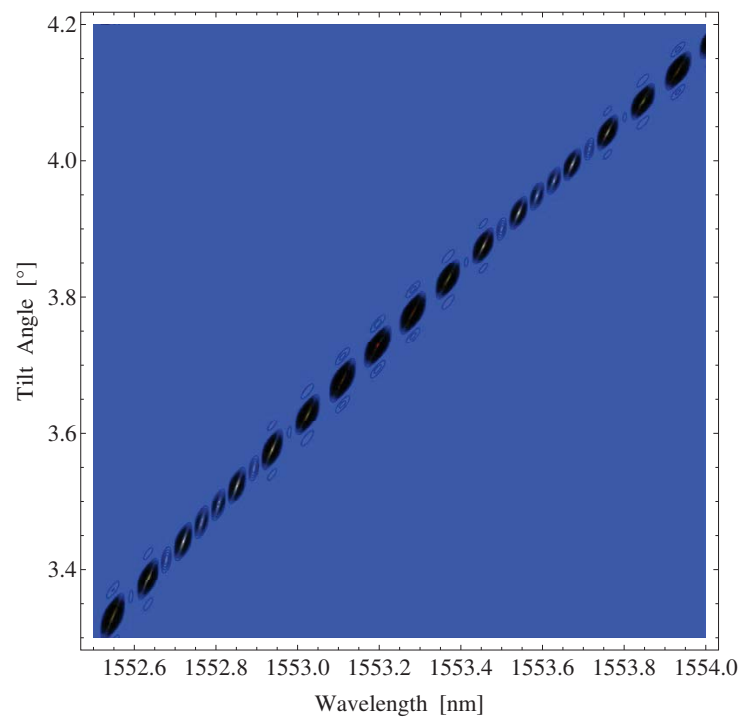

(e) uniform

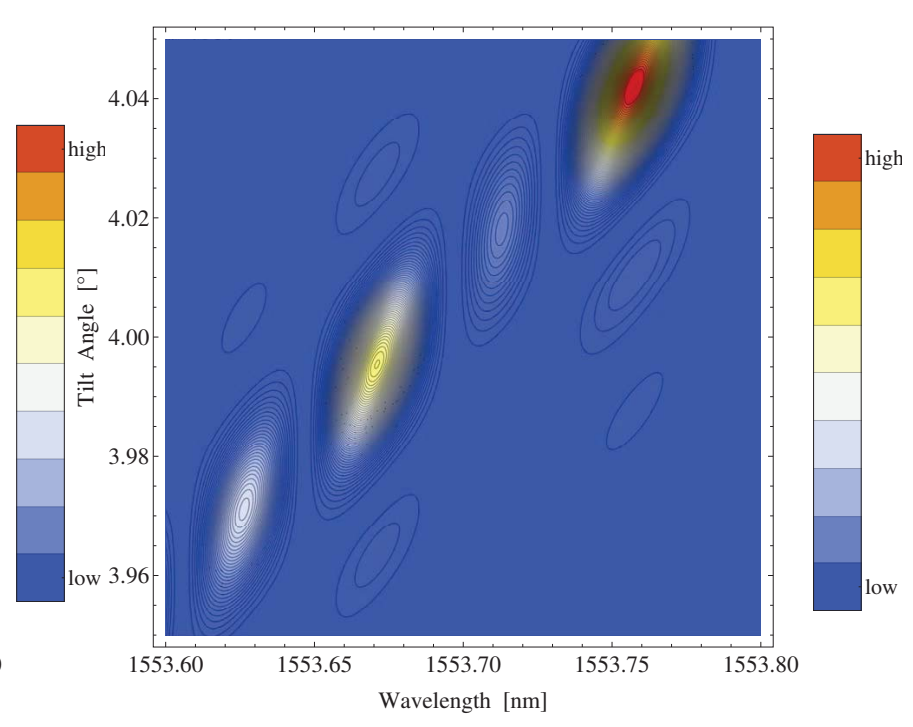

(f) uniform

Figure 6.18: Contour plot of power carried by plasmon excited by a (uniform and $\pi$-shifted) tilted grating in a fiber coated by Au distribution positioned in an index matching oil (with refractive index $n_{4}=1.42$ ) environment versus wavelength and tilt angle. Simulation parameters: azimuthal index of the mode $m=3$; wavelength $\lambda=1550 \mathrm{~nm}$; grating is tuned for coupling plasmon mode and core mode at resonance wavelength $\lambda_{\text {res }}=1550 \mathrm{~nm}$; thickness of $\mathrm{Au}$ distribution $d=100 \mathrm{~nm}$; angular width of distribution $\left(\theta_{\min }, \theta_{\max }\right)$ : $\theta_{\min }=-\pi / 10, \theta_{\max }=\pi / 10$, array length $2 z_{0}=2 \mathrm{~cm}$; distribution type: uniform array of patches; AC grating $\Delta \varepsilon_{A C}=3 \times 10^{-4}$. Au distribution parameters: $\delta z=0.9 \mathrm{~cm}$, $\Delta z=1 \mathrm{~cm}$. 


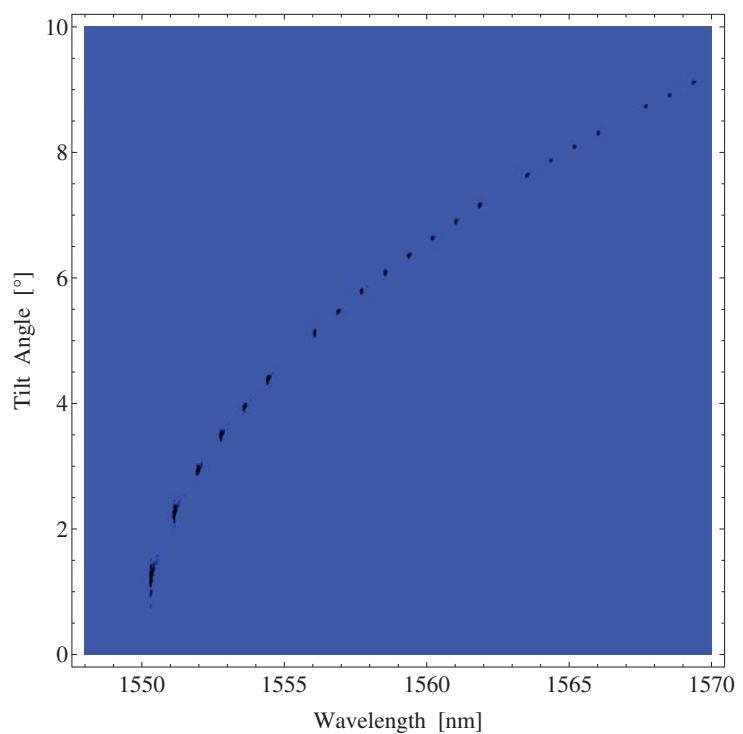

(a) $\pi$-shifted

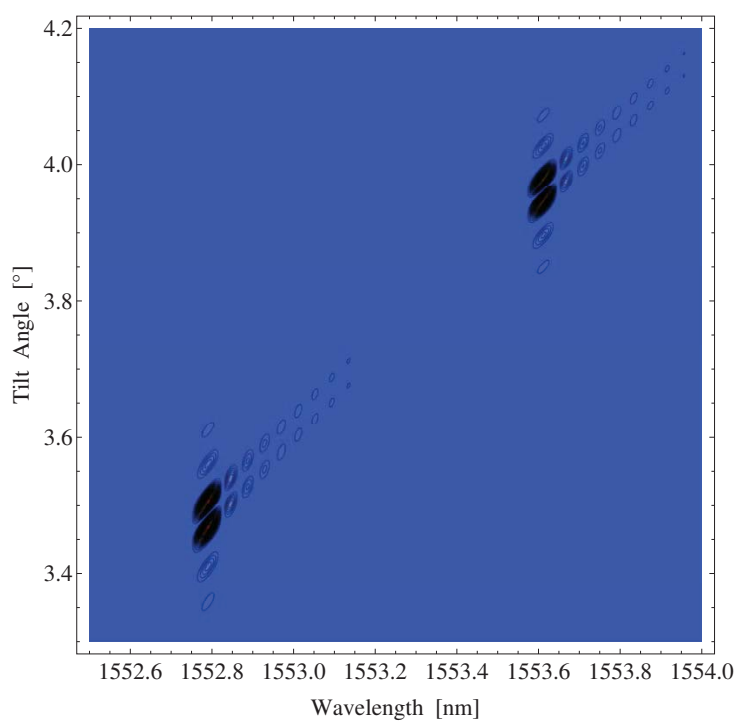

(c) $\pi$-shifted

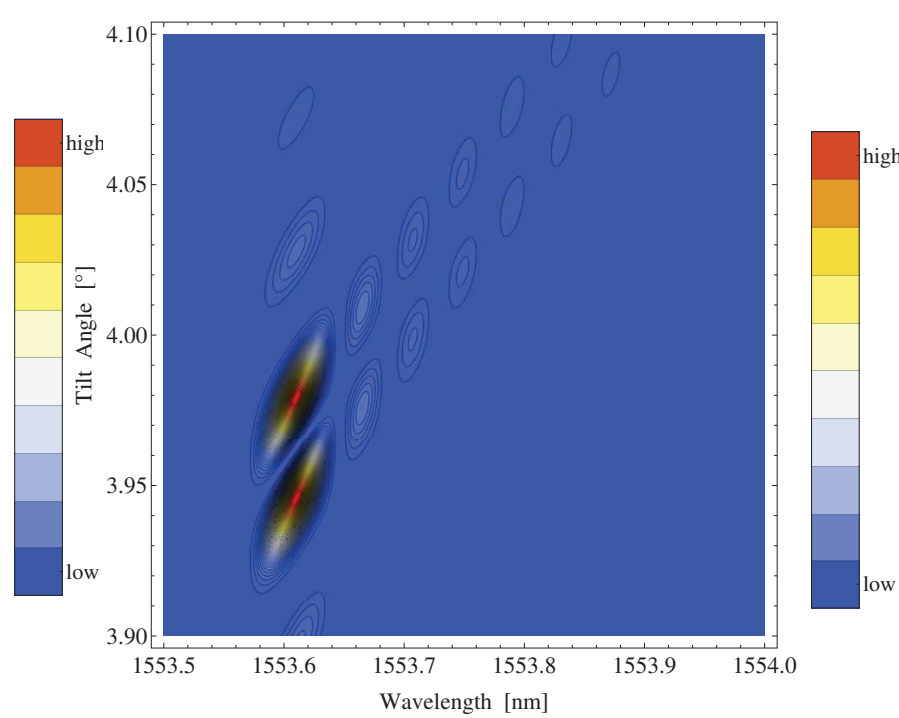

(b) $\pi$-shifted

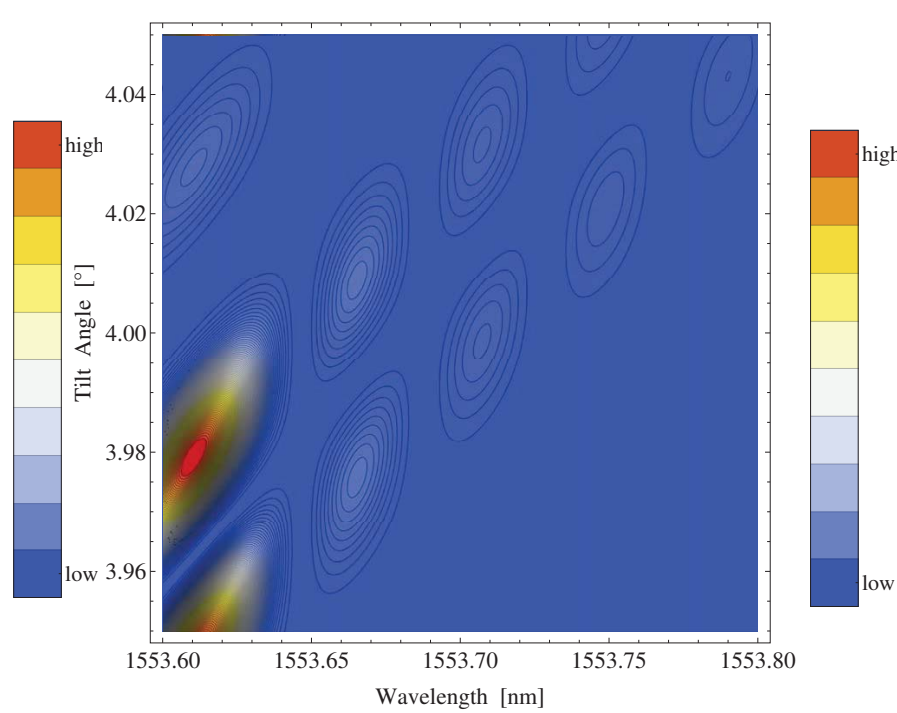

(d) $\pi$-shifted 


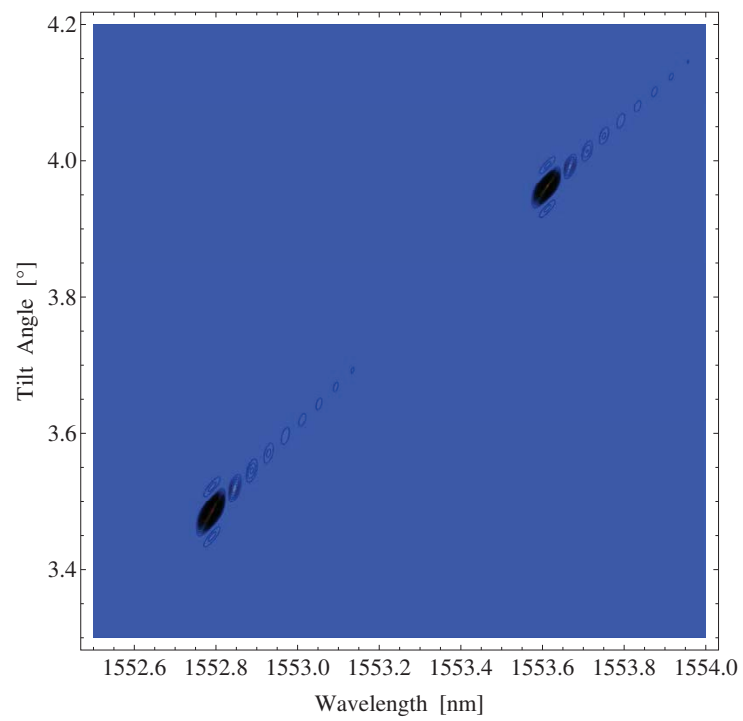

(e) uniform

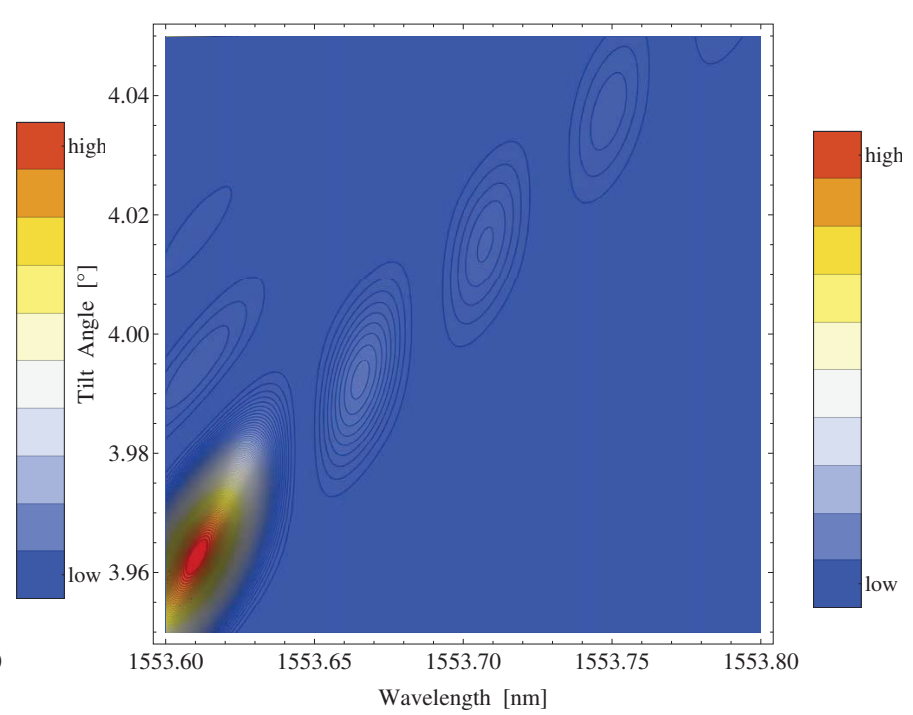

(f) uniform

Figure 6.19: Contour plot of power carried by plasmon excited by a (uniform and $\pi$-shifted) tilted grating in a fiber coated by Au distribution positioned in an index matching oil (with refractive index $n_{4}=1.42$ ) environment versus wavelength and tilt angle. Simulation parameters: azimuthal index of the mode $m=3$; wavelength $\lambda=1550 \mathrm{~nm}$; grating is tuned for coupling plasmon mode and core mode at resonance wavelength $\lambda_{\text {res }}=1550 \mathrm{~nm}$; thickness of $\mathrm{Au}$ distribution $d=100 \mathrm{~nm}$; angular width of distribution $\left(\theta_{\min }, \theta_{\max }\right)$ : $\theta_{\min }=-\pi / 10, \theta_{\max }=\pi / 10$, array length $2 z_{0}=2 \mathrm{~cm}$; distribution type: uniform array of patches; AC grating $\Delta \varepsilon_{A C}=3 \times 10^{-4}$. Au distribution parameters: $\delta z=\Delta z-60 \mathrm{~nm}$, $\Delta z=1000 \mu \mathrm{m}$. 


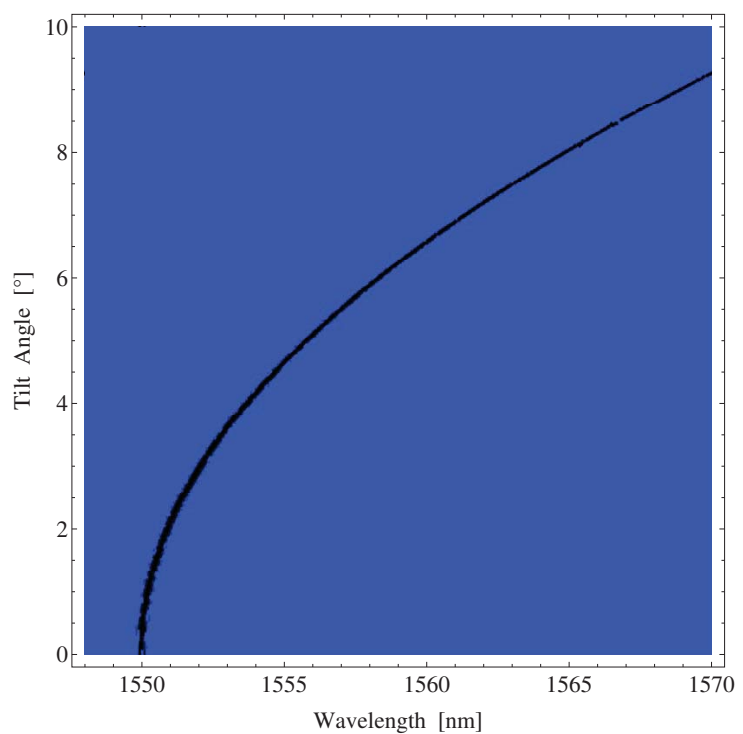

(a) $\pi$-shifted

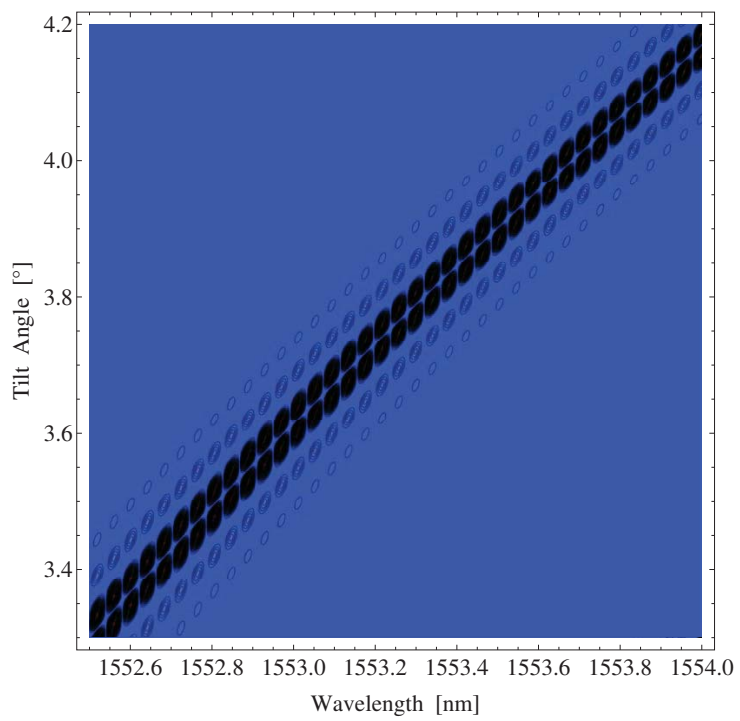

(c) $\pi$-shifted

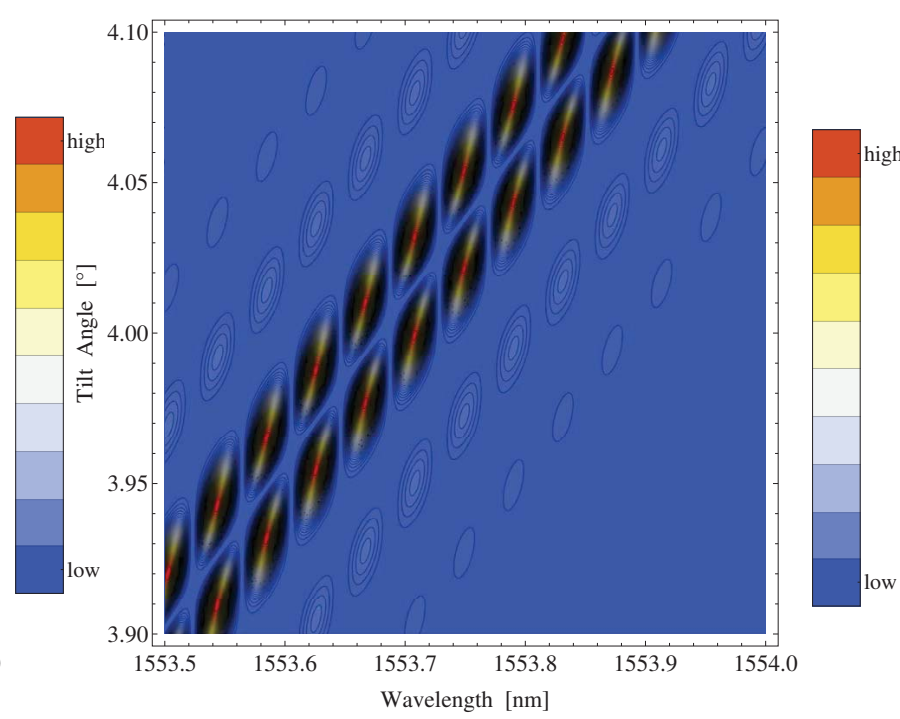

(b) $\pi$-shifted

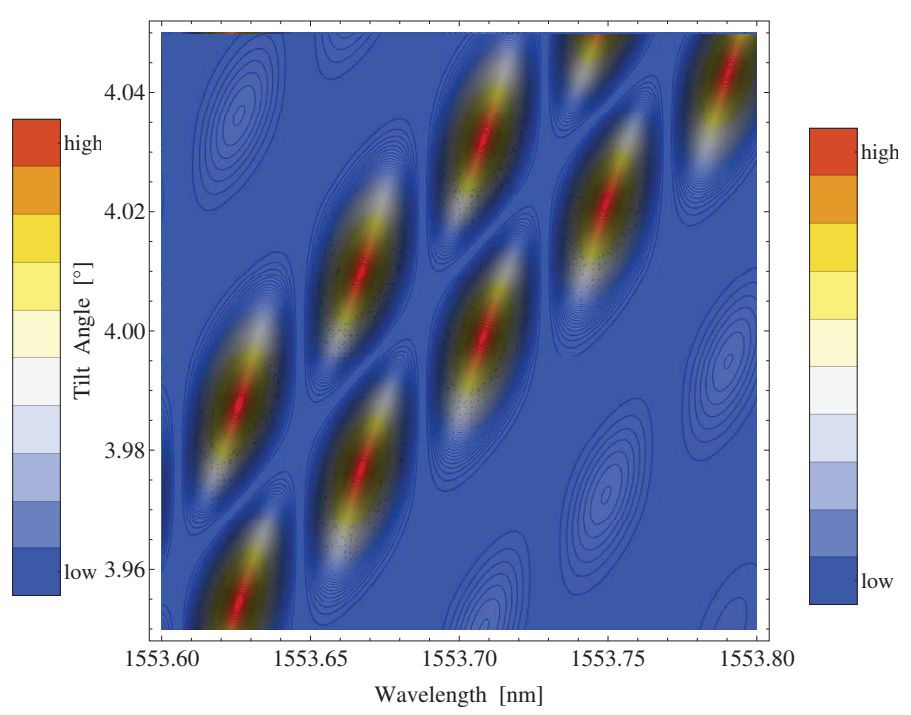

(d) $\pi$-shifted 


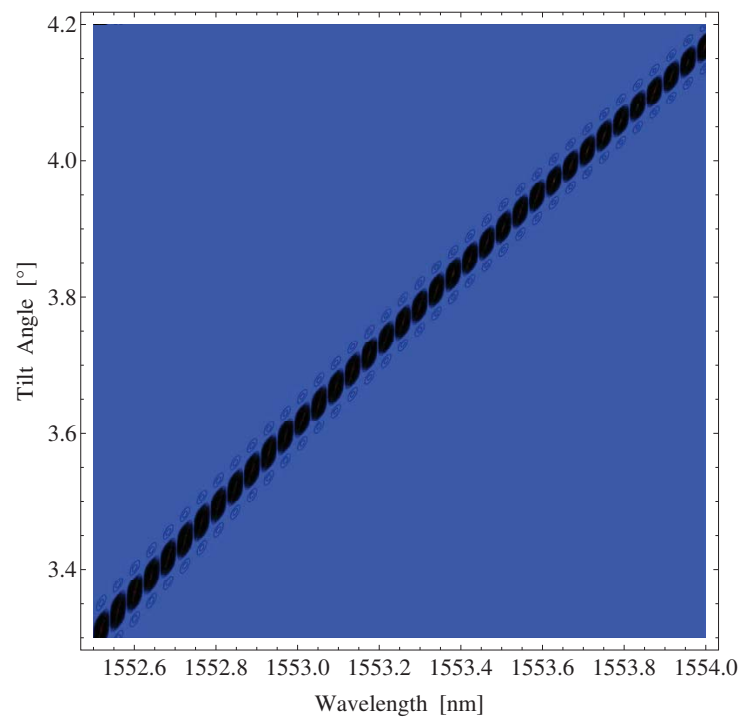

(e) uniform

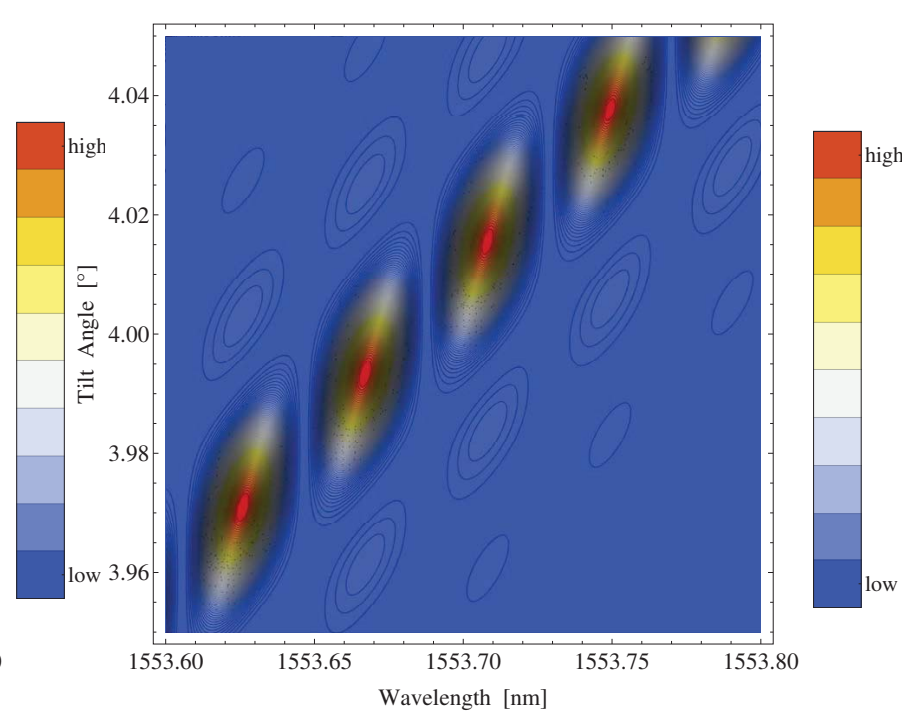

(f) uniform

Figure 6.20: Contour plot of power carried by plasmon excited by a (uniform and $\pi$-shifted) tilted grating in a fiber coated by Au distribution positioned in an index matching oil (with refractive index $n_{4}=1.42$ ) environment versus wavelength and tilt angle. Simulation parameters: azimuthal index of the mode $m=3$; wavelength $\lambda=1550 \mathrm{~nm}$; grating is tuned for coupling plasmon mode and core mode at resonance wavelength $\lambda_{\text {res }}=1550 \mathrm{~nm}$; thickness of Au distribution $d=100 \mathrm{~nm}$; angular width of distribution $\left(\theta_{\min }, \theta_{\max }\right): \theta_{\min }=$ $-\pi / 10, \theta_{\max }=\pi / 10$, array length $2 z_{0}=2 \mathrm{~cm}$; distribution type: uniform array of patches; $\mathrm{AC}$ grating $\Delta \varepsilon_{A C}=3 \times 10^{-4}$. Au distribution parameters: $\delta z=\Delta z, \Delta z=1000 \mu \mathrm{m}$. 


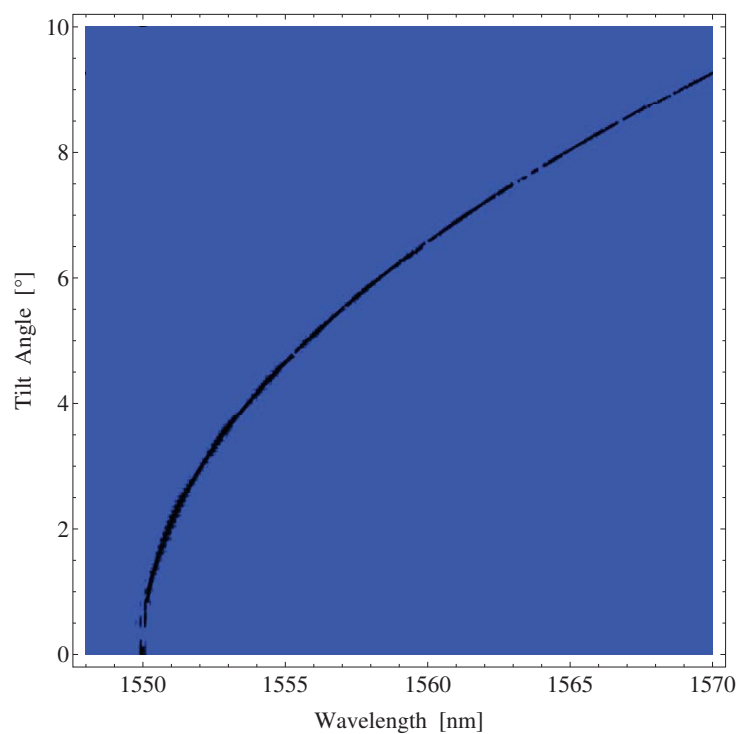

(a) $\pi$-shifted

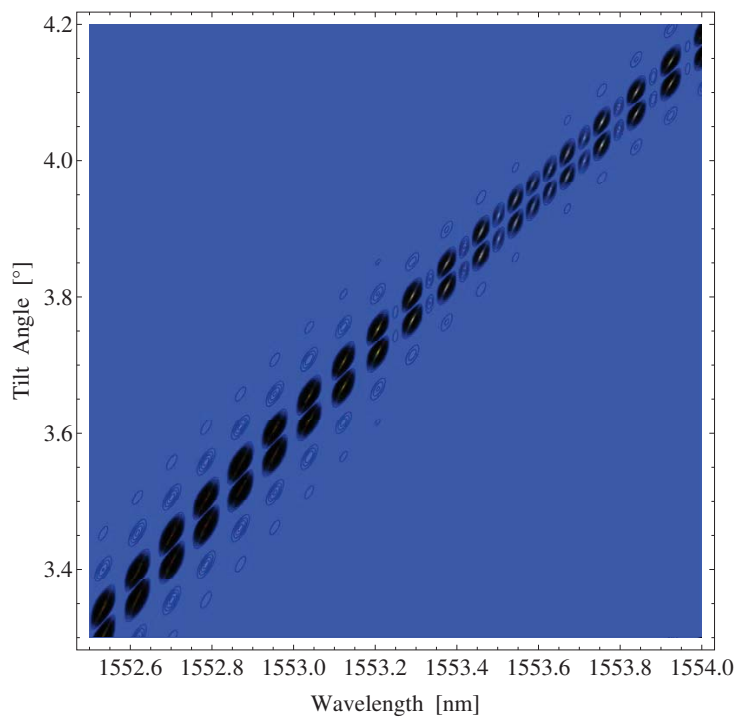

(c) $\pi$-shifted

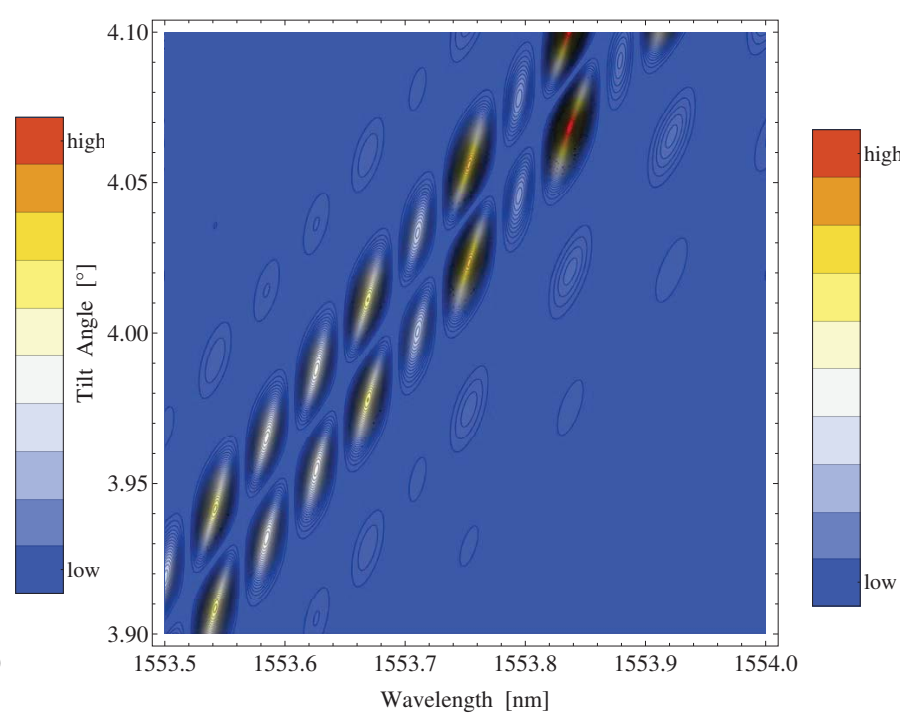

(b) $\pi$-shifted

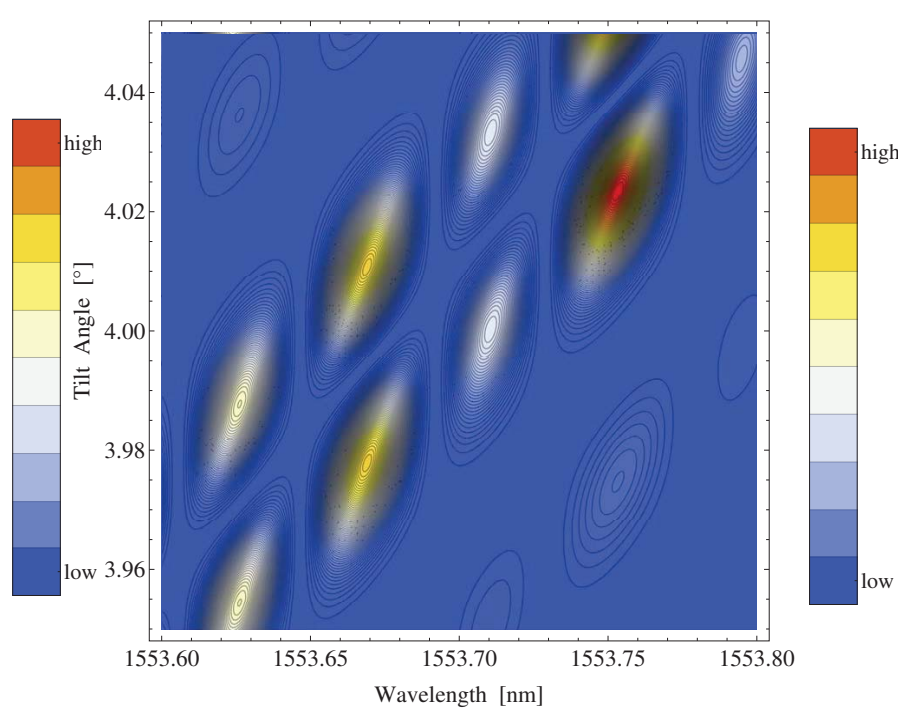

(d) $\pi$-shifted 


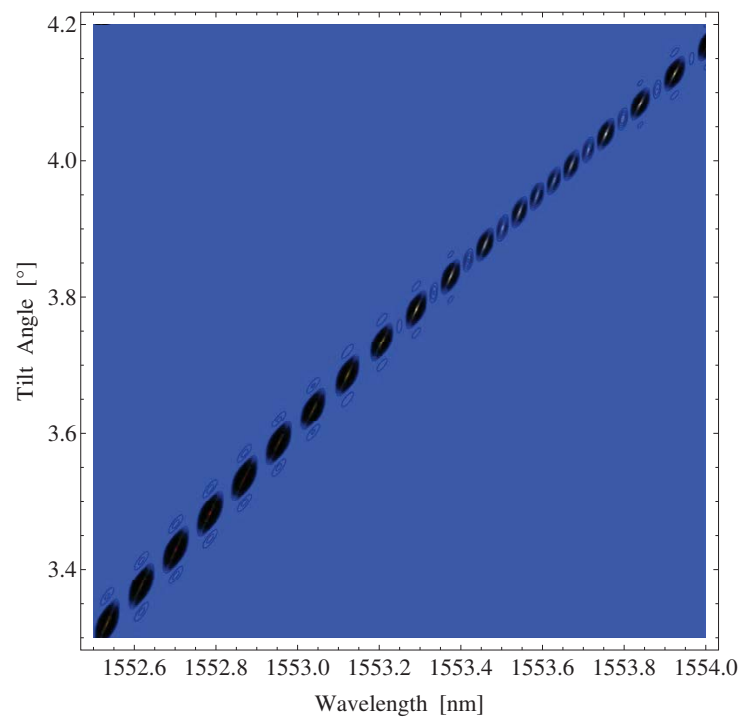

(e) uniform

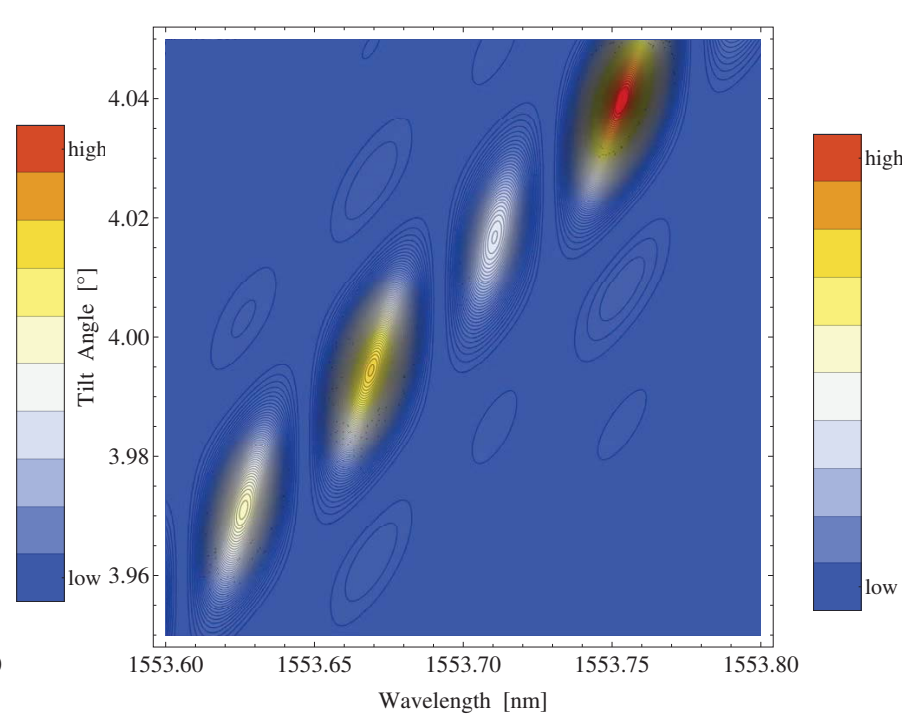

(f) uniform

Figure 6.21: Contour plot of power carried by plasmon excited by a (uniform and $\pi$-shifted) tilted grating in a fiber coated by Au distribution positioned in an index matching oil (with refractive index $n_{4}=1.42$ ) environment versus wavelength and tilt angle. Simulation parameters: azimuthal index of the mode $m=3$; wavelength $\lambda=1550 \mathrm{~nm}$; grating is tuned for coupling plasmon mode and core mode at resonance wavelength $\lambda_{\text {res }}=1550 \mathrm{~nm}$; thickness of $\mathrm{Au}$ distribution $d=100 \mathrm{~nm}$; angular width of distribution $\left(\theta_{\min }, \theta_{\max }\right)$ : $\theta_{\min }=-\pi / 10, \theta_{\max }=\pi / 10$, array length $2 z_{0}=2 \mathrm{~cm}$; distribution type: uniform array of patches; AC grating $\Delta \varepsilon_{A C}=3 \times 10^{-4}$. Au distribution parameters: $\delta z=0.95 \mathrm{~cm}$, $\Delta z=1 \mathrm{~cm}$.

It turns out that phase shift can be introduced not only in fiber grating but in metal distribution as well since it can be view as an array of metal patches. Power of plasmon excited by an uniform $\pi$-shifted fiber grating and computed for the case of metal distribution in the form of an uniform and $\pi$-shifted array of patches presented in Fig. 6.22, Fig. 6.23. Unlike for the case of phase shift in grating where the dip runs along the curved line of the power peak, the case of phase shift in an array patches leads to a dip that runs perpendicular to the $\lambda$ axis and divides all local peaks in two halves as shown in Fig. 6.22, Fig. 6.23. 


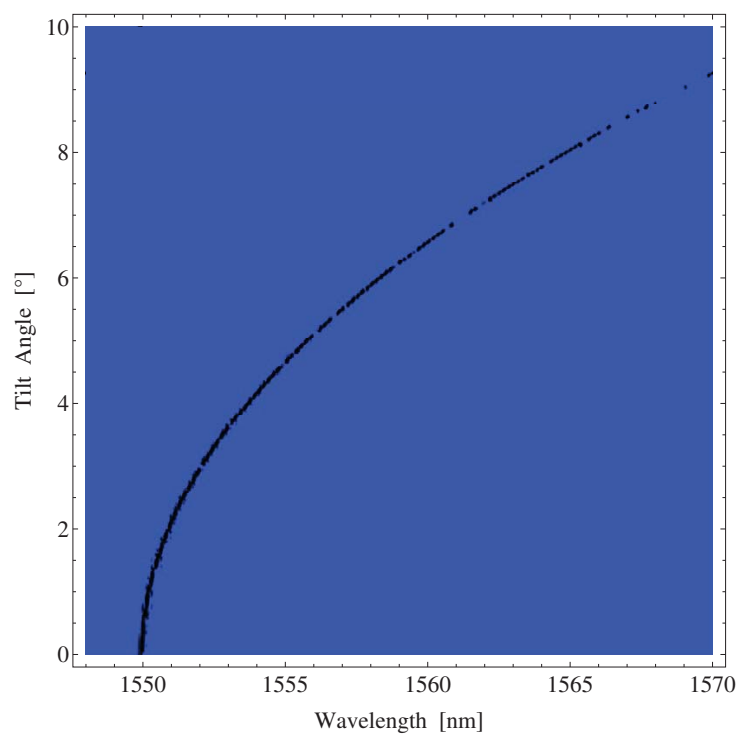

(a) $\pi$-shifted

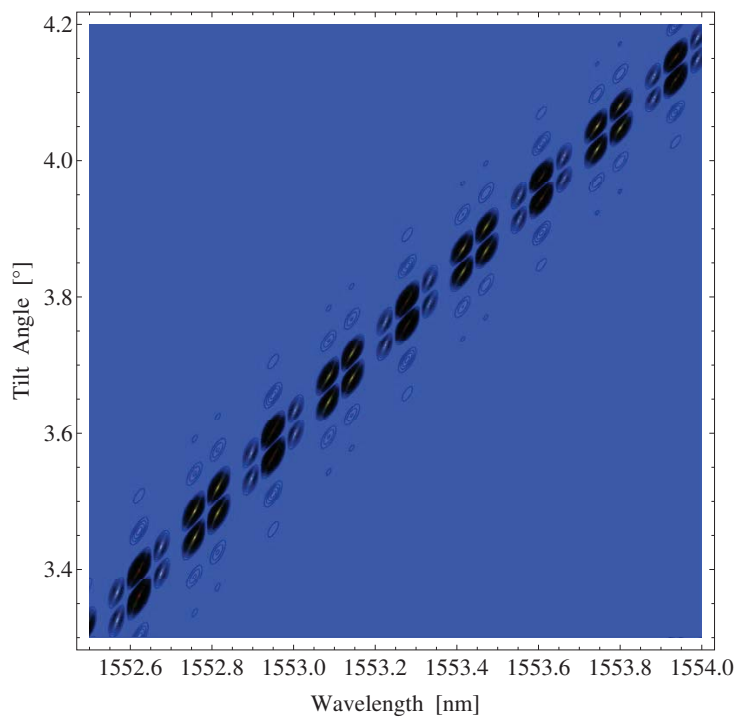

(c) $\pi$-shifted

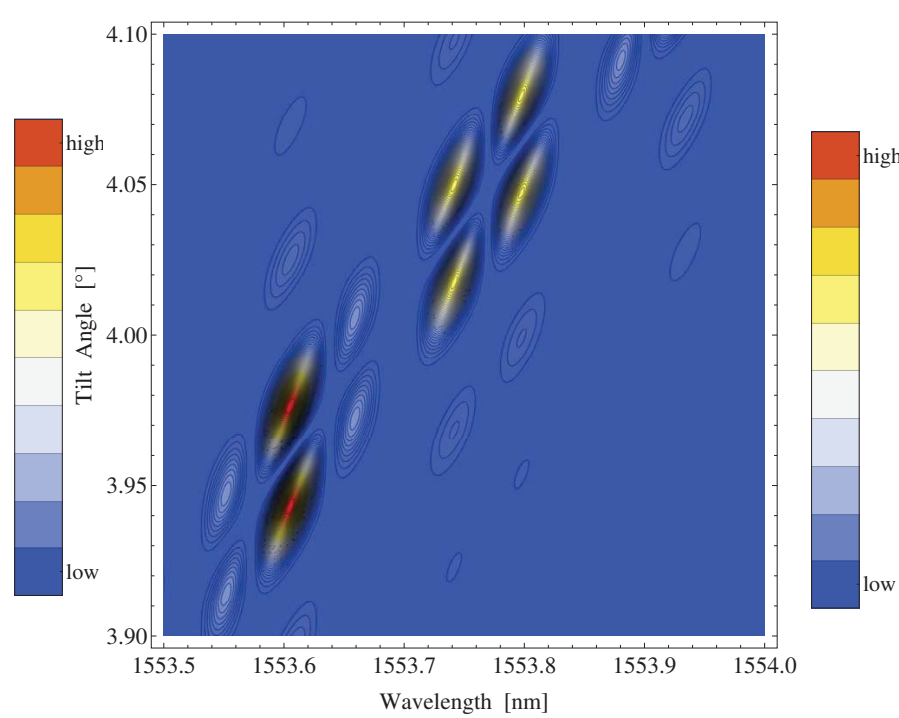

(b) $\pi$-shifted

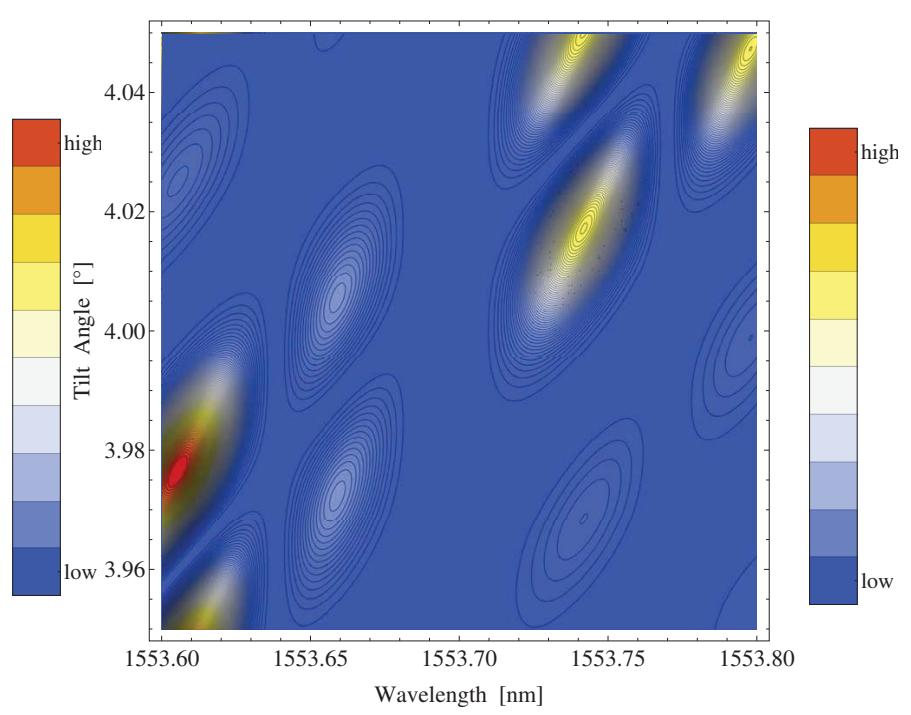

(d) $\pi$-shifted 


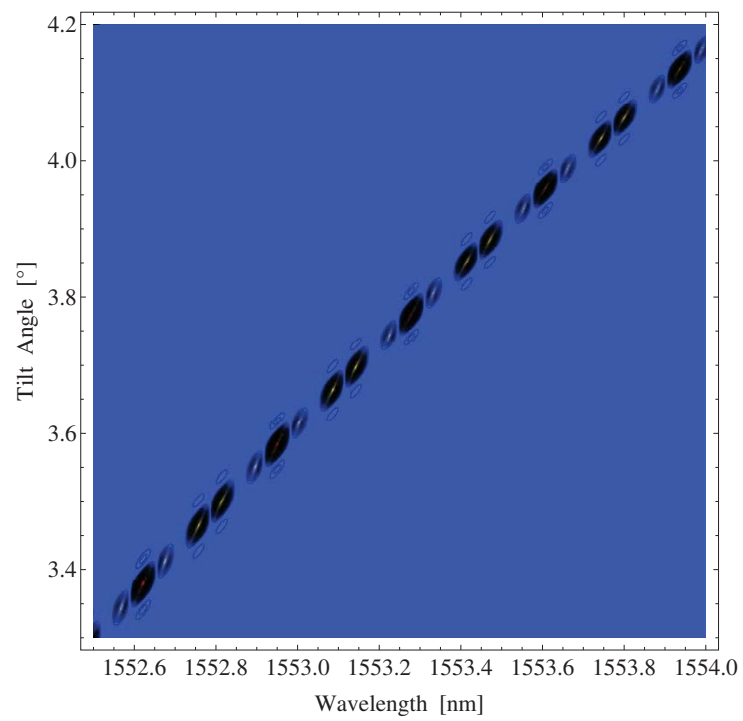

(e) uniform

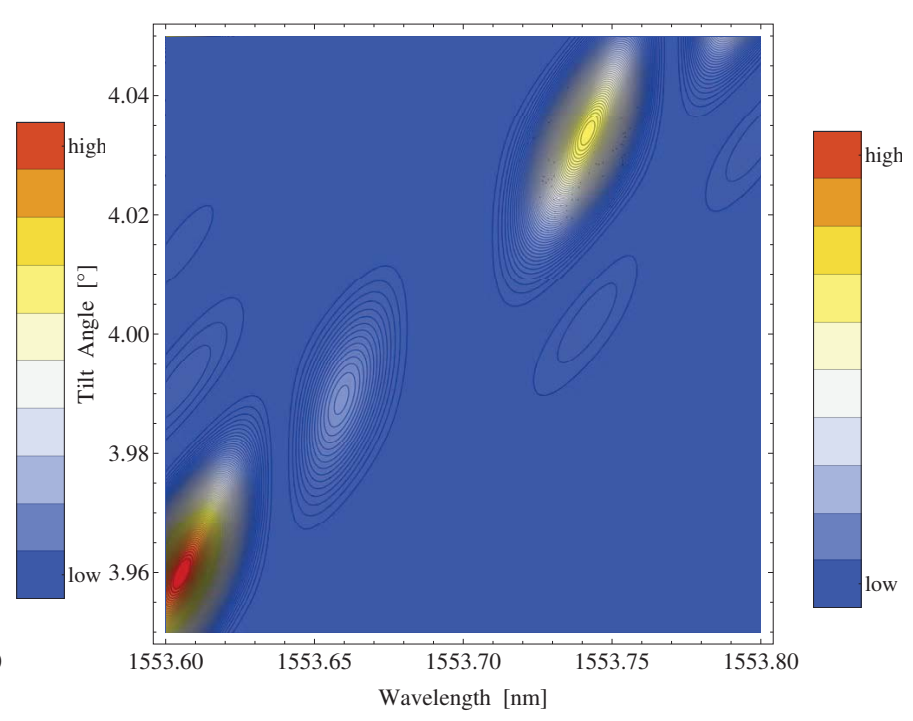

(f) uniform

Figure 6.22: Contour plot of power carried by plasmon excited by a (uniform and $\pi$-shifted) tilted grating in a fiber coated by Au distribution positioned in an index matching oil (with refractive index $n_{4}=1.42$ ) environment versus wavelength and tilt angle. Simulation parameters: azimuthal index of the mode $m=3$; wavelength $\lambda=1550 \mathrm{~nm}$; grating is tuned for coupling plasmon mode and core mode at resonance wavelength $\lambda_{\text {res }}=1550 \mathrm{~nm}$; thickness of $\mathrm{Au}$ distribution $d=100 \mathrm{~nm}$; angular width of distribution $\left(\theta_{\min }, \theta_{\max }\right)$ : $\theta_{\min }=-\pi / 10, \theta_{\max }=\pi / 10$, array length $2 z_{0}=2 \mathrm{~cm}$; distribution type: $\pi$-shifted array of patches; AC grating $\Delta \varepsilon_{A C}=3 \times 10^{-4}$. Au distribution parameters: $\delta z=50 \mathrm{~nm}$, $\Delta z=5000 \mu m$. 


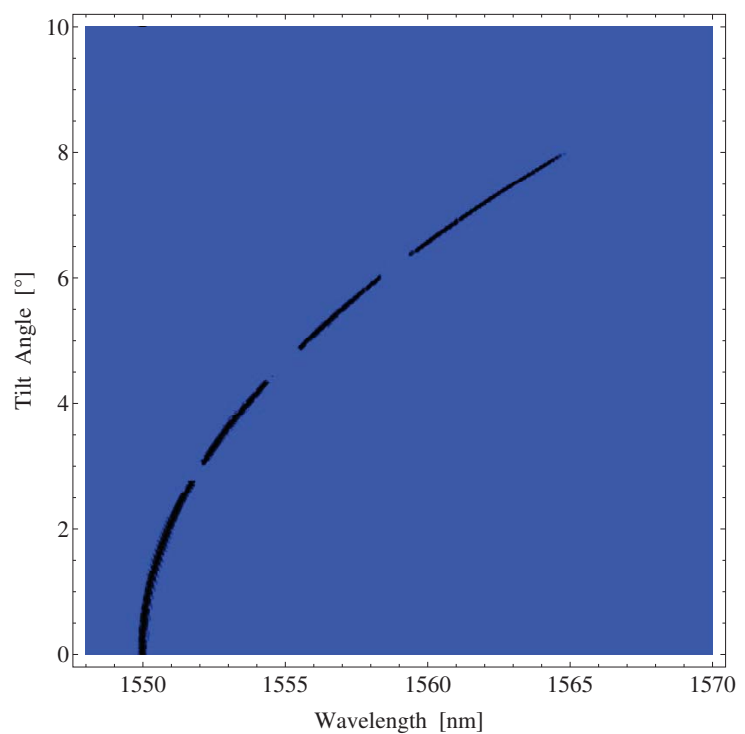

(a) $\pi$-shifted

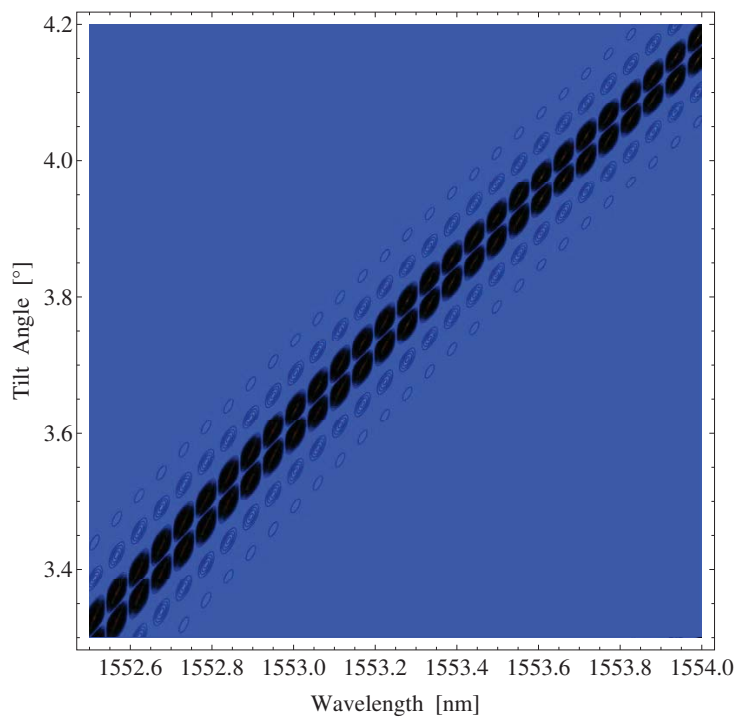

(c) $\pi$-shifted

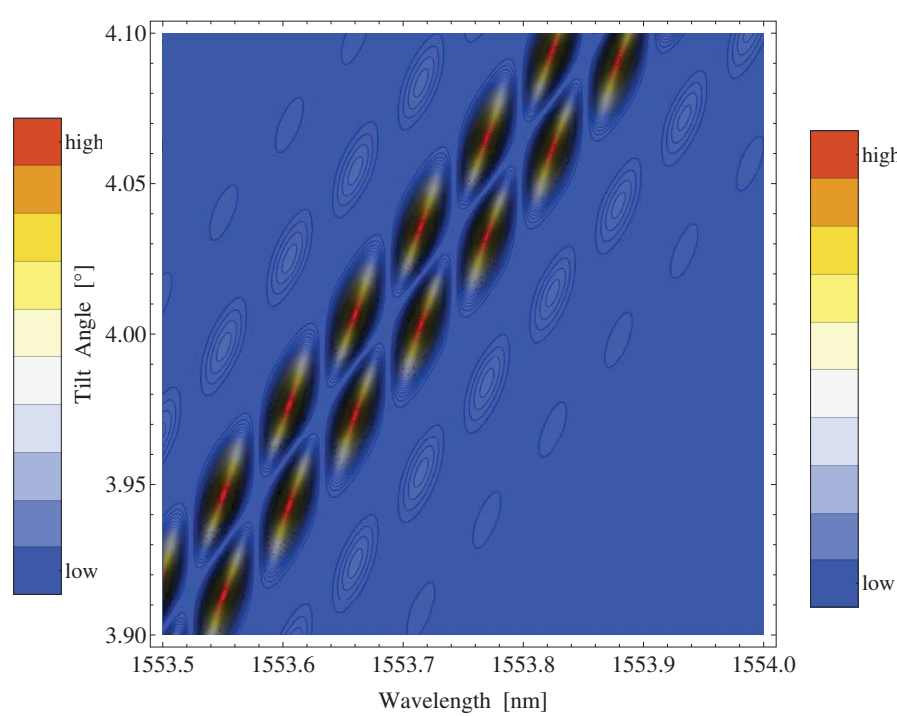

(b) $\pi$-shifted

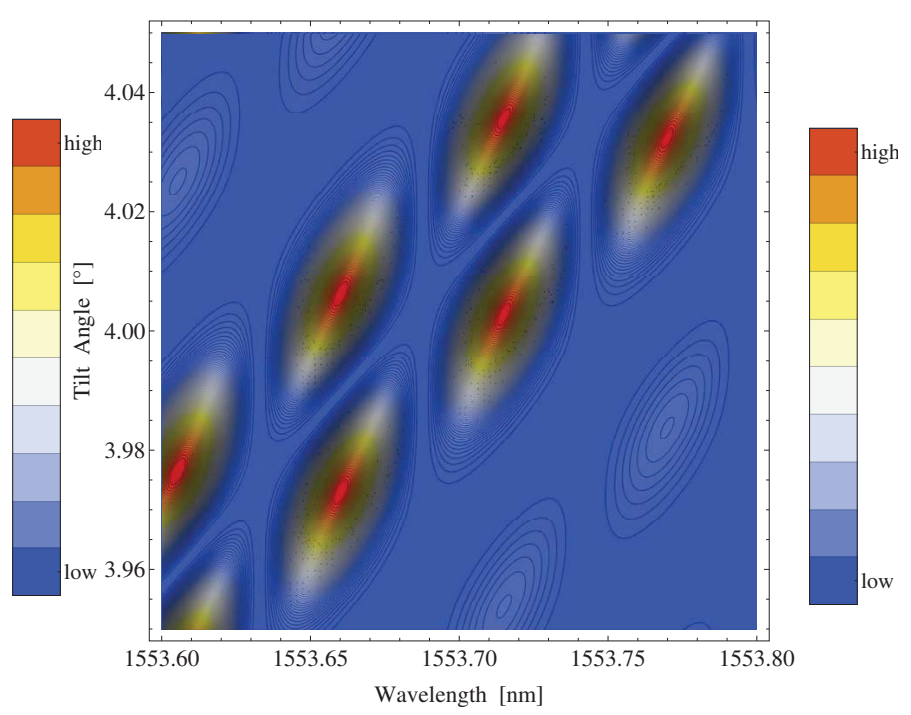

(d) $\pi$-shifted 


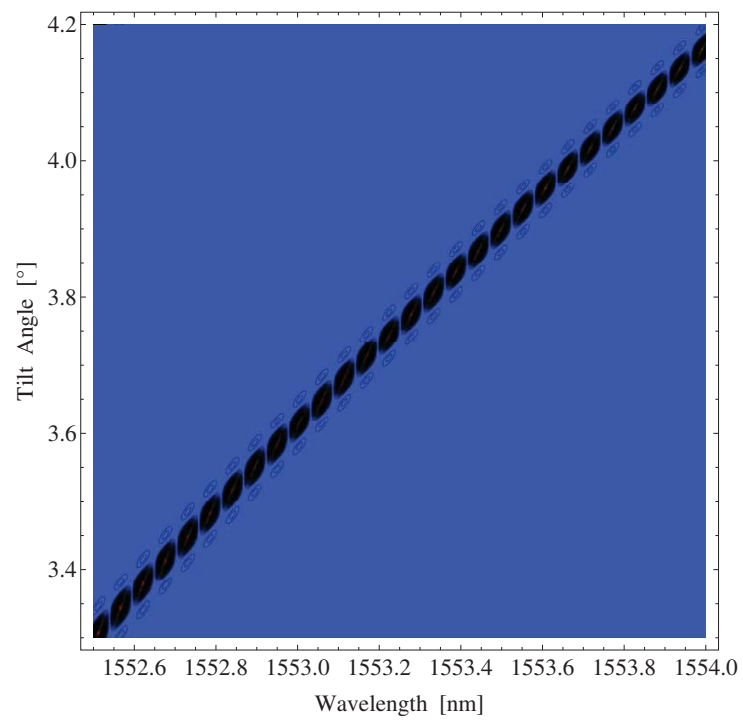

(e) uniform

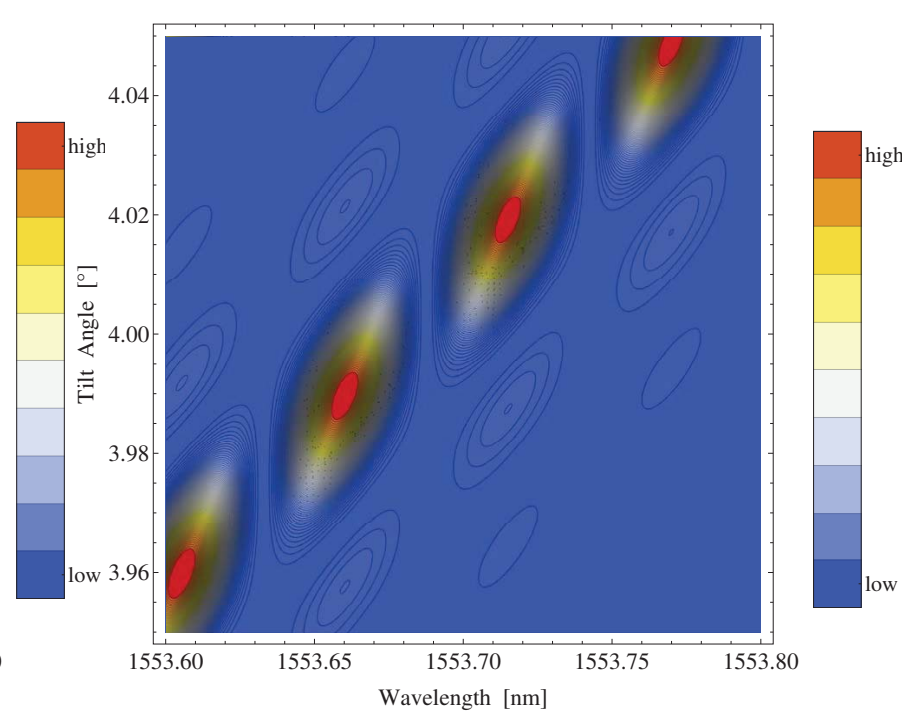

(f) uniform

Figure 6.23: Contour plot of power carried by plasmon excited by a (uniform and $\pi$-shifted) tilted grating in a fiber coated by Au distribution positioned in an index matching oil (with refractive index $n_{4}=1.42$ ) environment versus wavelength and tilt angle. Simulation parameters: azimuthal index of the mode $m=3$; wavelength $\lambda=1550 \mathrm{~nm}$; grating is tuned for coupling plasmon mode and core mode at resonance wavelength $\lambda_{\text {res }}=1550 \mathrm{~nm}$; thickness of $\mathrm{Au}$ distribution $d=100 \mathrm{~nm}$; angular width of distribution $\left(\theta_{\min }, \theta_{\max }\right)$ : $\theta_{\min }=-\pi / 10, \theta_{\max }=\pi / 10$, array length $2 z_{0}=2 \mathrm{~cm}$; distribution type: $\pi$-shifted array of patches; AC grating $\Delta \varepsilon_{A C}=3 \times 10^{-4}$. Au distribution parameters: $\delta z=50 \mathrm{~nm}$, $\Delta z=1 \mathrm{~cm}$.

By inspecting the following figures: Fig. 6.14(a-e) with Fig. 6.22(a-e) and Fig. 6.16(a-e) with Fig. 6.23(a-e), where Fig. 6.14, 6.16 represent an uniform array of patches distribution while Fig. 6.22, 6.23 represent a $\pi$-shifted array of patches distribution, it can be concluded that metal distribution with a certain symmetry in physical space is mapped into some symmetry of spectroscopy pattern in $(\lambda, \alpha)$-space due to an interplay of parameters $\delta z$, $\Delta z, z_{0}$.

Small perturbation of environment, i.e. perturbation of certain physical properties in the outer layer, causes a shift of an intensity plot along a wavelength axis. Detecting such shift is easier near the peak intensity rather than far outside of the peak, see Fig. 6.24(a). Therefore to characterize the outer perturbation it is sufficient to measure an intensity change at a given wavelength near the peak. A region with a greater slope $\left|\frac{\Delta I}{\Delta \lambda}\right|$ provides a larger precision in evaluating the outer perturbation. If an existing peak is divided in the middle in two pieces by a deep cut, the region near such cut has a greater slope than the slope near original peak, see Fig. 6.24(b). Since a $\pi$-shift in a series of metal patches distributed on a fiber cladding provides a spectroscopy pattern where every local peak is divided by a deep cut and thus favors the higher precision measurements of environmental perturbation. Therefore a $\pi$-shifted metal distributed is a useful tool in sensor like fiber 

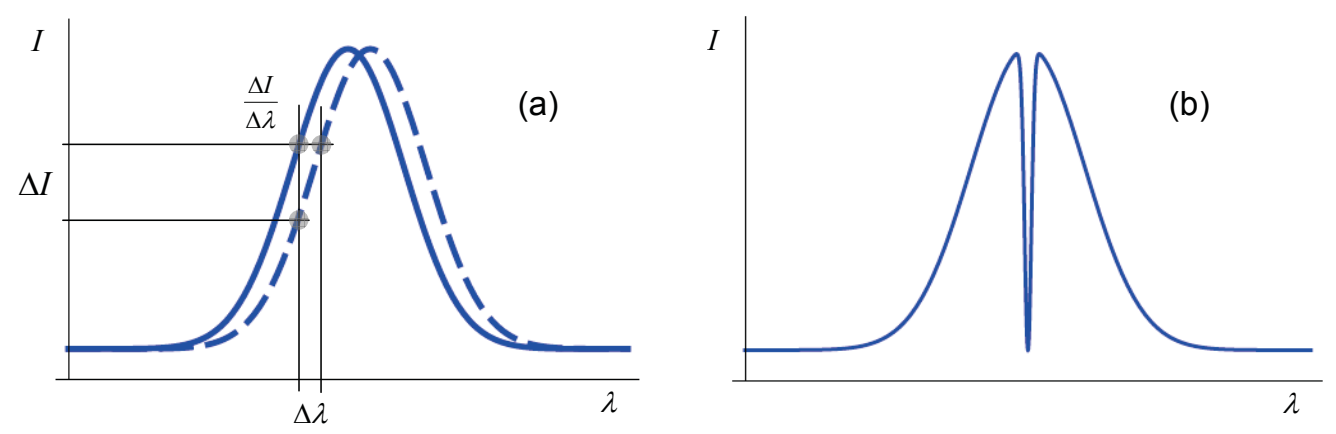

Figure 6.24: Dependence intensity vs. wavelength $I(\lambda)$ suitable for detecting small external perturbation.

application.

Assuming that tilted Bragg grating is written using the same photomask with spacing $\Lambda$, the grating period along $z$-axis is tilt angle dependent, $\Delta_{z}=\Lambda / \cos \alpha$. This makes azimuthal angle $\theta$ and tilt angle $\alpha$ coupled in an array factor $A F$ of sine grating unlike the case of an array of discrete current sources (set of rings or discs) where $\theta$ and $\alpha$ are not coupled, i.e. $A F$ is a product of two terms one depends on $\theta$ the other depends on $\alpha$. As a result small variation of a tilt angle $\alpha$ causes significant change of azimuthal dependence of the field. This could be useful in biosensor plasmon application provided tiny biosample can be precisely positioned at a particular azimuthal angle. The polar plot of normal component of cumulative electric field (its absolute value $\left|E_{r}(\theta)\right|$ ) over the four modes $(m=2 \ldots 5)$ is shown in Fig. 6.25. 


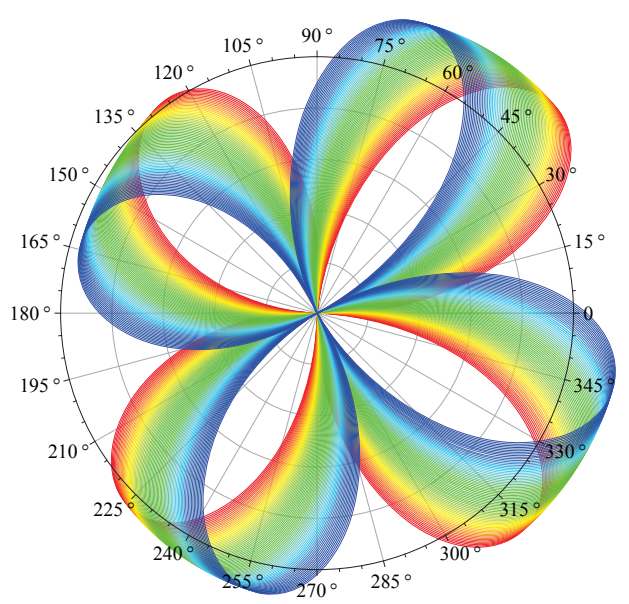

(a) $\lambda=1549 \mathrm{~nm}, d=29 \mathrm{~nm}$

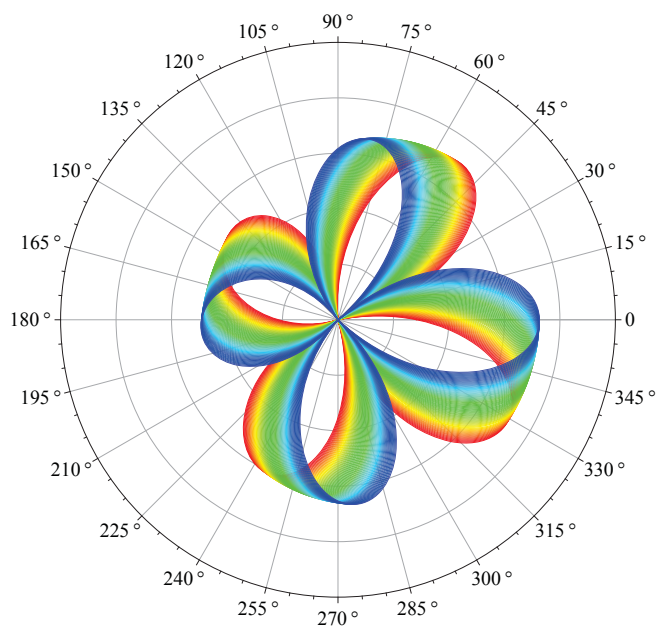

(c) $\lambda=1549 \mathrm{~nm}, d=31 \mathrm{~nm}$

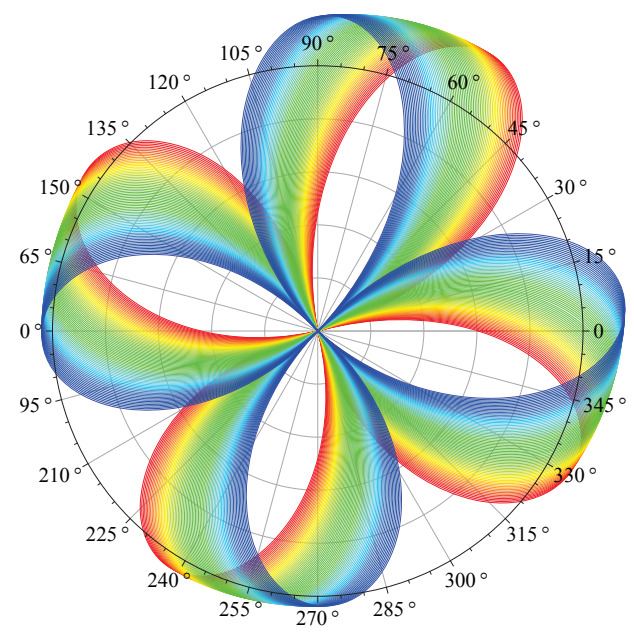

(e) $\lambda=1550 \mathrm{~nm}, d=30 \mathrm{~nm}$
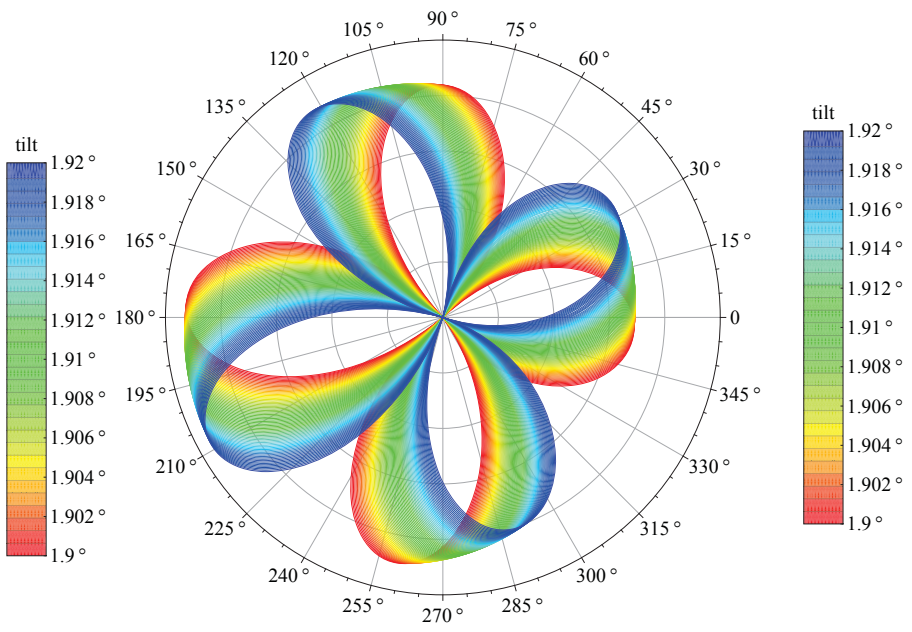

(b) $\lambda=1549 \mathrm{~nm}, d=30 \mathrm{~nm}$
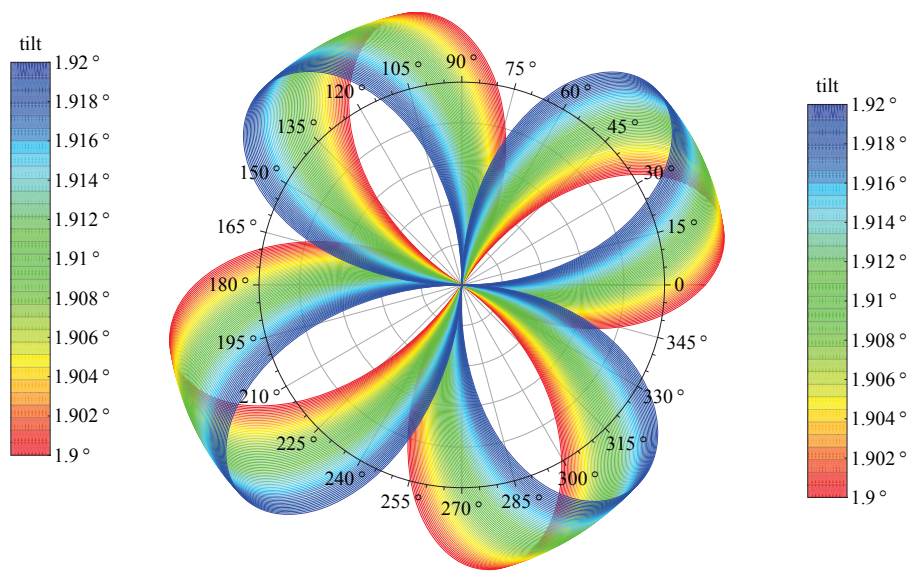

(d) $\lambda=1550 \mathrm{~nm}, d=29 \mathrm{~nm}$

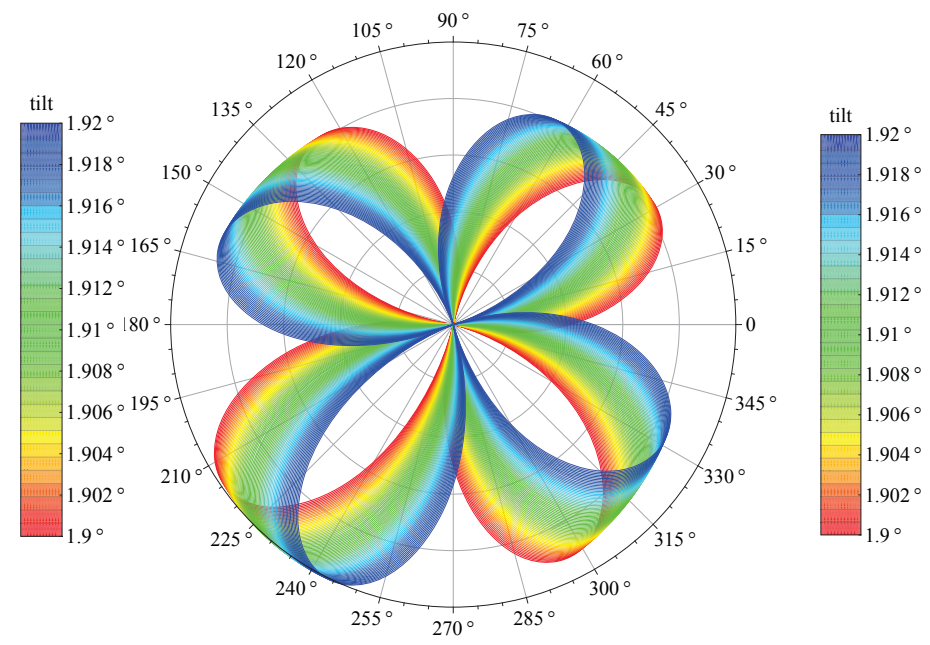

(f) $\lambda=1550 \mathrm{~nm}, d=31 \mathrm{~nm}$ 


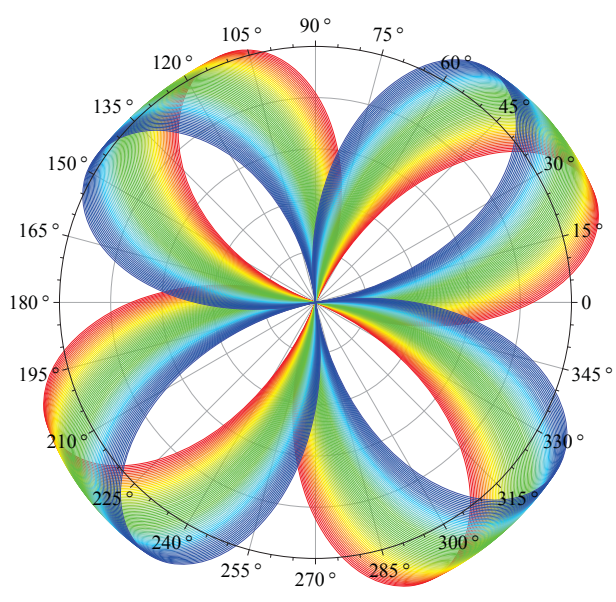

(g) $\lambda=1551 \mathrm{~nm}, d=29 \mathrm{~nm}$

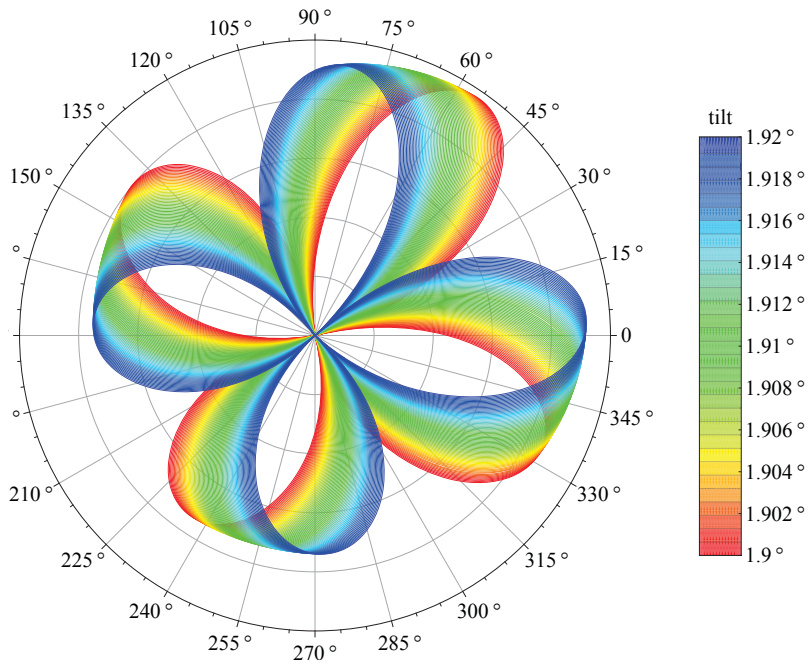

(i) $\lambda=1551 \mathrm{~nm}, d=31 \mathrm{~nm}$

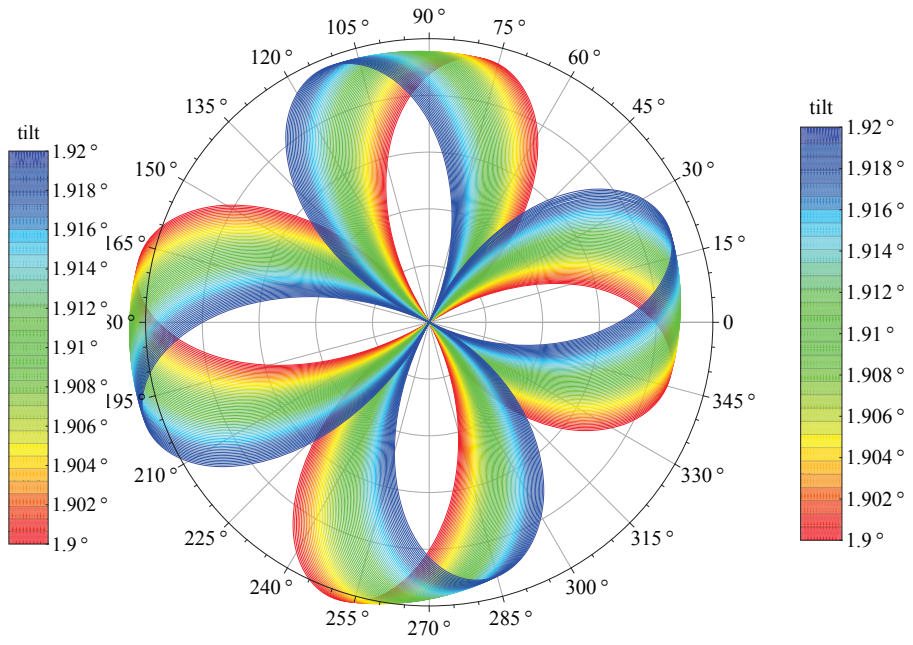

(h) $\lambda=1551 \mathrm{~nm}, \mathrm{~d}=30 \mathrm{~nm}$

Figure 6.25: Polar plot of an absolute value of a normal component of cumulative electric field $\left|E_{r}\right|$ over 4 azimuthal indices, $m=2 \ldots 5$ of plasmons excited by a source of $\pi$ shifted tilted grating of a fiber coated by thin $\mathrm{Au}$ layer positioned in air environment versus azimuthal angle $\theta$ with the following parameters: tilt angle $\alpha$, film thickness $d$ and wavelength $\lambda$. The grating is tuned for coupling plasmon mode with azimuthal index $m=3$ and core mode at resonance wavelength $\lambda_{\text {res }}=1550 \mathrm{~nm}$ and resonance film thickness $d_{\text {res }}=30 \mathrm{~nm}$. Field is measured at the middle of an the grating. Scale is arbitrary, but fixed within the same wavelength.

Throughout numerical simulation it was assumed that, as in part 1, refractive indices of the fiber core, cladding and metal layer are wavelength dependent, while refractive index of the outer layer is constant. The data for wavelength dependent refractive index of gold and platinum was taken from [52]. Wavelength dependent refractive index of fused silica calculated with Sellmeier coefficients from [91]. 


\subsection{Conclusion.}

Plasmon excitation problem due to a continuous, uniform or $\pi$-shifted, tilted Bragg grating in the core of a fiber coated either by thin metal film or metal distribution was solved from the Maxwell's equations as a scattering within first order approximation. Extension to higher order approximations is straightforward but computationally more expensive. In the end of a calculation a power carried by a plasmon mode along fiber is obtained which is a measurable quantity. Such model can found its use for a sensor like fiber application. 


\section{Chapter 7}

\section{Wavelet-Galerkin analysis of metal coated step-index fiber}

For a certain type of problems the prior knowledge of modal indices is crucial. Plasmon optical excitation in a waveguide when loss factor can not be ignored is one of them. Finding modal indices involves finding complex roots of a waveguide characteristic equation of the form $\operatorname{det} M=0$. Various root search algorithms can handle such task well, for example "Mathematica" routine "FindRoot" combines several search algorithms and is capable of finding complex root of transcendental equation for a 4-layer structure with an arbitrary precision, provided proper starting value of the root is given. One of the approach that can provide good initial guess value for a large number of modal indices consists of discretizing the original wave equation into either a matrix eigenvalue equation or a matrix generalized eigenvalue equation. The available methods are finite difference, finite element, spectral, Galerkin and some combinations of them. The standard Galerkin method with sine functions as orthogonal expansion functions applied to a vector wave equation for a lossless waveguide gives accurate results for both modal indices and field distributions within moderate number of grids, [94]. The drawback of trigonometric functions describing field distribution for guided modes is that they are not vanishing at infinity. The better choice is Hermit-Gauss (HG) functions, as they automatically satisfy required boundary condition for the fields at infinity. The HG functions were employed as the expansion functions in $[95,96]$; because of their better properties, there are fewer terms with the HG needed than with sine functions to satisfy given precision. Another possible choice of expansion is wavelets, the locally non-zero functions $[97,98]$. Many particular forms of wavelets suited for various application areas are discovered so far [98] and many kept being discovered on a regular basis. One of such recently presented function in [99] truncated Gaussian wavelet (TWG), demonstrated its ability to run generalized eigenvalue problem in a combination with the Galerkin method more efficiently than the HG functions defined over whole domain. For a smaller number of grids $N$, the TWG based test runs order of magnitude faster than the $\mathrm{HG}$ and it scales with $N$ linearly, whereas the HG scales exponentially. The speed advantage is attributed to a matrix property. Since the wavelet basis functions having non-zero value only locally while conventional basis functions being 
non-zero over the whole computational domain, the generated matrices are naturally different: sparse for wavelets, the TGW versus dense for non-wavelets, the HG. As pointed out in [99], the wavelet Galerkin method in comparison with finite difference and finite element methods was analyzed in [100]. It was found that accuracy of Galerkin method is superior for elliptic, parabolic, and hyperbolic problems.

\subsection{Piecewise cosine wavelets.}

The TGW at both of its ends is zero with non-zero derivative. This is an issue in application to a step index fiber where the fields and derivatives must be zero at the origin for most of the modes. To correct this issue a piecewise cosine wavelets are proposed. Function $y(x)$ made up of cosine spanning one cycle of oscillation from 0 to 1 and back to 0 when its argument running over the interval $\left(x_{a}, x_{b}\right)$ can be used as a basis function for wavelet; outside of the interval it is identical 0 and described by,

$$
y(x)=\left\{\begin{array}{cc}
\frac{1}{2}\left(1+\cos \left(\pi \frac{2 x-x_{b}-x_{a}}{x_{b}-x_{a}}\right)\right) & x_{a} \leq x \leq x_{b} \\
0 & x<x_{a}, \quad x>x_{b}
\end{array}\right.
$$

At the ends of the interval $\left(x_{a}, x_{b}\right)$ both function and first derivative equal to zero. Plot $y(x)$ is shown in Fig. 7.1 where the interval $\left(x_{a}, x_{b}\right)$ denoted as $\left(x_{i}, x_{i+s}\right)$, it covers $s$ grids or $(s+1)$ nodes of the computational domain. For a computational domain with $N$ grids or $(N+1)$ nodes, the total number of cosine wavelets needed to span this domain is $(N+1-s)$, see Fig. 7.2.

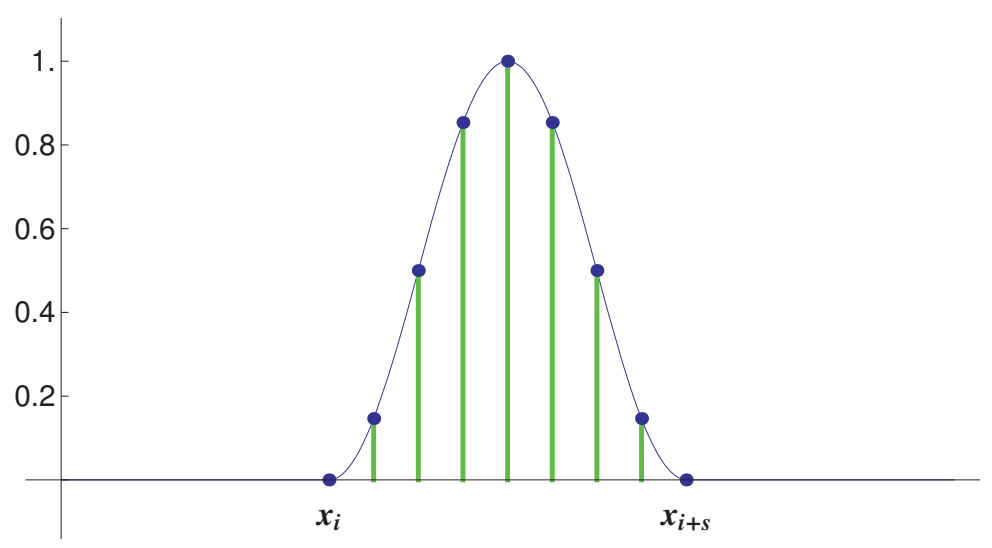

Figure 7.1: Wavelet based on cosine function where number of segments (grids) spanned by wavelet.

\subsection{Generalized eigenvalue wave equation.}

Maxwell's equations for an inhomogeneous media that has metal layer contain the source terms, i.e. charge density $\rho_{f}$ due to free charge of the metal and induced current density 


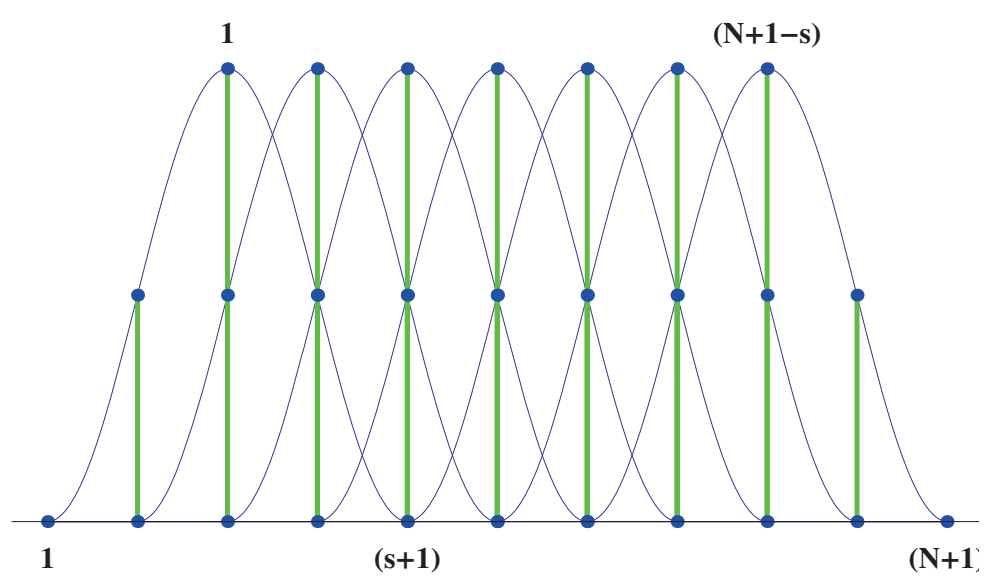

Figure 7.2: Train of wavelets over the computational domain where $N$ number of grids and $(N+1-s)$ number of wavelets of the whole domain.

$\vec{J}_{f}$. Permittivity of the media so far is a pure real quantity. The wave equations for electric and magnetic fields in an inhomogeneous non-magnetic media derived from the Maxwell's equations are,

$$
\begin{gathered}
\nabla^{2} \vec{E}+k^{2} \vec{E}-\nabla(\nabla \cdot \vec{E})=0 \\
\nabla^{2} \vec{H}+k^{2} \vec{H}+(\nabla \ln \varepsilon) \times(\nabla \times \vec{H})=0
\end{gathered}
$$

where permittivity is a complex quantity and the source terms are absorbed by the non-zero imaginary part of permittivity of a metal layer. It is assumed that time and $z$ dependence of the field is given by $e^{i(\beta z-\omega t)}$ for $z$ being direction of propagation and azimuthal dependence is given by $e^{i m \theta}$, where $\beta$ propagation constant, $m$ azimuthal modal index and $\theta$ azimuthal angle in the fiber. The vector wave equations split into the following scalar coupled wave equations,

$$
\begin{gathered}
\nabla^{2} E_{r}-\frac{1}{r^{2}} E_{r}-\frac{2}{r^{2}} \frac{\partial E_{\theta}}{\partial \theta}+k^{2} E_{r}+\frac{\partial}{\partial r}\left(E_{r} \frac{1}{\varepsilon} \frac{\partial \varepsilon}{\partial r}\right)=0 \\
\nabla^{2} E_{\theta}-\frac{1}{r^{2}} E_{\theta}+\frac{2}{r^{2}} \frac{\partial E_{r}}{\partial \theta}+k^{2} E_{\theta}+\frac{1}{r} \frac{\partial}{\partial \theta}\left(E_{r} \frac{1}{\varepsilon} \frac{\partial \varepsilon}{\partial r}\right)=0 \\
\nabla^{2} E_{z}+k^{2} E_{z}+\frac{\partial}{\partial z}\left(E_{r} \frac{1}{\varepsilon} \frac{\partial \varepsilon}{\partial r}\right)=0 \\
\nabla^{2} H_{r}-\frac{1}{r^{2}} H_{r}-\frac{2}{r^{2}} \frac{\partial H_{\theta}}{\partial \theta}+k^{2} H_{r}=0
\end{gathered}
$$




$$
\begin{gathered}
\nabla^{2} H_{\theta}-\frac{1}{r^{2}} H_{\theta}+\frac{2}{r^{2}} \frac{\partial H_{r}}{\partial \theta}+k^{2} H_{\theta}-\frac{1}{\varepsilon} \frac{\partial \varepsilon}{\partial r}\left(\frac{\partial H_{\theta}}{\partial r}+\frac{H_{\theta}}{r}-\frac{\partial H_{r}}{\partial \theta}\right)=0 \\
\nabla^{2} H_{z}+k^{2} H_{z}+\frac{1}{\varepsilon} \frac{\partial \varepsilon}{\partial r}\left(\frac{\partial H_{r}}{\partial z}-\frac{\partial H_{z}}{\partial r}\right)=0
\end{gathered}
$$

Equations involved only transverse field components, $E_{\theta}, E_{r}, H_{\theta}, H_{r}$ can be reduced to four scalar wave equations in terms of radial field functions $e_{\theta}(r), e_{r}(r), h_{\theta}(r), h_{r}(r)$,

$$
\begin{gathered}
\left(\frac{\partial^{2}}{\partial r^{2}}+\frac{1}{r} \frac{\partial}{\partial r}\right) e_{r}-\frac{m^{2}+1}{r^{2}} e_{r}-\frac{i 2 m}{r^{2}} e_{\theta}+\left(k^{2}-\beta^{2}\right) e_{r}+\frac{\partial}{\partial r}\left(e_{r} \frac{1}{\varepsilon} \frac{\partial \varepsilon}{\partial r}\right)=0 \\
\left(\frac{\partial^{2}}{\partial r^{2}}+\frac{1}{r} \frac{\partial}{\partial r}\right) e_{\theta}-\frac{m^{2}+1}{r^{2}} e_{\theta}+\frac{i 2 m}{r^{2}} e_{r}+\left(k^{2}-\beta^{2}\right) e_{\theta}+\frac{i m}{r} e_{r} \frac{1}{\varepsilon} \frac{\partial \varepsilon}{\partial r}=0 \\
\frac{\partial^{2} h_{r}}{\partial r^{2}}+\frac{1}{r} \frac{\partial h_{r}}{\partial r}-\frac{\left(m^{2}+1\right)}{r^{2}} h_{r}-i m \frac{2}{r^{2}} h_{\theta}+\left(k^{2}-\beta^{2}\right) h_{r}=0 \\
\frac{\partial^{2} h_{\theta}}{\partial r^{2}}+\frac{1}{r} \frac{\partial h_{\theta}}{\partial r}-\frac{\left(m^{2}+1\right)}{r^{2}} h_{\theta}+i m \frac{2}{r^{2}} h_{r}+\left(k^{2}-\beta^{2}\right) h_{\theta}-\frac{1}{\varepsilon} \frac{\partial \varepsilon}{\partial r}\left(\frac{\partial h_{\theta}}{\partial r}+\frac{h_{\theta}}{r}-i m h_{r}\right)=0
\end{gathered}
$$

where dielectric constant has radial dependence only, i.e. $\varepsilon=\varepsilon(r)$. Numerical solution of the wave equations in $r$-domain requires either simply truncating computational domain at some sufficiently large distance away from the metal coating [94] or imposing artificially absorbing boundary conditions, ABC ( $[101,102])$ or perfectly matching layer, PML ( [103, 104]) at a smaller distance to reduce / prevent reflection back to the computational domain. Both concepts $\mathrm{ABC}$ and PML proved to be quite effective tools for solving wave equations in electromagnetics, [105-112]. Another approach to abolish an unwanted reflection is the domain transformation where infinite $r$-domain $r:(0, \infty)$ is transformed/mapped into finite $\tau$-domain $\tau:(0, T)$ by some map function $u^{-1},[113-117]$. With substitutions for $r$ and first and second derivatives with respect to $r$,

$$
r=u(\tau), \quad \frac{d}{d r}=\frac{1}{u^{\prime}} \frac{d}{d \tau}, \quad \frac{d^{2}}{d r^{2}}=\frac{1}{\left(u^{\prime}\right)^{2}} \frac{d^{2}}{d \tau^{2}}-\frac{u^{\prime \prime}}{\left(u^{\prime}\right)^{3}} \frac{d}{d \tau}
$$

the wave equations in $\tau$-domain for electric fields become,

$$
\left(\frac{1}{\left(u^{\prime}\right)^{2}} \frac{d^{2}}{d \tau^{2}}-\frac{u^{\prime \prime}}{\left(u^{\prime}\right)^{3}} \frac{d}{d \tau}\right) e_{r}+\frac{1}{u} \frac{1}{u^{\prime}} \frac{d}{d \tau} e_{r}-\frac{m^{2}+1}{u^{2}} e_{r}-\frac{i 2 m}{u^{2}} e_{\theta}+\left(k^{2}-\beta^{2}\right) e_{r}+\frac{1}{u^{\prime}} \frac{d}{d \tau}\left(e_{r} \frac{1}{\varepsilon} \frac{1}{u^{\prime}} \frac{d \varepsilon}{d \tau}\right)=0
$$




$$
\left(\frac{1}{\left(u^{\prime}\right)^{2}} \frac{d^{2}}{d \tau^{2}}-\frac{u^{\prime \prime}}{\left(u^{\prime}\right)^{3}} \frac{d}{d \tau}\right) e_{\theta}+\frac{1}{u} \frac{1}{u^{\prime}} \frac{d}{d \tau} e_{\theta}-\frac{m^{2}+1}{u^{2}} e_{\theta}+\frac{i 2 m}{u^{2}} e_{r}+\left(k^{2}-\beta^{2}\right) e_{\theta}+\frac{i m}{u} e_{r} \frac{1}{\varepsilon} \frac{1}{u^{\prime}} \frac{d \varepsilon}{d \tau}=0
$$

Equations 7.15 and 7.16 can be written schematically as:

$$
\begin{aligned}
& L_{1} e_{r}+L_{2} e_{\theta}=\beta^{2} e_{r} \\
& L_{3} e_{r}+L_{4} e_{\theta}=\beta^{2} e_{\theta}
\end{aligned}
$$

where linear operators $L_{1}, L_{2}, L_{3}, L_{4}$ defined as,

$$
\begin{gathered}
L_{1} e_{r}=\left(\frac{1}{\left(u^{\prime}\right)^{2}} \frac{d^{2}}{d \tau^{2}}-\frac{u^{\prime \prime}}{\left(u^{\prime}\right)^{3}} \frac{d}{d \tau}\right) e_{r}+\frac{1}{u} \frac{1}{u^{\prime}} \frac{d}{d \tau} e_{r}-\frac{\left(m^{2}+1\right)}{u^{2}} e_{r}+k^{2} e_{r}+\frac{1}{u^{\prime}} \frac{d}{d \tau}\left(\frac{1}{u^{\prime}} \frac{1}{\varepsilon} \frac{d \varepsilon}{d \tau} e_{r}\right) \\
L_{2} e_{\theta}=-i m \frac{2}{u^{2}} e_{\theta} \\
L_{3} e_{r}=i m \frac{2}{u^{2}} e_{r}+i m \frac{1}{\varepsilon} \frac{1}{u} \frac{1}{u^{\prime}} \frac{d \varepsilon}{d \tau} e_{r} \\
L_{4} e_{\theta}=\left(\frac{1}{\left(u^{\prime}\right)^{2}} \frac{d^{2}}{d \tau^{2}}-\frac{u^{\prime \prime}}{\left(u^{\prime}\right)^{3}} \frac{d}{d \tau}\right) e_{\theta}+\frac{1}{u} \frac{1}{u^{\prime}} \frac{d}{d \tau} e_{\theta}-\frac{\left(m^{2}+1\right)}{u^{2}} e_{\theta}+k^{2} e_{\theta}
\end{gathered}
$$

Expanding fields in basis functions $\varphi_{i}(\tau)$ as follows,

$$
\begin{aligned}
& e_{r}(\tau)=\sum_{i=1}^{N} P_{1 i} \varphi_{i}(\tau) \\
& e_{\theta}(\tau)=\sum_{i=1}^{N} Q_{1 i} \varphi_{i}(\tau)
\end{aligned}
$$

and plugging in expansions for $e_{r}(\tau)$ and $e_{\theta}(\tau)$ the two equations for electric fields can be written as:

$$
\begin{aligned}
& L_{1} \sum_{i=1}^{N} P_{1 i} \varphi_{i}(\tau)+L_{2} \sum_{i=1}^{N} Q_{1 i} \varphi_{i}(\tau)=\beta^{2} \sum_{i=1}^{N} P_{1 i} \varphi_{i}(\tau) \\
& L_{3} \sum_{i=1}^{N} P_{1 i} \varphi_{i}(\tau)+L_{4} \sum_{i=1}^{N} Q_{1 i} \varphi_{i}(\tau)=\beta^{2} \sum_{i=1}^{N} Q_{1 i} \varphi_{i}(\tau)
\end{aligned}
$$


where $\varphi_{i}(\tau)$ is a cosine wavelet. Taking inner product by multiplying by $\varphi_{j}^{*}(\tau)$ and integrating over the whole interval of computational $\tau$-domain both equations take the following form,

$$
\begin{aligned}
& \int_{0}^{T} \varphi_{j}^{*}(\tau) L_{1} \sum_{i=1}^{N} P_{1 i} \varphi_{i}(\tau) d \tau+\int_{0}^{T} \varphi_{j}^{*}(\tau) L_{2} \sum_{i=1}^{N} Q_{1 i} \varphi_{i}(\tau) d \tau=\beta^{2} \int_{0}^{T} \varphi_{j}^{*}(\tau) \sum_{i=1}^{N} P_{1 i} \varphi_{i}(\tau) d \tau \\
& j=1, \ldots N
\end{aligned}
$$

$$
\begin{aligned}
& \int_{0}^{T} \varphi_{j}^{*}(\tau) L_{3} \sum_{i=1}^{N} P_{1 i} \varphi_{i}(\tau) d \tau+\int_{0}^{T} \varphi_{j}^{*}(\tau) L_{4} \sum_{i=1}^{N} Q_{1 i} \varphi_{i}(\tau) d \tau=\beta^{2} \int_{0}^{T} \varphi_{j}^{*}(\tau) \sum_{i=1}^{N} Q_{1 i} \varphi_{i}(\tau) d \tau \\
& j=1, \ldots N
\end{aligned}
$$

where $T$ is the upper limit of $\tau$-domain that corresponds to infinity in $r$-domain, $N$ is the number of grids in $\tau$-domain $(0, T)$. The above two sets of algebraic equations can be conveniently written in matrix form as,

$$
\left|\begin{array}{ll}
\widehat{L}_{1} & \widehat{L}_{2} \\
\widehat{L}_{3} & \widehat{L}_{4}
\end{array}\right|\left|\begin{array}{c}
P_{1} \\
Q_{1}
\end{array}\right|=\beta^{2}\left|\begin{array}{cc}
B & 0 \\
0 & B
\end{array}\right|\left|\begin{array}{c}
P_{1} \\
Q_{1}
\end{array}\right|
$$

where $P_{1}$ and $Q_{1}$ are column vectors each of size $N$ of expansion coefficients in $\varphi_{i}(\tau)$ for $e_{r}(\tau)$ and $e_{\theta}(\tau)$ respectively and $\widehat{L}_{1}, \widehat{L}_{2}, \widehat{L}_{3}, \widehat{L}_{4}, B$ are $N \times N$ matrices whose elements evaluated as,

$$
\begin{aligned}
& \int_{0}^{T} \varphi_{j}^{*} L_{1} \varphi_{i} d \tau=\widehat{L}_{1 j i} \\
& \int_{0}^{T} \varphi_{j}^{*} L_{2} \varphi_{i} d \tau=\widehat{L}_{2 j i} \\
& \int_{0}^{T} \varphi_{j}^{*} L_{3} \varphi_{i} d \tau=\widehat{L}_{3 j i} \\
& \int_{0}^{T} \varphi_{j}^{*} L_{4} \varphi_{i} d \tau=\widehat{L}_{4 j i} \\
& \int_{0}^{T} \varphi_{j}^{*} \varphi_{i} d \tau=B_{j i}
\end{aligned}
$$


Equations for magnetic fields in domain transformed as,

$$
\begin{gathered}
\left(\frac{1}{\left(u^{\prime}\right)^{2}} \frac{d^{2}}{d \tau^{2}}-\frac{u^{\prime \prime}}{\left(u^{\prime}\right)^{3}} \frac{d}{d \tau}\right) h_{r}+\frac{1}{u} \frac{1}{u^{\prime}} \frac{d}{d \tau} h_{r}-\frac{\left(m^{2}+1\right)}{u^{2}} h_{r}-i m \frac{2}{u^{2}} h_{\theta}+\left(k^{2}-\beta^{2}\right) h_{r}=0 \\
\left(\frac{1}{\left(u^{\prime}\right)^{2}} \frac{d^{2}}{d \tau^{2}}-\frac{u^{\prime \prime}}{\left(u^{\prime}\right)^{3}} \frac{d}{d \tau}\right) h_{\theta}+\frac{1}{u} \frac{1}{u^{\prime}} \frac{d}{d \tau} h_{\theta}-\frac{\left(m^{2}+1\right)}{u^{2}} h_{\theta}+i m \frac{2}{u^{2}} h_{r}+ \\
+\left(k^{2}-\beta^{2}\right) h_{\theta}-\frac{1}{\varepsilon} \frac{1}{u^{\prime}} \frac{d \varepsilon}{d \tau}\left(\frac{1}{u^{\prime}} \frac{d}{d \tau} h_{\theta}+\frac{h_{\theta}}{u}-i m h_{r}\right)=0
\end{gathered}
$$

Similarly the unified matrix form of both equation is,

$$
\left|\begin{array}{cc}
\widehat{L}_{5} & \widehat{L}_{6} \\
\widehat{L}_{7} & \widehat{L}_{8}
\end{array}\right|\left|\begin{array}{c}
P_{2} \\
Q_{2}
\end{array}\right|=\beta^{2}\left|\begin{array}{cc}
B & 0 \\
0 & B
\end{array}\right|\left|\begin{array}{c}
P_{2} \\
Q_{2}
\end{array}\right|
$$

where elements of matrices calculated by,

$$
\begin{aligned}
& \int_{0}^{T} \varphi_{j}^{*} L_{5} \varphi_{i} d \tau=\widehat{L}_{5 j i} \\
& \int_{0}^{T} \varphi_{j}^{*} L_{6} \varphi_{i} d \tau=\widehat{L}_{6 j i} \\
& \int_{0}^{T} \varphi_{j}^{*} L_{7} \varphi_{i} d \tau=\widehat{L}_{7 j i} \\
& \int_{0}^{T} \varphi_{j}^{*} L_{8} \varphi_{i} d \tau=\widehat{L}_{8 j i}
\end{aligned}
$$

magnetic fields expanded in wavelets as,

$$
\begin{aligned}
& h_{r}(\tau)=\sum_{i=1}^{N} P_{2 i} \varphi_{i}(\tau) \\
& h_{\theta}(\tau)=\sum_{i=1}^{N} Q_{2 i} \varphi_{i}(\tau)
\end{aligned}
$$

and linear operators $L_{5}, L_{6}, L_{7}, L_{8}$ defined as, 


$$
\begin{gathered}
L_{5} h_{r}=\left(\frac{1}{\left(u^{\prime}\right)^{2}} \frac{d^{2}}{d \tau^{2}}-\frac{u^{\prime \prime}}{\left(u^{\prime}\right)^{3}} \frac{d}{d \tau}\right) h_{r}+\frac{1}{u} \frac{1}{u^{\prime}} \frac{d}{d \tau} h_{r}-\frac{\left(m^{2}+1\right)}{u^{2}} h_{r}+k^{2} h_{r} \\
L_{6} h_{\theta}=-i m \frac{2}{u^{2}} h_{\theta} \\
L_{7} h_{r}=i m \frac{2}{u^{2}} h_{r}+i m \frac{1}{\varepsilon} \frac{1}{u^{\prime}} \frac{d \varepsilon}{d \tau} h_{r} \\
L_{8} h_{\theta}=\left(\frac{1}{\left(u^{\prime}\right)^{2}} \frac{d^{2}}{d \tau^{2}}-\frac{u^{\prime \prime}}{\left(u^{\prime}\right)^{3}} \frac{d}{d \tau}\right) h_{\theta}+\frac{1}{u} \frac{1}{u^{\prime}} \frac{d}{d \tau} h_{\theta}-\frac{\left(m^{2}+1\right)}{u^{2}} h_{\theta}+k^{2} h_{\theta}- \\
-\frac{1}{\varepsilon} \frac{1}{u^{\prime}} \frac{d \varepsilon}{d \tau}\left(\frac{1}{u^{\prime}} \frac{d}{d \tau} h_{\theta}+\frac{h_{\theta}}{u}\right)
\end{gathered}
$$

All the integrals $\widehat{L}_{j i}$ are technically taken over the whole computational domain $(0, T)$, however due to a piecewise nature of wavelet they are non-zero only over the overlap interval of two wavelets in the corresponding integrands. This is the reason the matrices in 7.29 and 7.37 are sparse. Such overlap interval schematically shown in Fig. 7.3. Matrices in left hand side of 7.29 and 7.37 have three bands: diagonal, upper subdiagonal and lower subdiagonal, while the matrix in right hand side has one diagonal band. The width of each band depends on parameter $s$, the number of grids spanned by a wavelet. Integral involving derivative of a piecewise function, needed for evaluation $\widehat{L}_{j i}$, is treated in Appendix E.

For a homogeneous waveguide with $s=1$ the matrices in the left hand side of 7.29, 7.37 are self-adjoint, therefore all the eigenvalues are real. Since step index fiber is only piecewise homogeneous, the non-zero terms $\frac{d \varepsilon}{d r}$ at interface between adjacent layers break down the self-adjointness of the above matrices. It follows, that eigenvalues of the matrices are no longer all real quantities even with the real permittivity in each layer. This leads to a conclusion, the lossless inhomogeneous waveguide possesses modal indices with non-zero imaginary parts. The topic is covered in great details and generality in [118].

\subsection{Generalized eigenvalue wave equation.}

Particular choice of mapping transformation affects the matrix elements 7.30-7.33, 7.387.41. Various map functions $u(\tau)$ are possible: algebraic map, logarithmical [119], trigonometric map [113]- [117]. The main limiting factor of the use of map function is that it complicates the wave equations (compare equations 7.10-7.13 and 7.15, 7.16, 7.35, 7.36) and integrands in 7.30-7.33 and 7.38-7.41 to the point where the corresponding integrals become difficult for analytical evaluation. Numerical integration on the other hand are both computationally expensive and an additional source of errors. However, the map function in the form, 

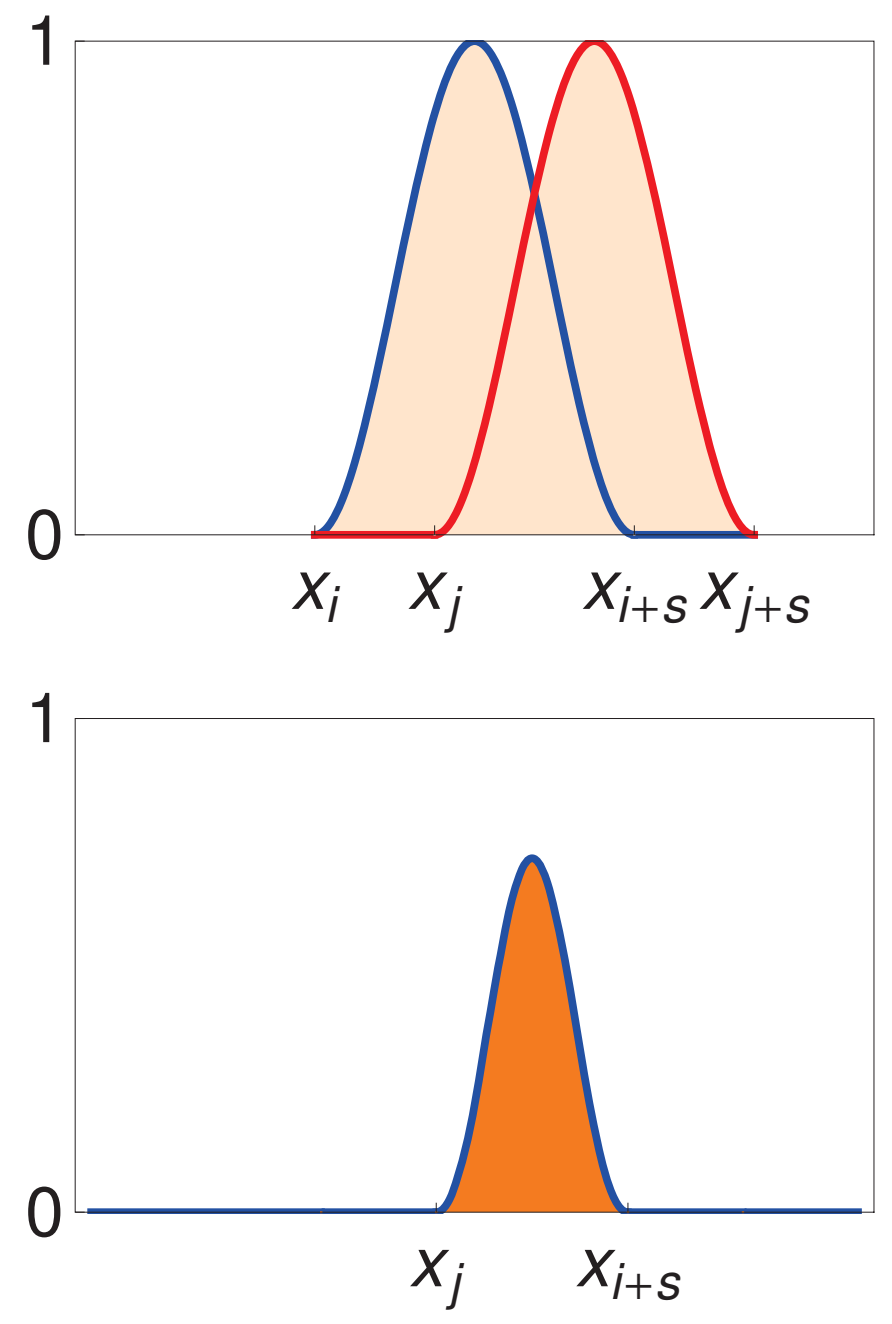

Figure 7.3: Overlap interval between two wavelets in the integrals $\widehat{L}_{j i}$.

$$
r=u(\tau)=a\left(\frac{\tau}{T-\tau}\right)^{\frac{1}{2}}
$$

where $a$ is a parameter allows performing integrations of 7.30-7.33 and 7.38-7.41 in closed forms with all the integrals expressed either through trigonometric functions or through sine integral $S i(x)$ and cosine integral $C i(x)$, tabulated in "Mathematica". The rest of the integrals have derivative of permittivity which is a piecewise constant function in a step index fiber, they evaluated as E.2 or E.3.

Another advantage of 7.48, it allows lift singularity of the term $\frac{1}{r} \frac{\partial}{\partial r}$ at the origin $r=0$ in wave equations 7.10-7.13. It also preserves the origin, i.e. $\tau=0$ mapped to $r=0$. For these reasons map 7.48 was employed. 


\subsection{Fields at boundaries.}

In order to properly solve generalized eigenvalue problem for $\beta^{2}$, the boundary condition for the fields at both ends of the computational domain has to be accurately imposed. The functional dependence of the fields in the step index fiber is well known, for the core layer the longitudinal fields are given by the Bessel function of the first kind $J_{m}(q r)$.

It follows that for the modes with $|m|>2$ both the transversal fields and their derivatives with respect to $r$ are zeros at the origin, for the modes with $m=0,2$ the transversal fields at the origin are zeros while their derivatives with respect to $r$ are non-zeros and for the modes with $m= \pm 1$ the transversal fields at the origin are non-zeros while their derivatives with respect to $r$ are zeros. In addition the following expressions hold for modes with $m= \pm 1$,

$$
\left[\frac{\left(m^{2}+1\right) e_{r}+i 2 m e_{\theta}}{r^{2}}\right]_{r=0}<\infty, \quad\left[\frac{\left(m^{2}+1\right) h_{r}+i 2 m h_{\theta}}{r^{2}}\right]_{r=0}<\infty
$$

which can be approximated as,

$$
\left[\frac{\left(m^{2}+1\right) e_{r}+i 2 m e_{\theta}}{r^{2}}\right]_{r=0}=0, \quad\left[\frac{\left(m^{2}+1\right) h_{r}+i 2 m h_{\theta}}{r^{2}}\right]_{r=0}=0
$$

to simplify the calculation.

Map function $u_{1}$ makes it possible to evaluate all the integrals involved in a closed form, i.e. evaluate analytically. It is best suited for the cases $|m|>2$ and $m= \pm 1$, however for remaining cases, i.e. for $m=0,2$ it is not applicable since non-zero fields derivative at the origin can not be preserved due to a factor $1 / u^{\prime}$ being zero identically at the origin with this map,

$$
\frac{d f(r)}{d r}=\frac{1}{u^{\prime}} \frac{d f(u(\tau))}{d \tau}, \quad\left[\frac{1}{u_{1}^{\prime}}\right]_{\tau=0}=0
$$

At the other end of computational domain, i.e. at $\tau=T$ which corresponds to $r=\infty$ for all guided modes regardless of azimuthal index value $m$ the fields and their first derivatives with respect to $r$ must be zero. The zero field condition is guaranteed by the choice of wavelet being zero at the second end, while the zero derivative is guaranteed by either map functions $u_{1}$ and $u_{2}$.

Corresponding wavelet and map function for each case based on azimuthal index are chosen in such a way as to preserve the appropriate values for the transversal fields and their first derivatives with respect to $r$ at the origin, $r=0(\tau=0)$ and at the end of computational domain $r=\infty(\tau=T)$. An additional requirements for the pair wavelet and map function is that they should keep all the integrals involved in the form that admits an analytical evaluation. The wavelets that accommodate all the features of the transversal fields at the origin for each case of azimuthal indices paired with the appropriate map function are given below. The plots of such wavelets are presented in Fig. 7.4. 


$$
\begin{aligned}
& |m|>2 \quad u_{1}=a\left(\frac{\tau}{T-\tau}\right)^{\frac{1}{2}} \\
& y(x)=\left\{\begin{array}{cc}
\sin ^{2}\left(\pi \frac{x-x_{i}}{x_{i+s}-x_{i}}\right) & x_{i} \leq x \leq x_{i+s} \\
0 & x<x_{i}, \quad x>x_{i+s}
\end{array}\right.
\end{aligned}
$$

wavelet for $|m|>2$ is equivalent to wavelet given by 7.1 .

$$
\begin{gathered}
m=0,2 \quad u_{2}=a \frac{\tau}{T-\tau} \\
y(x)=\left\{\begin{array}{cc}
\sin \left(\pi \frac{x-x_{i}}{x_{i+s}-x_{i}}\right) & x_{i} \leq x \leq x_{i+s} \\
0 & x<x_{i}, \quad x>x_{i+s}
\end{array}\right. \\
m= \pm 1 \quad u_{1}=a\left(\frac{\tau}{T-\tau}\right)^{\frac{1}{2}} \\
y(x)=\left\{\begin{array}{cc}
\frac{1}{2}\left(1+\cos \left(\pi \frac{x-x_{i}}{x_{i+s}-x_{i}}\right)\right) & x_{i} \leq x \leq x_{i+s} \\
0 & x<x_{i}, \quad x>x_{i+s}
\end{array}\right.
\end{gathered}
$$

\subsection{Uniform vs. adaptive mesh.}

To capture tiny geometrical features of the 4 layer fiber such as metal coating $(30 \mathrm{~nm})$ with sufficiently enough nodes (20-30) on it while keeping a uniform mesh throughout the whole computational domain $(100 \mu \mathrm{m})$ a prohibitively large $\left(10^{5} \times 10^{5}\right)$ matrix would be needed for a computing power of current PC. For this reason the partition of the computational domain has to be done by a non-uniform mesh with the smaller mesh size on a smaller layer followed by the larger mesh size on a larger layer. Another areas where the mesh size has to be smaller is the area near the interface between adjacent layers.

\subsection{Partitioning domain with Chebyshev nodes and Koslov map.}

Partitioning of each layer is done by Chebyshev nodes formula [120] applied to the interval $(a, b)$

$$
x_{i}=\frac{1}{2}\left((a+b)+(b-a) \cos \left(\frac{2 i-1}{2 N} \pi\right)\right), \quad i=1, \ldots, N
$$

followed by Koslov-Tal-Elzer map, [119],

$$
f\left(x_{i}\right)=\frac{\arcsin \left(\alpha x_{i}\right)}{\arcsin (\alpha)}
$$

Chebyshev nodes formula ensures the denser distribution of the nodes near the ends of the interval $(a, b)$ compare to its middle, so that the grid size near the ends scales as $O\left(N^{-2}\right)$ and as $O\left(N^{-1}\right)$ in the middle of $(a, b)$ for a large $N$, [120]. Denser nodes distribution near 

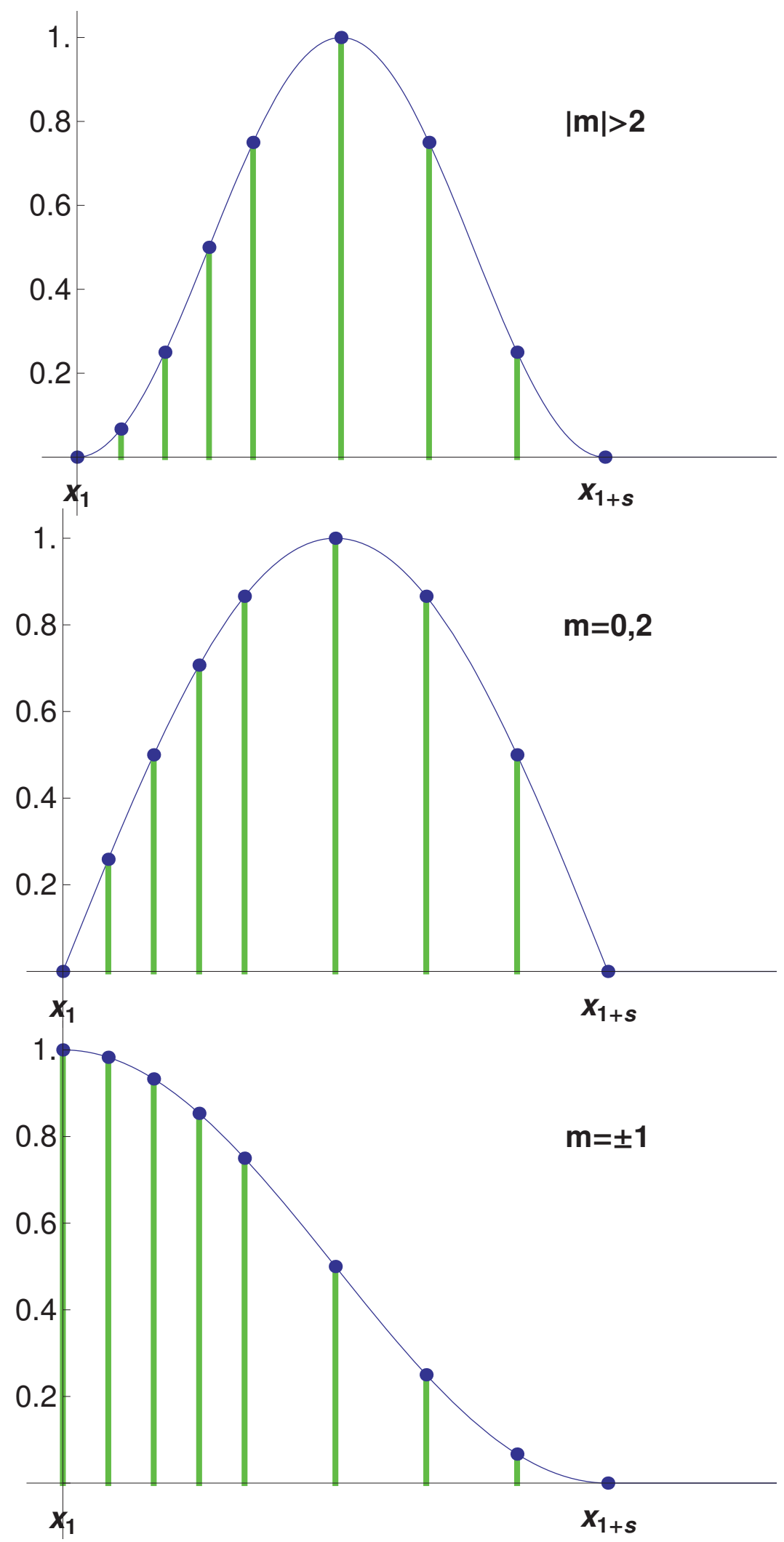

Figure 7.4: Wavelets adapted for different azimuthal index $m$. 
the ends allows work out properly boundary conditions at the interface between adjacent layers and at the ends of computational domain. Koslov-Tal-Elzer map on the other hand redisributes the nodes depending on parameter $\alpha_{i}, \quad(i=1,2,3,4)$ for each layer. When $\alpha_{i}=0$ the nodes distributed according to the Chebyshev formula, i.e. distribution remains untouched; when $\alpha_{i}=1$ the distribution (in a given layer) is equidistant. Having parameters that allow redistribute the nodes while keeping the total number of the nodes fixed is useful for the spurious modes monitoring.

\subsection{Simulation results and discussion.}

\subsubsection{Two layer fiber.}

The method was tested on a two layer step index fiber with the high refractive index contrast of a lossless media with the following parameters: layers refractive indices $n_{1}=$ $1.0036 \times n_{\mathrm{SiO}_{2}}, n_{2}=n_{\text {air }}$, core radius $r_{1}=4.15 \mu \mathrm{m}$, wavelength $1550 \mathrm{~nm}$. Refractive index of a fused silica at wavelength $1550 \mathrm{~nm}$ is assumed $n_{\mathrm{SiO}_{2}}=1.44402$. Effective indices obtained by the method are compared against the true value of indices calculated as real roots of characteristic equation of a fiber with the routine "RootSearch", [121] that attempts of finding all the real roots of a function $f(x)$ on a given interval $\left(x_{1}, x_{2}\right)$. Characteristic equation for a two layer fiber,

$$
\operatorname{det} M=0
$$

is relatively simple since the size of the matrix $M$ obtained from the boundary conditions on a single interface between two layers for tangential fields $E_{z}, E_{\theta}, H_{z}, H_{\theta}$ is only $(4 \times 4)$; therefore the roots of $\operatorname{det} M=0$ as well as the null space of the matrix $M$ can be easily found. Solutions of $\operatorname{det} M=0$ are considered as the true values of indices since such solutions found with the routine "RootSearch" can be evaluated with an arbitrary precision.

All results of solving generalized eigenvalue equations 7.29, 7.37 and solutions of characteristic equation 7.49 by routine "RootSearch" for azimuthal index $m=4$ presented in the Table 1. Precision of generalized eigenvalues in columns 1-4 of the Table 1 are limited by the matrix size in $7.29,7.37$, i.e. by the number of nodes of the computational domain, while precision of the roots of 7.49 in column 5 doesn't have such limitation.

The transversal fields for the first and last modal indices are plotted in Figs. 7.5, Figs. 7.6. The normal component of the electric field $E_{r}$ shows pretty steep disruption which imitates discontinuity at the interface while electric field $E_{\theta}$ and magnetic fields are continuous as expected. Theoretically, solution of the generalized eigenvalue equation for electric fields 7.29 is the same as solution of the generalized eigenvalue equation for magnetic fields 7.37. In practice though they are not identical as seen from the Table 1. However, they approaching the true value of indices when the grid size gets smaller.

The method has some trouble of finding the first modal index, in fact with the smaller number of nodes it doesn't catch it up, because this mode is close to a cut-off condition and 


\begin{tabular}{|c|c|c|c|c|c|}
\hline & $N_{1}=100$ & $N_{1}=100$ & $N_{1}=240$ & $N_{1}=240$ & \\
& $N_{2}=50$ & $N_{2}=50$ & $N_{2}=120$ & $N_{2}=120$ & $n_{e f}(\operatorname{det} M)$ \\
& $n_{e f}(\vec{E})$ & $n_{e f}(\vec{H})$ & $n_{e f}(\vec{E})$ & $n_{e f}(\vec{H})$ & \\
\hline 1 & & & 1.022637613 & 1.024571454 & 1.00139352633456446 \\
2 & 1.096846658 & 1.097378609 & 1.096087303 & 1.096353660 & 1.12070753986683500 \\
3 & 1.133744867 & 1.134106087 & 1.162197115 & 1.164063724 & 1.15137719541851874 \\
4 & 1.234179315 & 1.234539588 & 1.236106090 & 1.236359016 & 1.24483363524494966 \\
5 & 1.260564989 & 1.260618313 & 1.273515063 & 1.274405555 & 1.27093896410179030 \\
6 & 1.332625822 & 1.332824457 & 1.335143881 & 1.335368440 & 1.33759239818145829 \\
7 & 1.356428197 & 1.356417832 & 1.360745137 & 1.361208926 & 1.36121174984918470 \\
8 & 1.400637954 & 1.400719034 & 1.402263908 & 1.402514630 & 1.40255270493862138 \\
\hline
\end{tabular}

Table 7.1: Effective modal indices of two layer fiber for azimuthal index $m=4$ obtained by solving generalized eigenvalue equation for electric fields (columns 1,2), generalized eigenvalue equations for magnetic fields (columns 3,4) and by solving characteristic equation (column 5). $N_{1}, N_{2}$ are number of nodes in the core and outer layer respectively

it's confinement factor is large, therefore the field in the outer layer is not negligible and more points needed to properly describe it. In a second attempt with the larger number of nodes the mode was found. Poor confinement for the first mode compare to the last one can be seen on the plots of the transversal fields in Figs. 7.5- Figs. 7.6 and on the plots of z-components of the Poynting vectors for each mode in Figs. 7.7(a), (b).

It was verified, that the search algorithm coded by the routine "FindRoot" is capable using the initial guess values supplied either by 7.29 or 7.37 converge to a true set of indices calculated by characteristic equation 7.49. Therefore both solutions of 7.29 and 7.37 fall inside of the convergence circle of the search algorithm "FindRoot". This routine can find a complex root of a function $f(x)$ provided proper initial guess value is given. Whether or not the general solutions of generalized eigenvalue equations fall inside of the convergence circle of the search algorithm depends on a precision determined by the number of nodes of the computational domain.

To verify whether or not the set of the true indices in Table 1 column 5 is correct some independent test should be done. Such independent test can be just a checking the boundary conditions, since the wrong or imprecise modal indices wont make boundary conditions satisfied. For this test the constant coefficients of the tangential fields, i.e. the null space of the matrix $M$, were calculated for each mode and then were employed for evaluating the tangential fields of each layer at the interface. The fields in arbitrary units for the first and last mode are presented in the Table 2. It is clear from the table that the boundary conditions remain balanced within precision of 55 digits, i.e. the relative error in satisfying boundary conditions is better than $10^{-54}$. Thus it validates numerically the set of modal indices.

It follows from Table 1 that the indices in column 4 found from generalized eigenvalue equation for magnetic fields 7.37 seems slightly closer to the true values in column 5 than the indices in column 2 found from generalized eigenvalue equation for electric fields 7.29 

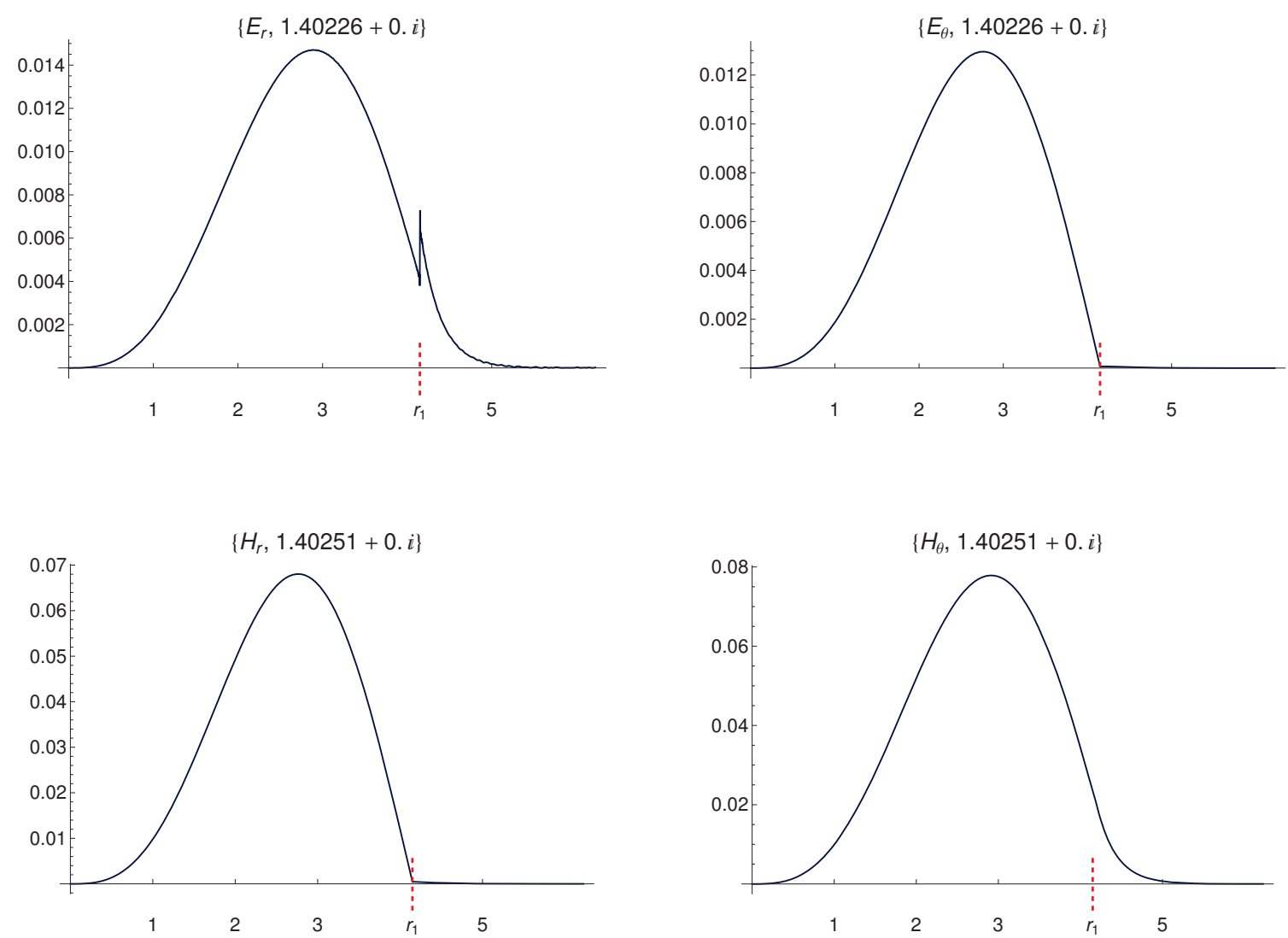

Figure 7.5: Transversal electric and magnetic fields calculated with generalized eigenvalue equations for the first mode of Table $7.1, r_{1}$ is a core radius in $\mu \mathrm{m}$.

\begin{tabular}{|c|c|}
\hline & $n_{e f}=1.00139352633456446525617486307477509826632348691161074180613$ \\
\hline$E_{z 1}\left(r_{1}\right)$ & -0.00139523949624507015926466751117763069323297748782103779536 \\
$E_{z 2}\left(r_{1}\right)$ & -0.00139523949624507015926466751117763069323297748782103779536 \\
$H_{z 1}\left(r_{1}\right)$ & $-7.8555221589502324761727853620069510961850694567510771316 \times 10^{-6}$ \\
$H_{z 2}\left(r_{1}\right)$ & $-7.8555221589502324761727853620069510961850694567510771316 \times 10^{-6}$ \\
$E_{\theta 1}\left(r_{1}\right)$ & -0.379469877389437309285706384158064339286480164989890609578 \\
$E_{\theta 2}\left(r_{1}\right)$ & -0.37946987738943730928570638415806433928648016498989060958 \\
$H_{\theta 1}\left(r_{1}\right)$ & -0.00099646927517779354517888462650112237948315651977225141847 \\
$H_{\theta 2}\left(r_{1}\right)$ & -0.00099646927517779354517888462650112237948315651977225141847 \\
\hline
\end{tabular}

Table 7.2: Tangential fields in arbitrary units of each layer at the interface for the first mode where within at least first 55 digits the boundary conditions are satisfied.

hence the remaining calculations done with equation 7.37. 

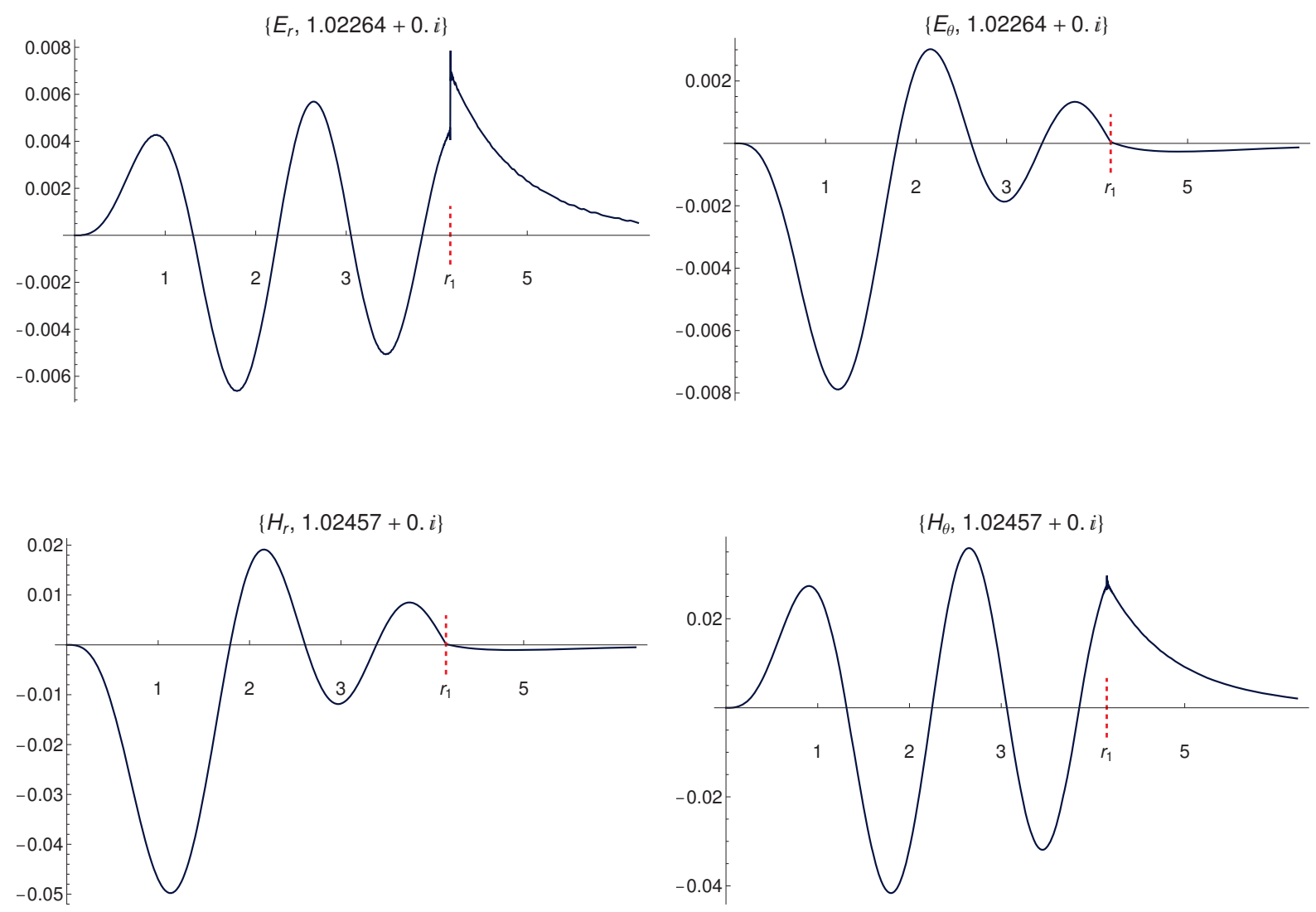

Figure 7.6: Transversal electric and magnetic fields calculated with generalized eigenvalue equations for the last mode of Table $7.1, r_{1}$ is a core radius in $\mu \mathrm{m}$.

\begin{tabular}{|c|c|}
\hline & $n_{e f}=1.40255270493862138922425697835247863234633226724042310083070$ \\
\hline$E_{z 1}\left(r_{1}\right)$ & $-3.1893773584282566460129580847534800797493238799771235929 \times 10^{-8}$ \\
$E_{z 2}\left(r_{1}\right)$ & $-3.1893773584282566460129580847534800797493238799771235930 \times 10^{-8}$ \\
$H_{z 1}\left(r_{1}\right)$ & $1.06379270466957746299950509531440385205225491259298568851 \times 10^{-10}$ \\
$H_{z 2}\left(r_{1}\right)$ & $1.06379270466957746299950509531440385205225491259298568850 \times 10^{-10}$ \\
$E_{\theta 1}\left(r_{1}\right)$ & $3.2079404605373902973060272243282473170570647851538762195 \times 10^{-8}$ \\
$E_{\theta 2}\left(r_{1}\right)$ & $3.2079404605373902973060272243282473170570647851538762195 \times 10^{-8}$ \\
$H_{\theta 1}\left(r_{1}\right)$ & $-5.4316780836011428254172106352610435859410562542218833830 \times 10^{-11}$ \\
$H_{\theta 2}\left(r_{1}\right)$ & $-5.4316780836011428254172106352610435859410562542218833831 \times 10^{-11}$ \\
\hline
\end{tabular}

Table 7.3: Tangential fields in arbitrary units of each layer at the interface for the last mode where within at least first 55 digits the boundary conditions are satisfied.

\subsubsection{SMF fiber coated by thin metal layer.}

Matrix generalized eigenvalue equation (24) was solved for a step index fiber SMF28 with the core and cladding refractive indices $n_{1}=1.0036 \times n_{\mathrm{SiO}_{2}}$ and coated by a thin metal 


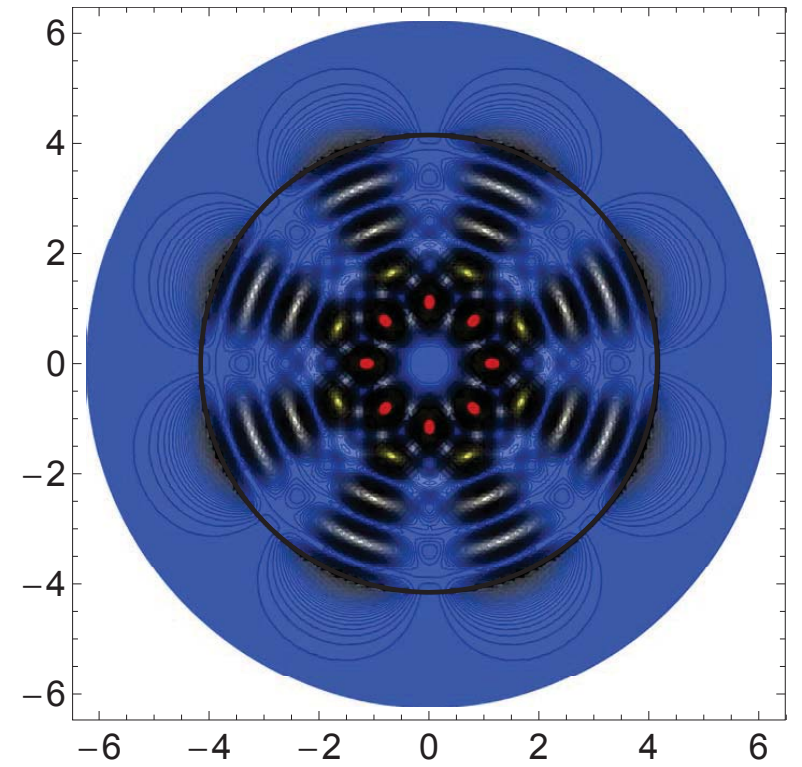

(a)

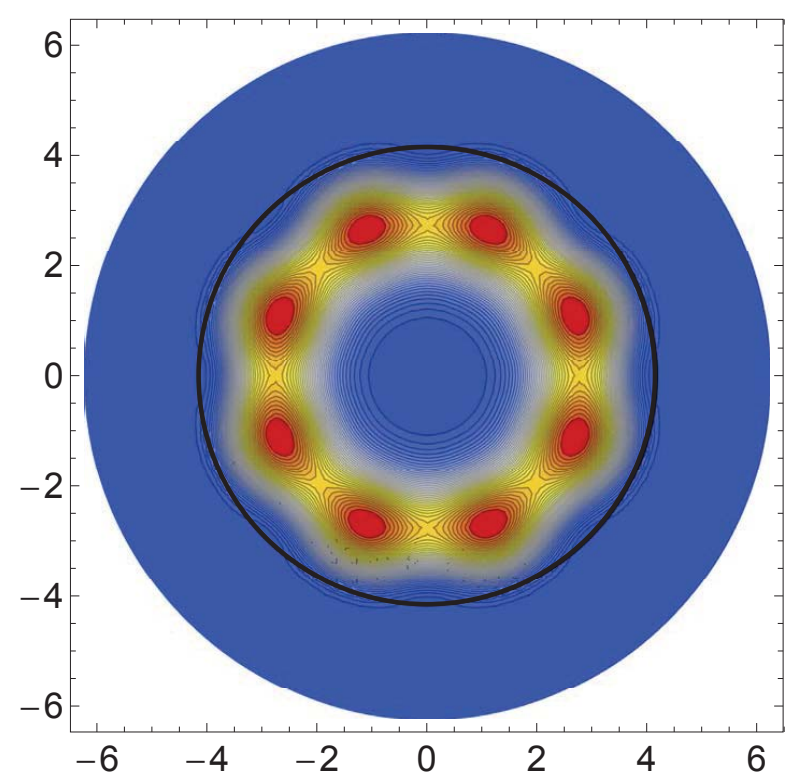

(b)

Figure 7.7: z-component of Poynting vector calculated with generalized eigenvalue equations (a) and (b) for the first and last modes of Table 1 respectively. Dimensions in microns.

layer of either gold or platinum $n_{3}=\left(n_{A u}, n_{P t}\right)$ whose thickness $\Delta$ lays in the range of $(30 \div 100) \mathrm{nm}$ and water as the outer layer $n_{4}=n_{H_{2} O}$. Refractive index of water at telecom range of wavelengths assumed to be $n_{H_{2} O}=1.315$, [122]. The core radius is $r_{1}=4.15 \mu \mathrm{m}$ and the cladding radius is $r_{2}=62.5 \mu \mathrm{m}$.

Since method is capable to find many modes in a single calculation, it can be used either for obtaining and further perfection of any given mode of interest through a more precise calculation with the search algorithm or it can be used for an evaluation the whole set of modes as a function of a certain waveguide parameter. In many cases the latter use is more interesting.

Complex modal indices were obtained for a fixed metal thickness $35 \mathrm{~nm}$ and wavelength $1550 \mathrm{~nm}$; the plots with gold as metal and azimuthal indices $m=5$ and $m=17$ presented in Fig.7.8(a), Fig.7.8(b), plots with platinum as metal and index $m=5$ presented in Fig.7.8(c).

The modal indices behavior with the metal thickness and wavelength as variable parameters is shown in Fig. 7.9 and Fig. 7.10 respectively. It was assumed that refractive indices of the core, cladding and metal layer are wavelength dependent, while refractive index of the outer layer is constant. The data for wavelength dependent refractive index of gold was taken from [52]. Wavelength dependent refractive index of fused silica calculated with Sellmeier coefficients from [91]. 


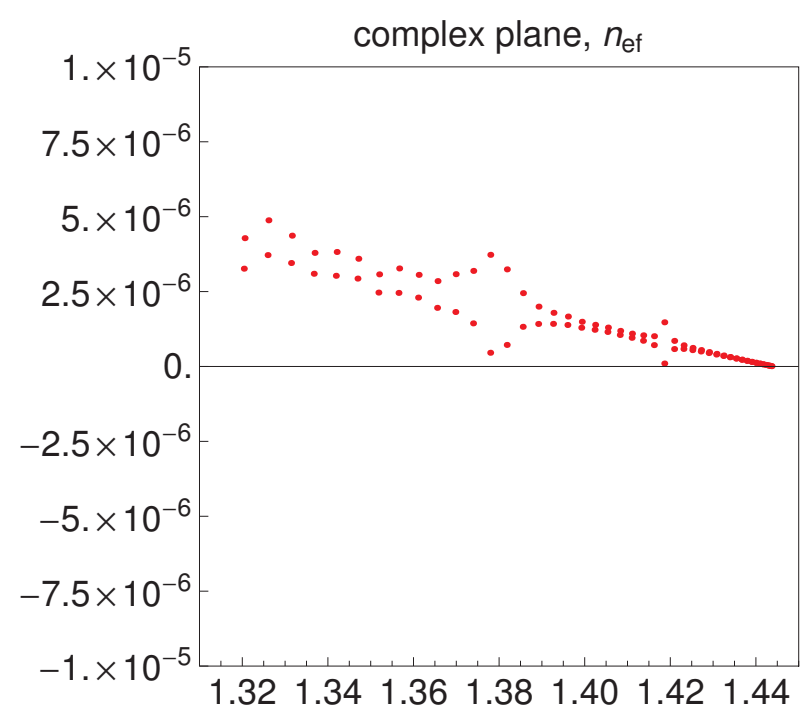

(a) metal Au, azimuthal index 5

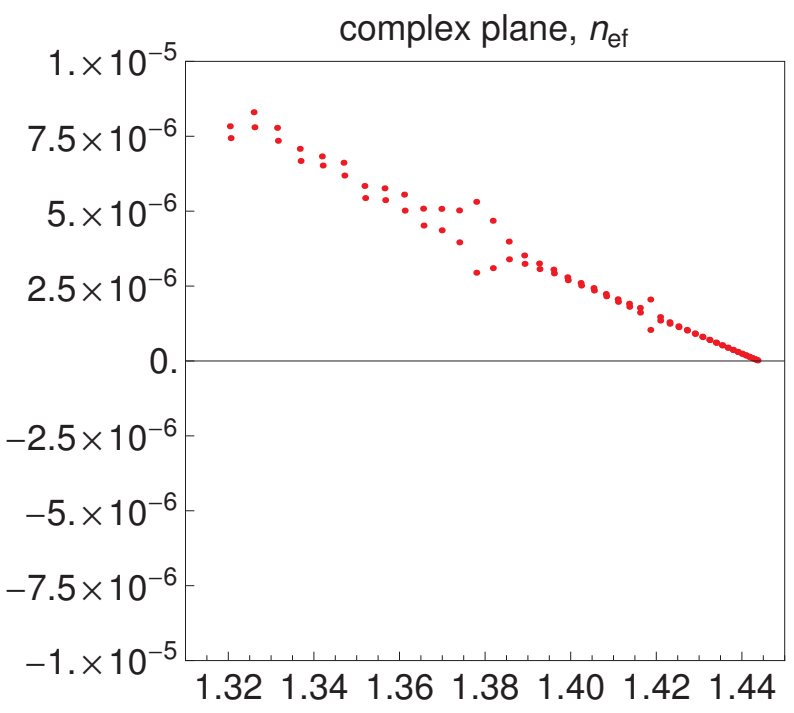

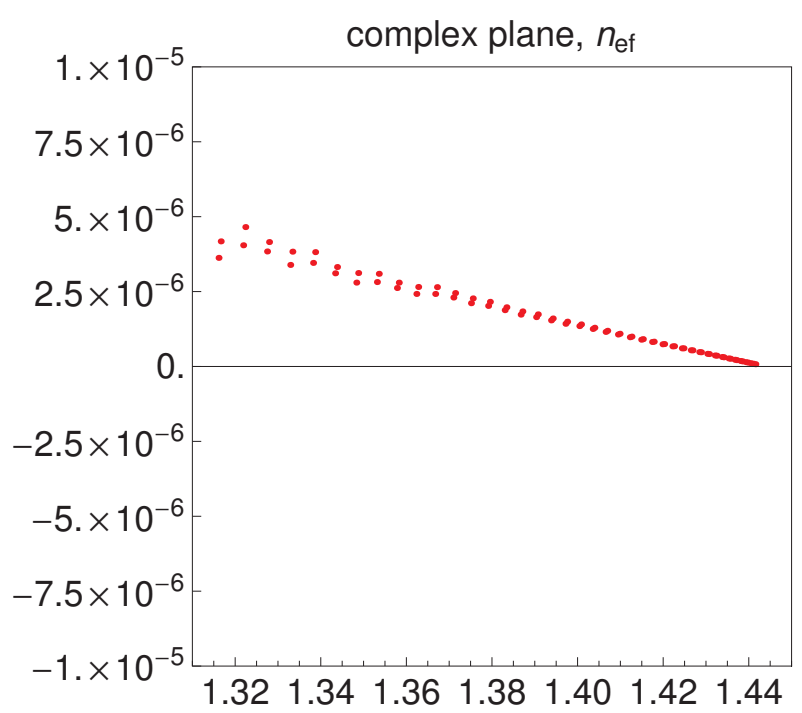

(b) metal $\mathrm{Au}$, azimuthal index 17

(c) metal Pt, azimuthal index 5

Figure 7.8: Modal indices in complex plane $\left(\operatorname{Re}\left(n_{e f}\right), \operatorname{Im}\left(n_{e f}\right)\right)$ for step index fiber SMF28 coated by $35 \mathrm{~nm}$ of metal in water environment at wavelength $1550 \mathrm{~nm}$.

\subsection{Treatment of spurious modes.}

In solving matrix eigenvalue equation some of the solutions turn out to be nonphysical or spurious. It was noticed that in application to a waveguide they usually are the ones not satisfying divergence equations either $\nabla \cdot(\mu \vec{H})=0$ or $\nabla \cdot(\varepsilon \vec{E})=0$, see [97, 123-125] for details. I.e. solutions of the wave equations for magnetic fields may have some spurious modes that do not satisfy condition $\nabla \cdot(\varepsilon \vec{E})=0$, while solutions of the wave equations 


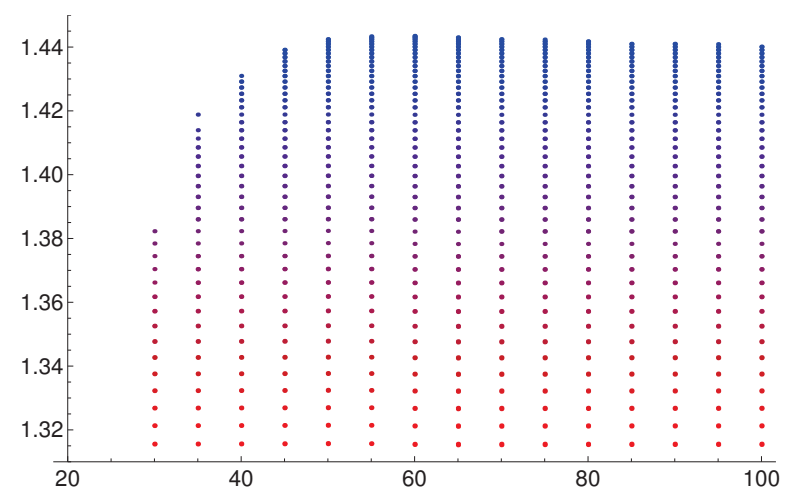

(a)

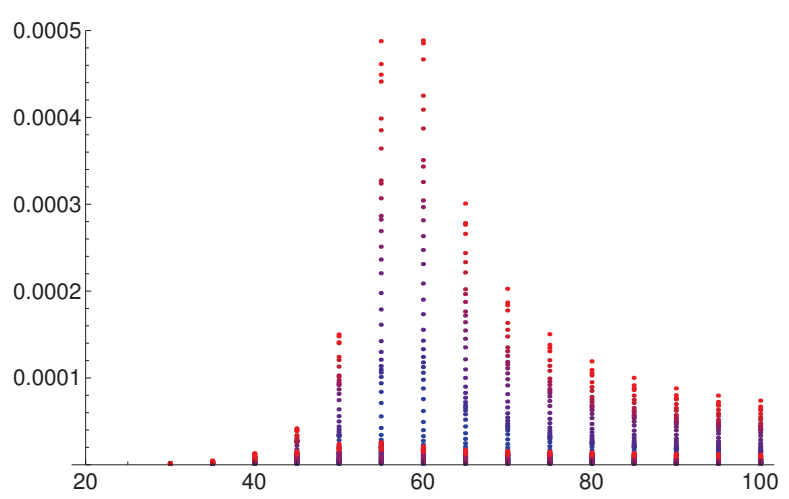

(b)

Figure 7.9: Modal indices of step index fiber SMF28 coated by $\mathrm{Au}$ in $\mathrm{H}_{2} \mathrm{O}$ environment with variable thickness of $A u$ layer at wavelength $1550 \mathrm{~nm}$ and azimuthal index $m=5$. (a) real parts vs. thickness in nanometers; (b) imaginary parts vs. thickness in nanometers. The real and imaginary parts of indices belonging to the same mode and the same metal thickness represented in (a) and (b) with the same color.

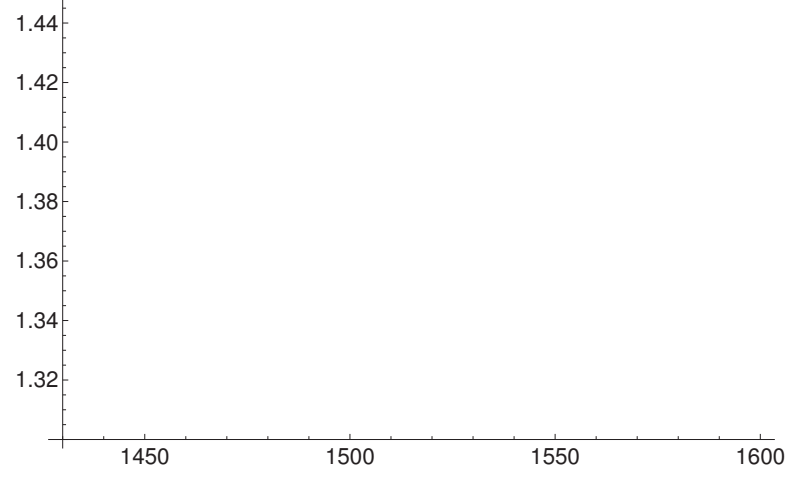

(a)

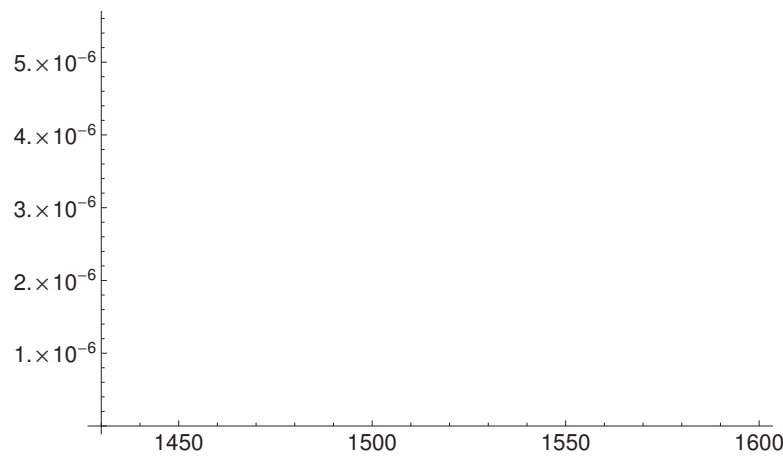

(b)

Figure 7.10: Modal indices of step index fiber SMF28 coated by $\mathrm{Au}$ in $\mathrm{H}_{2} \mathrm{O}$ environment with variable wavelength and fixed $A u$ layer at thickness $35 \mathrm{~nm}$ and azimuthal index $m=5$. (a) real parts vs. wavelength in nanometers; (b) imaginary parts vs. wavelength in nanometers. The real and imaginary parts of indices belonging to the same mode and the same wavelength represented in (a) and (b) with the same color.

for electric fields may have some spurious modes that do not satisfy condition $\nabla \cdot \vec{H}=0$. Therefore the mode present simultaneously among both solutions, i.e. solutions of the wave equations for electric fields, 7.29 and solutions of the wave equations for magnetic fields 7.37 should be non-spurious.

Some of the spurious modes might appear for a reason other than specified earlier, for example as a result of poor approximations to the exact eigenvalues due to possibly aliasing effect when current matrix size is too low to properly resolve oscillations of these 
particular modes, [119].

Certain spurious modes are easy to detect since they are outside of a valid range, i.e. real part or imaginary part or booth are way off the actual values for a guided or radiation modes. Others that masquerade themselves in the proper range of values can be detected by unusually strange behavior of the fields demonstrating presence of the source, region of positive divergence, or drain, region of negative divergence, [97].

Another remedy for eliminating spurious modes is to deviate slightly some auxiliary parameters of the model and monitor behavior of the eigenvalues. Small perturbation of any numerical scheme parameter usually causes a particular spurious mode to disappear with an emerging of a new spurious mode with different value; the genuine modes on the other hand are far more less sensitive to such perturbation. Therefore having parameters of numerical scheme that allow performing small perturbation of the solution of a generalized eigenvalue problem is seen as a pre-filtering tool of the waveguide modes, which is implemented by picking those modes that deviate only slightly with a small perturbation of the waveguide parameters. The eigenvalues that deviate significantly with the small perturbation of such parameters are suspicious and are subject for further verification.

Numerical parameters that perform the least invasive perturbation of the solution of generalized eigenvalue problem, i.e. the ones that do not affect the matrix size and matrix density/sparsity are:

- $\alpha_{1}, \alpha_{2}, \alpha_{3}, \alpha_{4}$ parameters of Koslov map that are responsible for the redistribution of the nodes keeping the total nodes of the computational domain the same and therefore do not affecting the matrix size,

- $a, T$ parameters of the map function between $r$-domain and $\tau$-domain.

Parameters that perform more invasive perturbation are:

- $s$ number of nodes spanned by single wavelet, it affects the density/sparsity of the matrix,

- $N_{1}, N_{2}, N_{3}, N_{4}$ number of nodes in each layer, they affect directly matrix size.

\subsection{The relevance of the method.}

1. The mapped Galerkin method can not handle leaky modes since the leaky modes grow exponentially in the outer layer even though they are not spurious, i.e. boundary conditions at infinity are not physical (such modes measured at a finite distance and found to be exist). Therefore to properly describe them the value of the wavelet at the last node should be infinitely large. This is not possible in numerical calculation, the method can not handle leaky modes. 
2. The mapped Galerkin method can not handle radiation modes, because towards the end of computational domain the spacial oscillation frequency in $r$-domain grow very fast which would require infinitely large matrix size to capture all the oscillation properly. The boundary conditions for the fields of radiation modes at infinity are physical as they vanish as $r^{-1 / 2}$, however the power remains finite at infinity, $\left[r^{-1 / 2} r^{-1 / 2} r\right]_{r \rightarrow \infty} \neq 0$, i.e. radiation modes are not square integrable. Therefore near the end of computational domain the fields remain finite as they decay slowly as, yet their frequency of spacial oscillation becomes infinitely large. Therefore to properly capture all the details of such oscillations the matrix size should be infinitely large as well. Since for numerical calculations this is not possible, the method can not handle radiation modes.

3. The mapped Galerkin method is capable to handle all the guided modes of the fiber that are well confined, i.e. the modes far from cut-off regime.

4. The mapped Galerkin method is also capable to handle those guided modes of the fiber that are not well confined, i.e. the modes near of cut-off regime, because they are decaying exponentially in the outer layer.

\subsection{Conclusion.}

1. The wave equations reduced by this method to a generalized eigenvalue equation which is computationally harder to deal with compare to eigenvalue equation due to built-in matrix inversion problem. In addition generalized eigenvalue equation is more prone to the spurious modes than eigenvalue equation.

2. Nevertheless the method is of practical usefulness because it allows in a single calculation determine many complex modes due to sparseness of the underlying matrices.

3. It can also be useful for evaluation of a functional dependence of a large number of guided modes versus certain parameter of the waveguide (thickness of metal layer or wavelength).

4. If the waveguide with a certain condition has a single mode, there is no guarantee the method can catch it up. However for a multimode waveguide with large number of modes like cladding modes in SMF28 fiber the method is capable of finding fair amount of these modes.

5. Precision of the method is limited by matrix size. Solutions of this method can be used as initial guess values supplied for filtering out and/or further refining to the search algorithm whose precision has lesser limitation.

6. Galerkin method is applicable to the guided modes of a step index fiber with thin metal coating. 


\section{Chapter 8}

\section{Summary and outlook}

Experimental part of this thesis is focused on fabricating a glass metal nanocomposite by forming silver nanoclusters in an ion-exchanged glass in a hydrogen atmosphere at elevated temperature followed by an investigation of a combined action of electric field and temperature on a glass metal nanocomposite and subsequent modification of its structure due to nanoclusters dissolution.

The main outcomes obtained in this part are:

- Fabrication of glass metal nanocomposite based on phosphate glass IOG-1 and silver nanoclusters was demonstrated.

- Alteration of nanoclusters results in manipulation of optical properties of glass metal nanocomposite via plasmon excitation in silver nanoclusters via plasmon resonance. Since plasmon resonance decreases with wavelength as Lorentzian type decay, the optical properties of glass metal nanocomposite are determined by plasmon excitation even at wavelengths far off plasmon resonance.

- Long period volume grating, as a proof-of-concept, was built in glass metal nanocomposite based on IOG-1 glass by means of activating electric field assisted dissolution in nanocomposite through spacially corrugated electrode.

In theoretical part of the thesis the standard approach used in an antenna theory, a wave equation with the source term, is applied for solving a problem of an optical waveguide coated by metal.

- A computational model of plasmon excitation in a metal-coated waveguide illuminated by an optical antenna, i.e. by a finite size of current source oscillating at optical frequencies, was developed and tested for basic waveguide geometry and well known experimental data. Then the model extended to a plasmon excitation by a tilted Bragg grating in a fiber coated with nanostructured metal. 
- It was shown that a $\pi$-phase shift in nanostructured metal coating permits precision measurements of small external perturbation and thus is a useful tool in fiber optics sensor application.

- A mode solver for finding complex roots of a fiber coated by metal based on Galerkin wavelet method was developed. The results obtained by mode solver are applicable to the main mode of plasmon excitation on fiber.

Future plan:

- Grating in glass metal nanocomposite achieved with periodically corrugated electrode via electric field assisted dissolution is not only a grating of refractive index change but grating of nonlinearity change due to periodic variation of silver nanocluster concentration and nonlinearity associated with periodically poled regions of glass matrix. By tuning parameters of glass metal nanocompopsite, cluster size and fill factor, its nonlinear properties can be optimized and waveguide with periodic nonlinearity can be implemented.

- Grating made by periodically varied concentration of silver nanoclusters can be considered as a saturable absorber since the fill factor of nanoclusters can be engineered at a stage of preparing glass metal nanocomposite.

- The volume long period grating in glass metal nanocompositebased on phosphate glass IOG-1 achieved with electric field assisted dissolution technique is demonstrated but the question of realization volume Bragg grating remains open. An alternative approach to build a volume Bragg grating in IOG-1 glass is to use femtosecond laser exposure that has ability permanently modification of glass structure in the form of self organizing nanocracks. Introducing nanocracks in glass makes glass locally anisotropic and thus makes diffusion process in this region anisotropic as well. Then procedure of making volume Bragg grating as follows: masking glass sample with pattern according to Bragg period, introducing nanocracks in the mask openings by femtosecond laser exposure, coating sample with silver film and performing ionexchange, cleaning surface and stacking two samples, prepared with the same mask, face to face and performing annealing in air, which leads to a bonding of two samples in a unified slice with combined waveguide and volume Bragg grating. 
APPENDICES 


\section{Appendix A}

\section{Conformal mapping of corrugated plate}

Conformal mapping of upper plane on periodically corrugated upper plane is done in three steps (see Fig.X17).

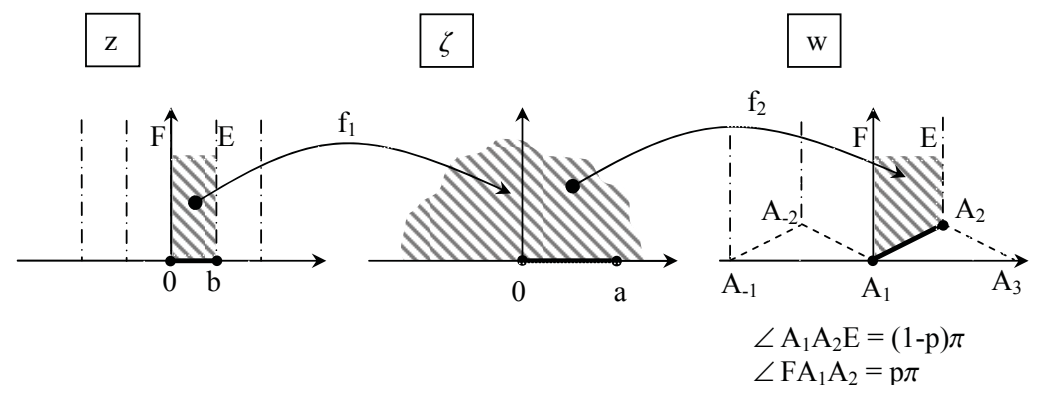

Figure A.1: Conformal mapping upper plane z to corrugated upper plane w (triangular saw tooth).

1. conformal map semi-infinite strip on upper plane

2. conformal map upper plane on corrugated upper strip

3. applying Schwarz symmetry principle to analytically extend mapping over the symmetry axes, i.e. extend from semi-infinite strip to upper plane.

\section{Implementation of steps 1-3. Triangular groove.}

Conformal mapping for groove angle $\pi / 2$ is given in [refY24], here is mapping for any angle from 0 to $\pi$.

1. $\zeta=f_{1}(z)$ map strip $(0, b, E, F)$ of width $(0, b)$ on upper plane in such a way that 0 maps to 0 and $b$ maps to $a$ :

$$
f_{1}(z)=a \sin ^{2}\left(\frac{\pi z}{2 b}\right)
$$


2. $w=f_{2}(\zeta)$ map upper plane to region $\left(A_{1}, A_{2}, E, F\right)$ by applying Schwarz-Christoffel transformation:

$$
\begin{gathered}
f_{2}(\zeta)=C_{1} \int_{0}^{\zeta}(t-0)^{p-1}(t-1)^{-p} d t+C_{2} \\
f_{2}(\zeta)=i(-1)^{-p} B(\zeta, p, 1-p)
\end{gathered}
$$

where $B(\zeta, p, 1-p)$ is incomplete beta function.

$p \pi$ is angle $\angle F A_{1} A_{2}, \quad 0 \leq p \leq 1 / 2$

Constants $C_{1}, C_{2}$ are to adjust scale and position of polygon $\left(A_{1}, A_{2}, E, F\right)$, we chose:

$$
\begin{gathered}
C_{1}=i, \quad C_{2}=0 \\
w=f(z)=f_{2}\left(f_{1}(z)\right)
\end{gathered}
$$

3. Vertical boundaries of strips $(0, b, E, F)$ and $\left(A_{1}, A_{2}, E, F\right)$ are axes of symmetry, therefore according to Schwarz symmetry principle the same mapping function $w=f(z)$ maps upper plane $z$ on periodically corrugated upper plane $w\left(\ldots A_{-1}, A_{-2}, A_{1}, A_{2}, A_{3} \ldots\right.$ on Fig. X17a)

\section{Implementation of steps 1-3. Rectangular groove.}

$($ see $[126])$

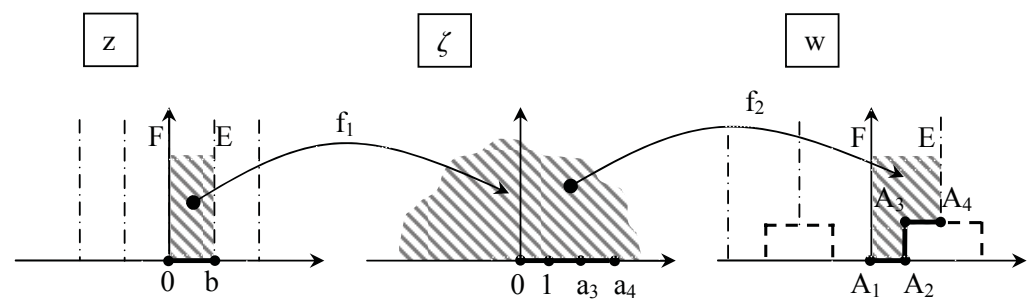

Figure A.2: Conformal mapping upper plane z to corrugated upper plane w (rectangular saw tooth).

1. $\zeta=f_{1}(z)$ map strip $(0, b, E, F)$ of width $(0, b)$ on upper plane in such a way that 0 maps to 0 and $b$ maps to $a_{4}$ :

$$
f_{1}(z)=a_{4} \sin ^{2}\left(\frac{\pi z}{2 b}\right)
$$

2. $w=f_{2}(\zeta)$ map upper plane to region $\left(A_{1}, A_{2}, A_{3}, A_{4}, E, F\right)$ by applying SchwarzChristoffel transformation: 


$$
\begin{gathered}
f_{2}(\zeta)=C_{1} \int_{0}^{\zeta}(t-0)^{-1 / 2}(t-1)^{-1 / 2}\left(t-a_{3}\right)^{1 / 2}\left(t-a_{4}\right)^{-1 / 2} d t+C_{2} \\
f_{2}(\zeta)=\frac{i 2}{\sqrt{a_{3}\left(a_{4}-1\right)}}\left(\left(a_{3}-1\right) F(\phi(\zeta), m)+\Pi(n, \phi(\zeta), m)\right)
\end{gathered}
$$

where

$$
\begin{gathered}
\phi(\zeta)=\arcsin \left(\frac{\sqrt{\left(a_{4}-1\right) \zeta}}{\sqrt{a_{4}(\zeta-1)}}\right) \\
m=\frac{\left(a_{3}-1\right) a_{4}}{\left(a_{4}-1\right) a_{3}} \\
n=\frac{a_{4}}{\left(a_{4}-1\right)}
\end{gathered}
$$

$F(\phi, m)$ is elliptic integral of the first kind

$\Pi(n, \phi, m)$ is incomplete elliptic integral

$w=f(z)=f_{2}\left(f_{1}(z)\right)$

Schwarz Christoffel integral for $f_{2}$ can be done with Mathematica integrator online.

3. Applying Schwarz symmetry multiple times analytically extends mapped region over axes of symmetry so that the same mapping function $w=f(z)$ maps upper plane $z$ on periodically corrugated upper plane $w$ (Fig.X17b). 


\section{Appendix B}

Show that differential operators $\nabla^{2}$ and $\nabla \nabla \cdot$ commute for some vector function $\vec{F}$,

$$
\nabla^{2} \nabla \nabla \cdot \vec{F}=\nabla \nabla \cdot \nabla^{2} \vec{F}
$$

Using the following two identities for some vector function and scalar function $s$,

$$
\begin{gathered}
\nabla \cdot(\nabla \times \vec{a})=0 \\
\nabla \times(\nabla s)=0
\end{gathered}
$$

and definition of Laplacian operator,

$$
\nabla^{2} \vec{a}=\nabla \nabla \cdot \vec{a}-\nabla \times \nabla \times \vec{a}
$$

working out $\nabla^{2} \nabla \nabla \cdot \vec{F}$ and $\nabla \nabla \cdot \nabla^{2} \vec{F}$ gives the following result,

$$
\begin{gathered}
\nabla^{2}(\nabla \nabla \cdot \vec{F})=\nabla \nabla \cdot(\nabla \nabla \cdot \vec{F})-\nabla \times \nabla \times(\nabla \nabla \cdot \vec{F})=\nabla \nabla \cdot(\nabla \nabla \cdot \vec{F}) \\
\nabla \nabla \cdot \nabla^{2} \vec{F}=\nabla \nabla \cdot(\nabla \nabla \cdot \vec{F})-\nabla \nabla \cdot(\nabla \times \nabla \times \vec{F})=\nabla \nabla \cdot(\nabla \nabla \cdot \vec{F})
\end{gathered}
$$

Therefore,

$$
\nabla^{2} \nabla \nabla \cdot \vec{F}=\nabla \nabla \cdot \nabla^{2} \vec{F}
$$




\section{Appendix C}

\section{Approximation of Bessel function through trigonometric series.}

A Bessel function expansion by trigonometric series based on [88] was used for an analytical evaluation of the integrals containing Bessel function. Utilizing an integral representation of a Bessel function of the first kind with an integer order $J_{n}(x)$,

$$
J_{n}(x)=\frac{1}{2 \pi} \int_{-\pi}^{+\pi} e^{-i(m \varphi-x \sin \varphi)} d \varphi
$$

an identity for Bessel function with negative argument

$$
J_{n}(-x)=(-1)^{n} J_{n}(x)
$$

and an approximation of Riemann integral by summation

$$
\int_{a}^{b} f(x) d x \approx \sum_{j=1}^{N} \frac{b-a}{N} f\left(x_{j}\right)
$$

we derive a trigonometric expansion of a Bessel function as follows, 


$$
\begin{array}{r}
J_{n}(x)=(-1)^{n} J_{n}(-x)=\frac{(-1)^{n}}{2 \pi} \int_{-\pi}^{+\pi} e^{-i(n \varphi+x \sin \varphi)} d \varphi \approx \\
\approx \frac{(-1)^{n}}{2 \pi} \sum_{j=1}^{N} \frac{2 \pi}{N} \exp \left[-i\left(n\left(-\pi+\frac{2 \pi}{N} j\right)+x \sin \left(-\pi+\frac{2 \pi}{N} j\right)\right)\right]= \\
=\frac{(-1)^{n}}{N} \sum_{j=1}^{N} \exp [i n \pi] \exp \left[-i\left(n \frac{2 \pi}{N} j-x \sin \left(\frac{2 \pi}{N} j\right)\right)\right]= \\
=\frac{1}{N} \sum_{j=1}^{N} \exp \left[-i\left(n \frac{2 \pi}{N} j-x \sin \left(\frac{2 \pi}{N} j\right)\right)\right]
\end{array}
$$

Therefore,

$$
J_{n}(x) \approx \frac{1}{N} \sum_{j=1}^{j=N} e^{-i\left(n \frac{2 \pi}{N} j-x \sin \left(\frac{2 \pi}{N} j\right)\right)}
$$

Such trigonometric expansion is an alternative to a Taylor expansion in power series,

$$
J_{n}(x) \approx \sum_{j=0}^{j=N} \frac{(-1)^{j}}{j !(j+n) !}\left(\frac{x}{2}\right)^{2 j+n}
$$




\section{Appendix D}

\section{Iterative scheme based on equivalence prin- ciple for finding scattered field in inhomo- geneous waveguide.}

(see [89], Ch. 7.7)

In a homogeneous waveguide the fields $\vec{E}^{h}, \vec{H}^{h}$, assuming a time dependence convention $e^{-i \omega t}$, obey Maxwell's equations,

$$
\begin{gathered}
\nabla \times \vec{E}^{h}=-\vec{J}_{m}+i \omega \mu_{1} \vec{H}^{h} \\
\nabla \times \vec{H}^{h}=\vec{J}_{e}-i \omega \varepsilon_{1} \vec{E}^{h}
\end{gathered}
$$

where $\vec{J}_{e}, \vec{J}_{m}$ are electric and magnetic impressed current sources that excite the fields. In an inhomogeneous waveguide with a spacial grating along $z$-axis the same current sources excite fields $\vec{E}^{g}, \vec{H}^{g}$ that satisfy the following Maxwell's equations,

$$
\begin{gathered}
\nabla \times \vec{E}^{g}=-\vec{J}_{m}+i \omega \mu_{g}(z) \vec{H}^{g} \\
\nabla \times \vec{H}^{g}=\vec{J}_{e}-i \omega \varepsilon_{g}(z) \vec{E}^{g}
\end{gathered}
$$

where $\varepsilon_{g}(z), \mu_{g}(z)$ permittivity and permeability of inhomogeneous waveguide, i.e. waveguide with grating,

$$
\begin{aligned}
\varepsilon_{g}(z) & =\varepsilon_{1}+\Delta \varepsilon(z) \\
\mu_{g}(z) & =\mu_{1}+\Delta \mu(z)
\end{aligned}
$$


Subtracting newly obtained Maxwell's equations D.3, D.4 from original ones D.1, D.2 leads to,

$$
\begin{aligned}
& \nabla \times \vec{E}^{s}=i \omega \Delta \mu \vec{H}^{g}+i \omega \mu_{1} \vec{H}^{s} \\
& \nabla \times \vec{H}^{s}=-i \omega \Delta \varepsilon \vec{E}^{g}-i \omega \varepsilon_{1} \vec{E}^{s}
\end{aligned}
$$

where the fields $\vec{E}^{s}, \vec{H}^{s}$ denote scattered fields,

$$
\begin{aligned}
& \vec{E}^{s}=\vec{E}^{g}-\vec{E}^{h} \\
& \vec{H}^{s}=\vec{H}^{g}-\vec{H}^{h}
\end{aligned}
$$

The first terms in both equations can be viewed as equivalent source terms,

$$
\begin{aligned}
& {\overrightarrow{J_{m}^{e q}}}^{e q}=-i \omega \Delta \mu(z) \vec{H}^{g}=-i \omega \Delta \mu(z)\left(\vec{H}^{h}+\vec{H}^{s}\right) \\
& \vec{J}_{e}^{e q}=-i \omega \Delta \varepsilon(z) \vec{E}^{g}=-i \omega \Delta \varepsilon(z)\left(\vec{E}^{h}+\vec{E}^{s}\right)
\end{aligned}
$$

which are identical zeros in inhomogeneous waveguide outside of a grating, i.e. where

$$
\begin{aligned}
& \Delta \varepsilon(z)=0 \\
& \Delta \mu(z)=0
\end{aligned}
$$

The Maxwell's equations for scattered fields then written as,

$$
\begin{gathered}
\nabla \times \overrightarrow{E^{s}}=-\overrightarrow{J_{m}^{e q}}+i \omega \mu_{1} \vec{H}^{s} \\
\nabla \times \overrightarrow{H^{s}}=\vec{J}_{e}^{e q}-i \omega \varepsilon_{1} \vec{E}^{s}
\end{gathered}
$$

Equations for scattered fields is difficult to solve since effective source terms themselves depend on the scattered fields. Replacing true value of effective source terms by approximations not involving scattered fields allows simplify these equations and solve them for approximate scattered fields, i.e. corresponding Maxwell's equations are,

$$
\begin{gathered}
\nabla \times \vec{E}^{s,(1)}=-\vec{J}_{m}^{e q,(0)}+i \omega \mu_{1} \vec{H}^{s,(1)} \\
\nabla \times \vec{H}^{s,(1)}=\vec{J}_{e}^{e q,(0)}-i \omega \varepsilon_{1} \vec{E}^{s,(1)}
\end{gathered}
$$


where $\vec{E}^{s,(1)}, \vec{H}^{s,(1)}$ are first order approximation of scattered fields and $\vec{J}_{e}^{e q,(0)}, \vec{J}_{m}^{e q,(0)}$ are zero order approximations of effective sources that do not depend on scattered fields,

$$
\begin{aligned}
& \vec{J}_{m}^{e q,(0)}=-i \omega \Delta \mu(z) \vec{H}^{h} \\
& \vec{J}_{e}^{e q,(0)}=-i \omega \Delta \varepsilon(z) \vec{E}^{h}
\end{aligned}
$$

The new equations can be solved for $\vec{E}^{s,(1)}, \vec{H}^{s,(1)}$ using for instance Fourier transform technique. The newly obtained fields $\vec{E}^{s,(1)}, \vec{H}^{s,(1)}$ which are the first approximation for true scattered fields, can be plugged in the effective source formulas for a further iteration,

$$
\begin{gathered}
\vec{J}_{m}^{e q,(1)}=-i \omega \Delta \mu(z)\left(\vec{H}^{h}+\vec{H}^{s,(1)}\right) \\
\vec{J}_{e}^{e q,(1)}=-i \omega \Delta \varepsilon(z)\left(\vec{E}^{h}+\vec{E}^{s,(1)}\right) \\
\nabla \times \vec{E}^{s,(2)}=-\vec{J}_{m}^{e q,(1)}+i \omega \mu_{1} \vec{H}^{s,(2)} \\
\nabla \times \vec{H}^{s,(2)}=\vec{J}_{e}^{e q,(1)}-i \omega \varepsilon_{1} \vec{E}^{s,(2)}
\end{gathered}
$$

Such iterative procedure is tedious, but straightforward,

$$
\begin{gathered}
\nabla \times \vec{E}^{s,(j+1)}=-\vec{J}_{m}^{e q,(j)}+i \omega \mu_{1} \vec{H}^{s,(j+1)} \\
\nabla \times \vec{H}^{s,(j+1)}=\vec{J}_{e}^{e q,(j)}-i \omega \varepsilon_{1} \vec{E}^{s,(j+1)}
\end{gathered}
$$

Schematically it is represented as,

$$
\vec{J}_{e}^{e q,(j)}, \vec{J}_{m}^{e q,(j)} \rightarrow \vec{E}^{s,(j+1)}, \vec{H}^{s,(j+1)} \rightarrow \vec{J}_{e}^{e q,(j+1)}, \vec{J}_{m}^{e q,(j+1)} \rightarrow \vec{E}^{s,(j+2)}, \vec{H}^{s,(j+2)} \rightarrow \ldots
$$

For non-magnetic material an equivalent magnetic current source term is identical zero,

$$
\overrightarrow{J_{m}^{e q}}=-i \omega \Delta \mu(z) \overrightarrow{H^{g}}=0
$$




\section{Appendix E}

Function $f(x)$ is a piecewise constant function defined as,

$$
f(x)=\left\{\begin{array}{cc}
f_{0}=f(0) & 0 \leq x<T_{1} \\
f_{1}=f\left(T_{1}\right) & T_{1} \leq x<T_{2} \\
f_{2}=f\left(T_{2}\right) & T_{2} \leq x<T_{3} \\
f_{3}=f\left(T_{3}\right) & T_{3} \leq x \leq T
\end{array}\right.
$$

Integral involving product derivative of such function and some continuous function evaluated with integration by parts.

$$
\begin{aligned}
& I_{1}=\int_{0}^{T} g(x) \frac{d f}{d x} d x=\left(f_{1}-f_{0}\right) g\left(T_{1}\right)+\left(f_{2}-f_{1}\right) g\left(T_{2}\right)+\left(f_{3}-f_{2}\right) g\left(T_{3}\right) \\
& I_{2}=\int_{0}^{T} w \frac{d}{d x}\left(g \frac{d f}{d x}\right) d x= \\
& =\left(f_{0}-f_{1}\right) w^{\prime}\left(T_{1}\right) g\left(T_{1}\right)+\left(f_{1}-f_{2}\right) w^{\prime}\left(T_{2}\right) g\left(T_{2}\right)+\left(f_{2}-f_{3}\right) w^{\prime}\left(T_{3}\right) g\left(T_{3}\right)
\end{aligned}
$$




\section{References}

[1] S Hüfner. Optical spectra of transparent rare earth compounds. Elsevier, 2012.

[2] Michel JF Digonnet. Rare-earth-doped fiber lasers and amplifiers, revised and expanded. CRC press, 2002.

[3] E.C. Le Ru, M Meyer, and P.G. Etchegoin. Proof of single-molecule sensitivity in surface enhanced raman scattering (SERS) by means of a two-analyte technique. The Journal of Physical Chemistry B, 110(4):1944-1948, 2006.

[4] G De Marchi, F Caccavale, F Gonella, G Mattei, P Mazzoldi, G Battaglin, and A Quaranta. Silver nanoclusters formation in ion-exchanged waveguides by annealing in hydrogen atmosphere. Applied Physics A, 63(4):403-407, 1996.

[5] C Mohr, M Dubiel, and H Hofmeister. Formation of silver particles and periodic precipitate layers in silicate glass induced by thermally assisted hydrogen permeation. Journal of Physics: Condensed Matter, 13(3):525, 2001.

[6] A Miotello, M Bonelli, G De Marchi, G Mattei, P Mazzoldi, C Sada, and F Gonella. Formation of silver nanoclusters by excimer-laser interaction in silver-exchanged soda-lime glass. Applied Physics Letters, 79(15):2456-2458, 2001.

[7] G Battaglin, E Cattaruzza, F Gonella, R Polloni, F D’Acapito, S Colonna, G Mattei, C Maurizio, P Mazzoldi, S Padovani, et al. Silver nanocluster formation in ion-exchanged glasses by annealing, ion beam and laser beam irradiation: an EXAFS study. Nuclear Instruments and Methods in Physics Research Section B: Beam Interactions with Materials and Atoms, 200:185-190, 2003.

[8] Nobuhito Takeshima, Yutaka Kuroiwa, Yoshihiro Narita, Shuhei Tanaka, and Kazuyuki Hirao. Precipitation of silver particles by femtosecond laser pulses inside silver ion doped glass. Journal of non-crystalline solids, 336(3):234-236, 2004.

[9] Quan-Zhong Zhao, Jian-Rong Qiu, Xiong-Wei Jiang, Chong-Jun Zhao, and CongShan Zhu. Controllable precipitation and dissolution of silver nanoparticles in ultrafast laser pulses irradiated $\mathrm{Ag}^{+}$-doped phosphate glass. Optics express, 12(17):40354040, 2004. 
[10] A Nahal, J Mostafavi-Amjad, A Ghods, MRH Khajehpour, SNS Reihani, and MR Kolahchi. Laser-induced dendritic microstructures on the surface of $\mathrm{Ag}^{+}$-doped glass. Journal of applied physics, 100(5):053503, 2006.

[11] M Kaempfe, G Seifert, K-J Berg, H Hofmeister, and H Graener. Polarization dependence of the permanent deformation of silver nanoparticles in glass by ultrashort laser pulses. The European Physical Journal D-Atomic, Molecular, Optical and Plasma Physics, 16(1):237-240, 2001.

[12] A Podlipensky, A Abdolvand, G Seifert, and H Graener. Femtosecond laser assisted production of dichroitic 3D structures in composite glass containing Ag nanoparticles. Applied Physics A, 80(8):1647-1652, 2005.

[13] A Podlipensky, A Abdolvand, G Seifert, H Graener, O Deparis, and PG Kazansky. Dissolution of silver nanoparticles in glass through an intense dc electric field. The Journal of Physical Chemistry B, 108(46):17699-17702, 2004.

[14] Olivier Deparis, Peter G Kazansky, Amin Abdolvand, Alexander Podlipensky, Gerhard Seifert, and Heinrich Graener. Poling-assisted bleaching of metal-doped nanocomposite glass. Applied physics letters, 85(6):872-874, 2004.

[15] Zhiyu Zou, Xiangjun Chen, Qiang Wang, Shiliang Qu, and Xinyu Wang. Electric field assisted dissolution of Au rods in gold-doped silicate glass. Journal of Applied Physics, 104(11):113113, 2008.

[16] Zhiyu Zou, Qiang Wang, Xiangjun Chen, and Shiliang Qu. Direct evidence for electric field assisted dissolution of au nanoparticles on glass surface. Journal of Applied Physics, 105(10):103114, 2009.

[17] M Leitner, H Peterlik, B Sepiol, H Graener, M Beleites, and G Seifert. Uniformly oriented, ellipsoidal nanovoids in glass created by electric-field-assisted dissolution of metallic nanoparticles. Physical Review B, 79(15):153408, 2009.

[18] David L Veasey, David S Funk, Norman A Sanford, and Joseph S Hayden. Arrays of distributed-Bragg-reflector waveguide lasers at $1536 \mathrm{~nm}$ in $\mathrm{Yb} /$ Er codoped phosphate glass. Applied Physics Letters, 74(6):789-791, 1999.

[19] David L Veasey, David S Funk, Philip M Peters, Norman A Sanford, Gregory E Obarski, Norman Fontaine, Matt Young, Adele P Peskin, Wei-Chih Liu, SN HoudeWalter, et al. Yb/Er-codoped and Yb-doped waveguide lasers in phosphate glass. Journal of non-crystalline solids, 263:369-381, 2000.

[20] J.F. Philipps, T. Töpfer, Heike Ebendorff-Heidepriem, D. Ehrt, and R. Sauerbrey. Spectroscopic and lasing properties of $\mathrm{Er}^{3+}: \mathrm{Yb}^{3+}$-doped fluoride phosphate glasses. Applied Physics B, 72(4):399-405, 2001.

[21] J.F. Philipps, T. Töpfer, Heike Ebendorff-Heidepriem, D. Ehrt, and R. Sauerbrey. Energy transfer and upconversion in erbium-ytterbium-doped fluoride phosphate glasses. Applied Physics B, 74(3):233-236, 2002. 
[22] Berton E Callicoatt, John B Schlager, Robert K Hickernell, Richard P Mirin, and Norman A Sanford. Compact solid-state waveguide lasers. Circuits and Devices Magazine, IEEE, 19(5):18-27, 2003.

[23] Pratheepan Madasamy, Seppo Honkanen, David F Geraghty, and Nasser Peyghambarian. Single-mode tapered waveguide laser in Er-doped glass with multimode-diode pumping. Applied physics letters, 82(9):1332-1334, 2003.

[24] K.O. Hill, B Malo, F Bilodeau, D.C. Johnson, and J Albert. Bragg gratings fabricated in monomode photosensitive optical fiber by UV exposure through a phase mask. Applied Physics Letters, 62(10):1035-1037, 1993.

[25] DZ Anderson, V Mizrahi, T Erdogan, and AE White. Production of in-fibre gratings using a diffractive optical element. Electronics Letters, 29(6):566-568, 1993.

[26] J-L Archambault and Stephen G Grubb. Fiber gratings in lasers and amplifiers. Lightwave Technology, Journal of, 15(8):1378-1390, 1997.

[27] Kenneth O Hill and Gerald Meltz. Fiber Bragg grating technology fundamentals and overview. Journal of lightwave technology, 15(8):1263-1276, 1997.

[28] Andreas Othonos. Fiber Bragg gratings. Review of scientific instruments, 68(12):4309-4341, 1997.

[29] S Suzuki, A Schulzgen, S Sabet, JV Moloney, and N Peyghambarian. Photosensitivity of Ge-doped phosphate glass to $244 \mathrm{~nm}$ irradiation. Applied physics letters, 89(17):171913-171913, 2006.

[30] Sanna Yliniemi, Jacques Albert, Qing Wang, and Seppo Honkanen. UV-exposed bragg gratings for laser applications in silver-sodium ion-exchanged phosphate glass waveguides. Optics express, 14(7):2898-2903, 2006.

[31] J Albert, A Schülzgen, VL Temyanko, S Honkanen, and N Peyghambarian. Strong bragg gratings in phosphate glass single mode fiber. Applied physics letters, 89(10):1127, 2006.

[32] Sanna Yliniemi, Seppo Honkanen, Anatoli Ianoul, Albane Laronche, and Jacques Albert. Photosensitivity and volume gratings in phosphate glasses for rare-earthdoped ion-exchanged optical waveguide lasers. JOSA B, 23(12):2470-2478, 2006.

[33] Jordi Sancho-Parramon, Vesna Janicki, J Arbiol, Hrvoje Zorc, and F Peiro. Electric field assisted dissolution of metal clusters in metal island films for photonic heterostructures. Applied physics letters, 92(16):163108, 2008.

[34] AA Lipovskii, VG Melehin, and VD Petrikov. Electric-field-induced bleaching of ion-exchanged glasses containing copper nanoparticles. Technical physics letters, $32(3): 275-277,2006$. 
[35] Andrey A Lipovskii, Vyacheslav V Rusan, and Dmitry K Tagantsev. Imprinting phase/amplitude patterns in glasses with thermal poling. Solid State Ionics, 181(17):849-855, 2010.

[36] Francesco P Mezzapesa, Isabel CS Carvalho, Peter G Kazansky, Olivier Deparis, Mitsuhiro Kawazu, and Koichi Sakaguchi. Bleaching of sol-gel glass film with embedded gold nanoparticles by thermal poling. Applied physics letters, 89(18):183121, 2006.

[37] Isabel Carvalho, Francesco P Mezzapesa, Peter G Kazansky, Olivier Deparis, Mitsuhiro Kawazu, and Koichi Sakaguchi. Dissolution of embedded gold nanoparticles in sol-gel glass film. Materials Science and Engineering: C, 27(5):1313-1316, 2007.

[38] Zhiyu Zou, Xiangjun Chen, Qiang Wang, Shiliang Qu, and Xinyu Wang. Electric field assisted dissolution of Au rods in gold-doped silicate glass. Journal of Applied Physics, 104(11):113113, 2008.

[39] Amy S Barnes, Carlo G Pantano, and Samuel D Conzone. Surface cleaning and etching of rare-earth-doped phosphate glass. In International Symposium on $\mathrm{Op}$ tical Science and Technology, pages 115-125. International Society for Optics and Photonics, 2001.

[40] Pratheepan Madasamy, G Nunzi Conti, Pekka Poyhonen, MM Morrell, N Peyghambarian, David F Geraghty, Y Hu, and S Honkanen. Waveguide distributed Bragg reflector laser arrays in erbium doped glass made by dry Ag film ion exchange. Optical Engineering, 41(5):1084-1086, 2002.

[41] F Gonella, P Canton, E Cattaruzza, A Quaranta, C Sada, and A Vomiero. Fieldassisted ion diffusion of transition metals for the synthesis of nanocomposite silicate glasses. Materials Science and Engineering: C, 26(5):1087-1091, 2006.

[42] Ricky W Chuang and Chin C Lee. Low-loss deep glass waveguides produced with dry silver electromigration process. Journal of lightwave technology, 20(8):1590, 2002.

[43] Y Ma, J Lin, LF Zhu, HY Wei, DW Li, and S Qin. Optical properties of Ag nanoparticle embedded silicate glass prepared by field-assisted diffusion. Applied Physics A, 102(3):521-525, 2011.

[44] Michael Quinten and Uwe Kreibig. Absorption and elastic scattering of light by particle aggregates. Applied optics, 32(30):6173-6182, 1993.

[45] PN Brunkov, VG Melekhin, VV Goncharov, AA Lipovskii, and MI Petrov. Submicron-resolved relief formation in poled glasses and glass-metal nanocomposites. Technical Physics Letters, 34(12):1030-1033, 2008.

[46] AA Lipovskii, M Kuittinen, P Karvinen, K Leinonen, VG Melehin, VV Zhurikhina, and $\mathrm{Yu} \mathrm{P}$ Svirko. Electric field imprinting of sub-micron patterns in glass-metal nanocomposites. Nanotechnology, 19(41):415304, 2008. 
[47] Pirkko Kuisma-Kursula. Accuracy, precision and detection limits of SEM-WDS, SEM-EDS and PIXE in the multielemental analysis of medieval glass. X-ray Spectrometry, 29(1):111-118, 2000.

[48] Ari Sihvola. Mixing rules with complex dielectric coefficients. Subsurface Sensing Technologies and Applications, 1(4):393-415, 2000.

[49] H Hövel, S Fritz, A Hilger, U Kreibig, and M Vollmer. Width of cluster plasmon resonances: bulk dielectric functions and chemical interface damping. Physical Review $B, 48(24): 18178,1993$.

[50] Vladimir P Drachev, Uday K Chettiar, Alexander V Kildishev, Hsiao-Kuan Yuan, Wenshan Cai, and Vladimir M Shalaev. The Ag dielectric function in plasmonic metamaterials. Optics express, 16(2):1186-1195, 2008.

[51] U Kreibig and L Genzel. Optical absorption of small metallic particles. Surface Science, 156:678-700, 1985.

[52] P.B. Johnson and R.W. Christy. Optical constants of the noble metals. Physical Review B, 6(12):4370, 1972.

[53] William T Doyle. Optical properties of a suspension of metal spheres. Physical review $B, 39(14): 9852,1989$.

[54] John Crank. The mathematics of diffusion. Oxford university press, 1979.

[55] IOG-1 phosphate laser glass. Available at http: //www.schott.com/advanced_optics/english/download/ schott-iog-1-phosphate-laser-glass-may-2013-eng.pdf.

[56] R Ramponi, R Osellame, M Marangoni, G Sorbello, P Laporta, S Jiang, Y Hu, and N Peyghambarian. New Er-doped phosphate glass for ion-exchange active waveguides: accurate determination of the refractive index. Optical Materials, 14(4):291$296,2000$.

[57] Prissana Thamboon and Denise M. Krol. Second-order optical nonlinearities in thermally poled phosphate glasses. Journal of Applied Physics, 93(1):32-37, 2003.

[58] M Mattarelli, M Montagna, K Vishnubhatla, A Chiasera, M Ferrari, and GC Righini. Mechanisms of Ag to Er energy transfer in silicate glasses: a photoluminescence study. Physical Review B, 75(12):125102, 2007.

[59] VK Tikhomirov, T Vosch, Eduard Fron, VD Rodríguez, JJ Velázquez, D Kirilenko, G Van Tendeloo, Johan Hofkens, Mark Van der Auweraer, and VV Moshchalkov. Luminescence of oxyfluoride glasses co-doped with $\mathrm{Ag}$ nanoclusters and $\mathrm{Yb}^{3+}$ ions. Rsc advances, 2(4):1496-1501, 2012.

[60] AA Lipovskii and VV Zhurikhina. Is it possible to use ion exchange in glasses for producing effective diffraction gratings? Journal of non-crystalline solids, 351(52):3784$3788,2005$. 
[61] Yasuhiko Shimotsuma, Kazuyuki Hirao, Peter G Kazansky, and Jiarong Qiu. Threedimensional micro-and nano-fabrication in transparent materials by femtosecond laser. Japanese journal of applied physics, 44(7R):4735, 2005.

[62] Rod Taylor, Cyril Hnatovsky, and Eli Simova. Applications of femtosecond laser induced self-organized planar nanocracks inside fused silica glass. Laser and Photonics Reviews, 2(1-2):26-46, 2008.

[63] Jeffrey F Herbstman and Alan J Hunt. High-aspect ratio nanochannel formation by single femtosecond laser pulses. Optics express, 18(16):16840-16848, 2010.

[64] Matthias Brinkmann, Ulrich Fotheringham, Joseph S Hayden, and Yoshio Okano. Glass modification techniques for photonic devices. In International Symposium on Photonic Glass, pages 96-102. International Society for Optics and Photonics, 2003.

[65] Erwin Kretschmann. Die bestimmung optischer konstanten von metallen durch anregung von oberflächenplasmaschwingungen. Zeitschrift für Physik, 241(4):313-324, 1971.

[66] Index of refraction. Available at http://www.cvimellesgriot.com/products/ Documents/GeneralInfo/Index_of_Refraction.pdf.

[67] J. J. Burke, G. I. Stegeman, and T. Tamir. Surface-polariton-like waves guided by thin, lossy metal films. Phys. Rev. B, 33:5186-5201, Apr 1986.

[68] J. Zhang, Z. H. Lu, and L. J. Wang. Precision refractive index measurements of air, $\mathrm{N}_{2}, \mathrm{O}_{2}$, Ar, and $\mathrm{CO}_{2}$ with a frequency comb. Appl. Opt., 47(17):3143-3151, Jun 2008.

[69] E. Hecht. Optics. Addison-Wesley, 4 edition, 1998.

[70] Hiroshi Kano. Excitation of surface plasmon polaritons by a focused laser beam. In Satoshi Kawata, editor, Near-Field Optics and Surface Plasmon Polaritons, volume 81 of Topics in Applied Physics, pages 189-206. Springer Berlin Heidelberg, 2001.

[71] Heinz Raether. Surface plasmons on smooth surfaces, volume 111 of Springer Tracts in Modern Physics. Springer Berlin Heidelberg, 1988.

[72] H. J. Simon, D. E. Mitchell, and J. G. Watson. Surface plasmons in silver films - a novel undergraduate experiment. American Journal of Physics, 43(7):630-636, 1975.

[73] Willes H. Weber and S. L. McCarthy. Anomalies in the surface plasmon resonance excitation at both surfaces of evaporated metal films. Applied Physics Letters, 25(7):396-398, 1974.

[74] R. Ulrich and W. Prettl. Planar leaky light-guides and couplers. Applied physics, 1(1):55-68, 1973. 
[75] Robert E Collin. Hertzian dipole radiating over a lossy earth or sea: some early and late 20th-century controversies. Antennas and Propagation Magazine, IEEE, 46(2):64-79, 2004.

[76] Weng Cho Chew. Waves and fields in inhomogeneous media, volume 522. IEEE press New York, 1995.

[77] Julius Adams Stratton. Electromagnetic Theory. International Series in Pure and Applied Physics. McGraw-Hill Book Company, 1941.

[78] J.S. Bagby and D.P. Nyquist. Dyadic Green's functions for integrated electronic and optical circuits. Microwave Theory and Techniques, IEEE Transactions on, 35(2):207-210, Feb 1987.

[79] Xingling Zhou. On independence, completeness of Maxwell's equations and uniqueness theorems in electromagnetics. Progress In Electromagnetics Research, 64:117$134,2006$.

[80] Xingling Zhou. On uniqueness theorem of a vector function. Progress In Electromagnetics Research, 65:93-102, 2006.

[81] XL Zhou. On Helmholtz's theorem and its interpretations. Journal of Electromagnetic Waves and Applications, 21(4):471-483, 2007.

[82] B Prade, JY Vinet, and A Mysyrowicz. Guided optical waves in planar heterostructures with negative dielectric constant. Phys. Rev. B, 44(24):13556-13572, 1991.

[83] H.P.W. Gottlieb. On the general solution of the vector Helmholtz equation in cylindrical polar coordinates. The Journal of the Acoustical Society of America, 81(5):16281629, 1987.

[84] G.F. Torres del Castillo. Solution of nonscalar equations in cylindrical coordinates. Revista Mexicana de Fisica, 38(1):19-39, 1992.

[85] G.F. Torres del Castillo. Solution of nonscalar equations in cylindrical coordinates. II. Revista Mexicana de Fisica, 40(6):833-845, 1994.

[86] Robert E Collin. Field theory of guided waves, volume 2. IEEE press New York, 1991.

[87] Corning SMF-28 optical fiber. product information. Available at http://www. corning.com/opticalfiber.

[88] Nelson M Blachman and S Hossein Mousavinezhad. Trigonometric approximations for Bessel functions. Aerospace and Electronic Systems, IEEE Transactions on, AES22(1):2-7, Jan 1986.

[89] Constantine A. Balanis. Advanced engineering electromagnetics. John Wiley and Sons, 1989. 
[90] Brian Cabral and Leith Casey Leedom. Imaging vector fields using line integral convolution. In Proceedings of the 20th annual conference on Computer graphics and interactive techniques, pages 263-270. ACM, 1993.

[91] IH Malitson. Interspecimen comparison of the refractive index of fused silica. JOSA, 55(10):1205-1208, 1965.

[92] SA Schelkunoff. Some equivalence theorems of electromagnetics and their application to radiation problems. Bell System Technical Journal, 15(1):92-112, 1936.

[93] Sembiam R Rengarajan and Yahya Rahmat-Samii. The field equivalence principle: Illustration of the establishment of the non-intuitive null fields. Antennas and Propagation Magazine, IEEE, 42(4):122-128, 2000.

[94] D. Marcuse. Solution of the vector wave equation for general dielectric waveguides by the galerkin method. Quantum Electronics, IEEE Journal of, 28(2):459-465, Feb 1992.

[95] A. Weisshaar, J. Li, R.L. GALLAWA, and I. C. Goyal. Vector and quasi-vector solutions for optical waveguide modes using efficient Galerkin's method with HermiteGauss basis functions. Lightwave Technology, Journal of, 13(8):1795-1800, Aug 1995.

[96] Alejandro Ortega-Monux, J Gonzalo Wangüemert-Pérez, and Inigo MolinaFernández. Adaptive Hermite-Gauss decomposition method to analyze optical dielectric waveguides. JOSA A, 20(3):557-568, 2003.

[97] George W Pan. Wavelets in electromagnetics and device modeling, volume 159. John Wiley \& Sons, 2003.

[98] Matthew NO Sadiku, Cajetan M Akujuobi, and Raymond C Garcia. An introduction to wavelets in electromagnetics. Microwave Magazine, IEEE, 6(2):63-72, 2005.

[99] Samit Barai and Anurag Sharma. Wavelet-Galerkin solver for the analysis of optical waveguides. JOSA A, 26(4):931-937, 2009.

[100] Roland Glowinski, Wayne Lawton, Michel Ravachol, and Eric Tenenbaum. Wavelet solutions of linear and nonlinear elliptic, parabolic and hyperbolic problems in one space dimension. Computing methods in applied sciences and engineering, pages 55-120, 1990.

[101] Björn Engquist and Andrew Majda. Absorbing boundary conditions for numerical simulation of waves. Proceedings of the National Academy of Sciences, 74(5):17651766, 1977.

[102] Allen Taflove and Susan C Hagness. Computational electrodynamics, volume 160. Artech house Boston, 2000.

[103] Jean-Pierre Berenger. A perfectly matched layer for the absorption of electromagnetic waves. Journal of computational physics, 114(2):185-200, 1994. 
[104] Zachary S Sacks, David M Kingsland, Robert Lee, and Jin-Fa Lee. A perfectly matched anisotropic absorber for use as an absorbing boundary condition. Antennas and Propagation, IEEE Transactions on, 43(12):1460-1463, 1995.

[105] Ning-Ning Feng, Gui-Rong Zhou, Chenglin Xu, and Wei-Ping Huang. Computation of full-vector modes for bending waveguide using cylindrical perfectlymatched layers. Journal of lightwave technology, 20(11):1976, 2002.

[106] Jiayuan Fang and Zhonghua Wu. Generalized perfectly matched layer for the absorption of propagating and evanescent waves in lossless and lossy media. Microwave Theory and Techniques, IEEE Transactions on, 44(12):2216-2222, 1996.

[107] Steven A Cummer. Perfectly matched layer behavior in negative refractive index materials. Antennas and Wireless Propagation Letters, IEEE, 3(1):172-175, 2004.

[108] XT Dong, XS Rao, YB Gan, B Guo, and WY Yin. Perfectly matched layer-absorbing boundary condition for left-handed materials. IEEE microwave and wireless components letters, 14(6):301-303, 2004.

[109] Peter G Petropoulos. Reflectionless sponge layers as absorbing boundary conditions for the numerical solution of maxwell equations in rectangular, cylindrical, and spherical coordinates. SIAM Journal on Applied Mathematics, 60(3):1037-1058, 2000.

[110] Po-jui Chiang and Hung-chun Chang. A high-accuracy pseudospectral full-vectorial leaky optical waveguide mode solver with carefully implemented upml absorbing boundary conditions. Optics express, 19(2):1594-1608, 2011.

[111] Chih-Hsien Lai and Hung-chun Chang. Effect of perfectly matched layer reflection coefficient on modal analysis of leaky waveguide modes. Optics express, 19(2):562$569,2011$.

[112] Frédéric Nataf. Absorbing boundary conditions and perfectly matched layers in wave propagation problems. Direct and Inverse problems in Wave Propagation and Applications, pages 219-231, 2013.

[113] Kai Ming Lo and E Herbert Li. Solutions of the quasi-vector wave equation for optical waveguides in a mapped infinite domains by the galerkin's method. Journal of lightwave technology, 16(5):937, 1998.

[114] Simon J Hewlett and Francois Ladouceur. Fourier decomposition method applied to mapped infinite domains: scalar analysis of dielectric waveguides down to modal cutoff. Lightwave Technology, Journal of, 13(3):375-383, 1995.

[115] Michele A Forastiere and Giancarlo C Righini. Improved scalar analysis of integrated optical structures by the mapped galerkin method and arnoldi iteration. JOSA A, 18(4):966-974, 2001. 
[116] Jinbiao Xiao, Xiaohan Sun, and Mingde Zhang. Vectorial analysis of optical waveguides by the mapped galerkin method based on E fields. JOSA B, 21(4):798-805, 2004.

[117] Jinbiao Xiao, Mingde Zhang, and Xiaohan Sun. Solutions of the full-and quasi-vector wave equations based on H-field for optical waveguides by using mapped galerkin method. Optics communications, 259(1):115-122, 2006.

[118] Tomasz F Jablonski. Complex modes in open lossless dielectric waveguides. JOSA A, 11(4):1272-1282, 1994.

[119] John P Boyd. Chebyshev and Fourier spectral methods. Courier Dover Publications, 2013.

[120] Lloyd N Trefethen. Spectral methods in MATLAB, volume 10. Siam, 2000.

[121] Ted Ersek. Rootsearch. Available at http://library.wolfram.com/infocenter/ Demos/4482/.

[122] Chang-Bong Kim and Chin B Su. Measurement of the refractive index of liquids at 1.3 and 1.5 micron using a fibre optic fresnel ratio meter. Measurement Science and Technology, 15(9):1683, 2004.

[123] Kazuya Hayata, Masanori Koshiba, Masashi Eguchi, and Michio Suzuki. Vectorial finite-element method without any spurious solutions for dielectric waveguiding problems using transverse magnetic-field component. Microwave Theory and Techniques, IEEE Transactions on, 34(11):1120-1124, 1986.

[124] Tuptim Angkaew, Masanori Matsuhara, and Nobuaki Kumagai. Finite-element analysis of waveguide modes: A novel approach that eliminates spurious modes. $M i$ crowave Theory and Techniques, IEEE Transactions on, 35(2):117-123, 1987.

[125] Masanori Koshiba. Optical waveguide analysis. McGraw-Hill New York etc., 1992.

[126] Yu A. Tsarin. Conformal mapping technique in the theory of periodic structures. Microwave and Optical Technology Letters, 26(1):57-61, 2000. 